GA-A 13827

UC-77

\title{
POSTIRRADIATION EXAMINATION OF CAPSULES P13R AND P13S
}

\author{
by \\ C. B. SCOTT, D. P. HARMON, and J. F. HOLZGRAF
}

Prepared under

Contract E(04-3)-167

Project Agreement No. 17

for the San Francisco Operations Office

U.S. Energy Research and Development Administration

DATE PUBLISHED: OCTOBER 8, 1976 


\section{NOTICE}

This report was prepared as an account of work sponsored by the United States Government. Neither the United States nor the United States Energy Research and Development Administration, nor any of their employees, nor any of their contractors, subcontractors, or their employees, makes any warranty, express or implied, or assumes any legal liability or responsibility for the accuracy, completeness or usefulness of any information, apparatus, product or process disclosed, or represents that its use would not infringe privately owned rights.

Printed in the United States of America Available from

National Technical Information Service

U.S. Department of Commerce

5285 Port Royal Road

Springfield, Virginia 22161

Price: Printed Copy $\$ 11.00$; Microfiche $\$ 2.25$ 


\section{DISCLAIMER}

This report was prepared as an account of work sponsored by an agency of the United States Government. Neither the United States Government nor any agency Thereof, nor any of their employees, makes any warranty, express or implied, or assumes any legal liability or responsibility for the accuracy, completeness, or usefulness of any information, apparatus, product, or process disclosed, or represents that its use would not infringe privately owned rights. Reference herein to any specific commercial product, process, or service by trade name, trademark, manufacturer, or otherwise does not necessarily constitute or imply its endorsement, recommendation, or favoring by the United States Government or any agency thereof. The views and opinions of authors expressed herein do not necessarily state or reflect those of the United States Government or any agency thereof. 


\section{DISCLAIMER}

Portions of this document may be illegible in electronic image products. Images are produced from the best available original document. 


\title{
POSTIRRADIATION EXAMINATION OF CAPSULES P13R AND P13S
}

\author{
by \\ C. B. SCOTT, D. P. HARMON, and J. F. HOLZGRAF
}

Prepared under

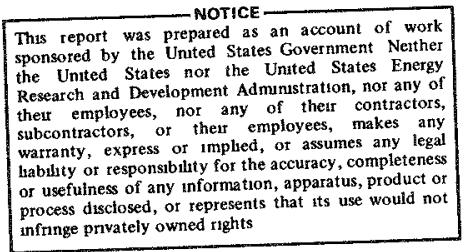

\author{
Contract E(04-3)-167 \\ Project Agreement No. 17 \\ for the San Francisco Operations Office \\ U.S. Energy Research and Development Administration
}




\section{ABSTRACT}

Capsules P13R and P13S were the seventh and eighth in a series of irradiation tests conducted under the ERDA-sponsored HTGR Fuels and Core Development Program. Reference type LHTGR fuel fabricated with a broad spectrum of property and process variables was irradiated to extreme temperature and fluence conditions. The purpose of these tests was to provide a technical basis for LHTGR fuel product specifications and to provide irradiation data to support and update fuel performance models used in design evaluations.

Postirradiation examination revealed that the bonded fuel rods exhibited good stability after irradiation to fast neutron fluences of $12.4 \times 10^{21}$ $\mathrm{n} / \mathrm{cm}^{2}$ ( $\left.\mathrm{E}>0.18 \mathrm{MeV}\right)_{\mathrm{HTGR}}$, which is $55 \%$ beyond the LHTGR peak design fast neutron fluence of $8.0 \times 10^{21} \mathrm{n} / \mathrm{cm}^{2}$. Thermal cycling to high temperatures did not adversely affect fuel rod integrity.

Particle batches with coating designs representative of the design requirements envisioned for the LHTGR exhibited excellent irradiation performance. Ten batches of fissile and fertile particles were irradiated without coating failure to fast neutron exposures which exceeded the LHTGR peak design exposure by 35 to $52 \%$.

A large number of particle batches was tested with coating designs or particle attributes that were not representative of LHTGR design requirements. The irradiation performance of these samples provided important data required to establish fuel particle specification limits.

Capsules P13R and P13S were considered to be very successful qualification tests of LHTGR fuel components. These results provided a substantial data base for the LHTGR Fuel Product Specification and performance models 
used in HTGR core design studies, and demonstrated the excellent irradiation performance of reference LHTGR fuel to well beyond peak design exposures. 


\section{ACKNOWLEDGMENTS}

The authors would like to acknowledge the contributions of the many people who assisted with the fabrication, irradiation, and postirradiation examination of capsules $\mathrm{P} 13 \mathrm{R}$ and P13S. In particular, they would like to acknowledge the Capsule Irradiation Branch for capsule design, fabrication, and irradiation; J. B. Wattier for irradiation data compilation and data reduction; the Equipment Engineering Department and Pilot Plant Engineering Department for fabrication of the fuel specimens; P. R. Macy, W. R. Ziegler, and M. S. Cronin of the Fuel Materials Branch for data reduction and analytical studies; P. T. Mattson, L. W. Burleigh, and M. N. Johnson of the Hot Cell Facility for capsule disassembly; W. E. Simpson of the Metallography Laboratory for hot cell metallography; T. B. Crockett and E. E. Anderson of the Analytical Chemistry Department for burnup and dosimetry analyses; J. N. Graves of the Fuel Chemistry Branch for fission gas release measurements; A. J. Zangari and J. M. McNair for capsule thermal analysis; J. Jayne and $B$. Yalof of the Publications Branch for editing of the manuscript. 


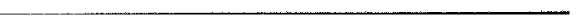
$-$ 
ABSTRACT - . . . . . . . . . . . . . . . . . . . . . . .

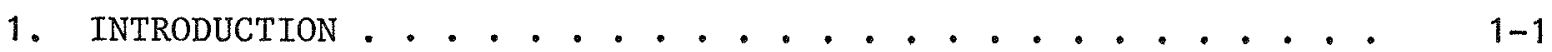

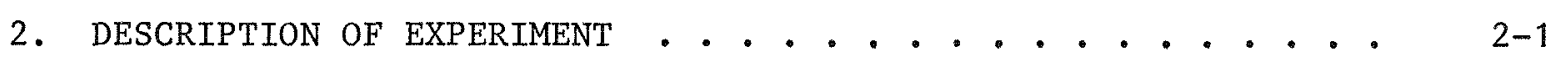

2.1. Objectives . . . . . . . . . . . . . . . . . . 2-1

2.2. Description of Fuel Specimens . . . . . . . . . . . . . 2-2

2.2.1. Coated Particles . . . . . . . . . . 2-2

2.2.2. Fuel Rods .................. 2-3

2.3. Capsule Design . . . . . . . . . . . . . . . . . . 2-5

References . . . . . . . . . . . . . . . . . . 2-6

3. CAPSULE OPERATION AND EVALUATION . . . . . . . . . . . . . . . 3-1

3.1. Operating History . . . . . . . . . . . . . . . 3-1

3.2. In-Pile Fission Gas Release Measurements . . . . . . 3-3

3.3. Analysis of F1ux Dosimeters . . . . . . . . . . . . 3-5

3.4. Heavy Metal Burnup Analysis . . . . . . . . . . . 3-8

3.5. Thermal Analysis . . . . . . . . . . . . . . . 3-12

3.5.1. Fuel Rod Temperatures . . . . . . . . . 3-13

3.5.2. Unbonded Fuel Particle Temperatures . . . . 3-15

3.5.3. Thermocouple Decalibration . . . . . . . 3-15

References . . . . . . . . . . . . . . . . . . . . . . 3-16

4. POSTIRRADIATION EXAMINATION RESULTS . . . . . . . . . . . . . 4-1

4.1. Capsule Disassemb1y . . . . . . . . . . . . . . . . . . . 4-1

4.2. Visual Examination . . . . . . . . . . . . . . . . 4-2

4.2.1. Fuel Rods . . . . . . . . . . . . 4-3

4.2.2. Unbonded Particle Samples . . . . . . . . 4-3

4.3. DIMENSIONAL CHANGE ANALYSES . . . . . . . . . . . . . . . 4-5

4.3.1. Fuel Rods ............. . . . . 4-5

4.3.2. Graphite Crucibles . . . . . . . . . . 4-8 
4.4. Fission Gas Release .............. 4-10

4.4.1. Postirradiation Fission Gas Release Measurements 4-10

4.4.2. Determination of Fuel Failure Levels . . . . 4-11

4.4.3. High-Temperature Fission Gas Release Measurements ......... 4-16

4.4.4. Comparison of In-pile and Postirradiation

Fission Gas Release Measurements . . . . 4-19

4.5. Gamma-Ray Spectrometry Analyses . . . . . . . . . 4-21

4.5.1. Fuel Rods . . . . . . . . . . . 4-21

4.5.2. Graphite Crucibles .......... 4-23

4.5.3. Unbonded Particle Samples......... . 4-23

4.6. Metallographic Examination . . . . . . . . . 4-24

4.6.1. Fuel Rod Matrix ............ 4-25

4.6.2. Fissile Particles........... 4-26

4.6.3. Fertile Particles........... 4-32

4.6.4. Inert Particles............. 4-34

4.7. OPyC Coating Permeability Studies ......... 4-35

4.8. Simulated Reprocessing Studies . . . . . . . . 4-38

4.9. Contact Microradiography . . . . . . . . . . 4-41

4.10. Density Measurements ............. 4 4-41

4.10.1 OPyC Coatings from Fissile Particles .... 4-41

4.10.2. Fertile Particles.......... 4-42

References ................. . . 4-42

5. DISCUSSION .................... . . . . . . . .

5.1. Coated Particle Irradiation Performance . . . . . . 5-1

5.1.1. Fissile Particles ........... . 5-1

5.1.2. Fertile Particles . . . . . . . . 5-15

5.1.3. Effects of High-Temperature Transients . . . 5-28

5.2. Fuel Rod Irradiation Performance . . . . . . . . 5-31

5.2.1. Integrity ........... 5-31

5.2.2. Influence of Product and Process Variables . . 5-32

5.3. Metallic Fission Product Behavior . . . . . . . 5-35

5.3.1. Fuel Rod Homogeneity .......... 5-35

5.3.2. Release from Failed Fuel . . . . . . . 5-36 
5.3.3. Fission Product Transport . . . . . . 5-39

References . . . . . . . . . . . . . . . . 5-39

6. IMPLICATIONS FOR LHTGR FUEL DESIGN AND PERFORMANCE . . . . . 6-1

6.1. Fuel Particle Irradiation Performance . . . . . . . . 6-1

6.2. PyC Coating Behavior ............. . . 6-2

6.3. Fuel Kerne1 Migration . . . . . . . . . . . 6-3

6.4. Fuel Rod Irradiation Performance .. . . . . . . . 6-4

6.5. Fuel Rod Dimensional Change Behavior ........ . 6-5

References .................... . . 6-6

7. SUMMARY . . . . . . . . . . . . . . . . . . 7-1

TABLES

1-1. Principal differences between Fort St. Vrain and large HTGR fuel ................... . . 1-3

2-1. General description of coated particles tested in capsule P13R ................... 2 . 2-7

2-2. General description of coated particles tested in capsule P13S .................... 2- 2-8

2-3. TRISO coated fissile particle variables tested in capsules P13R and P13S.................. 2-9

2-4. Fissile particle coating process data.......... . 2-10

2-5. Fertile particle variables tested in capsules $\mathrm{P} 13 \mathrm{R}$ and P13S ................... 2 . 2-11

2-6. Fertile particle coating process data . . . . . . . . 2-12

2-7. Description of fuel rods tested in capsule P13R . . . . . 2-13

2-8. Description of fuel rods tested in capsule P13S . . . . . 2-14

2-9. Fuel rod variables tested in capsules P13R and P13S . . . 2-15

3-1. Irradiation parameters for capsule P13R unbonded fuel particle samples ................. 3-18

3-2. Irradiation parameters for capsule P13S unbonded fuel particle samples .. . . . . . . . . . . 3-19

3-3. Irradiation parameters for capsule P13R fuel rods . . . . 3-20

3-4. Irradiation parameters for capsule P13S fuel rods . . . . . 3-21

3-5. Wire weights . . . . . . . . . . . . . 3-22

3-6. Measured Mn-54 activities . . . . . . . . . . 3-22 


\section{TABLES (Continued)}

3-7. Comparison of fast neutron fluences .......... 3-23

3-8. Thermal fission yields of cesium and zirconium isotopes . . 3-24

3-9. Results of heavy metal burnup determinations made at Genera1 Atomic

3-10. Fissile particle uranium isotopic composition determined by mass spectrometry ............... . 3-25

3-11. Fertile particle uranium isotopic composition determined by mass spectrometry

3-12. Comparison of fissile and fertile particle uranium isotopic composition determined by mass spectrometry and the Sigma 2 FISSIN computer program ..............

3-13. Methods evaluation data . . . . . . . . . . 3-28

3-14. Uranium isotopic analysis . . . . . . . . . . 3-29

3-15. Results of heavy metal burnup determinations made at oak Ridge National Laboratory . . . . . . . . . . 3-30

3-16. Irradiation temperatures of capsule P13R fuel rods . . . . 3-31

3-17. Irradiation temperatures of capsule P13S fuel rods . . . 3-32

3-18. Values used in estimating uncertainties in fuel rod centerline temperatures calculated by the CAPTEM code . . 3-33

3-19. Summary of unbonded fissile particle temperatures for samples irradiated in cells 3 and 4 of capsule P13R . . . . . . 3-34

3-20. Summary of unbonded fissile particle temperatures for samples irradiated in cells 3 and 4 of capsule P13S . . . . . 3-35

3-21. Summary of unbonded fertile particle temperatures for samples irradiated in cells 3 and 4 of capsule P13R . . . . . 3-36

3-22. Summary of unbonded fertile particle temperatures for samples irradiated in cells 3 and 4 of capsule P13S . . . . . 3-37

3-23. Decalibration factors derived for $W /$ Re thermocouples irradiated in capsules P13R and P13S . . . . . . . 3-38

4-1. Description and condition of fuel rods irradiated in capsule P13R .....................

4-2. Description and condition of fuel rods irradiated in capsule

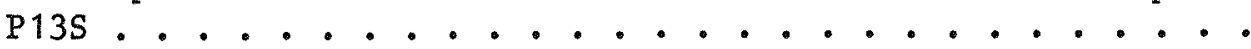

4-3. Results of visual examination of unbonded fissile particle samples irradiated in capsules P13R and P13S . . . . . 4-47

4-4. Results of visual examination of unbonded fertile particle samples irradiated in capsule P13R . . . . . . . . 4-48

4-5. Results of visual examination of unbonded fertile particle samples irradiated in capsule P13S ........... 
4-6. Fuel rod dimensional change data obtained from capsule P13R 4-50

4-7. Fuel rod dimensional change data obtained from capsule P13S 4-51

4-8. Measured diametral dimensional changes of fuel rod graphite crucibles . . . . . . . . . . . . . 4-52

4-9. Postirradiation radial gap between graphite crucibles and capsule P13R fuel rods . . . . . . . . . . . 4-55

4-10. Postirradiation radial gap between graphite crucibles and capsule P13s fuel rods . . . . . . . . . . . 4-56

4-11. TRIGA fission gas release data for capsule P13R fuel rods . 4-57

4-12. TRIGA fission gas release data for capsule P13S fuel rods . 4-58

4-13. TRIGA fission gas release data for unbonded fissile particle samples tested in capsules P13R and P13S . . . . . . . 4-59

4-14. TRIGA fission gas release data for unbonded fertile particle samples tested in capsules P13R and P13S . . . . . . . 4-60

4-15. Gaseous fission product release $(\mathrm{R} / \mathrm{B} \mathrm{Kr}-85 \mathrm{~m})$ resulting from in-service fuel particle failure in capsule $\mathrm{P} 13 \mathrm{R}$ fuel rods 4-61

4-16. Gaseous fission product release ( $\mathrm{R} / \mathrm{B} \mathrm{Kr}-85 \mathrm{~m}$ ) resulting from in-service fuel particle failure in capsule P13S fuel rods 4-62

4-17. Unbonded fissile particle failure levels determined by fission gas release measurements for capsules P13R and P13S . . . 4-63

4-18. Unbonded fertile particle failure levels determined by fission gas release measurements for capsules P13R and P13S . . . 4-64

4-19. Summary of high-temperature fission gas release measurements made on selected capsule P13R fuel rods . . . . . . . . 4-65

4-20. Summary of high-temperature fission gas release measurements made on selected capsule P13S fuel rods ......... 4-66

4-21. Comparison of capsule P13R EOL in-pile and TRIGA fission gas release data $(\mathrm{R} / \mathrm{B} \mathrm{Kr}-85 \mathrm{~m})$............ . . 4-67

4-22. Comparison of capsule P13S EOL in-pile and TRIGA fission gas release data $(\mathrm{R} / \mathrm{B} \mathrm{Kr}-85 \mathrm{~m})$............ . . 4-68

4-23. Comparison of capsule P13R BOL in-pile and TRIGA fission gas release data $(\mathrm{R} / \mathrm{B} \mathrm{Kr}-85 \mathrm{~m})$........... 4- . 4-69

4-24. Comparison of capsule P13S BOL in-pile and TRIGA fission gas release data $(\mathrm{R} / \mathrm{B} \mathrm{Kr}-85 \mathrm{~m})$. . . . . . . . . . 4-70

4-25. Fission product inventory of fuel rods irradiated in
capsule P13R . . . . . . . . . . . . . . . . 4-71

4-26. Fission product inventory of fuel rods irradiated in capsule P13S...................... 4-72 


\section{TABLES (Continued)}

4-27. Fission product isotope counting error . . . . . . . . . 4-73

4-28. Gamma scan results of capsule P13R fuel rod crucibles . . 4-74

4-29. Gamma scan results of capsule P13S fuel rod crucibles . • 4-75

4-30. Fission product isotope ratios of capsule P13R unbonded

fissile particle samples . . . . . . . . . . . . 4-76

4-31. Fission product isotope ratios of capsule P13S unbonded fissile particle samples . . . . . . . . . . . 4-77

4-32. Fission product isotope ratios of capsule P13R unbonded fertile particle samples . . . . . . . . . . . 4-78

4-33. Fission product isotope ratios of capsule P13S unbonded fertile particle samples . . . . . . . . . . . . 4-79

4-34. Gamma scan results of selected loose particle batches in capsules P13R and P13S . . . . . . . . . . . . . 4-80

4-35. Results of metallographic examination of fissile particles irradiated in capsules P13R and P13S . . . . . . . . 4-81

4-36. Results of metallographic examination of fertile particles irradiated in capsules P13R and P13S . . . . . . . . . 4-82

4-37. Results of metallographic examination of inert particles irradiated in P13R and P13S fuel rods . . . . . . . . . 4-83

4-38. Irradiation-induced change in macroporosity of $\mathrm{P} 13 \mathrm{R}$ and P13S fuel rods . . . . . . . . . . . . . . 4-84

4-39. Summary of metallic fission product interactions observed in TRISO $\mathrm{UC}_{2}$ particles... . . . . . . . . . . 4-85

4-40. Summary of fuel kernel migration data . . . . . . . . . 4-86

4-41. Summary of fission gas release measurements on unfailed BISO $\mathrm{ThO}_{2}$ particle batches . . . . . . . . . . . 4-87

4-42. Summary description of $\mathrm{ThO}_{2}$ particle samples selected for acid leaching and fission gas release measurements . . . . 4-88

4-43. Results of spectrophotometric analysis of leach solutions - 4-89

4-44. Results of visual and metallographic examinations of BISO coated particles after acid leaching. . . . . . . . . . 4-90

4-45. Chemical analysis of leach solutions . . . . . . . . . 4-91

4-46. Uranium isotopic composition of the leach solution . . . . 4-91

4-47. Uranium isotopic composition of the fuel rod . . . . . . 4-92

4-48. Postirradiation particle dimensions determined by contact microradiography ................. 4-93 
TABLES (Continued)

4-49. Irradiation-induced particle dimensional change determined by microradiography . . . . . . . . . . . . . . . 4-94

4-50. Results of OPyC density measurements of unbonded fissile particle samples irradiated in capsules P13R and P13S . . .

4-51. Results of density measurements of unbonded fertile particle samples irradiated in capsules P13R and P13S . . . . . . 4-96

5-1. Summary description and failure analysis of unbonded fissile particle samples .. . . . . . . . . . . 5-42

5-2. Comparison of fissile particle $\mathrm{OPyC}$ and total coating failure levels ................. . . 5-43

5-3. Analysis of metallic fission product attack of SiC coating in TRISO $\mathrm{UC}_{2}$ particles . . . . . . . . . . . . . 5-44

5-4. Summary description and failure analysis of unbonded fertile particle samples . . . . . . . . . . . 5 5-45

5-5. Summary description and failure analysis of fertile particles tested in fuel rods... . . . . . . . 5-46

5-6. Fertile particle OPyC coating density . . . . . . . . 5-47

5-7. Irradiation parameters and fuel failure levels in fuel rods tested in cell 1 of capsules P13R and P13S . . . . . . 5-48

5-8. Failure analysis of fuel rods heated to $1600^{\circ} \mathrm{C}$ in the TRIGA facility .. . . . . . . . . . . . . 5-49

5-9. Fission product isotope ratios of capsule P13R fuel rods . 5-50

5-10. Fission product isotope ratios of capsule P13S fuel rods . 5-51

5-11. Fractional $\mathrm{Cs}-137$ release from failed fuel in fuel rods . . 5-52

5-12. Comparison of $\mathrm{Cs}-137$ loss with fissile particle failure levels ................ 5-52

6-1. Performance of LHTGR reference type fissile particles . . . 6-8

6-2. Performance of LHTGR reference type fertile particles . . . 6-9

6-3. Recommended OPYC coating attribute specifications for HTGR fissile TRISO and fertile BISO particles . . . . . . 6-10 


\section{FIGURES}

2-1. Representative preirradiation photomicrographs of fuel rods tested in capsules $\mathrm{P} 13 \mathrm{R}$ and P13S: (a) fired at $1800^{\circ} \mathrm{C}$ in argon, which is the reference heat treatment for LHTGR fuel production, and (b) slug-injected fuel rod fired in packed bed of alumina

2-2. Representative preirradiation photomicrographs of fuel rods tested in capsules $\mathrm{P} 13 \mathrm{R}$ and P13S: (a) fired at $1500^{\circ} \mathrm{C}$ and (b) fired in a $\mathrm{N}_{2}$ atmosphere . . . . . . . . . . .

2-3. Schematic showing design of fuel rod cells $1,2,5$, and 6 of capsules P13R and P13S . . . . . . . . . . . 2-18

2-4. Sechmatic showing design of unbonded particle cells 3 and 4 of capsules P13R and P13S

3-1. GETR thermal flux profiles for three control rod bank positions . . . . . . . . . . . . . . . . . .

3-2. Design load-following transient for cell 1 of capsule P13S . . . . . . . . . . . . .....

3-3. Decalibration curves used to correct tungsten-rhenium thermocouple drift in cells $1,2,4$, and 6 in capsules $\mathrm{P} 13 \mathrm{R}$ and P13S .................

3-4. Decalibration curves used to correct tungsten-rhenium thermocouple drift in cell 5 in capsule P13R . . . . . . . 3-42

3-5. Decalibration curves used to correct tungsten-rhenium thermocouple drift in cell 5 in capsule P13S . . . . . 3-43

3-6. Operating history for cell 1 of capsule P13R . . . . . . 3-44

3-7. Operating history for cel1 2 of capsule P13R . . . . . 3-45

3-8. Operating history for cell 3 of capsule P13R . . . . . . 3-46

3-9. Operating history for cell 4 of capsule P13R . . . . . 3-47

3-10. Operating history for cell 5 of capsule P13R . . . . . 3-48

3-11. Operating history for ce11 6 of capsule P13R . . . . . 3-49

3-12. Operating history for cell 1 of capsule P13S . . . . . 3-50

3-13. Thermal cycle operating history for cell 1 of capsule P13S 3-51

3-14. Operating history for cell 2 of capsule P13S . . . . . 3-52

3-15. Operating history for cell 3 of capsule P13S . . . . . 3-53

3-16. Operating history for cel1 4 of capsule P13S . . . . . . 3-54

3-17. Operating history for cell 5 of capsule P13S . . . . . 3-55

3-18. Operating history for cell 6 of capsule P13s . . . . . 3-56 
3-19. Ratio of fission gas release to birth rate $(R / B)$ versus fast fluence $(E>0.18 \mathrm{MeV})_{\mathrm{HTGR}}$ for each cel1 in capsule P13R . 3-57

3-20. Ratio of fission gas release to birth rate $(R / B)$ versus fast fluence $(E>0.18 \mathrm{MeV})_{\text {HTGR }}$ for each cell in capsule P13S . 3-58

3-21. GETR fast and thermal neutron flux profiles for capsule P13R 3-59

3-22. GETR fast and thermal neutron flux profiles for capsule P13S 3-60

3-23. GETR fast and thermal neutron fluence profiles for capsule P13R ...................... 3-61

3-24. GETR fast and thermal neutron fluence profiles for capsule P13S.................. 3-62

3-25. Comparison between measured and calculated (TAC-2D) temperatures for capsule P13R at BOL and EOL . . . . . . . 3-63

3-26. Comparison between measured and calculated (TAC-2D) temperatures for capsule P13S at BOL and EOL . . . . . . . 3-64

3-27. Measured thermocouple readings of fuel and graphite for sample A, cell 2 of capsule P13R .......... 3-65

4-1. Photomicrographs showing P13S cell No. 2 fuel rods during removal from split graphite crucible... . . . . . 4-95

4-2. Representative photomicrographs showing (a) unbonded particle crucible assembly after removal from primary containment, and (b) unloading of unbonded particle samples from crucible.

4-3. Photomicrographs of fuel rod 7161-004-01-5 after irradiation in capsule P13R to a fast neutron fluence of $7.3 \times 10^{21} \mathrm{n} / \mathrm{cm}^{2}$ $(\mathrm{E}>0.18 \mathrm{MeV})_{\mathrm{HTGR}}$ at $1115^{\circ} \mathrm{C}$ and of fuel rod $7161-004-01-7$ after irradiation in capsule $\mathrm{P} 13 \mathrm{~S}$ to a fast neutron fluence of $7.0 \times 10^{21} \mathrm{n} / \mathrm{cm}^{2}$ ( $\left.\mathrm{E}>0.18 \mathrm{MeV}\right)_{\mathrm{HTGR}}$. . . . . . . .

4-4. Photomicrographs of fuel rod 7161-004-02-6 after irradiation in capsule P13R to a fast neutron fluence of $8.1 \times 10^{21} \mathrm{n} / \mathrm{cm}^{2}$ $(\mathrm{E}>0.18 \mathrm{MeV})_{\mathrm{HTGR}}$ at $1050^{\circ} \mathrm{C}$ and of fuel rod $7161-004-02-5$ after irradiation in capsule P13S to a fast neutron fluence of $7.7 \times 10^{21} \mathrm{n} / \mathrm{cm}^{2}(\mathrm{E}>0.18 \mathrm{MeV})_{\mathrm{HTGR}}$........ 4-98

4-5. Photomicrographs of fuel rod 7161-004-03-5 after irradiation in capsule P13R to a fast neutron fluence of $8.7 \times 10^{21} \mathrm{n} / \mathrm{cm}^{2}$ $(\mathrm{E}>0.18 \mathrm{MeV})_{\mathrm{HTGR}}$ at $1065^{\circ} \mathrm{C}$ and of fuel rod $7161-004-03-6$ after irradiation in capsule $\mathrm{P} 13 \mathrm{~S}$ to a fast neutron fluence of $8.5 \times 10^{21} \mathrm{n} / \mathrm{cm}^{2}(\mathrm{E}>0.18 \mathrm{MeV})_{\mathrm{HTGR}}$....... 
4-6. Photomicrographs of fuel rod 7161-004-04-6 after irradiation in capsule P13R to a fast neutron fluence of $9.3 \times 1021 \mathrm{n} / \mathrm{cm}^{2}$ $(\mathrm{E}>0.18 \mathrm{MeV})_{\mathrm{HTGR}}$ at $1115^{\circ} \mathrm{C}$ and of fuel $\operatorname{rod} 7161-004-04-5$ after irradiation in capsule P13S to a fast neutron fluence of $9.1 \times 10^{21} \mathrm{n} / \mathrm{cm}^{2}(\mathrm{E}>0.18 \mathrm{MeV})_{\mathrm{HTGR}}$........ 4-100

4-7. Photomicrographs of fuel rod 7161-004-05-6 after irradiation in capsule P13R to a fast neutron fluence of $9.9 \times 10^{21} \mathrm{n} / \mathrm{cm}^{2}$ $(\mathrm{E}>0.18 \mathrm{MeV})_{\mathrm{HTGR}}$ at $1125^{\circ} \mathrm{C}$ and of fuel rod $7161-004-05-5$ after irradiation in capsule $\mathrm{P} 13 \mathrm{~S}$ to a fast neutron fluence of $9.6 \times 10^{21} \mathrm{n} / \mathrm{cm}^{2}(\mathrm{E}>0.18 \mathrm{MeV})_{\mathrm{HTGR}}$....... 4-101

4-8. Photomicrographs of fuel rod 7161-004-06-5 after irradiation in capsule P13R to a fast neutron fluence of $11.8 \times 10^{21} \mathrm{n} / \mathrm{cm}^{2}$ $(\mathrm{E}>0.18 \mathrm{MeV})_{\mathrm{HTGR}}$ at $1050^{\circ} \mathrm{C}$ and of fuel rod $7161-004-11-6$ after irradiation in capsule $\mathrm{P} 13 \mathrm{~S}$ to a fast neutron fluence of $11.5 \times 10^{21} \mathrm{n} / \mathrm{cm}^{2}(\mathrm{E}>0.18 \mathrm{MeV})_{\mathrm{HTGR}}$ at $1040^{\circ} \mathrm{C} . . .4402$

4-9. Photomicrographs of fuel rod 7161-004-07-5 after irradiation in capsule P13R to a fast neutron fluence of $12.0 \times 10^{21} \mathrm{n} / \mathrm{cm}^{2}$ $(\mathrm{E}>0.18 \mathrm{MeV})_{\mathrm{HTGR}}$ at $1055^{\circ} \mathrm{C}$ and of fuel rod $7161-004-12-5$ after irradiation in capsule $\mathrm{P} 13 \mathrm{~S}$ to a fast neutron fluence

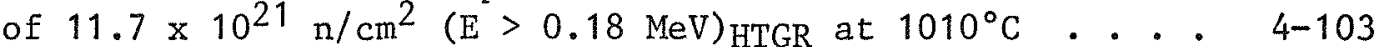

4-10. Photomicrographs of fuel rod 7161-004-08-013 after irradiation in capsule P13R to a fast neutron fluence of $12.2 \times 10^{21} \mathrm{n} / \mathrm{cm}^{2}$ $(E>0.18 \mathrm{MeV})_{\mathrm{HTGR}}$ at $1045^{\circ} \mathrm{C}$ and of fuel rod $7161-004-13-6$ after irradiation in capsule $\mathrm{P} 13 \mathrm{~S}$ to a fast neutron fluence of $11.9 \times 10^{21} \mathrm{n} / \mathrm{cm}^{2}(\mathrm{E}>0.18 \mathrm{MeV})_{\mathrm{HTGR}}$ at $975^{\circ} \mathrm{C}$. . . 4-104

4-11. Photomicrographs of fuel rod 7161-004-09-5 after irradiation in capsule P13R to a fast neutron fluence of $12.3 \times 10^{21} \mathrm{n} / \mathrm{cm}^{2}$ $(\mathrm{E}>0.18 \mathrm{MeV})_{\mathrm{HTGR}}$ at $1035^{\circ} \mathrm{C}$ and of fuel rod $7161-004-14-5$ after irradiation in capsule $\mathrm{P} 13 \mathrm{~S}$ to a fast neutron fluence of $12.0 \times 10^{21} \mathrm{n} / \mathrm{cm}^{2}$ (E $\left.>0.18 \mathrm{MeV}\right)_{\mathrm{HTGR}}$ at $985^{\circ} \mathrm{C}$. . . . 4-105

4-12. Photomicrographs of fuel rod 7161-004-10-5 after irradiation in capsule P13R to a fast neutron fluence of $12.4 \times 10^{21} \mathrm{n} / \mathrm{cm}^{2}$ $(\mathrm{E}>0.18 \mathrm{MeV})_{\mathrm{HTGR}}$ at $965^{\circ} \mathrm{C}$ and of fuel rod $7161-004-15-5$ after irradiation in capsule $\mathrm{P} 13 \mathrm{~S}$ to a fast neutron fluence of $12.1 \times 10^{21} \mathrm{n} / \mathrm{cm}^{2}(\mathrm{E}>0.18 \mathrm{MeV})_{\mathrm{HTGR}}$ at $960^{\circ} \mathrm{C}$... 4-106

4-13. Photomicrographs of fuel rod 7161-004-16-5 after irradiation in capsule P13R to a fast neutron fluence of $9.4 \times 10^{21} \mathrm{n} / \mathrm{cm}^{2}$ $(\mathrm{E}>0.18 \mathrm{MeV})_{\mathrm{HTGR}}$ at $1285^{\circ} \mathrm{C}$ and of fuel rod $7161-004-16-6$ after irradiation in capsule $\mathrm{P} 13 \mathrm{~S}$ to a fast neutron fluence

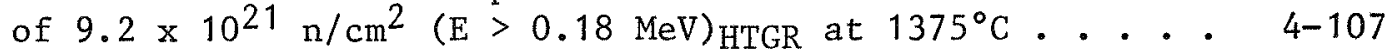

4-14. Photomicrographs of fuel rod 7161-004-17-5 after irradiation in capsule P13R to a fast neutron fluence of $9.0 \times 10^{21} \mathrm{n} / \mathrm{cm}^{2}$ $(\mathrm{E}>0.18 \mathrm{MeV})_{\mathrm{HTGR}}$ at $1225^{\circ} \mathrm{C}$ and of fuel rod $7161-004-17-6$ after irradiation in capsule $\mathrm{P} 13 \mathrm{~S}$ to a fast neutron fluence of $8.7 \times 10^{21} \mathrm{n} / \mathrm{cm}^{2}(\mathrm{E}>0.18 \mathrm{MeV})_{\mathrm{HTGR}}$ at $1330^{\circ} \mathrm{C} \cdot . \cdot 4-108$ 
4-15. Photomicrographs of fuel rod 7161-004-18-6 after irradiation in capsule P13R to a fast neutron fluence of $8.5 \times 10^{21} \mathrm{n} / \mathrm{cm}^{2}$ $(\mathrm{E}>0.18 \mathrm{MeV})_{\text {HTGR }}$ at $1235^{\circ} \mathrm{C}$ and of fuel rod $7161-004-18-5$ after irradiation in capsule P13S to a fast neutron fluence of $8.4 \times 10^{21} \mathrm{n} / \mathrm{cm}^{2}\left(\mathrm{E}>0.18 \mathrm{MeV}\right.$ ) HTGR at $1335^{\circ} \mathrm{C}$. . . 4-109

4-16. Photomicrographs of fuel rod 7161-004-19-7 after irradiation in capsule P13R to a fast neutron fluence of $8.2 \times 10^{21} \mathrm{n} / \mathrm{cm}^{2}$ $(\mathrm{E}>0.18 \mathrm{MeV})_{\text {HTGR }}$ at $1225^{\circ} \mathrm{C}$ and of fuel rod $7161-004-19-5$ after irradiation in capsule $\mathrm{P} 13 \mathrm{~S}$ to a fast neutron fluence of $7.9 \times 10^{21} \mathrm{n} / \mathrm{cm}^{2}$ (E $\left.>0.18 \mathrm{MeV}\right)_{\text {HTGR }}$ at $1325^{\circ} \mathrm{C}$... . 4-110

4-17. Photomicrographs of fuel rod 7161-004-20-6 after irradiation in capsule P13R to a fast neutron fluence of $7.7 \times 10^{21} \mathrm{n} / \mathrm{cm}^{2}$ $(\mathrm{E}>0.18 \mathrm{MeV})_{\mathrm{HTGR}}$ at $1280^{\circ} \mathrm{C}$ and of fuel rod $7161-004-20-5$ after irradiation in capsule P13S to a fast neutron fluence of $7.5 \times 10^{21} \mathrm{n} / \mathrm{cm}^{2}(\mathrm{E}>0.18 \mathrm{MeV})$ HTGR at $1350^{\circ} \mathrm{C} . . .4-111$

4-18. Photomicrographs of fuel rod 7161-004-21-5 after irradiation in capsule P13R to a fast neutron fluence of $5.4 \times 10^{21} \mathrm{n} / \mathrm{cm}^{2}$ $(E>0.18 \mathrm{MeV})_{\mathrm{HTGR}}$ at $1065^{\circ} \mathrm{C}$ and of fuel rod $7161-004-26-5$ after irradiation in capsule $P 13 S$ to a fast neutron fluence of $5.3 \times 10^{21} \mathrm{n} / \mathrm{cm}^{2}$ (E>0.18 MeV) HTGR at $1085^{\circ} \mathrm{C}$. . . $4-112$

4-19. Photomicrographs of fuel rod 7161-004-22-5 after irradiation in capsule P13R to a fast neutron fluence of $5.0 \times 10^{21} \mathrm{n} / \mathrm{cm}^{2}$ (E $>0.18 \mathrm{MeV})_{\text {HTGR }}$ at $1055^{\circ} \mathrm{C}$ and of fuel rod $7161-004-27-5$ after irradiation in capsule $\mathrm{P} 13 \mathrm{~S}$ to a fast neutron fluence of $4.8 \times 10^{21} \mathrm{n} / \mathrm{cm}^{2}(\mathrm{E}>0.18 \mathrm{MeV})_{\mathrm{HTGR}}$ at $1045^{\circ} \mathrm{C} . . .4-113$

4-20. Photomicrographs of fuel rod 7161-004-23-5 after irradiation in capsule P13R to a fast neutron fluence of $4.5 \times 10^{21} \mathrm{n} / \mathrm{cm}^{2}$ $(\mathrm{E}>0.18 \mathrm{MeV})_{\mathrm{HTGR}}$ at $1005^{\circ} \mathrm{C}$ and of fuel rod $7161-004-28-13$ after irradiation in capsule $\mathrm{P} 13 \mathrm{~S}$ to a fast neutron fluence of $4.4 \times 10^{21} \mathrm{n} / \mathrm{cm}^{2}(\mathrm{E}>0.18 \mathrm{MeV})_{\mathrm{HTGR}}$ at $1050^{\circ} \mathrm{C} . \cdots 4^{2}-114$

4-21. Photomicrographs of fuel rod 7161-004-24-5 after irradiation in capsule P13R to a fast neutron fluence of $4.1 \times 10^{21} \mathrm{n} / \mathrm{cm}^{2}$ $(E>0.18 \mathrm{MeV})_{\mathrm{HTGR}}$ at $1020^{\circ} \mathrm{C}$ and of fuel rod $7161-004-29-5$ after irradiation in capsule P13S to a fast neutron fluence of $3.9 \times 10^{21} \mathrm{n} / \mathrm{cm}^{2}(\mathrm{E}>0.18 \mathrm{MeV})$ HTGR at $1015^{\circ} \mathrm{C}$. . . 4-115

4-22. Photomicrographs of fuel rod 7161-004-25-5 after irradiation in capsule P13R to a fast neutron fluence of $3.6 \times 10^{21} \mathrm{n} / \mathrm{cm}^{2}$ $(E>0.18 \mathrm{MeV})_{\mathrm{HTGR}}$ at $1000^{\circ} \mathrm{C}$ and of fuel rod $7161-004-30-5$ after irradiation in capsule $\mathrm{P} 13 \mathrm{~S}$ to a fast neutron fluence of $3.5 \times 10^{21} \mathrm{n} / \mathrm{cm}^{2}(\mathrm{E}>0.18 \mathrm{MeV})$ HTGR at $995^{\circ} \mathrm{C} \cdot \ldots$. . 4-116

4-23. Photograph of TRISO ( $8 \mathrm{Th}, 1 \mathrm{U}$ ) $\mathrm{O}_{2}$ particles after irradiation in position 4-4 of capsule P13S to a fast neutron fluence of $11.1 \times 10^{21 \mathrm{n} / \mathrm{cm}^{2}(\mathrm{E}>0.18 \mathrm{MeV})_{\mathrm{HTGR}} \cdot . . . .}$

4-24. Representative photographs of LHTGR reference design type fissile particles ............... 4-118 
FIGURES (Continued)

4-25. Representative photographs of LHTGR reference design type fertile particles .............. 4-119

4-26. Representative photographs of LHTGR reference design type fertile particles with the OPyC coating deposited from mixed gas ................. . 44-120

4-27. Photographs of TRISO $\mathrm{UC}_{2}$ and $\mathrm{BISO} \mathrm{ThO}_{2}$ particles after irradiation in capsule $\mathrm{P} 13 \mathrm{R}$. . . . . . . . . . . 4-121

4-28. Photographs of TRISO $\mathrm{UC}_{2}$ and $\mathrm{BISO} \mathrm{ThO}_{2}$ particles after irradiation in capsule $\mathrm{P} 13 \mathrm{R}$. . . . . . . . . 4-122

4-29. Representative photographs of TRISO (Th,U) $\mathrm{O}_{2}$ fissile particles ................. 4-123

4-30. Representative photographs of TRISO $\mathrm{ThO}_{2}$ fertile particles 4-124

4-31. Photographs showing fuel rod dimensional measurements being made using (a) a barrel micrometer and (b) a dial gauge micrometer...................

4-32. Measured and predicted linear dimensional change data for $\mathrm{P} 13 \mathrm{R}$ and $\mathrm{P} 13 \mathrm{~S}$ fuel rods irradiated at $1100^{\circ} \mathrm{C}$. . . . . 4-126

4-33. Measured and predicted volume change data for P13R and P13S fuel rods irradiated at $1100^{\circ} \mathrm{C}$. . . . . . . . . .

4-34. Irradiation-induced diametral change of graphite crucibles as a function of fast neutron fluence . . . . . . . .

4-35. Fission gas release versus reciprocal temperature for fuel rod 7161-004-19-5 irradiated in capsule P13S . . . . .

4-36. Fission gas release versus reciprocal temperature for fuel rod 7161-004-17-6 irradiated in capsule P13S . . . . .

4-37. Fission gas release versus reciprocal temperature for fuel rod 7161-004-19-7 irradiated in capsule P13R . . . . . 4-131

4-38. Fission gas release versus reciprocal temperature for fuel rod 7161-004-03-5 irradiated in capsule P13R . . . . .

4-39. Fission gas release versus reciprocal temperature for fuel rod 7161-004-16-5 irradiated in capsule P13R . . . . .

4-40. Fission gas release versus reciprocal temperature for fuel rod 7161-004-14-5 irradiated in capsule P13S . . . . .

4-41. Fission gas release versus reciprocal temperature for fuel rod 7161-004-03-6 irradiated in capsule P13S . . . . .

4-42. Fission gas release versus reciprocal temperature for fuel rod 7161-004-17-5 irradiated in capsule P13R . . . . . 4-136

4-43. Single-channel $\mathrm{Cs}-137$ and $\mathrm{Zr}-95$ gamma-ray scans of fuel rods irradiated in cell 1 of capsule P13R . . . . . . 4-137 


\section{FIGURES (Continued)}

4-44. Single-channel $\mathrm{Cs}-137$ and $\mathrm{Zr}-95$ gamma-ray scans of fuel rods irradiated in cell 1 of capsule P13S . . . . . . 4-138

4-45. Single-channel $\mathrm{Cs}-137$ and $\mathrm{Zr}-95$ gamma-ray scans of fuel rods irradiated in cell 5 of capsule P13R . . . . . . . . 4-139

4-46. Single-channel $\mathrm{Cs}-137$ and $\mathrm{Zr}-95$ gamma-ray scans of fuel rods irradiated in cel1 5 of capsule P13S . . . . . . . . 4-140

4-47. Single-channel gamma scan of Cs-137 and location of multichannel gamma scan spectra for P13R graphite fuel rod crucibles . . . . . . . . . . . . . . . .

4-48. Single-channel gamma scan of $\mathrm{Cs}-137$ and location of multi-

channel gamma scan spectra for P13S graphite fuel rod crucibles

4-49. Photomicrographs of representative particles from fuel rod
7161-004-06-5 irradiated in capsule P13R to a fast neutron fluence of $11.8 \times 10^{21} \mathrm{n} / \mathrm{cm}^{2}(\mathrm{E}>0.18 \mathrm{MeV})$ HTGR at $1050^{\circ} \mathrm{C}$

4-50. Photomicrographs of representative particles from fuel rod 7161-004-07-5 irradiated in capsule P13R to a fast neutron fluence of $12.0 \times 10^{21} \mathrm{n} / \mathrm{cm}^{2}\left(\mathrm{E}>0.18 \mathrm{MeV}\right.$ ) $\mathrm{HTGR}$ at $1055^{\circ} \mathrm{C}$

4-51. Photomicrographs of representative particles from fuel rod 7161-004-08-13 irradiated in capsule P13R to a fast neutron fluence of $12.2 \times 10^{21} \mathrm{n} / \mathrm{cm}^{2}(\mathrm{E}>0.18 \mathrm{MeV})_{\mathrm{HTGR}}$ at $1045^{\circ} \mathrm{C}$

4-52. Photomicrographs of representative particles from fuel rod 7161-004-19-7 irradiated in capsule P13R to a fast neutron fluence of $8.2 \times 10^{21} \mathrm{n} / \mathrm{cm}^{2}(\mathrm{E}>0.18 \mathrm{MeV})_{\mathrm{HTGR}}$ at $1225^{\circ} \mathrm{C}$

4-53. Photomicrographs of representative particles from fuel rod 7161-004-20-6 irradiated in capsule P13R to a fast neutron fluence of $7.7 \times 10^{21} \mathrm{n} / \mathrm{cm}^{2}(\mathrm{E}>0.18 \mathrm{MeV})_{\mathrm{HTGR}}$ at $1280^{\circ} \mathrm{C}$

4-54. Photomicrographs of representative particles from fuel rod 7161-004-01-7 irradiated in capsule P13S to a fast neutron fluence of $7.0 \times 10^{21} \mathrm{n} / \mathrm{cm}^{2}$ ( $\mathrm{E}>0.18 \mathrm{MeV}$ ) HTGR . . .

4-55. Photomicrographs of representative particles from fuel rod 7161-004-12-5 irradiated in capsule P13S to a fast neutron fluence of $11.7 \times 10^{21} \mathrm{n} / \mathrm{cm}^{2}(\mathrm{E}>0.18 \mathrm{MeV})_{\mathrm{HTGR}}$ at $1010^{\circ} \mathrm{C}$

4-56. Photomicrographs of representative particles from fuel rod 7161-004-13-6 irradiated in capsule P13S to a fast neutron fluence of $11.9 \times 10^{21} \mathrm{n} / \mathrm{cm}^{2}(\mathrm{E}>0.18 \mathrm{MeV}) \mathrm{HTGR}$ at $975^{\circ} \mathrm{C}$

4-57. Photomicrographs of representative particles from fuel rod 7161-004-16-6 irradiated in capsule P13S to a fast neutron fluence of $9.2 \times 10^{21} \mathrm{n} / \mathrm{cm}^{2}$ (E > $\left.0.18 \mathrm{MeV}\right)_{\mathrm{HTGR}}$ at $1375^{\circ} \mathrm{C}$

4-58. Photomicrographs of representative particles from fuel rod 7161-004-19-5 irradiated in capsule P13S to a fast neutron fluence of $7.9 \times 10^{21} \mathrm{n} / \mathrm{cm}^{2}(\mathrm{E}>0.18 \mathrm{MeV})_{\mathrm{HTGR}}$ at $1325^{\circ} \mathrm{C}$

4-59. Photomicrographs of representative particles from fuel rod 7161-004-20-5 irradiated in capsule P13S to a fast neutron fluence of $7.5 \times 10^{21} \mathrm{n} / \mathrm{cm}^{2}(\mathrm{E}>0.18 \mathrm{MeV})_{\mathrm{HTGR}}$ at $1350^{\circ} \mathrm{C}$ 
FIGURES (Continued)

4-60. Photomicrographs of representative particles from fuel rod 7161-004-28-13 irradiated in capsule P13S to a fast neutron fluence of $4.4 \times 1021 \mathrm{n} / \mathrm{cm}^{2}(\mathrm{E}>0.18 \mathrm{MeV})_{\mathrm{HTGR}}$ at $1050^{\circ} \mathrm{C} \cdot 4-154$

4-61. Representative photomicrographs of fue1 rod 7161-004-03-5 after irradiation in capsule $P 13 R$ to a fast neutron fluence of $8.7 \times 10^{21} \mathrm{n} / \mathrm{cm}^{2}$ (E $>0.18 \mathrm{MeV}$ )HTGR. After irradiation this rod was heated to $1600^{\circ} \mathrm{C}$ in TRIGA for fission gas release measurements ................

4-62. Representative photomicrographs of fuel rod 7161-004-06-5 after irradiation in capsule $P 13 R$ to a fast neutron fluence of $11.8 \times 10^{21} \mathrm{n} / \mathrm{cm}^{2}$ (E $>0.18 \mathrm{MeV}$ ) HTGR at $1050^{\circ} \mathrm{C}$. . .

4-63. Representative photomicrographs of fuel rod 7161-004-07-5 after irradiation in capsule $P 13 R$ to a fast neutron fluence of $12.0 \times 10^{21} \mathrm{n} / \mathrm{cm}^{2}(\mathrm{E}>0.18 \mathrm{MeV})$ HTGR at $1055^{\circ} \mathrm{C}$. . . 4-157

4-64. Representative photomicrographs of fuel rod 7161-004-08-13 after irradiation in capsule $P 13 R$ to a fast neutron fluence of $12.2 \times 10^{21} \mathrm{n} / \mathrm{cm}^{2}(\mathrm{E}>0.18 \mathrm{MeV})$ HTGR at $1045^{\circ} \mathrm{C}$. . . 4-158

4-65. Representative photomicrographs of fuel rod 7161-004-16-5 after irradiation in capsule $\mathrm{P} 13 \mathrm{R}$ to a fast neutron fluence of $9.4 \times 10^{21} \mathrm{n} / \mathrm{cm}^{2}$ ( $\mathrm{E}>0.18 \mathrm{MeV}$ ) HTGR After irradiation this rod was heated to $1600^{\circ} \mathrm{C}$ in TRIGA for fission gas release measurements . . . . . . . . . . . . .

4-66. Representative photomicrographs of fuel rod 7161-004-19-7 after irradiation in capsule P13R to a fast neutron fluence of $8.2 \times 10^{21} \mathrm{n} / \mathrm{cm}^{2}(\mathrm{E}>0.18 \mathrm{MeV})_{\mathrm{HTGR}}$ at $1225^{\circ} \mathrm{C}$. . .

4-67. Representative photomicrographs of fuel rod 7161-004-20-6 after irradiation in capsule $\mathrm{P} 13 \mathrm{R}$ to a fast neutron fluence of $7.7 \times 1021 \mathrm{n} / \mathrm{cm}^{2}$ ( $\left.\mathrm{E}>0.18 \mathrm{MeV}\right)_{\mathrm{HTGR}}$ at $1280^{\circ} \mathrm{C}$. . .

4-68. Representative photomicrographs of fuel rod 7161-004-23-5 after irradiation in capsule $\mathrm{P} 13 \mathrm{R}$ to a fast neutron fluence of $4.5 \times 1021 \mathrm{n} / \mathrm{cm}^{2}$ ( $\mathrm{E}>0.18 \mathrm{MeV}$ ) $\mathrm{HTGR}$ at $1005^{\circ} \mathrm{C}$. . .

4-69. Representative photomicrographs of fuel rod 7161-004-01-7 after irradiation in capsule P13S to a fast neutron fluence

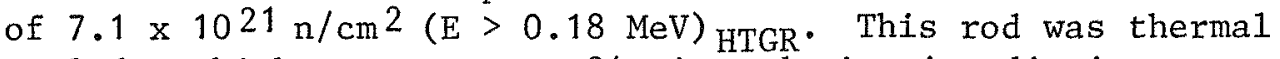
cycled to high temperatures 24 times during irradiation . 4-163

4-70. Representative photomicrographs of fuel rod 7161-004-12-5 after irradiation in capsule $P 13 S$ to a fast neutron fluence of $11.7 \times 1021 \mathrm{n} / \mathrm{cm}^{2}(\mathrm{E}>0.18 \mathrm{MeV}) \mathrm{HTGR}$ at $1010^{\circ} \mathrm{C} \cdot . \cdot 4-164$

4-71. Representative photomicrographs of fuel rod 7161-004-13-6 after irradiation in capsule P13S to a fast neutron fluence of $11.9 \times 10^{21} \mathrm{n} / \mathrm{cm}^{2}(\mathrm{E}>0.18 \mathrm{MeV})_{\mathrm{HTGR}}$ at $975^{\circ} \mathrm{C} \cdot . \cdot 4^{4}-165$ 
4-72. Representative photomicrographs of fuel rod 7161-004-15-5 after irradiation in capsule $\mathrm{P} 13 \mathrm{~S}$ to a fast neutron fluence of $12.1 \times 10^{21} \mathrm{n} / \mathrm{cm}^{2}(\mathrm{E}>0.18 \mathrm{MeV})_{\text {HTGR }}$ at $960^{\circ} \mathrm{C} \ldots$...

4-73. Representative photomicrographs of fuel rod 7161-004-16-6 after irradiation in capsule $P 13 S$ to a fast neutron fluence of $9.2 \times 10^{21} \mathrm{n} / \mathrm{cm}^{2}$ (E $>0.18 \mathrm{MeV}$ ) HTGR at $1375^{\circ} \mathrm{C}$. . . 4-167

4-74. Representative photomicrographs of fuel rod 7161-004-18-5 after irradiation in capsule P13S to a fast neutron fluence of $8.4 \times 1021 \mathrm{n} / \mathrm{cm}^{2}(\mathrm{E}>0.18 \mathrm{MeV})_{\mathrm{HTGR}}$ at $1335^{\circ} \mathrm{C}$. . . . 4-168

4-75. Representative photomicrographs of fuel rod 7161-004-19-5 after irradiation in capsule P13S to a fast neutron fluence of $7.9 \times 10^{21} \mathrm{n} / \mathrm{cm}^{2}$ (E $\left.>0.18 \mathrm{MeV}\right)_{\mathrm{HTGR}}$. After irradiation this rod was heated to $1500^{\circ} \mathrm{C}$ in TRIGA for fission gas release measurements ...............

4-76. Representative photomicrographs of fuel rod 7161-004-20-5 after irradiation in capsule P13S to a fast neutron fluence of $7.5 \times 10^{21} \mathrm{n} / \mathrm{cm}^{2}(\mathrm{E}>0.18 \mathrm{MeV})_{\mathrm{HTGR}}$ at $1350^{\circ} \mathrm{C}$. . . .

4-77. Representative photomicrographs of fuel rod 7161-004-28-13 after irradiation in capsule $\mathrm{P} 13 \mathrm{~S}$ to a fast neutron fluence of $4.4 \times 10^{21} \mathrm{n} / \mathrm{cm}^{2}(\mathrm{E}>0.18 \mathrm{MeV})_{\mathrm{HTGR}}$ at $1050^{\circ} \mathrm{C} \ldots .$.

4-78. Representative photomicrographs of TRISO $\mathrm{UC}_{2}$ particles irradiated in capsule P13S to a fast neutron fluence of $10.7 \times 10^{21} \mathrm{n} / \mathrm{cm}^{2}(\mathrm{E}>0.18 \mathrm{MeV})$ HTGR and burnup of $71 \%$ FIMA at $975^{\circ} \mathrm{C}$. . . . . . . . . . . .

4-79. Representative photomicrographs of TRISO $(8 \mathrm{Th}, 1 \mathrm{U}) \mathrm{O}_{2}$ particles irradiated in capsule P13S to a fast neutron fluence of $11.1 \times 10^{21} \mathrm{n} / \mathrm{cm}^{2}$ (E $>0.18 \mathrm{MeV}$ ) HTGR and burnup of $11 \%$ FIMA at $1090^{\circ} \mathrm{C}$..............

4-80. Representative photomicrographs of TRISO (Th,U) $\mathrm{O}_{2}$ particles irradiated in capsule P13S to a fast neutron fluence of $10.9 \times 10^{21} \mathrm{n} / \mathrm{cm}^{2}(\mathrm{E}>0.18 \mathrm{MeV}$ ) HTGR and burnup of $38 \%$ FIMA at $1090^{\circ} \mathrm{C}$

4-81. Photomicrographs of fissile and fertile particles from fuel rod 7161-004-01-7 that failed during irradiation in capsule P13S, which was thermal cycled to high temperatures 24 times during irradiation ...............

4-82. Photomicrographs showing a failed fissile particle from fuel rod 7161-004-035 irradiated in capsule P13R to a fast neutron fluence of $8.7 \times 10^{21} \mathrm{n} / \mathrm{cm}^{2}$ (E $>0.18 \mathrm{MeV}$ ) HTGR and burnup of $73 \%$ FIMA . . . . . . . . . . . . .

4-83. Photomicrographs showing OPyC coating failure of fissile particle in fuel rod 7161-004-18-5 that was irradiated in capsule P13S to a fast neutron fluence of $8.4 \times 10^{21} \mathrm{n} / \mathrm{cm}^{2}$ $(\mathrm{E}>0.18 \mathrm{MeV})_{\mathrm{HTGR}}$ at $1335^{\circ} \mathrm{C} . . . . . . . .$. 
FIGURES (Continued)

4-84. Photomicrographs showing the appearance of a typical

cracked SiC coating in a TRISO coated particle . . . . . 4-178

4-85. Photomicrograph showing a small laminar carbon inclusion in SiC coating of a TRISO $\mathrm{UC}_{2}$ particle irradiated in fuel

rod 7161-004-19-7 in capsule P13R . . . . . . . . . .

4-86. Photomicrographs showing an example of IPyC coating debonding that occurred in a TRISO $\mathrm{UC}_{2}$ particle during irradiation to a fast neutron fluence of $8.2 \times 10^{21} \mathrm{n} / \mathrm{cm}^{2}$ $(\mathrm{E}>0.18 \mathrm{MeV})$ HTGR

4-87. Photomicrographs showing a carbon deposit in the gap between the buffer and IPyC coating of a TRISO $\mathrm{UC}_{2}$ particle from fuel rod 7161-004-19-7 irradiated in capsule P13R at $1225^{\circ} \mathrm{C}$. . . . . . . . . . . . . .

4-88. Photomicrographs showing an example of a cracked buffer coating that occurred in a TRISO $\mathrm{UC}_{2}$ particle during irradiation to a fast neutron fluence of $11.8 \times 10^{2} \mathrm{n} / \mathrm{cm}^{2}$ $(\mathrm{E}>0.18 \mathrm{MeV})$

HTGR

4-89. Photomicrographs showing a TRISO $\mathrm{UC}_{2}$ particle from batch 6151-04-015-2 in which the structural coatings failed during irradiation leaving the buffer coating intact on the fuel kernel . . . . . . . . . . . . . .

4-90. Photomicrographs showing an example of $\mathrm{UC}_{2}$ fuel kernel migration that was observed in fuel rod 7161-004-18-5 irradiated in capsule $\mathrm{P} 13 \mathrm{~S}$ at $1335^{\circ} \mathrm{C} . . . . . .$.

4-91. Photomicrographs showing the microstructure of a $\mathrm{UC}_{2}$ fuel kernel irradiated in fuel rod $7161-004-07-5$ at $1055^{\circ} \mathrm{C}$. . 4-185

4-92. Photomicrographs showing the microstructure of a $\mathrm{UC}_{2}$ fuel kernel irradiated in fuel rod $7161-004-23-5$ at $1005^{\circ} \mathrm{C}$. $4-186$

4-93. Photomicrographs showing an example of $(1 \mathrm{Th}, 1 \mathrm{U}) \mathrm{O}_{2}$ kernel migration that was observed in fuel rod 7161-004-16-6 irradiated in capsule $\mathrm{P} 13 \mathrm{~S}$ at $1375^{\circ} \mathrm{C}$. . . . . . .

4-94. Photomicrographs showing metallic fission product attack of the SiC coating in a TRISO $\mathrm{UC}_{2}$ particle irradiated in fuel rod 7161-004-18-5 at an average centerline temperature of $1335^{\circ} \mathrm{C}$. . . . . . . . . . . . . . . 4-188

4-95. Photomicrographs of $\mathrm{BISO}^{\mathrm{ThO}} 2$ particles with an OPyC coating deposited from propylene . . . . . . . . . 4-189

4-96. Photomicrographs of $\mathrm{BISO}^{\mathrm{ThO}}{ }_{2}$ particles with an OPyC coating deposited from mixed gas . . . . . . . . . 4-190

4-97. Photomicrographs of severely faceted $\mathrm{BISO} \mathrm{ThO}_{2}$ particles after irradiation in capsule $P 13 R$ to a fast neutron fluence of $11.1 \times 10^{21} \mathrm{n} / \mathrm{cm}^{2}(\mathrm{E}>0.18 \mathrm{MeV})_{\mathrm{HTGR}}$ and burnup of $4.3 \%$ FIMA at $1000^{\circ} \mathrm{C}$. . . . . . . . . . . . 
FIGURES (Continued)

4-98. Photomicrographs of a severely faceted $\mathrm{BISO} \mathrm{ThO}_{2}$ particle from fuel rod 7161-004-06-5 that survived irradiation in capsule P13R to a fast neutron exposure of $11.8 \times 10^{21} \mathrm{n} / \mathrm{cm}^{2}$ $(\mathrm{E}>0.18 \mathrm{MeV})_{\mathrm{HTGR}}$ and burnup of $5.5 \%$ FIMA at $1050^{\circ} \mathrm{C} \cdot$. 4-192

4-99. Photomicrographs of $\mathrm{BISO}^{\mathrm{ThO}} 2$ particles with nonround fuel kernels after irradiation in capsule P13R to a fast neutron fluence of $10.9 \times 10^{21} \mathrm{n} / \mathrm{cm}^{2}(\mathrm{E}>0.18 \mathrm{MeV})_{\text {HTGR }}$ and burnup of $4.1 \%$ FIMA at $940^{\circ} \mathrm{C} \ldots \ldots$

4-100. Photomicrographs showing irradiation-induced failure in a BISO $\mathrm{ThO}_{2}$ particle with a high-density OPyC coating . . . 4-194

4-101. Photomicrographs showing irradiation-induced failure in a BISO $\mathrm{ThO}_{2}$ particle with a low-density OPyC coating . . . 4-195

4-102. Photomicrographs showing microcracks in OPyC coating of BISO $\mathrm{ThO}_{2}$ particle irradiated in capsule P13S . . . . . . . 4-196

4-103. Photomicrographs showing microcracks in OPyC coating of BISO $\mathrm{ThO}_{2}$ particle irradiated in capsule P13R . . . . . . . 4-197

4-104. Photomicrographs of a BISO $\mathrm{ThO}_{2}$ particle showing numerous small gaps formed at the buffer/OPyC coating interface where the seal coat cracked and densified during irradiation. .

4-105. Photomicrographs of $\mathrm{BISO} \mathrm{ThO}_{2}$ particles with no seal coat between the buffer and OPyC coatings . . . . . . . . 4-199

4-106. Photomicrographs of a BISO $\mathrm{ThO}_{2}$ particle where the buffer coating cracked and densified while the OPyC coating remained intact . . . . . . . . . . . . . . .

4-107. Photomicrograph of a BISO $\mathrm{ThO}_{2}$ particle which shows the propagation of a buffer coating crack into the adjacent OPyC coating and the partial conversion of a localized region of the fuel kernel to carbide . . . . . . . . .

4-108. Photomicrographs of BISO $\mathrm{ThO}_{2}$ particles with the buffer coating applied with $\mathrm{N}_{2}$ carrier gas . . . . . . . 4-202

4-109. Photomicrographs of TRISO $\mathrm{ThO}_{2}$ particles irradiated in capsule P13S to a fast neutron fluence of $11.5 \times 10^{21} \mathrm{n} / \mathrm{cm}^{2}$ $(\mathrm{E}>0.18 \mathrm{MeV})_{\mathrm{HTGR}}$ and burnup of $4.1 \%$ FIMA at $860^{\circ} \mathrm{C}$.

4-110. Photomicrographs showing reaction zone observed in TRISO $\mathrm{ThO}_{2}$ particle irradiated in capsule $\mathrm{P} 13 \mathrm{~S}$ to a fast neutron fluence of $11.5 \times 10^{21} \mathrm{n} / \mathrm{cm}^{2}$ ( $\mathrm{E}>0.18 \mathrm{MeV}$ ) HTGR and burnup of $4.1 \%$ FIMA at $860^{\circ} \mathrm{C}$. . . . . . . . . . .

4-111. Photomicrograph of a defective $\mathrm{BISO}^{\mathrm{ThO}_{2}}$ particle in fuel rod 7161-004-20-5 ...............

4-112. Photomicrographs showing the microstructure of a $\mathrm{ThO}_{2}$ kernel from particle batch 6542-01-010 irradiated in fuel rod 7161-004-08-13 of capsule P13R to a fast neutron fluence of $12.2 \times 10^{21} \mathrm{n} / \mathrm{cm}^{2}$ (E $\left.>0.18 \mathrm{MeV}\right)_{\mathrm{HTGR}}$ and burnup of $5.6 \%$ FIMA at $1045^{\circ} \mathrm{C}$. . . . . . . . . . . . . 
FIGURES (Continued)

4-113. Photomicrographs showing the microstructure of a $\mathrm{ThO}_{2}$ kerne1 from particle batch 6542-01-010 irradiated in fuel rod 7161-004-08-13 of capsule P13R . . . . . . . . . 4-207

4-114. Photomicrographs of TRISO inert particle irradiated in fuel rod 7161-004-08-13 of capsule P13R to a fast neutron fluence of $12.2 \times 10^{21 \mathrm{n} / \mathrm{cm}^{2}}$ (E $\left.>0.18 \mathrm{MeV}\right)_{\mathrm{HTGR}}$ at $1045^{\circ} \mathrm{C}$. . . 4-208

4-115. Postirradiation fission gas release versus unirradiated OPyC coating density for intact BISO $\mathrm{ThO}_{2}$ particles . . 4 4-209

4-116. Postirradiation fission gas release versus unirradiated OPyC coating density for intact $\mathrm{BISO} \mathrm{ThO}_{2}$ particles . . . 4-210

4-117. Amount of thorium leached from irradiated $\mathrm{BISO}^{\mathrm{ThO}} 2$ particle samples as a function of hot leaching time . . . . . . . 4-211

4-118. Photomicrographs showing the condition of irradiation BISO $\mathrm{ThO}_{2}$ particles after acid leaching. (a) Batch 6542-21-016-1 with an OPyC coating density of $1.73 \mathrm{~g} / \mathrm{cm}^{3}$ and a coating rate of $8.0 \mu \mathrm{m} / \mathrm{min}$ and (b) batch $6542-22-015-1$ with an OPyC coating density of $1.80 \mathrm{~g} / \mathrm{cm}^{3}$ and a coating rate of $4.0 \mu \mathrm{m} / \mathrm{min}$. . 4-212

4-119. Photomicrographs showing the condition of irradiated BISO $\mathrm{ThO}_{2}$ particles after acid leaching. (a) Batch 6542-24-015-1 with an OPyC coating density of $1.94 \mathrm{~g} / \mathrm{cm}^{3}$ and a coating rate of $6.6 \mathrm{\mu m} / \mathrm{min}$ and (b) batch 6542-02-020-2 with an OPyC coating density of $1.91 \mathrm{~g} / \mathrm{cm}^{3}$ and a coating rate of $8.5 \mu \mathrm{m} / \mathrm{min}$. . 4-213

4-120. Photomicrographs showing condition of irradiated $\mathrm{BISO} \mathrm{ThO}_{2}$ particles after acid leaching. (a) Batch 6542-09-010-2 with an $O P y C$ coating density of $1.93 \mathrm{~g} / \mathrm{cm}^{3}$ and a coating rate of $5.0 \mu \mathrm{m} / \mathrm{min}$ and (b) batch 6542-01-020-2 with an OPyC coating density of $1.82 \mathrm{~g} / \mathrm{cm}^{3}$ and a coating rate of $2.7 \mu \mathrm{m} / \mathrm{min} . .4-214$

4-121. Photomicrographs showing condition of irradiated $\mathrm{BISO} \mathrm{ThO}_{2}$ particles after acid leaching. (a) Batch 4252-06-018-1 with an $O P y C$ coating density of $1.82 \mathrm{~g} / \mathrm{cm}^{3}$ and a coating rate of $5.8 \mu \mathrm{m} / \mathrm{min}$ and (b) batch 6542-01-010-1 with an OPyC coating density of $1.80 \mathrm{~g} / \mathrm{cm}^{3}$ and a coating rate of $10.0 \mu \mathrm{m} / \mathrm{min}$. . 4-215

4-122. Photographs of fuel particles recovered from rod 7161-004-02-6 after it was burned in air at $950^{\circ} \mathrm{C}$ for $6 \mathrm{hr} \cdot 4-216$

4-123. Photographs showing $\mathrm{ThO}_{2}$ kernels and SiC coated fissile particles recovered from fuel rod 7161-004-02-6 . . . . 4 4-217

4-124. Photomicrograph showing condition of TRISO coated fissile particle from fuel rod 7161-004-11-6. . . . . . . . . 4-218

4-125. Photographs taken of microradiographs of fissile and fertile particles .................. . 4-219 
FIGURES (Continued)

5-1. OPyC coating failure versus coating rate and density for $\mathrm{UC}_{2}$ TRISO particles irradiated in capsules P13R and P13S to fast neutron fluences of 10.8 to $12.2 \times 10^{21} \mathrm{n} / \mathrm{cm}^{2}$ $(\mathrm{E}>0.18 \mathrm{MeV})_{\mathrm{HTGR}}$ at $1075^{\circ} \mathrm{C}$. . . . . . . . . 5-53

5-2. Optical anisotropy measured on individual OPyC coatings of $\mathrm{UC}_{2}$ TRISO particles versus apparent coating rate . . . . 5-54

5-3. OPyC coating failure versus optical anisotropy for $\mathrm{UC}_{2}$ TRISO particles irradiated in unbonded beds at $1075^{\circ} \mathrm{C}$ to fast neutron fluences of 10.7 to $12.1 \times 10^{21} \mathrm{n} / \mathrm{cm}^{2}$

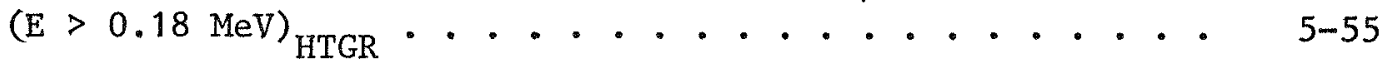

5-4. OPyC performance on 600- $\mu \mathrm{m}$-diameter TRISO particles irradiated in $\mathrm{P} 13 \mathrm{R}$ and $\mathrm{P} 13 \mathrm{~S}$ fuel rods . . . . . . . 5-56

5-5. Density change of OPyC coatings on TRISO fuel particles irradiated at $950^{\circ}$ to $1250^{\circ} \mathrm{C}$. . . . . . . . .

5-6. Measured and predicted $\mathrm{UC}_{2}$ fuel kernel migration distance as a function of radial location in fuel rod 7161-004-18-5 irradiated in capsule P13S . . . . . . . . . . .

5-7. Measured and predicted $\mathrm{UC}_{2}$ fuel kernel migration distance as a function of radial location in fuel rod 7161-004-19-7 irradiated in capsule $\mathrm{P} 13 \mathrm{R} . .$. . . . . . . . . . .

5-8. Measured and predicted $\mathrm{UC}_{2}$ fuel kernel migration distance as a function of radial location in fuel rod 7161-004-19-5 irradiated in capsule P13S . . . . . . . . . . .

5-9. Optical anisotropy measured on individual OPyC coatings of $\mathrm{ThO}_{2}$ BISO particles versus apparent coating rate.....

5-10. OPyC coating failure of $\mathrm{ThO}_{2}$ BISO particles versus (a) apparent coating rate and (b) optical anisotropy (BAF ${ }_{0}$ ) for coatings of various densities .............

5-11. Performance of OPyC coatings on $\mathrm{ThO}_{2}$ BISO particles irradiated in $\mathrm{P} 13 \mathrm{R}$ and $\mathrm{P} 13 \mathrm{~S}$ fuel rods ... . . . . . 5-63

5-12. Difference between sink-float and bulk density of fertile particle OPyC coatings as a function of coating rate . . 5-64

5-13. Capsule P13S cell 2 decalibrated control thermocouple temperature versus fast neutron fluence ... . . . 5-65

5-14. Release of Cs-137 versus fissile particle failure level calculated from $\mathrm{R} / \mathrm{B}$ values ........... 5-66

6-1. Irradiation strain of H-451 graphite . . . . . . . 6-11 


\section{INTRODUCTION}

Development of the high-temperature gas-cooled reactor (HTGR) has been under way in the United States since 1957. The initial fuel development effort for the Peach Bottom and Fort St. Vrain reactors was directed toward demonstrating the coated fuel particle concept and the multihole graphite fuel element design. Development of fuel for the large commercial HTGRs, termed LHTGRs, draws heavily upon General Atomic's experience in developing and manufacturing fuel for the early reactors, especially for the 330-MW(e) Fort St. Vrain power station. However, optimization of the LHTGR fuel has led to a number of changes from Fort St. Vrain fuel, which are summarized in Table 1-1.* These differences are principally the result of three major design goals: (1) complete separation of fissile and fertile material, (2) simplification of the fuel fabrication process, and (3) improved fuel performance.

LHTGR fuel consists of sma11, right-cylinder rods containing an intimate blend of coated fuel particles and isotropic graphite shim particles bonded together in a close-packed array. Fuel rods are formed by injecting the close-packed particle bed with a matrix material consisting of an organic binder and graphite filler, which when carbonized and heat treated yields a relatively strong carbonaceous bonding medium. The fuel rods are carbonized and heat treated in situ in the graphite fuel element. The use of isotropic graphite shim particles and the cure-in-place process for LHTGR rods represents design improvements that are attractive in both fresh fuel manufacturing and in the remote refabrication process. This commonality between fresh and recycle fuel will reduce overall development costs and reduce risks associated with the refabrication plant.

\footnotetext{
* Tables and figures appear at the end of each section.
} 
Two types of coated fuel particles are used in LHTGRs: fissile and fertile. The fissile particle has a dense $\mathrm{UC}_{2}$ fuel kernel consisting of highly enriched (93\%) uranium; the fertile particle has a Tho ${ }_{2}$ kernel. The design of the coated particles using TRISO coatings on fissile particles and BISO coatings on fertile particles facilitates separation of the bred U-233 from the nuclear poison U-236, generated primarily in the fissile kernel. During reprocessing, a burn-leach step is used to separate thorium and U-233 from the fissile particle.

Since the coatings also serve as the primary barrier to fission product release, it is important to ensure a high degree of coating integrity under all normal operating conditions. This is done in two ways: (1) design and specification of coated particle parameters to ensure a high probability of surviving irradiation-induced stresses, and (2) thermal design of the reactor core to eliminate significant failure due to thermochemical interactions between fuel and fission products and the structural coating layers.

Irradiation tests being conducted by General Atomic (GA) are designed to demonstrate reliable fuel performance to and beyond design LHTGR operating conditions. This report documents the postirradiation examination results for capsules $P 13 R$ and $P 13 S$ and discusses the impact of these results on the current fuel specifications and fuel performance models. 
TABLE $1-1$

PRINCIPAL DIFFERENCES BETWEEN FORT ST. VRAIN AND LARGE HTGR FUEL

\begin{tabular}{|c|c|c|c|}
\hline & Fort St. Vrain & LHTGR & Purpose of Change \\
\hline \multicolumn{4}{|l|}{ Fuel particles } \\
\hline Fissile kerne1 & $4: 1(\mathrm{Th}, \mathrm{U}) \mathrm{C}_{2}$ & $\mathrm{UC}_{2}$ (melted kernel) & Improve separability \\
\hline Fertile kernel & $\mathrm{ThC}_{2}$ & $\mathrm{ThO}_{2}$ & $\begin{array}{l}\text { Simplify process and } \\
\text { improve performance }\end{array}$ \\
\hline Fissile/fertile coatings & TRISO/TRISO & TRISO/BISO & Improve separability \\
\hline \multicolumn{4}{|l|}{ Fuel rods } \\
\hline Carbonization technique & In $\mathrm{Al}_{2} \mathrm{O}_{3}$ & In-block & Simplify process \\
\hline Rod shimming method & Fuel particle blending & Graphite shim particles & Simplify process \\
\hline Dimensions, $\mathrm{cm}$ & $\begin{array}{l}\text { Diameter: } 1.27 \\
\text { Length: } 5.08\end{array}$ & $\begin{array}{l}\text { Diameter: } 1.57 \\
\text { Length: } \quad 6.35\end{array}$ & $\begin{array}{l}\text { Improve core design } \\
\text { and simplify process }\end{array}$ \\
\hline
\end{tabular}





\section{DESCRIPTION OF EXPERIMENT}

\subsection{OBJECTIVES}

These tests have four primary objectives:

1. Characterize reference fresh fuel materials and processes (Ref. 2-1). Results of these tests will be used to support the HTGR fuel specifications (Ref. 2-2). Fuel rod tests are designed to characterize rod integrity and dimensional change as a function of irradiation conditions and rod variables (i.e., shim type and volume loading, heat-treatment temperature, filler type, macroporosity and microporosity, etc.). The series of unbonded particle tests conducted in cells 3 and 4 of capsules P13R and P13S were selected to provide information on effects of coating property variables and coating design on irradiation performance.

2. Determine the temperature-fluence dependence of in-pile fission gas release and relate it to coated particle integrity. Reference fresh fuel rods (200- $\mu \mathrm{m} \mathrm{UC} \mathrm{U}_{2}$ TRISO/500- $\left.\mu \mathrm{m} \mathrm{ThO}_{2} \mathrm{BISO}\right)$ were designed to be tested at $1075^{\circ}, 1300^{\circ}$, and $1500^{\circ} \mathrm{C}$. In-pile fission gas release data provide a means for monitoring fuel failure as a function of fluence and temperature. These results are used to support and update current fuel performance models (Ref. 2-3).

3. Determine the effect of thermal cycling on fuel integrity. Cell 1 of capsule P13S contained the first in-pile thermal cycling test of reference-type fuel. The thermal cycles simulated the most severe thermal transients to be experienced in an LHTGR during normal load-following conditions. A companion cell in P13R contained the same fuel and was not thermal cycled to provide a basis for performance comparison. 
4. Test alternative fuel materials. A number of alternative materials are included in these capsules. These materials include ( $\mathrm{Th}, \mathrm{U}) \mathrm{O}_{2}$ TRISO fissile particles, Lonza filler and shim particles, and nonimpregnated shim particles. At the time fuel for these capsules was being fabricated (early 1973), (Th,U) $0_{2}$ TRISO particles were considered an alternate candidate for the fissile fuel particle. Subsequently, the mixed oxide has been replaced by the weak-acidresin (WAR) fissile kernel.

\subsection{DESCRIPTION OF FUEL SPECIMENS}

Capsules P13R and P13S contained 40 fuel rod specimens and 96 unbonded particle samples that were designed to provide data on a broad spectrum of property and process variables for reference LHTGR fuel. Reference-type $\mathrm{UC}_{2}$ (VSM) TRISO fissile and $\mathrm{ThO}_{2}$ BISO fertile particles were used in the majority of the fuel samples. Most of the fuel rod specimens were fabricated by the hot-injection process and were carbonized in $\mathrm{H}-451$ graphite tubes to simulate in-block curing. A detailed description of the fuel specimens tested in capsules $\mathrm{P} 13 \mathrm{R}$ and $\mathrm{P} 13 \mathrm{~S}$ is given in Ref. 2-1.

\subsubsection{Coated Particles}

A general description of the coated particles is given in Tables 2-1 and 2-2. The reference-size 200- $\mu \mathrm{m}$-diameter $\mathrm{UC}_{2}$ (VSM) kernel was used in approximately $85 \%$ of the fissile particle batches. Most of the $\mathrm{UC}_{2}$ kernels selected for substrate material were from two large VSM production-size batches. One batch had approximately $1.0 \mathrm{wt} \%$ thorium added to improve spherodicity of the kernel. The other batch was not thorium doped; consequently, the kernels were "dimpled" and the coatings on these kernels were faceted. Two alternative fissile kernels, $250-\mu \mathrm{m}$-diameter $(\mathrm{Th}, \mathrm{U}) \mathrm{O}_{2}$ and $500-\mu \mathrm{m}$-diameter ( $8 \mathrm{Th}, \mathrm{U}) \mathrm{O}_{2}$ kernels, fabricated by the sol-gel process, were also tested in order to obtain additional irradiation data on these potential backup fuel kernels. All fissile particles were TRISO coated. The primary variables tested in each particle batch are summarized in Table 2-3, and the coating process data are summarized in Table 2-4. 
Reference-size 500- $\mu \mathrm{m}$-diameter $\mathrm{ThO}_{2}$ kernels were used in all fertile particle batches. Fuel kernels from a large number of pilot-scale equipment runs were tested. Many of the ThO 2 kernels were coated and "burned-back" one or more times. To evaluate the irradiation performance of fuel particles containing broken or misshapen kernels, irregularly shaped kernels from a parent batch were separated on a vibrating table and coated.

Fertile particle coating attributes investigated include OPyC coating rate, density, OPTAF, thickness, faceted coatings, no seal coat, and use of $\mathrm{N}_{2}$ carrier gas during the buffer coating deposition. The majority of oPyC coatings were deposited from propylene, although a number of OPyC coatings were deposited from propylene-acetylene (mixed-gas). Utilization of mixed-gas OPyC coatings is considered necessary in order to fabricate fertile particles on a scale envisioned for the LHTGR. One ThO 2 TRISO particle batch, which is the LHTGR backup fertile particle design, was tested for comparative purposes. A summary of fertile particle variables tested is given in Table 2-5, and the coating process data are summarized in Table 2-6.

\subsubsection{Fuel Rods}

The fuel rods contained a blend of TRISO coated fissile, BISO coated fertile, and graphite shim particles. Most of the fuel rods also contained varying amounts of TRISO coated inert resin particles in order to achieve close particle packing and the desired fuel particle and shim loadings. The fuel rods were approximately $1.242 \mathrm{~cm}(0.493 \mathrm{in}$.) in diameter by $1.905 \mathrm{~cm}$ ( $0.750 \mathrm{in.})$ long; 16 of the rods tested contained a central hole approximately $0.34 \mathrm{~cm}$ ( $0.13 \mathrm{in.})$ in diameter through the entire length of the rod to accommodate a thermocouple.

Normally only one attribute within the fuel rod was varied and all other attributes were held constant in order to isolate the effect of that variable on the irradiation performance of the fuel rod. Combining of some variables in given rods was necessary; however, the variables combined were judged not to interact with one another. A general description of the 
fuel rods irradiated is given in Tables $2-7$ and $2-8$, and a summary of the variables tested in each rod is given in Table 2-9.

Fuel rods were fabricated using a prototype LHTGR hot-injection press with the exception of two rods, which were fabricated by the slug-injection process. The majority of the rods were cured-in-place by placing five or six rods in $\mathrm{H}-451$ graphite tubes with Grafoil disks between the rods to prevent sticking. The tubes were heated in an induction furnace in a nitrogen atmosphere at $10^{\circ} \mathrm{C} / \mathrm{min}$ to $1000^{\circ} \mathrm{C}$. The final firing was done in an argon ox nitrogen atmosphere at $1500^{\circ}$ or $1800^{\circ} \mathrm{C}$. Two fuel rods were fired in packed beds of alumina at $1800^{\circ} \mathrm{C}$ in a nitrogen atmosphere. Preirradiation photomicrographs of fuel rods that are representative of the different fabrication and firing processes evaluated are shown in Figs. 2-1 and 2-2.

The graphite shim and filler materials used were comminuted, bulk, near-isotropic graphite supplied by candidate vendors for near-isotropic HTGR graphite. The GLCC $1099^{*}$ shim impregnated with furfuryl alcohol was used in 32 fuel rods. Four rods contained nonimpregnated GLCC 1099 shim and two rods contained impregnated Lonza ${ }^{* *}$ shim particles. The shim volume fraction in the rods tested was varied from 0.10 to 0.36 in order to study the effect of shim content on fuel rod dimensional change.

A matrix composition of 30 wt \% 6353 natural-flake graphite, ${ }^{\dagger} 65 \mathrm{wt} \%$ A240 pitch, ${ }^{+\dagger}$ and 5 wt \% SC011 (octadecanol) additive was used in 34 fuel rods. The remaining rods contained Lonza KS-44 graphite filler, SC003 (stearic acid), or $\mathrm{SC} 027$ (dimethyl terephthalate) matrix additives. The matrix additives were incorporated to improve the ease of fuel rod fabrication and to reduce the fuel rod pitch coke content after firing.

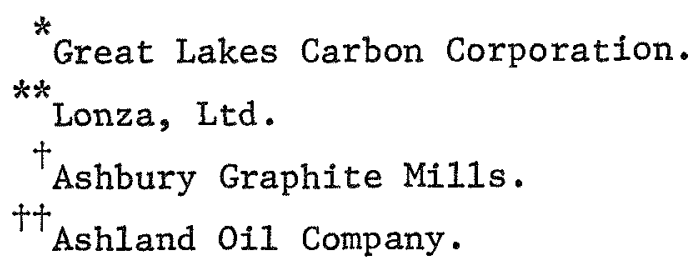




\subsection{CAPSULE DESIGN}

Capsules P13R and P13S were nearly identical in design and each capsule consisted of six individual cells. Fuel rod specimens were tested in cells $1,2,5$, and 6 and unbonded particle samples were tested in cells 3 and 4 . Fuel rod specimens were housed in split graphite crucibles inside Inconel cans which formed the primary containment for each cell, as shown in Fig. 2-3. Each unbonded particle cell contained a graphite crucible assembly, which consisted of 12 annular trays arranged on a threaded graphite stock, as shown in Fig. 2-4. The cells were arranged vertically in an outer containment tube and were numbered 1 through 6 from the bottom of the capsules. The outer containment tube was fabricated from type $304 \mathrm{~L}$ stainless steel and had an outside diameter of $3.18 \mathrm{~cm}$ (1.250 in.) and a wall thickness of $0.089 \mathrm{~cm}(0.035 \mathrm{in.})$. An aluminum band spacer was located between the Inconel cans and the outer containment tube. The outer portion of the spacer was slotted to guide the thermocouples and gas tubes to the lead tube.

Each cell was continuously purged with an inert neon/helium gas mixture. Primary temperature control gaps within the cells and secondary control gaps outside the cells were designed to provide the desired range of temperatures by varying the primary and secondary gas mixture composition.

Unbonded particle cells 3 and 4 and fuel rod cells 2 and 6 of both capsules and cell 1 of capsule P13R were designed to operate at a centerline temperature of $1075^{\circ} \mathrm{C}$. Cell 5 of capsules P13R and P13S was designed to operate at $1300^{\circ}$ and $1500^{\circ} \mathrm{C}$, respectively. Cell 1 of capsule P13S was designed to be thermal cycled between a nominal operating temperature of $1075^{\circ}$ and $1600^{\circ} \mathrm{C}$ periodically during capsule operation.

Each capsule contained 35 thermocouples located in or adjacent to the test specimens. Thermocouples used for measuring unfueled area temperatures below $1100^{\circ} \mathrm{C}$ were Inconel-sheathed Chromel-Alumel wire insulated with magnesium oxide. Fuel and high-temperature areas were monitored with tantalum-sheathed, tungsten-rhenium ( $\mathrm{W}-3 \% \mathrm{Re} / \mathrm{W}-25 \% \mathrm{Re}$ ) thermocouples 
insulated with beryllium oxide. The tungsten-rhenium thermocouples decalibrate under neutron-fluence-induced transmutation; therefore, ChromelAlumel thermocouples, which do not decalibrate, were placed in conjunction with the tungsten-rhenium thermocouples in the graphite crucible to establish decalibration rates for the experiment.

Standard dosimetry wires (Va - 0.216\% Co, V - 0.522\% Fe, and pure $\mathrm{Fe}$ ) were encapsulated in quartz ampoules and positioned along the capsules at seven axial locations at $0^{\circ}$ and $180^{\circ}$ radial orientations. In addition to the standard dosimetry wires, six ampoules containing purified Mn-54 were employed to evaluate the Mn-54 transmutation cross section.

REFERENCES

2-1. Young, C. A., and D. P. Harmon, "Preirradiation Report: Fuel Materials for P13R and P13S Irradiation Capsules," USAEC Report GA-A13026, General Atomic Company, November 1974.

2-2. Harmon, D. P., J. T. Ganley, and O. M. Stansfield, "HTGR Fuel Product Specification, Issue B," ERDA Report GA-A13464, General Atomic Company, January 1976.

2-3. Smith, C. L., "Fuel Particle Behavior Under Normal and Transient Conditions," USAEC Report GA-A12971 (GA-LTR-15), General Atomic Company, October 1, 1974 . 
GENERAL DESCRIPTION OF COATED PARTICLES TESTED IN CAPSTLE P13R

\begin{tabular}{|c|c|c|c|c|c|c|c|c|c|c|c|c|c|c|c|c|c|c|c|c|c|c|c|c|c|c|}
\hline \multirow[b]{3}{*}{ 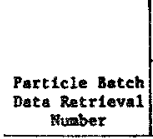 } & \multirow{2}{*}{\multicolumn{2}{|c|}{ Rerne1 }} & \multicolumn{13}{|c|}{ Coating } & \multirow{3}{*}{ 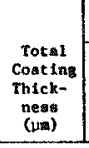 } & \multicolumn{8}{|c|}{ Total Coated Perticle } & \multirow{2}{*}{\multicolumn{2}{|c|}{\begin{tabular}{|c} 
Parrecle ploppositeion \\
Unead In
\end{tabular}}} \\
\hline & & & & Buff & fer & & & I Isotros & pic Pyc & s: & $1 \mathrm{c}$ & & Duter Iso & otrople PyC & & & \multirow[b]{2}{*}{$\begin{array}{c}\text { Mean } \\
\text { Ditamestar } \\
\text { (us) }\end{array}$} & \multirow[b]{2}{*}{ 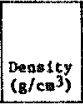 } & \multicolumn{2}{|c|}{ Meral Losding } & \multirow[b]{2}{*}{$\left|\begin{array}{c}\text { Pasaion } \\
\text { Gas } \\
\text { Release }(c)\end{array}\right|$} & \multicolumn{2}{|c|}{ Exposed Heavy Matra ${ }^{\text {(d) }}$} & \multirow[b]{2}{*}{ 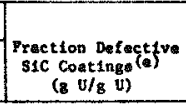 } & & \\
\hline & Type & 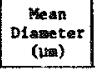 & Type ${ }^{(a)}$ & \begin{tabular}{|c|}
$\substack{\text { thick- } \\
\text { ness } \\
\text { ness } \\
\text { (Ham) }}$ \\
\end{tabular} & $\begin{array}{l}\text { Densitey } \\
\left(8 / \mathrm{cm}^{3}\right)\end{array}$ & $\begin{array}{l}\text { Seal } \\
\text { coat }\end{array}$ & \begin{tabular}{|c|} 
Thick- \\
ness \\
$(\mu \mathrm{m})$ \\
\end{tabular} & $\begin{array}{l}\text { Denostey } \\
\left(8 / \mathrm{cm}^{3}\right)\end{array}$ & $\operatorname{OPTAP}^{(b)}$ & $\begin{array}{c}\text { Thick- } \\
\text { nesa } \\
\text { (twas) }\end{array} \mid$ & \begin{tabular}{|l} 
Denst $5 y$ \\
$\left(g / \mathrm{cm}^{3}\right)$
\end{tabular} & $\begin{array}{c}\text { Thick- } \\
\text { ness } \\
\text { fesiag } \\
\text { tis }\end{array}$ & $\begin{array}{l}\text { Denestcy } \\
(\mathrm{g} / \mathrm{cm}\})\end{array}$ & 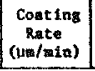 & optar ${ }^{\left({ }^{\prime}\right)} \mid$ & & & & $\begin{array}{l}\text { vrantum } \\
(\mathrm{ret} y)\end{array}$ & $\begin{array}{c}\text { Thoortus } \\
\text { (wt } k \text { ) }\end{array}$ & & 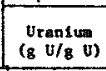 & $\begin{array}{c}\text { Thortum } \\
(\mathrm{g} / \mathrm{Th} / \mathrm{g} / \mathrm{m})\end{array}$ & & \begin{tabular}{|l|} 
Puat \\
Rods \\
\end{tabular} & $\begin{array}{c}\text { Unbooded } \\
\text { Tast }\end{array}$ \\
\hline \multicolumn{27}{|c|}{ Fisstle Particles $(5)$} \\
\hline S466-37 & $(\mathrm{Th}, \mathrm{v}) \mathrm{O}_{2}$ & 258 & TRISO & ${ }_{101}(\mathrm{~B})$ & 1.12 & Yes & 29 & 1.87 & 1.07 & 30 & 3.18 & $3 t$ & 1.79 & 6.0 & 1.10 & 197 & 619 & 2.37 & 12.7 & \begin{tabular}{|l|l|} 
& 12.30
\end{tabular} & $1 \times 10^{-6}$ & $2 \approx 10^{-7}$ & $6 \approx 10^{-5}$ & $S \approx 10^{-7}$ & $x$ & \\
\hline $6151-00-010$ & $\mathrm{uc}_{2}{ }^{(1)^{2}}$ & 199 & rRISO & $95^{(8)}$ & 1.18 & Yes & $3^{\pi}$ & 1.94 & 1.19 & 31 & 3.20 & 44 & 1.80 & 1.1 & 1.11 & 207 & 595 & 2.28 & 17.3 & 0.43 & $4 \times 10^{-7}$ & 0.00 & & $3 \times 10^{-4}$ & & $x$ \\
\hline S15t-00-025 & & 190 & IRISO & 90 (8) & 1.15 & Yes & 35 & 1.93 & 1.16 & 31 & 3.20 & 33 & 1.88 & 9.4 & 8.17 & 197 & 564 & 2.36 & 18.0 & $m a^{(h)}$ & $6 \times 10^{-8}$ & $4 \times 10^{-7}$ & $\mathrm{sm}^{(1)}$ & $2 \times 10^{-6}$ & & $x$ \\
\hline $615 t-00-035$ & $\mathrm{oc}_{2}^{2}(1)$ & 201 & TRISO & $87^{(8)}$ & 1.07 & Yes & 33 & 1.92 & 1.22 & 27 & $3.20^{\prime}$ & 37 & 1.85 & 3.9 & 1.17 & 191 & $5 n$ & 2.29 & 18.9 & $0.20^{(k)}$ & $3 \times 10^{-7}$ & $4 \times 10^{-7}$ & so & $2 \times 10^{-6}$ & $\mathrm{x}$ & $x$ \\
\hline $6151-00-045$ & $u_{c}^{2}$ & 196 & rRISO & $85^{(8)}$ & 1.15 & Yes & 35 & 1.93 & 1.17 & 29 & 3.22 & 40 & 1.81 & 3.8 & 8.13 & 192 & 578 & 2.33 & 17.8 & ma & $2 \times 10^{-7}$ & $1 \times 10^{-7}$ & so & $2 \times 10^{-6}$ & $x$ & $\mathrm{x}$ \\
\hline 6151-01-015 & $u c_{2}^{(1)}$ & 197 & TRISO & $99(8)$ & 8.15 & Yes & 38 & 1.94 & 1.20 & 25 & 3.21 & 42 & 1.90 & 0.7 & 1.15 & 203 & 590 & 2.28 & 37.4 & 0.22 & $1 \times 10^{-8}$ & $1 \times 10^{-7}$ & 0.00 & $4 \times 10^{-5}$ & & $x$ \\
\hline $6151-04-015$ & $u c_{2}^{(1)}$ & 202 & TRISO & $102(\mathrm{~g})$ & 0.87 & Yes & 33 & 1.88 & 1.23 & 28 & 3.20 & 36 & 1.82 & 1.4 & 1.14 & 202 & 599 & 2.45 & 17,4 & 0.22 & $8=10^{-7}$ & $1 \times 10^{-6}$ & $9 \times 10^{-4}$ & $7 \times 10^{-5}$ & & $\mathrm{x}$ \\
\hline $6151-08-015$ & $\mathrm{uc}_{2}^{2}(1)$ & 202 & Triso & $95^{(8)}$ & 1.07 & Yes & 33 & 1.92 & 1.25 & 26 & 3.21 & 36 & 1.76 & 3.6 & 1.09 & 190 & 593 & 2.28 & 18.6 & $0.20^{(k)}$ & $1 \times 10^{-7}$ & $9 \times 10^{-7}$ & ND & $2 \times 10^{-6}$ & $x$ & \\
\hline 6151-09-015 & $\mathrm{oc}_{2}^{2}(1)$ & 197 & TEISO & $95^{(8)}$ & 1.15 & Yes & 31 & 1.94 & 1.16 & 29 & 3.22 & 39 & 1.94 & 3.6 & 1.06 & $204^{\circ}$ & 587 & 2.30 & 17.8 & $0.20^{(k)}$ & $9 \times 10^{-8}$ & $4 \times 10^{-7}$ & mo & $A=10^{-6}$ & $\mathrm{x}$ & $x$ \\
\hline $6151-09-025$ & $\mathrm{uc}_{2}^{(1)}$ & 197 & rRiso & $8^{(\mathrm{B})}$ & 1.18 & Yes & 35 & 1.94 & 1.14 & 31 & 3.22 & 81 & 1.95 & 4.0 & 1.11 & 204 & 590 & 2.37 & 17.5 & $0.20^{(k)}$ & $2 \times 10^{-7}$ & $8 \times 10^{-7}$ & 0.00 & $3 \times 10^{-3}$ & & $\mathrm{x}$ \\
\hline
\end{tabular}

\begin{tabular}{|c|c|c|c|c|c|c|c|c|c|c|c|c|c|c|c|c|c|c|c|c|c|c|c|c|c|c|}
\hline \multicolumn{27}{|c|}{ Pertile Particles } \\
\hline $4252-06-018^{(m)}$ & $\mathrm{ThO}_{2}^{(n)}$ & 511 & BISO & 78 & 1.10 & Yes & SA & NA & Ma & NA & $\mathrm{sa}$ & 77 & 1.82 & 5.8 & 1.14 & iss & 826 & 2.72 & MA & 58.90 & ma & m & $7 \times 10^{-4}$ & & & $\mathrm{x}$ \\
\hline $6542-01-010$ & $\mathrm{TnO}_{2}(\mathrm{n})$ & 500 & BISO & 79 & 1.08 & Yes & NA & $\mathrm{NA}$ & nA & NA & MA & 85 & 1.80 & 10.0 & 1.06 & 164 & 828 & 3.59 & NA & 56.50 & $\mathrm{na}$ & $\mathrm{n}$ & $6 \times 10^{-6}$ & $\mathrm{ma}$ & \pm & $x$ \\
\hline $6542-01-020$ & $\mathrm{ThO}_{2}(0)$ & 504 & Biso & 81 & 1.17 & Yes & $\mathrm{NA}$ & MA & $\mathrm{Na}$ & na & HA & 74 & 1.82 & 2.7 & 1.10 & 155 & 813 & 3.55 & $\mathrm{su}$ & 57.50 & $\mathrm{nA}$ & หอ & $6 \times 10^{-6}$ & $\mathrm{NA}$ & $x$ & $\mathbf{x}$ \\
\hline $6542-02-020$ & $\mathrm{nho}_{2}^{2}(\mathrm{n})$ & 481 & siso & 87 & 1.08 & Yes & $\mathrm{WA}$ & NA & $\mathrm{NA}$ & NA & $\mathrm{NA}$ & 72 & 1.91 & 8.5 & 1.06 & 159 & 796 & 3.59 & $\mathrm{RA}$ & 56.20 & MA & no & $2 \times 10^{-5}$ & na & $x$ & $\mathrm{x}$ \\
\hline $6542-02-030$ & $\mathrm{moO}_{2}^{2}(\mathrm{n})$ & 481 & BIso & 79 & 1.18 & Yes & พA & MA & $\mathrm{xA}$ & ra & $\mathrm{xa}$ & 74 & 1.89 & 2.2 & 1.12 & 153 & 786 & 3.54 & na & 56.00 & ин & no & $8 \times 10^{-5}$ & Ka & & $\mathrm{x}$ \\
\hline $6542-11-015$ & $\mathrm{ThO}_{2}^{2}(0)$ & 497 & BISO & 87 & 1.12 & so & A & MA & sa & ra & HA & 74 & 1.83 & 2.6 & 1.29 & 161 & 819 & 3.35 & $\mathrm{xa}$ & 57.80 & nA & no & $6 \times 10^{-7}$ & $\mathbf{m}$ & & $\mathrm{x}$ \\
\hline $6542-18-013$ & $\mathrm{ThO}_{2}^{2}(0)$ & 476 & BIso & 84 & 1.11 & พo & $w$ & ma & sa & sA & $\mathrm{sh}$ & 70 & 1.84 & 6.5 & 1.13 & 154 & 791 & 3.53 & $\mathrm{na}$ & 58.80 & ma & so & $3 \times 10^{-5}$ & ma & & $\mathrm{x}$ \\
\hline $6542-19-015$ & $\mathrm{TnO}_{2}(0)$ & 486 & isso & 81 & 1.15 & Yes & wh & in & NA & $\mathrm{xa}$ & $\mathrm{na}$ & $n$ & 1.78 & 7.5 & 1.16 & 158 & 824 & 3.55 & $m$ & 58.90 & $m$ & 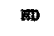 & $4 \times 10^{-6}$ & $m$ & $\pi$ & \\
\hline $6542-20-025$ & $\mathrm{TnO}_{2}^{2}(0)$ & 495 & BIso & 76 & 1.25 & Yes & MA & $\mathrm{NA}$ & Na & ка & na & 8 & 1.82 & 3.5 & 1.18 & 157 & sos & 3.57 & na & 58.20 & ma & $m$ & $3 \times 10^{-6}$ & $x$ & & $\mathrm{x}$ \\
\hline $6542-20-035$ & $\mathrm{ThO}_{2}^{2}(0)$ & $\$ 62$ & arso & 80 & 1.20 & Yes & $\mathbf{M A}$ & MA & $\mathrm{ma}$ & $\mathrm{sa}$ & na & 83 & 1.84 & 3.6 & 1.15 & 163 & 810 & 3.50 & $\mathrm{sa}$ & 57.40 & ma & $m$ & $1 \times 10^{-6}$ & $m$ & $x$ & $x$ \\
\hline $6542-25-015$ & $\mathrm{TnO}_{2}^{2}(0)$ & 489 & stso & 79 & 1.21 & Yes & 的 & m & MA & $\mathrm{ma}$ & na & 76 & 1.82 & 1.0 & 1.11 & 135 & 826 & 3.57 & m & 58.20 & $\mathrm{ma}$ & mo & $1 \times 10^{-6}$ & $m$ & & x \\
\hline \multicolumn{27}{|c|}{ Inert Pmanticlas } \\
\hline $6351-01-020$ & c & 253 & Triso & ma & sa & Ye:s & 29 & 1.76 & 1.09 & 29 & 3.22 & 36 & 1.75 & wo & 1.12 & 94 & 373 & 1.83 & m & $\mathrm{xA}$ & $\mathrm{mA}$ & $\mathrm{m}$ & A & ma & $\pi$ & \\
\hline
\end{tabular}

(a) rRiso denotes a coating design with an SiC layer and BISO denotes a particie with no SiC layer.

(a) TRISO denotes a coating design with an $S 1 C$
(b) Optical antaotropy factor, relative units.

(c) Release rata/birth tace for kr-85 at $1100^{\circ} \mathrm{c}$.

(d) Deteralined by leach teat.

(e) Deteratoratned by by burn-1each teat.

(f)

(8) calculated, 1.e., deteralned by subtracting the 1raer 18otrople thickness frow the meesured buffer plus inner toottople thicknese.

(b) na = not appliseable.

(3) Xexnels thorive doped.

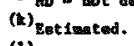

(1) Estianted.

(1) Density and stse separated from parent batch 4252-06-010.

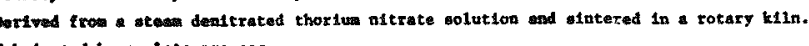

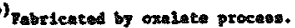


GENERAL DESCRIPTTON OF CAATED PARTICLES TESTEO IN CAPSULE P13S

\begin{tabular}{|c|c|c|c|c|c|c|c|c|c|c|c|c|c|c|c|c|c|c|c|c|c|c|c|c|c|c|}
\hline \multirow[b]{3}{*}{$\begin{array}{l}\text { Particie Batch } \\
\text { Data Rertseva1 } \\
\text { Number } \\
\end{array}$} & \multirow{2}{*}{\multicolumn{2}{|c|}{ ernel 1}} & \multicolumn{13}{|c|}{ Coarting } & \multirow{3}{*}{ 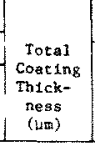 } & \multicolumn{8}{|c|}{ Total Coared Particle } & \multirow{2}{*}{\multicolumn{2}{|c|}{$\begin{array}{c}\text { Particle Diaposition } \\
\text { Uned In }\end{array}$}} \\
\hline & & & & & ffer & & & I Isot top & ic pyc & & Sic & & Outer Isc & otropic Py & & & \multirow[b]{2}{*}{$\mid \begin{array}{c}\text { Mean } \\
\text { Diameter } \\
(\omega \mathrm{m})\end{array}$} & \multirow[b]{2}{*}{$\begin{array}{l}\text { Density } \\
\left(\mathrm{g} / \mathrm{cm}^{3}\right)\end{array}$} & \multicolumn{2}{|c|}{ Metal Loading } & \multirow[b]{2}{*}{$\begin{array}{c}\text { Fission } \\
\text { Gas } \\
\text { Release }(c)\end{array}$} & \multicolumn{2}{|c|}{ Exposed Heavy Metal (d) } & \multirow[b]{2}{*}{ 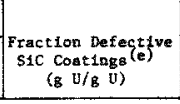 } & & \\
\hline & Type & 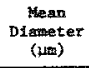 & Type & 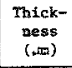 & 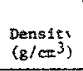 & $\begin{array}{l}\text { Sea1 } \\
\text { Coat }\end{array}$ & $\begin{array}{c}\text { Thick- } \\
\text { ness } \\
(-x) \\
\end{array}$ & 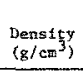 & $\mathrm{OPTAF}^{(\mathrm{b})}$ & $\begin{array}{c}\text { Thick- } \\
\text { ness } \\
(\mu \mathrm{m})\end{array}$ & $\begin{array}{l}\text { Density } \\
\left(\mathrm{g} / \mathrm{cm}^{3}\right)\end{array}$ & \begin{tabular}{|c|} 
Thyeks- \\
ness \\
(um)
\end{tabular} & $\begin{array}{l}\text { Dens } 1 \text { tev } \\
\left(\mathrm{g} / \mathrm{Cm}^{3} \mathrm{j}\right)\end{array}$ & \begin{tabular}{|} 
Coating \\
Rate \\
$(\mu \pi m / \min )$
\end{tabular} & ${ }_{\text {OPTAF }}^{(b)}$ & & & & \begin{tabular}{|l|} 
Urantums \\
(ut $\mathrm{z})$
\end{tabular} & 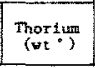 & & \begin{tabular}{|c|}
$\begin{array}{c}\text { Uranium } \\
(\mathrm{S} \cup \mathrm{ug})\end{array}$ \\
\end{tabular} & $\left(\begin{array}{c}\text { Thoritum } \\
(8 \mathrm{Th} / \mathrm{T} / \mathrm{T})\end{array}\right.$ & & \begin{tabular}{|l} 
Fuel \\
Rods
\end{tabular} & $\begin{array}{c}\text { Dabonded } \\
\text { Teat }\end{array}$ \\
\hline \multicolumn{27}{|c|}{ Fissile Particies ${ }^{(f)}$} \\
\hline $4161-01-021$ & vo & 189 & IRIso & 82 & 1.31 & Yes & 29 & 1.91 & $\longdiv { 1 . 1 6 }$ & 30 & 3.20 & 32 & 1.82 & 3.4 & 107 & 173 & 541 & 2.47 & 20.8 & $\mathrm{NA}_{\mathrm{A}}(\mathrm{g})$ & $5 \times 10^{-6}$ & $6 \times 10^{-7}$ & ${ }_{R D}^{(h)}$ & $1 \times 10^{-4}$ & & $x$ \\
\hline $4163-00-011$ & v) $\mathrm{O}_{2}$ & 253 & tso & $67^{(1)}$ & $\$ .17$ & Yes & 32 & 1.78 & 1.16 & 36 & 20 & 72 & 1.77 & 4.1 & 1. & 209 & 690 & 2.37 & 9.2 & 9.20 & $3 \times 10^{-9}$ & $6 \times 10^{-6}$ & $3 \times 10^{-5}$ & $2 \times$ & & $x$ \\
\hline $5466-37$ & $(\mathrm{Th}, \mathrm{v}) \mathrm{O}_{2}$ & 258 & TRISO & $108^{(1)}$ & 1.12 & Yes & 29 & 1.87 & 1.07 & 30 & 3.18 & 31 & 1.79 & 6.0 & 1.10 & 197 & 619 & 2.37 & 12.7 & 12.30 & $1 \times 10^{-6}$ & $2 \times 10^{-7}$ & $6 \times 10^{-5}$ & $5 \times 10^{-5}$ & $\mathbf{x}$ & $\mathrm{x}$ \\
\hline $6151 n 000035$ & $\mathrm{vc}_{2}(j)^{2}$ & 201 & TRIso & $87^{(1)}$ & 1.07 & Yes & 33 & 1.92 & 1.22 & 27 & 3.20 & 37 & 1.85 & 3.9 & 1.17 & 191 & 577 & 2.29 & 18.9 & $0.20^{(\mathrm{k})}$ & $3 \times 10^{-7}$ & $4 \times 10^{-7}$ & & $2 \times 10^{-6}$ & $\mathrm{x}$ & $\mathrm{x}$ \\
\hline $6151-0$ & & 196 & TRISO & $85^{(1)}$ & 0.15 & Yes & 35 & 1.93 & 1.17 & 29 & 3.22 & 40 & 1.81 & 3.8 & 1.13 & 192 & 578 & 2.33 & 17.8 & $\mathrm{NA}_{\mathrm{A}}$ & $2 \times 10^{-7}$ & $1 \times 10^{-7}$ & & $2 \times 10^{-6}$ & $\mathrm{x}$ & \\
\hline $02-025$ & $v c_{2}^{2}(j)$ & 204 & IRISO & $99^{(1)}$ & 1.07 & Yes & 33 & 1.92 & 1.25 & 27 & 3.22 & 43 & 1.50 & 0.7 & 1.1 & 202 & 608 & 2.19 & 28.4 & 0.22 & $2 \times 10^{-6}$ & $1 \times 10^{-6}$ & $8 \times 10^{-4}$ & $2 \times 10^{-3}$ & & $\mathrm{x}$ \\
\hline $6151-03-015$ & $\mathrm{uc}_{2}^{2}(j)$ & 199 & TRISO & $94^{(1)}$ & 3.10 & Yes & 31 & 1.90 & 1.24 & 29 & 3.22 & 45 & 1.27 & 1.5 & 1.12 & 204 & 594 & 2.40 & 17.6 & 0.23 & $2 \times 10^{-6}$ & $2 \times 10^{-6}$ & $7 \times 10^{-4}$ & $1 \times 10^{-4}$ & & $x$ \\
\hline $8151-08-$ & $\mathrm{vc}_{2}^{2}(1)$ & 202 & IRIso & $95^{(8)}$ & 1.07 & Yes & 33 & 1.92 & 1.25 & 26 & 3.21 & 36 & 1.76 & 3.6 & 1.09 & 190 & 593 & 2.28 & 18.6 & $0.20^{(\mathrm{k})}$ & $1 \times 10^{-7}$ & $9 \times 10^{-7}$ & ND & $2 \times 10^{-6}$ & $\mathrm{x}$ & $\approx$ \\
\hline $6151-09-015$ & $\mathrm{uc}_{2}^{2}(1)$ & 197 & TRISO & $95^{(1)}$ & 1.15 & Yes & 31 & 1.94 & 1.16 & 29 & 3.22 & 39 & 1.94 & 3.6 & 1. & 204 & 587 & 2.30 & 17.8 & $0.20^{(\mathrm{k})}$ & $9 \times 10^{-8}$ & $4 \times 10^{-7} \mid$ & & $4 \times 10^{-6}$ & $x$ & \\
\hline $6155-01-020$ & $(\mathrm{Th}, \mathrm{v}) \mathrm{O}_{2}$ & 502 & TRISO & ${ }_{86}{ }^{(i)}$ & 1.24 & Yes & 32 & 1.93 & 1.17 & 34 & 3.22 & 42 & 1.88 & 3.4 & 1.07 & 214 & 885 & 3.34 & 5.2 & 43.60 & $6 \times 10^{-7}$ & $6 \times 10^{-7}$ & $7 \times 10^{-5}$ & $1 \times 10^{-5}$ & & $\mathrm{x}$ \\
\hline
\end{tabular}

\begin{tabular}{|c|c|c|c|c|c|c|c|c|c|c|c|c|c|c|c|c|c|c|c|c|c|c|c|c|c|c|}
\hline \multicolumn{27}{|c|}{ Fertile Particies } \\
\hline $4252-02-010$ & $\mathrm{ThO}_{2}{ }^{(1)}$ & 488 & BIso & 83 & 1.08 & Yes & $\mathrm{M}$ & NA & M & NA & ra & 73 & 1.83 & 4.0 & 1.16 & 156 & 805 & 3.51 & $\mathrm{NA}$ & 58.80 & SA & $\mathrm{N}$ & $2 \times 10^{-5}$ & 跴 & & $x$ \\
\hline $4252-06-010$ & $\mathrm{ThO}_{2}^{2}(1)$ & 516 & BISO & 85 & 1.10 & Yes & $\mathrm{Na}$ & กล & $\mathrm{NA}$ & na & MA & 76 & 1.82 & 57 & 1.14 & 161 & 849 & 3.54 & $\mathrm{se}$ & 58.90 & WA & ND & $7 \times 10^{-4}$ & SA & & $x$ \\
\hline $6252-00-025$ & $\mathrm{ThO}_{2}^{2}(1)$ & 512 & TRISO & 60 & 1.16 & res & 30 & 1.87 & 1.25 & 31 & 3.21 & 42 & 1.81 & 42 & 1.24 & 164 & 833 & 3.77 & NA & 54.00 & $\mathrm{sA}_{\mathrm{A}}$ & ND & $9 \times 10^{-7}$ & $3 \times 10^{-4(\mathrm{~s})}$ & & $x$ \\
\hline $6542-01 \% 010$ & $\mathrm{ThO}_{2}^{2}(2)$ & 500 & BIsO & 79 & 1.08 & Yes & $\mathrm{ma}$ & NA & NA & $\mathrm{NA}$ & $\mathrm{NA}$ & 85 & 1.80 & 10.0 & 1.06 & 164 & 828 & 3.59 & NA & 56.50 & $\mathrm{Na}$ & ND & $6 \times 10^{-6}$ & ma & $\mathrm{x}$ & \\
\hline $6542-01-020$ & $\mathrm{ThO}_{2}^{2}(\mathrm{n})$ & 504 & BIso & 81 & 1.17 & yes & sa & $\mathrm{NA}$ & $\mathrm{NA}$ & $\mathrm{s}$ & NA & 74 & 1.82 & 2.7 & 1.10 & 155 & 813 & 3.55 & NA & 57.50 & Na & no & $6 \times 10^{-6}$ & us & $\mathrm{x}$ & \\
\hline $6542-02-020$ & $\mathrm{Tho}_{2}^{2}(1)$ & 481 & grso & 87 & 1.08 & Yes & 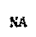 & $\mathrm{NA}$ & $\mathrm{NA}$ & $\mathrm{NA}$ & $\mathrm{min}$ & 72 & 1.91 & 8.5 & 1.06 & 159 & 796 & 3.59 & NA & 56.20 & NA & $m$ & $2 \times 10^{-5}$ & ma & $\mathrm{x}$ & \\
\hline $6542-09-010$ & $\mathrm{maO}_{2}^{2}(1)$ & 511 & BIsO & 84 & 1.06 & Yes & NA & $\mathrm{NA}$ & $\mathrm{NA}$ & ns & MA & 75 & 1.93 & 5.0 & 1.13 & 159 & 822 & 3.56 & NA & 57.60 & NA & wD & $1 \times 10^{-4}$ & HA & $x$ & \\
\hline $6542-12-025$ & $\mathrm{Tho}_{2}^{2}(\mathrm{~s})$ & sos & BIso & 84 & 1.10 & Yes & $\mathrm{SA}$ & $\mathbb{N A}$ & $\mathrm{NA}$ & พA & MA & 70 & 1.79 & 6.7 & 1.22 & 154 & 816 & 3.58 & $\mathrm{NA}$ & 58.00 & NA & D & $5 \times 10^{-7}$ & ma & & $\mathrm{x}$ \\
\hline $6542-16-010$ & $\mathrm{ThO}_{2}^{2}(\mathrm{~m})$ & 502 & BIsO & 39 & 1.08 & Yes & $\mathrm{MA}$ & NA & $\mathrm{NA}$ & NA & sh & 35 & ND & 3.7 & 1.07 & 94 & 687 & 4.84 & ns & 70.40 & $\mathrm{NA}$ & 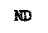 & $1 \times 10^{-4}$ & ma & & $\mathrm{x}$ \\
\hline $6562-17-010$ & $\operatorname{Tho}_{2}^{2}(\mathrm{a})$ & 502 & giso & 44 & 0.95 & Yes & $\$$ & NA & NA & $\mathrm{s}$ & $\mathrm{xA}$ & 122 & 1.86 & 2.8 & 1,27 & 166 & 829 & 3.55 & NA & 54.50 & NA & so & $6 \times 10^{-7}$ & ma & & $\mathbf{x}$ \\
\hline $6542-19-015$ & $\mathrm{ThO}_{2}{ }^{2(\mathrm{a})}$ & 486 & BIsO & 81 & 1.15 & Yes & NA & NA & NA & NA & sin & 77 & 1.78 & 7.5 & 1.16 & 158 & 824 & 3.55 & nA & 58.90 & NA & ND & $4 \times 10^{-6}$ & sa & $x$ & \# \\
\hline $6542-19-016^{(0, p)}$ & $\mathrm{ThO}_{2}{ }^{2}(\mathrm{n})$ & 486 & BIsO & 81 & 1.15 & Yes & $x_{A}$ & MA & $\mathrm{NA}$ & ns & NA & 77 & 1.78 & 7.5 & 1.16 & 158 & 824 & 3.55 & $\mathrm{NA}$ & 58.90 & NA & ND & $4 \times 10^{-6}$ & NA & & $\mathrm{x}$ \\
\hline $6542-20-039$ & $\mathrm{ThO}_{2}^{2}(\mathrm{n})$ & 462 & BISO & 80 & 1.20 & Yes & $\mathrm{M}$ & พA & $\mathrm{NA}$ & $\mathrm{NA}_{A}$ & $\mathrm{sa}$ & 83 & 1.84 & 3.6 & 1.15 & 163 & 810 & 3.50 & $\mathrm{NA}$ & 57.40 & NA & $m$ & $1 \times 10^{-6}$ & A & $\mathrm{x}$ & \\
\hline $6542-21-015$ & $\mathrm{ThO}_{2}^{2}$ (a) & 492 & BIsO & 79 & 1.13 & Yes & $\mathrm{NA}$ & ma & MA & NA & $\mathrm{sA}$ & 82 & 1.73 & 7.8 & 1.10 & 161 & 833 & 3.46 & NA & 57.40 & NA & אD & $4 \times 10^{-6}$ & M & & $\mathrm{x}$ \\
\hline $654 z-21-016^{(p, q)}$ & $\mathrm{ThO}_{2}^{2}(\mathrm{n})$ & 492 & BIsO & 79 & 1.13 & Yes & $\mathrm{kA}$ & HA & $\mathrm{NA}$ & NA & $\mathrm{MA}$ & 82 & 1.73 & 7.8 & 1.10 & 161 & 833 & 3.46 & $\mathrm{NA}$ & 57.40 & $\mathrm{NA}$ & ND & $4 \times 10^{-6}$ & a & & $\mathrm{x}$ \\
\hline $6542-22-015$ & $\mathrm{ThO}_{2}^{2}(\mathrm{n})$ & 503 & BISO & 85 & 1.12 & Yes & MA & ma & $\mathrm{KA}$ & NA & $\mathrm{ma}$ & 81 & 1.80 & 4.0 & 1.07 & 166 & 831 & 3.50 & NA & 58.00 & NA & NDD & $1 \times 10^{-6}$ & NA & & $x$ \\
\hline $6542-22-025$ & $\mathrm{ThO}_{2}^{2}(\mathrm{a})$ & 500 & BIso & 81 & 1.15 & res & $\mathrm{AA}$ & NA & พA & NA & $\mathrm{NA}$ & 80 & 9.88 & 3.6 & 8.10 & 161 & 833 & 3.53 & NA & 57.80 & $\mathrm{MA}$ & mo & $3 \times 10^{-6}$ & nA & & $x^{x}$ \\
\hline $6542-23-025$ & $\mathrm{ThO}_{2}^{2}(\mathrm{~s})$ & 486 & Btso & 82 & 1.19 & Yes & $\mathrm{MA}$ & ma & NA & $\mathrm{NA}$ & $\mathrm{rA}$ & 73 & 1.89 & 5.7 & 1.09 & 155 & 800 & 3.58 & NA & 57.50 & WA & $\mathrm{ND}$ & $4 \times 10^{-6}$ & NA & & $x^{2}-5$ \\
\hline $6542-24-015$ & $\mathrm{mho}_{2}^{2}(\mathrm{n})$ & 511 & BIso & 86 & .1 .13 & Yes & NA & NA & NA & NA & MA & 83 & 1.94 & 6.6 & 1.11 & 169 & 844 & 3.55 & NA & 57.10 & $\mathrm{NA}$ & $\mathrm{ND}$ & $1 \times 10^{-6}$ & MA & & $\mathrm{x}$ \\
\hline
\end{tabular}

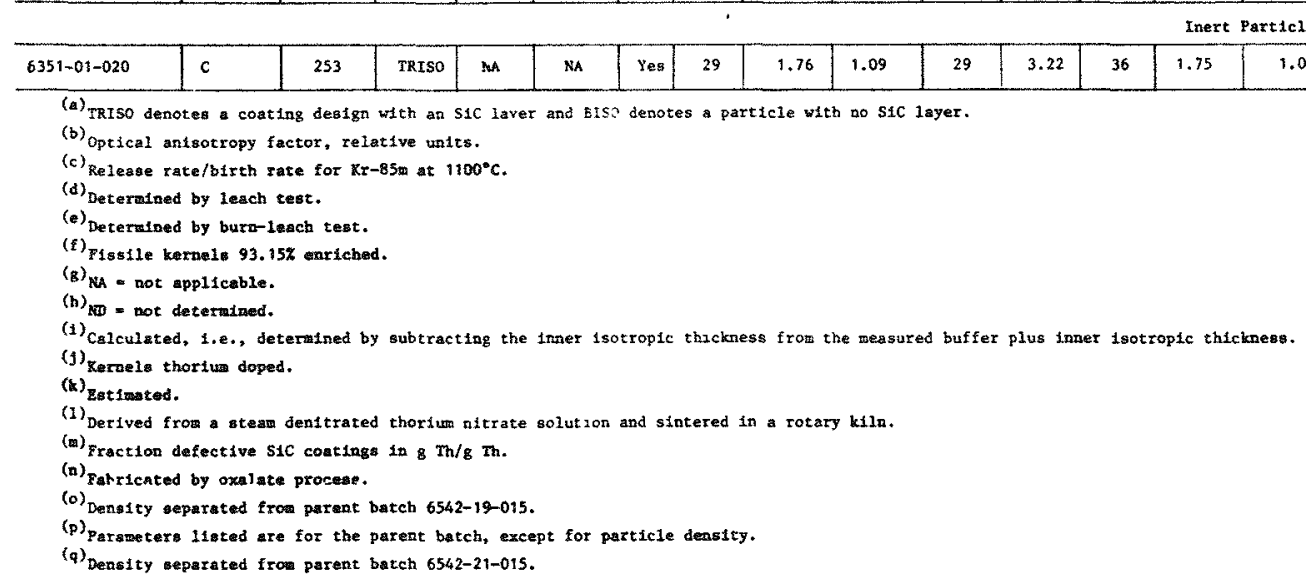


TABLE 2-3

TRISO COATED FISSILE PARTICLE VARIABLES TESTED IN CAPSULES P13R AND P13S

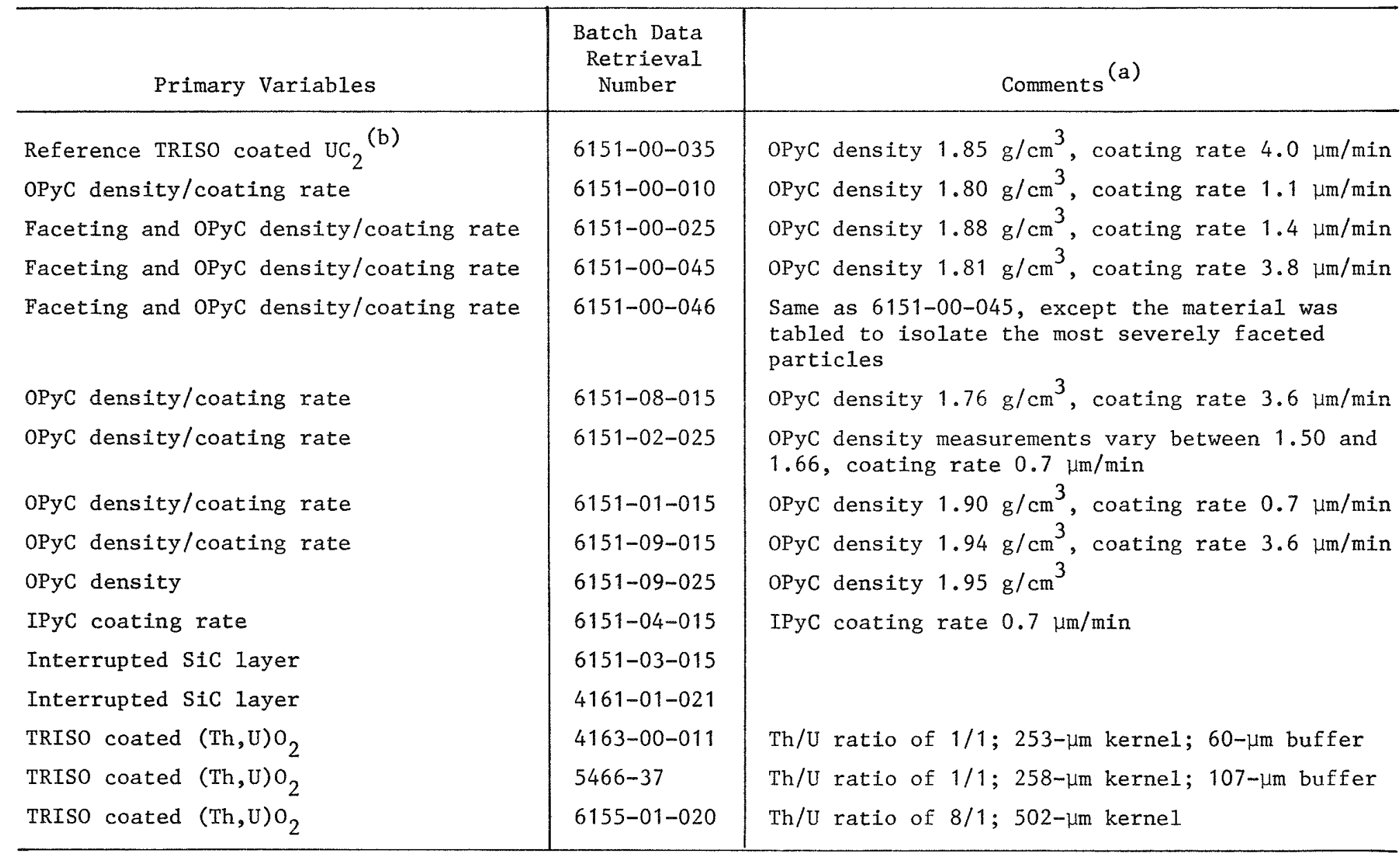

(a) Unless otherwise noted, all particles have 200- $\mu \mathrm{m} \mathrm{UC}_{2}$ kernels made via the VSM process.

(b) Reference coatings, are: buffer thickness $100 \mu \mathrm{m}$, density $1.1 \mathrm{~g} / \mathrm{cm}^{3}$; seal coat; IPyC thickness $30 \mu \mathrm{m}$, density $1.95 \mathrm{~g} / \mathrm{cm}^{3}$; SiC thickness $25 \mu \mathrm{m}$, density $>3.18 \mathrm{~g} / \mathrm{cm}^{3}$; OPyC thickness $40 \mu \mathrm{m}$, density $1.80 \mathrm{~g} / \mathrm{cm}^{3}$. 
TARLE 2-4
FISSILE PARTICLE COATYNG PROCLSS DATA

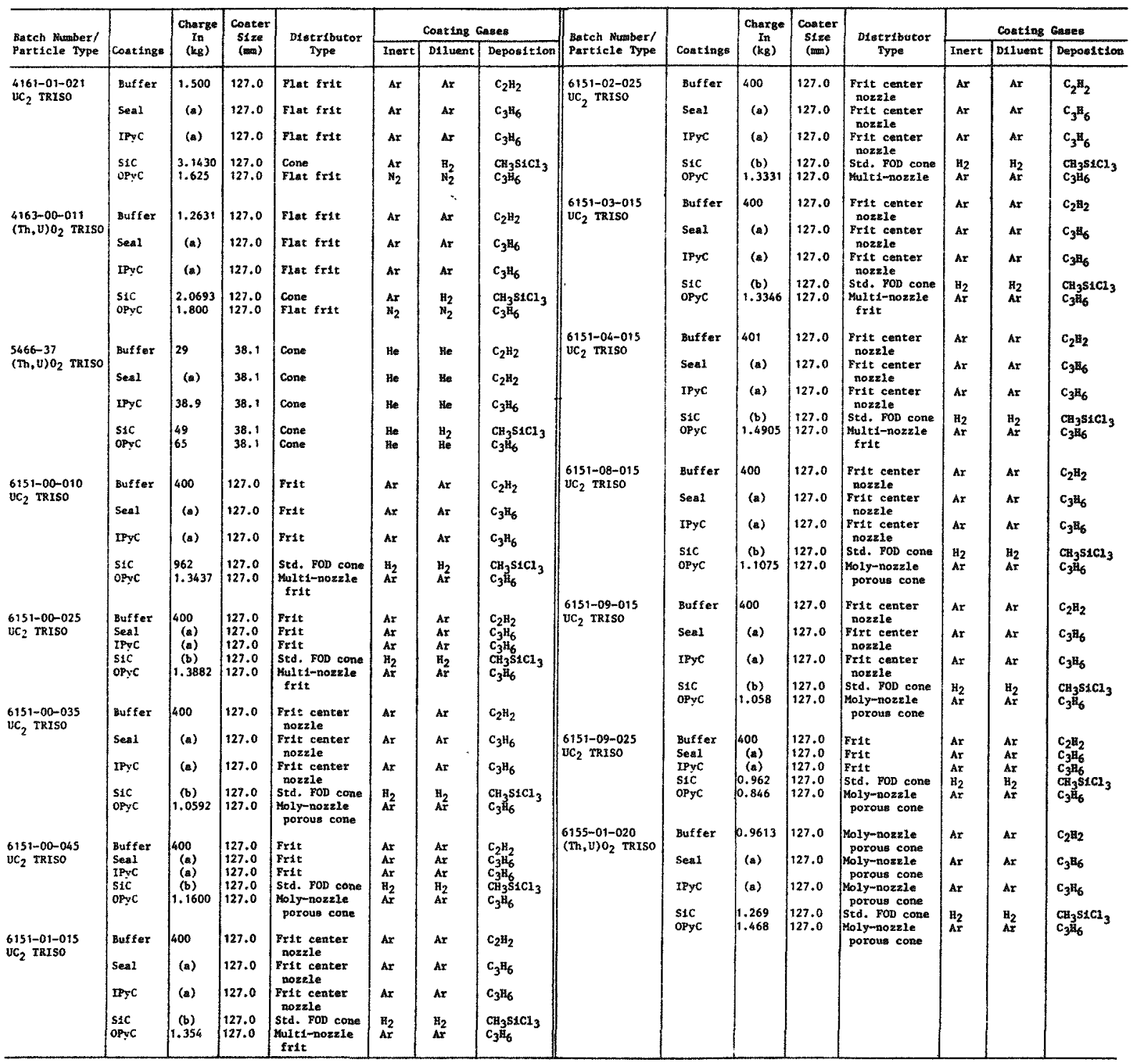

(a) Continuous frow buffer conting run.

(b) Date not avex.able. 
TABLE 2-5

FERTILE PARTICLE VARIAELES TESTED IN CAPSULES P13R AND P13S

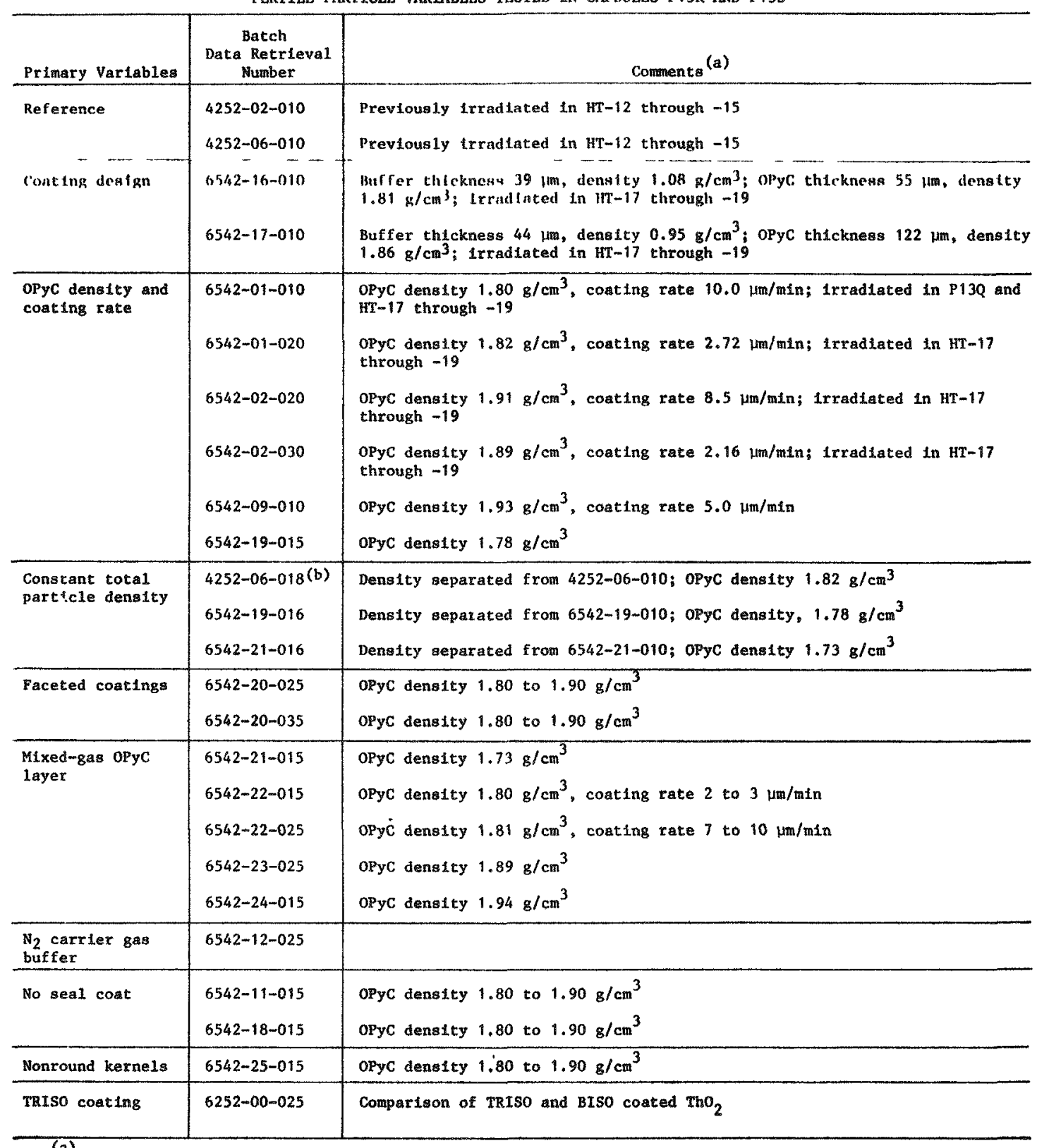

(a) Unless otherwloe Indicated, desired average particle attributes are: kexnel diatneter 480 to 520 um, density 9.5 to $10.0 \mathrm{~g} / \mathrm{cm}^{3}$; buffer thickness 80 to $90 \mu \mathrm{m}$, density 1.0 to $1.2 \mathrm{~g} / \mathrm{cm}^{3}$; seal coat; opyC thickness 70 to $80 \mu \mathrm{m}$, dens1ty 1.80 to $1.90 \mathrm{~g} / \mathrm{cm}^{3}$, OPTAF $\leq 1.20$,

(b) Also creened to opechic slze range. 
TABLE 2-6
FERTILE PARTICLE COATEMC PROCESS DATA

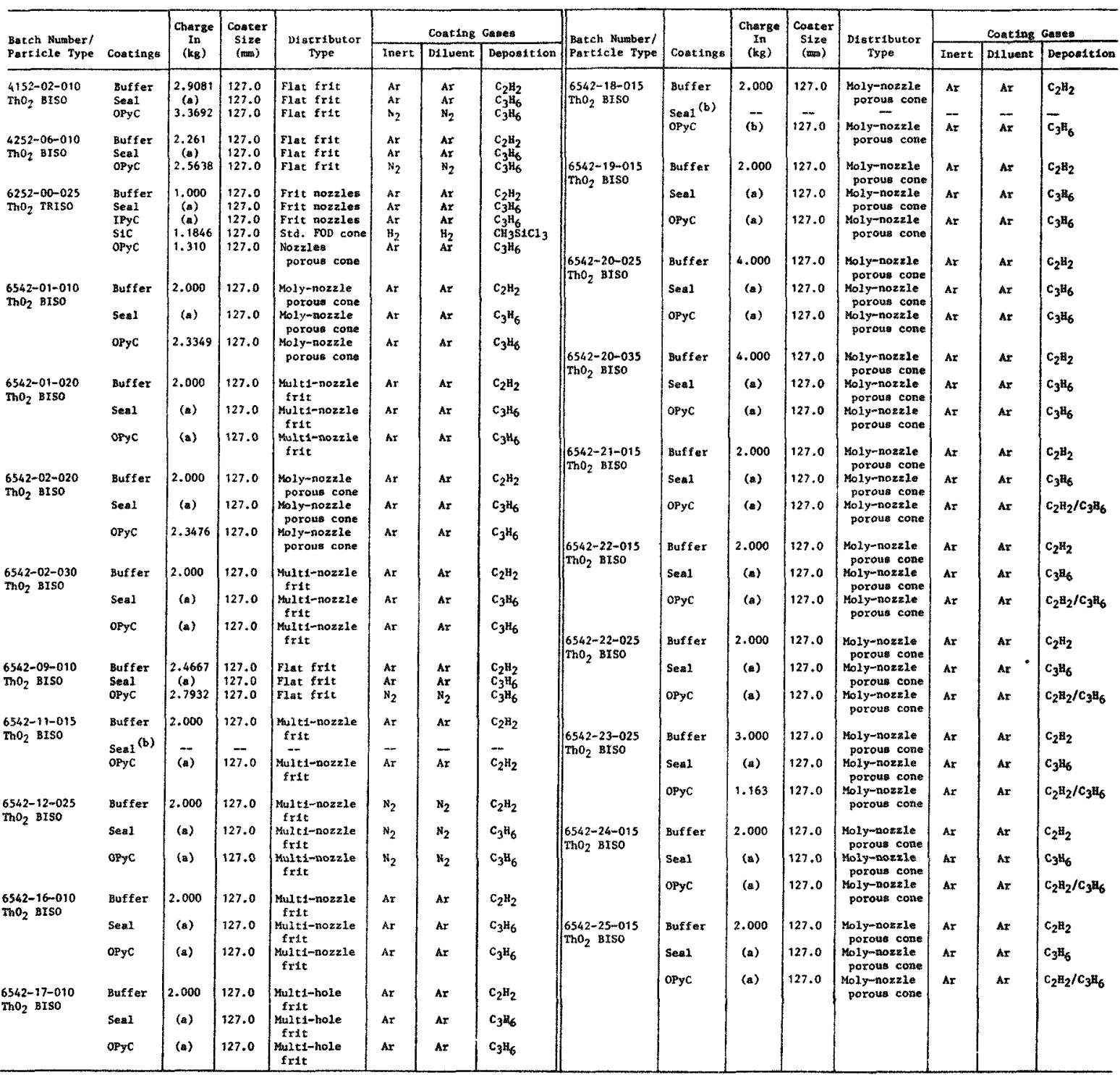

(a) Continuou from butfer costing rum.

(b) 1 o eal cout. 
TABI.E 2-7
DESCRIPTION OF FUEL RODS(a) TESTED IN CAPSULF P13R

\begin{tabular}{|c|c|c|c|c|c|c|c|c|c|c|c|c|c|c|c|c|c|c|}
\hline \multirow[b]{3}{*}{$\begin{array}{l}\text { Capsule } \\
\text { Pontelon } \\
\text { Celli }\end{array}$} & \multirow{3}{*}{$\begin{array}{c}\text { Data } \\
\text { Retrieval } \\
\text { Number } \\
\text { (7161-004-) }\end{array}$} & \multicolumn{4}{|c|}{$\ldots$ (onted Partir les ${ }^{(h)}$} & \multirow{2}{*}{\multicolumn{2}{|c|}{ Shim Particles }} & \multirow[b]{3}{*}{$\begin{array}{c}\text { Particle } \\
\text { Prcking(e, } f) \\
(z)\end{array}$} & \multirow[b]{3}{*}{$\left|\begin{array}{l}\text { Berch } \\
\text { Numlarer }\end{array}\right|$} & \multirow[b]{3}{*}{$\begin{array}{c}\text { Filer }(g) \\
\left(w x^{2}\right)\end{array}$} & \multicolumn{2}{|c|}{ Mntrix } & \multirow{2}{*}{\multicolumn{2}{|c|}{ Coke Content ${ }^{(f)}$}} & \multirow{3}{*}{\multicolumn{2}{|c|}{$\begin{array}{l}\text { Pued Loadtng } \\
\text { Uint formlty }(k)\end{array}$}} & \multirow{3}{*}{ 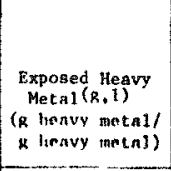 } & \multirow[b]{3}{*}{ 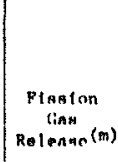 } \\
\hline & & \multicolumn{2}{|c|}{ FlasLle ${ }^{(\mathrm{c})}$} & \multicolumn{2}{|c|}{$\operatorname{tert11e^{(d)}}$} & & & & & & & & & & & & & \\
\hline & & rype & $\begin{array}{c}\text { Data } \\
\text { Retrtevn1 } \\
\text { Number }\end{array}$ & Inpm & \begin{tabular}{|c|} 
Data \\
Retrteval \\
Nitunly-1
\end{tabular} & $19 \mathrm{~m}$ & $\begin{array}{l}\text { Batch } \\
\text { Nitumluc }\end{array}$ & & & & $\begin{array}{c}\text { Apparenz } \\
\text { nena1ty }(\theta, f) \\
\left(R^{2} / \mathrm{cm}^{3}\right)\end{array}$ & $\begin{array}{l}\text { Macro- } \\
\text { porogltev }(h) \\
(z)\end{array}$ & $\begin{array}{l}\text { Vteld }(1) \\
(x)\end{array}$ & $\begin{array}{l}\text { Dens } f \text { ty } \\
\left(\mathrm{g} / \mathrm{em}^{3}\right)\end{array}$ & & {$\left[\begin{array}{ll}m 1 t y(k) \\
111-232\end{array}\right]$} & & \\
\hline $\operatorname{col1}_{A}^{(n)}$ & $01-5$ & $\mathrm{vc}_{2}$ & $6151-00-045$ & $\mathrm{ThO}_{2}$ & $6542-20-035$ & $1099-I^{(0)}$ & FO 147 & -. & Mo 269 & 37 & -- & 39 & -. & -- & 1.05 & 1.05 & $<8 \times 10^{-6}$ & $2 \times 10^{-6}$ \\
\hline B & $02-6$ & $\mathrm{vc}_{2}^{2}$ & $6151-00-035$ & $\mathrm{ThO}_{2}$ & $6542-02-020$ & $1099-\mathrm{N}^{(\mathrm{p})}$ & Fo 82 & in & mo 269 & 34 & 0.71 & 40 & 39 & 0.12 & 1.00 & 1.02 & $<8 \times 10^{-6}$ & $1 \times 10^{-6}$ \\
\hline c & $03-5$ & $\mathrm{vc}_{2}$ & $6151-00-035$ & $\mathrm{ThO}_{2}$ & $6542-01-010$ & $\mid 1099-1$ & FO 136 & 55 & MO 269 & 33 & 0.67 & 42 & 36 & 0.13 & 1.03 & 1.06 & $<8 \times 10^{-6}$ & $1 \times 10^{-6}$ \\
\hline D & $04-6$ & $\mathrm{uc}_{2}$ & $6151-00-035$ & $\mathrm{ThO}_{2}^{2}$ & $6542-01-020$ & 1099-1 & Fo 136 & 57 & Mo 269 & 34 & 0.68 & 35 & 36 & 0.14 & 1.04 & 1.12 & $<9 \times 10^{-6}$ & $3 \times 10^{-6}$ \\
\hline$E^{(n)}$ & $05-6$ & $(\mathrm{Th}, \mathrm{V}) \mathrm{O}_{2}$ & $5466-37$ & $\mathrm{ThO}_{2}$ & $6542-02-020$ & 1099-I & FO 147 & -- & MO 269 & 34 & -- & 32 & -- & -. & 1.03 & 2.07 & $<9 \times 10^{-6}$ & $4 \times 10^{-6}$ \\
\hline $\begin{array}{r}\text { Ce11 } 2 \\
A^{(n)}\end{array}$ & $06-5$ & $\mathrm{ve}_{2}$ & $6151-00-045$ & $\mathrm{ThO}_{2}$ & $6542-20-035$ & $\mid 1099-I$ & Fo 147 & $-\cdots$ & so 269 & 32 & - & 37 & -- & -- & 1.05 & 1.11 & $<9 \times 10^{-6}$ & $3 \times 10^{-6}$ \\
\hline B & $07-5$ & $\mathrm{vc}_{2}^{2}$ & $6151-00-035$ & $\mathrm{ThO}_{2}$ & $6542-02-020$ & 1099-I & FO 136 & 56 & \$O 270 & 42 & 0.77 & 28 & 41 & 0.13 & 1.08 & 1.10 & $<9 \times 10^{-6}$ & $3 \times 10^{-6}$ \\
\hline c & $08-13$ & $\mathrm{UC}_{2}$ & $6151-00-035$ & $\mathrm{ThO}_{2}$ & $6562201-010$ & 1099-I & FO 148 & 56 & MO 269 & 31 & 0.65 & 37 & 28 & 0.11 & 1.02 & 1.04 & $<9 \times 10^{-6}$ & $4 \times 10^{-6}$ \\
\hline D & $09-5$ & $\mathrm{uc}_{2}$ & $6151-00-035$ & $\mathrm{ThO}_{2}$ & $6542-01-020$ & Lonza & FO 149 & 57 & мо 267 & 33 & 0.66 & 36 & 31 & 0.12 & 1.02 & 1.04 & $<1 \times 10^{-5}$ & $3 \times 10^{-6}$ \\
\hline $\mathbf{E}^{(\mathrm{n})}$ & $10-5$ & $(\mathrm{Th}, \mathrm{U}) \mathrm{O}_{2}$ & $5466-37$ & $\mathrm{ThO}_{2}$ & $6542-01-010$ & $1099-\mathrm{I}$ & Fo 147 & -- & Mo 269 & ND ${ }^{(q)}$ & -- & 37 & -- & -- & 1.01 & 1.08 & $<1 \times 10^{-5}$ & $6 \times 10^{-6}$ \\
\hline $\begin{array}{r}\text { Ce11 } 5 \\
A^{(n)}\end{array}$ & $16-5$ & $(\mathrm{Th}, \mathrm{U}) \mathrm{O}_{2}$ & $5466-37$ & $\mathrm{ThO}_{2}$ & $6542-01-020$ & 1099-I & Fo 147 & -- & Mo 269 & 35 & -- & 37 & -- & -- & 3.01 & 1.00 & $<6 \times 10^{-6}$ & $4 \times 10^{-6}$ \\
\hline B & $17-5$ & $\mathrm{vC}_{2}$ & $6151-00-035$ & $\mathrm{ThO}_{2}$ & $6542-02-020$ & $1099-\mathrm{N}$ & Fo 82 & 56 & Mo 269 & 34 & 0.71 & 35 & 40 & 0.14 & 1.03 & 1.07 & $<>10^{-6}$ & $2 \times 10^{-6}$ \\
\hline c & $18-6$ & $\mathrm{UC}_{2}$ & $6151-00-035$ & $\mathrm{ThO}_{2}$ & $6542-01-010$ & $1099-I$ & $80 \quad 136$ & 55 & Mo 269 & 30 & 0.69 & 35 & 35 & 0.13 & 1.09 & 1.06 & $<7 \times 10^{-6}$ & $3 \times 10^{-6}$ \\
\hline D & $19-7$ & $u c_{2}$ & $6151-08-015$ & $\mathrm{ThO}_{2}$ & 6542-19-015 & 1099-I & Fo 136 & 56 & Mo 269 & 33 & 0.68 & 42 & 37 & 0.15 & 1.03 & 1.09 & $<>\times 10^{-6}$ & $2 \times 10^{-6}$ \\
\hline$E^{(n)}$ & $20-6$ & $u c_{2}$ & $6151-09-015$ & $\mathrm{ThO}_{2}$ & $6542-09-010$ & $1099-I$ & Fo 147 & - & мо 269 & 32 & -- & 27 & - & - & 1.04 & 1.08 & $<6 \times 10^{-6}$ & $3 \times 10^{-6}$ \\
\hline $\begin{array}{r}\operatorname{Cet12} 6 \\
\quad(n)\end{array}$ & $21-5$ & $\mathrm{uc}_{2}$ & $6151-08-015$ & $\mathrm{ThO}_{2}$ & 6542-19-015 & $1099-\mathrm{I}$ & Fo 147 & -- & MO 269 & 33 & -- & 34 & -- & -- & 9.07 & 1.10 & $<9 \times 10^{-6}$ & $4 \times 10^{-6}$ \\
\hline B & $22-5$ & $\mathrm{wc}_{2}^{2}$ & $6151-09-015$ & $\mathrm{ThO}_{2}^{2}$ & $6542-09-010$ & $1099-I$ & Fo 136 & 56 & M० 269 & 32 & 0.70 & 41 & 38 & 0.14 & 1.06 & 1.09 & $<8 \times 10^{-6}$ & $2 \times 10^{-6}$ \\
\hline c & $23-5$ & $U c_{2}^{*}$ & $6151-00-035$ & $\mathrm{ThO}_{2}^{2}$ & $6542+01-010$ & 1099-I & Fo 136 & 54 & Mo 271 & 30 & 0.57 & 43 & 25 & 0.09 & 1.07 & 1.12 & $2 \times 10^{-3(x)}$ & $2 \approx 10^{-6}$ \\
\hline D & $24-5$ & $\mathrm{wC}_{2}$ & $6151-00-035$ & $\mathrm{ThO}_{2} \mid$ & 6542-02-020 & Lonza & Fo 149 & 56 & ห० 267 & 31 & 0.67 & 40 & 31 & 0.12 & 1.10 & 1.13 & $<9 \times 10^{-6}$ & $6 \times 10^{-6}$ \\
\hline $\mathbb{E}^{(\mathfrak{n})}$ & $25-5$ & $\mathrm{uc}_{2}$ & $6151-00-045$ & $\mathrm{ThO}_{2}$ & $6542-20-035$ & $1099-1$ & po 147 & - & MO 269 & 33 & -- & 36 & - & - & 1.03 & 1.03 & $<1 \times 10^{-5}$ & $3 \times 10^{-6}$ \\
\hline
\end{tabular}

(a) A11 rods are approximately $1.25 \mathrm{~cm}(0.493 \mathrm{fn}$.) in d1ameter by $1.90 \mathrm{~cm}(0.75 \mathrm{tn}$.$) 1ong.$

(b) ${ }_{A 11}$ rods except cell 1, position B and position C, contain TRISO coated carbon 1nert particles (batch number 6351-02-020).

(c) All fissile particles are TRISO coated.

(d) All fertile particles are BISO coated.

(e) Calculated from fired rod and mean particle parameters.

(f) Not meaningful to deternine value for thermocouple rods due to some uncertainty in green and fired rod parameters.

(8) Measurement made on compantion green rod.

(h) Measurement made on companion fired rod.

(1) Determined from metallographic cross section.

(1) Calculated by dividing the coke weight by the initial pitch plus additive weight.

(k) Deterwined by ganta courting both ends of the rod and calculating the ratio of the maximum and mean values.

(1) Determined by hydrolysis test. "భ" denotes the amourt is below the 11 mit of detection of the apparatus. If a detectable quantity, the value 18 corrected for total conversion of tho ${ }_{2}$ to ThC ${ }_{2}$.

(n) Thernocouple rod - center hole [approximately $0.34 \mathrm{~cm}(0.13 \mathrm{ln}$.$) in diameter] through the ent1re length of the rod.$

(o) I atgnifles lapregnated with furfuryi alcohol.

$(p)_{\text {B }}$ algnifies nonimpregnated.

$(\mathrm{q})_{\mathrm{ND}}=$ not determined.

(x) Second rod from this betch had a value of $<9 \times 10^{-6}$. 
TABLL 2-8
DESCRIPTION OF FUEL RODS $(a)$ TESTED IN CAPSULE PI3S

\begin{tabular}{|c|c|c|c|c|c|c|c|c|c|c|c|c|c|c|c|c|c|c|}
\hline \multirow{4}{*}{$\begin{array}{l}\text { Capsule } \\
\text { Position }\end{array}$} & \multirow{4}{*}{$\begin{array}{c}\text { Data } \\
\text { Retrteval } \\
\text { Mutuber } \\
(7161-004-)\end{array}$} & \multicolumn{4}{|c|}{ Coated Particles (b) } & & & \multirow{4}{*}{$\begin{array}{c}\text { Particle } \\
\text { PackIng(e,f) } \\
(z)\end{array}$} & \multirow[b]{4}{*}{$\begin{array}{l}\text { Batch } \\
\text { Number }\end{array}$} & \multirow{4}{*}{$\underset{\text { (wt } \mathrm{X})}{\text { Fuler }}$} & \multirow{4}{*}{$\begin{array}{c}\text { Apparent } \\
\text { Density }(\mathrm{e}, \mathrm{f}) \\
\left(\mathrm{g} / \mathrm{cm}^{3}\right)\end{array}$} & \multirow{4}{*}{$\begin{array}{c}\text { Macro- } \\
\text { porostty }(\mathrm{h}, 1) \\
(\mathrm{z})\end{array}$} & & & \multirow{3}{*}{\multicolumn{2}{|c|}{$\begin{array}{l}\text { Fue l Loading } \\
\text { Uniformlty }(k) \\
\end{array}$}} & \multirow{4}{*}{$\begin{array}{l}\text { Exposed Heavy } \\
\text { Meta1 (g, 1) } \\
\text { (8 heavy meta1) } \\
\text { g heavy meta1) }\end{array}$} & \multirow{4}{*}{$\begin{array}{l}\text { Flasion } \\
\text { Gas } \\
\text { Rejease (m) }\end{array}$} \\
\hline & & \multicolumn{2}{|c|}{ F1salle $e^{(c)}$} & \multicolumn{2}{|c|}{ Fert1le } & \multicolumn{2}{|c|}{ Shim Particles } & & & & & & \multicolumn{2}{|c|}{ Coke Content ${ }^{(f)}$} & & & & \\
\hline & & & Data & & Data & & & & & & & & \multirow{2}{*}{$\underset{(z)}{\text { Yreld }^{\prime}(j)}$} & \multirow{2}{*}{$\left|\begin{array}{c}\text { Density } \\
\left(\mathrm{g} / \mathrm{cm}^{3}\right)^{3}\end{array}\right|$} & & & & \\
\hline & & Type & $\begin{array}{l}\text { Retrieval } \\
\text { Number }\end{array}$ & Type & $\begin{array}{l}\text { Retrieval } \\
\text { Number }\end{array}$ & Type & $\begin{array}{l}\text { Satch } \\
\text { Number }\end{array}$ & & & & & & & & v-235 & Th-232 & & \\
\hline $\operatorname{cec} 11(n)$ & & & & & & & & & & & & & & & & & & \\
\hline & $01-7$ & $\mathrm{vc}_{2}$ & $\mid 6151-00-045$ & $\mathrm{ThO}_{2}$ & $6542-20-035$ & $1099-I^{(0)}$ & Fo 147 & -- & MO 269 & 37 & -- & 31 & -- & - & 1.07 & 1.10 & $<8 \times 10^{-6}$ & $2 \times 10^{-6}$ \\
\hline B & $02-5$ & $\mathrm{vc}_{2}$ & $6151-00-035$ & $\mathrm{ThO}_{2}$ & $6542-02-020$ & $\mid 1099-\mathrm{N}^{(\mathrm{P})}$ & Fo 82 & 56 & หо 269 & 34 & 0.67 & 40 & 36 & 0.12 & 1.07 & 1.07 & $<8 \times 10^{-6}$ & $1 \times 10^{-6}$ \\
\hline $\begin{array}{l}\mathrm{C} \\
\mathrm{D}\end{array}$ & $\begin{array}{l}03-6 \\
04-5\end{array}$ & $\mathrm{UC}_{2}$ & $\left|\begin{array}{r}6151-00-035 \\
6151-00-035\end{array}\right|$ & $\mathrm{ThO}_{2}$ & 6542-01-010 & $1099-\mathrm{I}$ & FO 136 & 55 & Mn 269 & 33 & 0.66 & 42 & 34 & 0.13 & 1.10 & 1.07 & $<8 \times 10^{-6}$ & $1 \times 10^{-6}$ \\
\hline $\mathrm{E}^{(n)}$ & $05-5$ & $(\mathrm{Th}, \mathrm{v}) \mathrm{O}_{2}$ & & $\mathrm{ThO}_{2}$ & $\begin{array}{l}6542-01-020 \\
6542-02-020\end{array}$ & $\mid \begin{array}{l}1099-\mathrm{I} \\
3099-\mathrm{I}\end{array}$ & $\begin{array}{ll}\text { FO } & 136 \\
\text { Fo } & 147\end{array}$ & $\begin{array}{l}57 \\
--\end{array}$ & $\begin{array}{l}\text { MO } 269 \\
\text { MO } 269\end{array}$ & $\begin{array}{l}34 \\
34\end{array}$ & $\begin{array}{l}0.68 \\
--\end{array}$ & $\begin{array}{l}35 \\
32\end{array}$ & $\begin{array}{l}36 \\
\ldots\end{array}$ & 0.14 & $\begin{array}{l}1.11 \\
1.00\end{array}$ & $\begin{array}{l}1.09 \\
1.02\end{array}$ & $\begin{array}{l}9 \times 10^{-6} \\
<9 \times 10^{-6}\end{array}$ & $\begin{array}{l}2 \times 10^{-6} \\
2 \times 10^{-6}\end{array}$ \\
\hline Cell 2 & & & & & & & & & & & & & & & & & & \\
\hline$A^{(n)}$ & $11-6$ & $\mathrm{UC}_{2}$ & $6151-00-045$ & $\mathrm{ThO}_{2}$ & $6542-20-035$ & 1099-I & Fo 148 & -- & MO 269 & 29 & - & 36 & - & -- & 1.02 & 1.08 & $<9 \times 10^{-6}$ & $2 \times 10^{-7}$ \\
\hline B & $12-5$ & $\mathrm{vc}_{2}$ & $6151-09-015$ & $\mathrm{ThO}_{2}$ & 6542-09-010 & 1099-1 & Fo 136 & 55 & MO 269 & 32 & 0.70 & 32 & 35 & 0.14 & 1.02 & 1.07 & $6 \times 10^{-3(q)}$ & $2 \times 10^{-6}$ \\
\hline $\mathrm{c}$ & $13-6$ & $\mathrm{UC}_{2}$ & $6151-00-035$ & $\mathrm{ThO}_{2}$ & $6542-01-010$ & 1099-I & Fo 148 & 53 & мо 271 & 31 & 0.59 & 35 & 25 & 0.10 & 1.10 & 1.10 & $<9 \times 10^{-6}$ & $3 \times 10^{-6}$ \\
\hline$E^{(n)}$ & $14-5$ & $\mathrm{Uc}_{2}$ & $6151-00-035$ & $\mathrm{ThO}_{2}$ & $6542-02-020$ & $1099-\mathrm{N}$ & Fo 82 & 56 & Mo 269 & 36 & 0.69 & 36 & 36 & 0.14 & 1.00 & 1.02 & $<1 \times 10^{-5}$ & $2 \times 10^{-6}$ \\
\hline Cel1 5 & $15-5$ & $\mathrm{UC}_{2}$ & 5151-08-015 & $\mathrm{ThO}_{2}$ & $6542-19-015$ & 1099-I & Fo 147 & -- & Mo 269 & 36 & -- & 26 & -- & -- &, 1.05 & 1.03 & $1 \times 10^{-3}$ & $5 \times 10^{-6}$ \\
\hline$A^{(n)}$ & $16-6$ & $(\mathrm{Th}, \mathrm{W}) \mathrm{O}_{2}$ & $5466-37$ & $\mathrm{ThO}_{2}$ & $6542-01-020$ & $1099-I$ & Fo 147 & -- & Mo 269 & 35 & -- & 37 & -- & -- & 1.02 & 1.01 & $<6 \times 10^{-6}$ & $4 \times 10^{-6}$ \\
\hline B & $17-6$ & $u_{2}$ & $6151-00-035$ & $\mathrm{ThO}_{2}$ & $6542-02-020$ & 1099-N & Fo 82 & 56 & MO 269 & 34 & 0.67 & 35 & 38 & 0.14 & 1.08 & 1.06 & $<7 \times 10^{-6}$ & $1 \times 10^{-5}$ \\
\hline c & $18-5$ & $\mathrm{uc}_{2}^{2}$ & $6151-00-035$ & $\mathrm{ThO}_{2}$ & $6542-01-010$ & $1099-\mathrm{I}$ & Fo 136 & 55 & Mо 269 & 30 & 0.68 & 35 & 35 & 0.13 & 1.07 & 1.10 & $4 \times 10^{-6}$ & $2 \times 10^{-6}$ \\
\hline D & $19-5$ & $\mathrm{uc}_{2}$ & $6151-08-015$ & $\mathrm{ThO}_{2}$ & $6542-19-015$ & |1099-I & Fo 136 & 56 & мо 269 & 33 & 0.73 & 42 & 36 & 0.15 & 1.11 & 1.09 & $87 \times 10^{-6}$ & $3 \times 10^{-6}$ \\
\hline$E^{(n)}$ & $20-5$ & $\mathrm{wC}_{2}$ & $6151-09-015$ & $\mathrm{ThO}_{2}^{2}$ & $6542-09-010$ & $1099-I$ & For 147 & - & мо 269 & 32 & - & 27 & -- & - & 1.09 & 1.11 & $<6 \times 10^{-6}$ & $3 \times 10^{-6}$ \\
\hline $\begin{array}{r}\text { Ce11 } \\
\quad{ }_{A}^{(n)}\end{array}$ & $26-5$ & & $6151-00-035$ & $\mathrm{ThO}_{2}$ & $6542-01-020$ & | א א א & Fo 82 & -- & мо 269 & 33 & $\cdots$ & 40 & -- & 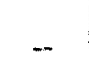 & ? 1.06 & 1.10 & $<9 \times 10^{-6}$ & $3 \times 10^{-6}$ \\
\hline$B$ & $27-5$ & $(\mathrm{Th}, \mathrm{U}) \mathrm{O}_{2}$ & $5466-37$ & $\mathrm{ThO}_{2}^{2}$ & $6542-02-020$ & $1099-1$ & Fo 136 & 55 & мо 269 & 32 & 0.71 & 29 & 35 & 0.15 & 1.00 & 1.08 & $<8 \times 10^{-6}$ & $1 \times 10^{-6}$ \\
\hline c & $28-13$ & $\mathrm{uc}_{2}$ & $6151-00-035$ & $\mathrm{ThO}_{2} \mathrm{~A}$ & $6542-01-010$ & $1099-\mathrm{I}$ & Fo 148 & 55 & 269 & 29 & 0.64 & 38 & 31 & 0.12 & 1.03 & 9.09 & $<B \times 10^{-6}$ & $4 \times 10^{-6}$ \\
\hline D & $29-5$ & $u c_{2}^{2}$ & $6151-00-035$ & $\mathrm{ThO}_{2}$ & $6542-01-020$ & 1099-I & Fo 136 & 57 & мо 270 & 44 & 0.79 & 32 & 39 & 0.13 & 1.06 & 1.11 & $<9 \times 10^{-6}$ & $1 \times 10^{-6}$ \\
\hline$f(n)$ & $30-5$ & $\mathrm{uc}_{2}$ & $6151-00-0<5$ & $\mathrm{ThO}_{2}^{2}$ & $6542-20-035$ & $1099-\mathrm{I}$ & Fo 147 & -- & Mo 269 & $\mathrm{ND}^{(\mathrm{r})}$ & -- & 30 & -- & - & 1.03 & 1.10 & $<1 \times 10^{-5}$ & $2 \times 10^{-6}$ \\
\hline
\end{tabular}

(a) All rods are approximately $1.25 \mathrm{~cm}(0.493 \mathrm{kn}$.$) in diameter by 1.90 \mathrm{~cm}(0.75 \mathrm{ln}$.) Iong.

(b) All rods except cell 1, position B and position C, contain TRISO coated carbon 1nett particles (batch number 6351-02-020).

(c) All fissile particles are TRISO coated.

(d) All fertile particles are BISO coated.

(e) Calculated from fired rod and mean particle parameters.

(f) Not meaningful to deternine value for thermocouple rods tue to some uncertainty in green and fired rod parameters.

(g) Measurement made on companion green rod.

(h) Measurement made on companion fired rod.

(i) Deternined from metallographic cross section.

(1) Calculated by dividing the coke weight by the 1nitial pitch pius additive weight.

(k) Determined by gamma counting both ends of the rod and calcuiating the ratio of maximum and mean values.

(1) Determined by hydrolysis test. "<" denotes the amount is below the 11 mit of detection of the spparatus. If a detectable quantity, the value 18 corrected for total co iverston of Tho 2 to The ${ }_{2}$.

(im)

(n) Thermocouple rod - center hole lapproximately 0.30

mately $0.34 \mathrm{~cm}(0.13 \mathrm{fn}$.$) in dameterl through the entire length of the rod.$

(0) I signif 1es impregnated with furfuryl alcoho1.

(p) $\mathrm{N}$ signtf tes nonimpregnated.

(q) Second rod from this batch had a value of $<9 \times 10^{-6}$.

(r) $\mathrm{ND}=$ not determined. 


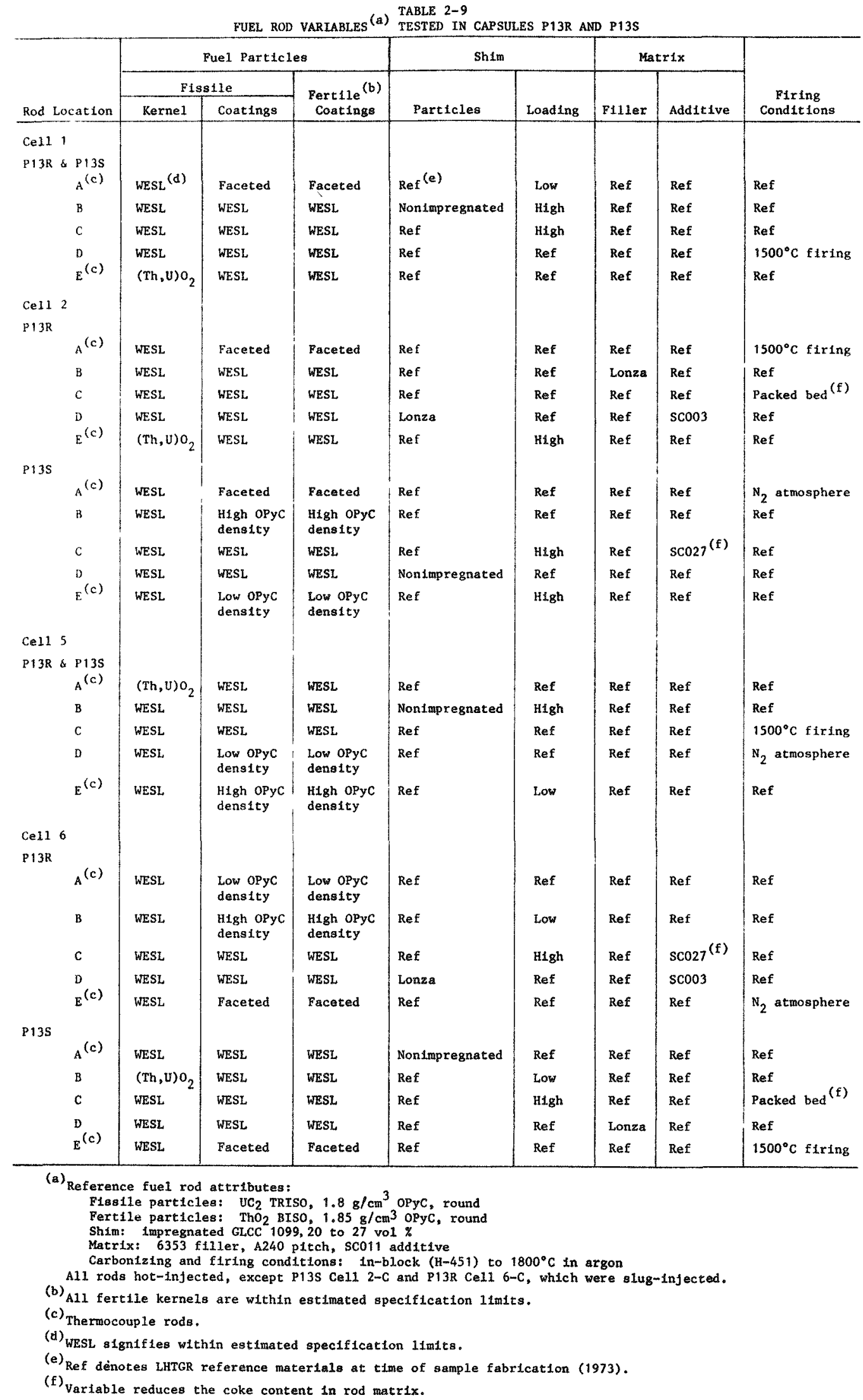




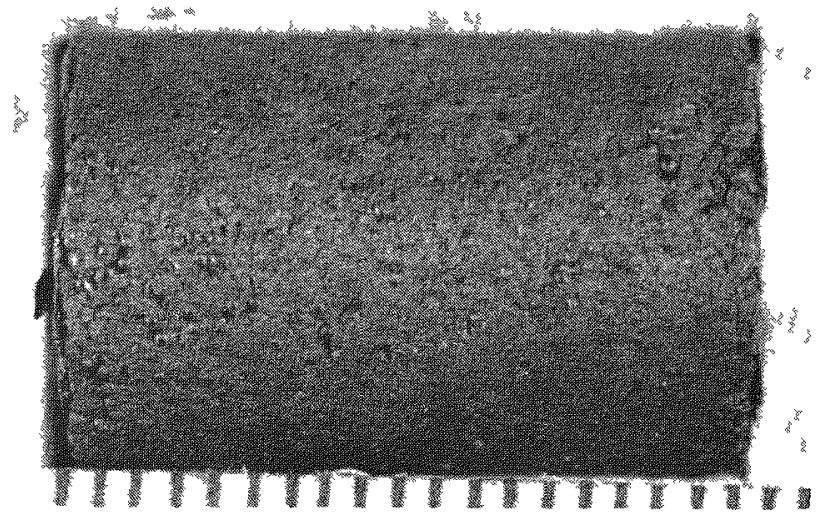

M40322-1

(a)

$$
0.5 \mathrm{~cm}
$$

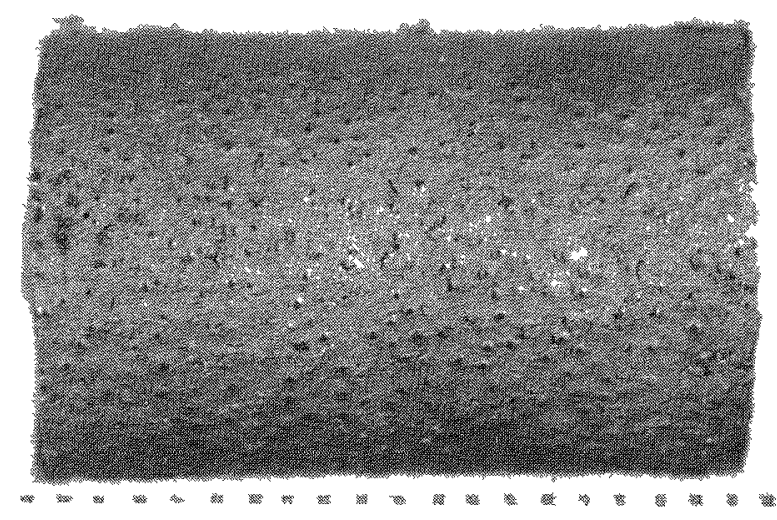

M40418-2

(b)

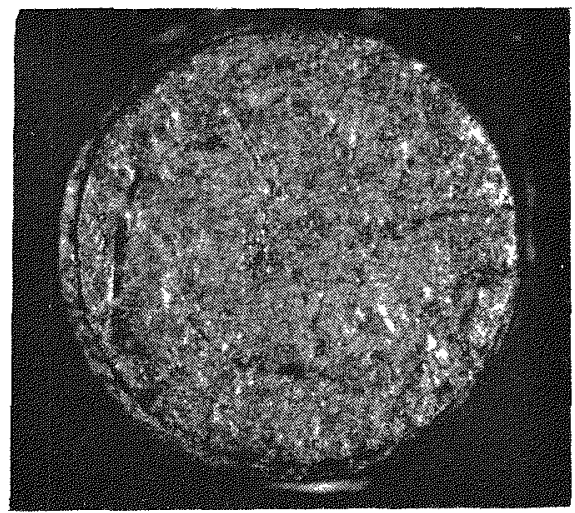

M40322-4

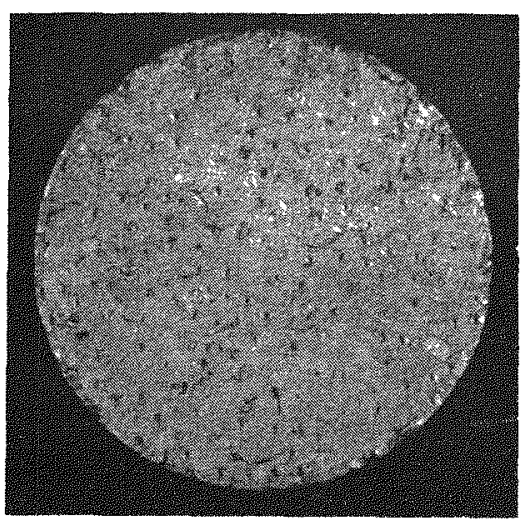

M40418-3

Fig. 2-1. Representative preirradiation photomicrographs of fuel rods tested in capsules $\mathrm{P} 13 \mathrm{R}$ and P13S: (a) fired at $1800^{\circ} \mathrm{C}$ in argon, which is the reference heat treatment for LHTGR fuel production, and (b) slug-injected fuel rod fired in packed bed of alumina 


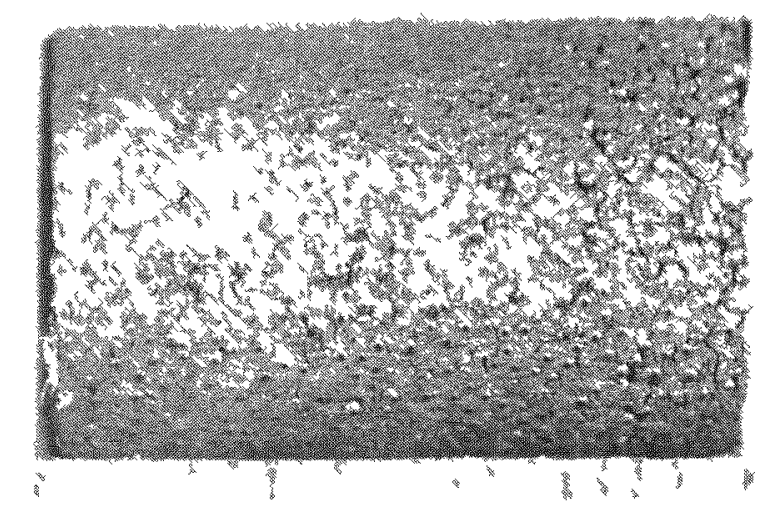

M40347-3 (a)

\section{$0.5 \mathrm{~cm}$}

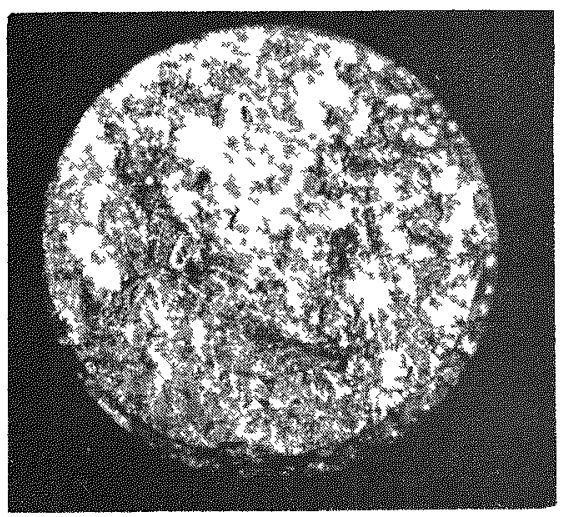

M40347-2
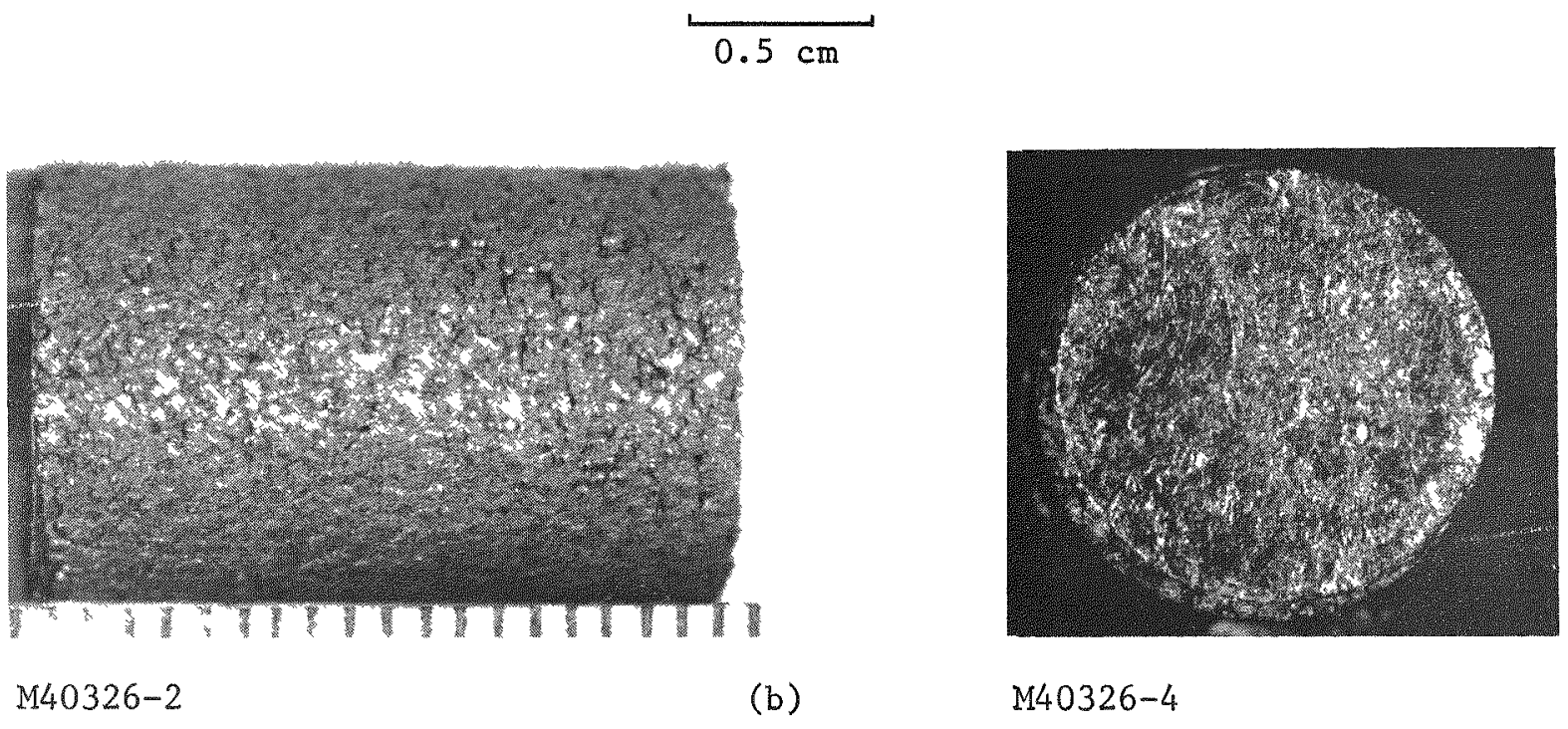

(b)

M40326-4

Fig. 2-2. Representative preirradiation photomicrographs of fuel rods tested in capsules $\mathrm{P} 13 \mathrm{R}$ and P13S: (a) fired at $1500^{\circ} \mathrm{C}$ and (b) fired in a $\mathrm{N}_{2}$ atmosphere. The Grafoil discs placed on the ends of each rod to prevent sticking during firing in $\mathrm{H}-451$ graphite tubes remained on the rods. 


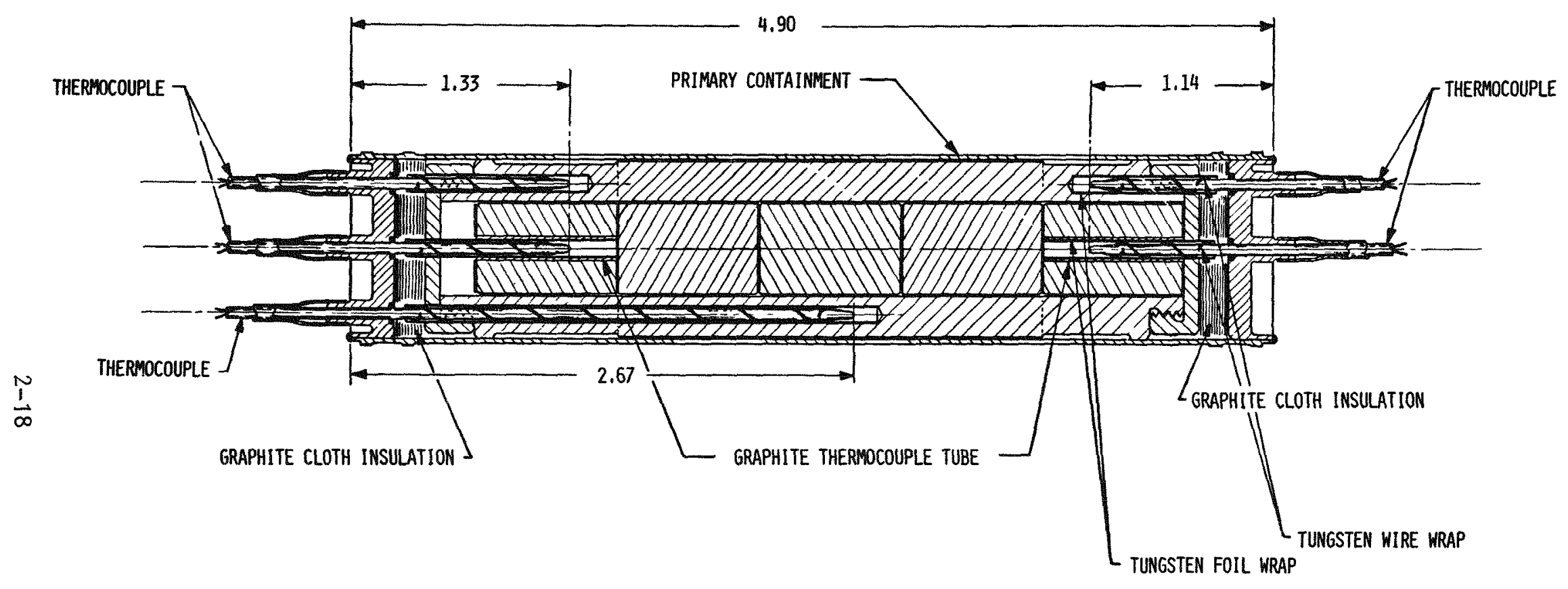

(NOTE: ALL DIMENSIONS ARE IN INCHES)

Fig. 2-3. Schematic showing design of fuel rod cells 1,2, 5, and 6 of capsules P13R and P13S 


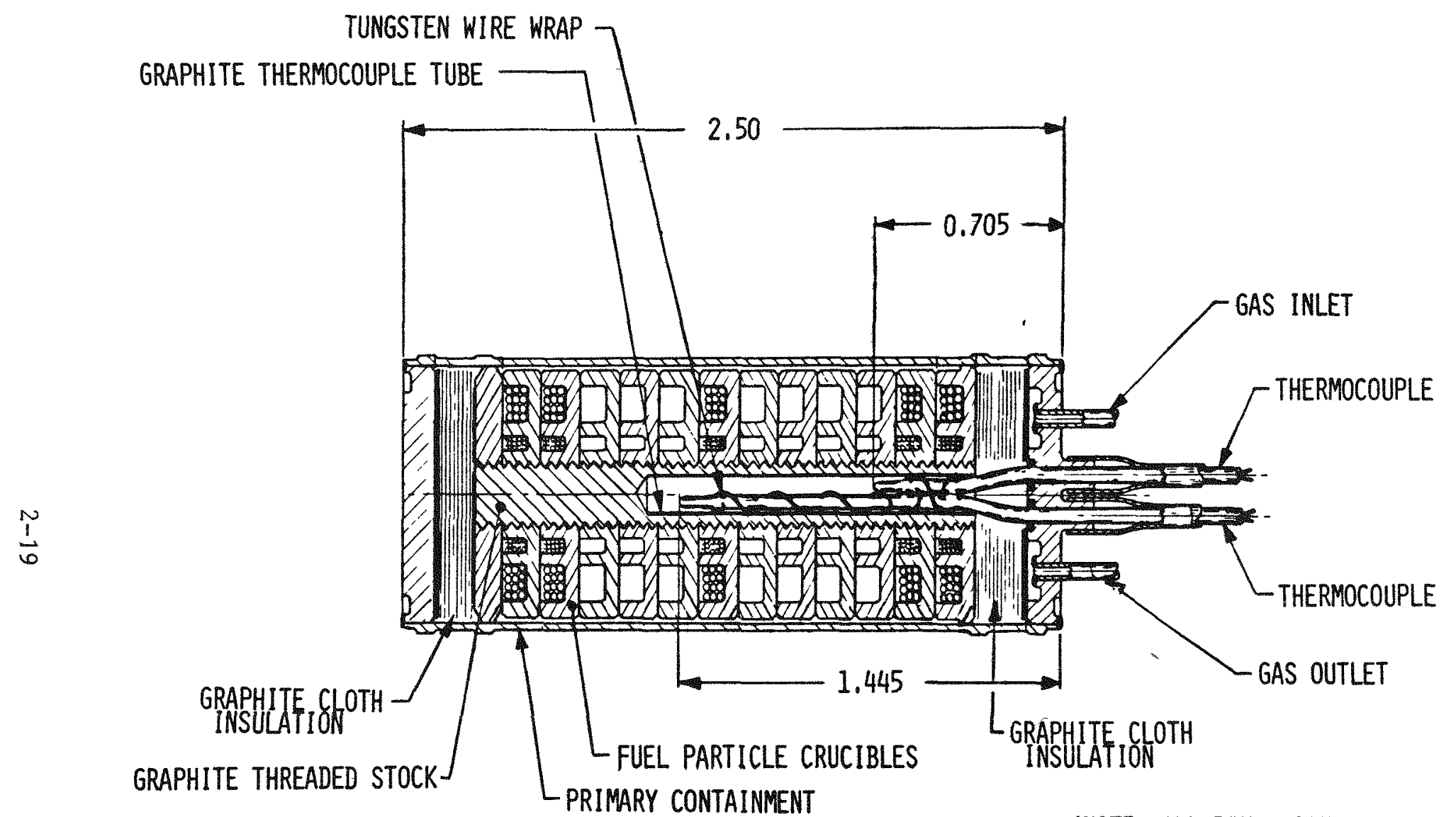

(NOTE: ALL DIMENSIONS ARE IN INCHES)

Fig. 2-4. Schematic showing design of unbonded particle ce11s 3 and 4 of capsules P13R and P13S 


\section{CAPSULE OPERATION AND EVALUATION}

\subsection{OPERATING HISTORY}

Capsules $\mathrm{P} 13 \mathrm{R}$ and $\mathrm{P} 13 \mathrm{~S}$ were inserted into the $\mathrm{E}-7$ position of the General Electric Test Reactor (GETR) at Pleasanton, California on December 14, 1973. Irradiation was completed on October 31, 1974 after 258 days of full power operation ( $50 \mathrm{MW}$ ). Peak fast neutron fluences of $12.4 \times 10^{21}$ and $12.1 \times 10^{21} \mathrm{n} / \mathrm{cm}^{2}$ ( $\mathrm{E}>0.18 \mathrm{MeV}$ ) ${ }_{\text {HTGR }}$ were reached by capsules $\mathrm{P} 13 \mathrm{R}$ and $\mathrm{P} 13 \mathrm{~S}$, respectively. The irradiation proceeded without incident for both capsules.

The capsules were irradiated for a total of 24 cycles. Each cycle was characterized by a gradual shift in the axial neutron flux profile resulting from reactor control rod withdrawal patterns. This necessitated periodic adjustments of the sweep gas mixtures to maintain the proper design temperature for each cell. Figure 3-1 shows the thermal flux spectrum as a function of the GETR control rod bank position. As can be seen in this figure, the greatest changes in flux levels occur in regions near the top and bottom of the reactor core. The cells in these regions (cells 1 and 6 ) required frequent gas-mixture adjustments during each cycle in order to maintain the desired design fuel temperature. The larger flux level shifts near the top of the core made it impossible to operate ce11 6 of both P13R and $\mathrm{P} 13 \mathrm{~S}$ at a constant temperature throughout irradiation. Fuel rods in these cells reached their design temperature only a fraction of the time, and as a result the time-average temperatures in cell 6 of both capsules were 1ow.

All cells in both capsules operated at a constant temperature, except ce11 6 in both P13R and P13S (for the above-mentioned reasons) and cel1 1 
of P13S. This latter cell was designed for cyclic operation to simulate extreme thermal transient conditions resulting from load-following transients in an HTGR. Immediately following each GETR refueling startup and after the temperatures were stabilized at the nominal design value, the temperature was increased by approximately $400^{\circ} \mathrm{C}$. The thermal transient test was performed at this point in the reactor cycle to take advantage of the high flux levels and hence high fuel power densities. The thermal cycle was accomplished in a series of steps of $100^{\circ} \mathrm{C}$ over a 20 -min period. The peak fuel centerline temperature was held at $\sim 1600^{\circ} \mathrm{C}$ for $1 \mathrm{hr}$ and then decreased to $1075^{\circ} \mathrm{C}$ over a period of $2 \mathrm{hr}$, using $100^{\circ} \mathrm{C}$ steps and spending approximately $30 \mathrm{~min}$ at each step. Figure 3-2 depicts the design conditions for thermal cycling fuel in cel1 1 of P13S. Measurements of fission gas release (R/B), gross gas activity, gas flow, time, and temperature were recorded during each thermal cycle test. Some problems were encountered in obtaining a fission gas release measurement at the peak of the thermal excursion during the latter part of the irradiation test because of the high activity levels (see Section 3.2).

Fuel temperatures were controlled with the aid of thermocouples which were located in each of the cel1s. Temperature control set point adjustments were made periodically to account for irradiation-induced emf drift in the tungsten-rhenium ( $\mathrm{W}-3 \% \mathrm{Re} / \mathrm{W}-25 \% \mathrm{Re}$ ) thermocouples. During the initial part of the irradiation, these corrections were made using empirical data derived from earlier capsule experiments (Refs. 3-1, 3-2). During the course of the irradiation, tests were conducted to compare measured and calculated temperatures. These tests were conducted once every GETR fuel cycle at a constant rod bank position, i.e., constant fuel power. Briefly, the test procedure was as follows. Once every fuel cycle at a 25-in. rod bank position, helium gas was flowed through the cells in a $100 \%$ mixture and when temperatures stabilized, they were recorded. This was repeated for $50 \%$ and $10 \%$ helium mixtures (neon being the other gas). Some problems were encountered in the test procedure in the initial stages, so only six cycles of data were used at the beginning of the study.

The temperature and design GETR thermal fluence data from the test runs were processed by GA's UNIVAC 1108 computer. The computer program 
was used to fit the temperature versus fluence data by the method of least squares to first-, second-, and third-order polynomials of the following form:

$$
\mathrm{T}_{i}=\mathrm{B}_{1}+\mathrm{B}_{2} \mathrm{~F}_{i}+\mathrm{B}_{3} \mathrm{~F}_{i}^{2}+\mathrm{B}_{4} \mathrm{~F}_{i}^{3} \quad i=1, \mathrm{~N} \text {, }
$$

where $B^{\prime} s=$ coefficients to be estimated from the data,

$$
\begin{aligned}
& F_{i}=i^{\text {th }} \text { fluence at time of test, } \\
& T_{i}=\text { thermocouple reading at the } i^{\text {th }} \text { fluence, } \\
& N=\text { number of data points. }
\end{aligned}
$$

Decalibration factors derived from these data were used to correct the W/Re drift during the last 90 days of irradiation. These curves and their uncertainty bands are shown in Figs. 3-3 through 3-5.

Periodic gas samples were taken from the primary gas exhaust lines of each cell and analyzed for gaseous fission products. These data were used to determine the fission gas release of the fuel in the six separate cells for each capsule, as discussed in Section 3.2 .

A summary of the operating history of each cell is presented in Figs. 3-6 through 3-18. These figures show fission gas release (Kr-85m), reference thermocouple control temperature, neutron fluence, burnup, reactor power, and rod bank profiles throughout the test. More detailed discussions of the first four parameters are presented in the following sections.

\subsection{IN-PILE FISSION GAS RELEASE MEASUREMENTS}

The inert sweep gas used to control fuel temperatures in each cell was periodically sampled to monitor the fission gas release from the fuel specimens during irradiation. Six short-lived gaseous fission product isotopes $(\mathrm{Kr}-85 \mathrm{~m}, \mathrm{Kr}-87, \mathrm{Kr}-88, \mathrm{Kr}-89, \mathrm{Xe}-135$, and $\mathrm{Xe}-138)$ were measured. These isotopes were of primary interest because they constitute the main source 
of gaseous activity released from failed fuel and thereby afford a direct means of determining fuel performance during irradiation.

Approximately 40 measurements were made on each of the six cells, with a total of about 240 measurements for each capsule. Samples were collected daily for the first week of the test and were monitored weekly for the remainder of the test. Additional samples were taken during the thermal cycle tests for cell 1 of P13S. The fission gas release was monitored just before and approximately $2 \mathrm{hr}$ after each thermal cycle. Samples were also obtained at the peak of the cycle, except during the last few cycles. The total gaseous activity of these samples exceeded permissible handling levels and made it impractical to continue making these particular measurements.

The procedure for taking gas samples for the $R / B$ determinations for the GETR was a conventional one similar to that practiced at other reactor sites. The sampling station consisted of a series of shunt lines which allowed the exhaust gas from any of the cells to flow through a line containing a calibrated length of tubing. The tubing was of known volume and could be isolated from the flow system at any time by a set of solenoid valves. The exhaust gas from the cells was normally purged through this calibrated section for 30 min prior to sampling to ensure that the system reached equilibrium. After the flow through the tube was shut off, the entire gas sample was withdrawn into an evacuated vial of special geometry for gamma counting using a Ge(Li) detector coupled with a multichannel analyzer. A standard decay time of $15 \mathrm{~min}$ was used to permit transport of the gas samples to the counting room. The daughter product, $\mathrm{Rb}-89$, was used for determining the yield of the short-1ived $\mathrm{Kr}-89$ isotope, and the longer-1ived isotopes, $\mathrm{Kr}-85 \mathrm{~m}, \mathrm{Kr}-87, \mathrm{Kr}-88, \mathrm{Xe}-135$, and $\mathrm{Xe}-138$, were directly counted. Absolute quantities of all isotopes were calculated to determine the amount of gas released at the surface of the samples. This was accomplished by knowing the flow rates of the sweep gas from each cell to the sampling station. Calibrated Hasting flowmeters were used to monitor the flow rates of the sweep gas entering the capsule. 
The resulting $R / B$ data for each of the six isotopes monitored throughout the irradiation period are presented in Figs. 3-19 and 3-20. The broad scatter in some of the data results from variations in reactor operating parameters and the inherent experimental error in making the fission gas release measurements. The gaseous releases showed an upward trend during irradiation, but the $R / B$ values remained within expected levels for the fluence-temperature conditions of the irradiation.

\subsection{ANALYSIS OF FLUX DOSIMETERS}

Dosimeter wires of $\mathrm{V}-0.216 \% \mathrm{Co}, \mathrm{V}-0.522 \% \mathrm{Fe}$, and pure $\mathrm{Fe}$ were employed in capsules P13R and P13S to monitor long-term thermal $(2200 \mathrm{~m} / \mathrm{sec})$ and fast $(E>0.18 \mathrm{MeV}$ ) neutron fluences. The wires were encapsulated in quartz ampoules and positioned along the capsules at seven axial locations at $0^{\circ}$ and $180^{\circ}$ radial orientations. In addition to the standard dosimetry wires, six ampoules containing purified $\mathrm{Mn}-54$ were employed to evaluate the Mn-54 transmutation cross section.

After removal from the capsule, each wire was cleaned and weighed. The gamma-ray spectrum of each wire was analyzed with a $\mathrm{Ge}(\mathrm{Li})$ detector and a Sigma 2 4096-channel computer analyzer. Due to the high radiation levels of the $\mathrm{Fe}$ and $\mathrm{V}$-Co dosimetry wires, it was necessary to dissolve and aliquot them prior to gamma-ray spectrometry analysis. Thermal and fast neutron fluxes and fluences were calculated for each axial position with the DOSE-1 computer program using the gamma-ray spectrometry analyses of the dosimeter wires. A third-order polynominal fit was made through the data points to obtain the axial thermal and fast neutron flux and fluence profiles shown in Figs. 3-21 through 3-24. A cobalt cross section of 36.8 barns $(2200 \mathrm{~m} / \mathrm{sec})$ was used to calculate the thermal fluences and a burnout cross section of $11.6 \pm 2.0$ barns $(696 \pm 120 \mathrm{~m} / \mathrm{sec})$ for $\mathrm{Mn}-54$, which was determined from analysis of the purified Mn-54 ampoules, was used to evaluate the fast neutron fluence levels.

Average values of the GETR fast and thermal neutron flux and fluence for each fuel specimen are given in Tables 3-1 through 3-4. These data were 
obtained from the flux and fluence profiles determined for the $0^{\circ}$ and $180^{\circ}$ capsule orientations. The neutron spectra of the E-7 position of the GETR experimental reactor and a typical HTGR are different, especially in the 50 - to $180-\mathrm{keV}$ range. To be consistent with HTGR design studies, the fast fluence data were converted to equivalent fast fluences in an HTGR environment $\left[(E>0.18 \mathrm{MeV})_{\mathrm{HTGR}}\right]$ using the following relationship (Ref. 3-3):

$$
\Phi(E>0.18 \mathrm{MeV})_{\mathrm{HTGR}}=0.89 \Phi \quad(\mathrm{E}>0.18 \mathrm{MeV})_{\mathrm{GETR}} .
$$

The equivalent fast neutron fluence in an HTGR environment for each fuel specimen is given in Tables 3-1 through 3-4.

In order to verify the dosimetry analysis, four $\mathrm{V}-\mathrm{Fe}$ dosimeter wires were submitted to Oak Ridge National Laboratory (ORNL) for analysis. The wires were weighed and the gamma-ray spectrum was analyzed to obtain the Mn-54 activity of each wire. The fast fluences required to produce the measured Mn-54 activities were then calculated using an ORNL computer code.

The weights of the wires obtained at GA and at ORNL are given in Table 3-5. With the exception of wire C-1632, the agreement in the measured weights was excellent. The weight determined by GA for this wire was selected as the preferred weight. The basis for this decision was that during initial fabrication, four 1-in. lengths had been cut simultaneously from parallel wires and their range of weights was 16.11 to $16.16 \mathrm{mg}$. Wire C-1632 was one of the four wires cut, and the ORNL weight of $16.639 \mathrm{mg}$ was not consistent with the other weights.

The gamma-ray spectrum of each wire was analyzed to obtain the $\mathrm{Mn}-54$ activity by four separate analytical chemistry groups at ORNL. The independent1y measured activities (in dps/mg) were corrected to June 5, 1975 and are given in Table 3-6. The average values are reported and the limits mark the range of values obtained within the four chemistry groups. The activities in $\mu \mathrm{Ci} / \mathrm{mg}$, corrected to October 31,1974 , are also given in 
Table 3-6. The activity reported by ORNL for wire C-1632 was normalized to the correct wire weight.

The activities were determined by GA for two different cases. Case I represents the original analyses completed for capsules $\mathrm{P} 13 \mathrm{R}$ and $\mathrm{P} 13 \mathrm{~S}$ in March 1975. The Sigma 2 computer/analyzer at GA was recalibrated in January 1976, which resulted in a reduction of approximately $6 \%$ in the computed activities. These Case II activities were still $\sim 4$ to $7 \%$ higher than the average values reported by ORNL. There are several sources of error in these analyses. It should be recognized that NBS standards have a total uncertainty of 2 to $3 \%$; also there is a 1 to $2 \%$ added error from preparation as Sigma 2 standards. Other sources of error include counting statistics for each wire (1 to $2 \%$ ) and a weighing error $(\leq 1 \%)$. Therefore, for these measurements the maximum total uncertainty would be $24.2 \%$. A similar uncertainty would be associated with the ORNL values.

The fast fluences required to produce the measured $\mathrm{Mn}-54$ activities in each wire are given in Table 3-7. The fast fluences calculated using the GA DOSE 1 computer program and the Mn-54 activities measured by ORNL were $\sim 2$ to $4 \%$ higher than those calculated by ORNL. This difference is slight and probably resulted from several sources, including: (1) uncertainties in the thermal fluence values used to correct for the iron and manganese burnout, (2) slight differences in the capsule power history used in the calculations, and (3) differences introduced by rounding off numerical values.

The differences between the fluences calculated by GA for Case I and Case II resulted from the Sigma 2 recalibration correction applied to the measured activities, as discussed previously. The fluences determined by GA for Case II are 25 to $7 \%$ greater than the fluences determined using the Mn-54 activities measured at ORNL, which is the result of differences in the activities measured at the two laboratories, as discussed previously. 
The neutron fluences given in Tables 3-1 through 3-4 and subsequently used in the analyses of the fuel specimens were determined using the Case I Mn-54 activities and are therefore high by $\sim 6 \%$.

\subsection{HEAVY METAL BURNUP ANALYSIS}

Heavy metal burnup is a measure of the total fissions which have occurred in a fuel sample during irradiation and is proportional to the amount of fission products generated. Heavy metal burnup analysis of capsules P13R and P13S was conducted by two techniques: (1) wet chemical analysis and mass spectroscopy and (2) calculations based on gamma-emitting fission product inventory and capsule irradiation history.

Determination of heavy metal burnups by chemical analysis was conducted in accordance with ASTM Procedure E-495-73T, "Spectrophotometric Determination of Fission Zirconium in Irradiated Nuclear Fuels;" ASTM Procedure E-244-69, "Atom Percent Fission in Uranium and Plutonium Fuel (Mass Spectrometric Method);" and General Atomic Procedure ACD-RC-001, "Atom Percent Fission in Fissile and Fertile Fuel Particles." Prior to chemical analysis all fuel particles were cleaned to remove external contamination. The gamma-ray spectrum of the individual fuel particles was analyzed to ensure that the fuel particles had not failed during irradiation, thus releasing a portion of their fission product inventory. The fuel particles were then crushed and dissolved in a perchloric - nitric acid solution. These solutions, which contained zirconium, uranium, and thorium in a strongly acidic chloride medium, were separated from plutonium and fission products by an anion exchange separation. Each fraction (Th, $\mathrm{Zr}$, and $U$ ) was determined spectrophotometrical1y by the absorbance of its respective Arsenazo-III complex.

Total heavy metal atom percent fissions (\% FIMA) were calculated from the results of the chemical analysis using the following equations:

$$
\% \text { FIMA }(U-235)=\frac{F}{U_{0}} \times 100=\frac{F}{U_{R}+F} \times 100
$$




$$
\text { \% FIMA }(U-233)=\frac{F}{T h_{0}} \times 100=\frac{F}{T h_{R}+U_{R}+F} \times 100,
$$

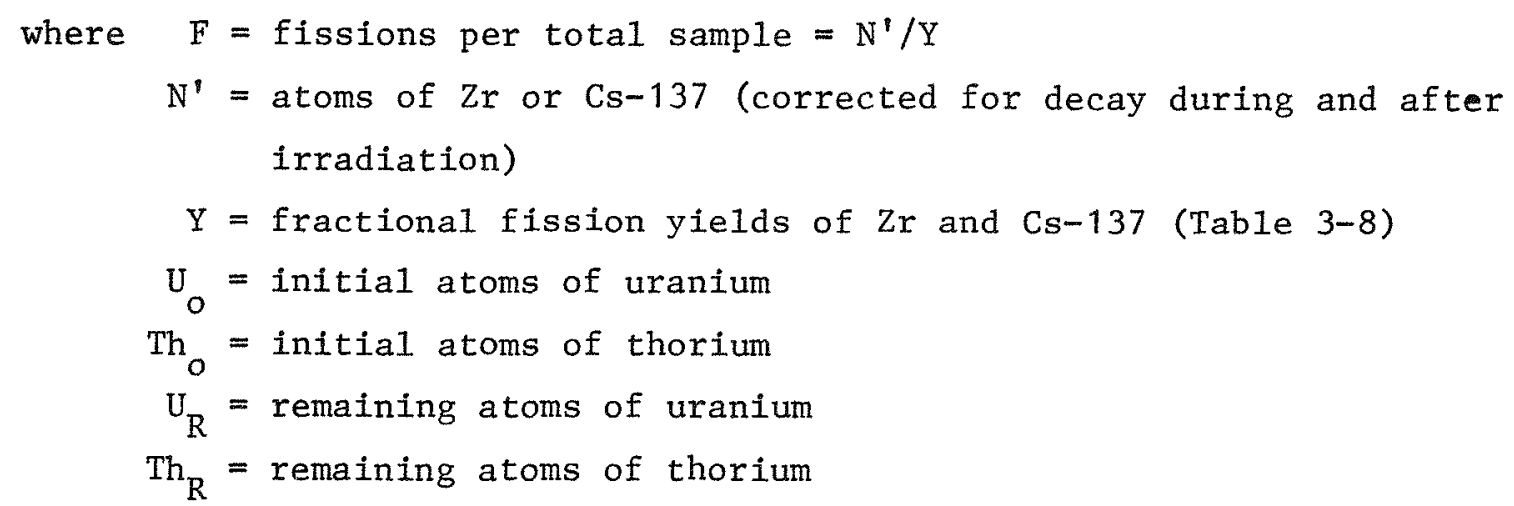

The heavy metal burnup values determined from the wet chemical analysis using Eqs. 3-2 and 3-3 are shown in Table 3-9 under the headings\% FIMA Fiss $\mathrm{Zr}$ and \% FIMA Fiss Cs-137.

In addition to using cesium and zirconium as fission product monitors, fissile particle burnups were also determined by a mass spectrometric uranium isotopic analysis method. This method measures burnup through changes in uranium isotopic composition and can only be applied to fuel particles that do not contain thorium before irradiation. The uranium isotopic compositions of the irradiated fissile, fertile, and reference archive fissile particle samples determined by mass spectrometry are given in Tables 3-10 and 3-11. The fissile particle burnups were calculated from the data given in Table 3-10 in accordance with ASTM Procedure E244-69, and the results are given in Table 3-9 for each of the three cases. A capture-to-fission cross-section ratio for U-235 of 0.1875 was used in the mass spectrometric calculations. These cross sections satisfied the FISSIN computer program discussed below.

The heavy metal burnup of each sample irradiated in capsules P13R and P13S was calculated using the Sigma 2 FISSIN computer code. This program calculates burnup for both U-235 and thorium (U-233) and the weight of uranium and thorium at the beginning of each reactor cycle, as well as the uranium isotopic inventory at the end of irradiation. Required inputs are 
reactor power history, average thermal fluences, initial uranium and thorium loadings, macroscopic cross sections, and fission yields. The cross sections used in the program were adjusted relative to the thermal fluence measured by the cobalt dosimetry in the GETR (see Section 3.3). The burnups calculated for the five particle samples using the FISSIN computer program are given in Table 3-9. Heavy metal burnup values were calculated for all fuel specimens using the FISSIN computer program and are presented in the following sections of this report.

The uranium isotopic compositions of the fissile and fertile samples determined by mass spectrometry and the Sigma 2 FISSIN computer code are compared in Table 3-12. Based on the results from these burnup analyses (Tables 3-9 and 3-12), wet chemistry burnup measurements of high burnup fissile particles overestimate the burnup by approximately 5\%. At lower burnups the error would be less. This overestimation occurred because smal1 amounts of U-236 and U-238 were transmuted to neptunium and plutonium and therefore were not recovered during the anion exchange separation. Modification of the chemistry procedure is planned to permit recovery and analysis of these transuranic species.

To obtain a comparison between laboratories, five particle samples were also submitted to ORNL for burnup analysis. Burnups were determined by wet chemistry in accordance with ASTM Procedure E-495-73T and by isotope dilution mass spectrometry (IDMS).

Two particles from each sample were analyzed separately using both techniques. Each particle was examined visually to ensure the coating was intact, and the $\mathrm{Zr}-95$ activity was measured by gamma-counting with a $\mathrm{Ge}(\mathrm{Li})$ detector. The particle was then dissolved in a $\mathrm{HNO}_{3}-\mathrm{HF}$ solution at $275^{\circ} \mathrm{C}$ using a Teflon-1ined steel container. After dissolution was effected, the solution was diluted and an aliquot was removed for IDMS measurements. The remaining solution was analyzed for $\mathrm{Zr}$, Th, and $\mathrm{U}$ using wet chemistry techniques. 
Thorium and uranium were analyzed by IDMS; however, zirconium activity could not be determined due to poor sensitivity. In the IDMS method, the total quantities of $T h$ and $U$ were measured by a double spike isotope dilution technique. Enriched Th-230 and U-238 were used as tracers. After the sample was dissolved, an aliquot was measured for isotopic composition; a second measured aliquot was then equilibrated with a double spike which contained known amounts of Th-230 and U-238. The newly established ratios were measured, and these ratios were then used to calculate the $T h$ and $U$ concentrations of the sample solution.

The above methods of analyses were evaluated using a standard solution which contained $2.32 \mu \mathrm{g} \mathrm{Zr} / \mathrm{ml}, 400 \mu \mathrm{g} \mathrm{Th} / \mathrm{ml}$, and $58.11 \mu \mathrm{g} \mathrm{U} / \mathrm{ml}$ in $4 \mathrm{M} \mathrm{HNO}_{3^{\circ}}$. An aliquot of the standard solution was analyzed in parallel with each particle sample analysis. The analysis of the standard solution included treatment in the Teflon-lined dissolution apparatus.

The precision and accuracy data obtained for the standard solution are presented in Table 3-13. Based on the $\mathrm{Zr}$ data, it was concluded that the precision and accuracy of the burnup measurements were $\pm 5.0 \%$ and the precision and accuracy of the Th and $U$ measurements by the wet chemistry or IDMS method were $\pm 2.0 \%$ 。

The uranium isotopic compositions determined for the fissile and fertile particles are given in Table 3-14. These data are in good agreement with the isotopic composition (atom \%) determined at GA (see Tables 3-10 and 3-11). The results of the heavy metal burnup analyses conducted using both techniques are given in Table 3-15. These data are in good agreement with the burnup values obtained at GA (Table 3-9).

Heavy metal burnup values reported for all fuel specimens in the following sections of this report were calculated using the GA FISSIN computer program. The fission and capture cross sections used in this program were adjusted relative to the thermal fluences measured for 
capsules $\mathrm{P} 13 \mathrm{R}$ and $\mathrm{P} 13 \mathrm{~S}$ using cobalt dosimetry. These burnup values were in good agreement with the values measured on individual fuel particles at both ORNL and GA by wet chemical analysis.

\subsection{THERMAL ANALYSIS}

One of the most important parameters influencing fuel irradiation behavior is the thermal environment of the fuel. Therefore, to evaluate fuel performance a good knowledge of the fuel's operating temperature history is essential. For this reason temperature profiles in capsules P13R and P13S were extensively monitored by thermocouples. However, difficulties arose in deducing fuel temperatures because the high-temperature thermocouples ( $W / R e)$ decalibrate as a function of neutron fluence. Furthermore, in a number of cases the fuel thermocouples failed during the irradiation. To better estimate the fuel temperatures, detailed numerical analyses were used to predict thermal histories for fuel rods and unbonded particle samples in these two capsules. In these analyses computer codes were used which take into account material geometry and properties, reactor operating parameters, irradiation-induced property changes, and the capsule's operational mode.

Capsule operating temperatures below $1100^{\circ} \mathrm{C}$ in unfueled regions were measured with Chromel-Alume1 (C/A) thermocouples. These thermocouples are not affected by the reactor's neutron environment (Ref. 3-1). Therefore, the C/A temperature measurements provide the bases for comparison with calculated values. Fuel and other high-temperature regions $\left(\mathrm{T}>1100^{\circ} \mathrm{C}\right)$ were monitored with tungsten/rhenium ( $\mathrm{W}-3 \% \operatorname{Re} / \mathrm{W}-25 \% \operatorname{Re}$ ) thermocouples. These thermocouples undergo irradiation-induced drift resulting from alloy compositional changes caused by transmutations in both metals. Empirical data are available to account for this emf drift; however, relatively large uncertainties are currently associated with the correction factors. One of the primary objectives of the thermal analysis was to more accurately predict irradiation-induced emf changes in the $W / \operatorname{Re}$ thermocouples. 


\subsubsection{Fuel Rod Temperatures}

Detailed TAC-2D models for each fuel rod cell were used to calculate beginning-of-1ife (BOL, first day of operation) and end-of-1ife (EOL, 258th day of operation) temperature profiles. All calculated fuel and graphite temperatures could be matched with the measured temperatures within $\pm 60^{\circ}$ at BOL and EOL except for cell 1 in both capsules, as shown in Figs. 3-25 and 3-26. End-of-Iife $W /$ Re thermocouple temperatures were corrected to account for emf drift according to procedures given in Section 3.1 .

To obtain agreement between measured and predicted values, a number of modifications had to be made to the data used in the calculations. Most of these changes were of the nature of refining the input data. However, the most significant problem in the initial calculations was the inability to obtain agreement with the measured primary containment temperature. In almost all cases, the calculated values were about $200^{\circ} \mathrm{C}$ higher than the measured values. This discrepancy apparently stems from uncertainties in the gap between the secondary containment and the aluminum thermal bond (see Section 2.3). Primary containment temperatures were found to be very sensitive to small changes in this gap; for example, the temperature drop across this gap could change by as much as $150^{\circ} \mathrm{C}$ for an error of $0.0015 \mathrm{~cm}$ in the gap width. It appears from the thermal predictions, as well as from capsule assembly information, that this gap could vary between approximately $0.0015 \mathrm{~cm}$ and the design value of $0.008 \mathrm{~cm}$. These gaps were adjusted in the model to make the calculated primary containment temperature agree with the measured value to within $5^{\circ} \mathrm{C}$.

The TAC-2D results are used as input to the SHAPE code (Ref. 3-4). This code determines the thermal resistance shape factor which will result in the same radial temperature distributions in the fuel samples when used in a one-dimensional model. A one-dimensional radial heat transfer code is then used to calculate fuel temperature histories on a daily basis. This model is called CAPTEM (Ref, 3-5). CAPTEM analyses were performed on each fuel rod cell (cells 1,2, 5, and 6) in capsules P13R and P13S. A detailed 
discussion of these results is presented in Ref. 3-6. The calculated fuel temperatures and temperature gradients are presented in Tables 3-16 and 3-17.

The uncertainties of the fuel centerline temperatures are calculated on the basis of estimated assigned uncertainties in selected variables. The estimated and calculated uncertainties shown in Table 3-18 (stated as two standard deviations assuming normal distributions) give half the range that is expected to contain $95 \%$ of the probable values. For example, if a fuel rod conductivity is predicted to be $7.0 \mathrm{Btu} / \mathrm{hr}-\mathrm{ft}-{ }^{\circ} \mathrm{F}$, an uncertainty of $10 \%$ would mean that $95 \%$ of the probable conductivity values for that rod lie within $7.0 \pm 0.7$. Calculations of fuel centerline uncertainties use the outer wall of the primary containment as the reference point (i.e., known temperature). These uncertainty calculations did not account for the fact that reliable temperature measurements were made in the graphite crucibles for most of the cells. Further uncertainties in the calculated temperature arise from: (1) the loss of radial gaps between the rods and graphite crucible and crucible bowing in cell 2 of capsules P13R and P13S, and (2) uncertainties in the gas mixtures in cell 6 of P13S. For item one, the model was modified to account for the fact that the rods contacted the crucibles in cell 2; however, slight differences between calculated and observed graphite crucible temperatures still remained. Regarding the second item, it was noted for cell 6 of P13S that from the 40 th to the 140th day of operation, the helium and neon gas flow data were apparently in error (Ref, 3-6). Relatively large discrepancies between calculated and measured temperatures were found during this period, and these discrepancies were the greatest when mixtures of the two gases were used. Good agreement between calculated and measured temperatures was obtained when only He or only Ne was used to control the gap conductivities. Data for this time period have been eliminated from the temperature histories reported in Table $3-17$.

Results of the thermal analysis have also shown that the control thermocouples for cell 5 of P13R and P13S were operated too low. The selection of the control temperature was based on preirradiation design studies which 
apparently improperly accounted for gamma heating of the W/Re fuel thermocouples. In addition, the fission powers in these cells varied marked1y from design conditions which led to different heat flow patterns. These two factors caused the cells to be controlled about $100^{\circ} \mathrm{C}$ lower than intended.

\subsubsection{Unbonded Fuel Particle Temperatures}

The CAPTEM code is presently not adaptable to thermal modeling of unbonded particle cells. Consequent1y, TAC-2D models used in the thermal design were used to evaluate temperature histories of fuel samples in cells 3 and 4 of P13R and P13S. The relationship between the central thermocouple temperatures and individual particle samples was determined as a function of fuel particle power and power distribution, conductivity gas mixture, absolute temperature, etc. The results of the TAC-2D analysis were used to determine the ratios of peak fuel temperatures to those at the reference thermocouple location. These ratios were fit to an empirical equation which accounted for the variables listed above. The indicated temperatures of the $\mathrm{W} / \mathrm{Re}$ thermocouples were corrected for irradiationinduced drift according to procedures outlined in sections 3.1 and 3.5.3.

There were twelve particle trays in each cell, each of which contained fissile and fertile samples. These twelve trays were divided into four fuel groups for analysis purposes. The criterion for grouping was to keep the temperature spread less than $\pm 25^{\circ} \mathrm{C}$ from nominal. Tables $3-19$ through 3-22 present the calculated average and maximum hot-spot temperatures for each unbonded fuel particle sample contained in capsules P13R and P13S.

\subsubsection{Thermocoup1e Decalibration}

In addition to providing fuel thermal histories, the temperature analysis provided information on the degree of irradiation-induced decalibration of the $W / \operatorname{Re}$ thermocouples. Direct evidence that the $W /$ Re thermocouples do decalibrate is shown in Fig. 3-27. The end-of-life indicated the fuel rod centerline temperature monitored by a $\mathrm{W} / \mathrm{Re}$ thermocouple was 
as low as $400^{\circ} \mathrm{C}$, or about $400^{\circ} \mathrm{C}$ lower than the surrounding graphite crucible which was monitored by C/A thermocouples. Thermocouple decalibration plots (i.e., difference between indicated and calculated temperatures) are presented in Ref. 3-6 for all W/Re thermocouples in each cell. A linear regression analysis was performed for each set of data and the resulting equations are given in Table 3-23. The slope of most of the curves for thermocouples operating at $\sim 1100^{\circ} \mathrm{C}$ was nearly constant, yielding an average value for the "decalibration constant" of $75^{\circ} \mathrm{C}$ per unit of thermal fluence. This value is slightly different from the ones used to control the capsules temperatures; however, these differences did not result in a significant error in maintaining the fuel at design temperatures. For the high-temperature cells (cell 5 of P13R and P13S), the slope of the curve is approximately $170^{\circ} \mathrm{C}$ per unit of thermal fluence for thermal fluences up to $2.5 \times 10^{21} \mathrm{n} / \mathrm{cm}^{2}$. After this time the degree of decalibration appeared to change, as did the fuel temperatures. These changes coincide with a change in the equation used to account for decalibration of the $W /$ Re thermocouples operating at the higher temperatures in these cells. The postirradiation thermal analysis indicated this means of control was incorrect, since the fuel temperatures in these cells were $300^{\circ}$ to $400^{\circ} \mathrm{C}$ below their design values at end-of-life (see Tables 3-16 and 3-17).

\section{REFERENCES}

3-1. Sandefur, N. L., J. S. Steibe1, and R. J. Grenda, "EMF Drift of Chromel/Alumel and $\mathrm{W}-3 \% \mathrm{Re} / \mathrm{W}-25 \%$ Re Thermocouples Measured In-Pile to High Neutron Exposures," USAEC Report Gulf-GA-A12501, Gulf General Atomic, May 24, 1973.

3-2. Steibel, J. S., General Atomic unpublished data.

3-3. "Pub1ic Service Company of Colorado 330-MW(e) High-Temperature Gas-Cooled Reactor Research and Development Report for the Period Ending September 30, 1974," USAEC Report GA-A13167, General Atomic, October 31, 1974.

3-4. Kearney, D. W., "SHAPE: A Computer Program to Calculate Shape Factors for the CAPTEM Program," USAEC Report GA-10602, Gulf General Atomic, April 16, 1971. 
3-5. Carosella, D. P., H. W. Chi, and D. W. Kearney, "CAPTEM - A Computer Code for the Thermal Analysis of Fuel Irradiation Capsules," USAEC Report Gu1f-GA-B10620, Gulf Genera1 Atomic, September 1971.

3-6. Zangari, A. J., "Thermal Analysis of Capsules P13R and P13S," General Atomic, to be published. 
TABLE 3-1

IRRADIATION PARAMETERS FOR CAPSULE P13R UNBONDED FUEL PARTICLE SAMPLES

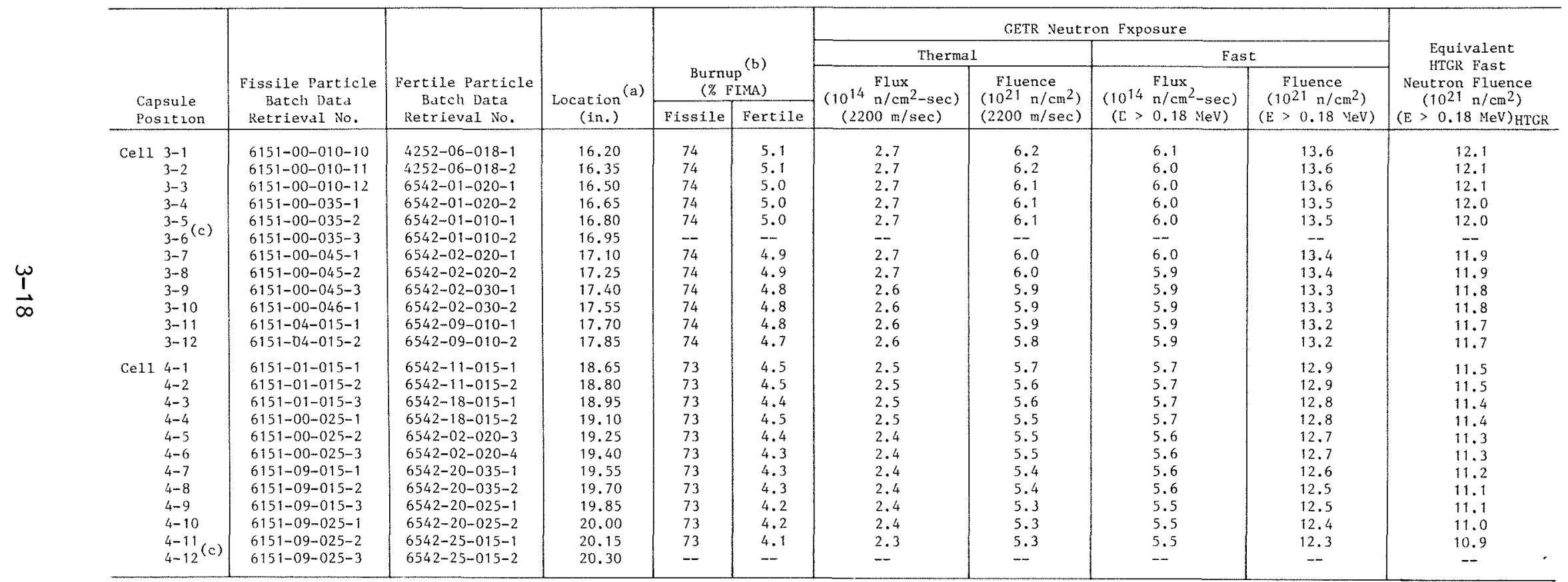

${ }^{(a)}$ Location in capsule measured from bottom of reactor core to midplane of sample tray.

(b) Heavy metal burnup calculated with FISSIN computer code.

(c) Sample tray was spilled during unloading. 
TABLE 3-2

TABLE $3-2$
IRRADIATION PARAMETERS FOR CAPSULE P13S UNBONDID FUEL PARTICLE SAYPLLTS

\begin{tabular}{|c|c|c|c|c|c|c|c|c|c|c|c|c|}
\hline \multirow{4}{*}{\multicolumn{2}{|c|}{$\begin{array}{l}\text { Capsule } \\
\text { Position } \\
\end{array}$}} & \multirow{4}{*}{$\begin{array}{c}\text { Fissile Particle } \\
\text { Batch Data } \\
\text { Retrieval No. } \\
\end{array}$} & \multirow{4}{*}{$\begin{array}{c}\text { Fertile Particle } \\
\text { Batch Data } \\
\text { Retrieval No. }\end{array}$} & \multirow{4}{*}{$\begin{array}{c}\text { Location (a) } \\
\text { (in.) }\end{array}$} & \multirow{3}{*}{\multicolumn{2}{|c|}{$\begin{array}{l}\text { Burnup }(\mathrm{b}) \\
(\% \text { FIMA) }\end{array}$}} & \multicolumn{5}{|c|}{ GETR Neutron Exposure } & \multirow{4}{*}{$\begin{array}{c}\text { Equivalent } \\
\text { HTGR Fast } \\
\text { veutron Fluence } \\
\left(10^{21} \mathrm{n} / \mathrm{cm}^{2}\right) \\
\text { (E) }>0.18 \mathrm{YeV})_{\text {HTGR }}\end{array}$} \\
\hline & & & & & & & \multicolumn{2}{|c|}{ Thermal } & \multicolumn{3}{|c|}{ Fast } & \\
\hline & & & & & & & \multirow{2}{*}{$\begin{array}{c}\text { Flux } \\
\left(10^{14} \mathrm{n} / \mathrm{cm}^{2}-\mathrm{sec}\right) \\
(2200 \mathrm{~m} / \mathrm{sec})\end{array}$} & \multirow{2}{*}{$\begin{array}{c}\text { Fiuence } \\
\left(1021 \mathrm{n} / \mathrm{cm}^{2}\right) \\
(2200 \mathrm{~m} / \mathrm{sec}) \\
\end{array}$} & \multirow{2}{*}{$\begin{array}{c}\left(10^{14}\right. \\
(\mathrm{C})\end{array}$} & \multirow{2}{*}{$\begin{array}{l}\text { Flux } \\
\text { n/cm } \\
0.18 \mathrm{Mev})\end{array}$} & \multirow{2}{*}{$\begin{array}{c}\text { Fiuence } \\
\left(10^{21} \mathrm{n} / \mathrm{cm}^{2}\right) \\
(1:=0.18 \mathrm{MeV})\end{array}$} & \\
\hline & & & & & Fissile & Fertile & & & & & & \\
\hline \multirow{12}{*}{ Cell } & $3-1$ & $6151-03-015-1$ & $4252-06-010-1$ & 16.20 & 73 & 4.3 & 2.4 & 5.4 & & 5.9 & 13.3 & 11.8 \\
\hline & $3-2$ & $6151-03-015-2$ & $4252-06-010-2$ & 16.35 & 73 & 4.3 & 2.4 & 5.4 & & 5.9 & 13.3 & 11.8 \\
\hline & $3-3$ & $6151-03-015-3$ & $6542-22-015-1$ & 16.50 & 73 & 4.3 & 2.4 & 5.4 & & 5.9 & 13.2 & 11.7 \\
\hline & $3-4$ & $4161-01-021-1$ & $6542-22-015-2$ & 16.65 & 73 & 4.2 & 2.4 & 5.4 & & 5.9 & 13.2 & 11.7 \\
\hline & $3-5$ & $4161-01-021-2$ & $6542-22-025-1$ & 16.80 & 73 & 4.2 & 2.4 & 5.3 & & 5.9 & 13.2 & 11.7 \\
\hline & $3-6$ & $4161-01-021-3$ & $6542-22-025-2$ & 16.95 & 73 & 4.2 & 2.4 & 5.3 & & 5.8 & 13.1 & 11.7 \\
\hline & $3-7$ & $6151-08-015-1$ & $6542-23-025-1$ & 17.10 & 73 & 4.2 & 2.4 & 5.3 & & 5.8 & 13.1 & 11.7 \\
\hline & $3-8$ & $6151-08-015-2$ & $6542-23-025-2$ & 17.25 & 73 & 4.1 & 2.3 & 5.3 & & 5.8 & 13.1 & 11.7 \\
\hline & $3-9$ & $6151-08-015-3$ & $6542-24-015-1$ & 17.40 & 73 & 4.1 & 2.3 & 5.3 & & 5.8 & 13.0 & 11.6 \\
\hline & $3-10(c)$ & $6151-02-025-1$ & $6542-24-015-2$ & 17.55 & 73 & 4.1 & 2.3 & 5.2 & & 5.8 & 13.0 & 11.6 \\
\hline & $3-11^{(c)}$ & $6151-02-025-2$ & $6252-00-025-1$ & 17.70 & - & -- & - & -- & & -- & - & -- \\
\hline & 3-12 & $6151-02-025-3$ & $6252-00-025-2$ & 17.85 & 72 & 4.1 & 2.3 & 5.2 & & 5.7 & 12.9 & 11.5 \\
\hline \multirow[t]{12}{*}{ Cell } & $4-1$ & $4163-00-011-3$ & $6542-17-010-1$ & 18.65 & 40 & 3.9 & 2.2 & 5.0 & & 5.6 & 12.6 & 11.2 \\
\hline & $4-2$ & $4163-00-011-4$ & $4252-02-010-1$ & 18.80 & 40 & 3.9 & 2.2 & 5.0 & & 5.6 & 12.6 & 11.2 \\
\hline & $4-3$ & $4163-00-011-5$ & $4252-02-010-2$ & 18.95 & 40 & 3.8 & 2.2 & 5.0 & & 5.6 & 12.5 & 11.1 \\
\hline & $4-4(c)$ & $6155-01-020-10$ & $6542-16-010-1$ & 19.10 & 11 & 3.8 & 2.2 & 5.0 & & 5.5 & 12.5 & 11.1 \\
\hline & $4-5^{(c)}$ & $6155-01-020-11$ & $6542-16-010-2$ & 19.25 & - & -- & - & -- & & -- & -- & -- \\
\hline & $4-6^{(c)}$ & $6155-01-020-12$ & $6542-16-010-3$ & 19.40 & -- & - & -- & -- & & - & -- & -- \\
\hline & $4-7$ & $5466-37-9$ & $6542-19-015-1$ & 19.55 & 38 & 3.7 & 2.2 & 4.9 & & 5.5 & 12.3 & 10.9 \\
\hline & $4-8$ & $5466-37-10$ & $6542-19-016-1$ & 19.70 & 38 & 3.7 & 2.2 & 4.8 & & 5.4 & 12.2 & 10.9 \\
\hline & $4-9$ & $5466-37-11$ & $6542-21-016-1$ & 19.85 & 38 & 3.7 & 2.1 & 4.8 & & 5.4 & 12.2 & 10.9 \\
\hline & $4-10$ & $6151-00-035-4$ & $6542-21-015-1$ & 20.00 & 71 & 3.6 & 2.1 & 4.7 & & 5.4 & 12.1 & 10.8 \\
\hline & $4-11$ & $6151-00-035-5$ & $6542-12-025-1$ & 20.15 & 71 & 3.6 & 2.1 & 4.7 & & 5.3 & 12.0 & 10.7 \\
\hline & $4-12$ & $6151-00-035-6$ & $6542-12-025-2$ & 20.30 & 71 & 3,6 & 2.1 & 4.7 & & 5.3 & 12.0 & 10.7 \\
\hline
\end{tabular}

(a) Location in capsule measured from bottom of reactor core to midplane of sample tray.

(b) Heavy metal burnup calculated with FISSIN computer code.

(c) Sample tray was spilled during unloading. 
TABLE 3-3

IRRADIATION PARAMETERS FOR CAPSULE P13R FUEL RODS

\begin{tabular}{|c|c|c|c|c|c|c|c|c|c|c|}
\hline \multirow{4}{*}{$\begin{array}{c}\text { Data } \\
\text { Retrieval } \\
\text { No. } \\
(7161-004-) \\
\end{array}$} & \multirow{4}{*}{$\begin{array}{l}\text { Capsule } \\
\text { Position }\end{array}$} & \multirow{4}{*}{$\begin{array}{c}\operatorname{Location}^{(a)} \\
\text { (in.) }\end{array}$} & \multirow{3}{*}{\multicolumn{2}{|c|}{$\begin{array}{l}\text { Burnup } \\
(\% \text { FIMA) } \\
\end{array}$}} & \multicolumn{5}{|c|}{ GETR Neutron Exposure } & \multirow{4}{*}{$\begin{array}{c}\text { Equivalent } \\
\text { HTGR Fast } \\
\text { Neutron Fluence } \\
\left(10^{21} \mathrm{n} / \mathrm{cm}^{2}\right) \\
(E>0.18 \mathrm{MeV}) \text { HTGR }\end{array}$} \\
\hline & & & & & \multicolumn{2}{|c|}{ Thermal } & \multicolumn{3}{|c|}{ Fast } & \\
\hline & & & & & \multirow{2}{*}{$\begin{array}{c}\text { F1ux } \\
\left(10^{14} \mathrm{n} / \mathrm{cm}^{2}-\mathrm{sec}\right) \\
(2200 \mathrm{~m} / \mathrm{sec})\end{array}$} & \multirow{2}{*}{$\begin{array}{c}\text { Fluence } \\
\left(10^{21} \mathrm{n} / \mathrm{cm}^{2}\right) \\
(2200 \mathrm{~m} / \mathrm{sec})\end{array}$} & \multirow{2}{*}{$\begin{array}{r}(1014 \\
(E> \\
\end{array}$} & \multirow{2}{*}{$\begin{array}{l}\text { F1ux } \\
\text { n/cm²-sec) } \\
0.18 \mathrm{MeV})\end{array}$} & \multirow{2}{*}{ 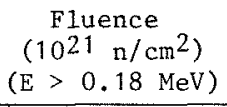 } & \\
\hline & & & Fissile & Fertile & & & & & & \\
\hline $01-5$ & $1 \mathrm{~A}$ & 2.9 & 71 & 3.5 & 2.1 & 4.7 & & 3.7 & 8.2 & 7.3 \\
\hline $02-6$ & $1 \mathrm{~B}$ & 3.6 & 72 & 3.9 & 2.2 & 5.0 & & 4.0 & 9.1 & 8.1 \\
\hline $03-5$ & $1 \mathrm{C}$ & 4.4 & 73 & 4.2 & 2.4 & 5.3 & & 4.4 & 9.8 & 8.7 \\
\hline $04-6$ & 10 & 5.1 & 73 & 4.5 & 2.5 & 5.6 & & 4.7 & 10.5 & 9.3 \\
\hline $05-6$ & $1 \mathrm{E}$ & 5.9 & 40 & 4.8 & 2.6 & 5.9 & & 5.0 & 11.1 & 9.9 \\
\hline $06-5$ & $2 \mathrm{~A}$ & 9.8 & 75 & 5.5 & 2.9 & 6.6 & & 5.9 & 13.3 & 11.8 \\
\hline $07-5$ & $2 B$ & 10.5 & 75 & 5.6 & 3.0 & 6.6 & & 6.0 & 13.5 & 12.0 \\
\hline $08-13$ & $2 \mathrm{C}$ & 11.3 & 75 & 5.6 & 3.0 & 6.7 & & 6.1 & 13.7 & 12.2 \\
\hline $09-5$ & $2 \mathrm{D}$ & 12.0 & 75 & 5.6 & 3.0 & 6.6 & & 6.1 & 13.8 & 12.3 \\
\hline $10-5$ & $2 \mathrm{E}$ & 12.8 & 41 & 5.5 & 2.9 & 6.6 & & 6.2 & 13.9 & 12.4 \\
\hline $16-5$ & $5 \mathrm{~A}$ & 23.7 & 36 & 3.1 & 1.9 & 4.2 & & 4.7 & 10.6 & 9.4 \\
\hline $17-5$ & $5 B$ & 24.4 & 68 & 2.8 & 1.8 & 4.0 & & 4.5 & 10.1 & 9.0 \\
\hline $18-6$ & $5 \mathrm{C}$ & 25.2 & 67 & 2.6 & 1,7 & 3.8 & & 4.3 & 9.6 & 8.5 \\
\hline $19-7$ & $5 D$ & 25.9 & 66 & 2.4 & 1.6 & 3.5 & & 4.1 & 9.2 & 8.2 \\
\hline $20-6$ & $5 \mathrm{E}$ & 26.7 & 64 & 2.1 & 1.5 & 3.3 & & 3.9 & 8.7 & 7.7 \\
\hline $21-5$ & $6 \mathrm{~A}$ & 30.6 & 54 & 1.1 & 1.0 & 2.2 & & 2.7 & 6.1 & 5.4 \\
\hline $22-5$ & $6 \mathrm{~B}$ & 31.3 & 51 & 1.0 & 0.9 & 2.0 & & 2.5 & 5.6 & 5.0 \\
\hline $23-5$ & $6 \mathrm{C}$ & 32.1 & 49 & 0.8 & 0.8 & 1.9 & & 2.3 & 5.1 & 4.5 \\
\hline $24-5$ & 6D & 32.8 & 47 & 0.7 & 0.8 & 1.7 & & 2.1 & 4.6 & 4.1 \\
\hline $25-5$ & $6 \mathrm{E}$ & 33.6 & 44 & 0.6 & 0.7 & 1.6 & & 1.8 & 4.1 & 3.6 \\
\hline
\end{tabular}

(a) Location in capsule measured from bottom of reactor core to fuel rod midplane.

(b) Heavy metal burnup calculated with FISSIN computer code. 
TABLE 3-4

IRRADIATION PARAMETERS FOR CAPSULE P13S FUEL RODS

\begin{tabular}{|c|c|c|c|c|c|c|c|c|c|c|}
\hline \multirow{4}{*}{$\begin{array}{c}\text { Data } \\
\text { Retrieval } \\
\text { No. } \\
(7161-004-) \\
\end{array}$} & \multirow{4}{*}{$\begin{array}{l}\text { Capsule } \\
\text { Position }\end{array}$} & \multirow{4}{*}{$\begin{array}{c}\text { Location } \\
\text { (in.) }\end{array}$} & & & \multicolumn{5}{|c|}{ GETR Neutron Exposure } & \multirow{4}{*}{$\begin{array}{c}\text { Equivalent } \\
\text { HTGR Fast } \\
\text { Neutron Fluence } \\
\left(10^{21} \mathrm{n} / \mathrm{cm}^{2}\right) \\
(\mathrm{E}>0.18 \mathrm{MeV}) \text { HTGR }\end{array}$} \\
\hline & & & \multirow{2}{*}{\multicolumn{2}{|c|}{$\begin{array}{l}\text { Burnup }(b) \\
(\% \text { FIMA })\end{array}$}} & \multicolumn{2}{|c|}{ Thermal } & \multicolumn{3}{|c|}{ Fast } & \\
\hline & & & & & \multirow{2}{*}{$\begin{array}{c}\text { Flux } \\
\left(10^{14} \mathrm{n} / \mathrm{cm}^{2}-\mathrm{sec}\right) \\
(2200 \mathrm{~m} / \mathrm{sec})\end{array}$} & \multirow{2}{*}{$\begin{array}{c}\text { Fluence } \\
\left(10^{21} \mathrm{n} / \mathrm{cm}^{2}\right) \\
(2200 \mathrm{~m} / \mathrm{sec})\end{array}$} & \multirow{2}{*}{$\begin{array}{r}(1014 \\
(E>\end{array}$} & \multirow{2}{*}{$\begin{array}{l}\text { Flux } \\
\left.\mathrm{n} / \mathrm{cm}^{2}-\mathrm{sec}\right) \\
0.18 \mathrm{MeV})\end{array}$} & \multirow{2}{*}{ 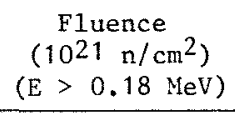 } & \\
\hline & & & Fissile & Fertile & & & & & & \\
\hline $\begin{array}{l}01-7 \\
02-5 \\
03-6 \\
04-5 \\
05-5\end{array}$ & $\begin{array}{l}1 \mathrm{~A} \\
1 \mathrm{~B} \\
1 \mathrm{C} \\
1 \mathrm{D} \\
1 \mathrm{E}\end{array}$ & $\begin{array}{l}2.9 \\
3.6 \\
4.4 \\
5.1 \\
5.9\end{array}$ & $\begin{array}{l}70 \\
71 \\
71 \\
72 \\
39\end{array}$ & $\begin{array}{l}3.2 \\
3.4 \\
3.7 \\
3.9 \\
4.1\end{array}$ & $\begin{array}{l}1.9 \\
2.0 \\
2.1 \\
2.2 \\
2.3\end{array}$ & $\begin{array}{l}4.4 \\
4.6 \\
4.8 \\
5.0 \\
5.2\end{array}$ & & $\begin{array}{l}3.5 \\
3.9 \\
4.2 \\
4.5 \\
4.8\end{array}$ & $\begin{array}{r}7.9 \\
8.7 \\
9.5 \\
10.2 \\
10.8\end{array}$ & $\begin{array}{l}7.0 \\
7.7 \\
8.5 \\
9.1 \\
9.6\end{array}$ \\
\hline $\begin{array}{l}11-6 \\
12-5 \\
13-6 \\
14-5 \\
15-5\end{array}$ & $\begin{array}{l}2 \mathrm{~A} \\
2 \mathrm{~B} \\
2 \mathrm{C} \\
2 \mathrm{D} \\
2 \mathrm{E}\end{array}$ & $\begin{array}{r}9.8 \\
10.5 \\
11.3 \\
12.0 \\
12.8\end{array}$ & $\begin{array}{l}74 \\
74 \\
74 \\
74 \\
74\end{array}$ & $\begin{array}{l}4.6 \\
4.6 \\
4.7 \\
4.6 \\
4.6\end{array}$ & $\begin{array}{l}2.5 \\
2.6 \\
2.6 \\
2.6 \\
2.6\end{array}$ & $\begin{array}{l}5.7 \\
5.8 \\
5.8 \\
5.8 \\
5.7\end{array}$ & & $\begin{array}{l}5.8 \\
5.9 \\
5.9 \\
6.0 \\
6.0\end{array}$ & $\begin{array}{l}12.9 \\
13.2 \\
13.4 \\
13.5 \\
13.6\end{array}$ & $\begin{array}{l}11.5 \\
11.7 \\
11.9 \\
12.0 \\
12.1\end{array}$ \\
\hline $\begin{array}{l}16-6 \\
17-6 \\
18-5 \\
19-5 \\
20-5\end{array}$ & $\begin{array}{l}5 \mathrm{~A} \\
5 \mathrm{~B} \\
5 \mathrm{C} \\
5 \mathrm{D} \\
5 \mathrm{E}\end{array}$ & $\begin{array}{l}23.7 \\
24.4 \\
25.2 \\
25.9 \\
26.7\end{array}$ & $\begin{array}{l}36 \\
67 \\
66 \\
65 \\
63\end{array}$ & $\begin{array}{l}2.8 \\
2.6 \\
2.4 \\
2.2 \\
2.0\end{array}$ & $\begin{array}{l}1.8 \\
1.7 \\
1.6 \\
1.5 \\
1.4\end{array}$ & $\begin{array}{l}3.9 \\
3.8 \\
3.6 \\
3.4 \\
3.2\end{array}$ & & $\begin{array}{l}4.6 \\
4.4 \\
4.2 \\
4.0 \\
3.8\end{array}$ & $\begin{array}{r}10.3 \\
9.8 \\
9.4 \\
8.9 \\
8.4\end{array}$ & $\begin{array}{l}9.2 \\
8.7 \\
8.4 \\
7.9 \\
7.5\end{array}$ \\
\hline $\begin{array}{l}26-5 \\
27-5 \\
28-13 \\
29-5 \\
30-5\end{array}$ & $\begin{array}{l}6 \mathrm{~A} \\
6 \mathrm{~B} \\
6 \mathrm{C} \\
6 \mathrm{D} \\
6 \mathrm{E}\end{array}$ & $\begin{array}{l}30.6 \\
31.3 \\
32.1 \\
32.8 \\
33.6\end{array}$ & $\begin{array}{l}54 \\
26 \\
48 \\
46 \\
42\end{array}$ & $\begin{array}{l}1.1 \\
1.0 \\
0.8 \\
0.7 \\
0.6\end{array}$ & $\begin{array}{l}1.0 \\
0.9 \\
0.8 \\
0.7 \\
0.7\end{array}$ & $\begin{array}{l}2.2 \\
2.0 \\
1.8 \\
1.6 \\
1.5\end{array}$ & & $\begin{array}{l}2.6 \\
2.4 \\
2.2 \\
1.9 \\
1.7\end{array}$ & $\begin{array}{l}5.9 \\
5.4 \\
4.9 \\
4.4 \\
3.9\end{array}$ & $\begin{array}{l}5.3 \\
4.8 \\
4.4 \\
3.9 \\
3.5\end{array}$ \\
\hline
\end{tabular}

(a) Location in capsule measured from bottom of reactor core to fuel rod midplane.

(b) Heavy metal burnup calculated with FISSIN computer code. 
TABLE 3-5

WIRE WEIGHTS

\begin{tabular}{|c|c|c|c|c|}
\hline $\begin{array}{c}\text { Wire } \\
\text { Ident. No. }\end{array}$ & Capsule & Location & $\begin{array}{l}\text { ORNL } \\
\begin{array}{l}\text { Wire Weight } \\
\text { (mg) }\end{array}\end{array}$ & $\begin{array}{l}\text { GA } \\
\begin{array}{l}\text { Wire Weight } \\
\text { (mg) }\end{array}\end{array}$ \\
\hline$C-1632$ & P13R & Cell $2 /$ hole $1 / 0^{\circ}$ & 16.639 & 16.16 \\
\hline$C-1635$ & P13R & Cell 5/hole $2 / 0^{\circ}$ & 22.411 & 22.40 \\
\hline$C-1675$ & P13S & $\operatorname{Cel1} 2 /$ hole $3 / 0^{\circ}$ & 16.100 & 16.11 \\
\hline$C-1679$ & P13S & Cell $5 /$ hole $4 / 0^{\circ}$ & 22.341 & 22.30 \\
\hline
\end{tabular}

TABLE 3-6

MEASURED Mn-54 ACTIVITIES

\begin{tabular}{c|c|c|c|c}
\hline \multirow{2}{*}{$\begin{array}{c}\text { Wire } \\
\text { Ident. No. }\end{array}$} & $\begin{array}{c}\text { ORNL } \\
\left(10^{5} \mathrm{dps} / \mathrm{mg}\right)\end{array}$ & $\begin{array}{c}\text { ORNL } \\
(\mu \mathrm{Ci} / \mathrm{mg})\end{array}$ & $\begin{array}{c}\text { GA - Case I } \\
(\mu \mathrm{Ci} / \mathrm{mg})\end{array}$ & $\begin{array}{c}\text { GA - Case II } \\
(\mu \mathrm{Ci} / \mathrm{mg})\end{array}$ \\
\hline C-1632 & $3.29 \pm 0.02$ & $14.81(\mathrm{a})$ & 16.92 & 15.98 \\
$\mathrm{C}-1635$ & $2.42 \pm 0.16$ & 10.58 & 11.79 & 11.16 \\
C-1675 & $3.32 \pm 0.23$ & 14.51 & 16.03 & 15.24 \\
$\mathrm{C}-1679$ & $2.31 \pm 0.09$ & 10.10 & 11.31 & 10.79 \\
\hline
\end{tabular}

(a) Value normalized to corrected weight. 
TABLE 3-7

COMPARISON OF FAST NEUTRON FLUENCES

\begin{tabular}{|c|c|c|c|c|}
\hline \multirow[b]{2}{*}{$\begin{array}{c}\text { Wire } \\
\text { Ident. No. }\end{array}$} & \multicolumn{4}{|c|}{$\begin{array}{l}\text { Calculated Fast Neutron Fluence } \\
\left(10^{21} \mathrm{n} / \mathrm{cm}^{2}\right)(\mathrm{E}>0.18 \mathrm{MeV})_{\text {GETR }}\end{array}$} \\
\hline & $\begin{array}{c}\text { ORNL } \\
\text { Program }\end{array}$ & $\begin{array}{c}\text { ORNL } \\
\text { Mn-54 Activity, } \\
\text { GA Dose } 1 \\
\text { Program } \\
\end{array}$ & $\begin{array}{c}\text { GA - Case I } \\
\text { Mn-54 Activity, } \\
\text { GA Dose } 1 \\
\text { Program }\end{array}$ & $\begin{array}{c}\text { GA - Case II } \\
\text { Mn-54 Activity, } \\
\text { GA Dose } 1 \\
\text { Program }\end{array}$ \\
\hline$C-1632$ & $12.5^{(a)}$ & $13.0^{(\mathrm{a})}$ & 14.9 & 14.0 \\
\hline$C-1635$ & 8.9 & 9.1 & 10.2 & 9.6 \\
\hline$C-1675$ & 12.2 & 12.7 & 14.0 & 13.4 \\
\hline$C-1679$ & 8.5 & 8.7 & 9.8 & 9.2 \\
\hline
\end{tabular}

(a) Activity was normalized to corrected wire weight.

TABLE 3-8

THERMAL FISSION YIELDS OF CESIUM AND ZIRCONIUM ISOTOPES

\begin{tabular}{l|c|c}
\hline & $\begin{array}{c}\mathrm{U}-233 \text { Yield } \\
\text { Isotope }\end{array}$ & $\begin{array}{c}\mathrm{U}-235 \text { Yield } \\
(\%)\end{array}$ \\
\hline Zr-91 & 6.52 & 5.92 \\
Zr-92 & 6.59 & 5.96 \\
Zr-93 & 7.01 & 6.37 \\
Zr-94 & 6.81 & 6.42 \\
Zr-96 & 5.68 & 6.26 \\
Cs-137 & 6.80 & 6.27 \\
\hline
\end{tabular}


TABLE 3-9

RESULTS OF HEAVY METAL BURNUP DETERMINATIONS MADE AT GENERAL ATOMIC

\begin{tabular}{|c|c|c|c|c|c|c|c|c|c|}
\hline \multirow[b]{2}{*}{ Capsule } & \multirow[b]{2}{*}{$\begin{array}{l}\text { Capsule } \\
\text { Position }\end{array}$} & \multirow[b]{2}{*}{ Batch No. } & \multirow[b]{2}{*}{$\begin{array}{c}\text { Particle } \\
\text { Type }\end{array}$} & \multicolumn{6}{|c|}{ Heavy Metal Burnup (\% FIMA) } \\
\hline & & & & $\begin{array}{l}\text { Fiss } \\
\mathrm{Cs}(\mathrm{a})\end{array}$ & $\begin{array}{l}\text { Fiss } \\
\mathrm{Zr}(\mathrm{b})\end{array}$ & Case $I^{(c)}$ & Case II ${ }^{(\mathrm{d})}$ & Case III $(\mathrm{e})$ & $\operatorname{FISSIN}^{(f)}$ \\
\hline P13R & $3-5$ & $6151-00-035-2$ & $\mathrm{UC}_{2}$ TRISO & 77.9 & 78.3 & 74.37 & 74.08 & 74.28 & 73.64 \\
\hline P13S & $4-12$ & $6151-00-035-6$ & $\mathrm{UC}_{2}$ TRISO & 76.9 & 75.7 & 71.83 & 71.65 & 71.76 & 71.12 \\
\hline P13R & $3-7$ & $6542-02-020-1$ & $\mathrm{ThO}_{2} \mathrm{BISO}$ & 4.21 & $\mathrm{ND}^{(\mathrm{g})}$ & $\mathrm{NA}^{(h)}$ & $\mathrm{NA}$ & NA & 4.33 \\
\hline P13S & $4-2$ & $4252-02-010-1$ & $\mathrm{ThO}_{2}$ BISO & 3.97 & ND & NA & NA & NA & 3.92 \\
\hline P13S & $3-12$ & $6252-00-025-2$ & $\mathrm{ThO}_{2}$ BISO & 4.01 & $\mathrm{ND}$ & NA & NA & NA & 4.10 \\
\hline
\end{tabular}

(a) Fiss $\mathrm{Cs}=$ atom percent fissions per initial heavy metal atom based on fission product cesium.

(b) Fiss $\mathrm{Zr}=$ atom percent fissions per initial heavy metal atom based on fission product zirconium.

(c) Case $I$ = atom percent fissions per initial heavy metal atom based on Case I of ASTM Procedure E244-69.

(d) Case II = atom percent fissions per initial heavy metal atom based on Case II of ASTM Procedure E244-69.

(e) Case III = atom percent fissions per initial heavy metal atom based on Case III of ASTM Procedure E244-69.

(f) FISSIN = atom percent fissions per initial heavy metal atom, Sigma 2 calculation, based on input thermal nvt, reactor power history, and macroscopic cross sections.
(g) ${ }_{\mathrm{ND}}=$ not determined.
${ }^{(h)}$ NA $=$ not applicable. 
TABLE $3-10$

FISSILE PARTICLE URANIUM ISOTOPIC COMPOSITION DETERMINED BY MASS SPECTROMETRY

\begin{tabular}{|c|c|c|c|c|c|c|c|c|c|c|c|c|}
\hline \multirow[b]{2}{*}{ Sample } & \multirow[b]{2}{*}{ Capsule } & \multirow{2}{*}{$\begin{array}{l}\text { Capsule } \\
\text { Position }\end{array}$} & \multicolumn{5}{|c|}{ Atom Percent } & \multicolumn{5}{|c|}{ Weight Percent } \\
\hline & & & 233 & 234 & 235 & 236 & 238 & 233 & 234 & 235 & 236 & 238 \\
\hline \multicolumn{13}{|c|}{ Irradiated Particles } \\
\hline $6151-00-035-2$ & P13R & $3-5$ & 0.183 & 1.222 & 24.04 & 54.48 & 20.08 & 0.181 & 1.211 & 23.93 & 54.45 & 20.23 \\
\hline $6151-00-035-2$ & P13R & $3-5$ & 0.172 & 1.232 & 23.11 & 55.33 & 20.15 & 0.170 & 1.220 & 23.00 & 55.30 & 20.31 \\
\hline $6151-00-035-6$ & P13S & $4-12$ & 0.135 & 1.180 & 31.52 & 48.09 & 19.08 & 0.134 & 1.170 & 31.38 & 48.08 & 19.23 \\
\hline $6151-00-035-6$ & P13S & $4-12$ & 0.132 & 1.178 & 32.46 & 47.81 & 18.41 & 0.130 & 1.168 & 32.32 & 47.81 & 18.57 \\
\hline \multicolumn{13}{|c|}{ Reference Archive Particles } \\
\hline $6151-00-035$ & -- & -- & $\begin{array}{l}\text { Not } \\
\text { detected }\end{array}$ & $\begin{array}{r}0.657 \\
\pm 0.003\end{array}$ & $\begin{array}{l}93.178 \\
\pm 0.011\end{array}$ & $\begin{array}{r}0.3977 \\
\pm 0.0028\end{array}$ & $\begin{array}{r}5.767 \\
\pm 0.010\end{array}$ & $\begin{array}{l}\text { Not } \\
\text { detected }\end{array}$ & $\begin{array}{r}0.654 \\
\pm 0.003\end{array}$ & $\begin{array}{l}93.111 \\
\pm 0.011\end{array}$ & $\begin{array}{r}0.3991 \\
\pm 0.0028\end{array}$ & $\begin{array}{r}5.836 \\
\pm 0.010\end{array}$ \\
\hline
\end{tabular}


TABLE $3-11$

FERTILE PARTICLE URANIUM ISOTOPIC COMPOSITION DETERMINED BY MASS SPECTROMETRY

\begin{tabular}{|c|c|c|c|c|c|c|c|c|c|c|c|c|}
\hline \multirow[b]{2}{*}{ Sample } & \multirow[b]{2}{*}{ Capsule } & \multirow{2}{*}{$\begin{array}{l}\text { Capsule } \\
\text { Position }\end{array}$} & \multicolumn{5}{|c|}{ Atom Percent } & \multicolumn{5}{|c|}{ Weight Percent } \\
\hline & & & 233 & 234 & 235 & 236 & 238 & 233 & 234 & 235 & 236 & 238 \\
\hline \multirow[t]{2}{*}{$6542-02-020-1$} & P13R & $3-7$ & 74.53 & 20.41 & 4.229 & 0.712 & 0.119 & 74.43 & 20.47 & 4.259 & 0.720 & 0.121 \\
\hline & & & 73.25 & 20.21 & 4.189 & 0.710 & 1.641 & 73.13 & 20.27 & 4.217 & 0.718 & 1.673 \\
\hline \multirow[t]{2}{*}{$6252-00-025-2$} & $\mathrm{P} 13 \mathrm{~S}$ & $3-12$ & 75.48 & 19.77 & 4.027 & 0.637 & 0.089 & 75.38 & 19.83 & 4.056 & 0.645 & 0.091 \\
\hline & & & 75.76 & 19.49 & 3.985 & 0.622 & 0.139 & 75.67 & 19.55 & 4.014 & 0.629 & 0.141 \\
\hline \multirow[t]{2}{*}{$4252-02-010-1$} & $\mathrm{P} 13 \mathrm{~S}$ & $4-2$ & 76.25 & 19.11 & 2.866 & 0.598 & 0.177 & 76.15 & 19.17 & 3.894 & 0.605 & 0.180 \\
\hline & & & 76.42 & 19.04 & 3.81 & 0.577 & 0.141 & 76.33 & 19.10 & 3.84 & 0.584 & 0.143 \\
\hline
\end{tabular}


TABLE $3-12$

COMPARISON OF FISSILE AND FERTILE PARTICLE URANIUM ISOTOPIC COMPOSITION DETERMINED BY MASS SPECTROMETRY AND THE SIGMA 2 FISSIN COMPUTER PROGRAM

\begin{tabular}{|c|c|c|c|c|c|c|c|}
\hline Sample & Capsule & $\begin{array}{l}\text { Capsule } \\
\text { Position }\end{array}$ & $\mathrm{U}-234$ & $\mathrm{U}-235$ & $\mathrm{U}-236$ & $\mathrm{U}-238$ & Method \\
\hline \multicolumn{8}{|c|}{ Fissile Weight Percent } \\
\hline \multirow[t]{2}{*}{$6151-00-035-2$} & P13R & $3-5$ & 1.218 & 23.50 & 54.97 & 20.30 & Mass Spec. (a) \\
\hline & & & 1.17 & 23.48 & 54.88 & 20.50 & Sigma 2 \\
\hline \multirow[t]{2}{*}{$6151-00-035-2$} & P13S & $4-12$ & 1.17 & 31.89 & 48.01 & 18.93 & Mass Spec. (a) \\
\hline & & & 1.18 & 31.91 & 48.18 & 18.73 & Sigma 2 \\
\hline \multicolumn{8}{|c|}{ Fertile Weight Percent } \\
\hline \multirow{3}{*}{$6542-02-020-1$} & & & $U-233$ & $U-234$ & $\mathrm{U}-235$ & $\mathrm{U}-236$ & \multirow{3}{*}{$\begin{array}{l}\text { Mass Spec. (b) } \\
\text { Sigma } 2\end{array}$} \\
\hline & P13R & $3-7$ & 74.45 & 20.56 & 4.28 & 0.726 & \\
\hline & & & 74.62 & 20.23 & 4.54 & 0.80 & \\
\hline \multirow[t]{2}{*}{$6252-00-025-2$} & P13S & $3-12$ & 75.61 & 19.71 & 4.04 & 0.638 & Mass Spec. (b) \\
\hline & & & 75.36 & 19.62 & 4.30 & 0.73 & Sigma 2 \\
\hline \multirow[t]{2}{*}{$4252-02-010-1$} & P13S & $4-2$ & 76.36 & 19.17 & 3.87 & 0.596 & Mass Spec. (b) \\
\hline & & & 76.09 & 19.13 & 4.11 & 0.67 & Sigma 2 \\
\hline
\end{tabular}

(a) U-233 normalized out.

(b) U-238 normalized out. 
TABLE $3-13$

METHODS EVALUATION DATA

\begin{tabular}{|c|c|c|c|c|c|c|c|}
\hline \multirow{3}{*}{$\begin{array}{c}\text { Standard } \\
\text { Sample No. (a) }\end{array}$} & \multicolumn{5}{|c|}{ Metal Concentration Found $(\mu \mathrm{g} / \mathrm{ml})$} & \multirow{2}{*}{\multicolumn{2}{|c|}{$\begin{array}{c}\text { Weight Ratio, } \\
\mathrm{Th} / \mathrm{U}\end{array}$}} \\
\hline & \multirow{2}{*}{$\begin{array}{c}\mathrm{Zr} \\
\text { Wet Chem. }\end{array}$} & \multicolumn{2}{|l|}{ Th } & \multicolumn{2}{|l|}{$\mathrm{U}$} & & \\
\hline & & Wet Chem. & IDMS & Wet Chem. & IDMS & Wet Chem. & IDMS \\
\hline 1 & 3.2 & 418 & 389 & 56.1 & 56.4 & 7.45 & 6.89 \\
\hline 2 & 3.5 & 400 & 396 & 59.8 & 57.6 & 6.94 & 6.87 \\
\hline 3 & 3.4 & 416 & 397 & 59.8 & 57.7 & 6.96 & 6.88 \\
\hline 4 & 3.4 & 406 & 399 & 59.3 & 59.1 & 6.84 & 6.75 \\
\hline 5 & 3.2 & 411 & 400 & 59.1 & 60.4 & 6.95 & 6.62 \\
\hline 6 & 3.5 & 401 & 422 & 59.1 & 61.3 & 6.78 & 6.89 \\
\hline 7 & 3.9 & 416 & (b) & 59.3 & (b) & 7.01 & (b) \\
\hline 8 & 3.7 & 400 & (b) & 61.7 & (b) & 6.48 & (b) \\
\hline 9 & 3.2 & 400 & (b) & 57.9 & (b) & 6.90 & (b) \\
\hline 10 & 3.6 & 411 & (b) & 59.6 & (b) & 6.90 & (b) \\
\hline Average & 3.46 & 408 & 400 & 59.2 & 58.8 & 6.92 & 6.82 \\
\hline $\begin{array}{l}\text { Relative } \\
\text { Std. Dev., \% }\end{array}$ & \pm 6.4 & \pm 1.7 & \pm 2.5 & \pm 2.4 & \pm 2.9 & \pm 3.2 & \pm 1.5 \\
\hline $\begin{array}{l}\text { Relative } \\
\text { error, \% }\end{array}$ & +4.2 & +2.0 & 0 & +1.9 & +1.2 & +0.6 & -0.9 \\
\hline
\end{tabular}

(a) Composition of standard: $\mathrm{Zr}=3.32 \mu \mathrm{g} / \mathrm{ml}, \mathrm{Th}=400 \mu \mathrm{g} / \mathrm{mI}, \mathrm{U}=$ $58.1 \mu \mathrm{g} / \mathrm{ml}$, and $\mathrm{Th} / \mathrm{U}=6.88$. 
TABLE $3-14$

URANIUM ISOTOPIC ANALYSIS

\begin{tabular}{|c|c|c|c|c|c|c|c|}
\hline \multirow[b]{2}{*}{ Sample } & \multirow{2}{*}{$\begin{array}{l}\text { Sample } \\
\text { Code (a) }\end{array}$} & \multicolumn{5}{|c|}{ Isotopic Abundance (atom \%) } & \multirow{2}{*}{$\begin{array}{l}\text { Avg. Gram } \\
\text { Atomic } \\
\text { Weight (b) }\end{array}$} \\
\hline & & 233 & 234 & 235 & 236 & 238 & \\
\hline \multirow[t]{2}{*}{$6151-00-035-2$} & $3-5-(2)$ & 0.473 & 1.217 & 25.99 & 52.77 & 19.55 & 236.1 \\
\hline & $-(8)$ & 0.220 & 1.216 & 23.55 & 55.00 & 20.02 & 236.1 \\
\hline \multirow[t]{2}{*}{$6542-02-020-1$} & $3-7-(1)$ & 73.08 & 19.93 & 5.49 & 0.778 & 0.728 & 233.4 \\
\hline & $-(6)$ & 74.34 & 20.42 & 4.42 & 0.739 & 0.074 & 233.3 \\
\hline \multirow[t]{2}{*}{$6252-00-025-2$} & $3-12-(4)$ & 74.93 & 19.56 & 4.62 & 0.730 & 0.160 & 233.3 \\
\hline & $-(5)$ & 74.83 & 19.49 & 4.72 & 0.784 & 0.185 & 233.3 \\
\hline \multirow[t]{2}{*}{$4252-02-010-1$} & $4-2-(2)$ & 75.97 & 19.39 & 4.01 & 0.588 & 0.044 & 233.3 \\
\hline & $-(6)$ & 75.51 & 19.01 & 4.70 & 0.662 & 0.108 & 233.4 \\
\hline \multirow[t]{2}{*}{$6151-00-035-6$} & $4-12-(2)$ & 0.197 & 1.202 & 31.61 & 48.59 & 18.39 & 236.0 \\
\hline & $-(4)$ & 0.222 & 1.194 & 32.60 & 47.88 & 18.10 & 235.5 \\
\hline
\end{tabular}

(a) Number in parentheses denotes the particle number assigned for the designated particle sample.

(b) Average gram atomic weight for uranium used in the calculation of FIMA. 
TABLE $3-15$

RESULTS OF HEAVY METAL BURNUP DETERMINATIONS MADE AT OAK RIDGE NATIONAL LABORATORY

\begin{tabular}{|c|c|c|c|c|c|}
\hline \multirow[b]{2}{*}{ Capsule } & \multirow{2}{*}{$\begin{array}{l}\text { Capsule } \\
\text { Position }\end{array}$} & \multirow{2}{*}{$\begin{array}{l}\text { Batch } \\
\text { Number }\end{array}$} & \multirow{2}{*}{$\begin{array}{c}\text { Particle } \\
\text { Type }\end{array}$} & \multicolumn{2}{|c|}{$\begin{array}{c}\text { Heavy Metal Burnup } \\
(\% \text { FIMA })\end{array}$} \\
\hline & & & & Wet Chem. & IDMS \\
\hline P13R & $3-5$ & $6151-00-035-2$ & $\mathrm{UC}_{2}$ TRISO & $\begin{array}{l}75.3 \\
74.6\end{array}$ & $\begin{array}{l}78.3 \\
75.4\end{array}$ \\
\hline P13R & $3-7$ & $6542-02-020-1$ & $\mathrm{ThO}_{2} \mathrm{BISO}$ & $\begin{array}{l}4.72 \\
4.70\end{array}$ & $\begin{array}{l}4.96 \\
--\end{array}$ \\
\hline P13S & $3-12$ & $6252-00-025-2$ & $\mathrm{ThO}_{2}$ TRISO & $\begin{array}{l}4.29 \\
4.44\end{array}$ & $\begin{array}{l}4.39 \\
4.36\end{array}$ \\
\hline P13S & $4-2$ & $4252-02-010-1$ & $\mathrm{ThO}_{2}$ BISO & $\begin{array}{l}3.83 \\
3.52\end{array}$ & -- \\
\hline P13S & $4-12$ & $6151-00-035-6$ & $\mathrm{UC}_{2}$ TRISO & $\begin{array}{l}71.6 \\
72.5\end{array}$ & $\begin{array}{l}71.4 \\
72.7\end{array}$ \\
\hline
\end{tabular}


TABLE $3-16$

IRRADIATION TEMPERATURES OF CAPSULE P13R FUEL RODS

\begin{tabular}{|c|c|c|c|c|c|c|c|c|c|c|c|c|}
\hline \multirow{2}{*}{$\begin{array}{c}\text { Data } \\
\text { Retrieval } \\
\text { No. } \\
(7161-004-)\end{array}$} & \multirow{2}{*}{$\begin{array}{l}\text { Capsule } \\
\text { Position }\end{array}$} & \multicolumn{3}{|c|}{$\begin{array}{c}\text { Centerline Temperature } \\
\left({ }^{\circ} \mathrm{C}\right)\end{array}$} & \multicolumn{3}{|c|}{$\begin{array}{l}\text { Volume Average } \\
\text { Temperature }\left({ }^{\circ} \mathrm{C}\right)\end{array}$} & \multicolumn{3}{|c|}{$\begin{array}{c}\text { Fuel Surface } \\
\text { Temperature }\left({ }^{\circ} \mathrm{C}\right)\end{array}$} & \multicolumn{2}{|c|}{$\begin{array}{c}\text { Fuel Surface } \\
\text { Temperature } \\
\text { Gradient } \\
\left({ }^{\circ} \mathrm{C} / \mathrm{cm}\right)\end{array}$} \\
\hline & & Maximum & Average & EOL & Maximum & Average & EOL (a) & Maximum & Average & EOL & Maximum & Average \\
\hline $01-5$ & $1 \mathrm{~A}$ & 1340 & 1115 & 1050 & 1255 & 1050 & 910 & 1175 & 985 & 950 & 725 & 455 \\
\hline $02-6$ & $1 \mathrm{~B}$ & 1225 & 1050 & 1010 & 1175 & 1000 & 890 & 1135 & 950 & 925 & 480 & 320 \\
\hline $03-5$ & $1 C$ & 1235 & 1065 & 1020 & 1190 & 1015 & 900 & 1150 & 965 & 940 & 465 & 315 \\
\hline $04-6$ & $1 D$ & 1290 & 1115 & 1050 & 1210 & 1045 & 915 & 1155 & 975 & 930 & 695 & 450 \\
\hline $05-6$ & $1 \mathrm{E}$ & 1305 & 1125 & 1040 & 1250 & 1070 & 920 & 1205 & 1010 & 945 & 600 & 405 \\
\hline $06-5$ & $2 \mathrm{~A}$ & 1225 & 1050 & 1170 & 1135 & 975 & 1045 & 1050 & 900 & 1040 & 665 & 515 \\
\hline $07-5$ & $2 B$ & 1220 & 1055 & 1170 & 1135 & 985 & 1050 & 1065 & 910 & 1040 & 585 & 470 \\
\hline $08-13$ & $2 \mathrm{C}$ & 1195 & 1045 & 1130 & 1135 & 980 & 1025 & 1085 & 920 & 1020 & 475 & 395 \\
\hline $09-5$ & 20 & 1180 & 1035 & 1150 & 1110 & 965 & 1035 & $\uparrow 050$ & 895 & 1025 & 550 & 455 \\
\hline $10-5$ & $2 \mathrm{E}$ & 1110 & 965 & 1110 & 1060 & 915 & 995 & 1015 & 860 & 1015 & 410 & 365 \\
\hline $16-5$ & $5 \mathrm{~A}$ & 1515 & 1285 & 1050 & 1460 & 1230 & 1010 & 1400 & 1180 & 935 & 450 & 345 \\
\hline $17-5$ & $5 B$ & 1460 & 1225 & 1020 & 1400 & 1175 & 970 & 1345 & 1130 & 915 & 420 & 295 \\
\hline $18-6$ & $5 \mathrm{C}$ & 1475 & 1235 & 1055 & 1410 & 1180 & 985 & 1350 & 1125 & 930 & 510 & 350 \\
\hline $19-7$ & $5 \mathrm{D}$ & -1480 & 1225 & 1050 & 1410 & 1170 & 980 & 1345 & 1120 & 925 & 505 & 335 \\
\hline $20-6$ & $5 E$ & 1590 & 1280 & 1055 & 1505 & 1215 & 980 & 1425 & 1155 & 920 & 685 & 415 \\
\hline $21-5$ & $6 \mathrm{~A}$ & 1220 & 1065 & 1015 & 1185 & 1035 & 925 & 1145 & 1000 & 940 & 350 & 215 \\
\hline $22-5$ & $6 \mathrm{~B}$ & 1215 & 1055 & 1050 & 1170 & 1020 & 950 & 1125 & 980 & 955 & 395 & 235 \\
\hline $23-5$ & $6 \mathrm{C}$ & 1160 & 1005 & 985 & 1125 & 975 & 895 & 1090 & 950 & 915 & 300 & 175 \\
\hline $24-5$ & $6 \mathrm{D}$ & 1175 & 1020 & 1005 & 1135 & 985 & 905 & 1095 & 950 & 920 & 355 & 210 \\
\hline $25-5$ & $6 \mathrm{E}$ & 1155 & 1000 & 940 & 1120 & 970 & 855 & 1085 & 940 & 865 & 350 & 205 \\
\hline
\end{tabular}

(a) Volume average irradiation temperature for the last cycle of operation. 
TABLE $3-17$

IRRADIATION TEMPERATURES OF CAPSULE P13S FUEL RODS

\begin{tabular}{|c|c|c|c|c|c|c|c|c|c|c|c|c|}
\hline \multirow{2}{*}{$\begin{array}{c}\text { Data } \\
\text { Retrieval } \\
\text { No. } \\
(7161-004-) \\
\end{array}$} & \multirow{2}{*}{$\begin{array}{l}\text { Capsule } \\
\text { Position }\end{array}$} & \multicolumn{3}{|c|}{$\begin{array}{c}\text { Centerline Temperature } \\
\left({ }^{\circ} \mathrm{C}\right)\end{array}$} & \multicolumn{3}{|c|}{$\begin{array}{l}\text { Volume Average } \\
\text { Temperature }\left({ }^{\circ} \mathrm{C}\right)\end{array}$} & \multicolumn{3}{|c|}{$\begin{array}{l}\text { Fuel Surface } \\
\text { Temperature }\left({ }^{\circ} \mathrm{C}\right)\end{array}$} & \multicolumn{2}{|c|}{$\begin{array}{c}\text { Fuel Surface } \\
\text { Temperature } \\
\text { Gradient } \\
\left({ }^{\circ} \mathrm{C} / \mathrm{cm}\right)\end{array}$} \\
\hline & & Maximum & Average & EOL & Maximum & Average & $\mathrm{EQL}^{\text {(a) }}$ & Maximum & Average & EOL & Maximum & Average \\
\hline $\begin{array}{l}01-7 \\
02-5 \\
03-6 \\
04-5 \\
05-5\end{array}$ & $\begin{array}{l}1 \mathrm{~A} \\
1 \mathrm{~B} \\
1 \mathrm{C} \\
1 \mathrm{D} \\
1 \mathrm{E}\end{array}$ & $\begin{array}{l}1545^{(\mathrm{b})} \\
1470(\mathrm{~b}) \\
1505^{(\mathrm{b})} \\
1555^{(\mathrm{b})} \\
1575^{(\mathrm{b})}\end{array}$ & $\begin{array}{r}1030 \\
975 \\
995 \\
1035 \\
1035\end{array}$ & $\begin{array}{r}1015 \\
975 \\
1000 \\
1015 \\
1010\end{array}$ & $\begin{array}{l}1465 \\
1405 \\
1445 \\
1470 \\
1505\end{array}$ & $\begin{array}{l}965 \\
925 \\
950 \\
965 \\
980\end{array}$ & $\begin{array}{l}930 \\
915 \\
935 \\
940 \\
935\end{array}$ & $\begin{array}{l}1380 \\
1345 \\
1380 \\
1385 \\
1430\end{array}$ & $\begin{array}{l}900 \\
880 \\
905 \\
900 \\
925\end{array}$ & $\begin{array}{l}915 \\
900 \\
920 \\
910 \\
920\end{array}$ & $\begin{array}{l}695 \\
435 \\
435 \\
635 \\
555\end{array}$ & $\begin{array}{l}445 \\
300 \\
300 \\
420 \\
385\end{array}$ \\
\hline $\begin{array}{l}11-6 \\
12-5 \\
13-6 \\
14-5 \\
15-5\end{array}$ & $\begin{array}{l}2 A \\
2 B \\
2 C \\
2 D \\
2 E\end{array}$ & $\begin{array}{l}1170 \\
1135 \\
1090 \\
1100 \\
1095\end{array}$ & $\begin{array}{r}1040 \\
1010 \\
975 \\
985 \\
960\end{array}$ & $\begin{array}{l}885 \\
880 \\
860 \\
870 \\
830\end{array}$ & $\begin{array}{l}1105 \\
1065 \\
1040 \\
1045 \\
1050\end{array}$ & $\begin{array}{l}970 \\
945 \\
915 \\
920 \\
910\end{array}$ & $\begin{array}{l}895 \\
895 \\
875 \\
880 \\
850\end{array}$ & $\begin{array}{r}1050 \\
1015 \\
995 \\
995 \\
1010\end{array}$ & $\begin{array}{l}905 \\
880 \\
860 \\
855 \\
860\end{array}$ & $\begin{array}{l}765 \\
770 \\
760 \\
760 \\
745\end{array}$ & $\begin{array}{l}595 \\
530 \\
435 \\
480 \\
385\end{array}$ & $\begin{array}{l}460 \\
420 \\
360 \\
400 \\
335\end{array}$ \\
\hline $\begin{array}{l}16-6 \\
17-6 \\
18-5 \\
19-5 \\
20-5\end{array}$ & $\begin{array}{l}5 \mathrm{~A} \\
5 \mathrm{~B} \\
5 \mathrm{C} \\
5 \mathrm{D} \\
5 \mathrm{E}\end{array}$ & $\begin{array}{l}1645 \\
1605 \\
1625 \\
1610 \\
1650\end{array}$ & $\begin{array}{l}1375 \\
1330 \\
1335 \\
1325 \\
1350\end{array}$ & $\begin{array}{l}1125 \\
1110 \\
1140 \\
1130 \\
1110\end{array}$ & $\begin{array}{l}1595 \\
1560 \\
1570 \\
1560 \\
1585\end{array}$ & $\begin{array}{l}1330 \\
1290 \\
1285 \\
1280 \\
1295\end{array}$ & $\begin{array}{l}1055 \\
1035 \\
1040 \\
1035 \\
1010\end{array}$ & $\begin{array}{l}1545 \\
1515 \\
1510 \\
1505 \\
1525\end{array}$ & $\begin{array}{l}1285 \\
1250 \\
1235 \\
1230 \\
1240\end{array}$ & $\begin{array}{r}1030 \\
1025 \\
1030 \\
1030 \\
995\end{array}$ & $\begin{array}{l}430 \\
405 \\
535 \\
530 \\
670\end{array}$ & $\begin{array}{l}290 \\
255 \\
310 \\
300 \\
370\end{array}$ \\
\hline $\begin{array}{l}26-5 \\
27-5 \\
28-13 \\
29-5 \\
30-5\end{array}$ & $\begin{array}{l}6 \mathrm{~A} \\
6 \mathrm{~B} \\
6 \mathrm{C} \\
6 \mathrm{D} \\
6 \mathrm{E}\end{array}$ & $\begin{array}{l}1250 \\
1215 \\
1215 \\
1185 \\
1165\end{array}$ & $\begin{array}{r}1085 \\
1045 \\
1050 \\
1015 \\
995\end{array}$ & $\begin{array}{l}850 \\
860 \\
845 \\
825 \\
765\end{array}$ & $\begin{array}{l}1210 \\
1170 \\
1185 \\
1150 \\
1130\end{array}$ & $\begin{array}{r}1050 \\
1010 \\
1020 \\
985 \\
965\end{array}$ & $\begin{array}{l}955 \\
935 \\
935 \\
900 \\
855\end{array}$ & $\begin{array}{l}1175 \\
1130 \\
1150 \\
1110 \\
1095\end{array}$ & $\begin{array}{r}1020 \\
975 \\
995 \\
955 \\
935\end{array}$ & $\begin{array}{l}770 \\
775 \\
780 \\
750 \\
695\end{array}$ & $\begin{array}{l}355 \\
360 \\
285 \\
310 \\
315\end{array}$ & $\begin{array}{l}215 \\
215 \\
170 \\
190 \\
195\end{array}$ \\
\hline
\end{tabular}

(a) Volume average irradiation temperature for the last cycle of operation.

(b) Does not include maximum temperature achieved during thermal cycling. 
TABLE $3-18$

VALUES USED IN ESTIMATING UNCERTAINTIES IN FUEL ROD CENTERLINE TEMPERATURES CALCULATED BY THE CAPTEM CODE

\begin{tabular}{|c|c|}
\hline Variable & $\begin{array}{l}\text { Assigned } \\
\text { Uncertainty } \\
(\%)\end{array}$ \\
\hline Fuel rod thermal conductivity & 20 \\
\hline $\begin{array}{l}\text { Fuel heat generation rate (fission and } \\
\text { gamma) }\end{array}$ & 5 \\
\hline $\begin{array}{l}\text { Gamma heating of cell components } \\
\text { Gap widths }\end{array}$ & 10 \\
\hline Fuel - graphite crucible & 10 \\
\hline $\begin{array}{l}\text { Graphite crucible - primary container } \\
\text { Gap conductivities }\end{array}$ & 50 \\
\hline Fuel - graphite crucible & 10 \\
\hline Graphite crucible - primary container & 10 \\
\hline Primary container thermocouple reading & 2 \\
\hline
\end{tabular}

(a) Values give half the range that is expected to contain $95 \%$ of the probable values.

(b) The total uncertainty is the root mean square of the individual uncertainties. 
TABLE 3-19

SUMMARY OF UNBONDED FISSILE PARTICLE TEMPERATURES FOR SAMPLES IRRADIATED IN CELLS 3 AND 4 OF CAPSULE P13R

\begin{tabular}{c|c|c|c}
\hline & & \multicolumn{2}{|c}{$\begin{array}{c}\mid c \\
\text { Ceak Fue1 Temperature }\end{array}$} \\
Capsule & $\begin{array}{c}\text { Data } \\
\text { Retrieval }\end{array}$ & \multicolumn{2}{|c}{ Tray $\left({ }^{\circ} \mathrm{C}\right)$} \\
\cline { 2 - 4 } Position & Number & Time Average & Maximum \\
\hline $3-1$ & $6151-00-010-10$ & 1015 & 1360 \\
$3-2$ & $6151-00-010-11$ & 1015 & 1360 \\
$3-3$ & $6151-00-010-12$ & 1075 & 1400 \\
$3-4$ & $6151-00-035-1$ & 1075 & 1400 \\
$3-5$ & $6151-00-035-2$ & 1075 & 1400 \\
$3-6$ & $6151-00-035-3$ & 1075 & 1400 \\
$3-7$ & $6151-00-045-1$ & 1075 & 1400 \\
$3-8$ & $6151-00-045-2$ & 1075 & 1400 \\
$3-9$ & $6151-00-045-3$ & 1075 & 1400 \\
$3-10$ & $6151-00-046-1$ & 1025 & 1345 \\
$3-11$ & $6151-04-015-1$ & 1025 & 1345 \\
$3-12$ & $6151-04-015-2$ & 960 & 1325 \\
$4-1$ & $6151-01-015-1$ & 1010 & 1455 \\
$4-2$ & $6151-01-015-2$ & 1070 & 1400 \\
$4-3$ & $6151-01-015-3$ & 1070 & 1400 \\
$4-4$ & $6151-00-025-1$ & 1070 & 1400 \\
$4-5$ & $6151-00-025-2$ & 1070 & 1400 \\
$4-6$ & $6151-00-025-3$ & 1070 & 1400 \\
$4-7$ & $6151-09-015-1$ & 1070 & 1400 \\
$4-8$ & $6151-09-015-2$ & 1070 & 1400 \\
$4-9$ & $6151-09-015-3$ & 1070 & 1345 \\
$4-10$ & $6151-09-025-1$ & 1005 & 1315 \\
$4-11$ & $6151-09-025-2$ & 1005 & \\
$4-12$ & $6151-09-025-3$ & 935 & \\
\hline
\end{tabular}


TABLE $3-20$

SUMMARY OF UNBONDED FISSILE PARTICLE TEMPERATURES FOR SAMPLES IRRADIATED IN CELLS 3 AND 4 OF CAPSULE P13S

\begin{tabular}{|c|c|c|c|}
\hline \multirow{2}{*}{$\begin{array}{l}\text { Capsule } \\
\text { Position }\end{array}$} & \multirow{2}{*}{$\begin{array}{c}\text { Data } \\
\text { Retrieva1 } \\
\text { Number }\end{array}$} & \multicolumn{2}{|c|}{$\begin{array}{c}\text { Peak Fuel Temperature } \\
\text { in Tray }\left({ }^{\circ} \mathrm{C}\right)\end{array}$} \\
\hline & & Time Average & Maximum \\
\hline Ce11 $\begin{array}{l}3-1 \\
3-2 \\
3-3 \\
3-4 \\
3-5 \\
3-6 \\
3-7 \\
3-8 \\
3-9 \\
3-10 \\
3-11 \\
3-12\end{array}$ & $\begin{array}{l}6151-03-015-1 \\
6151-03-015-2 \\
6151-03-015-3 \\
4161-01-021-1 \\
4161-01-021-2 \\
4161-01-021-3 \\
6151-08-015-1 \\
6151-08-015-2 \\
6151-08-015-3 \\
6151-02-025-1 \\
6151-02-025-2 \\
6151-02-025-3\end{array}$ & $\begin{array}{r}970 \\
970 \\
1020 \\
1020 \\
1020 \\
1020 \\
1020 \\
1020 \\
1020 \\
980 \\
980 \\
920\end{array}$ & $\begin{array}{l}1255 \\
1255 \\
1280 \\
1280 \\
1280 \\
1280 \\
1280 \\
1280 \\
1280 \\
1230 \\
1230 \\
1200\end{array}$ \\
\hline $\begin{array}{c}\text { Ce11 } 4-1 \\
4-2 \\
4-3 \\
4-4 \\
4-5 \\
4-6 \\
4-7 \\
4-8 \\
4-9 \\
4-10 \\
4-11 \\
4-12\end{array}$ & $\begin{array}{l}4163-00-011-3 \\
4163-00-011-4 \\
4163-00-011-5 \\
6155-01-020-10 \\
6155-01-020-11 \\
6155-01-020-12 \\
5466-37-9 \\
5466-37-10 \\
5466-37-11 \\
6151-00-035-4 \\
6151-00-035-5 \\
6151-00-035-6\end{array}$ & $\begin{array}{r}1045 \\
1090 \\
1090 \\
1090 \\
1090 \\
1090 \\
1090 \\
1090 \\
1090 \\
1035 \\
1035 \\
975\end{array}$ & $\begin{array}{l}1385 \\
1430 \\
1430 \\
1430 \\
1430 \\
1430 \\
1430 \\
1430 \\
1430 \\
1370 \\
1370 \\
1345\end{array}$ \\
\hline
\end{tabular}


TABLE $3-21$

SUMMARY OF UNBONDED FERTILE PARTICLE TEMPERATURES FOR SAMPLES IRRADIATED IN CELLS 3 AND 4 OF CAPSULE P13R

\begin{tabular}{c|c|c|c}
\hline & \multirow{2}{*}{$\begin{array}{c}\text { Data } \\
\text { Retrieval } \\
\text { Nupsule }\end{array}$} & \multicolumn{2}{|c}{$\begin{array}{c}\text { Peak Fuel Temperature } \\
\text { in Tray }\end{array}$} \\
\cline { 3 - 4 } Position & \multicolumn{2}{|c}{$\mathrm{C})$} \\
\cline { 3 - 4 } Cell $3-1$ & $4252-06-018-1$ & 960 & 1055 \\
$3-2$ & $4252-06-018-2$ & 960 & 1055 \\
$3-3$ & $6542-01-020-1$ & 1010 & 1105 \\
$3-4$ & $6542-01-020-2$ & 1010 & 1105 \\
$3-5$ & $6542-01-010-1$ & 1010 & 1105 \\
$3-6$ & $6542-01-010-2$ & 1010 & 1105 \\
$3-7$ & $6542-02-020-1$ & 1010 & 1105 \\
$3-8$ & $6542-02-020-2$ & 1010 & 1105 \\
$3-9$ & $6542-02-030-1$ & 1010 & 1105 \\
$3-10$ & $6542-02-030-2$ & 970 & 1065 \\
$3-11$ & $6542-09-010-1$ & 970 & 1065 \\
$3-12$ & $6542-09-010-2$ & 895 & 975 \\
$4-1$ & $6542-11-015-1$ & 945 & 1020 \\
$4-2$ & $6542-11-015-2$ & 1000 & 1115 \\
$4-3$ & $6542-18-015-1$ & 1000 & 1115 \\
$4-4$ & $6542-18-015-2$ & 1000 & 1115 \\
$4-5$ & $6542-02-020-3$ & 1000 & 1115 \\
$4-6$ & $6542-02-020-4$ & 1000 & 1115 \\
$4-7$ & $6542-20-035-1$ & 1000 & 1115 \\
$4-8$ & $6542-20-035-2$ & 1000 & 1115 \\
$4-9$ & $6542-20-025-1$ & 1000 & 1115 \\
$4-10$ & $6542-20-025-2$ & 940 & 1050 \\
$4-11$ & $6542-25-015-1$ & 940 & 1050 \\
$4-12$ & $6542-25-015-2$ & 870 & 970 \\
\hline
\end{tabular}


TABLE 3-22

SUMMARY OF UNBONDED FERTILE PARTICLE TEMPERATURES FOR SAMPLES IRRADIATED IN CELLS 3 AND 4 OF CAPSULE P13S

\begin{tabular}{c|c|c|c}
\hline & \multirow{2}{*}{$\begin{array}{c}\text { Data } \\
\text { Retrieval } \\
\text { Capsule }\end{array}$} & \multicolumn{2}{|c}{$\begin{array}{c}\text { Peak Fuel Temperature } \\
\text { in Tray }\end{array}$} \\
\cline { 3 - 4 } Position & $\left.{ }^{\circ} \mathrm{C}\right)$ \\
\cline { 3 - 4 } Ce11 $3-1$ & $4252-06-010-1$ & Time Average & Maximum \\
$3-2$ & $4252-06-010-2$ & 925 & 1035 \\
$3-3$ & $6542-22-015-1$ & 925 & 1035 \\
$3-4$ & $6542-22-015-2$ & 955 & 1075 \\
$3-5$ & $6542-22-025-1$ & 955 & 1075 \\
$3-6$ & $6542-22-025-2$ & 955 & 1075 \\
$3-7$ & $6542-23-025-1$ & 955 & 1075 \\
$3-8$ & $6542-23-025-2$ & 955 & 1075 \\
$3-9$ & $6542-24-015-1$ & 955 & 1075 \\
$3-10$ & $6542-24-015-2$ & 955 & 1075 \\
$3-11$ & $6252-00-025-1$ & 925 & 1035 \\
$3-12$ & $6252-00-025-2$ & 925 & 1035 \\
Ce11 & 860 & 960 \\
$4-1$ & $6542-17-010-1$ & 910 & 1040 \\
$4-2$ & $4252-02-010-1$ & 950 & 1085 \\
$4-3$ & $4252-02-010-2$ & 950 & 1085 \\
$4-4$ & $6542-16-010-1$ & 950 & 1085 \\
$4-5$ & $6542-16-010-2$ & 950 & 1085 \\
$4-6$ & $6542-16-010-3$ & 950 & 1085 \\
$4-7$ & $6542-19-015-1$ & 950 & 1085 \\
$4-8$ & $6542-19-016-1$ & 950 & 1085 \\
$4-9$ & $6542-21-016-1$ & 950 & 1085 \\
$4-10$ & $6542-21-015-1$ & 900 & 1025 \\
$4-11$ & $6542-12-025-1$ & 900 & 1025 \\
$4-12$ & $6542-12-025-2$ & 830 & 940 \\
& & &
\end{tabular}


TABLE 3-23

DECALIBRATION FACTORS DERIVED FOR W/Re THERMOCOUPLES IRRADIATED IN CAPSULES P13R AND P13S

\begin{tabular}{|c|c|c|c|c|c|}
\hline \multirow[b]{2}{*}{ Capsule-Ce11 } & \multirow{2}{*}{$\begin{array}{c}\text { Thermocouple } \\
\text { Location }\end{array}$} & \multirow{2}{*}{$\begin{array}{c}\text { Average } \\
\text { Thermocouple } \\
\text { Temperature, } \overline{\mathrm{T}} \\
\left({ }^{\circ} \mathrm{C}\right)\end{array}$} & \multicolumn{2}{|c|}{$\begin{array}{l}\text { Coefficients for } \\
\text { Linear Equation } \\
\Delta \mathrm{T}=\mathrm{a} \phi_{\mathrm{Th}}+\mathrm{b}\end{array}$} & \multirow{2}{*}{$\begin{array}{c}\text { Decalibration Factor, } \mathrm{m} \\
(\mathrm{m}=\mathrm{b} / \overline{\mathrm{T}}) \\
\left(\mathrm{n} / \mathrm{cm}^{2}\right)^{-1}\end{array}$} \\
\hline & & & a $\left({ }^{\circ} \mathrm{C}\right)$ & $\mathrm{b}\left({ }^{\circ} \mathrm{C} \mathrm{cm}^{2} / \mathrm{n}\right)$ & \\
\hline P13R-1 & Fue1 & 1150 & 17 & -71 & -0.0614 \\
\hline $\mathrm{P} 13 \mathrm{R}-2$ & Fuel & 1095 & 77 & -70 & -0.0636 \\
\hline P13R-5 & Fue1 & 1305 & -76 & -112 & -0.0855 \\
\hline$P 13 R-6$ & Fue1 & 980 & -69 & -73 & -0.0747 \\
\hline P13S-1 & Fue1 & 1140 & 79 & -69 & -0.0604 \\
\hline P13S-2 & Fue1 & 1100 & 59 & -65 & -0.0594 \\
\hline $\mathrm{P} 13 \mathrm{~S}-5$ & Fue1 & 1450 & -19 & -135 & -0.0926 \\
\hline P13S-6 & Fue1 & 1010 & -57 & -97 & -0.0963 \\
\hline
\end{tabular}




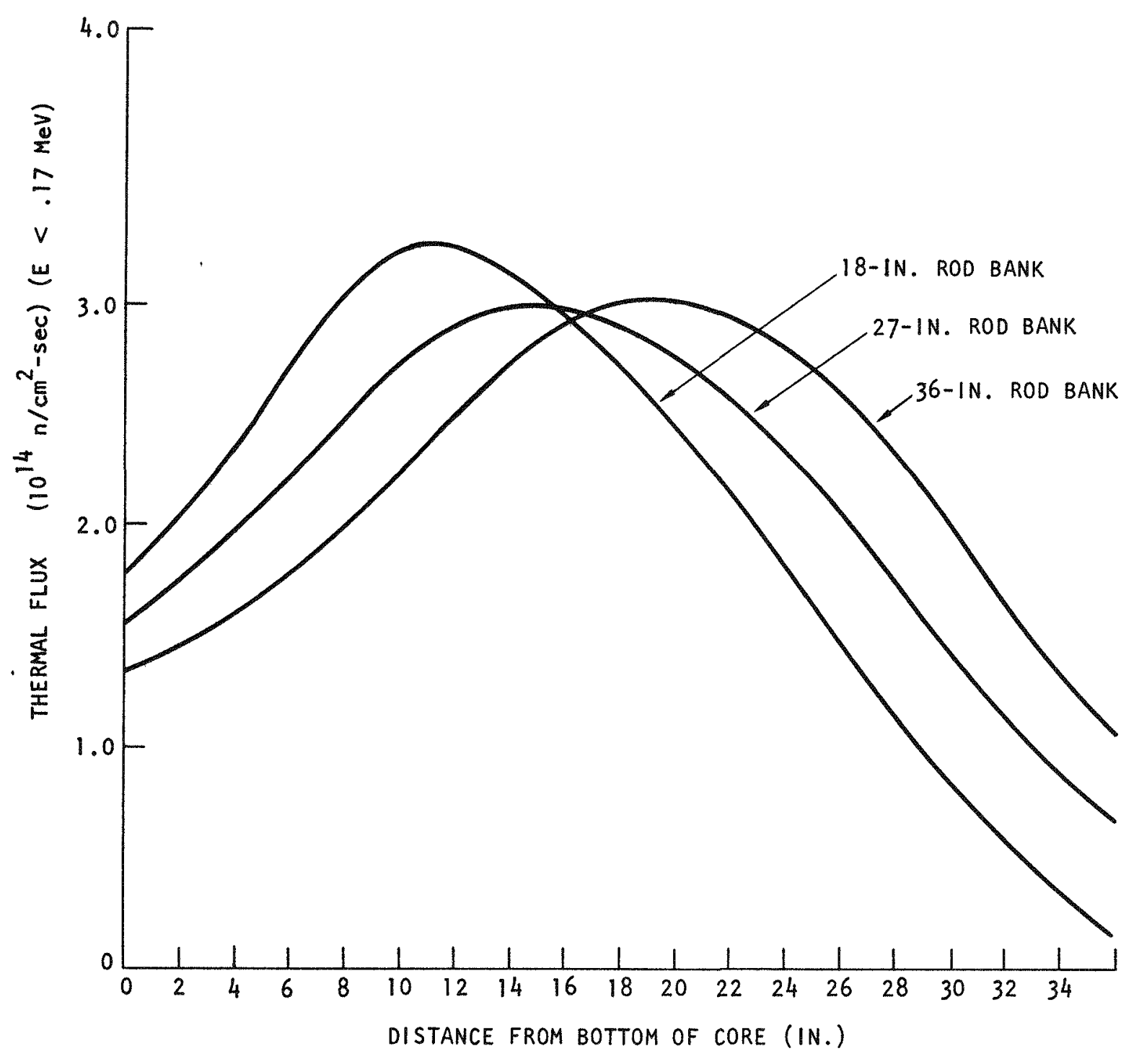

Fig. 3-1. GETR thermal flux profiles for three control rod bank positions 


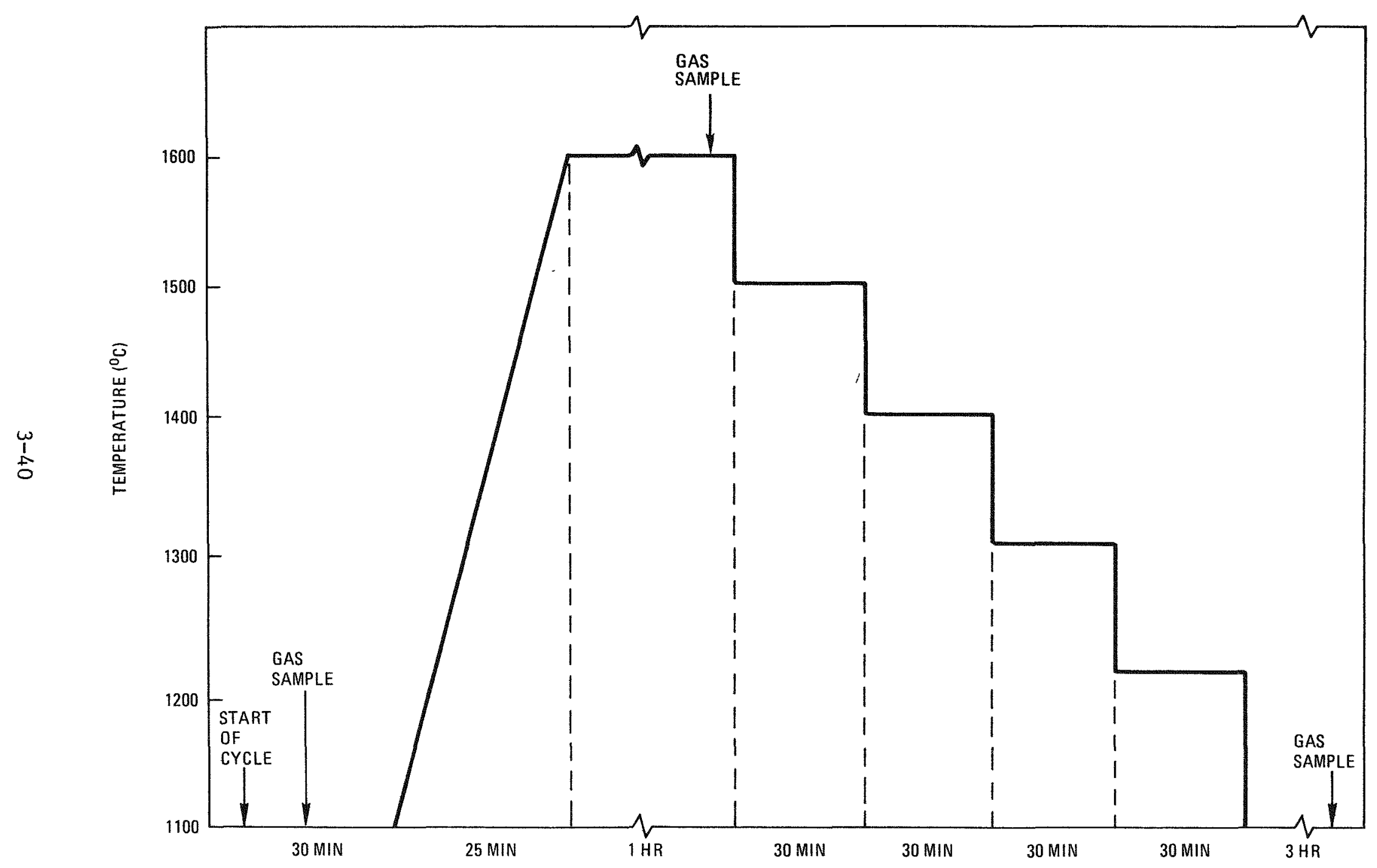

Fig. 3-2. Design 1oad-following transient for cell 1 of capsule P13S 


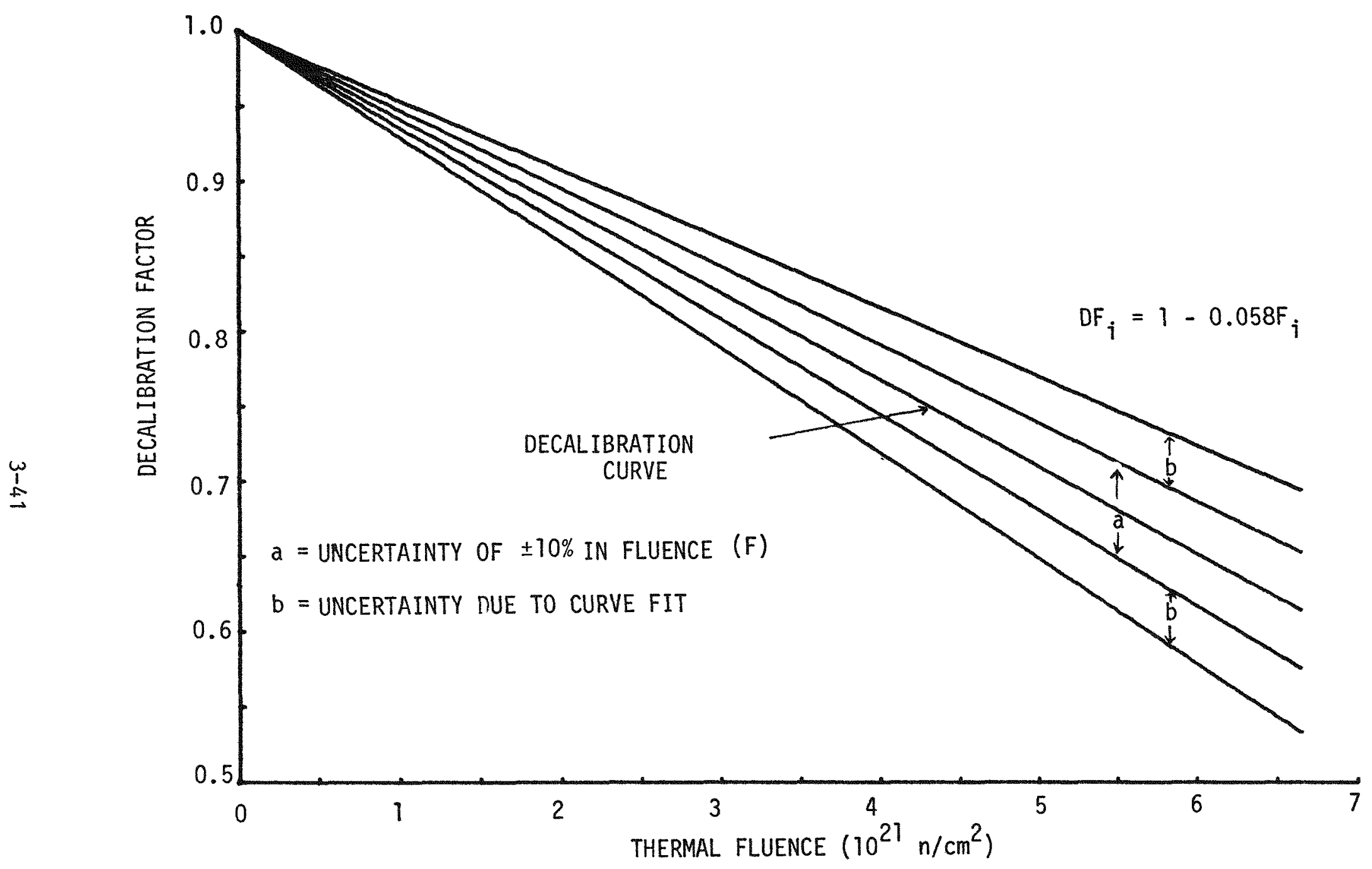

Fig. 3-3. Decalibration curves used to correct tungsten-rhenium thermocouple drift in cells 1 , 2 , 4, and 6 in capsules P13R and P13S. Data derived from ce11 2 of capsule P13S. 


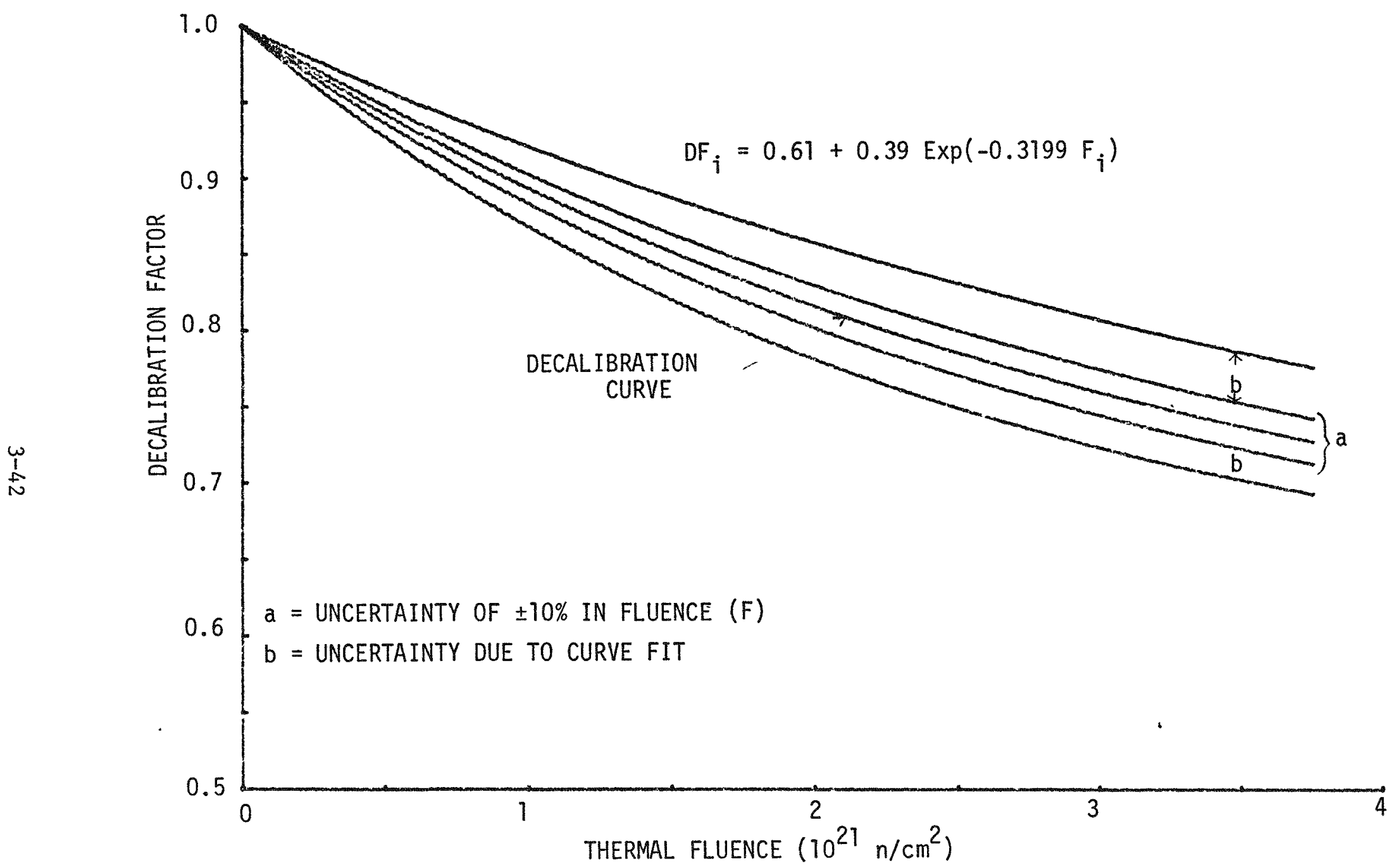

Fig. 3-4. Decalibration curves used to correct tungsten-rhenium thermocouple drift in cell 5 in capsule P13R 


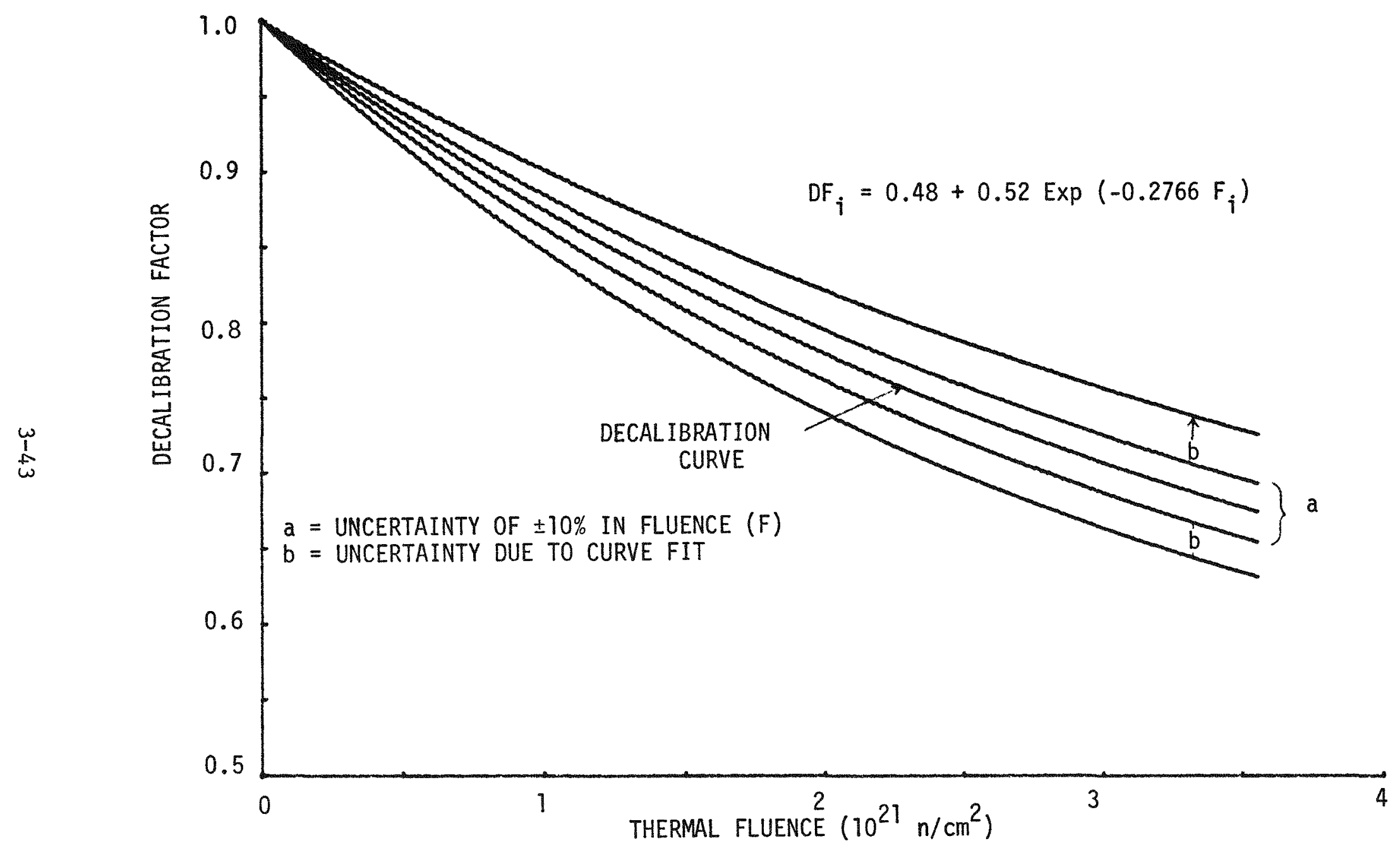

Fig. 3-5. Decalibration curves used to correct tungsten-rhenium thermocouple drift in cell 5 in capsule P13S 


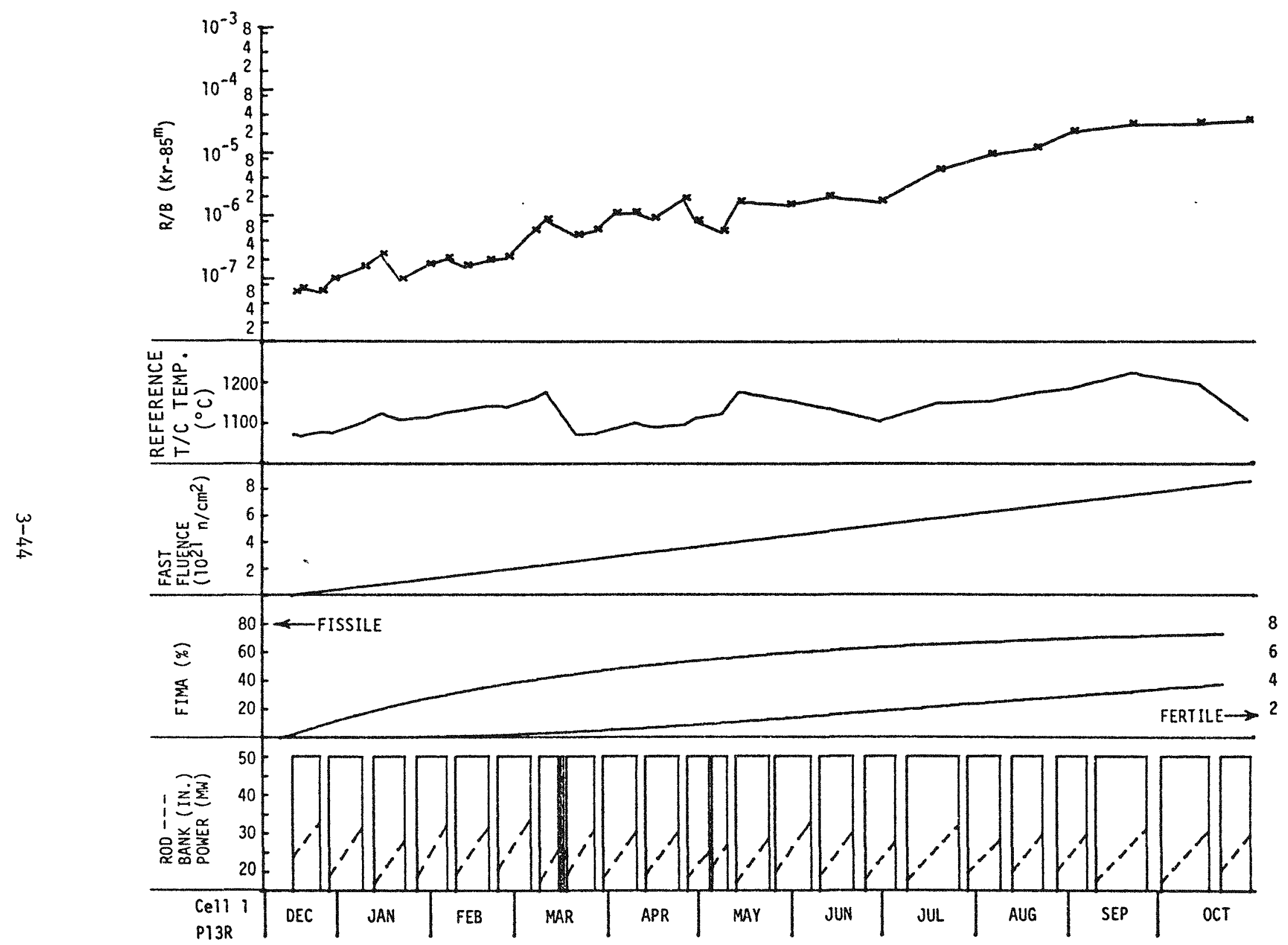

Fig. 3-6. Operating history for cel1 1 of capsule P13R 


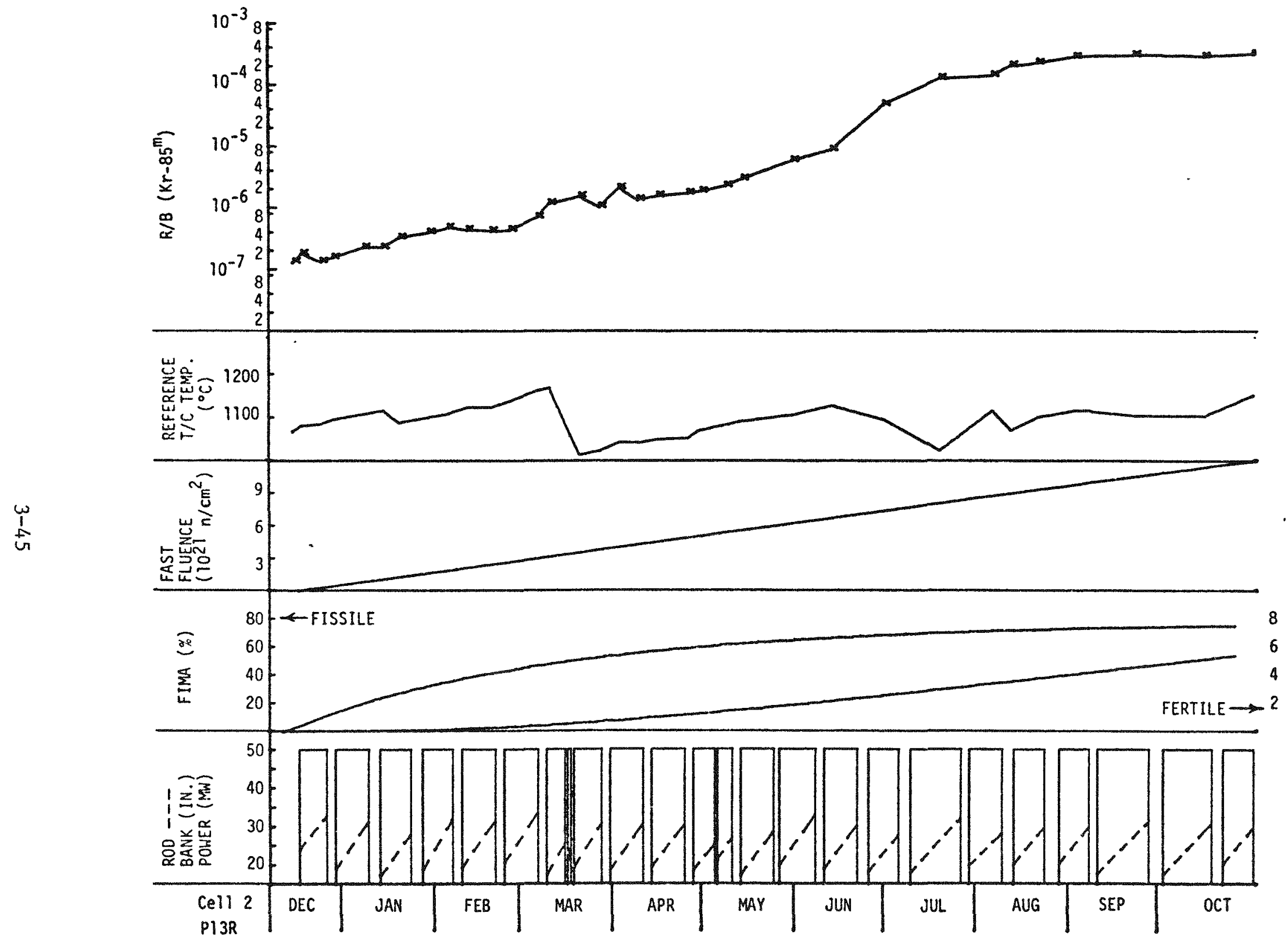

Fig. 3-7. Operating history for cell 2 of capsule P13R 


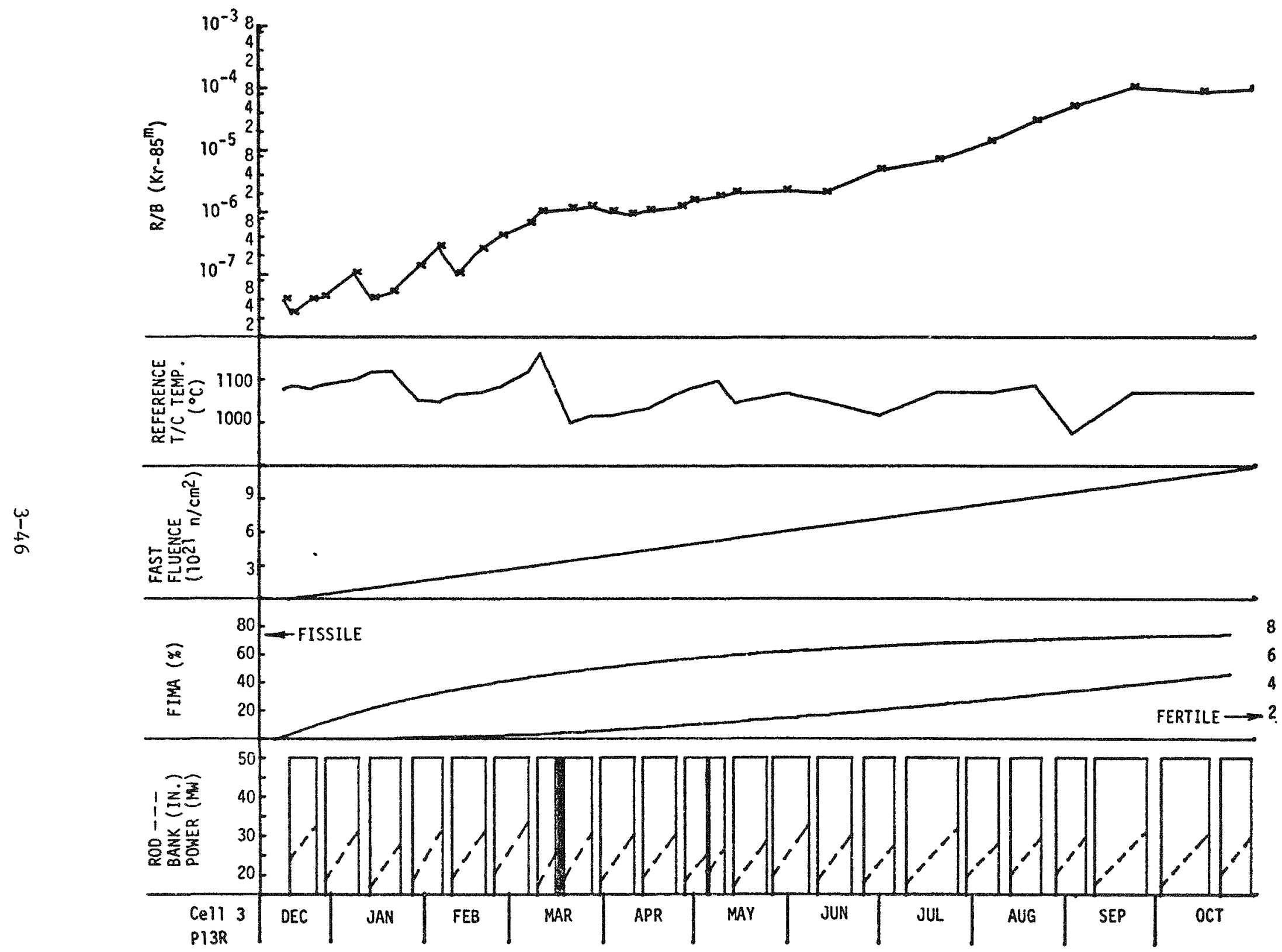

Fig. 3-8. Operating history for cell 3 of capsule P13R 


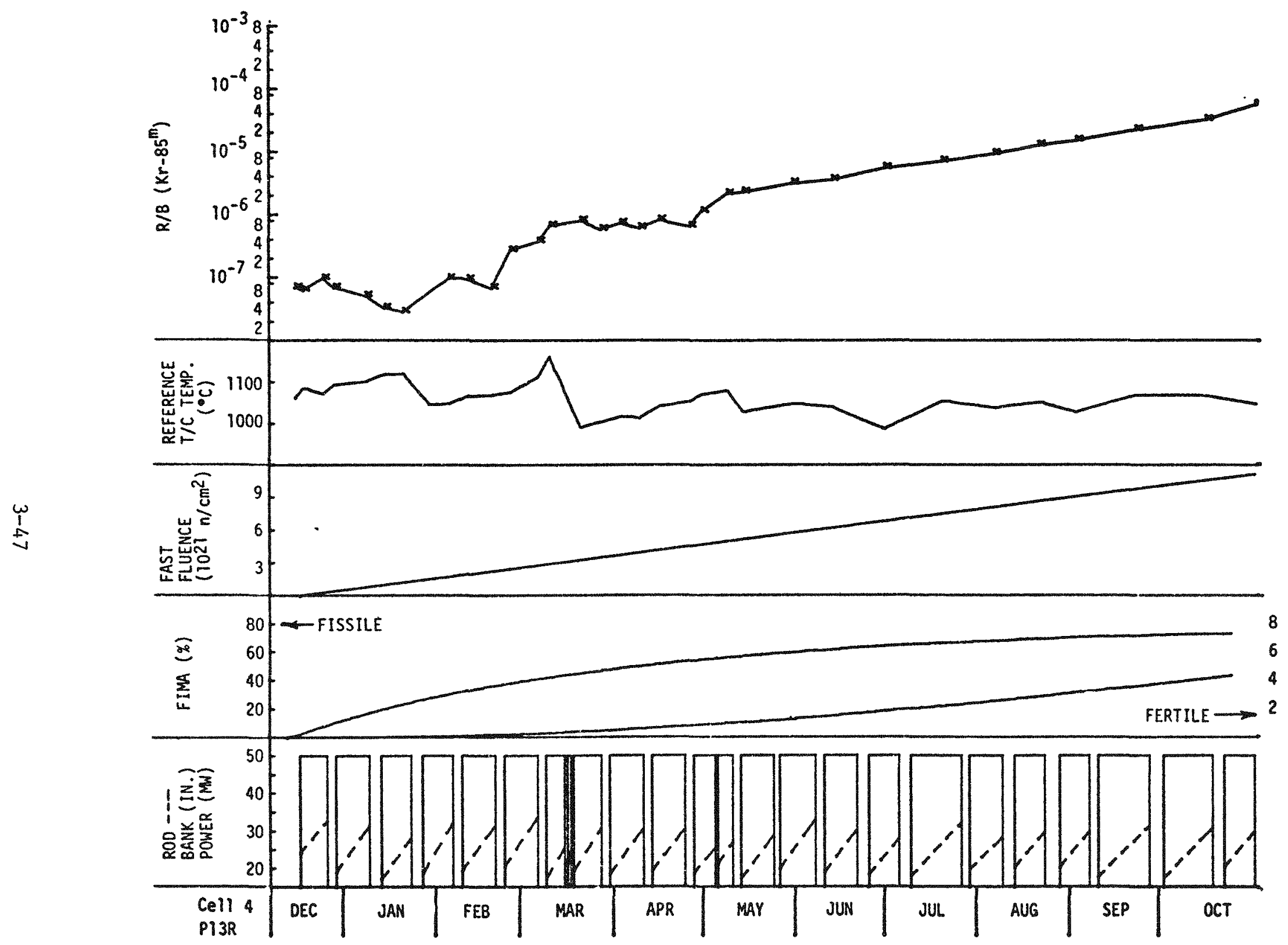

Fig. 3-9. Operating history for cel1 4 of capsule P13R 


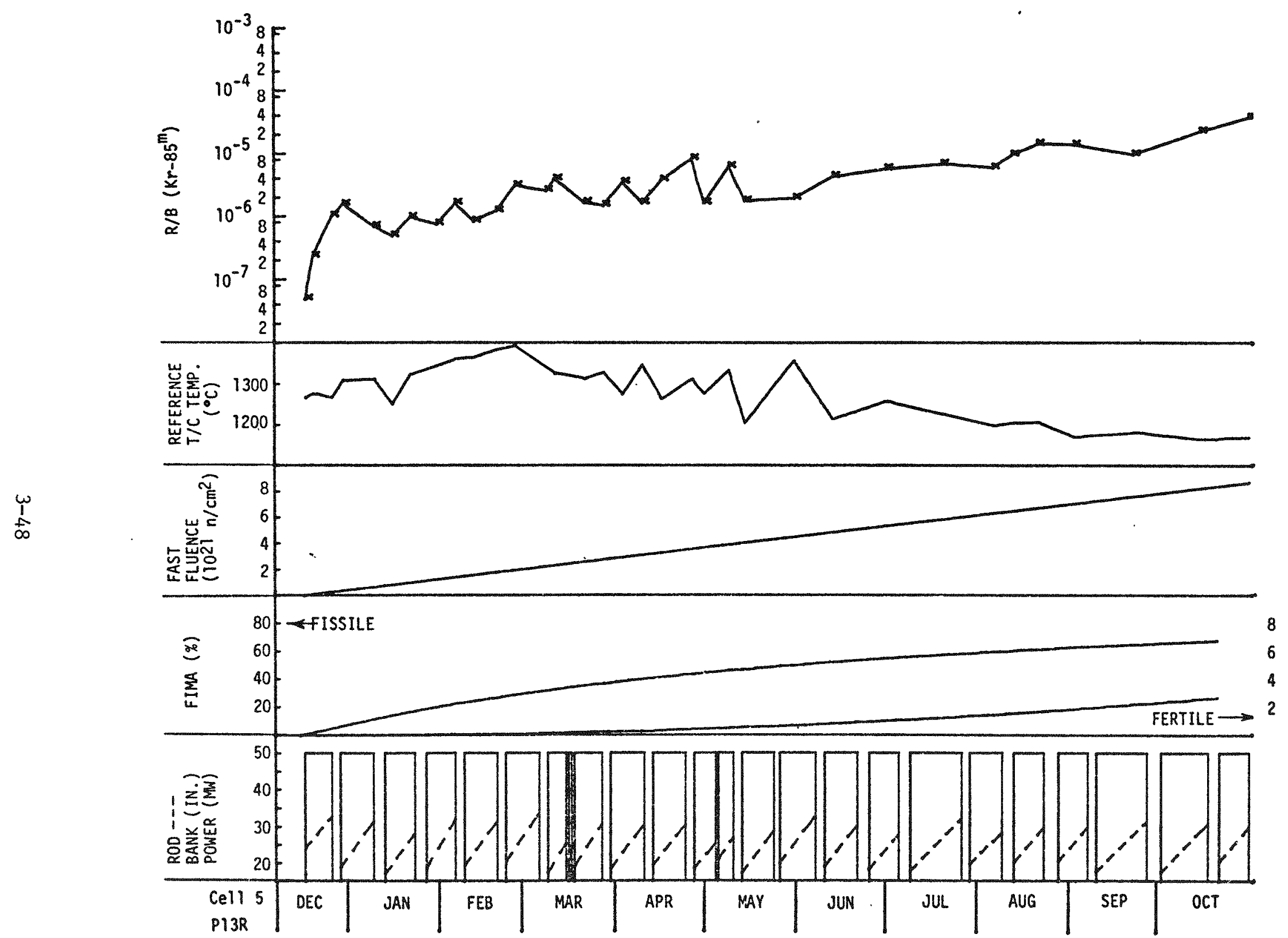

Fig. 3-10. Operating history for cell 5 of capsule P13R 


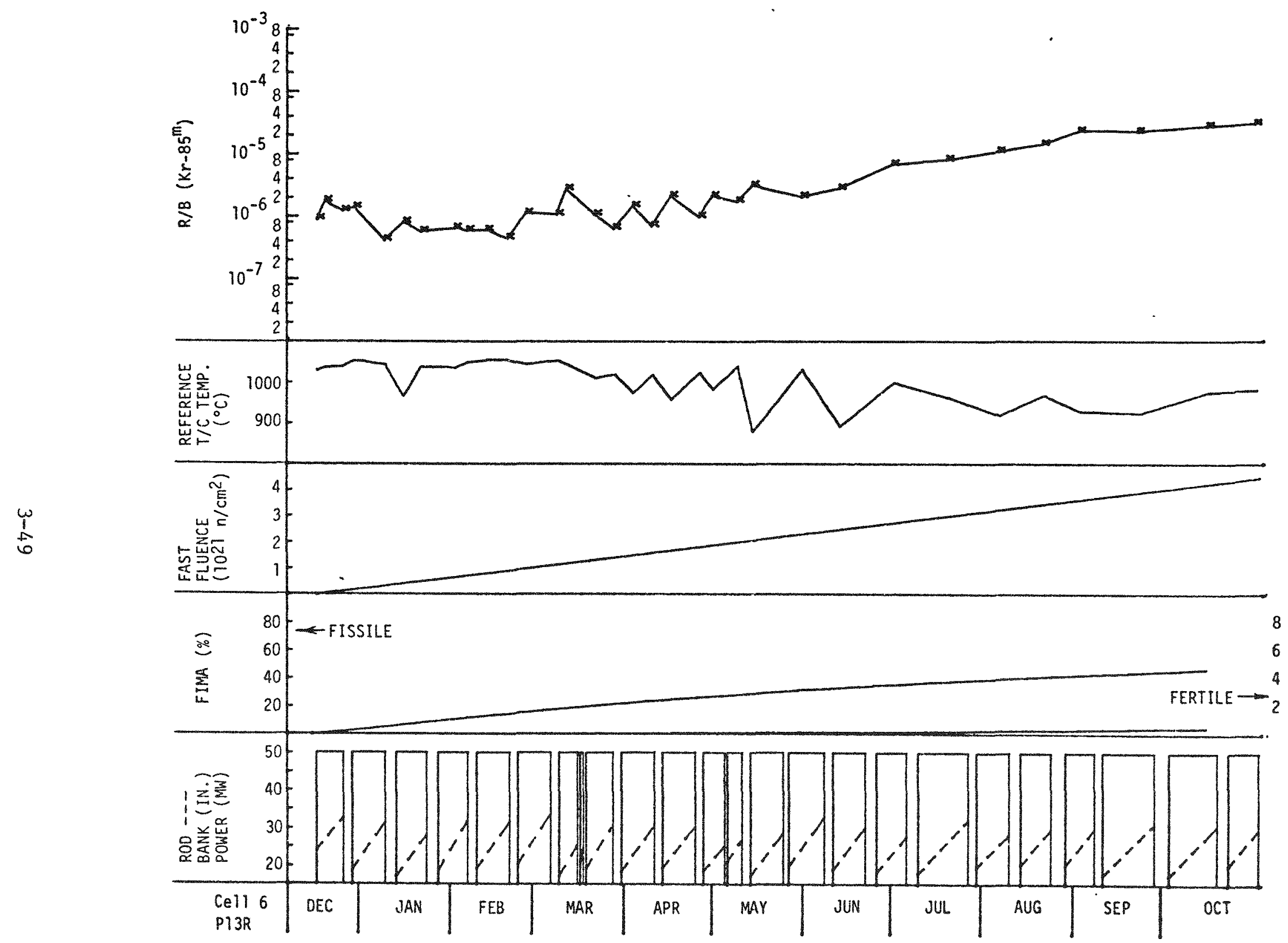

Fig. 3-11. Operating history for cel1 6 of capsule P13R 


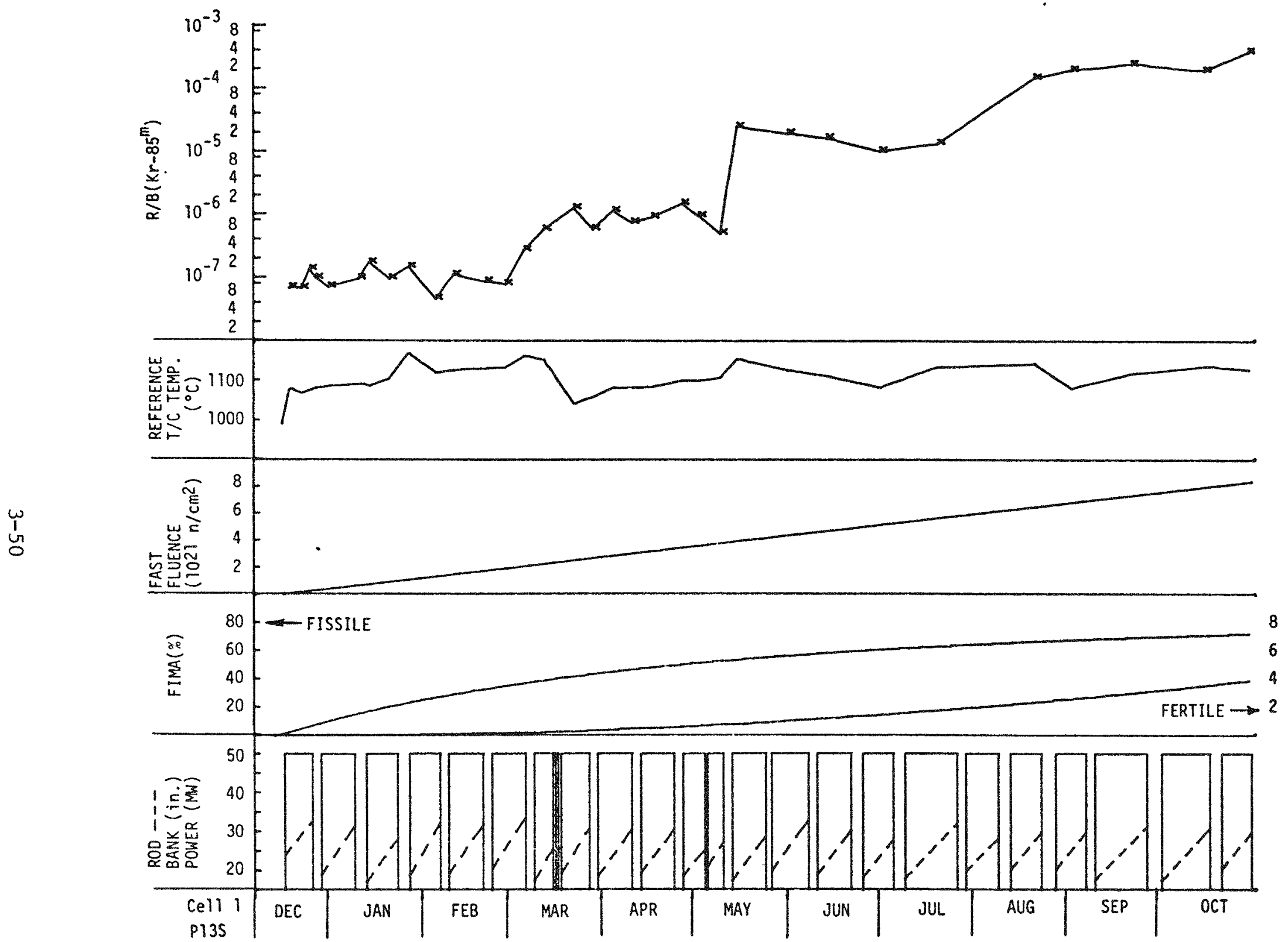

Fig. 3-12. Operating history for cell 1 of capsule P13S 


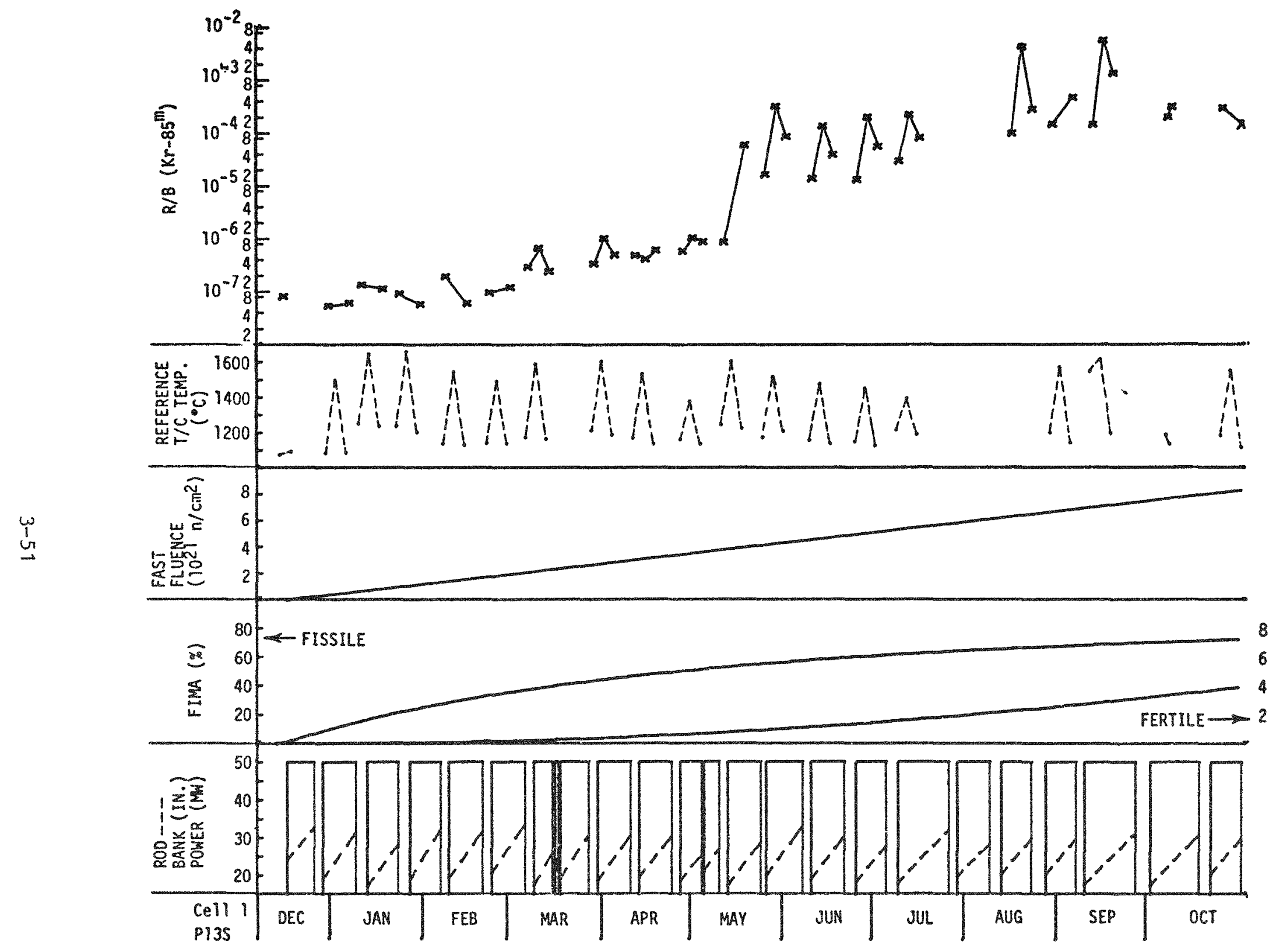

Fig. 3-13. Thermal cycle operating history for cell 1 of capsule P13S 


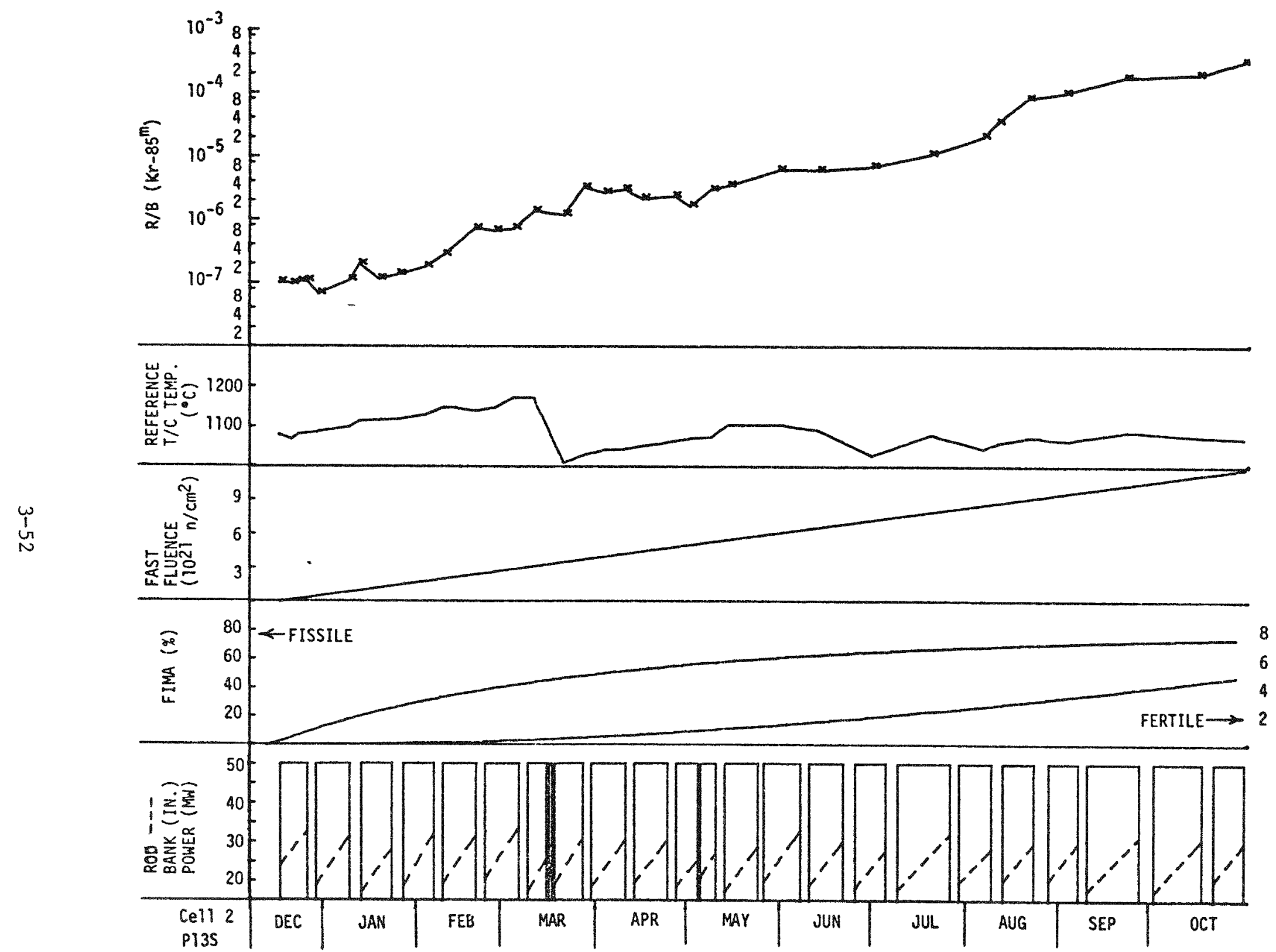

Fig. 3-14. Operating history for cell 2 of capsule P13S 


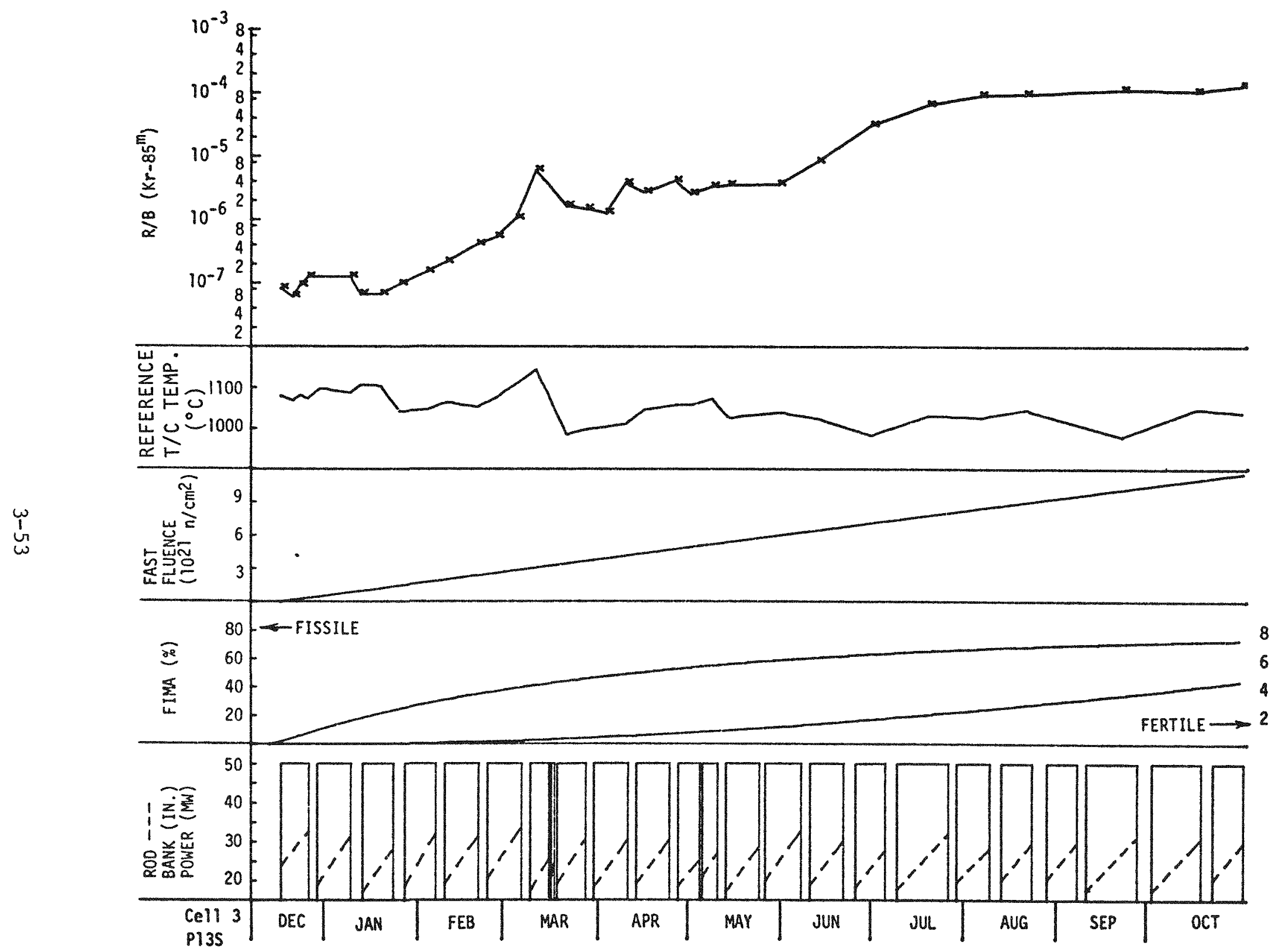

Fig. 3-15. Operating history for cell 3 of capsule P13S 


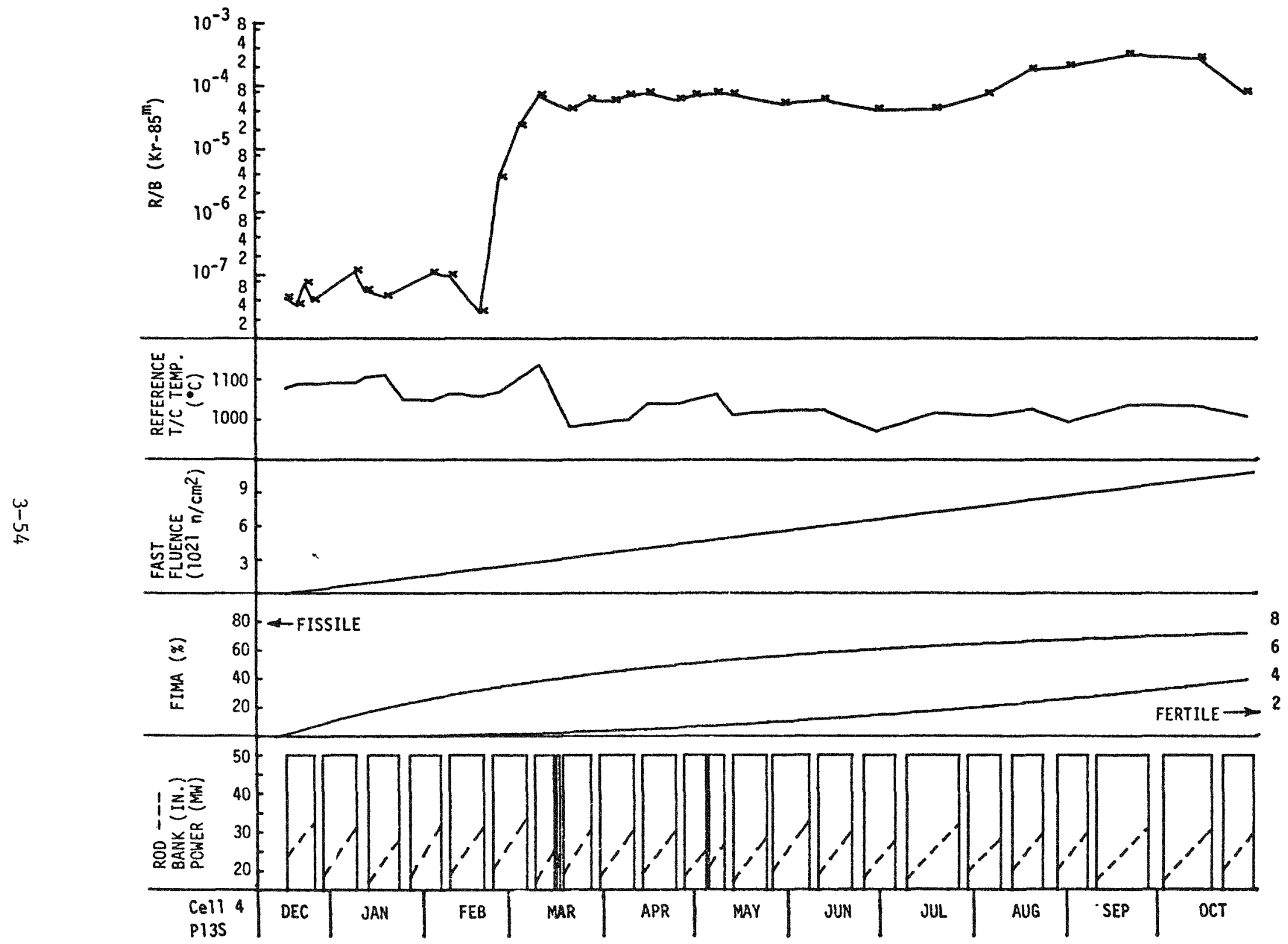

Fig. 3-16. Operating history for cell 4 of capsule P13S 


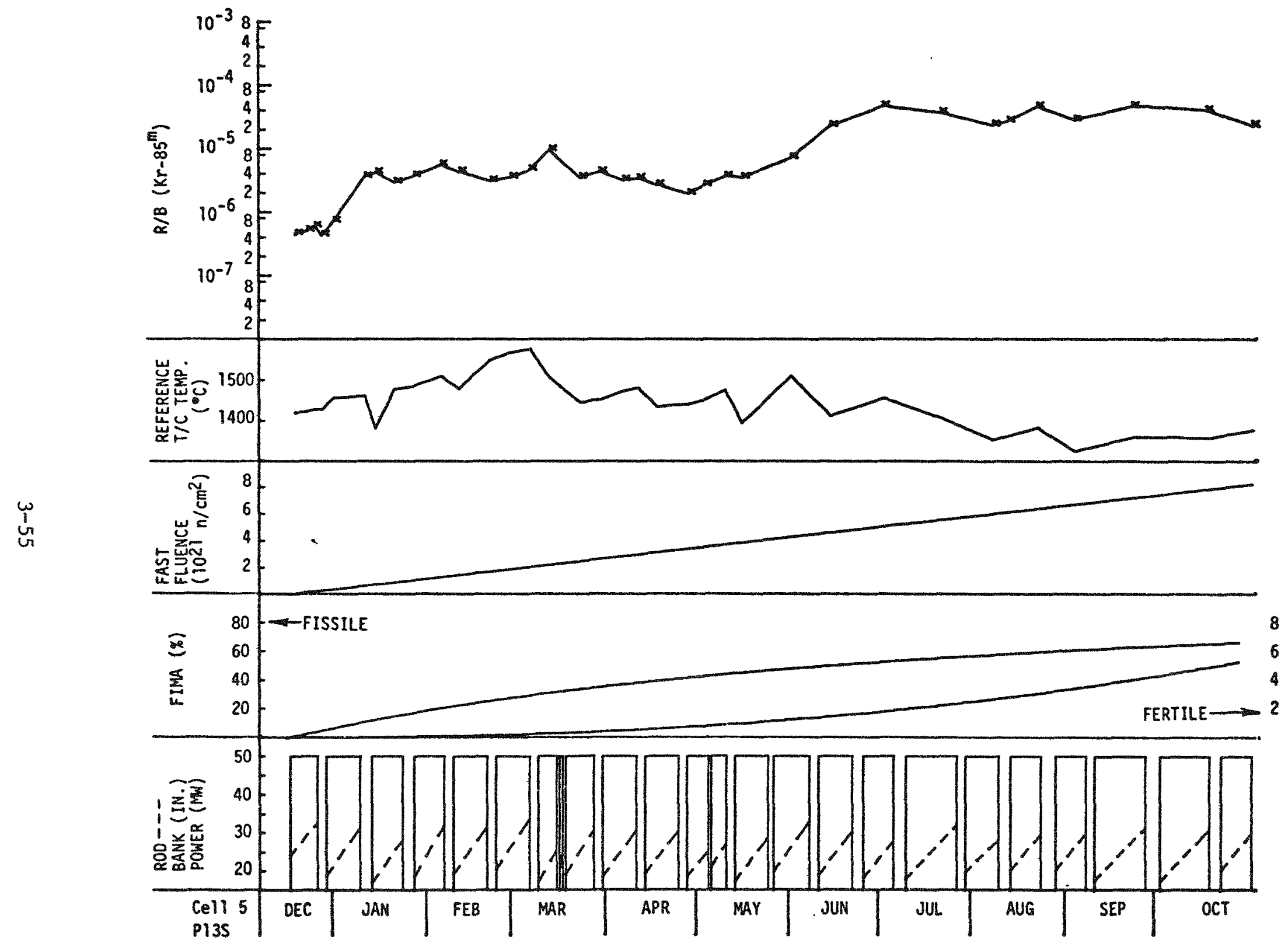

Fig. 3-17. Operating history for cell 5 of capsule P13S 


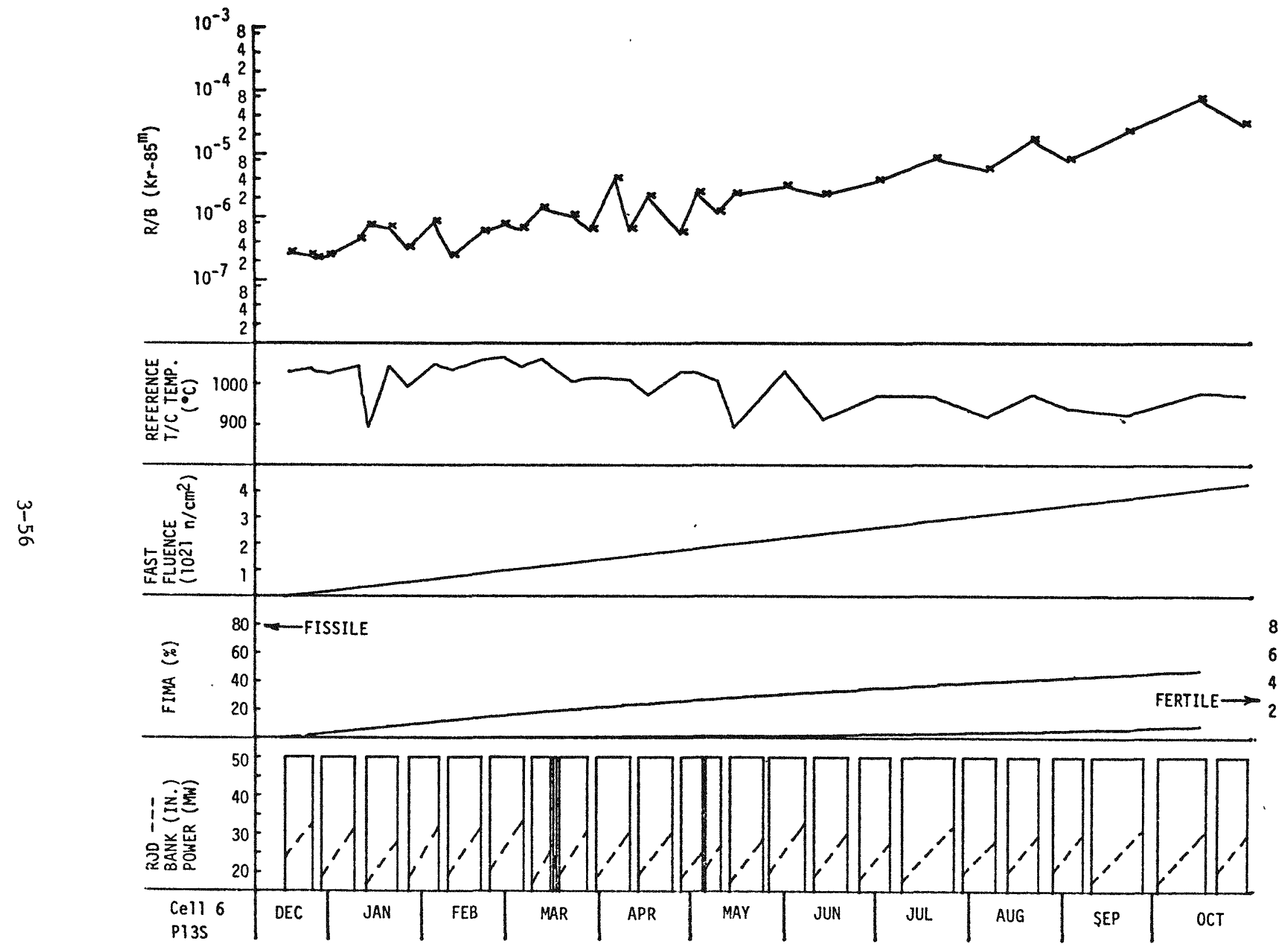

Fig. 3-18. Operating history for cell 6 of capsule P13S 

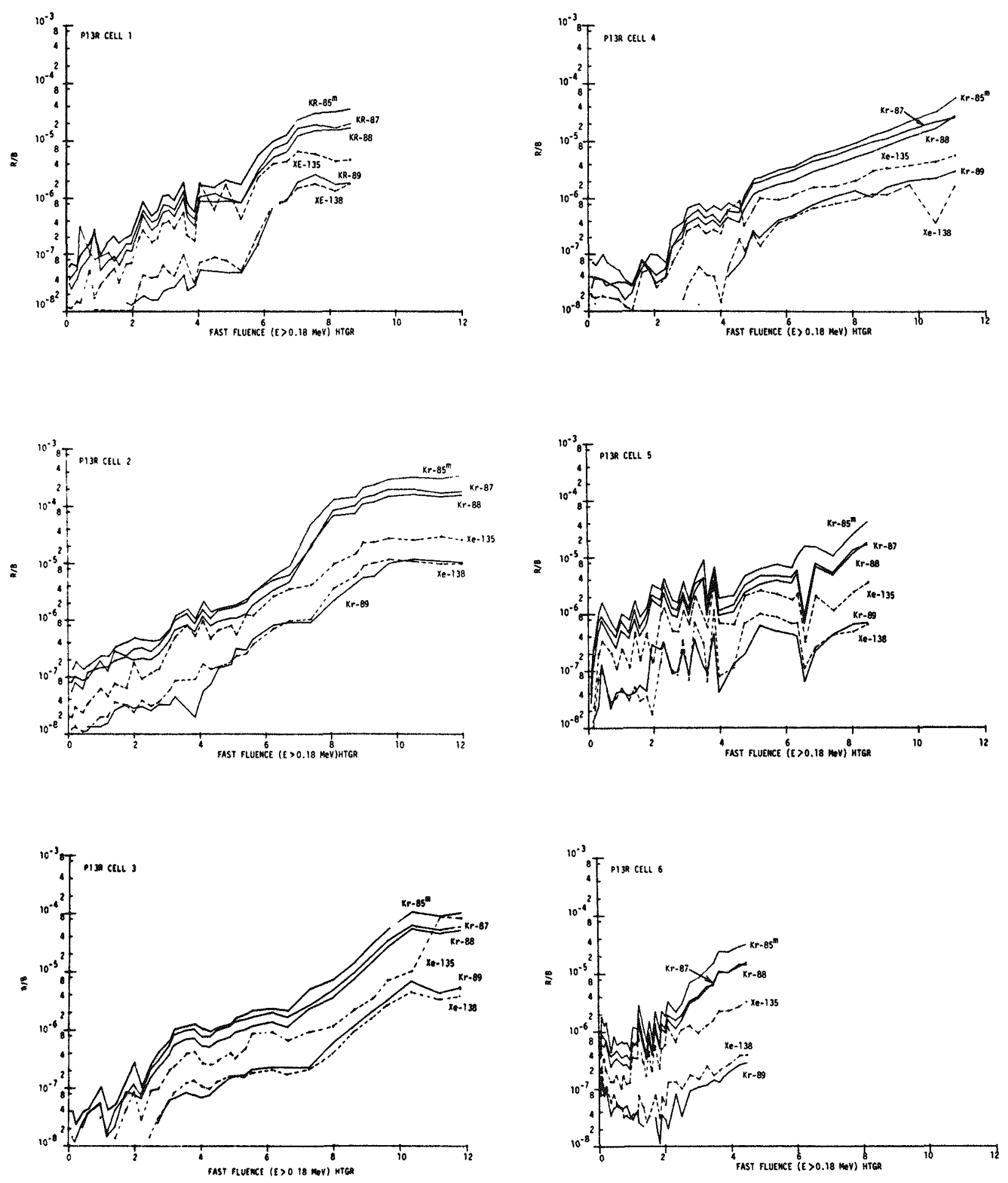

Fig. 3-19. Ratio of fission gas release to birth rate $(R / B)$ versus fast fluence $(E>0.18 \mathrm{MeV})_{\mathrm{HTGR}}$ for each cell in capsule P13R 

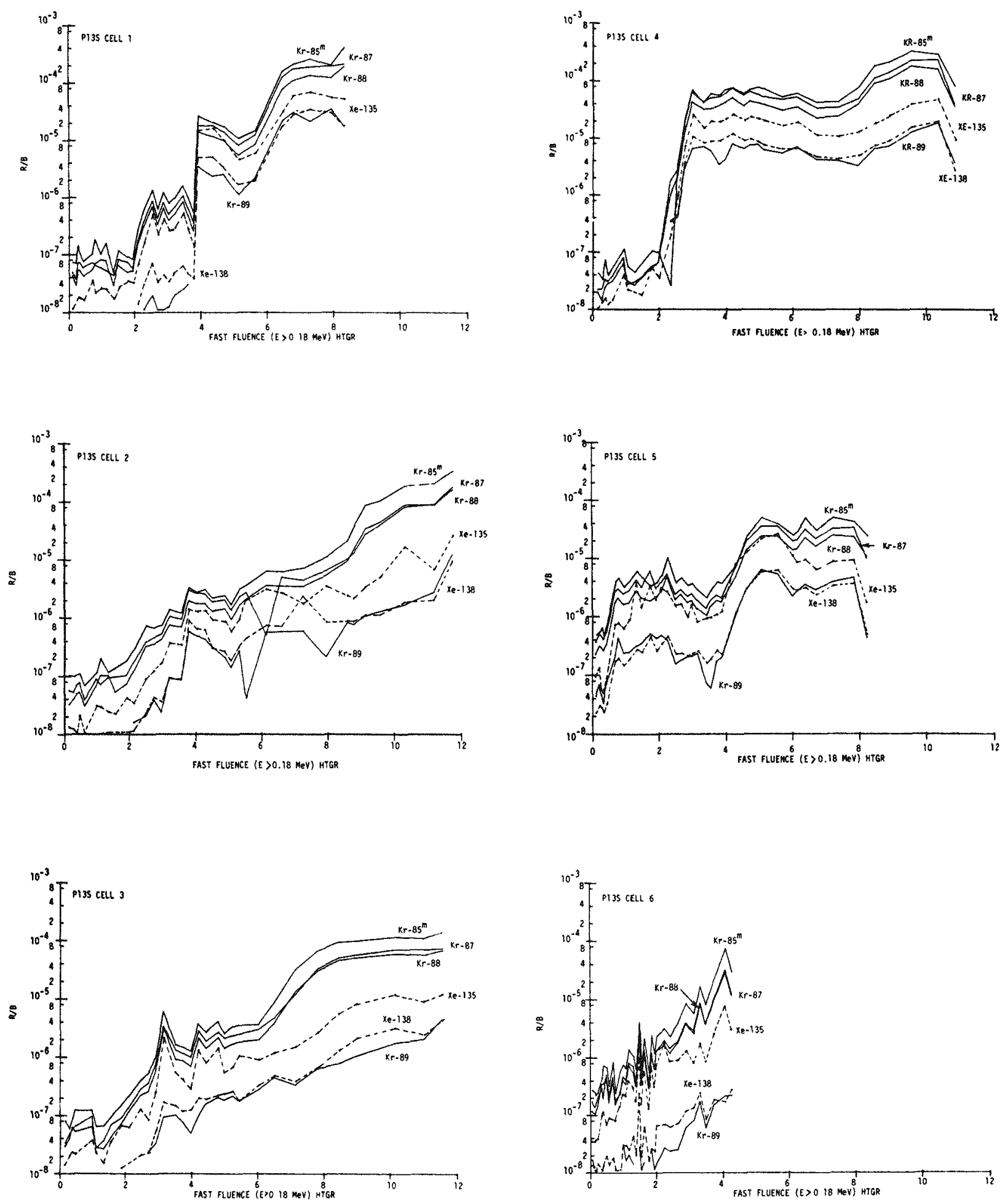

Fig. 3-20. Ratio of fission gas release to birth rate (R/B) versus fast fluence $(E>0.18 \mathrm{MeV})_{\text {HTGR }}$ for each cell in capsule P13S 


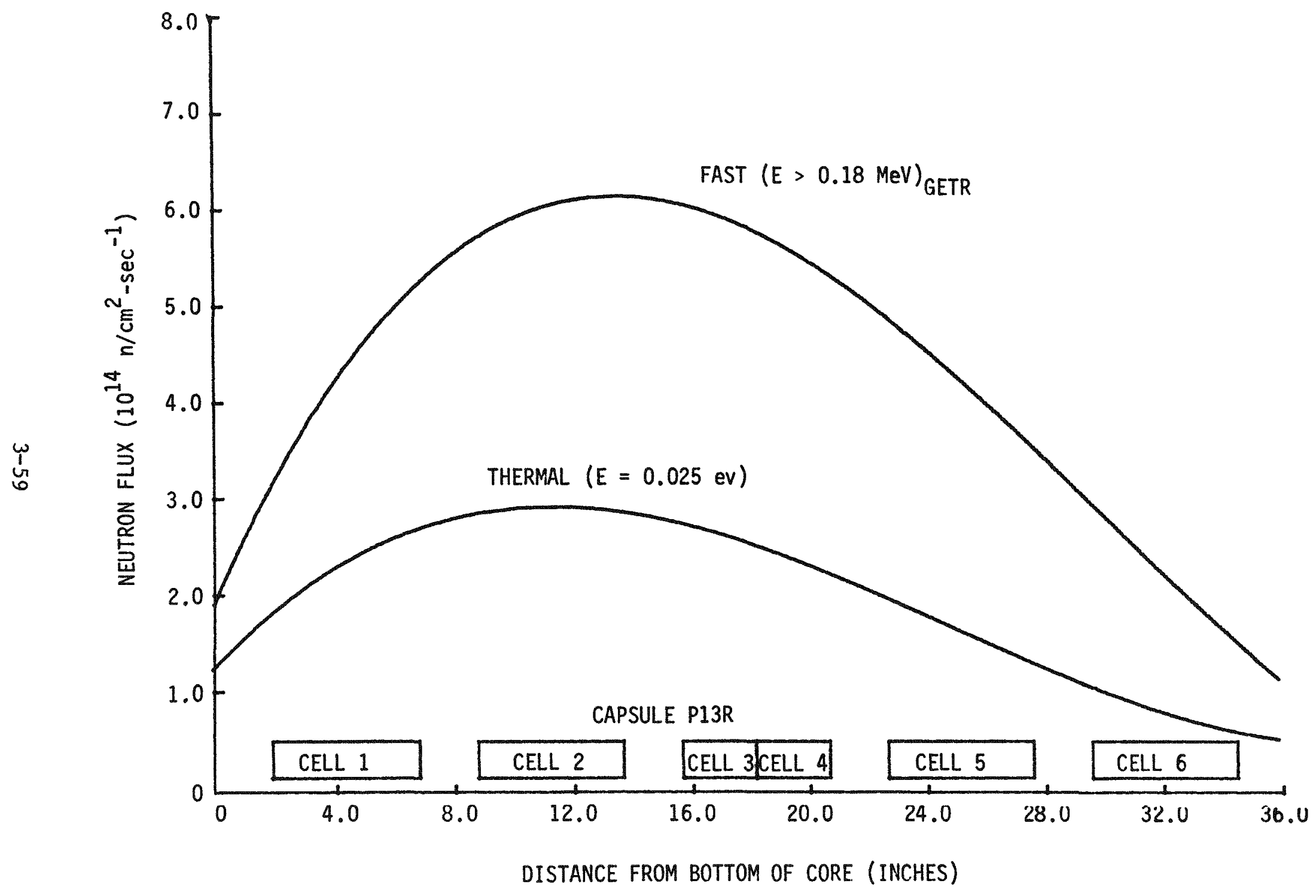

Fig. 3-21. GETR fast and thermal neutron flux profiles for capsule P13R 


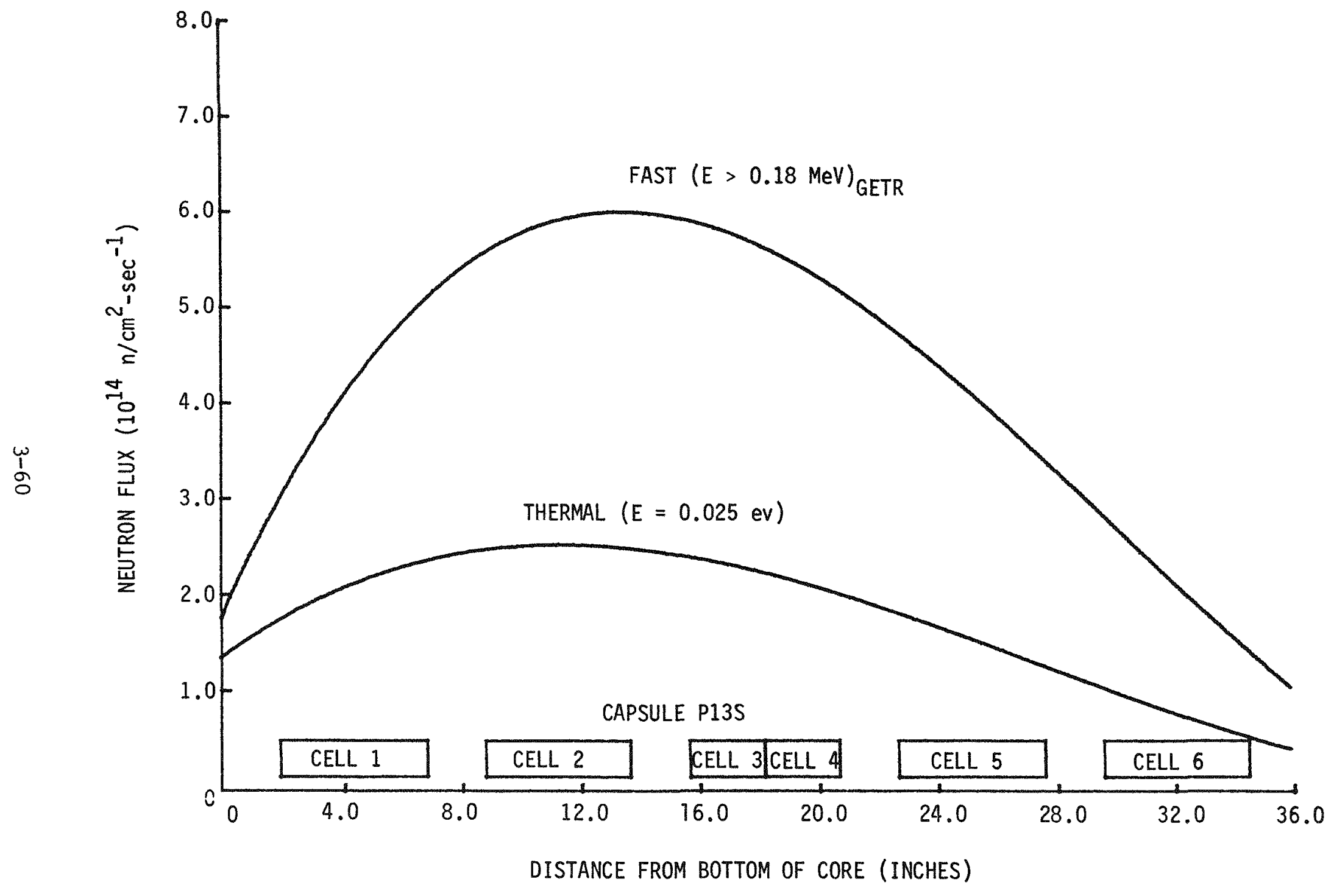

Fig. 3-22. GETR fast and thermal neutron flux profiles for capsule P13S 


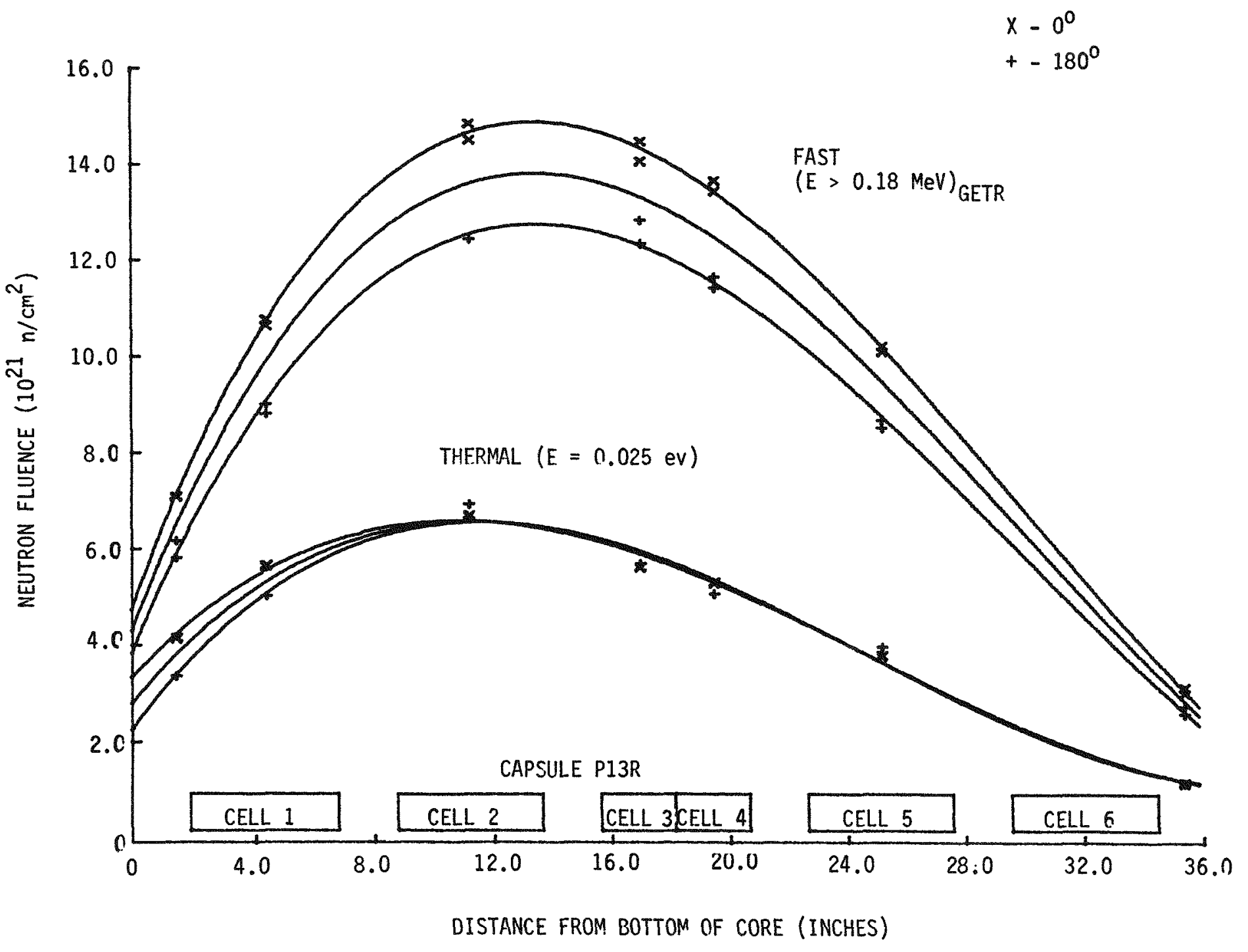

Fig. 3-23. GETR fast and thermal neutron fluence profiles for capsule P13R 


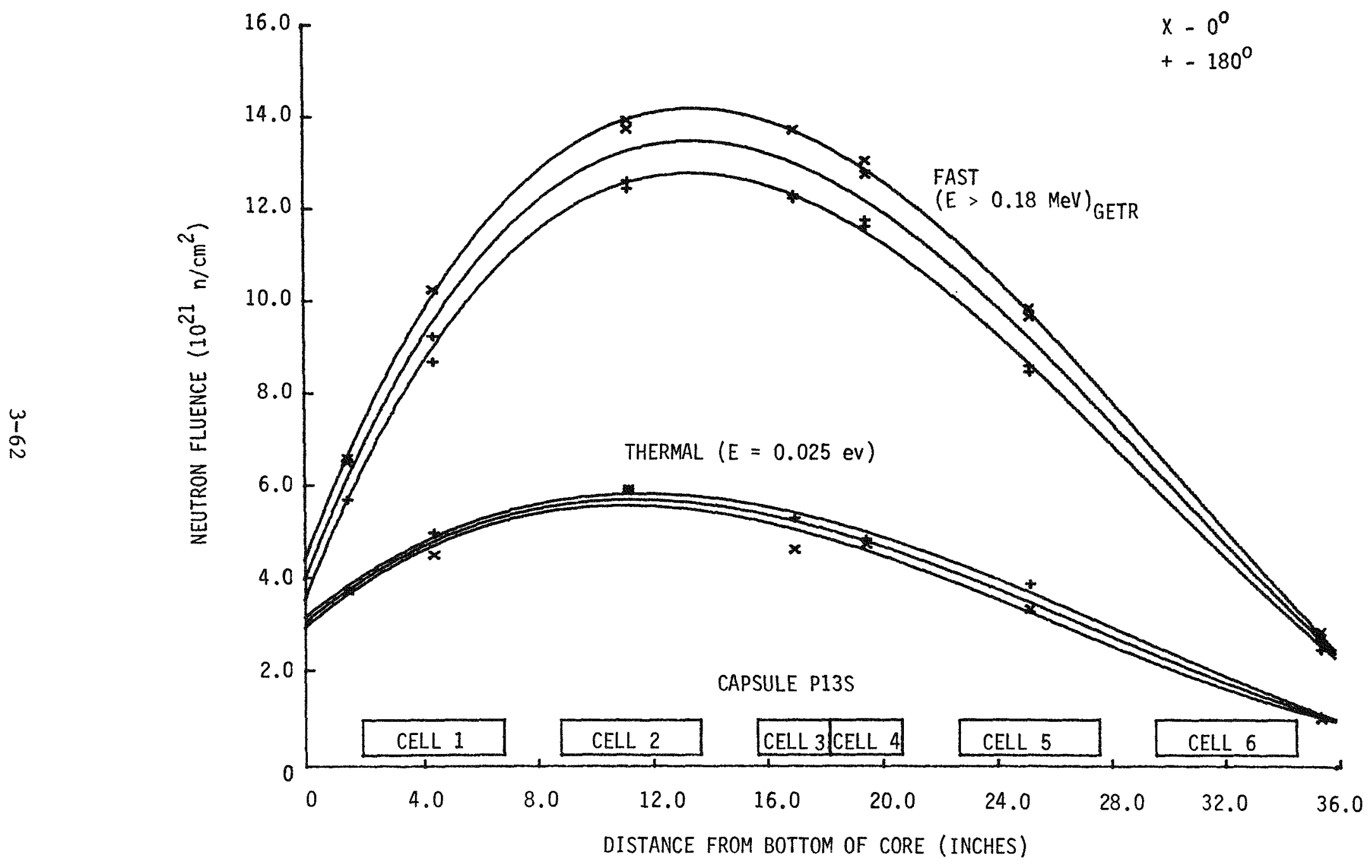

Fig. 3-24. GETR fast and thermal neutron fluence profiles for capsule P13S 


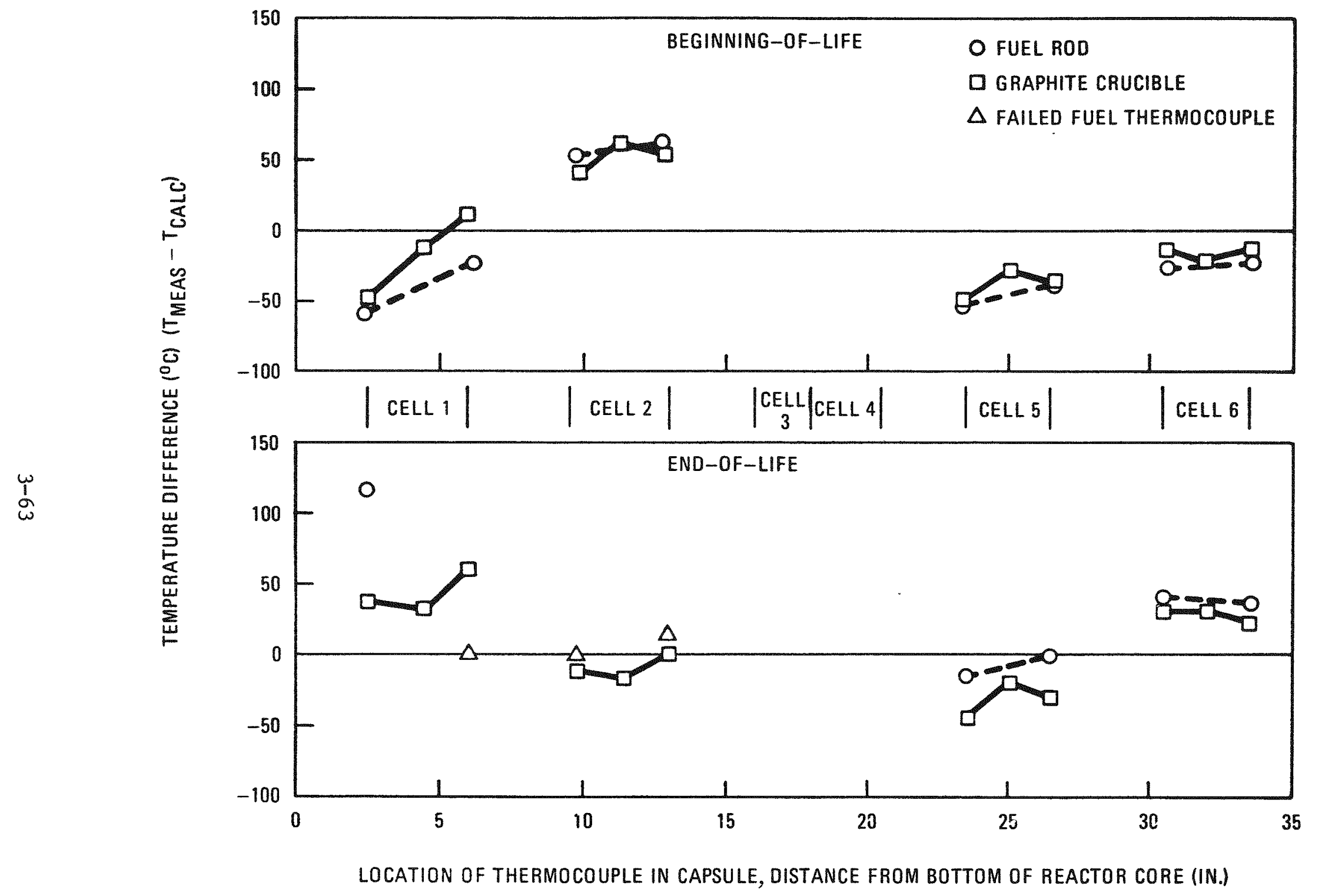

Fig. 3-25. Comparison between measured and calculated (TAC-2D) temperatures for capsule P13R at BOL and EOL 


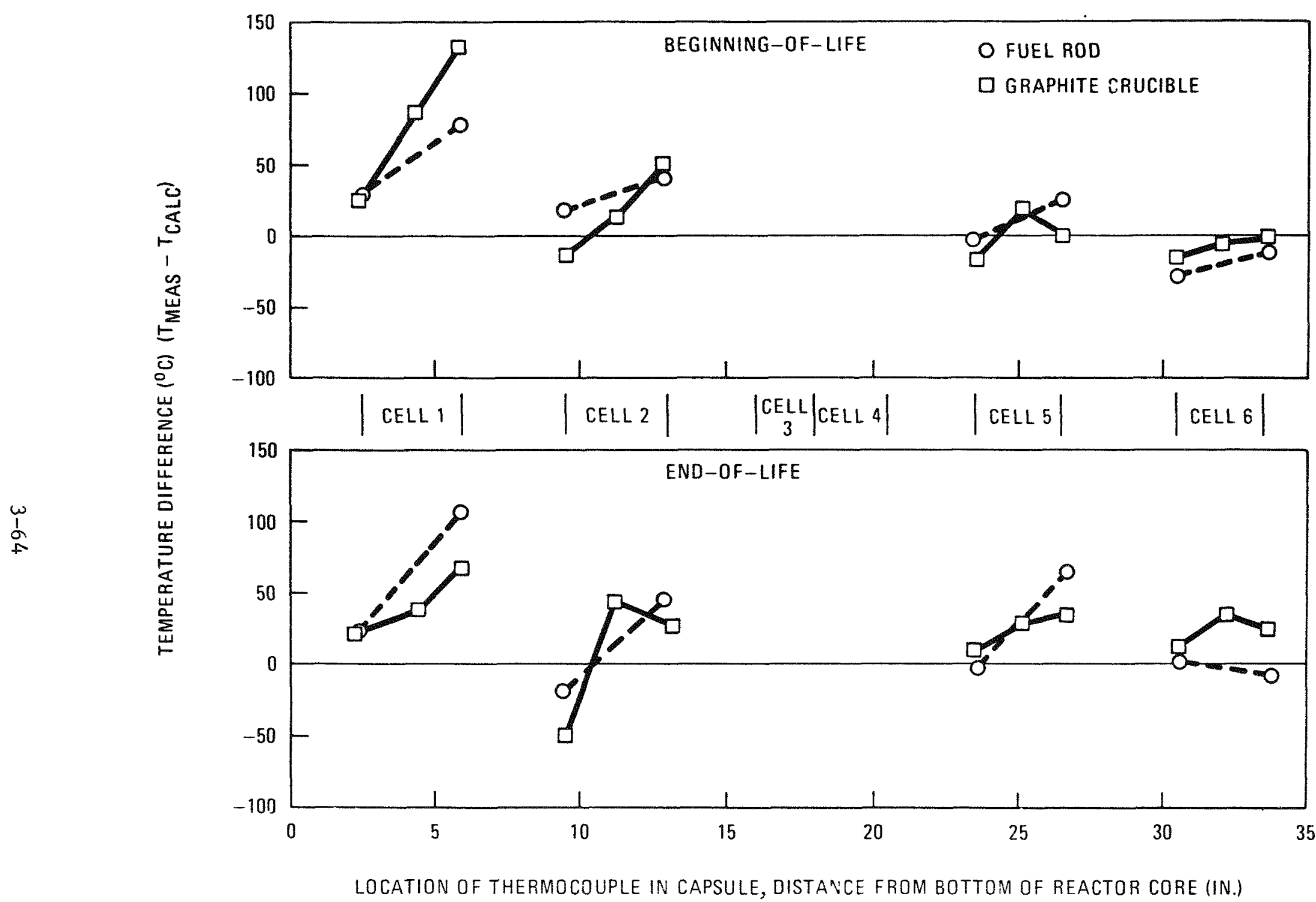

Fig. 3-26. Comparison between measured and calculated (TAC-2D) temperatures for capsule P13S at BOL and EOL 


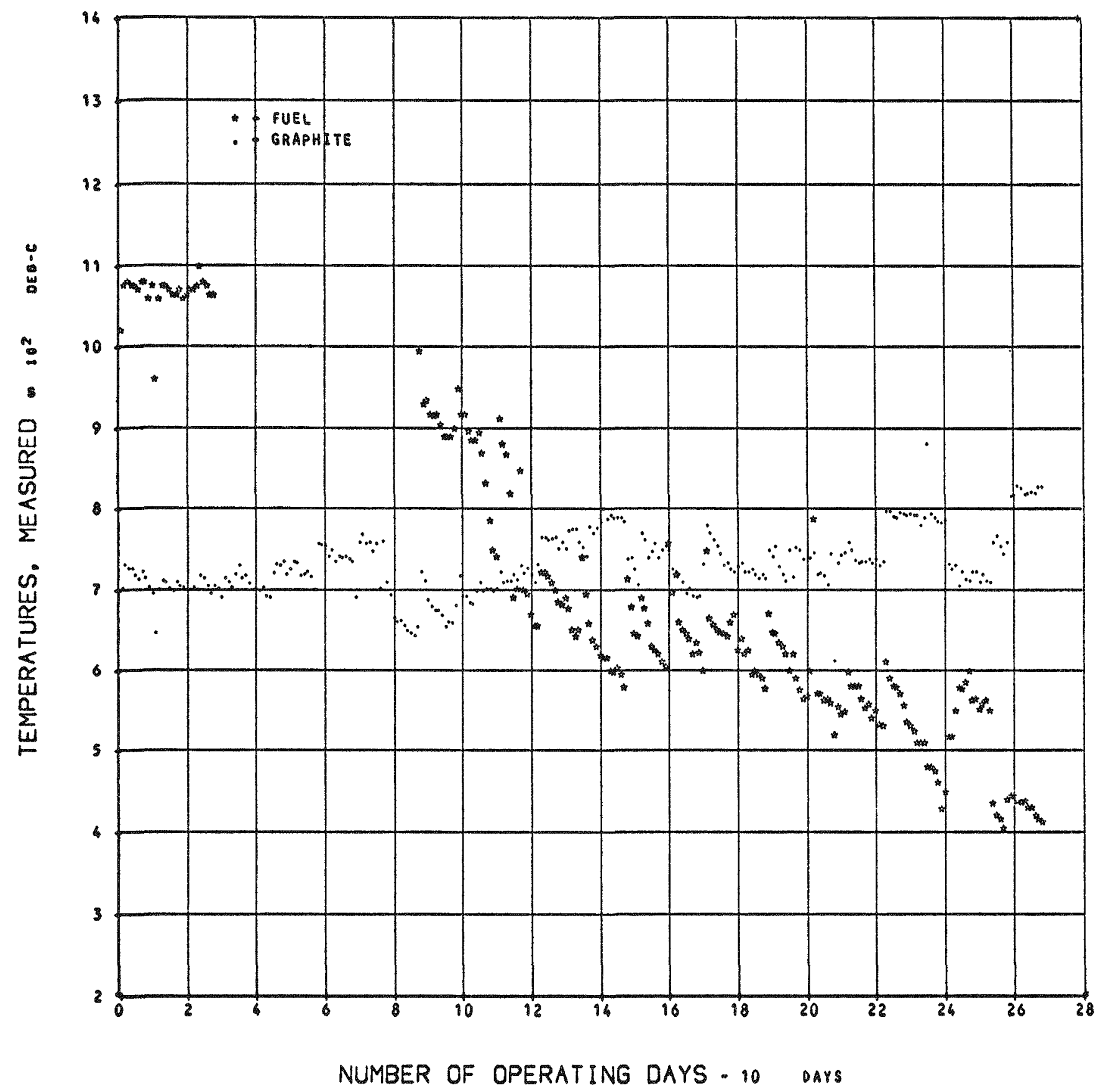

Fig. 3-27. Measured thermocouple readings of fuel and graphite for sample A, ce11 2 of capsule P13R 



\section{POSTIRRADIATION EXAMINATION RESULTS}

After removal from the graphite crucibles, the unbonded particle samples and fuel rods were examined visually and dimensional measurements were made on all fuel rods. Fission gas retention of all fuel specimens was evaluated by neutron activation in the TRIGA reactor facility. Gammaray spectrometry analysis was performed on all fuel specimens and capsule components to evaluate metallic fission product release and transport during irradiation. Metallographic examination was performed on selected fuel rod and unbonded particle specimens to evaluate irradiation-induced changes in the fuel components. Contact microradiography and measurements of fuel particle and OPyC coating density were performed on selected unbonded particle samples. The results obtained from these examinations are presented in Sections 4.1 through 4.10 .

\subsection{CAPSULE DISASSEMBLY}

The secondary containments were slit at $0^{\circ}$ and $180^{\circ}$ orientations and removed to expose the aluminum thermal bond spacers. All dosimeter vials, located in grooves of the thermal bond spacers, were recovered with the exception of two secondary dosimeter vials from capsule P13S. Transverse and longitudinal cracking of the protective tantalum thermocouple sheathing was observed, as expected. A11 purge gas lines were intact. The therma1 bond spacers, purge gas lines, and thermocouples were removed, which exposed the primary containment of each cell. Dimensional measurements made on the thermal bond spacers and the primary and secondary containments showed no measurable change in these components during irradiation.

Fuel specimens were housed in interlocking graphite crucibles inside Inconel cans, which formed the primary containment for each ce11. After removal of the Inconel cans, the fuel rods were exposed by lifting off the top segment of the graphite crucibles as shown in Fig. 4-1. The fuel rods 
were removed by sliding them out of the graphite crucibles into a padded plastic box or by lifting them out with master-slave manipulators. All fuel rods were removed intact and irradiation-induced damage to the fuel rods was considered to be minimal.

Slight bowing of the graphite crucibles in cell 2 of both capsules was observed. The fuel rods were wedged in the graphite crucibles and although the fuel specimens were not damaged, they were removed with considerable difficulty. Subsequent dimensional measurements revealed the fuel specimens were restrained by the graphite crucibles as a result of shrinkage of the graphite crucibles and slight expansion of the fuel rods after irradiation to the severe fast neutron fluences $\left[11.5\right.$ to $12.4 \times 10^{21} \mathrm{n} / \mathrm{cm}^{2}$ $\left.(\mathrm{E}>0.18 \mathrm{MeV})_{\mathrm{HTGR}}\right]$ experienced by these cells.

Each unbonded particle cell contained a graphite crucible assembly, which consisted of twelve annular trays arranged on a threaded graphite stock as shown in Fig. 4-2. Neutron radiographs taken of the capsules after irradiation revealed that all four of the crucible assemblies were intact. However, at least one sample tray from each cell was spilled during capsule disassembly or the fuel unloading operation because the threaded graphite stock broke. The sample trays spilled were positions 3-6, 4-1, and 4-12 in capsule P13R and positions 3-11, 4-5, and 4-6 in capsule P13S. Subsequent failure analyses were not performed on these specific samples because only a portion of the fuel particles in each sample was recovered. However, duplicate samples of each particle type were tested in companion trays and were examined.

\subsection{VISUAL EXAMINATION}

General irradiation performance and integrity of the unbonded coated particle and fuel rod samples after irradiation were assessed by visual examination. The examination was conducted using an in-cell Bausch and Lomb stereomicroscope with a magnification range of 4 to $30 \mathrm{X}$ and a camera attachment for photography. 


\subsubsection{Fue1 Rods}

The criteria used during the visual examination for judging fuel rod integrity after irradiation are that the fuel rods remain intact and experience negligible particle debonding. The general irradiation performance of the fuel rods was good and excellent fuel rod integrity was demonstrated after irradiation to fast neutron fluences significant1y exceeding LHTGR design limits. Very little irradiation-induced damage was observed, even in fuel rods irradiated to a fast neutron fluence of $12.4 \times 10^{21} \mathrm{n} / \mathrm{cm}^{2}$ $(E>0.18 \mathrm{MeV})_{\text {HTGR }}$, which is $55 \%$ beyond the peak LHTGR design fast neutron exposure. The visual appearance of the fuel rods after irradiation is summarized in Tables 4-1 and 4-2. Representative photomicrographs of each rod taken during the visual examination are shown in Figs. 4-3 through 4-22.

\subsubsection{Unbonded Particle Samples}

Each particle sample was examined visually and a measure of the OPyC coating integrity and pressure vessel failure was determined by counting the number of particles with defective coatings. The irradiation parameters and the results of the visual examination of each particle batch are given in Tables 4-3 through 4-5.

Visual examination of unbonded particle samples provides an accurate assessment of pressure vessel failure levels in BISO coated fertile particles and OPyC coating failure levels in TRISO coated fissile particles. In TRISO coated particles the fragments of failed OPyC coatings often remain attached to the $\mathrm{SiC}$ coating substrate as shown in Fig. 4-23. This made an accurate assessment of the pressure vessel failure levels in some TRISO coated particle batches impossible by visual examination. The pressure vessel failure levels reported in Table 4-3 are compared with failure levels determined by fission gas release measurements, which provide a more accurate assessment of TRISO coating failure (see Section 4.4.2). 
Particle batches with coating designs representative of the design requirements envisioned for the LHTGR exhibited excellent irradiation performance as shown in Figs. 4-24 through 4-26. A number of fissile and fertile particle batches exhibited $0 \%$ failure levels after irradiation to fast neutron exposures which exceeded the LHTGR peak design exposure by 34 to 51\%. BISO coated fertile particles with OPyC coatings deposited from acetylene-propylene (mixed gas) exhibited irradiation performance comparable to similarly designed particles with OPyC coatings deposited from propylene.

A large number of particle batches with coating designs or particle attributes that were not representative of LHTGR design requirements were tested. These samples were tested to investigate the effect of variables such as coating deposition rate, density, and faceting on fuel particle irradiation performance.

Fissile particles tested with OPyC coating densities in the range of 1.76 to $1.95 \mathrm{~g} / \mathrm{cm}^{3}$ exhibited good integrity after irradiation to fast neutron fluences up to $12.1 \times 10^{21} \mathrm{n} / \mathrm{cm}^{2}(\mathrm{E}>0.18 \mathrm{MeV})_{\mathrm{HTGR}}$. One fissile particle batch (6151-02-025), which had an OPyC coating density of 1.50 $\mathrm{g} / \mathrm{cm}^{3}$, exhibited an OPYC coating failure level of $46.3 \%$. The poor irradiation stability of this OPyC coating was probably due to the low deposition rate $(0.7 \mu \mathrm{m} / \mathrm{min})$ rather than the low density. OPyC coatings with densities as low as $1.53 \mathrm{~g} / \mathrm{cm}^{3}$ have been irradiated successfully to low fast neutron exposures in previous capsule tests (Ref. 4-1). BISO coated fertile particles exhibited good integrity during irradiation to the severe fast neutron exposures with $\mathrm{OPyC}$ coating densities in the range of 1.73 to 1.94 $\mathrm{g} / \mathrm{cm}^{3}$.

For the fissile and fertile particle designs tested, the OPyC coating deposition rate (anisotropy) had a greater influence on particle survival than OPyC coating density or thickness. In UC $\mathrm{U}_{2}$ TRISO batches where the primary variable tested was OPyC coating rate, no failure occurred in OPyC coatings deposited at rates greater than $3.8 \mu \mathrm{m} / \mathrm{min}$. As the deposition 
rate decreased below $1.5 \mu \mathrm{m} / \mathrm{min}$, the OPyC coating failure levels increased significantly. The onset of failure shifted to a lower coating rate as the OPyC coating density decreased. BISO coated fertile particles also exhibited similar irradiation performance as a function of OPyC coating deposition rate. As the deposition rate decreased below $4.0 \mathrm{\mu m} / \mathrm{min}$, the fertile particle OPyC coating failure levels increased. Photographs of fissile and fertile particles with OPyC coatings deposited at relatively low rates are shown in Fig. 4-27.

Fuel particles with severely faceted coatings, nonround fuel kernels, or interrupted SiC layers exhibited higher failure rates than similarly designed fuel particles that did not have these attributes. Photographs of fissile and fertile particles that exhibited failure due to severely faceted coatings are shown in Fig. 4-28.

One TRISO coated $\mathrm{ThO}_{2}$ fertile particle batch and two alternative TRISO coated fissile kernels, 250- $\mu \mathrm{m}$-diameter (Th,U) $\mathrm{O}_{2}$ and $500-\mu \mathrm{m}$-diameter $(8 \mathrm{Th}, 1 \mathrm{U}) \mathrm{O}_{2}$, were tested to obtain additional irradiation data on these potential backup fuel particles. The TRISO coated $250-\mu \mathrm{m}$-diameter (Th,U) $\mathrm{O}_{2}$ fuel particles (Fig. 4-29) exhibited no coating failure during irradiation to a fast neutron fluence of $11.2 \times 10^{21} \mathrm{n} / \mathrm{cm}^{2}$ (E $>0.18 \mathrm{MeV}$ ) ${ }_{\text {HTGR }}$. However, the larger diameter (8Th, $1 U$ ) $\mathrm{O}_{2}$ fuel particles (Fig. 4-23) exhibited a significant level of OPyC coating failure after irradiation to a fast neutron fluence of $11.1 \times 10^{21} \mathrm{n} / \mathrm{cm}^{2}$ ( $\left.\mathrm{E}>0.18 \mathrm{MeV}\right)_{\mathrm{HTGR}}$. The TRISO coated fertile particle batch (Fig. 4-30) exhibited OPyC coating and pressure vessel failure levels of $0.4 \%$ and $0 \%$, respectively, after irradiation to a fast neutron exposure of $11.5 \times 10^{21} \mathrm{n} / \mathrm{cm}^{2}$ (E $>0.18 \mathrm{MeV}$ ) $\mathrm{HTGR}$.

\subsection{DIMENSIONAL CHANGE ANALYSES}

\subsubsection{Fuel Rods}

Preirradiation fuel rod dimensional measurements were made using calibrated micrometers. One length and six diametral measurements were made on. each fuel rod to tolerances of $\pm 0.0013 \mathrm{~cm}$. The diameter measurements were 
made at $0^{\circ}$ and $90^{\circ}$ points along the rod; the selection of the $0^{\circ}$ and $90^{\circ}$ orientation was arbitrary (Ref. 4-2). For a fuel rod having a nominal diameter of $1.250 \mathrm{~cm}$, the precision of the measurements was $\pm 0.1 \%$.

The majority of the fuel rods were cured in graphite tubes; to prevent the rods from sticking to one another, pieces of Grafoil were placed on their ends. Normally only a portion of the Grafoil disc remained attached to the rods, although in some instances intact discs adhered to each end of a rod. Preirradiation length measurements included these discs. The nominal thickness of a disc was $0.013 \mathrm{~cm}$.

Postirradiation fuel rod dimensional measurements were performed using a calibrated dial gauge micrometer that could be interpreted to $\pm 0.0013 \mathrm{~cm}$. For comparative purposes selected rods also were measured using a ratcheted barrel micrometer similar to the one used to make the preirradiation measurements. Both micrometers were specially mounted on platforms to facilitate hot cell measurements (Fig. 4-31). Again six diametral measurements were made at randomly selected $0^{\circ}$ and $90^{\circ}$ orientations. Two length measurements were made on most of the fuel rods. Typically the standard deviation for the diametral measurements was $0.005 \mathrm{~cm}$, i.e., $\pm 0.4 \%$.

Irradiation-induced fuel rod dimensional changes were calculated to show the percent difference between the unirradiated and irradiated states. This was done for diametral, length, and volume changes. These data are summarized in Table 4-6 for capsule P13R and Table 4-7 for capsule P13S. The linear dimensional changes for rods irradiated at $1100^{\circ} \mathrm{C}$ are plotted in Fig. 4-32 and volumetric dimensional changes for these rods are shown in Fig. 4-33. The length and volume changes for rod $1 \mathrm{~B}$ in P13R are not plotted in these figures since they appear to be erroneous measurements. The range for predicted dimensional change is also shown on each of these plots. The irradiation exposure for rods tested in cel1 2 of P13R and P13S exceeds the limits of current dimensional change data for pyrocarbon and graphite shim; therefore, dimensional changes were not predicted for these rods. 
Dimensional changes were predicted using the SHRINK computer code. The model assumes that the particles in the fuel rod are in point-to-point contact (close-packed array) and the fuel rod dimensional change is isotropic. It is also assumed that the percent volume change of a fuel rod during irradiation is equal to the percent volume change of the constituent particle types. The percent linear dimensional change of the rod is therefore equal to one-third the percent volume change and can be calculated using Eq. 4-1 if the dimensional change behavior of the constituent particle types as a function of neutron fluence and temperature is known:

$$
\% \Delta \mathrm{D} / \mathrm{D}_{\mathrm{o}}=1 / 3 \sum_{i} \mathrm{x}_{i}\left(\% \Delta \mathrm{V} / \mathrm{v}_{\mathrm{o}}\right) \mathrm{i}
$$

where $\% \Delta D / D_{0}=$ percent diameter change of the fuel rod

$$
\begin{aligned}
\% \Delta \mathrm{V} / \mathrm{V}_{0} & =\text { percent volume change of the } i^{\text {th }} \text { particle type } \\
\mathrm{X}_{i} & =\text { particle volume fraction of the } i^{\text {th }} \text { particle type }
\end{aligned}
$$

Two general trends can be seen from the plots presented in Figs. 4-32 and 4-33: (1) the rods do not shrink as much as predicted and (2) the rod dimensional changes are anisotropic. Regarding the first point, this trend is similar to that noted in capsules $\mathrm{HRB}-4,-5$, and -6 (Ref. 4-3) where the average fuel rod diametral shrinkage was $36 \%$ less than predicted using the SHRINK code. These results are in reasonable agreement with the P13R and P13S results, which show the average diametral shrinkage to be $48 \% 1$ ess than predicted. The HRB irradiations were the first tests of cured-inplace rods and graphite-shimmed rods. More recently, similar results for LHTGR type rods have been seen in capsule P13Q (Ref. 4-4).

Regarding the second point, this is the first GA capsule in which the rods exhibited notable anisotropic behavior. In experiments HRB-4, -5 , and -6 (Ref. 4-3), as well as all previous tests (Ref. 4-1), the rods behaved isotropically. Although the general profile for the diametral dimensional changes for the $\mathrm{P} 13 \mathrm{R}$ and $\mathrm{P} 13 \mathrm{~S}$ rods irradiated at $1100^{\circ} \mathrm{C}$ is consistent (Fig. 4-32), the axial dimensional changes appear more random. This 
scattering of the axial data could be caused in part by the Grafoil discs attached to the ends of the rods. The Grafoil was noted to have partially flaked off on some of the rods; furthermore, this material is suspected to undergo volumetric expansion, especially in the axial direction. However, more recent irradiation tests (Ref. 4-4) also have shown LHTGR type rods to exhibit anisotropic behavior, and these results support the observed anisotropic behavior of the $\mathrm{P} 13 \mathrm{R}$ and $\mathrm{P} 13 \mathrm{~S}$ fuel rods. The pair of rods that was cured in a packed $\mathrm{Al}_{2} \mathrm{O}_{3}$ bed behaved nearly isotropically. Measured and predicted dimensions were also in good agreement for the packed-bed rods. However, Grafoil discs were not placed on the ends of these rods.

The degree of anisotropy for rods irradiated in cell 2 of P13R and P13S to fluences of $>10.5 \times 10^{21} \mathrm{n} / \mathrm{cm}^{2}$ partially resulted from the closing of the radial gap between the graphite crucible and the fuel rod (see Section 4.3.2). The interference between these components apparently caused the rods to expand axially as a result of the lack of accommodation of radial growth of the rod. It was noted that the two highest exposure rods in cell 1 of P13R and P13S (1D and $1 \mathrm{E}$ ) had significantly greater axial expansions than the other rods in these cells. This may be an indication of interference between the rods and crucible wall at temperature, although room temperature gaps of 0.002 to $0.007 \mathrm{~cm}$ were measured for these rods.

\subsubsection{Graphite Crucibles}

Characterization of the irradiation-induced dimensional change behavior of the H-451 graphite crucibles was required for capsule thermal analysis. Prior to removal of the fuel rods, dimensional measurements were made on the graphite crucibles. Axial and radial measurements both perpendicular and parallel to the plane of the split in the crucibles were made with a dial gauge micrometer.

The measured diametral dimensional changes of the fuel rod graphite crucibles are reported in Table 4-8. Because some of the crucibles had bowed, data obtained by measurement parallel to the crucible split most accurately reflected the irradiation-induced dimensional change of the 
crucibles; these data are plotted in Fig. 4-34 as a function of fast neutron fluence. Irradiation-induced dimensional changes ranged from -0.2 to $-1.5 \%$. The data were fit to a third-order polynomial given by:

$$
\Delta \ell / \ell(\%)=A \phi+B \phi^{2}+C \phi^{3},
$$

where $A=-1.86 \times 10^{-2}$

$$
\begin{aligned}
& B=-2.49 \times 10^{-2} \\
& C=1.57 \times 10^{-3} \\
& \phi=\text { fast neutron fluence }(E>0.18 \mathrm{MeV})_{\text {HTGR }}
\end{aligned}
$$

Also shown in Fig. 4-34 are the predicted dimensional change curves for $\mathrm{H}-451$ graphite in the radial direction for irradiation at $650^{\circ}$ and $1050^{\circ} \mathrm{C}$ (Ref. 4-5). The observed dimensional change of the graphite crucibles (within the precision of the measurements) was in fair agreement with the predicted dimensional change behavior of H-451 graphite. In the capsule thermal analysis, Eq. 4-2 was used to calculate the gaps between the fuel rods and graphite crucibles because the predicted dimensional change curves for $\mathrm{H}-451$ graphite only covered fast neutron fluences to $8.0 \times 10^{21} \mathrm{n} / \mathrm{cm}^{2}$.

The radial gap between each fuel rod and the wall of the graphite crucible was calculated from the crucible and fuel rod dimensions; the results are given in Tables 4-9 and 4-10. The fuel rods tested in cell 2 of both capsules were wedged in the graphite crucibles after irradiation. The results shown in Tables 4-9 and 4-10 indicate the fuel rods in cell 2 of each capsule were restrained by the graphite crucibles after irradiation as a result of shrinkage of the crucibles and slight expansion of the fuel rods. Since the magnitude of the coefficient of thermal expansion is similar for the fuel rods and $\mathrm{H}-451$ graphite, these fuel rods were probably also in contact with the wall of the graphite crucible during the latter part of the irradiation. No damage to the fuel rods or graphite crucibles was observed as a result of this mechanical interaction. It should be noted that this interaction only occurred in the cell 2 assemblies, which 
were irradiated to fast neutron fluences considerably beyond the LHTGR peak design fast neutron fluence of $8.0 \times 10^{21} \mathrm{n} / \mathrm{cm}^{2}(\mathrm{E}>0.18 \mathrm{MeV})_{\mathrm{HTGR}}$.

\subsection{FISSION GAS RELEASE}

\subsubsection{Postirradiation Fission Gas Release Measurements}

Fission gas retention characteristics of fuel rod and unbonded particle samples were evaluated by fission gas release measurements conducted in the GA TRIGA reactor facility. Preirradiation measurements were made as part of the quality control evaluation to determine the amount of heavy metal contamination in the fuel specimens prior to irradiation. The primary objectives of the postirradiation measurements were (1) to cross check the in-pile R/B measurements made at the GETR during capsule irradiation, (2) to obtain $R / B$ values for each fuel sample to better define fuel failure as a function of irradiation conditions, and (3) to compare the more statistically significant particle failure fractions predicted from $R / B$ data with those determined by other postirradiation examination techniques.

Fission gas release measurements were made by irradiating fuel specimens at $1100^{\circ}, 1300^{\circ}, 1500^{\circ}$, or $1600^{\circ} \mathrm{C}$ in a graphite tube furnace designed to fit into a fuel element position of the TRIGA reactor core (Ref. 4-6). The furnace allowed measurements to be made at high temperatures, independent of the power generated in the fuel. The rods were irradiated at appropriate power levels for $0.5 \mathrm{hr}$ to produce approximately $10^{14}$ fissions in each fuel specimen. During irradiation, the released fission gases were swept with helium into a liquid-nitrogen-cooled charcoal trap. The trapped gases were assayed for fission product isotopes by gamma-ray spectroscopy (Ref. 4-7) using a $\mathrm{Ge}(\mathrm{L} i)$ detector and a 4096-channel analyzer. The fission product isotope birth fractions were calculated using the neutron flux in the TRIGA core and the end-of-life (EOL) fissionable material loadings. The amount of fissionable material remaining in each fuel rod was calculated using the Sigma 2 FISSIN computer code, which takes into account initial fuel loadings, capsule irradiation conditions, and the GETR power history and cross sections. The adjusted EOL fissile fuel loading for each specimen was 
determined by expressing the sum of the weights of all remaining fissionable material (U-233 and U-235) in terms of U-235, producing the equivalent $\mathrm{Kr}-85 \mathrm{~m}$ yield (Ref. 4-8).

The results of the preirradiation and postirradiation fission gas release measurements in terms of $\mathrm{R} / \mathrm{B}$ for $\mathrm{Kr}-85 \mathrm{~m}$ are reported in Tables 4-11 through 4-14. The $\mathrm{Kr}-85 \mathrm{~m}$ isotope was selected as the reference isotope because it has a short half-Iife $(4.4 \mathrm{hr})$ and its gamma-ray energy peak can easily be resolved. A reference temperature of $1100^{\circ} \mathrm{C}$ was used for analysis of fuel failure levels; however, fission gas release measurements were also made at temperatures up to $1600^{\circ} \mathrm{C}$ on selected fuel rods. The preirradiation $R / B$ data obtained under non-steady-state conditions were corrected to steady state (multiplied by a factor of 1.9). However, the postirradiation $R / B$ values were not corrected to steady state because a study showed that the $R / B$ values obtained for irradiated fuel in the 0.5-hr TRIGA irradiation were essentially steady-state values (Ref. 4-9).

\subsubsection{Determination of Fuel Failure Levels}

Fuel particle failure levels for each specimen were determined from the fission gas release data. The fission gas released during TRIGA activation comes from two sources: (1) heavy metal contamination outside the particle coatings and (2) failed fuel particles, as shown by the following equation:

$$
\mathrm{R}_{\mathrm{BOL}}=\mathrm{R} / \mathrm{B}_{\text {failed fuel }}+\mathrm{R}_{\mathrm{B}} \mathrm{B}_{\mathrm{c}(\mathrm{U})}+\mathrm{R}_{\mathrm{B}} \mathrm{B}_{\mathrm{c}(\mathrm{Th})} \text {, }
$$

where

$$
\begin{aligned}
\mathrm{R} / \mathrm{B}_{\mathrm{EOL}} & =\text { measured postirradiation fission gas release } \\
\mathrm{R}_{\mathrm{B}} \mathrm{B}_{\text {failed fuel }}= & \text { fission gas release from failed fuel particles } \\
\mathrm{R} / \mathrm{B}_{\mathrm{C}(\mathrm{U})}= & \text { fission gas release from uranium contamination } \\
\mathrm{R} / \mathrm{B}_{\mathrm{C}(\mathrm{Th})}= & \text { fission gas release from thorium (U-233) } \\
& \text { contamination }
\end{aligned}
$$

A certain amount of uranium and thorium contamination in the fuel rods and on the surface of the coated particles is unavoidable during fabrica- 
tion. Uranium and thorium contamination levels were determined for each fuel specimen prior to irradiation using fission gas release, thorium hydrolysis, or acid-leach tests (Ref. 4-2). The difference between the fission gas release resulting from contamination and the measured postirradiation fission gas release $\left(R / B_{E O L}\right)$ is the fission gas released from fuel particles that failed during irradiation.

4.4.2.1. Fuel Rod Specimens. The gaseous fission product release resulting from in-service fuel particle failure was calculated for each fuel rod specimen using the equation

$$
R / B_{\text {failed fuel }}=R / B_{E O L}-\left[R / B_{c(U)}+R / B_{c(T h)}\right] \text {. }
$$

The values obtained are given in Tables 4-15 and 4-16 for P13R and P13S, respectively. The fission gas release arising from heavy metal contamination was calculated using the following equations:

$$
\begin{aligned}
\mathrm{R}_{\mathrm{C}(\mathrm{U})} & =\left(\mathrm{R}_{\mathrm{BOL}}\right)\left(\mathrm{x}_{\mathrm{U}-235}\right) \\
\mathrm{R}_{\mathrm{C}} \mathrm{B}_{\mathrm{C}(\mathrm{Th})} & =\left(\mathrm{Th} \mathrm{C}_{\mathrm{C}}\right)\left(\mathrm{r} / \mathrm{b}_{\mathrm{C}}\right)\left(\mathrm{X}_{\mathrm{U}-233}\right),
\end{aligned}
$$

where $R / B_{B O L}=$ measured preirradiation fission gas release

$$
\begin{aligned}
\mathrm{Th}_{\mathrm{C}} & =\text { level of thorium contamination }(\mathrm{g} \mathrm{Th} / \mathrm{g} \mathrm{Th}) \\
\mathrm{r} / \mathrm{b}_{\mathrm{c}} & =\text { fractional gaseous release from heavy metal contamination } \\
\mathrm{X}_{\mathrm{U}-235} & =\text { fraction of fissions that occurred in } \mathrm{U}-235 \text { at EOL } \\
\mathrm{X}_{\mathrm{U}-233} & =\text { fraction of fissions that occurred in U-233 at EOL }
\end{aligned}
$$

A value for the fractional gaseous release from heavy metal contamination of 0.30 was used in these calculations (Ref. 4-9). The fraction of fissions occurring in each isotope at EOL was determined by summing the weights of all remaining fissionable material (U-233 and U-235) normalized to the thermal fission yields of $\mathrm{Kr}-85 \mathrm{~m}$. 
When the postirradiation fission gas release $\left(R / B_{E O L}\right)$ of nine of the fuel rods was corrected for heavy metal contamination, the irradiationinduced change in the $R / B$ value became negative. This indicates that no fuel failure occurred during irradiation and the level of thorium contamination measured on a companion unirradiated fuel rod was slightly higher than in the fuel rod actually tested.

An equivalent level of in-service fuel failure (F) which would release the amount of fission gas attributed to failed fuel was calculated for each fuel rod using the following equation:

$$
F=\frac{R / B_{\text {failed fuel }}}{r / b_{f}}
$$

The values obtained are given in Tables 4-15 and 4-16 for P13R and P13S, respectively. All available data (Ref. 4-9) indicate that the mean fractional release of $\mathrm{Kr}-85 \mathrm{~m}$ at $1100^{\circ} \mathrm{C}$ from failed fuel particles $\left(\mathrm{r} / \mathrm{b}_{\mathrm{f}}\right)$ constrained in a fuel rod is $5.0 \times 10^{-3}$. and this value was used in these calculations. However, it should be noted that for conservatism in LHTGR inventory calculations, a value of 0.02 is used (Ref.4-10). The calculated in-service particle failure levels ranged from 0 to $24 \%$. Twentyfive of the forty rods tested exhibited particle failure levels of less than $1.0 \%$.

Extremely low levels of particle failure can be detected by fission gas release measurements. Each fuel rod contained approximately 2500 coated particles.' Assuming a fractional release from failed fuel of $5.0 \times 10^{-3}$, an $\mathrm{R} / \mathrm{B}$ of $2.0 \times 10^{-6}$ corresponds to one failed particle (a failure level of $0.04 \%$ ) in a rod.

Although low levels of particle failure in fuel rods can be detected, it is not possible to accurately differentiate between fissile and fertile particle failure using postirradiation $R / B$ data alone. The maximum possible fissile and fertile particle failure levels were calculated for each fuel rod by assuming that all the fission gas recovered was released from 
either the fissile or the fertile particles. The fraction of fissions occurring in each particle type, i.e., $x_{\text {fissile }}$ or $x_{\text {fertile, were }}$ used to calculate the particle failure fractions. Dividing the gaseous release from failed fuel by $x_{\text {fissile }}$ in one case and $x_{\text {fertile }}$ in the other case gave the maximum R/B for each particle type. For this reason, the particle failure fractions calculated from the $R / B$ data using Eqs. 4-8 and 4-9 are termed maximum failure fractions:

$$
\begin{aligned}
& F_{\text {fissile }}=\frac{R / B_{\text {failed fuel }}}{\left(x_{\text {fissile }}\right)\left(r / b_{f}\right)}, \\
& F_{\text {fertile }}=\frac{R / B_{\text {failed fuel }}}{\left(x_{\text {fertile }}\right)\left(r / b_{f}\right)} .
\end{aligned}
$$

The maximum in-service fertile particle failure levels calculated using Eq. 4-9 ranged from 0 to $26.3 \%$ (Tables $4-15$ and 4-16). Since the failure levels calculated from the $R / B$ data represent the maximum possible in-service failure, these failure levels are considered to be an upper limit for fertile particle failure in the fuel rods rather than the upper $95 \%$ confidence 1 imit obtained during the metallographic examination (see Section 4.6.3).

Assuming that no fertile particle failure occurred, the maximum possible fissile particle failure levels calculated for the fuel rods using Eq. 4-8 ranged from 0 to $280 \%$. These data were not reported in Tables 4-15 and 4-16 because failure levels greater than $100 \%$ indicate that the fissile particles did not contain sufficient fissionable material at EOL to account for all the measured postirradiation fission gas release. When the amount of fissionable material in the fissile particles is low relative to the fertile particles, a much smaller fraction of fissions occurs in the fissile particles than in the fertile particles at EOL, as shown in Tables 4-15 and 4-16. When this is the case, a low level of fertile particle failure gives an $R / B$ equivalent to a much higher fissile particle failure fraction. Unless the fraction of fissions occurring in the fissile particles represents a significant fraction of the total fissions occurring 
in the fuel rod at EOL, postirradiation fission gas release data cannot be used to accurately determine fissile particle failure levels.

\subsubsection{Unbonded Particle Samples. Gaseous fission product release} resulting from failed particles was calculated for the unbonded particles in a manner similar to the fuel rods; the values obtained are reported in Tables 4-17 and 4-18. The R/B failed fuel for $\mathrm{UC}_{2}$ TRISO particles was calculated using the equation

$$
R / B_{\text {failed fuel }}=R / B_{E O L}-R / B_{B O L} \text {, }
$$

where $R / B_{B O L}$ represents the contribution from uranium contamination. For the $(\mathrm{Th}, \mathrm{U}) \mathrm{O}_{2}$ TRISO particles, the $\mathrm{R} / \mathrm{B}_{\text {failed fuel }}$ was calculated using Eq. 4-4, which takes into account both thorium and uranium contamination. The $\mathrm{R} / \mathrm{B}_{\text {failed fuel }}$ for the $\mathrm{ThO}_{2}$ fertile particles was calculated using the following equations:

$$
\begin{aligned}
& R / B_{\text {failed fuel }}=R / B_{E O L}-R / B_{c(T h),} \\
& R / B_{c(T h)}=\left(T h_{c}\right)\left(r / b_{c}\right),
\end{aligned}
$$

where $\mathrm{Th}_{\mathrm{c}}$ is the level of thorium contamination measured prior to irradiation and $\mathrm{r} / \mathrm{b}_{\mathrm{c}}=0.30$.

The in-service particle failure level was calculated for each sample using Eq. 4-7. The results are reported in Tables 4-17 and 4-18. The failure levels for all particle samples except selected $\mathrm{UC}_{2}$ TRISO particle batches were calculated using a value of $2 \times 10^{-2}$ for the fractional release from failed fuel $\left(r / b_{f}\right)$ in an unconstrained geometry (Ref. 4-9). This value is applicable only when the particle coating is severely cracked yet remains attached to the fuel kernel. In a number of $\mathrm{UC}_{2}$ TRISO particle batches, the observed coating failures resulted in bare kernels, i.e., complete loss of coating integrity. In these samples an $\mathrm{r} / \mathrm{b} f$ of $3.5 \times 10^{-2}$ for bare kernels (Ref. 4-9) was used to calculate the failure levels. 


\subsubsection{High-Temperature Fission Gas Release Measurements}

Fission gas release measurements were made on all fuel specimens at a reference temperature of $1100^{\circ} \mathrm{C}$ to evaluate fuel integrity. Additional measurements at temperatures up to $1600^{\circ} \mathrm{C}$ were made on eight fuel rods to gain additional data on the temperature dependence of fission gas release from reference-type LHTGR fuel rods and to compare the data obtained at these high temperatures with the fission gas release data obtained from fuel rods in cell 1 of capsule P13S that were thermal cycled to high temperatures periodically during irradiation.

The temperature dependence for steady-state fission gas release of short-lived isotopes can be represented by:

$$
\frac{(R / B)_{T}}{(R / B)_{0}}=F_{\ell}^{0} \exp \left[\frac{Q_{\ell}}{R_{g}}\left(\frac{1}{T_{o}}-\frac{1}{T}\right)\right]+F_{h}^{o} \exp \left[\frac{Q_{h}}{R_{g}}\left(\frac{1}{T_{o}}-\frac{1}{T}\right)\right],
$$

where $(R / B)_{T},(R / B)_{0}=$ fission gas release at temperature $T\left({ }^{\circ} \mathrm{K}\right)$ and at the reference temperature $\left(1373^{\circ} \mathrm{K}\right)$

$$
\mathrm{F}_{\ell}^{O}, \mathrm{~F}_{\mathrm{h}}^{\mathrm{O}}=\text { pre-exponential constants; for } \mathrm{Kr}-85 \mathrm{~m} \mathrm{~F}_{\ell}^{\mathrm{O}}=0.08 \text { and }
$$
$\mathrm{F}_{\mathrm{h}}^{\mathrm{O}}=0.92$

$Q_{\ell}, Q_{h}=$ activation energy (kcal/mole) for the low-temperature and high-temperature processes, respectively; for $\mathrm{Kr}-85 \mathrm{~m} \mathrm{Q}_{\ell}=0.7$ and $\mathrm{Q}_{\mathrm{h}}=16.5$

$R_{g}=$ gas constant $\left(1.987 \times 10^{-3} \mathrm{kcal} / \mathrm{mole}^{\circ} \mathrm{K}\right)$

This equation is an empirical fit to fission gas release data (steady state) obtained during long-time, high-temperature anneals of Fort St. Vrain fuel particles. Equation 4-13 has been superseded by a semi-empirical equation given in Ref. 4-9. For short-lived isotopes in the temperature range of $1100^{\circ}$ to $1500^{\circ} \mathrm{C}$, the two equations yield $\mathrm{R} / \mathrm{B}$ values which are not significantly different. 
A summary of the high-temperature fission gas release measurements, including the $1100^{\circ} \mathrm{C}$ data, obtained for the eight fuel rods irradiated in capsules P13R and P13S is given in Tables 4-19 and 4-20. The data are also plotted as a function of reciprocal temperature in Figs. 4-35 through 4-42. In these figures the closed points indicate the fission gas release measurement was the first or only measurement made during the day and the open points indicate previous measurements had been made on the same day. The arrows indicate the order in which the measurements were made.

The fission gas release data for the three fuel rods in which only two measurements were made are plotted in Figs. 4-35 through 4-37. The dashed line is the predicted temperature dependence for steady-state fission gas release given by $\mathrm{Eq} \cdot 4-13$ and was calculated using the first measurement made on each rod. The agreement between the predicted and measured temperature dependence for fuel rods 7161-004-17-6 and 7161-004-19-5 was good. The fission gas release measured for fuel rod 7161-004-19-7 at $1100^{\circ} \mathrm{C}$ was slightly higher than the value predicted from $1300^{\circ} \mathrm{C}$ measurement. This may be due, in part, to the residual gas inventory remaining after the first measurement was made. This effect will be discussed in more detail later in this section.

Fission gas release data obtained for fuel rods $7161-004-03-5$ and 7161-004-16-5 at temperatures up to $1600^{\circ} \mathrm{C}$ are shown in Figs. 4-38 and 4-39, respectively. The two reference $R / B$ values (closed points) obtained for fuel rod $7161-004-16-5$ at $1100^{\circ} \mathrm{C}$ are in good agreement. The fission gas release values obtained for both rods during successive measurements above $1100^{\circ} \mathrm{C}$ were significantly higher than the predicted levels of fission gas release based on a temperature dependence extrapolation from the $1100^{\circ} \mathrm{C}$ data. A portion of the observed increase in $R / B$ at the high temperatures was attributed to particle failure induced during the TRIGA measurements.

Fission gas release data obtained for fuel rods 7161-004-14-5 and 7161-004-03-6 at temperatures up to $1600^{\circ} \mathrm{C}$ are shown in Figs. 4-40 and 4-41, respectively. The two reference $R / B$ values (closed points) obtained for fuel rod $7161-004-14-5$ at $1100^{\circ} \mathrm{C}$ are in good agreement. The $\mathrm{R} / \mathrm{B}$ values 
obtained for both rods at $1300^{\circ} \mathrm{C}$ were in excellent agreement with the predicted values based on a temperature dependence extrapolation from the $1100^{\circ} \mathrm{C}$ data. At temperatures above $1300^{\circ} \mathrm{C}$ the measured $\mathrm{R} / \mathrm{B}$ values were significantly higher than the predicted values. After the high-temperature measurements, two measurements were again made at $1100^{\circ} \mathrm{C}$. The final measurements (closed points) were made several weeks after the $1100^{\circ} \mathrm{C}$ measurements made upon completion of the high-temperature series (open points). The difference between the two $R / B$ values was attributed to residual fission gases generated during the previous measurements. When the final measurements were made several weeks later, the residual gas inventory would have decayed away. The difference in the $R / B$ values obtained during the final measurements and the original reference value at $1100^{\circ} \mathrm{C}$ was attributed to $28.2 \%$ particle failure induced in both fuel rods during the heating in TRIGA to $1600^{\circ} \mathrm{C}$.

The fission gas release data obtained for fuel rod 7161-004-17-5 at temperatures up to $1600^{\circ} \mathrm{C}$ are shown in Fig. 4-42. The first measurement was made at $1300^{\circ} \mathrm{C}$ (point 1) followed by an $1100^{\circ} \mathrm{C}$ measurement (point 2) on the same day. The $R / B$ value obtained at $1100^{\circ} \mathrm{C}$ was higher than the predicted value based on the temperature dependence extrapolation. After several weeks a second measurement was made, again at $1100^{\circ} \mathrm{C}$ (point 3); this value was in excellent agreement with the predicted value. Successive measurements were then made at higher temperatures. The $R / B$ measured at $1300^{\circ} \mathrm{C}$ (point 4) was almost identical to the value measured previously. At $1500^{\circ} \mathrm{C}$ the measured $\mathrm{R} / \mathrm{B}$ (point 5) was close to the predicted value. However, the value obtained at $1600^{\circ} \mathrm{C}$ (point 6) was significantly higher than the predicted value, which indicates that particle failure occurred between the $1500^{\circ}$ and $1600^{\circ} \mathrm{C}$ measurements. Measurements were made at $1100^{\circ} \mathrm{C}$ immediately after the high-temperature series (point 7) and several weeks later (point 8 ). The difference between the two $R / B$ values was attributed to the residual gas inventory. The difference between the final $R / B$ value (point 8 ) and the reference $1100^{\circ} \mathrm{C}$ value (point 3) was attributed to $\checkmark 4.8 \%$ particle failure induced during the high-temperature measurements. 
Several observations were made from these data. There was excellent agreement between the measured fission gas release for the eight fuel rods and the $R / B$ values predicted from the temperature dependence for steadystate fission gas release (Eq. 4-13). Significant levels of fuel failure can be induced by heating highly irradiated fuel samples to $1600^{\circ} \mathrm{C}$ in TRIGA. A discussion of the fuel failure induced during in-pile thermal cycling and the TRIGA heat treatments is given in Section 5.1.3. If two or more successive fission gas release measurements are made on a fuel specimen within an $8-\mathrm{hr}$ period, residual $\mathrm{Kr}-85 \mathrm{~m}$ fission gas generated during the previous measurement can contribute to the release obtained during the subsequent measurements.

\subsubsection{Comparison of In-pile and Postirradiation Fission Gas Release}

\section{Measurements}

In-pile fission gas release measurements (see Section 3.2) were used to evaluate fuel performance during irradiation. One of the objectives of the postirradiation fission gas release measurements was to verify the in-pile data. To accomplish this, an EOL in-pile fission gas release value for each cell containing fuel rods (cells 1, 2, 5, and 6) was determined from the TRIGA postirradiation fission gas release data. The contribution from each fuel rod to the total cell R/B was calculated using the equation

$$
R / B_{i}=\left(R / B_{E O L}\right)\left(T_{c o r r}\right)\left(x_{i}\right)
$$

where $R / B_{i}=$ contribution to the cell $R / B$ from the $i^{\text {th }}$ fuel rod

$$
\begin{aligned}
\mathrm{R} / \mathrm{B}_{\text {EOL }}= & \text { postirradiation TRIGA fission gas release value }(\mathrm{R} / \mathrm{B} \mathrm{Kr}-85 \mathrm{~m} \\
& \text { at } \left.1100^{\circ} \mathrm{C}\right) \text { for the } i^{\text {th }} \text { fuel rod } \\
\mathrm{T}_{\text {corr }}= & \text { temperature correction factor for } \mathrm{Kr}-85 \mathrm{~m} \text { determined from } \\
& \mathrm{Eq} \cdot 4-13 \\
\mathrm{X}_{i}= & \text { fraction of fissions in cell occurring in the } i^{\text {th }} \text { fuel rod }
\end{aligned}
$$

The TRIGA fission gas release data $\left(R / B_{E O L}\right)$ were corrected to the volume-average irradiation temperature at which the fuel rods operated 
when the in-pile fission gas release measurements were made. The contribution from each fuel rod was then added to obtain the calculated TRIGA EOL $R / B$ for each cell. The results of these calculations are given in Tables 4-21 and 4-22.

The cell EOL R/B values calculated from the TRIGA postirradiation data and the EOL cell R/B values monitored in-pile were in good agreement for cel1s 1, 2, and 5 of P13R and for cells 1 and 5 of P13S. However, the EOL cell $R / B$ values determined from the TRIGA data were significantly lower than the in-pile data measured for cell 6 of P13R and for cells 2 and 6 of P135.

There are several possible reasons for the large discrepancy between the calculated and measured EOL R/B values for the three cells. The TRIGA $R / B$ data are believed to be the most valid measure of fission gas release from the fuel specimens based on previous experience (Refs. 4-1, 4-9). It is possible the EOL volume-average fuel temperatures used in the calculations were lower than the actual operating temperatures of the fuel specimens at the time the in-pile measurements were made. However, even a temperature difference of $100^{\circ} \mathrm{C}$ would only increase the calculated TRIGA cell $\mathrm{R} / \mathrm{B}$ values by a factor of two. When the $\mathrm{R} / \mathrm{B}$ values were normalized to the fraction of fissions occurring in each fuel rod, a constant neutron flux across the cell was assumed. Although a steep flux gradient existed across cell 6 in each capsule, the effect could not account for such a large discrepancy between the calculated and measured $R / B$ values.

It is possible that a systematic or calibration error was made for some of the cells at the GETR when the in-pile fission gas release was monitored. To evaluate this possibility, a comparison was made between the BOL cell R/B values measured in-pile and those calculated from TRIGA preirradiation data. This calculation was performed in the same manner as the EOL analysis; the results are given in Tables 4-23 and 4-24. At $B O L$ the $R / B$ value monitored in-pile for each cell was lower than the value calculated from the TRIGA fission gas release data. However, at these low 
activity levels (in the $10^{-7}$ range), the differences between the measured and calculated values were not considered significant.

Based on these results, fission gas release data obtained from in-pile measurements at the GETR gave a qualitative indication of fuel performance throughout irradiation. However, to quantitatively evaluate levels of irradiation-induced fuel failure, postirradiation fission gas release measurements were required.

\subsection{GAMMA-RAY SPECTROMETRY ANALYSES}

Gamma-ray spectrometry was utilized to study fuel rods, unbonded particle samples, and fuel rod graphite crucibles. The analyses were conducted to provide information on fuel rod homogeneity, fuel specimen fission product inventories, and fission product transport.

Gamma counting was performed using a lithium-drifted germanium (Ge-Li) gamma-spectrometer coupled to a 4096-channe1 Sigma 2 computer analyzer. Single channel analyzers were used to print out relative profiles of individual isotopes. All specimens were counted in the low-level hot cell through a 42-in.-1ong collimator. Each type of specimen required a different size collimator cross section because of the different sample activities and geometries. Unbonded particle samples were positioned using a Nuclear Chicago automatic sample changer. Fuel rods and graphite crucibles were scanned axially using a special traversing unit that allowed a slow and continuous movement of the sample past the collimator.

\subsubsection{Fuel Rods}

Fuel rods were gamma counted using a $0.023-i n$. by $0.875-i n$. collimator that was positioned with the long axis perpendicular to the long axis of the fuel rod. A column of rods was moved past the collimator continuously at a speed of $0.05 \mathrm{in.} / \mathrm{min}$. Single channel scans for $\mathrm{Cs}-137$ and $\mathrm{Zr}-95$ using their respective $661-$ and $724-\mathrm{keV}$ energy peaks were plotted continuously. These scans are shown in Figs. 4-43 through 4-46 for the fuel rods irradia- 
ted in cells 1 and 5. Multichannel scans of 7-min duration over the 0 to 2000-keV energy range were made on 25 fuel rods. This ensured that one complete scan averaged over a 0.35 -in. (0.05 in. $x 7 \mathrm{~min})$ long fuel zone was obtained for each fuel rod. The fission product isotope inventory for each rod obtained from the multichannel scans and calculated using the FISS-PROD computer program is given in Tables 4-25 and 4-26. The counting error of each fission product isotope is given in Table 4-27. A quantitative fission product isotope inventory was determined from the multichannel scans using the following equations:

$$
\mu \mathrm{Ci}=\frac{\mathrm{DPM}}{2.22 \times 10^{6}}
$$

and

$$
\mathrm{DPM}=\frac{\mathrm{CPM}}{(\mathrm{CE})(\mathrm{AI})}
$$

where $D P M=$ disintegrations per minute of each isotope

$C P M=$ counts per minute of each isotope, measured by the detector

$\mathrm{CE}=$ counting efficiency of the particular counting geometry

AI $=$ absolute intensity of the various isotopes

Fuel rods 7161-004-18-6 (P13R, 5C) and 7161-004-18-5 (P13S, 5C) were used as internal standards. The theoretical DPM of each isotope in these rods was calculated using the FISS-PROD computer program. A counting efficiency versus peak energy calibration curve was constructed using the measured isotope CPM, the known values of the absolute intensities, and Eq. 4-16. A quantitative value for each isotope was calculated using this calibration curve and the measured CPM. The measured and theoretical fission product isotope inventories are in agreement within $\pm 20 \%$. The differences between the two inventories are a composite of the counting error for each isotope and the error involved in the calibration technique used. 


\subsubsection{Graphite Crucibles}

The fuel rod graphite crucibles were gamma counted to aid in the evaluation of metallic fission product release and transport from the fuel rods. The graphite crucibles were counted in a manner similar to the fuel rods except a $0.100-i n$. by $0.875-i n$. collimator was used. Plots of the Cs-137 profiles obtained for the crucibles and the positions of the multichannel scans are shown in Figs. 4-47 and 4-48. The only significant isotope activities detected were $\mathrm{Ta}-182, \mathrm{Cs}-134$, and $\mathrm{Cs}-137$; these data are presented in Tables 4-28 and 4-29. The concentration of the cesium isotopes was calculated using Eq. 4-15 and Eq. 4-16. The relative activity of Ta-182 (1189 keV) was reported because the calibration curve used was only valid over the energy range of 300 to $750 \mathrm{keV}$.

The crucible from cell 1 of P13S, which was thermal cycled up to $1675^{\circ} \mathrm{C}$ during irradiation, exhibited the highest cesium activity. Low cesium levels were detected in the other crucibles, which indicated negligible fission product release from the fuel rods during irradiation.

\subsubsection{Unbonded Particle Samples}

All unbonded particle batches were gamma counted using an automatic sample changer to position plastic vials containing the particles in front of the collimator. The particle batches were counted using a $0.346-i n$. by 0.738-in. collimator. Due to the high activity of the particle batches, a 2-in.-thick lead shield was placed between the particle samples and the collimator during counting. Because of the lead shield, a reliable calibration for quantitative analysis of the data could not be made. Instead, a qualitative evaluation of metallic fission product retention of each particle batch was made by comparing the measured and calculated Cs-137/ $\mathrm{Zr}-95$ isotope ratios. The $\mathrm{Zr}-95$ isotope was chosen as the reference isotope because of its physical stability and ease of measurement. The theoretical fission product inventory for each batch was calculated with the FISS-PROD computer program. 
The measured and calculated $\mathrm{Cs}-137 / \mathrm{Zr}-95$ isotope ratios of each particle batch examined are given in Tables 4-30 through 4-33. The BISO and TRISO coated fertile particle batches did not exhibit any measurable cesium 1oss. The measured Cs $-137 / \mathrm{Zr}-95$ isotope ratios of each particle batch were within $10 \%$ of the calculated theoretical ratio; however, they were systematically higher. This systematic difference was attributed to uncertainties in the input data to the FISS-PROD computer program used to calculate the theoretical ratios. The TRISO coated fissile particle batches retained from 80 to $100 \%$ of their cesium inventory. The relationship between metallic fission product loss and particle failure is discussed in more detail in section 5.3 .1 .

The majority of particles from each of six different batches were split into groups of one to nine particles each and recounted to evaluate the variation of the Cs-137/Zr-95 ratio from particle to particle. The Cs-137/ Zr-95 isotope ratio for each particle aliquot from these six batches is given in Table 4-34. The variation in the isotope ratio between the particle aliquots of most of the particle batches was low, as evidenced by the narrow standard deviation. The measured $\mathrm{Cs}-137 / \mathrm{Zr}-95$ ratio for scan number 1170 of batch 6151-00-046-1 was approximately 20\% 1ower than the mean isotope ratio. This indicates that at least one of the three particles in the aliquot had lost a significant portion of its Cs-137 inventory. If preparation of the particle aliquots could be automated, gamma-ray spectrometry would be a viable technique for determining failure levels in large particle batches.

\subsection{METALLOGRAPHIC EXAMINATION}

Metallographic examination was performed on 17 fuel rods and 14 unbonded particle samples to evaluate the irradiation-induced changes in the fuel particle coatings and fuel kernel microstructure and to observe the condition of the carbonaceous fuel rod matrix. Metallographic specimens containing 34 to 210 unbonded coated particles or bonded fuel rods were prepared in the metallographic hot cell using special techniques developed for 
high-radiation metallography. After grinding, most specimens were reimpregnated with mounting compound to reduce kernel pullout during polishing. A second polished section of some fuel rods was taken to increase the population size of the fuel particles examined. Prior to examination, al1 polished specimens were passivated with a solution of $50 / 50 \mathrm{HNO}_{3}-\mathrm{H}_{2} \mathrm{O}$ to impede hydrolysis of the $\mathrm{UC}_{2}$ fuel kernels. All specimens were examined under bright-field illumination and polarized light with a Lietz metallograph. Photographs of representative particles from some of the fuel rods examined metallographically are shown in Figs. 4-49 through 4-60.

An evaluation of the fissile, fertile, and inert particle coating failure observed in the fuel rods and unbonded particle samples was made during the metallographic examination; the results are summarized in Tables 4-35 through 4-37. The corresponding 95\% confidence intervals for the failure levels, determined using a binomial distribution (Ref. 4-11), are also reported. The $95 \%$ confidence statement, which was based on the number of particles examined, means that an infinite population of particles with attributes of the particle batch tested to these conditions will have a failure level at the $95 \%$ confidence level bounded by this interval.

\subsubsection{Fuel Rod Matrix}

The carbonaceous matrix of all 17 fuel rods examined was judged to be in good condition after irradiation. No significant matrix cracking or other deleterious irradiation effects were observed. Representative photomicrographs of the matrix graphite and composite radial cross sections of the fuel rods are shown in Figs. 4-61 through 4-77.

Matrix macroporosity is made up of the large voids in the injected matrix of a fired rod. The voids are characterized by an absence of continuous matrix and a physical dimension in excess of about $100 \mu \mathrm{m}$. Macroporosity, or the lack of matrix, influences the amount of surface area available for bonding between the particles and matrix and thus is related to the strength and thermal conductivity of the fuel rod. Fuel rod matrix 
macroporosities, determined by the point-count method (Ref. 4-12) from the diametral composite metallographic cross sections, are reported in Table 4-38.

The postirradiation fuel rod macroporosities ranged from 38 to 60 vol \% of the matrix phase. Densification of the carbonaceous matrix during irradiation is reflected by an increase in the fuel rod macroporosity. Irradiation-induced change in the fuel rod macroporosities ranged from 0 to $112 \%$. The precision of the measurements was estimated to be $\pm 2.5 \%$ (absolute) at the $95 \%$ confidence level based on six measurements made on the same polished section of two different fuel rods. The uncertainty in the fuel rod macroporosity is probably greater than this, however, since macroporosity varies with location in a rod.

\subsubsection{Fissile Particles}

The condition of the TRISO coated fissile particles was considered to be excellent after irradiation to the severe fast neutron exposures capsules $\mathrm{P} 13 \mathrm{R}$ and $\mathrm{P} 13 \mathrm{~S}$ received. Photomicrographs of representative fissile particles after irradiation are shown in Figs. 4-49 through 4-60 and Figs. 4-78 through 4-80.

A number of particle batches with coating parameters representative of LHTGR design requirements did not exhibit any failure. "No coating failure was observed that could be attributed to thermochemical effects such as fuel kernel migration or fission product attack. A majority of the particle batches that exhibited significant failure levels were tested to evaluate the effect of a particular variable such as faceted coatings, PyC deposition rate, or high-temperature transients on fuel particle irradiation performance. Photomicrographs of fuel particles that suffered pressure vessel failure as a result of thermal cycling to $1675^{\circ} \mathrm{C}$ in-pile and during postirradiation heating to $1600^{\circ} \mathrm{C}$ in the TRIGA facility are shown in Figs. 4-81 and 4-82, respectively. 
An example of fissile particle OPyC coating failure is shown in Fig. 4-83. In particles that did not suffer complete pressure vessel failure the OPyC coating failure was attributed to irradiation-induced dimensional change of the coatings during irradiation to the severe fast neutron exposures. No obvious occurrences of matrix-coating interaction were observed in the fuel rods examined metallographically.

Most of the SiC coating failure appeared mechanical in nature and occurred in the form of fine radial cracks, as shown in Fig. 4-84. It has been observed in previous examinations that the brittle SiC coatings are subject to a finite amount of damage during preparation of the metallographic specimens (Refs. 4-3, 4-13). This effect is illustrated by two unbonded particle samples [batch 6155-01-020 tested in P13S (4-4) and batch 6151-04-015 tested in P13R (3-12)]. The in-service particle failure levels determined by fission gas release measurements for these samples were $2.6 \%$ and $0.6 \%$ (see Section 4.4.2). These low particle failure levels indicate that the SiC coatings in most of the particles were intact. However, total coating (OPyC plus SiC coating) failure levels of 50 and $95 \%$ were recorded for batches 6155-01-020 and 6151-04-015, respectively, during the metallographic examination. These results indicate that a large fraction of the cracked SiC coatings observed was an artifact of sample preparation. For these reasons, the level of cracked SiC coatings observed in the fissile particles was not reported in Table 4-35.

Smal1 laminar inclusions in the SiC coating, of the type shown in Fig. 4-85, were observed in 2 to $3 \%$ of the TRISO $\mathrm{UC}_{2}$ particles examined metallographically. Since only one polished cross section of a particle was examined, the actual population of the inclusions would be greater. The presence of this type of inclusion in the SiC coating is usually observed in most batches of TRISO coated fuel particles. Previous electron microprobe analysis of TRISO particles revealed these inclusions consist almost entirely of carbon (Ref. 4-13). Although no SiC coating failure directly attributable to the presence of these inclusions was observed, if the inclusions were large or occurred frequently enough, they would be expected to adversely affect the mechanical integrity of the SiC coatings. 
Levels of IPyC coating debonding and failure observed in the fissile particles are reported in Table 4-35. Relatively high levels of IPyC debonding, an example of which is shown in Fig. 4-86, were observed in most fissile particles. However, actual failure of the IPyC coating, with the exception of particles that suffered complete pressure vessel failure as a result of high-temperature postirradiation heating, was only observed in a few particle batches.

In particles where the IPyC coating remained bonded to the SiC, the buffer coating decoupled from the IPyC coating at the seal coat and densified onto the fuel kerne1. In most particles the buffer remained attached to the IPyC coating only in a small localized area, thereby creating a gap at the buffer-IPyC interface which was predominantly on one side of the particle. Although the formation of a gap at the buffer-IPyC interface can affect the temperature profile within a particle (Ref. 4-14), the location of the gap was random with respect to the hot or cold side of the particle.

In one TRISO $\mathrm{UC}_{2}$ particle, a deposit of carbon was present in the gap formed between the buffer and IPyC coating, as shown in Fig. 4-87. The deposit was optically active and had a columnar-type structure when viewed under polarized 1ight. Similar carbon deposits observed in irradiated TRISO $\mathrm{UO}_{2}$ particles were attributed to gas phase carbon transport.

Only a small fraction $(<2 \%)$ of the buffer coatings of the TRISO UC particles were cracked. This occurred only in particles where the fuel kernel had segregated into what appeared to be a fuel phase and regions of rejected carbon, as shown in Fig. 4-88. When the buffer coating cracked, the fuel kernel did not extrude into the resulting gap. In particles where the fuel kernels appeared to have segregated into a fuel phase and regions of rejected carbon, the fission product recoil zone at the bufferkernel interface was often perforated with holes up to $30 \mu \mathrm{m}$ in diameter, as shown in Figs. 4-88 and 4-89. The holes in the recoil zone may have resulted from a combination of fission recoil damage and interaction with fragments of the fuel kernel. In some unbonded TRISO $\mathrm{UC}_{2}$ particle samples, 
the structural coatings of a few particles failed, leaving the buffer coating on the fuel kernel as shown in Fig. 4-89.

Less than $1 \%$ of the buffer coatings were cracked in the TRISO $(1 \mathrm{Th}, 1 \mathrm{U}) \mathrm{O}_{2}$ particles (5466-37); however, $88 \%$ of the buffer coatings were cracked in the larger-diameter TRISO ( $8 \mathrm{Th}, 1 \mathrm{U}) \mathrm{O}_{2}$ particles (6155-01-020). In some of the particles the fuel kernel extruded into the gaps formed in the buffer coating; however, interaction between the kernel and IPyC coating was not observed.

Three distinct types of microstructures were observed in the $\mathrm{UC}_{2}$ fuel kernels. The kernels that operated in the high-temperature positions (average temperature above $1200^{\circ} \mathrm{C}$ ) were spherical and had a homogeneous appearance, as shown in Fig. 4-90. Under high magnification small flakes of 1ight-colored material with a particle size of $\sim 1.0 \mu \mathrm{m}$ could be seen evenly dispersed throughout the fuel kernels. Fuel kernels that operated at intermediate temperatures of $1000^{\circ}$ to $1100^{\circ} \mathrm{C}$ appeared to have segregated into a fuel phase and regions of rejected carbon, as shown in Fig. 4-91. The kernels were irregular in shape and the high-carbon regions appeared optica1ly anisotropic when viewed under polarized light.

The fuel kernels that operated at average temperatures of less than $1000^{\circ} \mathrm{C}$ were more spherical and contained large fission gas bubbles, as shown in Fig. 4-92. In many cases a substance filled the smaller fission gas voids or coated the inner surface of the larger voids. This material was probably carbon, and it appeared optically active under polarized 1ight. The presence of $1 \mathrm{wt} \%$ thorium in most of the $\mathrm{UC}_{2}$ fuel kernels did not have any noticeable effect on the kernel microstructure.

The appearance of the $(8 \mathrm{Th}, 1 \mathrm{U}) \mathrm{O}_{2}$ and $(1 \mathrm{Th}, 1 \mathrm{U}) \mathrm{O}_{2}$ fuel kernels irradiated as unbonded particle samples at an average temperature of $1090^{\circ} \mathrm{C}$ was similar to the low-temperature $U_{2}$ particles, as shown in Figs. $4-79$ and 4-80. The kernels were spherical and contained small fission gas bubbles dispersed evenly throughout the kernel. The appearance of the $(1 \mathrm{Th}, 1 \mathrm{U}) \mathrm{O}_{2}$ 
fuel kernels irradiated in fuel rod $7161-004-16-6$ at $1375^{\circ} \mathrm{C}$ was similar to the unbonded particle samples; however, the fission gas bubbles had coalesced into large voids as shown in Fig. 4-93. Small white inclusions observed in the kernels are typically found in high burnup oxide fuels and probably consist of molybdenum, ruthenium, and technetium.

Significant diffusion of metallic fission products into the IPyC coating of the TRISO (Th,U) $\mathrm{O}_{2}$ particles was not observed. Localized concentrations of metallic fission products were observed in the IPyC coatings of T'RISO $\mathrm{UC}_{2}$ particles tested as unbonded particle samples, but attack of the SiC coating did not occur. However, slight degradation of the SiC coating by metallic fission products was observed in TRISO $\mathrm{UC}_{2}$ particles irradiated in fuel rods at average temperatures above $1050^{\circ} \mathrm{C}$. A summary of the metallic fission product interactions observed in the TRTSO $\mathrm{UC}_{2}$ particles during the metallographic examination is given in Table 4-39.

A localized concentration of fission products was observed in the IPyC coating on the cool side, away from the fuel rod centerline, of most particles. If the buildup of fission products at the IPyC/SiC coating interface was large enough, the IPyC coating was forced away from the SiC coating as shown in Fig. 4-94. Reaction of metallic fission products with the SiC coatings was slight. Maximum penetrations of 2 to $5 \mu \mathrm{m}$ were genera11y observed, except for two particles in fuel rod 7161-004-18-5 (capsule $\mathrm{P} 13 \mathrm{~S}$, position $5 \mathrm{C}$ ) where the penetration was observed to be slight1y less than one-half the thickness of the SiC coating.

Fuel kernel migration was observed in TRISO $\mathrm{UC}_{2}$ and TRISO (Th, U) $\mathrm{O}_{2}$ particles irradiated in the high-temperature (cell 5) fuel rods. The direction of migration was toward the hot side of the fuel particles (in a direction perpendicular to the fuel rod centerline), although in a few particles the migration direction was almost parallel to the fuel rod centerline. This deviation probably resulted from a change in the temperature profile within the particles due to localized fuel rod inhomogeneities or fuel rod end effects. The location of the buffer/IPyC coating gap did not have any noticeable effect on the kernel migration direction. 
Many of the $\mathrm{UC}_{2}$ particles had a region of what appeared to be rejected carbon on the cool side of the kernel, as shown in Fig. 4-90. This region was optically active under polarized light and contained small white globules, which were probably a fuel-rich phase.

Photomicrographs of $(\mathrm{Th}, \mathrm{U}) \mathrm{O}_{2}$ fuel kernel migration are shown in Fig. 4-93. A crescent-shaped deposit of material, which appeared to be carbon, was present on the cool side of many of the kernels. A carbon deposit with a granular-type structure was also observed in the gap formed between the buffer and IPyC coating.

The magnitude of $\mathrm{UC}_{2}$ and $(\mathrm{Th}, \mathrm{U}) \mathrm{O}_{2}$ fuel kernel migration was measured in six of the fuel rods; the data are presented in Table 4-40. The 1ocation of each particle with respect to the fuel rod centerline was established. Migration distances were determined from high-magnification photomicrographs by subtracting the diameter of the fuel kernel perpendicular to the temperature gradient from the distance of the hot kernel surface to the original position of the cool kernel surface. This measurement technique was developed from analyzing microradiographs of $\mathrm{UC}_{2}$ fuel kernel migration and takes into account migration which has occurred as a result of kernel elongation and rejection of carbon. The difficulty with this measurement technique is that the original position of the cool surface of the fuel kernel cannot be established accurately, and measurements cannot be made if the fuel kernel is irregularly shaped. However, this technique provides a better estimate of the kernel migration distance for small-diameter fissile kernels than a technique where the distances from the hot and cool surfaces of the fuel kernel are measured relative to the SiC coating. In the fissile particles the formation of a gap at the buffer/IPyC coating interface often results in the translation of the fuel kernel toward one side of the particle, which would erroneously influence the migration distance measured.

The observed $(\mathrm{Th}, \mathrm{U}) \mathrm{O}_{2}$ kernel migration rate was faster than the $\mathrm{UC}_{2}$ migration rate over the temperature range $1275^{\circ}$ to $1375^{\circ} \mathrm{C}$. This result was 
expected based on available kernel migration data. The $\mathrm{UC}_{2}$ kernel migration data presented in Table 4-40 are compared with predicted migration rates based on HTGR core design data in Section 5.1.1.2.

\subsubsection{Fertile Particles}

Photomicrographs of representative fertile particles irradiated in fuel rods are shown in Figs. 4-49 through 4-60. A number of particle batches with coating parameters representative of LHTGR design requirements did not exhibit any failure. A summary of the coating failure analysis obtained during the metallographic examination is given in Table 4-36. The majority of fertile particle batches that exhibited significant failure levels were tested to evaluate the effect of a particular variable such as faceted coatings, OPyC coating deposition rate, or high-temperature transients on fuel particle irradiation performance.

The BISO OPyC coatings of all particle batches appeared relatively isotropic under polarized 1ight. Photomicrographs of BISO fertile particles with OPyC coatings deposited from propylene and from mixed gas are shown in Figs. 4-95 and 4-96, respectively. No differences were observed in the appearance of the two types of coatings.

The highest levels of OPyC coating failure were observed in particle batch 6542-20-035 (fue1 rod 7161-004-01-7) which had faceted coatings and was thermal cycled in-pile (Fig. 4-81) and in particle batch 6542-01-020 (fuel rod 7161-004-16-5) which was heated in TRIGA to $1600^{\circ} \mathrm{C}$ after irradiation. Particles with severely faceted coatings (Figs. 4-97 and 4-98) exhibited slightly higher failure levels than particles without faceted coatings. Fuel particles with nonround fuel kernels (Fig. 4-99) also exhibited higher failure. Examples of irradiation-induced failure in BISO $\mathrm{ThO}_{2}$ particles with high- and low-density OPyC coatings are shown in Figs. 4-100 and 4-101, respectively.

Very fine microcracks were observed in the OPyC coatings of two unbonded particle samples (4252-06-010-1 and 6542-20-035-2), as shown in 
Figs. 4-102 and 4-103. Microcracks of the size observed in these two particles would probably not be observed during the visual examination.

In all but two BISO $\mathrm{ThO}_{2}$ particle batches, a thin high-density seal coat was applied between the buffer and OPyC coating. During irradiation the seal coat cracked and densified, leaving numerous small gaps at the buffer/OPyC coating interface as shown in Fig. 4-104. The breakup of the seal coat did not initiate any cracks in the adjacent buffer or OPyC coatings. One BISO $\mathrm{ThO}_{2}$ particle batch (6542-18-015) was examined that did not have a seal coat, as shown in Fig. 4-105. The absence of the thin seal coat did not have any apparent effect on the BISO coating irradiation performance. In some particles the buffer coating delaminated and shrank away from the OPyC coating.

The buffer coatings densified during irradiation and appeared slightly optically active under polarized light. High levels of buffer cracking were noted in most particle batches; however, this did not appear to be detrimental to the stability of the BISO coatings during irradiation. In many particles the buffer coatings cracked and densified while the OPyC coating remained intact, as shown in Fig. 4-106. Propagation of buffer coating cracks into the adjacent OPyC coating, as shown in Fig. 4-107, was only observed in a few particles. The buffer coatings deposited using $\mathrm{N}_{2}$ carrier gas (Fig. 4-108) appeared similar to coatings deposited using $\mathrm{Ar}$ as the carrier gas. Although a significant fraction of the buffer coatings deposited using $\mathrm{N}_{2}$ carrier gas (6542-12-025-2) were broken, no apparent differences between the coatings applied using $\mathrm{Ar}$ or $\mathrm{N}_{2}$ carrier gases could be ascertained.

One batch of TRISO $\mathrm{ThO}_{2}$ particles, shown in Fig. 4-109, was irradiated for comparative purposes. Thirty-seven particles were examined meta11ographically. The PyC coatings appeared relatively isotropic under polarized 1ight. The OPyC coating of one particle was failed; however, the SiC coating was intact. The SiC coating of one particle was cracked; this was the only instance where the IPyC coating had also debonded from the SiC coating. The buffer coating debonded and shrank away from the IPyC coating in $~ 250 \%$ of 
the particles, and in four of the particles the buffer coating was cracked. A reaction zone was observed on the inner surface of the SiC coating of one particle, as shown in Fig. 4-110. The IPyC coating was intact although a portion of the buffer coating pulled out of the particle during polishing. No signs of metallic fission products were observed in the IPyC coating and the reaction probably was a result of oxidation.

Only one defective particle was observed in the fuel rods during the metallographic examination. The BISO fertile particle shown in Fig. 4-111 had a broken OPyC coating during fuel rod fabrication. Matrix material was bonded to the buffer coating in the region where the OPYC coating is missing. During irradiation the coating broke and the fuel kernel converted to carbide and subsequently hydrolyzed.

In some fertile particles where irradiation-induced coating failure occurred early in life, the fuel kernels also partially converted to carbide. An example of this is shown in Fig. 4-107 where partial conversion of a localized region of the $\mathrm{ThO}_{2}$ kernel near a crack in the OPyC coating has occurred.

Irradiation-induced changes in the $\mathrm{ThO}_{2}$ fuel kernel microstructure were quite apparent. At low burnup ( $<3 \%$ FIMA) the kernel grain structure was still visible under polarized light. After irradiation to higher burnup, ( $>5.6 \%$ FIMA) formation of pores in the $\mathrm{ThO}_{2}$ grains occurred as shown in Fig. 4-112. In many particles the $\mathrm{ThO}_{2}$ grain structure was completely obliterated, although large variations were observed in the microstructures of particles within a given sample. In one particle significant grain boundary thickening occurred on the hot side of the particle, as shown in Fig. 4-113. The grain boundary region was optically active under polarized 1ight, which indicates carbon may have been present (Ref. 4-15).

\subsubsection{Inert Particles}

A11 but two fuel rods contained TRISO carbon inert particles in order to achieve close particle packing and the desired fuel particle and shim 
loadings. Photomicrographs of a representative inert particle are shown in Fig. 4-114. The OPyC coating of the TRISO inert particles was deposited at a low coating rate of $1.1 \mu \mathrm{m} / \mathrm{min}$. Consequently significant levels of irradiation-induced OPYC coating failure occurred in the particles, as shown in Table 4-37. Many of the particles exhibited cracked SiC coatings. The broken SiC coatings were attributed to metallographic polishing damage. Since the particles contain inert kernels, irradiation-induced failure resulting from the effects of high burnup would not occur.

\subsection{OPYC COATING PERMEABILITY STUDIES}

A design requirement for BISO coated particles, in addition to irradiation performance requirements, is that the OPyC coating must be sufficiently dense and impermeable to retain fission gases. Previous results on unirradiated $\mathrm{BISO} \mathrm{ThO}_{2}$ particles without seal coats have indicated that low-density OPyC coatings are permeable to fission gases; however, the fission gas release through OPyC coatings with densities of $1.90 \mathrm{~g} / \mathrm{cm}^{3}$ or higher was immeasurably low (Refs. 4-1, 4-9).

Fission gas release measurements were made on all fuel specimens after irradiation in capsules $\mathrm{P} 13 \mathrm{R}$ and $\mathrm{P} 13 \mathrm{~S}$ (see Section 4.4). A description of the BISO $\mathrm{ThO}_{2}$ particle batches in which no coating failure was observed during the visual examination and the results of the fission gas release measurements are summarized in Table 4-41. The measurements revealed a large variation in the amount of gaseous release from the unfailed BISO coated particles. A low level of gaseous release $\left(R / B<1 \times 10^{-6}\right.$ ) from intact fuel particles results from heavy metal contamination. However, the higher levels of gaseous release indicate the PyC coatings are permeable to fission gases. The permeability may result from diffusion through a porous coating microstructure or through microcracks that are not visible with a 20X microscope. The fission gas release values obtained for the ten particle batches are plotted in Fig. 4-115 as a function of preirradiation OPyC coating density. The data show an increase in gaseous release as the OPyC coating density decreases, which indicates the leve1 of gaseous release is 
probably related to the PyC coating microstructure rather than to the existence of microcracks.

In addition to the results discussed above, the OPyC coating permeability of several BISO $\mathrm{ThO}_{2}$ particle batches described in Table 4-42 was evaluated using acid-leach and fission gas release measurements. The bathes were selected to provide a range of OPyC coating densities and coating rates. Each batch was examined visually using an in-cell stereomicroscope and approximately 200 intact particles were selected. The particle samples were cleaned with diluted nitric acid in an ultrasonic cleaner and rinsed with acetone to remove any surface contamination. Fission gas release measurements were then made on each sample; the results are given in Table 4-42. Visual examination revealed that a low level of coating failure had occurred in two of the particle samples during the fission gas release measurements. The measured fission gas release is plotted in Fig. 4-116 as a function of preirradiation OPyC coating density. The data are consistent with the results obtained for entire particle batches (Fig. 4-115) and indicate that gaseous release increases as the OPyC coating density decreases.

The particles were then leached in an acid ( $13 \mathrm{M} \mathrm{HNO}, 0.1 \mathrm{M} \mathrm{HF}$ ) solution. Aliquots of the acid solutions were taken at regular intervals and analyzed for thorium using a spectrophotometric technique. Results of the analysis, with the exception of batch 6542-01-010-1 in which the acid solution was contaminated during leaching, are presented in Table 4-43. To allow a comparison of data between samples, the leach results from batch 6542-01-020-2 had to be normalized to the other samples since it only contained 55 particles. This was done by multiplying the micrograms of thorium leached from the sample by $200 / 55$.

The condition of the particle samples after leaching was evaluated by visual and metallographic examination; the results are summarized in Table 4-44. Several of the samples, particularly batches 6542-22-015-1 and 6542-01-020-2, exhibited significant levels of OPyC coating failure after leaching. The failure was attributed to the attack of the OPyC coatings 
by the acid solution after extended periods of exposure. The rapid rate at which thorium was leached from batch 6542-22-015-1 was attributed to the onset of particle breakage early in the leaching process. To minimize the effect of particle failure contributing to the amount of thorium leached from the particle samples, only the data from aliquots taken during the first $21 \mathrm{hr}$ of hot leaching were analyzed.

Results of the acid leaching experiments, with the exception of particle batches 6542-01-010-1 and 6542-22-015-1, are shown in Fig. 4-117, where the amount of thorium leached is plotted as a function of hot leaching time. The effect of OPyC coating density on the leachability is significant. The entire thorium content of batch 6542-21-016-1, which had an OPyC coating density of $1.73 \mathrm{~g} / \mathrm{cm}^{3}$, was leached out within $11 \mathrm{hr}$, whereas only $0.7 \%$ of the thorium content of batch 6542-24-015-1, which had an oPyC coating density of $1.94 \mathrm{~g} / \mathrm{cm}^{3}$, was leached out after $15 \mathrm{hr}$.

The OPyC coating rate (numbers shown in parentheses in Fig. 4-117) also had an effect on the leachability of the particles, but it was not as pronounced as the effect of OPyC coating density. By comparing the results from particle batch 6542-01-020-2 with 4252-06-018-1 and batch 6542-09-010-2 with 6542-02-020-2 it can be seen that for approximately the same OPyC coating density, the leachability increases slightly as the coating rate increases.

Representative photomicrographs taken during the metallographic examination of these particle batches after acid leaching are shown in Figs. 4-118 through 4-121. The $\mathrm{ThO}_{2}$ fuel kernels were completely leached from the particle batches with low-density OPyC coatings. In the particle batches with high-density OPyC coatings, approximately $90 \%$ of the fuel kernels showed no signs of degradation from the acid leaching, which indicates the PyC coatings were impermeable to the leach solution. The OPyC coatings were affected by the acid leaching. The low-density OPyC coatings, with the exception of batch 6542-01-020-2, appeared more porous and pitted relative to the higher-density coatings, which showed little sign of degradation. The OPyC coating of batch 6542-01-020-2, which had a density of $1.80 \mathrm{~g} / \mathrm{cm}^{3}$ 
and a coating rate of $2.7 \mu \mathrm{m} / \mathrm{min}$, had a smooth appearance, similar to the high-density OPyC coatings, except all the fuel kernels were heavily Ieached.

As mentioned previously the particle samples exhibited varying levels of coating failure after leaching; consequently, only leach aliquots taken during the first $21 \mathrm{hr}$ of hot leaching were analyzed. Coating failure undoubtedly contributed to the levels of thorium leached from the samples, particularly batches 6542-02-020-2, 6542-01-020-2, and 4252-06-018-1. However, in most particle samples the amount of thorium recovered greatly exceeded the amount that would be exposed to the leach solution as a result of the observed level of coating failure.

The leachability of the particle samples correlated with the fission gas release measurements made prior to the acid leaching. The particle samples with low-density OPyC coatings that exhibited the highest thorium leaching rates also exhibited the highest fission gas release.

\subsection{SIMULATED REPROCESSING STUDIES}

After a 4-year irradiation, spent fuel elements will be removed from the reactor and reprocessed for fuel recovery and fission product removal. Utilization of a TRISO coated all-uranium fissile kernel and a BISO coated fertile particle will improve the recovery and separability of U-233 from U-236 during reprocessing, which is an integral part of the thorium fuel cycle (Ref. 4-16). During reprocessing, the elements will be mechanically crushed and burned to eliminate the fuel element graphite and remove the OPyC coatings from the fuel particles. A leach - solvent extraction process will permit recovery of the U-233 bred in the fertile particles. The fissile particle SiC coating, which remains intact during the burning and leaching operations, prevents the recovered U-233 from being significantly contaminated with U-236 (a neutron poison) that was bred in the fissile particles. To ensure that a sufficient fraction of the fissile particles remain intact, the following criterion has been established (Ref. 4-17): the fissile (TRISO) fuel coating failure from all effects 
after irradiation at $\leq 1250^{\circ} \mathrm{C}$ to $8.0 \times 10^{21} \mathrm{n} / \mathrm{cm}^{2}(\mathrm{E}>0.18 \mathrm{MeV})_{\mathrm{HTGR}}$ and/or $78 \%$ FIMA shall be $<10 \%$ after removal of the OPyC coating by oxidation at $\leq 1000^{\circ} \mathrm{C}$ in the reprocessing facility. To provide data for support of this criterion, two fuel rods were burned in air and fissile particle failure fractions were determined by visual examination and acid leaching.

The fuel rods selected for the burn-back studies were 7161-004-02-6 (P13R, 1B) and 7161-004-11-6 (P13S, 2A). The rods were burned separately in the high-level hot cell using a small muffle furnace. Each rod was placed in an alumina crucible and burned in air at $950^{\circ} \mathrm{C}$ for $6 \mathrm{hr}$. After burning the crucibles were allowed to cool overnight before they were removed from the furnace.

Remnants of fuel rod 7161-004-02-6 after the burn-back are shown in Fig. 4-122. All of the fuel rod matrix graphite and fuel particle oPyC coatings were completely oxidized. The remaining fuel particles, $\mathrm{ThO}_{2}$ kernels and TRISO coated fissile particles, were covered with a white ash. The particles were cleaned in an ultrasonic bath using concentrated nitric acid, rinsed several times in acetone, and air dried. The $\mathrm{ThO}_{2}$ kernels and TRISO coated fissile particles were then segregated in a separatory funnel using tetrabromoethane with a density of $2.97 \mathrm{~g} / \mathrm{cm}^{3}$ as the solution. The fissile particles were the $10 \mathrm{w}$-density $\left(2.6 \mathrm{~g} / \mathrm{cm}^{3}\right)$ fraction, and the $\mathrm{ThO}_{2}$ kernels were the high-density $\left(>10.0 \mathrm{~g} / \mathrm{cm}^{3}\right)$ fraction. Photographs of the $\mathrm{ThO}_{2}$ kernels and TRISO fissile particles recovered from the separatory funnel are shown in Fig. 4-123. During burn-back, fissile particles with failed coatings will completely oxidize leaving SiC coating hulls. Visual examination of the recovered particles revealed $1401 \mathrm{ThO}_{2}$ kernels, 1007 intact fissile particles, and very few SiC coating fragments. Based on the original particle weights, approximately 1120 fissile and 1400 fertile particles were estimated to be in the fuel rod. These data indicate that $100 \%$ of the $\mathrm{ThO}_{2}$ kernels were recovered and approximately $90 \%$ of the fissile particles were recovered intact. The remaining $10 \%$ of the fissile particles were assumed to be failed after the fuel rod burn-back. 
The remnants of fuel rod 7161-004-11-6 (P13S, 2A) were boiled for 15 hr in an acid solution consisting of $13 \mathrm{M} \mathrm{HNO}_{3}$ and $0.1 \mathrm{M} \mathrm{HF}$. The chemical analysis of the leach solutions is given in Table 4-45. The results indicate that the end point of the leach was reached in a very short time and all the exposed uranium had been dissolved after $3.5 \mathrm{hr}$ of leaching. The fissile particle failure fraction was determined using isotopic dilution mass spectroscopy by the standard additions technique of aliquots of the acid solution taken at regular intervals. The fourth aliquot of the leach solution was submitted for isotopic dilution mass spectroscopy to determine the uranium isotopic composition. These results are given in Table 4-46. The theoretical uranium isotopic composition of the fuel rod at EOL +120 days was calculated using the FISSIN computer code, and the results are given in Table 4-47. Because the U-238 isotope is unique to the fissile particles, the fissile particle failure fraction $(x)$ can be determined by:

$$
\mathrm{x}=\frac{\text { weight } \mathrm{U}-238 \text { in leach solution }}{\text { weight } \mathrm{U}-238 \text { in fuel rod }} .
$$

Using Eq. 4-17 and the data given in Tables 4-46 and 4-47, the calculated fissile particle failure fraction for rod 7161-004-11-6 after the burn-back was $4.2 \%$.

After leaching, the particles were examined visually and metallographically. Only three failed TRISO particles were observed out of approximately 2600 TRISO coated fissile and carbon inert particles recovered from the leach solution. Metallographic examination of a portion of the recovered particles revealed no Sic coating attack or failure due to the acid leaching (Fig. 4-124).

In summary, two fuel rods that had been irradiated to fast neutron fluences of $8.2 \times 10^{21}$ and $11.6 \times 10^{21} \mathrm{n} / \mathrm{cm}^{2}(\mathrm{E}>0.18 \mathrm{MeV})_{\mathrm{HTGR}}$ at $1050^{\circ}$ and $1040^{\circ} \mathrm{C}$, respective1y, were burned in air at $950^{\circ} \mathrm{C}$. After the fuel rod matrix graphite and OPyC coatings were completely oxidized, fissile particle failure levels of $10 \%$ and $4.2 \%$ were ascertained by visual examination and acid leaching, respectively. These results indicate that the design 
criterion required for reprocessing, namely that $\geq 90 \%$ of the TRISO coated fissile fuel maintains structural integrity after irradiation to peak conditions followed by oxidation of the fuel rods, can be achieved.

\subsection{CONTACT MICRORADIOGRAPHY}

Contact $\mathrm{x}$-ray microradiographs of five unbonded particle samples were obtained by exposing high-resolution photographic plates to an $x$-ray pulse from a Picker $110-\mathrm{kV}$ x-ray unit. The particle images on the radiograph plates were then examined to determine the postirradiation particle dimensions, which are given in Table 4-48. Photographs taken of microradiographs of representative fissile and fertile particles are shown in Fig. 4-125. The light regions within the SiC coating of the fissile particles are concentrations of metallic fission products that have diffused out of the fuel kernels. The irradiation-induced change in the particle dimensions are given in Table 4-49. These data are used to determine kernel swelling and irradiation-induced particle dimensional changes, which must be known for fuel rod dimensional change and coated particle design studies.

\subsection{DENSITY MEASUREMENTS}

\subsubsection{OPyC Coatings from Fissile Particles}

Postirradiation density measurements were made on OPyC coatings from nine fissile particle batches irradiated in capsules P13R and P13S to extend the data base on irradiation-induced PyC densification to fast

neutron fluences up to $12.1 \times 10^{21} \mathrm{n} / \mathrm{cm}^{2}(\mathrm{E}>0.18 \mathrm{MeV})_{\mathrm{HTGR}}$. Coating fragments were stripped from intact TRISO particles and were recovered from failed particles. The fragments were cleaned in an ultrasonic bath and examined by x-ray microradiography. All fragments that contained pieces of SiC coating were removed from the samples. A density column technique similar to the one described in Ref. 4-18 was employed to measure the density of the OPyC coating fragments. Benzene and tetrabromoethane were blended to provide a uniform density gradient column medium, and a density gradient curve for the liquid column was established 
with calibrated glass density floats. Postirradiation OPyC coating densities determined for the nine batches of coated particles are reported in Table 4-50.

\subsubsection{Fertile Particles}

Postirradiation particle density measurements were made on four unbonded fertile particle batches. Sample preparation and density measurements were conducted in the hot cell facility. Small samples were split from the irradiated particle batches and examined using an in-cell stereomicroscope to ensure that all the particles were intact. The samples were cleaned in an ultrasonic bath to remove any surface contamination. A density column technique similar to the one described in Ref. 4-17 was employed to measure the density of the coated particles. Two concentrations of aqueous thallium malonate were blended to provide a uniform density gradient column medium, and a density gradient curve for the liquid column was established with calibrated glass density floats.

Results of the density measurements are given in Table 4-51. Irradiation-induced densification of the BISO coated particles ranged from 7.0 to $15.5 \%$. These data will be used in future BISO particle model and design studies.

\section{REFERENCES}

4-1. Harmon, D. P., and C. B. Scott, "Development and Irradiation Performance of LHTGR Fue1," ERDA Report GA-A13173, General Atomic, October 31, 1975.

4-2. Young, C. A., and D. P. Harmon, "Preirradiation Report: Fuel Materials for P13R and P13S Irradiation Capsules," USAEC Report GA-A13026, Genera1 Atomic, November 1974.

4-3. Scott, C. B., and D. P. Harmon, "Postirradiation Examination of Capsules HRB-4, HRB-5, and HRB-6," ERDA Report GA-A13267, Genera1 Atomic, November 28, 1975. 
4-4. "HTGR Fuels and Core Development Program Quarterly Progress Report for the Period Ending August 31, 1975," ERDA Report GA-A13592, General Atomic, September 30, 1975.

4-5. Price, R. J., and L. A. Beavan, "Final Report on Graphite Irradiation Test OG-2," ERDA Report GA-A13556, Genera1 Atomic, December 15, 1975.

4-6. Anderson, E. E., et a1., "An In-Core Furnace for the High-Temperature Irradiation Testing of Reactor Fuels," Nuc1. Techno1. 11, 259 (1971).

4-7. Lofing, D. R., E. E. Anderson, and L. R. Zunwalt, "The Use of Gamma-Ray Spectroscopy in the Study of Fission-Product Release From Nuclear Fuels," General Atomic Division of General Dynamics Report GA-4615, October 8, 1963.

4-8. Meek, M. E., and B. F. Rider, "Compilation of Fission Product Yields," Vallecitos Nuclear Center Report NEDO-12154, 1972.

4-9. Myers, B. F., et al., "The Behavior of Fission Product Gases in HTGR Fuel Material," ERDA Report GA-A13723, Genera1 Atomic, to be published.

4-10. Haire, M. J., and D. W. McEachern, "Gaseous Radioactivity Levels in the Primary Coolant of an HTGR," General Atomic Report GA-A12946, (GA-LTR-14), October 1, 1974.

4-11. Burington, R. S., and D. C. May, Jr., Handbook of Probability and Statistics With Tables, 2nd Ed., McGraw-Hi11, New York (1970), pp. 245-255.

4-12. Rostoker, W., and J. R. Dvorak, Interpretation of Metallographic Structures, Academic Press, New York (1965), pp. 195-200.

4-13. Scott, C. B., and D. P. Harmon, "Postirradiation Examination of Capsule F-30," General Atomic Report GA-A13208, Apri1 1, 1975.

4-14. McElmury, S. S., and O. M. Stansfield, "An Analysis of the Effect of Buffer-IPyC Coating Separation on the Temperature Distribution in TRISO UC 2 Fuel Particles," General Atomic Report GA-A13500, December 1, 1975.

4-15. "HTGR Fuels and Core Development Program Quarter1y Progress Report for the Period Ending May 31, 1975," ERDA Report GA-A13444, General Atomic, June 30, 1975. 
4-16. Dahlberg, R. C., R. R. Turner, and W. V. Goedde1, "HTGR Fuel and Fuel Cycle Summary Description," General Atomic Report GA-A12801 (Rev.), January 21, 1974.

4-17. "Fuel Development Program P1an for the Steam Cycle HTGR," ERDA Report GA-A13660, General Atomic, to be published.

4-18. Canada, D. C., and W. R. Laing, "Use of a Density Gradient Column to Measure the Density of Microspheres," Ana1. Chem. 39, 691 (1967). 
TABLE 4-1

DESCRIPTION AND CONDITION OF FUEL RODS IRRADIATED IN CAPSLLE P13R

\begin{tabular}{|c|c|c|c|c|c|c|c|c|c|c|}
\hline \multicolumn{7}{|c|}{ Fue1 Rod Description } & \multicolumn{2}{|c|}{ Irradiation Parameters } & \multirow{2}{*}{\multicolumn{2}{|c|}{$\begin{array}{l}\text { Postirradiation } \\
\text { Condition }\end{array}$}} \\
\hline \multirow[b]{2}{*}{$\begin{array}{l}\text { Capsule } \\
\text { Position }\end{array}$} & \multirow{2}{*}{$\begin{array}{l}\text { Fuel Rod Data } \\
\text { Retrieval No. } \\
(7161-004-)\end{array}$} & \multicolumn{2}{|c|}{ Shim } & \multicolumn{3}{|c|}{ Matrix } & \multirow{2}{*}{$\begin{array}{l}\text { Average } \\
\text { Centerline } \\
\text { Cemperature } \\
\text { Teme } \\
1 \quad\left({ }^{\circ} \mathrm{C}\right)\end{array}$} & \multirow{2}{*}{$\begin{array}{c}\text { Fast Fluence } \\
\left(10^{21} \mathrm{n} / \mathrm{cm}^{2}\right) \\
(\mathrm{E}>0.18 \cdot \mathrm{leV})_{\text {HTGR }}\end{array}$} & & \multirow[b]{2}{*}{$\begin{array}{c}\text { Visual } \\
\text { Appearance (b) }\end{array}$} \\
\hline & & $\begin{array}{c}\text { Particle } \\
\text { Type }\end{array}$ & $\begin{array}{l}\text { Loading } \\
(\operatorname{vol} \%)\end{array}$ & Filler & Additive & $\begin{array}{c}\text { Firing } \\
\text { Conditions }\end{array}$ & & & $\begin{array}{l}\text { Number of } \\
\text { Particles } \\
\text { Debonded }\end{array}$ & \\
\hline $\begin{array}{l}1 \mathrm{~A} \\
1 \mathrm{~B} \\
1 \mathrm{C} \\
1 \mathrm{D} \\
1 \mathrm{E}\end{array}$ & $\begin{array}{l}01-5(c) \\
02-6 \\
03-5 \\
04-6 \\
05-6\end{array}$ & $\begin{array}{ll}\text { GLCCC } & 1099 \\
\text { GLCC } & 1099 \\
\text { GLCC } & 1099 \\
\text { GLCC } & 1099 \\
\text { GLCC } & 1099\end{array}$ & $\begin{array}{l}10.0 \\
35.3 \\
36.3 \\
20.0 \\
25.0\end{array}$ & $\begin{array}{l}6353 \\
6353 \\
6353 \\
6353 \\
6353\end{array}$ & $\begin{array}{l}\text { SCO11 } \\
\text { SCO11 } \\
\text { SCO11 } \\
\text { SCO11 } \\
\text { SCO11 }\end{array}$ & $\begin{array}{l}\text { Ref. } \\
\text { Ref. } \\
\text { Ref. } \\
1500^{\circ} \mathrm{C} \\
\text { Ref. }\end{array}$ & $\begin{array}{l}1115 \\
1050 \\
1065 \\
1115 \\
1125\end{array}$ & $\begin{array}{l}7.3 \\
8.1 \\
8.7 \\
9.3 \\
9.9\end{array}$ & $\begin{array}{r}50 \\
0 \\
8 \\
5 \\
0\end{array}$ & $\begin{array}{l}\text { Fair } \\
\text { Excellent } \\
\text { Excellent } \\
\text { Excellent } \\
\text { Excellent }\end{array}$ \\
\hline $\begin{array}{l}2 \mathrm{~A} \\
2 \mathrm{~B} \\
2 \mathrm{C} \\
2 \mathrm{D} \\
2 \mathrm{E}\end{array}$ & $\begin{array}{l}06-5(\mathrm{c}) \\
07-5 \\
08-13 \\
09-5(\mathrm{~d}) \\
10-5\end{array}$ & $\begin{array}{ll}\text { GLCC } & 1099 \\
\text { GLCC } & 1099 \\
\text { GLCC } & 1099 \\
\text { Lonza } & \\
\text { GLCC } & 1099\end{array}$ & $\begin{array}{l}20.0 \\
25.0 \\
30.0 \\
25.0 \\
33.0\end{array}$ & $\begin{array}{l}6353 \\
\mathrm{KS}-44 \\
6353 \\
6353 \\
6353\end{array}$ & $\begin{array}{l}\text { SCO11 } \\
\text { SC011 } \\
\text { SCO11 } \\
\text { SC003 } \\
\text { SCO11 }\end{array}$ & $\begin{array}{l}1500^{\circ} \mathrm{C} \\
\text { Ref. } \\
\text { Packed sed } \\
\text { Ref. } \\
\text { Ref. }\end{array}$ & $\begin{array}{r}1050 \\
1055 \\
1045 \\
1035 \\
965\end{array}$ & $\begin{array}{l}11.8 \\
12.0 \\
12.2 \\
12.3 \\
12.4\end{array}$ & $\begin{array}{r}0 \\
0 \\
0 \\
35 \\
0\end{array}$ & $\begin{array}{l}\text { Excellent } \\
\text { Excellent } \\
\text { Excellent } \\
\text { Fair } \\
\text { Excellent }\end{array}$ \\
\hline $\begin{array}{l}5 \mathrm{~A} \\
5 \mathrm{~B} \\
5 \mathrm{C} \\
5 \mathrm{D} \\
5 \mathrm{E}\end{array}$ & $\begin{array}{l}16-5(c) \\
17-5 \\
18-6 \\
19-7 \\
20-6\end{array}$ & $\begin{array}{ll}\text { GLCC } & 1099 \\
\text { GLCC } & 1099 \\
\text { GLCC } & 1099 \\
\text { GLCC } & 1099 \\
\text { GLCC } & 1099\end{array}$ & $\begin{array}{l}25.0 \\
30.0 \\
20.0 \\
20.0 \\
10.0\end{array}$ & $\begin{array}{l}6353 \\
6353 \\
6353 \\
6353 \\
6353\end{array}$ & $\begin{array}{l}\text { SCO11 } \\
\text { SCO11 } \\
\text { SCO11 } \\
\text { SCO11 } \\
\text { SC011 }\end{array}$ & $\begin{array}{l}\text { Ref. } \\
\text { Ref. } \\
1500^{\circ} \mathrm{C} \\
\mathrm{N}_{2} \text { atmospnere } \\
\text { Ref. }\end{array}$ & $\begin{array}{l}1285 \\
1225 \\
1235 \\
1225 \\
1280\end{array}$ & $\begin{array}{l}9.4 \\
9.0 \\
8.5 \\
8.2 \\
7.7\end{array}$ & $\begin{array}{r}21 \\
0 \\
10 \\
10 \\
20\end{array}$ & $\begin{array}{l}\text { Good } \\
\text { Excellent } \\
\text { Good } \\
\text { Good } \\
\text { Good }\end{array}$ \\
\hline $\begin{array}{l}6 \mathrm{~A} \\
6 \mathrm{~B} \\
6 \mathrm{C} \\
6 \mathrm{D} \\
6 \mathrm{E}\end{array}$ & $\begin{array}{l}21-5(c) \\
22-5 \\
23-5 \\
24-5 \\
25-5 \\
(d)\end{array}$ & $\begin{array}{ll}\text { GLCC } & 1099 \\
\text { GLCC } & 1099 \\
\text { GLCC } & 1099 \\
\text { Lonza } & \\
\text { GLCC } & 1099\end{array}$ & $\begin{array}{l}25.0 \\
15.0 \\
30.0 \\
20.0 \\
25.0\end{array}$ & $\begin{array}{l}6353 \\
6353 \\
6353 \\
6353 \\
6353\end{array}$ & $\begin{array}{l}\text { SCO11 } \\
\text { SCO11 } \\
\text { SC027 } \\
\text { SCO03 } \\
\text { SC011 }\end{array}$ & $\begin{array}{l}\text { Ref. } \\
\text { Ref. } \\
\text { Ref. } \\
\text { Ref. } \\
N_{2} \text { atmosprere }\end{array}$ & $\begin{array}{l}1065 \\
1055 \\
1005 \\
1020 \\
1000\end{array}$ & $\begin{array}{l}5.4 \\
5.0 \\
4.5 \\
4.1 \\
3.6\end{array}$ & $\begin{array}{r}0 \\
8 \\
0 \\
30 \\
0\end{array}$ & $\begin{array}{l}\text { Excellent } \\
\text { Excellent } \\
\text { Excellent } \\
\text { Fair } \\
\text { Excellent }\end{array}$ \\
\hline
\end{tabular}

(a) Reference firing conditions are carbonization to $1800^{\circ} \mathrm{C}$ in an argon atmosphere in an H-451 graphite tube to simulate the LHTGR cure-in-place process. Firing conditions that vary from this process are noted.

(b) Relative condition of fuel rod based on visual appearance; e.g., excellent condition indicates rod exhibited no significant matrix spalling, matrix cracking, or particle debonding.

(c) Thermocouple fuel rods.

(d) Fuel rods were fabricated by slug-injection process. 
TABLE 4-2

DESCRIPTION AND CONDITION OF FUEL RODS IRRADIATED IN CAPSULE P13S

\begin{tabular}{|c|c|c|c|c|c|c|c|c|c|c|}
\hline \multicolumn{7}{|c|}{ Fuel Rod Description } & \multicolumn{2}{|c|}{ Irradiation Parameters } & \multicolumn{2}{|c|}{$\begin{array}{c}\text { Postirradiation } \\
\text { Condition } \\
\end{array}$} \\
\hline \multirow[b]{2}{*}{$\begin{array}{l}\text { Capsule } \\
\text { Position }\end{array}$} & \multirow{2}{*}{$\begin{array}{c}\text { Fuel Rod Data } \\
\text { Retrieval No. } \\
(7161-004-)\end{array}$} & \multicolumn{2}{|c|}{ Shim } & \multicolumn{3}{|c|}{ Matrix } & \multirow{2}{*}{$\begin{array}{l}\text { Average } \\
\text { Centerline } \\
\text { Temperature } \\
\left({ }^{\circ} \mathrm{C}\right)\end{array}$} & \multirow{2}{*}{ 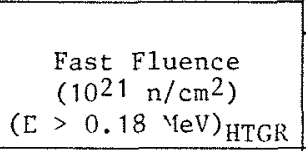 } & \multirow{2}{*}{\begin{tabular}{l|} 
Number of \\
Particles \\
Debonded
\end{tabular}} & \multirow[b]{2}{*}{$\begin{array}{c}\text { Visual } \\
\text { Appearance (b) }\end{array}$} \\
\hline & & $\begin{array}{c}\text { Particle } \\
\text { Type }\end{array}$ & $\begin{array}{l}\text { Loading } \\
(\text { vol \%) }\end{array}$ & Filler & Additive & $\begin{array}{c}\text { Firing } \\
\text { Conditions }(a) \\
\end{array}$ & & & & \\
\hline $\begin{array}{l}1 \mathrm{~A} \\
1 \mathrm{~B} \\
1 \mathrm{C} \\
1 \mathrm{D} \\
1 \mathrm{E}\end{array}$ & $\begin{array}{l}01-7(c) \\
02-5 \\
03-6 \\
04-5 \text { (c) } \\
05-5\end{array}$ & \begin{tabular}{ll|} 
GLCC & 1099 \\
GLCC & 1099 \\
GLCC & 1099 \\
GLCC & 1099 \\
GLCC & 1099
\end{tabular} & $\begin{array}{l}10.0 \\
35.3 \\
36.3 \\
20.0 \\
25.0\end{array}$ & $\begin{array}{l}6353 \\
6353 \\
6353 \\
6353 \\
6353\end{array}$ & $\begin{array}{l}\text { SC011 } \\
\text { SC011 } \\
\text { SC011 } \\
\text { SC011 } \\
\text { SC011 }\end{array}$ & $\begin{array}{l}\text { Ref. } \\
\text { Ref. } \\
\text { Ref. } \\
1500^{\circ} \mathrm{C} \\
\operatorname{Ref} \text {. }\end{array}$ & $\begin{array}{r}1030(\mathrm{~d}) \\
975(\mathrm{~d}) \\
995(\mathrm{~d}) \\
1035(\mathrm{~d}) \\
1035^{(\mathrm{d})}\end{array}$ & $\begin{array}{l}7.0 \\
7.7 \\
8.5 \\
9.1 \\
9.6\end{array}$ & $\begin{array}{r}15 \\
0 \\
2 \\
3 \\
1\end{array}$ & $\begin{array}{l}\text { Fair } \\
\text { Excellent } \\
\text { Excellent } \\
\text { Excellent } \\
\text { Excellent }\end{array}$ \\
\hline $\begin{array}{l}2 \mathrm{~A} \\
2 \mathrm{~B} \\
2 \mathrm{C} \\
2 \mathrm{D} \\
2 \mathrm{E}\end{array}$ & $\begin{array}{l}11-6^{(c)} \\
12-5 \\
13-6 \\
14-5 \\
15-5\end{array}$ & $\begin{array}{lc}\text { GLCC } & 1099 \\
\text { GLCC } & 1099 \\
\text { GLCC } & 1099 \\
\text { GLCC } & 1099 \\
\text { GLCC } & 1099\end{array}$ & $\begin{array}{l}20.0 \\
25.0 \\
30.0 \\
25.0 \\
33.0\end{array}$ & $\begin{array}{l}6353 \\
6353 \\
6353 \\
6353 \\
6353\end{array}$ & $\begin{array}{l}\text { SC011 } \\
\text { SC011 } \\
\text { SC027 } \\
\text { SC011 } \\
\text { SC011 }\end{array}$ & $\begin{array}{l}\mathrm{N}_{2} \text { atmosphere } \\
\text { Ref. } \\
\text { Ref. } \\
\text { Ref. } \\
\text { Ref. }\end{array}$ & $\begin{array}{r}1040 \\
1010 \\
975 \\
985 \\
960\end{array}$ & $\begin{array}{l}11.5 \\
11.7 \\
11.9 \\
12.0 \\
12.1\end{array}$ & $\begin{array}{l}0 \\
2 \\
2 \\
0 \\
8\end{array}$ & $\begin{array}{l}\text { Excellent } \\
\text { Excellent } \\
\text { Excellent } \\
\text { Excellent } \\
\text { Good }\end{array}$ \\
\hline $\begin{array}{l}5 \mathrm{~A} \\
5 \mathrm{~B} \\
5 \mathrm{C} \\
5 \mathrm{D} \\
5 \mathrm{E}\end{array}$ & $\begin{array}{l}16-6 \\
17-6 \\
18-5 \\
19-5 \\
20-5\end{array}$ & $\begin{array}{lr}\text { GLCC } & 1099 \\
\text { GLCC } & 1099 \\
\text { GLCC } & 1099 \\
\text { GLCC } & 1099 \\
\text { GLCC } & 1099\end{array}$ & $\begin{array}{l}25.0 \\
30.0 \\
20.0 \\
20.0 \\
10.0\end{array}$ & $\begin{array}{l}6353 \\
6353 \\
6353 \\
6353 \\
6353\end{array}$ & $\begin{array}{l}\text { SC011 } \\
\text { SC011 } \\
\text { SC011 } \\
\text { SC011 } \\
\text { SC011 }\end{array}$ & $\begin{array}{l}\text { Ref. } \\
\text { Ref. } \\
1500^{\circ} \mathrm{C} \\
\mathrm{N}_{2} \text { atmosphere } \\
\text { Ref. }\end{array}$ & $\begin{array}{l}1375 \\
1330 \\
1335 \\
1325 \\
1350\end{array}$ & $\begin{array}{l}9.2 \\
8.7 \\
8.4 \\
7.9 \\
7.5\end{array}$ & $\begin{array}{r}6 \\
0 \\
4 \\
13 \\
0\end{array}$ & $\begin{array}{l}\text { Excellent } \\
\text { Excellent } \\
\text { Good } \\
\text { Fair } \\
\text { Excellent }\end{array}$ \\
\hline $\begin{array}{l}6 \mathrm{~A} \\
6 \mathrm{~B} \\
6 \mathrm{C} \\
6 \mathrm{D} \\
6 \mathrm{E}\end{array}$ & $\begin{array}{l}26-5(c) \\
27-5 \\
28-13 \\
29-5(c) \\
30-5\end{array}$ & $\begin{array}{ll}\text { GLCC } & 1099 \\
\text { GLCC } & 1099 \\
\text { GLCC } & 1099 \\
\text { GLCC } & 1099 \\
\text { GLCC } & 1099\end{array}$ & $\begin{array}{l}25.0 \\
15.0 \\
30.0 \\
20.0 \\
25.0\end{array}$ & $\begin{array}{l}6353 \\
6353 \\
6353 \\
\text { KS-44 } \\
6353\end{array}$ & $\begin{array}{l}\text { SC011 } \\
\text { SC011 } \\
\text { SC011 } \\
\text { SC011 } \\
\text { SC011 }\end{array}$ & $\begin{array}{l}\text { Ref. } \\
\text { Ref. } \\
\text { Packed bed } \\
\text { Ref. } \\
1500^{\circ} \mathrm{C}\end{array}$ & $\begin{array}{r}1085 \\
1045 \\
1050 \\
1015 \\
995\end{array}$ & $\begin{array}{l}5.3 \\
4.8 \\
4.4 \\
3.9 \\
3.5\end{array}$ & $\begin{array}{r}4 \\
50 \\
0 \\
0 \\
2\end{array}$ & $\begin{array}{l}\text { Excellent } \\
\text { Fair } \\
\text { Excellent } \\
\text { Excellent } \\
\text { Good }\end{array}$ \\
\hline
\end{tabular}

(a) Reference firing conditions are carbonization to $1800^{\circ} \mathrm{C}$ in an argon atmosphere in an H-451 graphite tube to simulate the LHTGR cure-in-place process. Firing conditions that vary from this process are noted.

(b) Relative condition of fuel rod based on visual appearance; e.g., excellent condition indicates rod exhibited no significant matrix spalling, matrix cracking, or particle debonding.

(c) Thermocouple fuel rods.

${ }^{\text {(d) }}$ Cell 1 was thermal cycled from $1075^{\circ}$ to $1600^{\circ} \mathrm{C}$ (design) twenty-four times during capsule operation. 


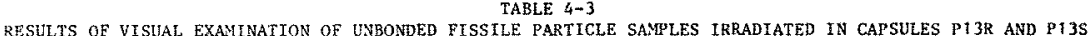

\begin{tabular}{|c|c|c|c|c|c|c|c|c|c|c|c|c|c|c|c|}
\hline \multirow[b]{3}{*}{ Capsule } & \multicolumn{6}{|c|}{ Sample Description ${ }^{(a)}$} & & & & \multicolumn{5}{|c|}{ Visual Examination } & \multirow[b]{3}{*}{ Renarhs } \\
\hline & \multirow[b]{2}{*}{$\begin{array}{l}\text { Data } \\
\text { Retrieval } \\
\text { Number }\end{array}$} & \multirow[b]{2}{*}{$\begin{array}{c}\text { opyc } \\
\text { Thick- } \\
\text { ness } \\
\text { (Hm) }\end{array}$} & \multirow[b]{2}{*}{$\begin{array}{c}\text { OPyC } \\
\text { Density (b) } \\
\left(8 / \mathrm{cm}^{3}\right)\end{array}$} & \multirow[b]{2}{*}{$\begin{array}{c}\text { opyc } \\
\text { Coating } \\
\text { Rate } \\
(\text { rm/min })\end{array}$} & \multirow[b]{2}{*}{$\begin{array}{c}\text { OPyC } \\
\text { Anisotropy } \\
\left(\mathrm{BAF}_{\mathrm{O}}\right)\end{array}$} & \multirow{2}{*}{$\begin{array}{c}\text { Total } \\
\text { Coating } \\
\text { Thick- } \\
\text { ness } \\
\text { (um) }\end{array}$} & \multicolumn{3}{|c|}{ Irradiation Conditions } & \multirow[b]{2}{*}{$\begin{array}{c}\text { No. of } \\
\text { Par- } \\
\text { ticles }\end{array}$} & \multirow{2}{*}{$\begin{array}{c}\text { opyc } \\
\text { Coating } \\
\text { Fal lure } \\
(\%)\end{array}$} & \multirow[b]{2}{*}{$\begin{array}{l}\text { 95\% Conf idence } \\
\text { Interval, P(c) } \\
(\%)\end{array}$} & \multirow{2}{*}{$\begin{array}{c}\text { Pressure } \\
\text { Vessel } \\
\text { Faliure } \\
\text { (\%) } \\
\end{array}$} & \multirow[b]{2}{*}{$\begin{array}{c}\text { 95: Conf } 2 \text { dence } \\
\text { Interval, } P(c) \\
(\%)\end{array}$} & \\
\hline & & & & & & & $\begin{array}{l}\text { Capsule } \\
\text { Position }\end{array}$ & 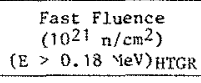 & $\begin{array}{l}\text { Burnup } \\
(\% \text { FTMA })\end{array}$ & & & & & & \\
\hline $\mathrm{P} 13 \mathrm{R}$ & $6151-00-010$ & 44 & 1.80 & 1.1 & 1.042 & 200 & $3-1,2,3$ & 12.1 & 73 & 682 & 32.8 & $31 \leq \mathrm{P} \leq 33$ & 0.7 & $0.3 \leq \mathrm{P} \leq 1.7$ & \\
\hline & $615 t-00-035$ & 37 & 1.85 & 3.9 & 1.030 & 191 & $3^{-4,4} 5^{(d)}$ & 12.0 & 74 & 459 & 0 & $0 \leq P \leq 0.8$ & 0 & $0 \leq \mathrm{P} \leq 0.8$ & \\
\hline & $6151-00-045$ & 40 & 1.81 & 3.8 & 1.024 & 192 & $3-7,8,9$ & 11.9 & 74 & 749 & 6.8 & $5.1 \leq P \leq 8.1$ & 6.3 & $4.8 \leq P \leq 7.7$ & Faceted coatings \\
\hline & $6151-00-046$ & 40 & 1.81 & 3.3 & 1.024 & 191 & $3-10$ & 11.8 & 74 & 254 & 3.5 & $1.9 \leq P \leq 6.6$ & 2.0 & $0.8 \leq P \leq 4.5$ & Faceted coatings \\
\hline & $6151-04-015$ & 36 & 1.82 & 1.4 & 1.038 & 199 & $3-11,12$ & 11.7 & 73 & 492 & 49.0 & $44.6 \leq P \leq 53.4$ & 0 & $0 \leq P \leq 0.8$ & \\
\hline & $6151-01-015$ & 42 & 1.90 & 0.7 & 1.077 & 197 & $4-2,3^{(d)}$ & 11.5 & 73 & 464 & 100 & $99.2 \leq \mathrm{P} \leq 100$ & 2.8 & $1.6 \leq \mathrm{P} \leq 4.7$ & \\
\hline & $615 i-00-025$ & 33 & 1.88 & 1.4 & 1.052 & 188 & $4-4,5,6$ & 11.3 & 73 & 819 & 83.0 & $81 \leq P \leq 85$ & 0 & $0 \leq \mathrm{P} \leq 0.4$ & Faceted coatings \\
\hline & $6151-09-015$ & 39 & 1.94 & 3.6 & 1.028 & 195 & $4-7,8,9$ & 11.1 & 73 & 785 & 0 & $0 \leq P \leq 0.5$ & 0 & $0 \leq P \leq 0.5$ & \\
\hline & 6151-09-025 & 41 & 1.95 & 4.0 & 1.033 & 194 & $4-10,11^{(d)}$ & 11.0 & 73 & 491 & 0 & $0 \leq P \leq 0.8$ & $\theta$ & $0 \leq p \leq 0.8$ & \\
\hline piss & 6151-03-015 & 45 & 1.77 & 1,5 & 1.020 & 198 & $3-1,2,3$ & 11.8 & 72 & 729 & 4.3 & $3.0 \leq P \leq 6.0$ & 0.1 & $0.01 \leq P \leq 0.7$ & Interrupted SiC laver \\
\hline & $4161-01-021$ & 32 & 1.82 & 3.4 & $\mathrm{ND}(\mathrm{e})$ & 173 & $3-4,5,6$ & 11.7 & 73 & 718 & 3.9 & $2.7 \leq P \leq 5.6$ & 3.3 & $2.2 \leq P \leq 4.9$ & Interrupted $S_{2} C$ laver \\
\hline & $6151-08-015$ & 36 & 1.76 & 3.6 & 1.018 & 190 & $3-7,8,9$ & 11.7 & 73 & 688 & 0 & $0 \leq P \leq 0.6$ & 0 & $0 \leq P \leq 0.6$ & \\
\hline & $6151-02-025$ & 43 & 1.50 & 0.7 & 1.039 & 202 & $3-10,12^{(d)}$ & 11.6 & 72 & 501 & 46.3 & $42.0 \leq P \leq 50.7$ & 0.2 & $0.04 \leq P \leq 1.1$ & \\
\hline & $4163-00-011$ & 72 & 1.77 & 4.1 & ND & 238 & $4-1,2,3$ & 11.2 & .27 & 767 & 0 & $0 \leq P \leq 0.5$ & 0 & $0 \leq P \leq 0.5$ & $250 m_{4}(\mathrm{mh}, \mathrm{L}) \mathrm{O}_{2}, \mathrm{~m} / \mathrm{l}=1: 1$ \\
\hline & 6155-01-020 & 42 & 1.81 & 3.4 & 1.025 & 194 & $14-4^{(d)}$ & 11.1 & 11 & 163 & 76.7 & $596.6 \leq \mathrm{P} \leq 82.5$ & 1.8 & $0.6 \leq P \leq>.3$ & $500-$ ufl $(h,+1) 0_{2}$, in/l $=8: 1$ \\
\hline & $5466-37-9$ & 31 & 1.79 & 6.0 & No & 182 & $4-7,8,9$ & 10.9 & 38 & 801 & 0 & $0 \leq P \leq 0.4$ & 0 & $0 \leq P \leq 0.4$ & $250-\ldots \mathrm{Nl}(\mathrm{Th}, \mathrm{L}) \mathrm{u}_{2}, \mathrm{Th} / \mathrm{C}=1.1$ \\
\hline & $6151-00-035$ & 37 & 1.85 & 3.9 & 1.030 & 191 & $4-10,11,12$ & 10.7 & 72 & 809 & 0 & $0 \leq P \leq 0.4$ & 0 & $0 \leq \mathrm{B}^{3} \leq 0.4$ & \\
\hline
\end{tabular}

(a) ${ }_{A 11}$ fissile particle samples are TRISO UC $\mathrm{C}_{2}$ particles with a nominal kernel diameter of 200 im, except where noted.

(b) Yeasured using a density gradient column.

(c) An infanite sample population with attributes of the particle batch tested to these conditions will have a failure level at the $95 /$ confadence level bounded by this 1 interval.

(d) Companion sample was spilled during unioading.

$(e)_{\text {vis }}=$ not determined. 
TABLE $4-4$

RESULTS OF VISUAL EXAMTNATION OF UNBONDED FERTILE PARTICLE SALPLES IRRADTATED IN CAPSULE P13R

\begin{tabular}{|c|c|c|c|c|c|c|c|c|c|c|c|c|c|}
\hline \multicolumn{7}{|c|}{ Sample Description (a) } & \multicolumn{3}{|c|}{ Irradiation Conditions } & \multicolumn{3}{|c|}{ Visual Examination } & \multirow[b]{2}{*}{ Remarks } \\
\hline $\begin{array}{c}\text { Data } \\
\text { Retrieval } \\
\text { Number } \\
\end{array}$ & $\begin{array}{c}\text { Buffer } \\
\text { Thick- } \\
\text { ness } \\
(\mu \mathrm{m}) \\
\end{array}$ & $\begin{array}{l}\text { Opyc } \\
\text { Thick- } \\
\text { ness } \\
(\mathrm{mm})\end{array}$ & $\begin{array}{c}\text { OPyC } \\
\text { Density }(b) \\
\left(\mathrm{g} / \mathrm{cm}^{3}\right) \\
\end{array}$ & $\begin{array}{c}\text { opyc } \\
\text { Coating } \\
\text { Rate } \\
(\mathrm{mm} / \mathrm{min}) \\
\end{array}$ & $\begin{array}{c}\text { OPvC } \\
\text { Anisotropy } \\
\text { (BAE }) \\
\end{array}$ & $\begin{array}{c}\text { Particle } \\
\text { Densitv (c) } \\
\left(\mathrm{g} / \mathrm{cm}^{3}\right) \\
\end{array}$ & $\begin{array}{l}\text { Capsule } \\
\text { Position } \\
\end{array}$ & $\begin{array}{c}\text { Eradiation Condition } \\
\text { Fast Fluence } \\
\left(10^{21} \mathfrak{n} / \mathrm{cm}^{2}\right) \\
(\mathrm{E}-0.18 \mathrm{Yel}) \text { HTGR }\end{array}$ & $\begin{array}{r}\text { Burnup } \\
(\text {; FINA) }\end{array}$ & $\begin{array}{c}\text { Yo. of } \\
\text { Par- } \\
\text { ticles }\end{array}$ & \begin{tabular}{|c|} 
OPyC \\
Coating \\
Failure \\
$(\%)$ \\
\end{tabular} & $\begin{array}{l}95 \% \text { Confidence } \\
\text { Interval, } \mathrm{P}(d) \\
(\%)\end{array}$ & \\
\hline $4252-05-018$ & 78 & 77 & 1.82 & 5.8 & 1.029 & 3.34 & $3-1,2$ & 12.1 & 5.1 & $8 \div 0$ & 0.1 & $0.01 \leq P \leq 0.8$ & \\
\hline $6542-01-020$ & 81 & 74 & 1.82 & 2.7 & 1.028 & 3.35 & $3-3,4$ & 12.1 & 5.0 & 1004 & 7.8 & $6.5 \leq P \leq 9.0$ & \\
\hline $6542-01-010$ & 79 & 85 & 1.80 & 10.0 & 1.028 & 3.59 & $3-5^{(e)}$ & 12.0 & 5.0 & 455 & 0.2 & $0.04 \leq \mathrm{P} \leq 1.2$ & \\
\hline $6542-02-020$ & 87 & 72 & 1.91 & 8.5 & 1.036 & 3.59 & $3-7,8$ & 11.9 & 4.9 & 1072 & 0 & $0 \leq \mathrm{P} \leq 0.5$ & \\
\hline $6542-02-030$ & 79 & 74 & 1.89 & 2.2 & 1.042 & 3.54 & $3-9,10$ & 11.8 & 4.8 & 1148 & 10.2 & $10.0 \leq P \leq 10.3$ & \\
\hline $6542-09-010$ & 84 & 75 & 1.93 & 5.0 & 1.042 & 3.56 & $3-11,12$ & 11.7 & 4.8 & 998 & 0 & $0 \leq p \leq 0.5$ & \\
\hline $6592-11-015$ & 87 & 74 & 1.83 & 2.6 & $\mathrm{WD}(\mathrm{E})$ & 3.35 & $4-2^{(e)}$ & 11.5 & 4.5 & 492 & 0.4 & $0.1 \leq P \leq 1.5$ & So seal coat \\
\hline $6542-18-015$ & 84 & 70 & 1.84 & 6.5 & $\mathrm{~W}$ & 3.53 & $4-3,4$ & 11.4 & 4.4 & 953 & 0 & $0 \leq \mathrm{P} \leq 0.6$ & $\therefore$ seal coat \\
\hline $6542-02-020$ & 87 & 72 & 1.91 & 8.5 & 1.028 & 3.59 & $4-5,6$ & 11.3 & 4.4 & 1149 & 0 & $0 \leq \mathrm{P} \leq 0.5$ & \\
\hline $6542-20-035$ & 80 & 83 & 1.84 & 3.6 & SD & 3.50 & $4-7,8$ & 11.2 & 4.3 & 1012 & 0.6 & $0.2 \leq P \leq 1.2$ & Faceted coatings \\
\hline $6542-20-025$ & 76 & 81 & 1.82 & 3.5 & 1.047 & 3.57 & $4-9,10$ & 11.1 & 4.2 & 1040 & 1.0 & $0.6 \leq \mathrm{P} \leq 1.7$ & Faceted coatings \\
\hline $6542-25-015$ & 79 & 76 & 1.82 & 10.8 & VD & 3.57 & $4-11^{(e)}$ & 10.9 & 4.1 & 539 & 1.7 & $0.9 \leq P \leq 3.1$ & Nonround kernels \\
\hline
\end{tabular}

(a) All particle samples are BlSO coated Tho, particles with a nominal kernel diameter of 500 um.

(b) Measured using a density gradient column,

(c) Measured using an air pycnometer.

(d) An infinite sample population with attributes of the particle batch tested to these conditions will have a failure level at the $95 \%$ confidence level bounded by this interval.

(e) Companion sample spilled during unloading.

${ }^{(f)} \mathrm{ND}=$ not determined. 
TABIE $4-5$

RESULTS OF VISUAL EXAMINATION OF LXBONDED FER:- PARTICLE SAMPLES IRRADIATED IN CAPSULE P13S

\begin{tabular}{|c|c|c|c|c|c|c|c|c|c|c|c|c|c|}
\hline \multicolumn{7}{|c|}{ Sample Description(a) } & \multicolumn{3}{|c|}{ Irradiation Conditions } & \multicolumn{3}{|c|}{ Visual Examination } & \multirow[b]{2}{*}{ Remarks } \\
\hline $\begin{array}{c}\text { Data } \\
\text { Retrieval } \\
\text { Number }\end{array}$ & \begin{tabular}{|c|} 
Buffer \\
Thick- \\
ness \\
$(\mu \mathrm{m})$ \\
\end{tabular} & $\begin{array}{c}\text { OPyC } \\
\text { Thick- } \\
\text { ness } \\
(\mu \mathrm{m})\end{array}$ & $\begin{array}{l}\text { OPyC } \\
\text { Densiry (b) } \\
\left(\mathrm{g} / \mathrm{cm}^{3}\right)\end{array}$ & $\begin{array}{c}\text { OPyC } \\
\text { Coating } \\
\text { Rate } \\
(\mu \mathrm{m} / \mathrm{min}) \\
\end{array}$ & $\begin{array}{c}\text { OPyC } \\
\text { Anisotropy } \\
\left(\mathrm{BAF}_{\mathrm{O}}\right) \\
\end{array}$ & $\begin{array}{c}\text { Particle } \\
\text { Density }(\mathrm{c}) \\
\left(\mathrm{g} / \mathrm{cm}^{3}\right) \\
\end{array}$ & $\begin{array}{l}\text { Capsule } \\
\text { Position }\end{array}$ & $\begin{array}{c}\text { Eradiation Conditio } \\
\text { Fast Fluence } \\
\left(1021 \mathrm{n} / \mathrm{cm}^{2}\right) \\
E>0.18 \mathrm{MeV}) \mathrm{HTGR} \\
\end{array}$ & $\begin{array}{c}\text { Burnup } \\
\text { (\% FIMA) }\end{array}$ & $\begin{array}{l}\text { No. of } \\
\text { Par- } \\
\text { ticles }\end{array}$ & $\begin{array}{c}\text { OPyC } \\
\text { Coating } \\
\text { Failure } \\
(\%) \\
\end{array}$ & $\begin{array}{c}95 \% \text { Conitidence } \\
\text { Interval, } ?(d) \\
\left({ }^{*}\right)\end{array}$ & \\
\hline $4252-06-010$ & 85 & 76 & 1.82 & 5.8 & 1.029 & 3.54 & $3-1,2$ & 11.8 & 4.3 & 872 & 0 & $0 \leq P \leq 0.6$ & \\
\hline $6542-22-015$ & 85 & 81 & 1.80 & 4.0 & 1.020 & 3.50 & $3-3,4$ & 11.7 & 4.3 & 979 & 1.0 & $0.5 \leq P \leq 1.5$ & Mixed-gas OPyC layer \\
\hline $6542-22-025$ & 81 & 80 & 1.81 & 7.6 & 1.028 & 3.53 & $3-5,6$ & 11.7 & 4.2 & 964 & 0.3 & $0.1 \leq \mathrm{P} \leq 0.9$ & Mixed-gas OPyC layer \\
\hline $6542-23-025$ & 82 & 73 & 1.89 & 5.8 & & 3.58 & $3-7,8$ & 11.7 & 4.2 & 992 & 0 & $0 \leq P \leq 0.5$ & Mixed-gas OPyC layer \\
\hline $6542-24-015$ & 86 & 83 & 1.94 & 6.6 & 1.032 & 3.55 & $3-9,10$ & 11.6 & 4.1 & 942 & 0 & $0 \leq P \leq 0.6$ & Mixed-gas opyC layer \\
\hline $6252-00-025$ & 60 & 42 & 1.81 & 4.2 & 1.015 & 3.77 & $3-12^{(e)}$ & 11.5 & 4.1 & 528 & 0.4 & $0.1 \leq P \leq 1.4$ & TRISO coated \\
\hline $6542-17-010$ & 44 & 122 & 1.86 & 2.8 & $\mathrm{ND}(f)$ & 3.55 & $4-1$ & 11.2 & 3.9 & 458 & 2.6 & $1.5 \leq P \leq 4.5$ & \\
\hline $4252-02-010$ & 83 & 73 & 1.83 & 4.0 & 1.032 & 3.51 & $4-2,3$ & 11.2 & 3.9 & 925 & 0 & $0 \leq P \leq 0.6$ & \\
\hline $6542-16-010$ & 39 & 55 & XD & 3.7 & $\mathbb{N}$ & 4.84 & $4-4^{(e)}$ & 11.1 & 3.8 & 349 & 5.4 & $3.5 \leq P \leq 8.3$ & \\
\hline $6542-19-015$ & 81 & 77 & 1.78 & 7.6 & 1.025 & 3.55 & $4-7$ & 10.9 & 3.7 & 495 & 0.6 & $0.2 \leq P \leq 1.8$ & \\
\hline $6542-19-016$ & 82 & 71 & 1.78 & 7.0 & 1.026 & 3.45 & $4-8$ & 10.9 & 3.7 & 502 & 0.2 & $0.04 \leq P \leq 1.1$ & \\
\hline $6542-21-016$ & 81 & 84 & 1.73 & 8.0 & 1.020 & 3.41 & $4-9$ & 10.9 & 3.6 & 508 & 0 & $0 \leq \mathrm{P} \leq 0.8$ & Mixed-gas OPyC layer \\
\hline $6542-21-015$ & 79 & 82 & 1.73 & 7.8 & 1.020 & 3.46 & $4-10$ & 10.8 & 3.6 & 513 & 0 & $0 \leq P \leq 0.7$ & Mixed-gas OPyC layer \\
\hline $6542-12-025$ & 84 & 70 & 1.79 & 6.7 & $\mathrm{ND}$ & 3.58 & $4-11,12$ & 10.7 & 3.6 & 1214 & 0.2 & $0.1 \leq P \leq 0.8$ & $\mathrm{~N}_{2}$ buffer carrier gas \\
\hline
\end{tabular}

(a) All particle samples are BISO coated Tho ${ }_{2}$ particles with a nominal kernel diazeter of $500 \mathrm{um}$, except where noted.

(b) Measured using a density gradient column.

(c) Measured using an air pycnometer.

(d) An infinite sample population with attributes of the particle batch tested to these conditions will have a failure level at the $95 \%$ confidence level bounded by this interval.

(e) Companion sample spilled during unloading.

(f) $\mathrm{ND}=$ not determined. 
TABLE 4-6

FUEL ROD DIMENSIONAL CHANGE DATA OBTAINED FROM CAPSULE P13R

\begin{tabular}{|c|c|c|c|c|c|c|c|c|c|c|c|}
\hline \multirow{4}{*}{$\begin{array}{l}\text { Capsule } \\
\text { Position }\end{array}$} & \multirow{4}{*}{$\begin{array}{c}\text { Fuel Rod } \\
\text { Data Retrieval } \\
\text { Number } \\
(7161-004-) \\
\end{array}$} & \multicolumn{2}{|c|}{ Irradiation Conditions } & \multicolumn{8}{|c|}{ Dimensional Change } \\
\hline & & \multirow{3}{*}{$\begin{array}{c}\text { Volume } \\
\text { Average } \\
\text { Temperature } \\
\left({ }^{\circ} \mathrm{C}\right)\end{array}$} & \multirow{3}{*}{$\begin{array}{c}\text { Fast Fluence } \\
\left(10^{21} \mathrm{n} / \mathrm{cm}^{2}\right) \\
(\mathrm{E}>0.18 \mathrm{MeV})_{\text {HTGR }}\end{array}$} & \multicolumn{4}{|c|}{ Measured $^{(a)}$} & \multicolumn{2}{|c|}{ Ca1culated $^{(b)}$} & \multirow{2}{*}{\multicolumn{2}{|c|}{ Difference ${ }^{(c)}$}} \\
\hline & & & & \multirow{2}{*}{$\begin{array}{c}\Delta \mathrm{D} / \mathrm{D}_{\mathrm{O}} \\
(\%)\end{array}$} & \multirow{2}{*}{$\begin{array}{l}\text { Std. } \\
\text { Dev. }\end{array}$} & \multirow{2}{*}{$\begin{array}{c}\Delta \mathrm{L} / \mathrm{L}_{\mathrm{O}} \\
(\%)\end{array}$} & \multirow{2}{*}{$\begin{array}{c}\Delta \mathrm{V} / \mathrm{V}_{\mathrm{O}} \\
(\%)\end{array}$} & \multirow{2}{*}{$\begin{array}{c}\Delta \mathrm{D} / \mathrm{D}_{\mathrm{O}} \\
(\%)\end{array}$} & \multirow{2}{*}{$\begin{array}{c}\Delta \mathrm{V} / \mathrm{V}_{\mathrm{O}} \\
(\%)\end{array}$} & & \\
\hline & & & & & & & & & & Diameter & Volume \\
\hline $\begin{array}{l}1 \mathrm{~A} \\
1 \mathrm{~B} \\
1 \mathrm{C} \\
1 \mathrm{D} \\
1 \mathrm{E}\end{array}$ & $\begin{array}{l}01-5 \\
02-6 \\
03-5 \\
04-6 \\
05-6\end{array}$ & $\begin{array}{l}1050 \\
1000 \\
1015 \\
1045 \\
1070\end{array}$ & $\begin{array}{l}7.3 \\
8.1 \\
8.7 \\
9.3 \\
9.9\end{array}$ & $\begin{array}{l}-0.87 \\
-1.54 \\
-1.52 \\
-0.33 \\
-0.60\end{array}$ & $\begin{array}{l}0.08 \\
0.34 \\
0.09 \\
0.12 \\
0.23\end{array}$ & $\begin{array}{l}\text { ND }(d) \\
+6.82(e) \\
-0.26 \\
+0.93 \\
+0.29\end{array}$ & $\begin{array}{l}\text { ND } \\
\text { ND } \\
-3.30 \\
+0.27 \\
-0.91\end{array}$ & $\begin{array}{l}-2.53 \\
-2.65 \\
-2.28 \\
-1.83 \\
-1.92\end{array}$ & $\begin{array}{l}-- \\
-7.95 \\
-6.84 \\
-5.49 \\
-5.76\end{array}$ & $\begin{array}{l}-65.6 \\
-41.9 \\
-33.3 \\
-82.0 \\
-68.8\end{array}$ & $\begin{array}{c}-- \\
-- \\
-96.2 \\
-104.9 \\
-84.2\end{array}$ \\
\hline $\begin{array}{l}2 A \\
2 B \\
2 C \\
2 D \\
2 E\end{array}$ & $\begin{array}{l}06-5 \\
07-5 \\
08-13 \\
09-5 \\
10-5\end{array}$ & $\begin{array}{l}975 \\
985 \\
980 \\
965 \\
915\end{array}$ & $\begin{array}{l}11.8 \\
12.0 \\
12.2 \\
12.3 \\
12.4\end{array}$ & $\begin{array}{l}-0.27 \text { (f) } \\
+1.13^{(f)} \\
-0.48 \\
+1.61 \text { (f) } \\
-0.12 \text { (f) }\end{array}$ & $\begin{array}{l}0.07 \\
0.60 \\
0.16 \\
0.22 \\
0.13\end{array}$ & $\begin{array}{l}-0.21 \\
+1.97 \\
-0.40 \\
+4.75 \\
+3.89\end{array}$ & $\begin{array}{l}-0.75 \\
+4.23 \\
-1.36 \\
+7.97 \\
+3.65\end{array}$ & $\begin{array}{l}(\mathrm{g}) \\
(\mathrm{g}) \\
(\mathrm{g}) \\
(\mathrm{g}) \\
(\mathrm{g})\end{array}$ & $\begin{array}{l}(\mathrm{g}) \\
(\mathrm{g}) \\
(\mathrm{g}) \\
(\mathrm{g}) \\
(\mathrm{g})\end{array}$ & $\begin{array}{l}-- \\
-- \\
-- \\
-- \\
--\end{array}$ & $\begin{array}{l}-- \\
-- \\
-- \\
--\end{array}$ \\
\hline $\begin{array}{l}5 A \\
5 B \\
5 C \\
5 D \\
5 E\end{array}$ & $\begin{array}{l}16-5 \\
17-5 \\
18-6 \\
19-7 \\
20-6\end{array}$ & $\begin{array}{l}1230 \\
1175 \\
1180 \\
1170 \\
1215\end{array}$ & $\begin{array}{l}9.4 \\
9.0 \\
8.5 \\
8.2 \\
7.7\end{array}$ & $\begin{array}{l}-1.19 \\
-1.33 \\
-1.53 \\
-1.33 \\
-1.35\end{array}$ & $\begin{array}{l}0.24 \\
0.44 \\
0.15 \\
0.13 \\
0.16\end{array}$ & $\begin{array}{l}+2.34 \\
+0.89 \\
-0.15 \\
+2.62 \\
+0.68\end{array}$ & $\begin{array}{l}-0.04 \\
-1.77 \\
-3.21 \\
-0.04 \\
-2.02\end{array}$ & $\begin{array}{l}-2.73 \\
-2.62 \\
-2.79 \\
-2.90 \\
-3.87\end{array}$ & $\begin{array}{r}-8.19 \\
-7.86 \\
-8.37 \\
-8.70 \\
-11.61\end{array}$ & $\begin{array}{l}-56.4 \\
-49.2 \\
-45.2 \\
-54.1 \\
-65.1\end{array}$ & $\begin{array}{l}-99.5 \\
-77.5 \\
-61.6 \\
-99.5 \\
-82.6\end{array}$ \\
\hline $\begin{array}{l}6 \mathrm{~A} \\
6 \mathrm{~B} \\
6 \mathrm{C} \\
6 \mathrm{D} \\
6 \mathrm{E}\end{array}$ & $\begin{array}{l}21-5 \\
22-5 \\
23-5 \\
24-5 \\
25-5\end{array}$ & $\begin{array}{r}1035 \\
1020 \\
975 \\
985 \\
970\end{array}$ & $\begin{array}{l}5.4 \\
5.0 \\
4.5 \\
4.1 \\
3.6\end{array}$ & $\begin{array}{l}-2.01 \\
-1.63 \\
-1.77 \\
-1.44 \\
-1.63\end{array}$ & $\begin{array}{l}0.22 \\
0.13 \\
0.12 \\
0.93 \\
0.17\end{array}$ & $\begin{array}{l}\mathrm{ND} \\
-0.37 \\
-0.67 \\
-1.09 \\
-0.10\end{array}$ & $\begin{array}{l}\text { ND } \\
-3.63 \\
-4.21 \\
-3.97 \\
-3.36\end{array}$ & $\begin{array}{l}-2.85 \\
-2.40 \\
-2.71 \\
-2.25 \\
-2.47\end{array}$ & $\begin{array}{l}-8.55 \\
-7.20 \\
-8.13 \\
-6.75 \\
-7.41\end{array}$ & $\begin{array}{l}-29.5 \\
-32.1 \\
-34.7 \\
-36.0 \\
-34.0\end{array}$ & $\begin{array}{l}-- \\
-49.6 \\
-48.2 \\
-41.2 \\
-54.7\end{array}$ \\
\hline
\end{tabular}

(a) Measurements made with dial gauge micrometer.

(b) Dimensional changes calculated using SHRINK computer code.

(c) Percent difference between calculated and measured dimensional change: $\frac{\Delta \mathrm{x} / \mathrm{x}_{\mathrm{o}_{\text {meas }}}-\Delta \mathrm{x} / \mathrm{x}_{\mathrm{o}_{\mathrm{calc}}} \mathrm{x} 100=\mathrm{difference}}{\Delta \mathrm{x} / \mathrm{x}_{\mathrm{o}_{\mathrm{calc}}}}$

(d) $\mathrm{ND}=$ not determined.

(e) Length measurement appears to be erroneous datum point.

(f) Fuel rods were constrained by graphite crucibles at end of the irradiation; therefore, dimensional change of rods may not be representative.

(g) Fast neutron exposure received by these rods exceeds limits of pyrocarbon and graphite shim dimensional change data; therefore, fuel rod dimensional changes cannot be predicted using SHRINK code. 
TABLE 4-7

FUEL ROD DIMENSIONAL CHANGE DATA OBTAINED FROM CAPSULE P13S

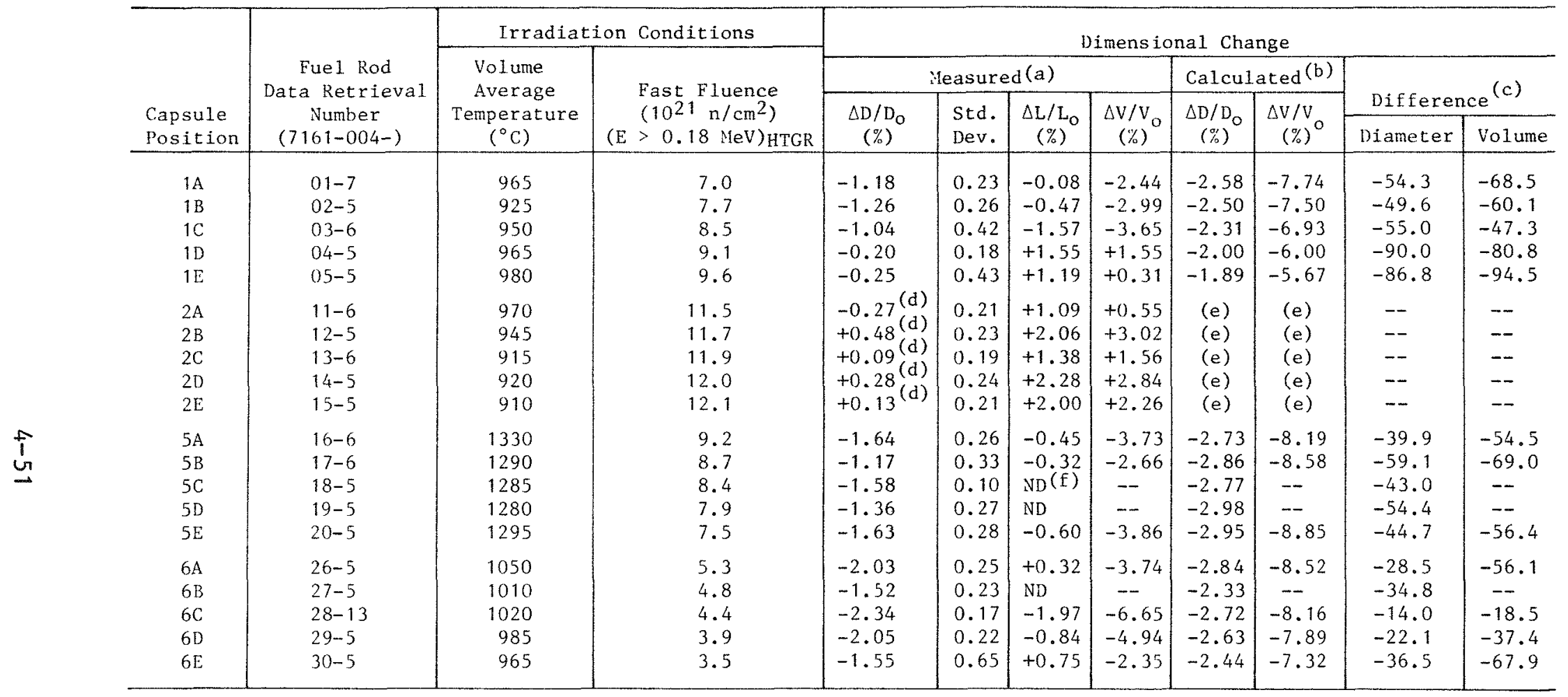

(a) Measurements made with dial gauge micrometer.

(b) Dimensional changes calculated using SHRINK computer code.

(c) Percent difference between calculated and measured dimensional change: $\frac{\Delta x / x_{0} o_{\text {meas }}-\Delta x / x_{o}}{\Delta x / x_{0}{ }_{c a l c}} x 100=$ difference.

(d) Fuel rods were constrained by graphite crucibles at end of irradiation; therefore, dimensional change of rods may not be representative.

(e) Fast neutron exposure received by these rods exceeds limits of pyrocarbon and graphite shim dimensional change data; therefore, fuel rod dimensional changes cannot be predicted using SHRINK code.

(f) ND = not determined. 
TABLE 4-8

MEASURED DIAMETRAL DIMENSIONAL CHANGES OF FUEL ROD GRAPHITE CRUCIBLES

\begin{tabular}{|c|c|c|c|c|c|c|}
\hline \multirow[b]{2}{*}{ Capsule } & \multirow[b]{2}{*}{ Cel1 } & \multirow[b]{2}{*}{ Position } & \multicolumn{2}{|c|}{$\begin{array}{c}\text { Dimensional Change, } \\
\Delta \mathrm{L} / \mathrm{L}_{\mathrm{O}}(\%)\end{array}$} & \multirow{2}{*}{ 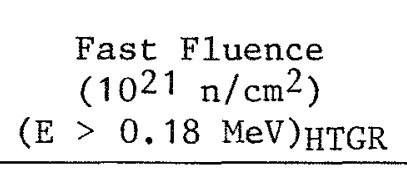 } & \multirow{2}{*}{$\begin{array}{c}\text { Nominal } \\
\text { Temperature } \\
\left({ }^{\circ} \mathrm{C}\right)\end{array}$} \\
\hline & & & $\perp$ to Split & II to Split & & \\
\hline P13R & 1 & $\begin{array}{l}\text { Top } \\
\text { Center } \\
\text { Bottom }\end{array}$ & $\begin{array}{l}-0.8 \\
-0.4 \\
-0.5\end{array}$ & $\begin{array}{l}-1.2 \\
-1.1 \\
-0.8\end{array}$ & $\begin{array}{l}9.9 \\
8.7 \\
7.3\end{array}$ & 780 \\
\hline P13R & 2 & $\begin{array}{l}\text { Top } \\
\text { Center } \\
\text { Bottom }\end{array}$ & $\begin{array}{l}-0.2 \\
+0.6 \\
-0.5\end{array}$ & $\begin{array}{l}-1.2 \\
-0.8 \\
-0.7\end{array}$ & $\begin{array}{l}12.4 \\
12.2 \\
11.8\end{array}$ & 730 \\
\hline P13R & 5 & $\begin{array}{l}\text { Top } \\
\text { Center } \\
\text { Bottom }\end{array}$ & $\begin{array}{l}-0.9 \\
-0.9 \\
-1.1\end{array}$ & $\begin{array}{l}-1.2 \\
-1.5 \\
-1.2\end{array}$ & $\begin{array}{l}7.7 \\
8.6 \\
9.4\end{array}$ & 960 \\
\hline $\mathrm{P} 13 \mathrm{R}$ & 6 & $\begin{array}{l}\text { Top } \\
\text { Center } \\
\text { Bottom }\end{array}$ & $\begin{array}{l}-0.2 \\
-0.4 \\
-0.6\end{array}$ & $\begin{array}{l}-0.2 \\
-0.4 \\
-0.5\end{array}$ & $\begin{array}{l}3.6 \\
4.5 \\
5.4\end{array}$ & 765 \\
\hline P13S & 1 & $\begin{array}{l}\text { Top } \\
\text { Center } \\
\text { Bottom }\end{array}$ & $\begin{array}{l}-0.6 \\
-0.4 \\
-0.5\end{array}$ & $\begin{array}{l}-0.8 \\
-0.7 \\
-0.6\end{array}$ & $\begin{array}{l}9.6 \\
8.4 \\
7.0\end{array}$ & 840 \\
\hline P13S & 2 & $\begin{array}{l}\text { Top } \\
\text { Center } \\
\text { Bottom }\end{array}$ & $\begin{array}{l}-0.7 \\
-0.3 \\
-0.8\end{array}$ & $\begin{array}{l}-1.2 \\
-1.3 \\
-1.3\end{array}$ & $\begin{array}{l}12.1 \\
11.9 \\
11.5\end{array}$ & 750 \\
\hline P13S & 5 & $\begin{array}{l}\text { Top } \\
\text { Center } \\
\text { Bottom }\end{array}$ & $\begin{array}{l}-0.8 \\
-0.6 \\
-0.6\end{array}$ & $\begin{array}{l}-0.7 \\
-0.6 \\
-1.1\end{array}$ & $\begin{array}{l}7.5 \\
8.4 \\
9.2\end{array}$ & 1150 \\
\hline P13S & 6 & $\begin{array}{l}\text { Top } \\
\text { Center } \\
\text { Bottom }\end{array}$ & $\begin{array}{l}-0.2 \\
-0.5 \\
-0.6\end{array}$ & $\begin{array}{l}-0.2 \\
-0.6 \\
-0.9\end{array}$ & $\begin{array}{l}3.5 \\
4.4 \\
5.3\end{array}$ & 780 \\
\hline
\end{tabular}


TABLE 4-9

POSTIRRADIATION RADIAL GAP BETWEEN GRAPHITE CRUCIBLES AND CAPSULE P13R FUEL RODS

\begin{tabular}{c|c|c|c|c}
\hline \multirow{2}{*}{$\begin{array}{c}\text { Fue1 Rod } \\
\text { Data } \begin{array}{c}\text { Retrieval } \\
\text { No. }\end{array}\end{array}$} & \multicolumn{3}{|c}{ Postirradiation Dimensions $(\mathrm{cm})$} \\
\cline { 3 - 5 }$(7161-004-)$ & $\begin{array}{c}\text { Capsule } \\
\text { Position }\end{array}$ & $\begin{array}{c}\text { Crucible } \\
\text { I.D. }\end{array}$ & $\begin{array}{c}\text { Fuel Rod } \\
\text { O.D. }\end{array}$ & $\begin{array}{c}\text { Radial } \\
\text { Gap }\end{array}$ \\
\hline $01-5$ & 1A & 1.258 & 1.241 & 0.009 \\
$02-6$ & 1B & 1.258 & 1.236 & 0.011 \\
$03-5$ & 1C & 1.254 & 1.232 & 0.011 \\
$04-6$ & 1D & 1.254 & 1.250 & 0.002 \\
$05-6$ & 1E & 1.254 & 1.242 & 0.006 \\
$06-5$ & 2A & 1.259 & 1.250 & 0.005 \\
$07-5$ & 2B & 1.259 & 1.263 & 0 \\
$08-13$ & 2C & 1.258 & 1.241 & 0.009 \\
$09-5$ & 2D & 1.256 & 1.270 & 0 \\
$10-5$ & 2E & 1.250 & 1.252 & 0 \\
$16-5$ & 5A & 1.253 & 1.236 & 0.009 \\
$17-5$ & 5B & 1.251 & 1.238 & 0.007 \\
$18-6$ & 5C & 1.249 & 1.235 & 0.007 \\
$19-7$ & 5D & 1.251 & 1.235 & 0.008 \\
$20-6$ & 5E & 1.253 & 1.234 & 0.010 \\
$21-5$ & 6A & 1.262 & 1.225 & 0.019 \\
$22-5$ & 6B & 1.263 & 1.232 & 0.016 \\
$23-5$ & 6C & 1.263 & 1.230 & 0.017 \\
$24-5$ & 6D & 1.265 & 1.224 & 0.020 \\
$25-5$ & 6E & 1.266 & 1.230 & 0.018 \\
\hline
\end{tabular}


TABLE $4-10$

POSTIRRADIATION RADIAL GAP BETWEEN GRAPHITE CRUCIBLES AND CAPSULE P13S FUEL RODS

\begin{tabular}{|c|c|c|c|c|}
\hline \multirow{2}{*}{$\begin{array}{c}\text { Fuel Rod } \\
\text { Data Retrieval } \\
\text { No. } \\
(7161-004-) \\
\end{array}$} & \multirow[b]{2}{*}{$\begin{array}{l}\text { Capsule } \\
\text { Position }\end{array}$} & \multicolumn{3}{|c|}{ Postirradiation Dimensions $(\mathrm{cm})$} \\
\hline & & $\begin{array}{c}\text { Crucible } \\
\text { I.D. }\end{array}$ & $\begin{array}{c}\text { Fuel Rod } \\
\text { O.D. }\end{array}$ & $\begin{array}{c}\text { Radial } \\
\text { Gap }\end{array}$ \\
\hline $\begin{array}{l}01-7 \\
02-5 \\
03-6 \\
04-5 \\
05-5\end{array}$ & $\begin{array}{l}1 \mathrm{~A} \\
1 \mathrm{~B} \\
1 \mathrm{C} \\
1 \mathrm{D} \\
1 \mathrm{E}\end{array}$ & $\begin{array}{l}1.261 \\
1.261 \\
1.260 \\
1.260 \\
1.259\end{array}$ & $\begin{array}{l}1.238 \\
1.238 \\
1.237 \\
1.253 \\
1.246\end{array}$ & $\begin{array}{l}0.012 \\
0.012 \\
0.012 \\
0.004 \\
0.007\end{array}$ \\
\hline $\begin{array}{l}11-6 \\
12-5 \\
13-6 \\
14-5 \\
15-5\end{array}$ & $\begin{array}{l}2 A \\
2 B \\
2 C \\
2 D \\
2 E\end{array}$ & $\begin{array}{l}1.252 \\
1.252 \\
1.252 \\
1.252 \\
1.253\end{array}$ & $\begin{array}{l}1.247 \\
1.258 \\
1.254 \\
1.256 \\
1.253\end{array}$ & $\begin{array}{l}0.003 \\
0 \\
0 \\
0 \\
0\end{array}$ \\
\hline $\begin{array}{l}16-6 \\
17-6 \\
18-5 \\
19-5 \\
20-5\end{array}$ & $\begin{array}{l}5 \mathrm{~A} \\
5 \mathrm{~B} \\
5 \mathrm{C} \\
5 \mathrm{D} \\
5 \mathrm{E}\end{array}$ & $\begin{array}{l}1.254 \\
1.258 \\
1.261 \\
1.261 \\
1.259\end{array}$ & $\begin{array}{l}1.230 \\
1.237 \\
1.233 \\
1.234 \\
1.232\end{array}$ & $\begin{array}{l}0.012 \\
0.011 \\
0.014 \\
0.014 \\
0.014\end{array}$ \\
\hline $\begin{array}{l}26-5 \\
27-5 \\
28-13 \\
29-5 \\
30-5\end{array}$ & $\begin{array}{l}6 \mathrm{~A} \\
6 \mathrm{~B} \\
6 \mathrm{C} \\
6 \mathrm{D} \\
6 \mathrm{E}\end{array}$ & $\begin{array}{l}1.257 \\
1.259 \\
1.261 \\
1.264 \\
1.266\end{array}$ & $\begin{array}{l}1.227 \\
1.233 \\
1.212 \\
1.225 \\
1.230\end{array}$ & $\begin{array}{l}0.015 \\
0.013 \\
0.025 \\
0.020 \\
0.018\end{array}$ \\
\hline
\end{tabular}


TABLE $4-11$

TRIGA FISSION GAS RELEASE DATA FOR CAPSULE P13R FUEL RODS

\begin{tabular}{|c|c|c|c|c|}
\hline \multirow{2}{*}{$\begin{array}{l}\text { Data } \\
\text { Retrieva1 } \\
\text { Number } \\
(7161-004-)\end{array}$} & \multirow{2}{*}{$\begin{array}{l}\text { Capsule } \\
\text { Position }\end{array}$} & \multirow{2}{*}{$\begin{array}{l}\text { EOL Fissile } \\
\text { Loading(a) } \\
\text { (mg U-235) }\end{array}$} & \multicolumn{2}{|c|}{$\begin{array}{c}\text { TRIGA Fission } \\
\text { Gas Release Data } \\
\left(\mathrm{R} / \mathrm{B} \mathrm{Kr}-85 \mathrm{~m} \text { at } 1100^{\circ} \mathrm{C}\right)\end{array}$} \\
\hline & & & BOL & EOL \\
\hline $01-5$ & $1 \mathrm{~A}$ & 45.1 & $2 \times 10^{-6}$ & $2.4 \times 10^{-4}$ \\
\hline $02-6$ & $1 \mathrm{~B}$ & 44.7 & $1 \times 10^{-6}$ & $3.1 \times 10^{-5}$ \\
\hline $03-5$ & $1 \mathrm{C}$ & 43.8 & $1 \times 10^{-6}$ & $3.3 \times 10^{-6}$ \\
\hline $04-6$ & 1D & 43.4 & $3 \times 10^{-6}$ & $1.1 \times 10^{-4}$ \\
\hline $05-6$ & $1 \mathrm{E}$ & 41.3 & $4 \times 10^{-6}$ & $3.0 \times 10^{-6}$ \\
\hline $06-5$ & $2 \mathrm{~A}$ & 43.2 & $3 \times 10^{-6}$ & $2.5 \times 10^{-4}$ \\
\hline $07-5$ & $2 B$ & 42.6 & $3 \times 10^{-6}$ & $1.6 \times 10^{-5}$ \\
\hline $08-13$ & $2 \mathrm{C}$ & 41.4 & $4 \times 10^{-6}$ & $1.6 \times 10^{-5}$ \\
\hline $09-5$ & 2D & 39.5 & $3 \times 10^{-6}$ & $3.9 \times 10^{-4}$ \\
\hline $10-5$ & $2 \mathrm{E}$ & 37.7 & $6 \times 10^{-6}$ & $4.1 \times 10^{-5}$ \\
\hline $16-5$ & $5 \mathrm{~A}$ & 50.5 & $4 \times 10^{-6}$ & $4.0 \times 10^{-5}$ \\
\hline $17-5$ & $5 B$ & 49.2 & $2 \times 10^{-6}$ & $3.3 \times 10^{-5(b)}$ \\
\hline $18-6$ & $5 \mathrm{C}$ & 52.5 & $3 \times 10^{-6}$ & $4.7 \times 10^{-7}$ \\
\hline $19-7$ & 5D & 53.2 & $2 \times 10^{-6}$ & $1.3 \times 10^{-5}$ \\
\hline $20-6$ & $5 E$ & 57.1 & $3 \times 10^{-6}$ & $6.3 \times 10^{-6}$ \\
\hline $21-5$ & $6 \mathrm{~A}$ & 39.1 & $4 \times 10^{-6}$ & $3.1 \times 10^{-6}$ \\
\hline $22-5$ & $6 \mathrm{~B}$ & 40.8 & $2 \times 10^{-6}$ & $3.9 \times 10^{-6}$ \\
\hline $23-5$ & $6 \mathrm{C}$ & 41.4 & $2 \times 10^{-6}$ & $5.0 \times 10^{-6}$ \\
\hline $24-5$ & $6 \mathrm{D}$ & 44.4 & $6 \times 10^{-6}$ & $1.8 \times 10^{-5}$ \\
\hline $25-5$ & $6 \mathrm{E}$ & 45.8 & $3 \times 10^{-6}$ & $7.7 \times 10^{-6}$ \\
\hline
\end{tabular}

(a) EOL fissile loading is the weight of U-235 which would produce the equivalent $\mathrm{Kr}-85 \mathrm{~m}$ yield of all fissionable material (U-233 and $U-235$ ) calculated to be in the fuel rod.

(b) Value extrapolated from $1300^{\circ} \mathrm{C}$ measurement using Eq. 4-13. 
TABLE $\quad 4-12$

TRIGA FISSION GAS RELEASE DATA FOR CAPSULE P13S FUEL RODS

\begin{tabular}{|c|c|c|c|c|}
\hline \multirow{2}{*}{$\begin{array}{l}\text { Data } \\
\text { Retrieva1 } \\
\text { Number } \\
(7161-004-)\end{array}$} & \multirow{2}{*}{$\begin{array}{l}\text { Capsule } \\
\text { Position }\end{array}$} & \multirow{2}{*}{$\begin{array}{l}\text { EOL Fissile } \\
\text { Loading (a) } \\
\text { (mg U-235) }\end{array}$} & \multicolumn{2}{|c|}{$\begin{array}{c}\text { TRIGA Fission } \\
\text { Gas Release Data } \\
\left(\mathrm{R} / \mathrm{B} \mathrm{Kr}-85 \mathrm{~m} \text { at } 1100^{\circ} \mathrm{C}\right)\end{array}$} \\
\hline & & & BOL & EOL \\
\hline $01-7$ & $1 \mathrm{~A}$ & 44.8 & $2 \times 10^{-6}$ & $1.1 \times 10^{-3}$ \\
\hline $02-5$ & $1 \mathrm{~B}$ & 44.3 & $1 \times 10^{-6}$ & $5.9 \times 10^{-5}$ \\
\hline $03-6$ & $1 \mathrm{C}$ & 43.2 & $1 \times 10^{-6}$ & $1.1 \times 10^{-4}$ \\
\hline $04-5$ & $1 \mathrm{D}$ & 42.7 & $2 \times 10^{-6}$ & $1.2 \times 10^{-3}$ \\
\hline $05-5$ & $1 \mathrm{E}$ & 40.6 & $2 \times 10^{-6}$ & $2.2 \times 10^{-4}$ \\
\hline $11-6$ & $2 \mathrm{~A}$ & 42.2 & $2 \times 10^{-7}$ & $1.5 \times 10^{-4}$ \\
\hline $12-5$ & $2 B$ & 41.6 & $2 \times 10^{-6}$ & $3.4 \times 10^{-7}$ \\
\hline $13-6$ & $2 \mathrm{C}$ & 40,4 & $3 \times 10^{-6}$ & $3.5 \times 10^{-5}$ \\
\hline $14-5$ & $2 \mathrm{D}$ & 38.6 & $2 \times 10^{-6}$ & $1.2 \times 10^{-5}$ \\
\hline $15-5$ & $2 \mathrm{E}$ & 36.8 & $5 \times 10^{-6}$ & $1.0 \times 10^{-4}$ \\
\hline $16-6$ & $5 \mathrm{~A}$ & 49.9 & $4 \times 10^{-6}$ & $1.8 \times 10^{-4}$ \\
\hline $17-6$ & $5 B$ & 48.7 & $1 \times 10^{-6}$ & $1.0 \times 10^{-4(b)}$ \\
\hline $18-5$ & $5 \mathrm{C}$ & 52.0 & $2 \times 10^{-6}$ & $2.6 \times 10^{-5}$ \\
\hline $19-5$ & $5 \mathrm{D}$ & 52.9 & $3 \times 10^{-6}$ & $7.4 \times 10^{-5}$ \\
\hline $20-5$ & $5 E$ & 56.8 & $3 \times 10^{-6}$ & $1.4 \times 10^{-4}$ \\
\hline $26-5$ & $6 \mathrm{~A}$ & 39.1 & $3 \times 10^{-6}$ & $2.1 \times 10^{-6}$ \\
\hline $27-5$ & $6 B$ & 40.7 & $1 \times 10^{-6}$ & $<10^{-7}$ \\
\hline $28-13$ & $6 \mathrm{C}$ & 41.4 & $4 \times 10^{-6}$ & $8.4 \times 10^{-7}$ \\
\hline $29-5$ & $6 \mathrm{D}$ & 44.5 & $1 \times 10^{-6}$ & $3.6 \times 10^{-7}$ \\
\hline $30-5$ & $6 \mathrm{E}$ & 46.4 & $2 \times 10^{-6}$ & $1.3 \times 10^{-6}$ \\
\hline
\end{tabular}

(a) EOL fissile loading is the weight of U-235 which would produce the equivalent $\mathrm{Kr}-85 \mathrm{~m}$ yield of all fissionable material (U-233 and U-235) calculated to be in the fuel rod.

(b) Value extrapolated from $1500^{\circ} \mathrm{C}$ measurement using $\mathrm{Eq} \cdot 4-13$. 
TABLE $4-13$

TRIGA FISSION GAS RELEASE DATA FOR UNBONDED FISSILE PARTICLE SAMPLES TESTED IN CAPSULES P13R AND P13S

\begin{tabular}{|c|c|c|c|c|c|}
\hline \multirow[b]{2}{*}{ Batch No. } & \multirow[b]{2}{*}{ Capsule } & \multirow{2}{*}{$\begin{array}{l}\text { Capsule } \\
\text { Position }\end{array}$} & \multirow{2}{*}{$\begin{array}{l}\text { EOL } \\
\text { Fissile } \\
\text { Loading }(a) \\
\text { (mg U-235) }\end{array}$} & \multicolumn{2}{|c|}{$\begin{array}{c}\text { TRIGA Fission } \\
\text { Gas Release Data } \\
\left(\mathrm{R} / \mathrm{B} \mathrm{Kr}-85 \mathrm{~m} \text { at } 1100^{\circ} \mathrm{C}\right)\end{array}$} \\
\hline & & & & BOL & EOL \\
\hline $6151-00-010$ & P13R & $3-1,2,3$ & 1.09 & $4 \times 10^{-7}$ & $1.4 \times 10^{-3}$ \\
\hline $6151-00-035$ & P13R & $3-4,5$ & 0.71 & $3 \times 10^{-7}$ & $1.2 \times 10^{-4}$ \\
\hline $6151-00-035$ & P13S & $4-10,11,12$ & 2.58 & $3 \times 10^{-7}$ & $5.9 \times 10^{-5}$ \\
\hline $6151-00-045$ & P13R & $3-7,8,9$ & 1.14 & $2 \times 10^{-7}$ & $6.7 \times 10^{-3}$ \\
\hline $6151-00-046$ & $\mathrm{P} 13 \mathrm{R}$ & $3-10$ & 0.39 & $2 \times 10^{-7}$ & $4.9 \times 10^{-3}$ \\
\hline $6151-04-015$ & P13R & $3-11,12$ & 0.88 & $8 \times 10^{-7}$ & $1.2 \times 10^{-4}$ \\
\hline $6151-01-015$ & P13R & $4-2,3$ & 0.93 & $1 \times 10^{-8}$ & $5.0 \times 10^{-4}$ \\
\hline $6151-00-025$ & P13R & $4-4,5,6$ & 1.55 & $6 \times 10^{-8}$ & $1.3 \times 10^{-3}$ \\
\hline $6151-09-015$ & P13R & $4-7,8,9$ & 1.68 & $9 \times 10^{-8}$ & $3.5 \times 10^{-4}$ \\
\hline $6151-09-025$ & P13R & $4-10,11$ & 1.19 & $2 \times 10^{-7}$ & $2.4 \times 10^{-5}$ \\
\hline $6151-03-015$ & P13S & $3-1,2,3$ & 1.52 & $2 \times 10^{-6}$ & $6.7 \times 10^{-5}$ \\
\hline $4161-01-021$ & P13S & $3-4,5,6$ & 1.58 & $5 \times 10^{-6}$ & $9.4 \times 10^{-3}$ \\
\hline $6151-08-015$ & P13S & $3-7,8,9$ & 1.64 & $1 \times 10^{-7}$ & $1.4 \times 10^{-5}$ \\
\hline $6151-02-025$ & P13S & $3-10,12$ & 1.23 & $2 \times 10^{-6}$ & $1.5 \times 10^{-3}$ \\
\hline $4163-00-011$ & P13S & $4-1,2,3$ & 5.11 & $3 \times 10^{-9}$ & $5.8 \times 10^{-6}$ \\
\hline $6155-01-020$ & P13S & $4-4$ & 5.23 & $6 \times 10^{-7}$ & $5.3 \times 10^{-4}$ \\
\hline $5466-37$ & P13S & $4-7,8,9$ & 3.99 & $1 \times 10^{-6}$ & $3.0 \times 10^{-6}$ \\
\hline
\end{tabular}

(a) EOL fissile loading is the weight of U-235 which would produce the equivalent $\mathrm{Kr}-85 \mathrm{~m}$ yield of all fissionable material calculated to be in the sample. 
TABLE $4-14$

TRIGA FISSION GAS RELEASE DATA FOR UNBONDED FERTILE PARTICLE SAMPLES TESTED IN CAPSULES P13R AND P13S

\begin{tabular}{|c|c|c|c|c|}
\hline Batch No. & Capsule & $\begin{array}{l}\text { Capsule } \\
\text { Position }\end{array}$ & $\begin{array}{c}\text { EOL } \\
\text { Fissile } \\
\text { Loading (a) } \\
\text { (mg U-235) }\end{array}$ & $\begin{array}{l}\text { TRIGA Fission } \\
\text { Gas Release Data, EOL } \\
\left(\mathrm{R} / \mathrm{B} \mathrm{Kr}-85 \mathrm{~m} \text { at } 1100^{\circ}\right)\end{array}$ \\
\hline $4252-06-018$ & P13R & $3-1,2$ & 31.97 & $1.4 \times 10^{-3}$ \\
\hline $6542-01-020$ & P13R & $3-3,4$ & 31.85 & $1.7 \times 10^{-4}$ \\
\hline $6542-01-010$ & P13R & $3-5$ & 32.31 & $1.2 \times 10^{-3}$ \\
\hline $6542-25-015$ & P13R & $4-11$ & 32.31 & $1.2 \times 10^{-3}$ \\
\hline $6542-02-020$ & P13R & $3-7,8$ & 31.76 & $4.4 \times 10^{-5}$ \\
\hline $6542-02-020$ & P13R & $4-5,6$ & 33.20 & $3.3 \times 10^{-5}$ \\
\hline $6542-02-030$ & P13R & $3-9,10$ & 31.67 & $2.9 \times 10^{-3}$ \\
\hline $6542-09-010$ & P13R & $3-11,12$ & 34.47 & $2.4 \times 10^{-5}$ \\
\hline $6542-11-015$ & P13R & $4-2$ & 46.16 & $2.4 \times 10^{-3}$ \\
\hline $6542-19-015$ & P13S & $4-7$ & 46.16 & $2.4 \times 10^{-3}$ \\
\hline $6542-19-016$ & P13S & $4-8$ & 46.16 & $2.4 \times 10^{-3}$ \\
\hline $6542-18-015$ & P13R & $4-3,4$ & 31.19 & $2.2 \times 10^{-6}$ \\
\hline $6542-20-035$ & P13R & $4-7,8$ & 32.95 & $6.9 \times 10^{-4}$ \\
\hline $6542-20-025$ & P13R & $4-9,10$ & 32.98 & $3.7 \times 10^{-4}$ \\
\hline $4252-06-010$ & P13S & $3-1,2$ & 30.77 & $6.2 \times 10^{-5}$ \\
\hline $6542-22-015$ & P13S & $3-3,4$ & 30.79 & $2.7 \times 10^{-4}$ \\
\hline $6542-22-025$ & P13S & $3-5,6$ & 30.73 & $2.4 \times 10^{-4}$ \\
\hline $6542-23-025$ & P13S & $3-7,8$ & 30.62 & $2.8 \times 10^{-6}$ \\
\hline $6542-24-015$ & P13S & $3-9,10$ & 30.59 & $3.9 \times 10^{-6}$ \\
\hline $6252-00-025$ & P13S & $3-12$ & 18.00 & $3.8 \times 10^{-5}$ \\
\hline $6542-17-010$ & P13s & $4-1$ & 24.44 & $4.7 \times 10^{-3}$ \\
\hline $6542-16-010$ & P13S & $4-4$ & 24.44 & $4.7 \times 10^{-3}$ \\
\hline $4252-02-010$ & P13S & $4-2,3$ & 27.86 & $1.1 \times 10^{-3}$ \\
\hline $6542-21-016$ & P13S & $4-9$ & 31.30 & $2.1 \times 10^{-3}$ \\
\hline $6542-21-015$ & P13S & $4-10$ & 31.30 & $2.1 \times 10^{-3}$ \\
\hline $6542-12-025$ & P13S & $4-11,12$ & 35.50 & $2.0 \times 10^{-4}$ \\
\hline
\end{tabular}

(a) EOL fissile loading is the weight of U-235 which would produce the equivalent $\mathrm{Kr}-85 \mathrm{~m}$ yield of all fissionable material calculated to be in the sample. 
TABLE 4-15

GASEOUS FISSION PRODUCI RELEASE (R/B Kr-85m) RESULTING FROM IN-SERVICE FUEL PARTICLE FAILURE IN CAPSULE P13R FUEL RODS

\begin{tabular}{|c|c|c|c|c|c|c|c|c|c|c|c|}
\hline \multirow{2}{*}{$\begin{array}{c}\text { Fuel lod Data } \\
\text { Retrieval No. } \\
(7161-004-)\end{array}$} & \multirow{2}{*}{$\begin{array}{l}\text { Capsule } \\
\text { Position }\end{array}$} & \multirow{2}{*}{$\begin{array}{l}\text { Thoriug } \\
\text { Cont. (a) } \\
(\mathrm{g} \mathrm{Th} / \mathrm{g} \mathrm{Th})\end{array}$} & \multirow[b]{2}{*}{$\mathrm{R} / \mathrm{B}_{\mathrm{BOL}}$} & \multicolumn{2}{|c|}{$\begin{array}{c}\text { Fraction of } \\
\text { Fissions at } \\
\text { EOL (b) }\end{array}$} & \multirow[b]{2}{*}{$\mathrm{R} / \mathrm{B}_{\mathrm{c}}(\mathrm{Th})^{(\mathrm{c})}$} & \multirow[b]{2}{*}{$R / B_{C}(U)$} & \multirow[b]{2}{*}{$\mathrm{R} / \mathrm{B}_{\mathrm{EOL}}$} & \multirow{2}{*}{$\begin{array}{c}\mathrm{R} / \mathrm{B}_{\mathrm{fa1led}}^{(\mathrm{d})} \\
\quad \text { Euel } \\
\end{array}$} & \multirow{2}{*}{$\begin{array}{c}\text { In-Service } \\
\text { Particle } \\
\text { Failure (e) } \\
(\%)\end{array}$} & \multirow{2}{*}{$\begin{array}{c}\text { Maximum } \\
\text { Fertile } \\
\text { Particle } \\
\text { Failure }(e, f) \\
(\%)\end{array}$} \\
\hline & & & & Fissile & Fertile & & & & & & \\
\hline $01-3$ & $1 \Lambda$ & $8 \times 10^{-6}$ & $2 \times 10^{-6}$ & 0.108 & 0.892 & $2.1 \times 10^{-6}$ & $2.2 \times 10^{-7}$ & $2.4 \times 10^{-4}$ & $2.4 \times 10^{-4}$ & 4.8 & 5.4 \\
\hline $02-6$ & 1B & $8 \times 10^{-6}$ & $1 \times 10^{-6}$ & 0.092 & 0.908 & $2.2 \times 10^{-6}$ & $9.2 \times 10^{-8}$ & $3.1 \times 10^{-5}$ & $2.9 \times 10^{-5}$ & 0.6 & 0.6 \\
\hline $03-5$ & 10 & $8 \times 10^{-6}$ & $1 \times 10^{-6}$ & 0.081 & 0.919 & $2.2 \times 10^{-6}$ & $8.1 \times 10^{-8}$ & $3.3 \times 10^{-6}$ & $1.0 \times 10^{-6}$ & 0 & 0 \\
\hline $0 / 4-6$ & 11) & $9 \times 10^{-6}$ & $3 \times 10^{-6}$ & 0.074 & $0.9 \%$ & $2.5 \times 10^{-6}$ & $2.2 \times 10^{-7}$ & $1.1 \times 10^{-4}$ & $1.1 \times 10^{-4}$ & 2.2 & 2.4 \\
\hline $05-6$ & $1 \mathrm{l}$ & $9 \times 10^{-6}$ & $4 \times 10^{-6}$ & 0.094 & 0.906 & $2.4 \times 10^{-6}$ & $3.8 \times 10^{-7}$ & $3.0 \times 10^{-6}$ & $2.2 \times 10^{-7}$ & 0 & 0 \\
\hline $06-5$ & $2 A$ & $9 \times 10^{-6}$ & $3 \times 10^{-6}$ & 0.062 & 0.938 & $2.5 \times 10^{-6}$ & $1.9 \times 10^{-7}$ & $2.5 \times 10^{-4}$ & $2.5 \times 10^{-4}$ & 5.0 & 5.3 \\
\hline $07-5$ & $2 B$ & $9 \times 10^{-6}$ & $3 \times 10^{-6}$ & 0.061 & 0.939 & $2.5 \times 10^{-6}$ & $1.8 \times 10^{-7}$ & $1.6 \times 10^{-5}$ & $1.3 \times 10^{-5}$ & 0.3 & 0.3 \\
\hline $08-13$ & $2 C$ & $9 \times 10^{-6}$ & $4 \times 10^{-6}$ & 0.061 & 0.939 & $2.5 \times 10^{-6}$ & $2.4 \times 10^{-7}$ & $1.6 \times 10^{-5}$ & $1.3 \times 10^{-5}$ & 0.3 & 0.3 \\
\hline $09-5$ & $21)$ & $1 \times 10^{-5}$ & $3 \times 10^{-6}$ & 0.062 & 0.938 & $2.8 \times 10^{-6}$ & $1.9 \times 10^{-7}$ & $3.9 \times 10^{-4}$ & $3.9 \times 10^{-4}$ & 7.8 & 8.3 \\
\hline $10-5$ & $2 \mathrm{r}$ & $1 \times 10^{-3}$ & $6 \times 10^{-6}$ & 0.075 & 0.925 & $2.8 \times 10^{-6}$ & $4.5 \times 10^{-7}$ & $4.1 \times 10^{-5}$ & $3.8 \times 10^{-5}$ & 0.8 & 0.8 \\
\hline $16-5$ & $5 \Lambda$ & $6 \times 10^{-i}$ & $4 \times 10^{-6}$ & 0.117 & 0.883 & $1.1 \times 10^{-6}$ & $4.7 \times 10^{-7}$ & $4.0 \times 10^{-5}$ & $3.8 \times 10^{-5}$ & 0.8 & 0.9 \\
\hline $17-5$ & $5 B$ & $7 \times 10^{-6}$ & $2 \times 10^{-6}$ & 0.109 & 0.891 & $2.1 \times 10^{-6}$ & $2.2 \times 10^{-7}$ & $3.3 \times 10^{-5}$ & $3.1 \times 10^{-5}$ & 0.6 & 0.7 \\
\hline $18-6$ & $5 \mathrm{C}$ & $7 \times 10^{-6}$ & $3 \times 10^{-6}$ & 0.118 & 0.882 & $1.9 \times 10^{-6}$ & $3.5 \times 10^{-7}$ & $4.7 \times 10^{-7}$ & 0 & 0 & 0 \\
\hline $19-7$ & 5 i) & $7 \times 10^{-6}$ & $2 \times 10^{-6}$ & 0.133 & 0.867 & $1.8 \times 10^{-6}$ & $2.7 \times 10^{-7}$ & $1.3 \times 10^{-5}$ & $1.1 \times 10^{-5}$ & 0.2 & 0.2 \\
\hline $20-6$ & 5 & $6 \times 10^{-6}$ & $3 \times 10^{-63}$ & 0.157 & 0.843 & $1.5 \times 10^{-6}$ & $4.7 \times 10^{-7}$ & $6.3 \times 10^{-6}$ & $4.3 \times 10^{-6}$ & 0.09 & 0.1 \\
\hline $21-5$ & $6 A$ & $9 \times 10^{-6}$ & $4 \times 10^{-6}$ & 0.345 & 0.655 & $1.8 \times 10^{-6}$ & $1.4 \times 10^{-6}$ & $3.1 \times 10^{-6}$ & 0 & 0 & 0 \\
\hline $22-5$ & $6 B$ & $8 \times 10^{-6}$ & $2 \times 10^{-6}$ & 0.350 & 0.650 & $1.6 \times 10^{-6}$ & $7.0 \times 10^{-7}$ & $3.9 \times 10^{-6}$ & $1.6 \times 10^{-6}$ & 0 & 0 \\
\hline $23-5$ & $6 \mathrm{C}$ & $9 \times 10^{-6}$ & $2 \times 10^{-6}$ & 0.418 & 0.582 & $1.6 \times 10^{-6}$ & $8.4 \times 10^{-7}$ & $5.0 \times 10^{-6}$ & $2.6 \times 10^{-6}$ & 0.05 & 0.09 \\
\hline $24-5$ & 60 & $9 \times 10^{-6}$ & $6 \times 10^{-6}$ & 0.464 & 0.536 & $1.4 \times 10^{-6}$ & $7.8 \times 10^{-6}$ & $1.8 \times 10^{-5}$ & $1.4 \times 10^{-5}$ & 0.3 & 0.5 \\
\hline $25-5$ & $6 \mathrm{E}$ & $1 \times 10^{-5}$ & $3 \times 10^{-6}$ & 0.619 & 0.381 & $1.1 \times 10^{-6}$ & $1.8 \times 10^{-6}$ & $7.7 \times 10^{-6}$ & $4.8 \times 10^{-6}$ & 0.1 & 0.3 \\
\hline
\end{tabular}

(a) Wetermined by hydrolysis test on companion unirradiated fuel rods (Ref. 4-2).

(b) Normalized to $\mathrm{Kr}-85 \mathrm{~m}$ thermal fission yields.

(c) Calculated assuming a fractional release for contamination of 0.30 (Ref. 4-9).

${ }^{\text {(d) }}{ }_{R / B_{\text {falled fuel }}}=R_{\text {EOL }}-B_{E}-\left[R / B_{C(T h)}+R / B_{C(U)}\right]$.

(e) Calculated assuming a fractional release of $5 \times 10^{-3}$ for failed fuel in a constrained geometry (Ref. 4-9).

(f) Calculated assuming all fission gas released came from failed fertile particles. 
TABLE 4-16

GASEOUS FISSION PRODUCT RELEASE (R/B $\mathrm{Kr}-85 \mathrm{~m}$ ) RESULTING FROM IN-SERVICE FUEL PARTICLE FAILURE IN CAPSULE P13S FUEL RODS

\begin{tabular}{|c|c|c|c|c|c|c|c|c|c|c|c|}
\hline \multirow{2}{*}{$\begin{array}{l}\text { Fuel Rod Data } \\
\text { Retrieval No. } \\
(7161-004-)\end{array}$} & \multirow{2}{*}{$\begin{array}{l}\text { Capsule } \\
\text { Position } \\
\end{array}$} & \multirow{2}{*}{$\begin{array}{l}\text { Thorium } \\
\text { Cont. (a) } \\
\text { (g Th/g Th) }\end{array}$} & \multirow[b]{2}{*}{$\mathrm{R} / \mathrm{B}_{\mathrm{BOL}}$} & \multicolumn{2}{|c|}{$\begin{array}{c}\text { Fraction of } \\
\text { Fissions at } \\
\text { EOL (b) }\end{array}$} & \multirow[b]{2}{*}{$\mathrm{R} / \mathrm{B}_{\mathrm{C}}(\mathrm{Th})^{(\mathrm{c})}$} & \multirow[b]{2}{*}{$\mathrm{R} / \mathrm{B}_{\mathrm{c}}(\mathrm{U})$} & \multirow[b]{2}{*}{$\mathrm{R} / \mathrm{B}_{\mathrm{EOL}}$} & \multirow{2}{*}{$\begin{array}{l}\mathrm{R} / \mathrm{B}_{\text {failed }}^{(\mathrm{d})} \\
\quad \text { fue1 } \\
\end{array}$} & \multirow{2}{*}{$\begin{array}{c}\text { In-Service } \\
\text { Particle } \\
\text { Failure (e) } \\
(\%)\end{array}$} & \multirow{2}{*}{$\begin{array}{c}\text { Maximum } \\
\text { Fertile } \\
\text { Particle } \\
\text { Failure }(\mathrm{e}, \mathrm{f}) \\
(\%)\end{array}$} \\
\hline & & & & Fissile & Fertile & & & & & & \\
\hline $01-7$ & $1 \mathrm{~A}$ & $8 \times 10^{-6}$ & $2 \times 10^{-6}$ & 0.121 & 0.879 & $2.1 \times 10^{-6}$ & $2.4 \times 10^{-7}$ & $1.1 \times 10^{-3}$ & $1.1 \times 10^{-3}$ & 22.0 & 24.9 \\
\hline $02-5$ & $1 \mathrm{~B}$ & $8 \times 10^{-6}$ & $1 \times 10^{-6}$ & 0.106 & 0.894 & $2.1 \times 10^{-6}$ & $1.1 \times 10^{-7}$ & $5.9 \times 10^{-5}$ & $5.7 \times 10^{-5}$ & 1.1 & 1.3 \\
\hline $03-6$ & $1 \mathrm{C}$ & $8 \times 10^{-6}$ & $1 \times 10^{-6}$ & 0.094 & 0.906 & $2.2 \times 10^{-6}$ & $9.4 \times 10^{-8}$ & $1.1 \times 10^{-4}$ & $1.1 \times 10^{-4}$ & 2.2 & 2.4 \\
\hline $04-5$ & $1 \mathrm{D}$ & $9 \times 10^{-6}$ & $2 \times 10^{-6}$ & 0.086 & 0.914 & $2.5 \times 10^{-6}$ & $1.7 \times 10^{-7}$ & $1.2 \times 10^{-3}$ & $1.2 \times 10^{-3}$ & 24.0 & 26.3 \\
\hline $06-5$ & $1 \mathrm{E}$ & $9 \times 10^{-6}$ & $2 \times 10^{-6}$ & 0.111 & 0.889 & $2.4 \times 10^{-6}$ & $2.2 \times 10^{-7}$ & $2.2 \times 10^{-4}$ & $2.2 \times 10^{-4}$ & $4 \cdot 4$ & 4.9 \\
\hline $11-6$ & $2 \mathrm{~A}$ & $9 \times 10^{-6}$ & $2 \times 10^{-7}$ & 0.069 & 0.931 & $2.5 \times 10^{-6}$ & $1.4 \times 10^{-8}$ & $1.5 \times 10^{-4}$ & $1.5 \times 10^{-4}$ & 3.0 & 3.2 \\
\hline $12-5$ & $2 \mathrm{~B}$ & $9 \times 10^{-6}$ & $2 \times 10^{-6}$ & 0.068 & 0.932 & $2.5 \times 10^{-6}$ & $1.4 \times 10^{-7}$ & $3.4 \times 10^{-7}$ & 0 & 0 & 0 \\
\hline $13-6$ & $2 \mathrm{C}$ & $9 \times 10^{-6}$ & $3 \times 10^{-6}$ & 0.068 & 0.932 & $2.5 \times 10^{-6}$ & $2.0 \times 10^{-7}$ & $3.5 \times 10^{-5}$ & $3.2 \times 10^{-5}$ & 0.6 & 0.7 \\
\hline $14-5$ & $2 \mathrm{D}$ & $1 \times 10^{-5}$ & $2 \times 10^{-6}$ & 0.068 & 0.932 & $2.8 \times 10^{-6}$ & $1.4 \times 10^{-7}$ & $1.2 \times 10^{-5}$ & $9.1 \times 10^{-6}$ & 0.2 & 0.2 \\
\hline $15-5$ & $2 \mathrm{~F}$ & $1 \times 10^{-5}$ & $5 \times 10^{-6}$ & 0.069 & 0.931 & $2.8 \times 10^{-6}$ & $3.4 \times 10^{-7}$ & $1.0 \times 10^{-4}$ & $9.7 \times 10^{-5}$ & 1.9 & 2.1 \\
\hline $16-6$ & $5 \mathrm{~A}$ & $6 \times 10^{-6}$ & $4 \times 10^{-6}$ & 0.130 & 0.870 & $1.6 \times 10^{-6}$ & $5.2 \times 10^{-7}$ & $1.8 \times 10^{-4}$ & $1.8 \times 10^{-4}$ & 3.6 & 4.1 \\
\hline $17-6$ & $5 B$ & $7 \times 10^{-6}$ & $1 \times 10^{-6}$ & 0.121 & 0.879 & $1.8 \times 10^{-6}$ & $1.2 \times 10^{-7}$ & $1.0 \times 10^{-4}$ & $9.8 \times 10^{-5}$ & 2.0 & 2.2 \\
\hline $18-5$ & $5 \mathrm{C}$ & $7 \times 10^{-6}$ & $2 \times 10^{-6}$ & 0.129 & 0.871 & $1.8 \times 10^{-6}$ & $2.6 \times 10^{-7}$ & $2.6 \times 10^{-5}$ & $2.4 \times 10^{-5}$ & 0.5 & 0.6 \\
\hline $19-5$ & $5 \mathrm{D}$ & $7 \times 10^{-6}$ & $3 \times 10^{-6}$ & 0.144 & 0.856 & $1.8 \times 10^{-6}$ & $4.3 \times 10^{-7}$ & $7.4 \times 10^{-5}$ & $7.1 \times 10^{-5}$ & 1.4 & 1.7 \\
\hline $20-5$ & $5 \mathrm{E}$ & $6 \times 10^{-6}$ & $3 \times 10^{-6}$ & 0.167 & 0.833 & $1.5 \times 10^{-6}$ & $5.0 \times 10^{-7}$ & $1.4 \times 10^{-4}$ & $1.4 \times 10^{-4}$ & 2.8 & 3.3 \\
\hline $26-5$ & $6 \mathrm{~A}$ & $9 \times 10^{-6}$ & $3 \times 10^{-6}$ & 0.350 & 0.650 & $1.8 \times 10^{-6}$ & $1.0 \times 10^{-6}$ & $2.1 \times 10^{-6}$ & 0 & 0 & 0 \\
\hline $27-5$ & $6 \mathrm{~B}$ & $8 \times 10^{-6}$ & $1 \times 10^{-6}$ & 0.390 & 0.610 & $1.5 \times 10^{-6}$ & $3.9 \times 10^{-7}$ & $<10^{-7}$ & 0 & 0 & 0 \\
\hline $28-13$ & $6 \mathrm{C}$ & $8 \times 10^{-6}$ & $4 \times 10^{-6}$ & 0.425 & 0.575 & $1.4 \times 10^{-6}$ & $1.7 \times 10^{-6}$ & $8.4 \times 10^{-7}$ & 0 & 0 & 0 \\
\hline $29-5$ & $6 D$ & $9 \times 10^{-6}$ & $1 \times 10^{-6}$ & 0.478 & 0.522 & $1.4 \times 10^{-6}$ & $4.8 \times 10^{-7}$ & $3.6 \times 10^{-7}$ & 0 & 0 & 0 \\
\hline $30-5$ & $6 \mathrm{E}$ & $1 \times 10^{-5}$ & $2 \times 10^{-6}$ & 0.637 & 0.363 & $1.1 \times 10^{-6}$ & $1.3 \times 10^{-6}$ & $1.3 \times 10^{-6}$ & 0 & 0 & 0 \\
\hline
\end{tabular}

(a) Determined by hydrolysis test on companion unirradiated fuel rods (Ref. 4-2).

(b) Normalized to $\mathrm{Kr}-85 \mathrm{~m}$ thermal fission yields.

(c) Calculated assuming a fractional release for contamination of 0.30 (Ref. 4-9).

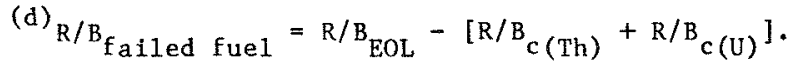

(e) Calculated assuming a fractional release of $5 \times 10^{-3}$ for failed fuel in a constrained geometry (Ref. 4-9).

${ }^{\text {(f) }}$ Calculated assuming all fission gas released came from falled fertile particles. 
TABLE $\quad 4-17$

UNBONDED FISSILE PARTICLE FAILURE LEVELS DETERMINED BY FISSION GAS RELEASE MEASUREMENTS FOR CAPSULES P13R AND P13S

\begin{tabular}{|c|c|c|c|c|c|c|c|}
\hline \multirow[b]{2}{*}{ Batch No. } & \multirow{2}{*}{$\begin{array}{l}\text { Particle } \\
\text { Type(a) }\end{array}$} & \multirow[b]{2}{*}{ Capsule } & \multirow{2}{*}{$\begin{array}{l}\text { Capsule } \\
\text { Position }\end{array}$} & \multicolumn{2}{|c|}{$\begin{array}{c}\text { TRIGA Fission } \\
\text { Gas Release }\end{array}$} & \multirow{2}{*}{$\begin{array}{l}\text { R/B } \\
\text { Failed } \\
\text { Fuel(b) }\end{array}$} & \multirow{2}{*}{$\begin{array}{c}\text { In-Service } \\
\text { Particle } \\
\text { Failure (c) } \\
(\%)\end{array}$} \\
\hline & & & & BOL & EOL & & \\
\hline $6151-00-010$ & $\mathrm{UC}_{2}$ & P13R & $3-1,2,3$ & $4 \times 10^{-7}$ & $1.4 \times 10^{-3}$ & $1.4 \times 10^{-3}$ & $4.0^{(\mathrm{d})}$ \\
\hline $6151-00-035$ & $\mathrm{UC}_{2}^{2}$ & P13R & $3-4,5$ & $3 \times 10^{-7}$ & $1.2 \times 10^{-4}$ & $1.2 \times 10^{-4}$ & 0.6 \\
\hline $6151-00-035$ & $\mathrm{UC}_{2}^{2}$ & P13S & $4-10,11,12$ & $3 \times 10^{-7}$ & $5.9 \times 10^{-5}$ & $5.9 \times 10^{-5}$ & 0.3 \\
\hline $6151-00-045$ & $\mathrm{UC}_{2}^{2}$ & P13R & $3-7,8,9$ & $2 \times 10^{-7}$ & $6.7 \times 10^{-3}$ & $6.7 \times 10^{-3}$ & $19.1^{(d)}$ \\
\hline $6151-00-046$ & $\mathrm{UC}_{2}$ & P13R & $3-10$ & $2 \times 10^{-7}$ & $4.9 \times 10^{-3}$ & $4.9 \times 10^{-3}$ & $14.0^{(d)}$ \\
\hline $6151-04-015$ & $\mathrm{UC}_{2}$ & P13R & $3-11,12$ & $8 \times 10^{-7}$ & $1.2 \times 10^{-4}$ & $1.2 \times 10^{-4}$ & 0.6 \\
\hline $6151-01-015$ & $\mathrm{UC}_{2}^{2}$ & P13R & $4-2,3$ & $1 \times 10^{-8}$ & $5.0 \times 10^{-4}$ & $5.0 \times 10^{-4}$ & 1.4 \\
\hline $6151-00-025$ & $\mathrm{UC}_{2}^{2}$ & P13R & $4-4,5,6$ & $6 \times 10^{-8}$ & $1.3 \times 10^{-3}$ & $1.3 \times 10^{-3}$ & 6.5 \\
\hline $6151-09-015$ & $\mathrm{UC}_{2}^{2}$ & P13R & $4-7,8,9$ & $9 \times 10^{-8}$ & $3.5 \times 10^{-4}$ & $3.5 \times 10^{-4}$ & 1.8 \\
\hline $6151-09-025$ & $\mathrm{UC}_{2}^{2}$ & P13R & $4-10,11$ & $2 \times 10^{-7}$ & $2.4 \times 10^{-5}$ & $2.4 \times 10^{-5}$ & 0.12 \\
\hline $6151-03-015$ & $\mathrm{UC}_{2}{ }_{2}$ & P13S & $3-1,2,3$ & $2 \times 10^{-6}$ & $6.7 \times 10^{-5}$ & $6.5 \times 10^{-5}$ & $0.2^{(\mathrm{d})}$ \\
\hline $4161-01-021$ & $\mathrm{UC}_{2}^{2}$ & P13S & $3-4,5,6$ & $5 \times 10^{-6}$ & $9.4 \times 10^{-3}$ & $9.4 \times 10^{-3}$ & $26.9^{(\mathrm{d})}$ \\
\hline $6151-08-015$ & $\mathrm{UC}_{2}^{2}$ & P13S & $3-7,8,9$ & $1 \times 10^{-7}$ & $1.4 \times 10^{-5}$ & $1.4 \times 10^{-5}$ & 0 \\
\hline $6151-02-025$ & $\mathrm{UC}_{2}$ & P13S & $3-10,12$ & $2 \times 10^{-6}$ & $1.5 \times 10^{-3}$ & $1.5 \times 10^{-3}$ & $4.3^{(d)}$ \\
\hline $4163-00-011$ & $(\mathrm{Th}, \mathrm{U}) \mathrm{O}_{2}$ & P13S & $4-1,2,3$ & $3 \times 10^{-9}$ & $5.8 \times 10^{-6}$ & 0 & 0 \\
\hline $6155-01-020$ & $(\mathrm{Th}, \mathrm{U}) \mathrm{O}_{2}$ & P13S & $4-4$ & $6 \times 10^{-7}$ & $5.3 \times 10^{-4}$ & $5.3 \times 10^{-4}$ & 2.6 \\
\hline $5466-37$ & $(\mathrm{Th}, \mathrm{U}) \mathrm{O}_{2}$ & P13S & $4-7,8,9$ & $1 \times 10^{-6}$ & $3.0 \times 10^{-6}$ & 0 & 0 \\
\hline
\end{tabular}

(a) All fissile particles were TRISO coated.

(b) For UC, particles $R / B_{\text {failed fuel }}=\mathrm{R} / \mathrm{B}_{\mathrm{EOL}}-\mathrm{R}_{2} \mathrm{~B}_{\mathrm{BOL}}$; for $(\mathrm{Th}, \mathrm{U}) \mathrm{O}_{2}$ particles $R / B_{\text {failed fuel }}=R_{E O L}-\left[R / B_{c(T h)}-R / B_{c(U)}\right]$.

(c) Calculated assuming a fractional release of $2 \times 10^{-2}$ for unconstrained failed particles, except where noted.

(d) Calculated assuming a Eractional release of $3.5 \times 10^{-2}$ for bare fuel kernels. 
TABLE $4-18$

UNBONDED FERTILE PARTICLE FATLURE LEVELS DETERMINED BY FISSION GAS RELEASE MEASUREMENTS FOR CAPSULES P13R AND P13S

\begin{tabular}{|c|c|c|c|c|c|c|c|}
\hline Batch No. & Capsule & $\begin{array}{l}\text { Capsule } \\
\text { Position }\end{array}$ & $\begin{array}{l}\text { Thorium } \\
\text { Cont. (a) } \\
\text { (g Th/g Th) }\end{array}$ & $R / B_{C}(T h)^{(b)}$ & $\mathrm{R} / \mathrm{BEOL}$ & $\begin{array}{c}\text { R/B } \\
\text { Failed } \\
\text { Fuel (c) }\end{array}$ & $\begin{array}{c}\text { In-Service } \\
\text { Particle } \\
\text { Failure }(\mathrm{d}) \\
(\%)\end{array}$ \\
\hline 4252-06-018 & P13R & $3-1,2$ & $7 \times 10^{-4}$ & $2.1 \times 10^{-4}$ & $1.4 \times 10^{-3}$ & $1.2 \times 10^{-3}$ & 6.0 \\
\hline $6542-01-020$ & P13R & $3-3,4$ & 0 & 0 & $1.7 \times 10^{-4}$ & $1.7 \times 10^{-4}$ & 0.9 \\
\hline $6542-01-010$ & $\mathrm{P} 13 \mathrm{R}$ & $3-5$ & $6 \times 10^{-6}$ & $1.1 \times 10^{-6}$ & $1.2 \times 10^{-3}$ & $1.2 \times 10^{-3}$ & 6.0 \\
\hline $6542-25-015$ & P13R & $4-11$ & $1 \times 10^{-6}$ & $1.1 \times 10^{-6}$ & $1.2 \times 10^{-3}$ & $1.2 \times 10^{-3}$ & 6.0 \\
\hline $6542-02-020$ & P13R & $3-7,8$ & 0 & 0 & $4.4 \times 10^{-5}$ & $4.4 \times 10^{-5}$ & 0.2 \\
\hline $6542-02-020$ & P13R & $4-5,6$ & 0 & 0 & $3.3 \times 10^{-5}$ & $3.3 \times 10^{-5}$ & 0.17 \\
\hline $6542-02-030$ & P13R & $3-9,10$ & $8 \times 10^{-5}$ & $2.4 \times 10^{-5}$ & $2.9 \times 10^{-3}$ & $2.9 \times 10^{-3}$ & 14.5 \\
\hline $6542-09-010$ & P13R & $3-11,12$ & $1 \times 10^{-4}$ & $3.0 \times 10^{-5}$ & $2.4 \times 10^{-5}$ & 0 & 0 \\
\hline $6542-11-015$ & P13R & $4-2$ & $6 \times 10^{-7}$ & $8.6 \times 10^{-7}$ & $2.4 \times 10^{-3}$ & $2.4 \times 10^{-3}$ & 12.0 \\
\hline $6542-19-015$ & P13S & $4-7$ & $4 \times 10^{-6}$ & $8.6 \times 10^{-7}$ & $2.4 \times 10^{-3}$ & $2.4 \times 10^{-3}$ & 12.0 \\
\hline $6542-19-016$ & P13S & $4-8$ & $4 \times 10^{-6}$ & $8.6 \times 10^{-7}$ & $2.4 \times 10^{-3}$ & $2.4 \times 10^{-3}$ & 12.0 \\
\hline $6542-18-015$ & P13R & $4-3,4$ & $3 \times 10^{-6}$ & $9.0 \times 10^{-7}$ & $2.2 \times 10^{-6}$ & $1.3 \times 10^{-6}$ & 0 \\
\hline $6542-20-035$ & $\mathrm{P} 13 \mathrm{R}$ & $4-7,8$ & $1 \times 10^{-6}$ & $3.0 \times 10^{-7}$ & $6.9 \times 10^{-4}$ & $6.9 \times 10^{-4}$ & 3.5 \\
\hline $6542-20-025$ & P13R & $4-9,10$ & $3 \times 10^{-6}$ & $9.0 \times 10^{-7}$ & $3.7 \times 10^{-4}$ & $3.7 \times 10^{-4}$ & 1.9 \\
\hline $4252-06-010$ & P13S & $3-1,2$ & $7 \times 10^{-4}$ & $2.1 \times 10^{-4}$ & $6.2 \times 10^{-5}$ & 0 & 0 \\
\hline $6542-22-015$ & P13S & $3-3,4$ & $1 \times 10^{-6}$ & $3.0 \times 10^{-7}$ & $2.7 \times 10^{-4}$ & $2.7 \times 10^{-4}$ & 1.4 \\
\hline $6542-22-025$ & P13S & $3-5,6$ & $3 \times 10^{-6}$ & $9.0 \times 10^{-7}$ & $2.4 \times 10^{-4}$ & $2.4 \times 10^{-4}$ & 1.2 \\
\hline $6542-23-025$ & P13s & $3-7,8$ & $4 \times 10^{-6}$ & $1.2 \times 10^{-6}$ & $2.8 \times 10^{-6}$ & $1.6 \times 10^{-6}$ & 0 \\
\hline $6542-24-015$ & P13S & $3-9,10$ & $1 \times 10^{-6}$ & $3.0 \times 10^{-7}$ & $3.9 \times 10^{-6}$ & $3.6 \times 10^{-6}$ & 0 \\
\hline $6252-00-025^{(e)}$ & P13S & $3-12$ & 0 & 0 & $3.9 \times 10^{-5}$ & $3.9 \times 10^{-5}$ & 0.2 \\
\hline $6542-17-010$ & P13S & $4-1$ & 0 & 0 & $4.7 \times 10^{-3}$ & $4.7 \times 10^{-3}$ & 23.5 \\
\hline $6542-16-010$ & P13S & $4-4$ & 0 & 0 & $4.7 \times 10^{-3}$ & $4.7 \times 10^{-3}$ & 23.5 \\
\hline $4252-02-010$ & P13S & $4-2,3$ & $1 \times 10^{-4}$ & $3.0 \times 10^{-5}$ & $1.1 \times 10^{-3}$ & $1.1 \times 10^{-3}$ & 5.5 \\
\hline $6542-21-016$ & P13S & $4-9$ & $4 \times 10^{-6}$ & $1.2 \times 10^{-6}$ & $2.1 \times 10^{-3}$ & $2.1 \times 10^{-3}$ & 10.5 \\
\hline $6542-21-015$ & P13S & $4-10$ & $4 \times 10^{-6}$ & $1.2 \times 10^{-6}$ & $2.1 \times 10^{-3}$ & $2.1 \times 10^{-3}$ & 10.5 \\
\hline $6542-12-025$ & P13S & $4-11,12$ & 0 & 0 & $2.0 \times 10^{-4}$ & $2.0 \times 10^{-4}$ & 1.0 \\
\hline
\end{tabular}

(a) Determined by acid-1each test.

(b) Fission gas release from heavy metal contamination, $R / B_{c}(T h)=\left(T h_{c}\right)\left(r / b_{c}\right)$ where $r / b_{C}=0.30$.

${ }^{(c)}{ }_{R / B}$ failed fuel $=R / B_{E O L}-R / B_{c}(T h)$.

(d) Calculated assuming a fractional release of $2 \times 10^{-2}$ for unconstrained failed particles.

(e) TRISO coated. 
TABLE 4-19

SUMMARY OF HIGH-TEMPERATURE FISSION GAS RELEASE MEASUREMENTS MADE ON SELECTED CAPSULE P13R FUEL RODS

\begin{tabular}{|c|c|c|c|c|}
\hline $\begin{array}{c}\text { Data } \\
\text { Retrieval } \\
\text { Number } \\
\text { (7161-004-) }\end{array}$ & $\begin{array}{l}\text { Capsule } \\
\text { Position }\end{array}$ & $\begin{array}{c}\text { Date of } \\
\text { Measurement }\end{array}$ & $\begin{array}{l}\text { Measurement } \\
\text { Temperature } \\
\left({ }^{\circ} \mathrm{C}\right)\end{array}$ & $\begin{array}{l}\text { Fission Gas } \\
\text { Release } \\
\text { (R/B Kr-85m) }\end{array}$ \\
\hline \multirow[t]{5}{*}{$03-5$} & \multirow[t]{5}{*}{$1 \mathrm{C}$} & Feb. 21, 1975 & 1100 & $3.3 \times 10^{-6}$ \\
\hline & & July 9, 1975 & 1300 & $1.9 \times 10^{-4}$ \\
\hline & & July 9, 1975 & 1500 & $2.0 \times 10^{-2}$ \\
\hline & & July 9,1975 & 1600 & $2.9 \times 10^{-2}$ \\
\hline & & July 9, 1975 & 1100 & $4.3 \times 10^{-3}$ \\
\hline \multirow[t]{6}{*}{$16-5$} & \multirow[t]{6}{*}{$5 \mathrm{~A}$} & March 6, 1975 & 1100 & $3.9 \times 10^{-5}$ \\
\hline & & Oct. 16,1975 & 1100 & $1.7 \times 10^{-5}$ \\
\hline & & Oct. 16,1975 & 1300 & $5.9 \times 10^{-4}$ \\
\hline & & Oct. 16,1975 & 1500 & $1.5 \times 10^{-3}$ \\
\hline & & Oct. 16,1975 & 1600 & $5.1 \times 10^{-3}$ \\
\hline & & oct. 16,1975 & 1100 & $7.3 \times 10^{-4}$ \\
\hline \multirow[t]{8}{*}{$17-5$} & \multirow[t]{8}{*}{$5 B$} & March 6, 1975 & 1300 & $6.8 \times 10^{-5}$ \\
\hline & & March 6, 1975 & 1100 & $7.6 \times 10^{-5}$ \\
\hline & & Oct. 10,1975 & 1100 & $2.0 \times 10^{-5}$ \\
\hline & & oct. 10,1975 & 1300 & $6.9 \times 10^{-5}$ \\
\hline & & Oct. 10,1975 & 1500 & $2.6 \times 10^{-4}$ \\
\hline & & oct. 10,1975 & 1600 & $9.0 \times 10^{-3}$ \\
\hline & & Oct. 10,1975 & 1100 & $9.1 \times 10^{-4}$ \\
\hline & & Dec. 19,1975 & 1100 & $2.9 \times 10^{-4}$ \\
\hline \multirow[t]{2}{*}{$19-7$} & \multirow[t]{2}{*}{$5 \mathrm{D}$} & March 12, 1975 & 1300 & $2.5 \times 10^{-5}$ \\
\hline & & March 12, 1975 & 1100 & $3.4 \times 10^{-5}$ \\
\hline
\end{tabular}


TABLE 4-20

SUMMARY OF HIGH-TEMPERATURE FISSION GAS RELEASE MEASUREMENTS MADE ON SELECTED CAPSULE P13S FUEL RODS

\begin{tabular}{|c|c|c|c|c|}
\hline $\begin{array}{c}\text { Data } \\
\text { Retrieval } \\
\text { Number } \\
(7161-004-)\end{array}$ & $\begin{array}{l}\text { Capsule } \\
\text { Position } \\
\end{array}$ & $\begin{array}{c}\text { Date of } \\
\text { Measurement }\end{array}$ & $\begin{array}{c}\text { Measurement } \\
\text { Temperature } \\
\left({ }^{\circ} \mathrm{C}\right)\end{array}$ & $\begin{array}{l}\text { Fission Gas } \\
\text { Release } \\
\text { (R/B Kr-85m) }\end{array}$ \\
\hline \multirow[t]{6}{*}{$03-6$} & \multirow[t]{6}{*}{$1 \mathrm{C}$} & April 11, 1975 & 1100 & $1.1 \times 10^{-4}$ \\
\hline & & July 9, 1975 & 1300 & $3.1 \times 10^{-4}$ \\
\hline & & July 9, 1975 & 1500 & $2.2 \times 10^{-3}$ \\
\hline & & Ju1y 9, 1975 & 1600 & $1.2 \times 10^{-2}$ \\
\hline & & July 9, 1975 & 1100 & $1.8 \times 10^{-3}$ \\
\hline & & Dec. 17,1975 & 1100 & $5.2 \times 10^{-4}$ \\
\hline \multirow[t]{7}{*}{$14-5$} & \multirow[t]{7}{*}{$2 \mathrm{D}$} & May 7, 1975 & 1100 & $1.2 \times 10^{-5}$ \\
\hline & & Oct. 13,1975 & 1100 & $1.7 \times 10^{-5}$ \\
\hline & & oct. 13,1975 & 1300 & $3.2 \times 10^{-5}$ \\
\hline & & Oct. 13,1975 & 1500 & $1.6 \times 10^{-3}$ \\
\hline & & Oct. 13,1975 & 1600 & $1.6 \times 10^{-2}$ \\
\hline & & Oct. 13,1975 & 1100 & $1.0 \times 10^{-3}$ \\
\hline & & Dec. 17,1975 & 1100 & $4.2 \times 10^{-4}$ \\
\hline \multirow[t]{2}{*}{$17-6$} & \multirow[t]{2}{*}{$5 B$} & May 7,1975 & 1500 & $3.0 \times 10^{-4}$ \\
\hline & & May 7, 1975 & 1100 & $5.9 \times 10^{-5}$ \\
\hline \multirow[t]{2}{*}{$19-5$} & \multirow[t]{2}{*}{$5 \mathrm{D}$} & May 7, 1975 & 1500 & $4.8 \times 10^{-4}$ \\
\hline & & May 8, 1975 & 1100 & $7.4 \times 10^{-5}$ \\
\hline
\end{tabular}


TABLE 4-21

CO:PARISON OF CAPSLLE P13R EOL IN-PILE AND TRIGA FISSION GAS RELEASE DATA ( $\mathrm{R} / \mathrm{B} \mathrm{Kr}-85 \mathrm{~m}$ )

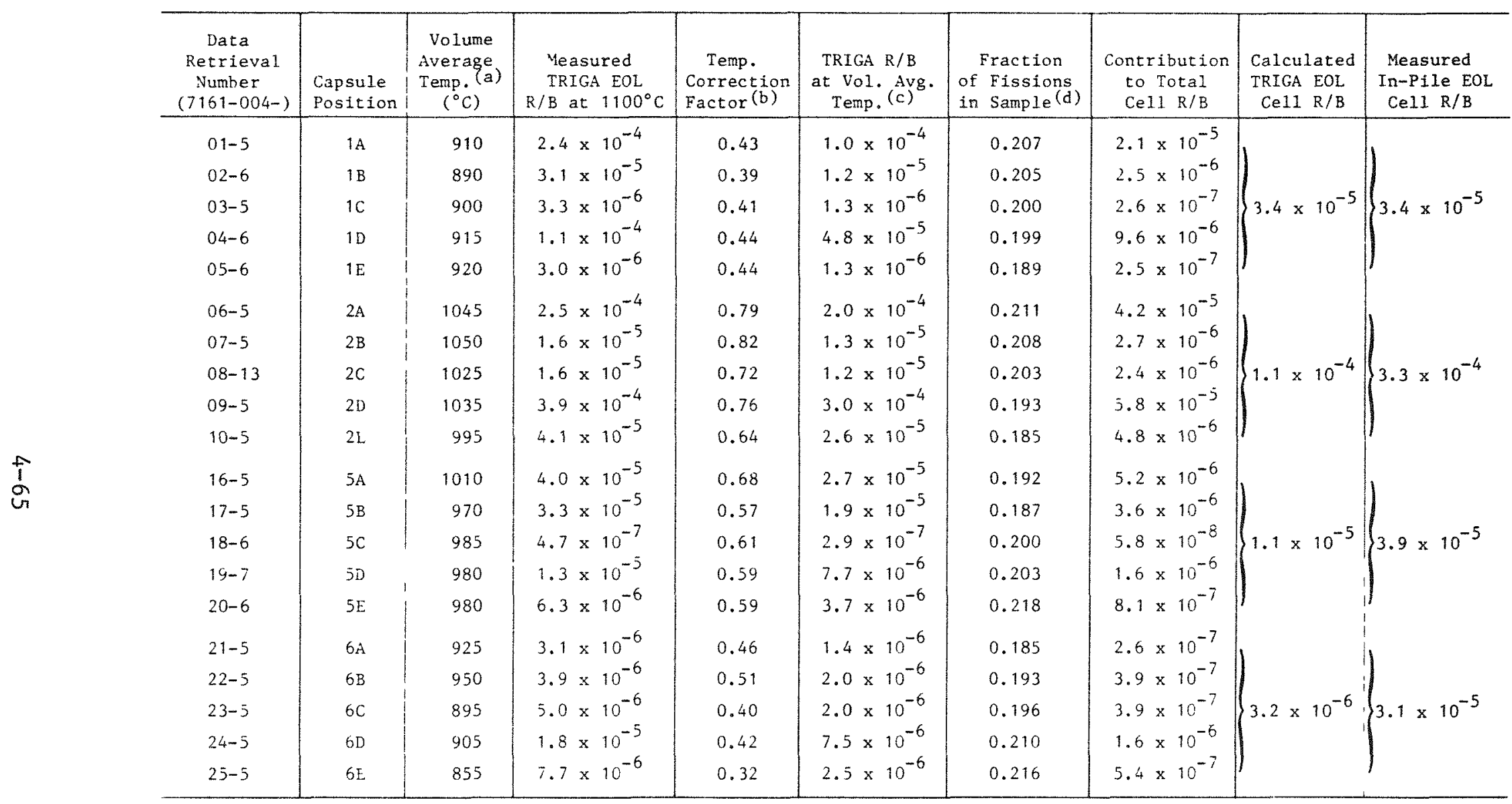

(a) Volume average irradiation temperature for the last cycle of operation.

(b) Temperature correction factor was calculated using Eq. 4-13.

(c) Measured TRIGA fission gas release data corrected to volume average irradiation temperature.

(d) Fraction of fissions in individual fuel rods equals the amount of fissionable material in the fuel rod divided by the total amount of fissionable material in the cell at EOL. 
TABLE 4-22

COMPARISON OF CAPSULE P13S EOL IN-PILE AND TRIGA FISSION GAS RELEASE DATA (R/B Kr-85m)

\begin{tabular}{|c|c|c|c|c|c|c|c|c|c|}
\hline $\begin{array}{c}\text { Data } \\
\text { Retrieval } \\
\text { Number } \\
(7161-004-) \\
\end{array}$ & $\begin{array}{l}\text { Capsule } \\
\text { Position } \\
\end{array}$ & $\begin{array}{c}\text { Volume } \\
\text { Average } \\
\text { Temp. (a) } \\
\left({ }^{\circ} \mathrm{C}\right)\end{array}$ & $\begin{array}{c}\text { Measured } \\
\text { TRIGA EOL } \\
\text { R/B at } 1100^{\circ} \mathrm{C} \\
\end{array}$ & $\begin{array}{l}\text { Temp. } \\
\text { Correction } \\
\text { Factor(b) } \\
\end{array}$ & $\begin{array}{c}\text { TRIGA R/B } \\
\text { at Vol. Avg. } \\
\text { Temp. (c) }\end{array}$ & $\begin{array}{c}\text { Fraction } \\
\text { of Fissions } \\
\text { in Sample(d) }\end{array}$ & $\begin{array}{c}\text { Contribution } \\
\text { to Total } \\
\text { Cell R/B } \\
\end{array}$ & $\begin{array}{l}\text { Calculated } \\
\text { TRIGA EOL } \\
\text { Cell R/B } \\
\end{array}$ & $\begin{array}{c}\text { Measured } \\
\text { In-Pile EOL } \\
\text { Ce11 R/B }\end{array}$ \\
\hline $01-7$ & $1 \mathrm{~A}$ & 930 & $1.1 \times 10^{-3}$ & 0.47 & $5.2 \times 10^{-4}$ & 0.208 & $1.1 \times 10^{-4}$ & \multirow{6}{*}{$2.7 \times 10^{-4}$} & \multirow{6}{*}{$3.9 \times 10^{-4}$} \\
\hline $02-5$ & $1 B$ & 915 & $5.9 \times 10^{-5}$ & 0.43 & $2.5 \times 10^{-5}$ & 0.206 & $5.2 \times 10^{-6}$ & & \\
\hline $03-6$ & 10 & 935 & $1.1 \times 10^{-4}$ & 0.48 & $5.3 \times 10^{-5}$ & 0.200 & $1.1 \times 10^{-5}$ & & \\
\hline $04-5$ & $1 \mathrm{D}$ & 940 & $1.2 \times 10^{-3}$ & 0.50 & $6.0 \times 10^{-4}$ & 0.198 & $1.2 \times 10^{-4}$ & & \\
\hline $05-5$ & $I E$ & 935 & $2.2 \times 10^{-4}$ & 0.48 & $1.1 \times 10^{-4}$ & 0.188 & $2.1 \times 10^{-5}$ & & \\
\hline $11-6$ & $2 \mathrm{~A}$ & 895 & $1.5 \times 10^{-4}$ & 0.39 & $5.9 \times 10^{-5}$ & 0.212 & $1.3 \times 10^{-5}$ & & \\
\hline $12-5$ & $2 \mathrm{~B}$ & 895 & $3.4 \times 10^{-7}$ & 0.39 & $1.3 \times 10^{-7}$ & 0.208 & $2.7 \times 10^{-8}$ & \multirow{4}{*}{$2.2 \times 10^{-5}$} & \multirow{4}{*}{$3.2 \times 10^{-4}$} \\
\hline $13-6$ & $2 \mathrm{C}$ & 875 & $3.5 \times 10^{-5}$ & 0.36 & $1.3 \times 10^{-5}$ & 0.203 & $2.6 \times 10^{-6}$ & & \\
\hline $14-5$ & $2 \mathrm{D}$ & 880 & $1.2 \times 10^{-5}$ & 0.37 & $4.4 \times 10^{-6}$ & 0.193 & $8.5 \times 10^{-7}$ & & \\
\hline $15-5$ & $2 \mathrm{E}$ & 850 & $1.0 \times 10^{-4}$ & 0.31 & $3.1 \times 10^{-5}$ & 0.184 & $5.7 \times 10^{-6}$ & & \\
\hline $16-6$ & $5 \mathrm{~A}$ & 1055 & $1.8 \times 10^{-4}$ & 0.84 & $1.5 \times 10^{-4}$ & 0.192 & $2.9 \times 10^{-5}$ & \multirow{5}{*}{$7.9 \times 10^{-5}$} & \multirow{5}{*}{$2.4 \times 10^{-5}$} \\
\hline $17-6$ & $5 B$ & 1035 & $1.0 \times 10^{-4}$ & 0.75 & $7.5 \times 10^{-5}$ & 0.187 & $1.4 \times 10^{-5}$ & & \\
\hline $18-5$ & $5 c$ & 1040 & $2.6 \times 10^{-5}$ & 0.78 & $2.0 \times 10^{-5}$ & 0.200 & $4.0 \times 10^{-6}$ & & \\
\hline $19-5$ & $5 \mathrm{D}$ & 1035 & $7.4 \times 10^{-5}$ & 0.76 & $5.6 \times 10^{-5}$ & 0.203 & $1.1 \times 10^{-5}$ & & \\
\hline $20-5$ & $5 \mathrm{E}$ & 1010 & $1.4 \times 10^{-4}$ & 0.68 & $9.5 \times 10^{-5}$ & 0.218 & $2.1 \times 10^{-5}$ & & \\
\hline $26-5$ & $6 \mathrm{~A}$ & 955 & $2.1 \times 10^{-6}$ & 0.53 & $1.1 \times 10^{-6}$ & 0.184 & $2.0 \times 10^{-7}$ & \multirow{5}{*}{$4.1 \times 10^{-7}$} & \multirow{5}{*}{$2.9 \times 10^{-5}$} \\
\hline $27-5$ & $6 B$ & 935 & $<10^{-7}$ & 0.48 & $4.9 \times 10^{-8}$ & 0.192 & $9.4 \times 10^{-9}$ & & \\
\hline $28-13$ & $6 \mathrm{C}$ & 935 & $8.4 \times 10^{-7}$ & 0.48 & $4.0 \times 10^{-7}$ & 0.195 & $7.8 \times 10^{-8}$ & & \\
\hline $29-5$ & $6 \mathrm{D}$ & 900 & $3.6 \times 10^{-7}$ & 0.41 & $1.5 \times 10^{-7}$ & 0.210 & $3.2 \times 10^{-8}$ & & \\
\hline $30-5$ & $6 E$ & 855 & $1.3 \times 10^{-6}$ & 0.32 & $4.2 \times 10^{-7}$ & 0.219 & $9.2 \times 10^{-8}$ & & \\
\hline
\end{tabular}

(a) Volume average irradiation temperature for the last cycle of operation.

(b) Temperature correction factor was calculated using Eq. 4-13.

(c) Measured TRIGA fission gas release data corrected to volume average irradiation temperature.

(d) Fraction of fissions in individual tuel rods equals the amount of fissionable material in the fuel rod divided by the total amount of fissionable material in the cell at EOL. 
TABLE 4-23

COMPARISON OF CAPSULE P13R BOL IN-PILE AND TRIGA FISSION GAS RELEASE DATA (R/B Kr-85m)

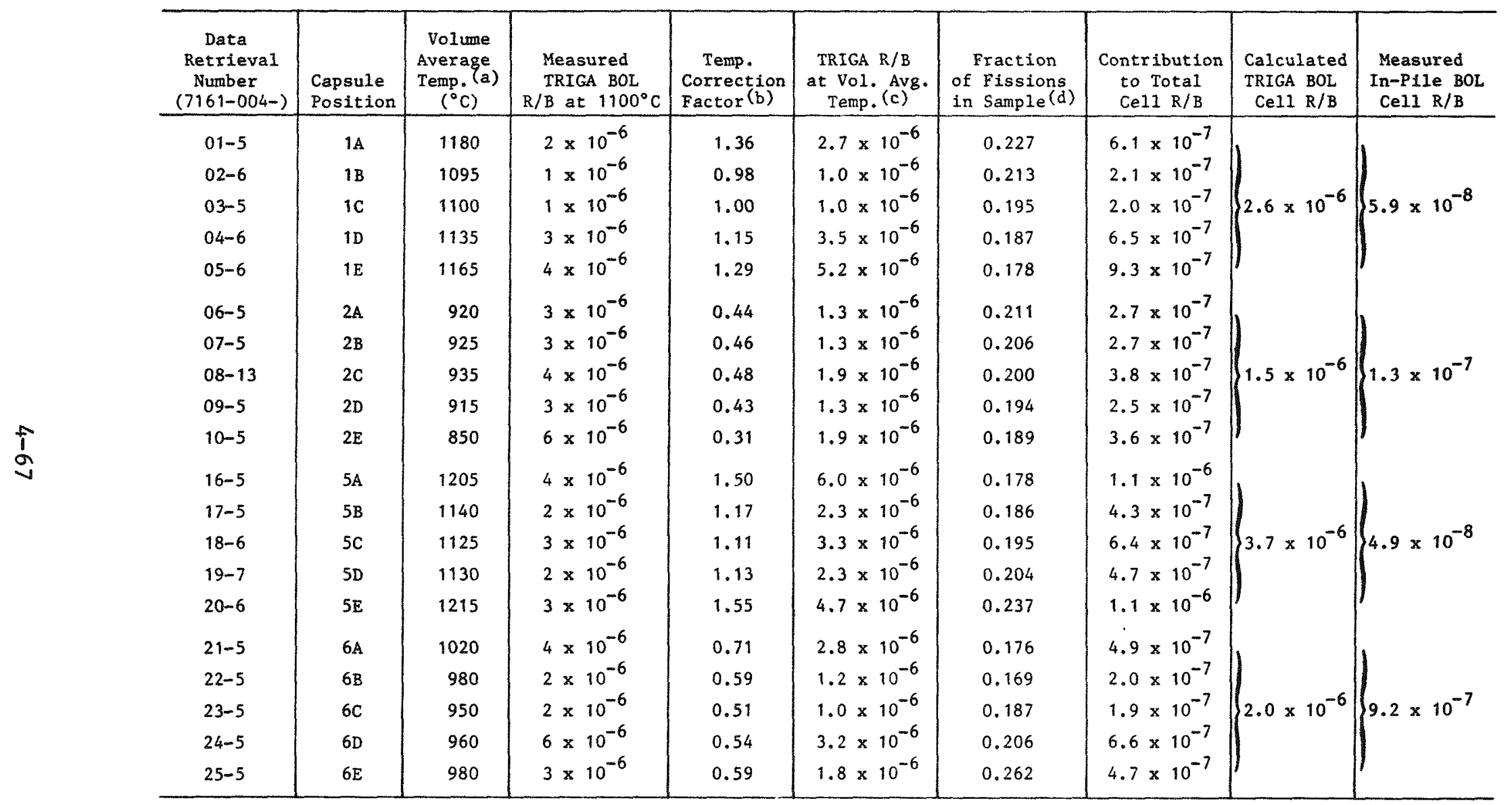

(a) Volume average irradiation temperature at the time the in-pile fission gas release measurement was made.

(b) Temperature correction factor was calculated using Eq. 4-13.

(c) Measured TRIGA fission gas release data corrected to volume average irradiation temperature.

(d) Fraction of fissions in individual fuel rods equals the amount of fissionable material in the fuel rod divided by the total amount of fissionable material in the cell at BOL. 
TABLE $\quad 4-24$

COMPARISON OF CAPSULE P13S BOL IN-PILE AND TRIGA FISSION GAS RELEASE DATA (R/B Kr-85m)

\begin{tabular}{|c|c|c|c|c|c|c|c|c|c|}
\hline $\begin{array}{c}\text { Data } \\
\text { Retrieval } \\
\text { Number } \\
(7161-004-)\end{array}$ & $\begin{array}{l}\text { Capsule } \\
\text { Position }\end{array}$ & $\begin{array}{c}\text { Volume } \\
\text { Average } \\
\text { Temp. (a) } \\
\left({ }^{\circ} \mathrm{C}\right) \\
\end{array}$ & $\begin{array}{c}\text { Measured } \\
\text { TRIGA BOL } \\
\text { R/B at } 1100^{\circ} \mathrm{C} \\
\end{array}$ & $\begin{array}{l}\text { Temp. } \\
\text { Correction } \\
\text { Factor (b) }\end{array}$ & $\begin{array}{l}\text { TRIGA R/B } \\
\text { at Vol. Avg. } \\
\text { Temp. (c) }\end{array}$ & $\begin{array}{c}\text { Fraction } \\
\text { of Fissions } \\
\text { in Sample(d) }\end{array}$ & $\begin{array}{c}\text { Contribution } \\
\text { to Total } \\
\text { Cell R/B } \\
\end{array}$ & $\begin{array}{c}\text { Calculated } \\
\text { TRIGA BOL } \\
\text { Cell R/B } \\
\end{array}$ & $\begin{array}{c}\text { Measured } \\
\text { In-Pile BOI } \\
\text { CelI R/B } \\
\end{array}$ \\
\hline $01-7$ & $1 \mathrm{~A}$ & 1000 & $2 \times 10^{-6}$ & 0.65 & $1.3 \times 10^{-6}$ & 0.227 & $3.0 \times 10^{-7}$ & & \\
\hline $02-5$ & 1B & 905 & $1 \times 10^{-6}$ & 0.42 & $4.2 \times 10^{-7}$ & 0.213 & $8.9 \times 10^{-8}$ & & \\
\hline $03-6$ & $1 \mathrm{C}$ & 925 & $1 \times 10^{-6}$ & 0.46 & $4.6 \times 10^{-7}$ & 0.195 & $9.0 \times 10^{-8}$ & $8.7 \times 10^{-7}$ & $6.9 \times 10^{-8}$ \\
\hline $04-5$ & 10 & 935 & $2 \times 10^{-6}$ & 0.48 & $9.6 \times 10^{-7}$ & 0.187 & $1.8 \times 10^{-7}$ & & \\
\hline $05-5$ & $1 \mathrm{E}$ & 975 & $2 \times 10^{-6}$ & 0.58 & $1.2 \times 10^{-6}$ & 0.178 & $2.1 \times 10^{-7}$ & & \\
\hline $11-6$ & $2 \mathrm{~A}$ & 955 & $2 \times 10^{-7}$ & 0.53 & $1.1 \times 10^{-7}$ & 0.211 & $2.3 \times 10^{-8}$ & & \\
\hline $12-5$ & $2 B$ & 915 & $2 \times 10^{-6}$ & 0.43 & $8.6 \times 10^{-7}$ & 0.206 & $1.8 \times 10^{-7}$ & & \\
\hline $13-6$ & $2 \mathrm{C}$ & 870 & $3 \times 10^{-6}$ & 0.35 & $1.1 \times 10^{-6}$ & 0.200 & $2.2 \times 10^{-7}$ & $9.5 \times 10^{-7}$ & $1.0 \times 10^{-7}$ \\
\hline $14-5$ & 2D & 885 & $2 \times 10^{-6}$ & 0.38 & $7.6 \times 10^{-7}$ & 0.194 & $1.5 \times 10^{-7}$ & & \\
\hline $15-5$ & $2 E$ & 890 & $5 \times 10^{-6}$ & 0.39 & $2.0 \times 10^{-6}$ & 0.189 & $3.8 \times 10^{-7}$ & & \\
\hline $16-6$ & $5 \mathrm{~A}$ & 1330 & $4 \times 10^{-6}$ & 2.27 & $9.1 \times 10^{-6}$ & 0.178 & $1.6 \times 10^{-6}$ & & \\
\hline $17-6$ & $5 B$ & 1295 & $1 \times 10^{-6}$ & 2.03 & $2.0 \times 10^{-6}$ & 0.186 & $3.7 \times 10^{-7}$ & & \\
\hline $18-5$ & $5 C$ & 1280 & $2 \times 10^{-6}$ & 1.94 & $3.9 \times 10^{-6}$ & 0.195 & $7.6 \times 10^{-7}$ & $5.5 \times 10^{-6}$ & $4.6 \times 10^{-7}$ \\
\hline $19-5$ & $5 D$ & 1280 & $3 \times 10^{-6}$ & 1.94 & $5.8 \times 10^{-6}$ & 0.204 & $1.2 \times 10^{-6}$ & & \\
\hline $20-5$ & $5 E$ & 1320 & $3 \times 10^{-6}$ & 2.20 & $6.6 \times 10^{-6}$ & 0.237 & $1.6 \times 10^{-6}$ & & \\
\hline $26-5$ & $6 A$ & 960 & $3 \times 10^{-6}$ & 0.54 & $1.6 \times 10^{-6}$ & 0.176 & $2.8 \times 10^{-7}$ & & \\
\hline $27-5$ & $6 B$ & 900 & $1 \times 10^{-6}$ & 0.41 & $4.1 \times 10^{-7}$ & 0.169 & $6.9 \times 10^{-8}$ & & \\
\hline $28-13$ & $6 \mathrm{C}$ & 935 & $4 \times 10^{-6}$ & 0.48 & $1.9 \times 10^{-6}$ & 0.187 & $3.6 \times 10^{-7}$ & $1.0 \times 10^{-6}$ & $2.7 \times 10^{-7}$ \\
\hline $29-5$ & $6 \mathrm{D}$ & 890 & $1 \times 10^{-6}$ & 0.39 & $3.9 \times 10^{-7}$ & 0.206 & $8.0 \times 10^{-8}$ & & \\
\hline $30-5$ & $6 \mathrm{E}$ & 900 & $2 \times 10^{-6}$ & 0.41 & $8.2 \times 10^{-7}$ & 0.262 & $2.1 \times 10^{-7}$ & & \\
\hline
\end{tabular}

(a) Volume average irradiation temperature at the time the in-pile fission gas release measurement was made.

(b) Temperature correction factor was calculated using Eq. 4-13.

(c) Measured TRIGA fission gas release data corrected to volume average irradiation temperature.

(d) Fraction of fissions in individual fuel rods equals the amount of fissionable material in the fuel rod divided by the total amount of fissionable material in the cell at BOL. 
TABLE 4-25

FISSION PRODUCT INVENTORY OF FUEL RODS IRRADIATED IN CAPSULE P13R

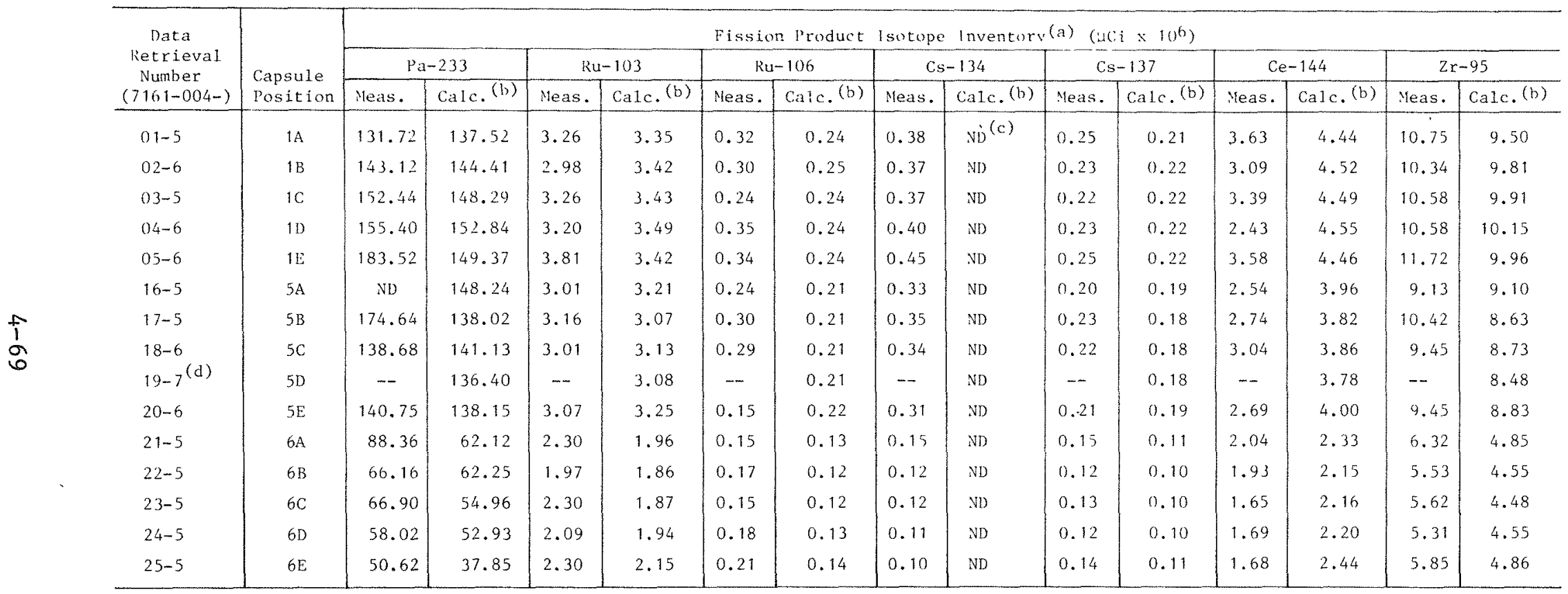

(a) Fission product isotope inventory was decayed back to EOL.

(b) Fission product isotope inventory was calculated using FISS-PROD computer code.

(c) $\mathrm{ND}=$ not determined.

(d) Fuel rod was not gamma counted. 
TABLE 4-26

FISSION PRODUCT INVENTORY OF FUEL RODS IRRADIATED IN CAPSULE P13S

\begin{tabular}{|c|c|c|c|c|c|c|c|c|c|c|c|c|c|c|c|}
\hline \multirow{3}{*}{$\begin{array}{l}\text { Data } \\
\text { Retrieval } \\
\text { Number } \\
(7161-004-)\end{array}$} & \multirow{3}{*}{$\begin{array}{l}\text { Capsule } \\
\text { Position }\end{array}$} & \multicolumn{14}{|c|}{ Fission Product Isotope Inventory (a) ( $\mathrm{HC} i \times 10^{6}$ ) } \\
\hline & & \multicolumn{2}{|c|}{$\mathrm{Pa}-233$} & \multicolumn{2}{|c|}{$\mathrm{Ru}-103$} & \multicolumn{2}{|c|}{$\mathrm{Ru}-106$} & \multicolumn{2}{|c|}{$\operatorname{cs}-134$} & \multicolumn{2}{|c|}{$\operatorname{cs}-137$} & \multicolumn{2}{|c|}{$\mathrm{Ce}-144$} & \multicolumn{2}{|c|}{$2 x-95$} \\
\hline & & Yeas. & Calc. (b) & Meas. & Calc. (b) & Meas. & Calc. (b) & Meas. & CaIc. (b) & Meas. & Calc. (b) & Meas. & Calc. (b) & Meas. & Calc. (b) \\
\hline $01-7$ & $1 d$ & 119.58 & 129.87 & 3.10 & 3.21 & 0.31 & 0.23 & 0.30 & $\mathrm{ND}(\mathrm{c})$ & 0.20 & 0.20 & 4.44 & 4.23 & 9.80 & 9.01 \\
\hline $02-5$ & $1 \mathrm{~B}$ & 152.44 & 134.28 & 3.53 & 3.22 & 0.25 & 0.23 & 0.35 & ND & 0.23 & 0.20 & 4.21 & 4.24 & 10.61 & 9.14 \\
\hline $03-6$ & $1 \mathrm{C}$ & 145.78 & 136.26 & 3.32 & 3.18 & 0.22 & 0.23 & 0.39 & ND & 0.23 & 0.20 & 4.95 & 4.15 & 10.20 & 9.10 \\
\hline $04-5$ & 10 & 167.24 & 139.10 & 3.26 & 3.20 & 0.29 & 0.23 & 0.33 & ND & 0.22 & 0.20 & 4.71 & 4.16 & 11.34 & 9.21 \\
\hline $05-5$ & $1 E$ & 182.04 & 119.19 & 3.50 & 2.84 & 0.18 & 0.21 & 0.44 & ND & 0.26 & 0.18 & 5.26 & 3.79 & 11.50 & 8.18 \\
\hline $16-6$ & $5 \mathrm{~A}$ & 156.88 & 139.32 & 3.44 & 3.03 & 0.37 & 0.20 & 0.31 & ND & 0.23 & 0.18 & 4.55 & 3.73 & 10.45 & 8.52 \\
\hline $17-6$ & $5 \mathrm{~B}$ & 102.42 & 130.40 & 2.64 & 2.92 & 0.24 & 0.20 & 0.34 & ND & 0.21 & 0.17 & 4.03 & 3.62 & 9.64 & 8.14 \\
\hline $18-5$ & $5 \mathrm{C}$ & 110.41 & 134.55 & 2.73 & 3.00 & 0.25 & 0.20 & 0.30 & ND & 0.18 & 0.17 & 3.89 & 3.68 & 7.92 & 8.26 \\
\hline $19-5$ & 5D & 112.48 & 130.86 & 2.77 & 2.97 & 0.26 & 0.20 & 0.30 & No & 0.19 & 0.17 & 4.71 & 3.63 & 7.98 & 8.14 \\
\hline $20-5$ & $5 \mathrm{E}$ & 155.45 & 133.29 & 3.23 & 3.15 & 0.32 & 0.21 & 0.30 & ND & 0.21 & 0.18 & 4.10 & 3.87 & 9.96 & 8.53 \\
\hline
\end{tabular}

(a) Fission product isotope inventory was decayed back to EOL.

(b) Fission product isotope inventory was calculated using FISS-PROD computer code.

(c) $\mathrm{ND}=$ not determined. 
TABLE 4-27

FISSION PRODUCT ISOTOPE COUNTING ERROR

\begin{tabular}{l|c}
\hline Isotope & Counting Error, ${ }_{(\%)}^{(a)} 2 \sigma$ \\
\hline Pa-233 & 5.0 \\
Ru-103 & 15.0 \\
Ru-106 & 10.0 \\
Cs-134 & 1.6 \\
Cs-137 & 1.6 \\
Ce-144 & 6.0 \\
Zr-95 & 1.6 \\
\hline
\end{tabular}

(a) The $2 \sigma$ counting error is calculated by:

$2 \sigma= \pm \frac{1.96}{\mathrm{PEAK} \cdot \mathrm{LT}}\left\{\mathrm{CNT}+(1-\mathrm{CH} / 2)^{2} \cdot(\mathrm{LC}-\mathrm{RC}) / \sqrt{\mathrm{PTS}}\right\}^{1 / 2} \cdot 100(\%)$

where $\mathrm{PEAK}=$ counts in the peak $\left(\mathrm{min}^{-1}\right)$

$$
\begin{aligned}
\mathrm{CNT} & =\text { total counts in peak (peak + background) } \\
\mathrm{CH} & =\text { number of channels in peak, } \\
\mathrm{LC} & =\text { counts in lowest-energy channel } \\
\mathrm{RC} & =\text { counts in highest-energy channel } \\
\mathrm{PTS} & =\text { number of smoothing points } \\
\mathrm{LT} & =\text { analog-to-digital converter lifetime (min) }
\end{aligned}
$$


TABLE $4-28$

GAMMA SCAN RESULTS OF CAPSULE P13R FUEL ROD CRUCIBLES

\begin{tabular}{|c|c|c|c|c|}
\hline \multirow[b]{2}{*}{$\begin{array}{c}\text { Spectrum } \\
\text { No. (a) }\end{array}$} & \multicolumn{2}{|c|}{$\begin{array}{c}\text { Isotope Concentration } \\
\left(\mu \mathrm{Ci} \times 10^{2}\right)\end{array}$} & \multirow{2}{*}{$\begin{array}{c}\text { Relative } \\
\text { Activity (b) } \\
\text { (cpm) } \\
\text { Ta-182 } \\
(1189 \mathrm{keV})\end{array}$} & \multirow{2}{*}{$\begin{array}{r}\text { Isotop } \\
\text { Ratio } \\
\text { Cs-137 } \\
\text { Cs-134 }\end{array}$} \\
\hline & $\begin{array}{l}\mathrm{Cs}-134 \\
(605 \mathrm{keV})\end{array}$ & $\begin{array}{c}\mathrm{Cs}-137 \\
(661 \mathrm{keV})\end{array}$ & & \\
\hline 1 & 6.50 & 5.29 & 150 & 0.81 \\
\hline 2 & 9.96 & 6.17 & 146 & 0.62 \\
\hline 3 & 7.93 & 5.84 & 152 & 0.74 \\
\hline 4 & 5.59 & 4.40 & 150 & 0.79 \\
\hline 5 & 6.71 & 5.73 & 163 & 0.85 \\
\hline 6 & 8.44 & 8.37 & 106 & 0.99 \\
\hline 7 & 7.83 & 6.61 & 104 & 0.88 \\
\hline 8 & 13.92 & 7.27 & 123 & 0.52 \\
\hline 9 & 30.29 & 15.31 & 103 & 0.51 \\
\hline 10 & 14.53 & 9.14 & 98 & 0.63 \\
\hline 11 & 16.26 & 10.79 & 84 & 0.66 \\
\hline 12 & 17.99 & 11.34 & 69 & 0.63 \\
\hline 13 & 11.89 & 9.36 & 43 & 0.79 \\
\hline 14 & 12.71 & 9.36 & 34 & 0.74 \\
\hline 15 & 3.35 & 4.73 & 0 & 1.41 \\
\hline 16 & 2.34 & 2.09 & 82 & 0.89 \\
\hline 17 & 1.83 & 2.20 & 62 & 1.20 \\
\hline 18 & 0 & 1.54 & 60 & -- \\
\hline 19 & 0 & 1.98 & 56 & -- \\
\hline 20 & 0 & 0 & 50 & -- \\
\hline 21 & 0 & 1.65 & 37 & -- \\
\hline 22 & 0 & 1.54 & 81 & -- \\
\hline 23 & 0 & 0 & 106 & - \\
\hline 24 & 0 & 1.54 & 104 & -- \\
\hline 25 & 0 & 2.20 & 74 & -- \\
\hline 26 & 0 & 1.32 & 85 & -- \\
\hline 27 & 0 & 1.98 & 110 & -- \\
\hline 28 & 0 & 2.09 & 90 & -- \\
\hline
\end{tabular}

(a) Location of spectrum number is given in Fig. 4-47. Activity was decayed back to EOL.

(b) Relative activity includes background counts. 
TABLE $4-29$

GAMMA SCAN RESULTS OF CAPSULE P13S FUEL ROD CRUCIBLES

\begin{tabular}{|c|c|c|c|c|}
\hline \multirow[b]{2}{*}{$\begin{array}{l}\text { Spectrum } \\
\text { No. (a) }\end{array}$} & \multicolumn{2}{|c|}{$\begin{array}{l}\text { Isotope Concentration } \\
\left(\mu \mathrm{C} i \times 10^{2}\right) \\
\end{array}$} & \multirow{2}{*}{$\begin{array}{c}\text { Relative } \\
\text { Activity (b) } \\
\text { (cpm) } \\
\text { Ta-182 } \\
(1189 \mathrm{keV})\end{array}$} & \multirow{2}{*}{$\begin{array}{l}\text { Isotope } \\
\text { Ratio } \\
\text { Cs-137 } \\
\text { Cs-134 }\end{array}$} \\
\hline & $\begin{array}{c}\mathrm{Cs}-134 \\
(605 \mathrm{keV})\end{array}$ & $\begin{array}{c}\mathrm{Cs}-137 \\
(661 \mathrm{keV})\end{array}$ & & \\
\hline 1 & 85.16 & 44.04 & 0 & 0.52 \\
\hline 2 & 193.28 & 96.46 & 115 & 0.50 \\
\hline 3 & 107.92 & 51.64 & 114 & 0.48 \\
\hline 4 & 81.91 & 38.10 & 302 & 0.47 \\
\hline 5 & 119.10 & 53.07 & 214 & 0.45 \\
\hline 6 & 212.28 & 90.84 & 166 & 0.43 \\
\hline 7 & 125.81 & 56.93 & 156 & 0.45 \\
\hline 8 & 19.00 & 8.81 & 55 & 0.46 \\
\hline 9 & 14.13 & 8.15 & 72 & 0.58 \\
\hline 10 & 14.33 & 9.14 & 113 & 0.64 \\
\hline 11 & 12.30 & 7.82 & 71 & 0.64 \\
\hline 12 & 14.43 & 9.58 & 54 & 0.66 \\
\hline 13 & 11.58 & 7.27 & 73 & 0.63 \\
\hline 14 & 29.67 & 13.65 & 0 & 0.46 \\
\hline 15 & 27.13 & 13.32 & 0 & 0.49 \\
\hline 16 & 22.25 & 11.34 & 0 & 0.51 \\
\hline 17 & 14.73 & 8.04 & 0 & 0.57 \\
\hline 18 & 7.93 & 4.95 & 0 & 0.62 \\
\hline 19 & 2.95 & 2.42 & 0 & 0.82 \\
\hline 20 & 2.03 & 3.41 & 0 & 1.68 \\
\hline 21 & 0 & 0 & 0 & -- \\
\hline 22 & 0 & 0 & 94 & -- \\
\hline 23 & 0 & 0 & 0 & -- \\
\hline 24 & 0 & 1.21 & 37 & - \\
\hline 25 & 0 & 1.43 & 74 & -- \\
\hline 26 & 0 & 5.84 & 79 & -- \\
\hline 27 & 0 & 0 & 153 & -- \\
\hline
\end{tabular}

(a) Location of spectrum number is given in Fig. 4-48. Activity was decayed back to EOL.

(b) Relative activity includes background counts. 
TABLE $4-30$

FISSION PRODUCT ISOTOPE RATIOS OF CAPSULE P13R UNBONDED FISSILE PARTICLE SAMPLES

\begin{tabular}{|c|c|c|c|c|c|c|c|}
\hline \multirow[b]{2}{*}{ Batch No. } & \multirow{2}{*}{\multicolumn{2}{|c|}{$\begin{array}{l}\text { Capsule } \\
\text { Position } \\
\end{array}$}} & \multirow[b]{2}{*}{$\begin{array}{l}\text { Kerne1 } \\
\text { Type (a) }\end{array}$} & \multirow{2}{*}{$\begin{array}{l}\text { Number } \\
\quad \text { of } \\
\text { Parti- } \\
\text { cles }\end{array}$} & \multicolumn{3}{|c|}{$\begin{array}{c}\text { Fission Product Isotope Ratio (b) } \\
(\mathrm{Cs}-137 / \mathrm{Zr}-95)\end{array}$} \\
\hline & & & & & Meas. (c) & Calc. (d) & $\underset{(\%)}{\operatorname{Difference}(e)}$ \\
\hline $6151-00-010-10$ & CelI & $3-1$ & $\mathrm{UC}_{2}$ & 225 & 0.0231 & 0.0253 & -8.7 \\
\hline $6151-00-010-11$ & & $3-2$ & $\mathrm{UC}_{2}$ & 233 & 0.0228 & 0.0253 & -10.0 \\
\hline $6151-00-010-12$ & & $3-3$ & $\mathrm{UC}_{2}$ & 224 & 0.0236 & 0.0253 & -6.7 \\
\hline $6151-00-035-1$ & & $3-4$ & $\mathrm{UC}_{2}$ & 231 & 0.0267 & 0.0249 & +7.2 \\
\hline $6151-00-035-2$ & & $3-5$ & $\mathrm{UC}_{2}$ & 228 & 0.0235 & 0.0249 & -5.6 \\
\hline $6151-00-045-1$ & & $3-7$ & $\mathrm{UC}_{2}$ & 251 & 0.0208 & 0.0250 & -16.8 \\
\hline $6151-00-045-2$ & & $3-8$ & $\mathrm{UC}_{2}$ & 246 & 0.0228 & 0.0250 & -8.8 \\
\hline $6151-00-045-3$ & & $3-9$ & $\mathrm{UC}_{2}$ & 252 & 0.0212 & 0.0250 & -15.2 \\
\hline $6151-00-046-1$ & & $3-10$ & $\mathrm{UC}_{2}$ & 254 & 0.0223 & 0.0246 & -9.3 \\
\hline $6151-04-015-1$ & & $3-11$ & $\mathrm{UC}_{2}$ & 225 & 0.0226 & 0.0244 & -7.4 \\
\hline $6151-04-015-2$ & & $3-12$ & $\mathrm{UC}_{2}$ & 267 & 0.0240 & 0.0244 & -1.6 \\
\hline $6151-01-015-2$ & Cel1 & $4-2$ & $\mathrm{UC}_{2}$ & 232 & 0.0209 & 0.0238 & -12.2 \\
\hline $6151-01-015-3$ & & $4-3$ & $\mathrm{UC}_{2}$ & 232 & 0.0217 & 0.0238 & -8.8 \\
\hline $6151-00-025-1$ & & $4-4$ & $\mathrm{UC}_{2}$ & 260 & 0.0215 & 0.0234 & -8.1 \\
\hline $6151-00-025-2$ & & $4-5$ & $\mathrm{UC}_{2}$ & 284 & 0.0212 & 0.0234 & -9.4 \\
\hline $6151-00-025-3$ & & $4-6$ & $\mathrm{UC}_{2}$ & 275 & 0.0240 & 0.0234 & -2.6 \\
\hline $6151-09-015-1$ & & $4-7$ & $\mathrm{UC}_{2}$ & 261 & 0.0225 & 0.0228 & -1.3 \\
\hline $6151-09-015-2$ & & $4-8$ & $\mathrm{UC}_{2}$ & 251 & 0.0210 & 0.0228 & -7.9 \\
\hline $6151-09-015-3$ & & $4-9$ & $\mathrm{UC}_{2}$ & 273 & 0.0225 & 0.0228 & -1.3 \\
\hline $6151-09-025-1$ & & $4-10$ & $\mathrm{UC}_{2}$ & 246 & 0.0214 & 0.0225 & -4.9 \\
\hline $6151-09-025-2$ & & $4-11$ & $\mathrm{UC}_{2}$ & 245 & 0.0211 & 0.0225 & -6.2 \\
\hline
\end{tabular}

(a) All particle samples were TRISO coated.

(b) Fission product isotope inventory was decayed back to EOL.

(c) The measured isotope ratios were normalized using intrinsic efficiency curve factors and absolute intensities of the isotopes.

(d) Calculated ratio was determined using the FISS-PROD computer code and attenuation calculations.

(e) Difference between the measured and theoretical isotope ratio. 
TABLE $\quad 4-31$

FISSION PRODUCT ISOTOPE RATIOS OF CAPSULE P13S UNBONDED FISSILE PARTICLE SAMPLES

\begin{tabular}{|c|c|c|c|c|c|c|c|}
\hline \multirow[b]{2}{*}{ Batch No. } & \multirow{2}{*}{\multicolumn{2}{|c|}{$\begin{array}{l}\text { Capsule } \\
\text { Position }\end{array}$}} & \multirow[b]{2}{*}{$\begin{array}{l}\text { Kerne1 } \\
\text { Type (a) }\end{array}$} & \multirow{2}{*}{$\begin{array}{l}\text { Number } \\
\quad \text { of } \\
\text { Parti- } \\
\text { cles }\end{array}$} & \multicolumn{3}{|c|}{$\begin{array}{c}\text { Fission Product Isotope Ratio (b) } \\
(\mathrm{Cs}-137 / \mathrm{Zr}-95)\end{array}$} \\
\hline & & & & & Meas. (c) & Calc. (d) & $\begin{array}{c}\text { Difference }(e) \\
(\%)\end{array}$ \\
\hline $6151-03-015-1$ & $\mathrm{Cel1}$ & $3-1$ & $\mathrm{UC}_{2}$ & 245 & 0.0221 & 0.0229 & -3.5 \\
\hline $6151-03-015-2$ & & $3-2$ & $\mathrm{UC}_{2}$ & 239 & 0.0226 & 0.0229 & -1.3 \\
\hline $6151-03-015-3$ & & $3-3$ & $\mathrm{UC}_{2}$ & 245 & 0.0236 & 0.0229 & -3.1 \\
\hline $4161-01-021-1$ & & $3-4$ & $\mathrm{UC}_{2}$ & 238 & 0.0190 & 0.0229 & -17.0 \\
\hline $4161-01-021-2$ & & $3-5$ & $\mathrm{UC}_{2}$ & 239 & 0.0188 & 0.0229 & -17.9 \\
\hline $4161-01-021-3$ & & $3-6$ & $\mathrm{UC}_{2}$ & 241 & 0.0181 & 0.0229 & -21.0 \\
\hline $6151-08-015-1$ & & $3-7$ & $\mathrm{UC}_{2}$ & 229 & 0.0226 & 0.0225 & +0.4 \\
\hline $6151-08-015-2$ & & $3-8$ & $\mathrm{UC}_{2}$ & 234 & 0.0238 & 0.0225 & +5.8 \\
\hline $6151-08-015-3$ & & $3-9$ & $\mathrm{UC}_{2}$ & 225 & 0.0238 & 0.0225 & +5.8 \\
\hline $6151-02-025-1$ & & $3-10$ & $\mathrm{UC}_{2}$ & 236 & 0.0230 & 0.0222 & +3.6 \\
\hline $6151-02-025-3$ & & $3-12$ & $\mathrm{UC}_{2}$ & 265 & 0.0235 & 0.0222 & +5.6 \\
\hline $4163-00-011-3$ & $\mathrm{Cell}$ & $4-1$ & $(\mathrm{Th}, \mathrm{U}) \mathrm{O}_{2}$ & 255 & 0.0212 & 0.0186 & +14.0 \\
\hline $4163-00-011-4$ & & $4-2$ & $(\mathrm{Th}, \mathrm{U}) \mathrm{O}_{2}$ & 254 & 0.0201 & 0.0186 & +8.0 \\
\hline $4163-00-011-5$ & & $4-3$ & $(\mathrm{Th}, \mathrm{U}) \mathrm{O}_{2}$ & 258 & 0.0217 & 0.0186 & +16.7 \\
\hline $6155-01-020-10$ & & $4-4$ & $(\mathrm{Th}, \mathrm{U}) \mathrm{O}_{2}$ & 163 & 0.0152 & 0.0141 & +7.8 \\
\hline $5466-37-9$ & & $4-7$ & $(\mathrm{Th}, \mathrm{U}) \mathrm{O}_{2}$ & 269 & 0.0206 & 0.0186 & +10.8 \\
\hline $5466-37-10$ & & $4-8$ & $(\mathrm{Th}, \mathrm{U}) \mathrm{O}_{2}$ & 269 & 0.0204 & 0.0186 & +9.7 \\
\hline $5466-37-11$ & & $4-9$ & $(\mathrm{Th}, \mathrm{U}) \mathrm{O}_{2}$ & 263 & 0.0198 & 0.0186 & +6.5 \\
\hline $6151-00-035-4$ & & $4-10$ & $\mathrm{UC}_{2}$ & 247 & 0.0233 & 0.0210 & +11.0 \\
\hline $6151-00-035-5$ & & $4-11$ & $\mathrm{UC}_{2}$ & 246 & 0.0214 & 0.0210 & +1.9 \\
\hline $6151-00-035-6$ & & $4-12$ & $\mathrm{UC}_{2}$ & 316 & 0.0206 & 0.0210 & -1.9 \\
\hline
\end{tabular}

(a) All particle samples were TRISO coated.

(b) Fission product inventory was decayed back to EOL.

(c) The measured isotope ratios were normalized using intrinsic efficiency curve factors and absolute intensities of the isotopes.

${ }^{(d)}$ Calculated ratio was determined using the FISS-PROD computer code and attenuation calculations.

(e) Difference between the measured and theoretical isotope ratio. 
TABLE 4-32

FISSION PRODUCT ISOTOPE RATIOS OF CAPSULE P13R UNBONDED

FERTILE PARTICLE SAMPLES

\begin{tabular}{c|c|c|c|c|c}
\hline & Capsule & Kerne1 & $\begin{array}{c}\text { Number } \\
\text { of } \\
\text { Parti- } \\
\text { c1es }\end{array}$ & $\begin{array}{c}\text { Measured Fission } \\
\text { Product Isotope } \\
\text { Ratio(b) } \\
\text { (Cs-137/Zr-95) }\end{array}$ & $\begin{array}{c}\text { Difference } \\
(\mathrm{c})\end{array}$ \\
\hline Position
\end{tabular}

(a) All particle samples are BISO coated.

(b) Fission product isotope inventory was decayed back to EOL. The isotope ratios were normalized using intrinsic efficiency curve factors and absolute intensities of the isotopes.

(c) Difference between the measured and calculated isotope ratio. The calculated isotope ratio for all particle samples was 0.0086 . This ratio was determined using the FISS-PROD computer code and attenuation calculations. 
TABLE $4-33$

FISSION PRODUCT ISOTOPE RATIOS OF CAPSULE P13S UNBONDED FERTILE PARTICLE SAMPLES

\begin{tabular}{|c|c|c|c|c|c|c|}
\hline Batch No. & $\begin{array}{l}\text { Capsu } \\
\text { Posit }\end{array}$ & $\begin{array}{l}\text { le } \\
\text { ion }\end{array}$ & $\begin{array}{l}\text { Kernel } \\
\text { Type (a) }\end{array}$ & $\begin{array}{l}\text { Number } \\
\quad \text { of } \\
\text { Parti- } \\
\text { cles }\end{array}$ & $\begin{array}{c}\text { Measured Fission } \\
\text { Product Isotope } \\
\text { Ratio (b) } \\
(\mathrm{Cs}-137 / 2 r-95) \\
\end{array}$ & $\begin{array}{c}\text { Difference } \\
(\%)\end{array}$ \\
\hline $4252-06-010-1$ & \multirow[t]{11}{*}{ Cell } & $3-1$ & $\mathrm{ThO}_{2}$ & 436 & 0.0088 & +2.3 \\
\hline $4252-06-010-2$ & & $3-2$ & $\mathrm{ThO}_{2}$ & 436 & 0.0094 & +9.3 \\
\hline $6542-22-015-1$ & & $3-3$ & $\mathrm{ThO}_{2}$ & 491 & 0.0086 & 0 \\
\hline $6542-22-015-2$ & & $3-4$ & $\mathrm{ThO}_{2}$ & 488 & 0.0090 & +4.7 \\
\hline $6542-22-025-1$ & & $3-5$ & $\mathrm{ThO}_{2}$ & 483 & 0.0086 & 0 \\
\hline $6542-22-025-2$ & & $3-6$ & $\mathrm{ThO}_{2}$ & 481 & 0.0087 & +1.2 \\
\hline $6542-23-025-1$ & & $3-7$ & $\mathrm{ThO}_{2}$ & 496 & 0.0094 & +9.3 \\
\hline $6542-23-025-2$ & & $3-8$ & $\mathrm{ThO}_{2}$ & 496 & 0.0090 & +4.7 \\
\hline $6542-24-015-1$ & & $3-9$ & $\mathrm{ThO}_{2}$ & 475 & 0.0091 & +5.8 \\
\hline $6542-24-015-2$ & & $3-10$ & $\mathrm{ThO}_{2}$ & 467 & 0.0093 & +8.1 \\
\hline $6252-00-025-2^{(d)}$ & & $3-12$ & $\mathrm{ThO}_{2}$ & 528 & 0.0087 & +1.2 \\
\hline $6542-17-010-1$ & \multirow[t]{10}{*}{ Cell } & $4-1$ & $\mathrm{ThO}_{2}$ & 458 & 0.0095 & +10.5 \\
\hline $4252-02-010-1$ & & $4-2$ & $\mathrm{ThO}_{2}$ & 462 & 0.0093 & +8.1 \\
\hline $4252-02-010-2$ & & $4-3$ & $\mathrm{ThO}_{2}$ & 463 & 0.0089 & +3.5 \\
\hline $6542-16-010-1$ & & $4-4$ & $\mathrm{ThO}_{2}$ & 349 & 0.0094 & +9.3 \\
\hline $6542-19-015-1$ & & $4-7$ & $\mathrm{ThO}_{2}$ & 495 & 0.0090 & +4.7 \\
\hline $6542-19-016-1$ & & $4-8$ & $\mathrm{ThO}_{2}$ & 502 & 0.0091 & +5.8 \\
\hline $6542-21-016-1$ & & $4-9$ & $\mathrm{ThO}_{2}$ & 508 & 0.0091 & +5.8 \\
\hline $6542-21-015-1$ & & $4-10$ & $\mathrm{ThO}_{2}$ & 513 & 0.0094 & +9.3 \\
\hline $6542-12-025-1$ & & $4-11$ & $\mathrm{ThO}_{2}$ & 538 & 0.0089 & +3.5 \\
\hline $6542-12-025-2$ & & $4-12$ & $\mathrm{ThO}_{2}$ & 676 & 0.0088 & +2.3 \\
\hline
\end{tabular}

(a) All particle samples were BISO coated except where noted.

(b) Fission product isotope inventory was decayed back to EOL. The isotope ratios were normalized using intrinsic efficiency curve factors and absolute intensities of the isotopes.

(c) Difference between the measured and calculated isotope ratio. The calculated isotope ratio for all particle samples was 0.0086 . This ratio was determined using the FISS-PROD computer code and attenuation calculations.

(d) TRISO coated particle batch. 
GAMMA SCAN RESULTS OF SELECTED LOOSE PARTICLE BATChes IN CAPSULES P13R AND P135

\begin{tabular}{|c|c|c|c|c|c|c|c|c|c|c|c|c|c|c|c|c|c|}
\hline \multicolumn{3}{|c|}{$\begin{array}{l}\text { 6151-09-025-1 } \\
\text { P13R Cel1 4-10 } \\
\text { UC TRISO }\end{array}$} & \multicolumn{3}{|c|}{$\begin{array}{l}6151-00-035-4 \\
\text { P13S Cell1 4-10 } \\
\text { UC } 2 \text { TRISO }\end{array}$} & \multicolumn{3}{|c|}{$\begin{array}{c}6151-09-015-2 \\
\text { P13R Cel1 } 4-8 \\
\mathrm{UC}_{2} \text { TRISO }\end{array}$} & \multicolumn{3}{|c|}{$\begin{array}{l}\text { 6151-00-046-1 } \\
\text { P13R Cel1 3-10 } \\
\text { UC } \mathrm{C}_{2} \text { TRISO }\end{array}$} & \multicolumn{3}{|c|}{ 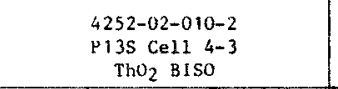 } & \multicolumn{3}{|c|}{$\begin{array}{c}6252-00-025-2 \\
\mathrm{P}^{2} 3 \mathrm{~S} \text { Cell } 3-12 \\
\text { Tho }\end{array}$} \\
\hline $\begin{array}{c}\text { Scan } \\
\text { Number }\end{array}$ & $\begin{array}{c}\text { No. of } \\
\text { Particles } \\
\text { Scanned }\end{array}$ & ${\frac{\mathrm{Cs}-137^{(a)}}{\mathrm{Zr}-95}}^{(\mathrm{s})}$ & $\begin{array}{c}\text { Scan } \\
\text { Number }\end{array}$ & $\begin{array}{c}\text { No. of } \\
\text { Particles } \\
\text { Scanned }\end{array}$ & $\frac{C s-137}{2 r-95}^{(a)}$ & $\begin{array}{c}\text { Scan } \\
\text { Number }\end{array}$ & \begin{tabular}{|c|} 
No. of \\
Particles \\
Scanned \\
\end{tabular} & $\frac{\mathrm{Cs}-137}{2 r-95}^{(a)}$ & $\begin{array}{c}\text { Scan } \\
\text { Number }\end{array}$ & \begin{tabular}{|c|} 
No. of \\
Particles \\
Scanned
\end{tabular} & $\frac{\mathrm{Cs}-137}{2 \mathrm{r}-95}^{(\mathrm{a})}$ & $\begin{array}{c}\text { Scan } \\
\text { Number }\end{array}$ & $\begin{array}{c}\text { :o. of } \\
\text { Particles } \\
\text { Scanned }\end{array}$ & $\frac{\mathrm{Cs}^{-137}}{\mathrm{Zr}_{\mathrm{r}-95}^{(a)}}$ & $\begin{array}{c}\text { Scan } \\
\text { Number }\end{array}$ & \begin{tabular}{|c|} 
No. of \\
Particles \\
Scanned
\end{tabular} & $\frac{C_{s-137}}{2 r-95}$ \\
\hline 2043 & 3 & 0.037 & 1184 & 3 & 0.038 & 2070 & 3 & 0.037 & 1157 & 1 & 0.042 & 1104 & 9 & 0.016 & 1130 & 3 & 0.016 \\
\hline 2045 & 3 & 0.038 & 1185 & 3 & 0.038 & 2071 & 3 & 0.038 & 1158 & 3 & 0.039 & 1105 & 3 & 0.017 & 1131 & 3 & 0.016 \\
\hline 2046 & 3 & 0.038 & 1186 & 3 & 0.037 & 2072 & 3 & 0.039 & 1159 & 2 & 0.040 & 1106 & 1 & 0.016 & 1132 & 3 & 0.016 \\
\hline 2047 & 3 & 0.035 & 1187 & 3 & 0.039 & 2073 & 3 & 0.038 & 1160 & 3 & 0.038 & 1107 & 1 & 0.017 & 1134 & 3 & 0.010 \\
\hline 2048 & 3 & 0.038 & 1188 & 3 & 0.038 & 2075 & 3 & 0.039 & 1161 & 2 & 0.044 & 1108 & 1 & 0.016 & 1135 & 3 & 0.016 \\
\hline 2049 & 3 & 0.039 & 1189 & 3 & 0.039 & 2076 & 3 & 0.039 & 1162 & 6 & 0.039 & 1109 & 1 & 0.017 & 1136 & 3 & 0.016 \\
\hline 2050 & 3 & 0.038 & 1190 & 3 & 0.036 & 2077 & 3 & 0.037 & 1164 & 3 & 0.040 & 1110 & 1 & 0.015 & 1137 & 3 & 0.036 \\
\hline 2051 & 3 & 0.038 & 1191 & 3 & 0.036 & 2078 & 3 & 0.039 & 1165 & 1 & 0.043 & 1111 & 1 & 0.017 & 1138 & 3 & 0.017 \\
\hline 2052 & 3 & 0.039 & 1192 & 3 & 0.038 & 2079 & 3 & 0.040 & 1166 & 1 & 0.043 & 1112 & 1 & 0.016 & 1139 & 3 & 0.016 \\
\hline 2053 & 3 & 0.038 & 1194 & 3 & 0.039 & 2080 & 3 & 0.040 & 1167 & 3 & 0.042 & 1114 & 3 & 0.016 & 1140 & 3 & 0.016 \\
\hline 2055 & 3 & 0.038 & 3195 & 3 & 0.037 & 2081 & 3 & 0.038 & 1168 & 3 & 0.040 & 1113 & 3 & $0.01 \mathrm{~b}$ & 1141 & 3 & 0.016 \\
\hline 2056 & 3 & 0.038 & 1196 & 3 & 0.038 & 2082 & 3 & 0.037 & 1169 & 3 & 0.041 & 1116 & 3 & 0.016 & 1142 & 3 & 0.017 \\
\hline 2057 & 3 & 0.038 & 1197 & 3 & 0.040 & 2083 & 3 & 0.037 & 1170 & 3 & 0.033 & 1117 & 5 & 0.016 & 1144 & 3 & 0.016 \\
\hline 2058 & 3 & 0.038 & 1198 & 3 & 0.037 & 2085 & 3 & 0.039 & 1171 & 3 & 0.041 & 1118 & 4 & 0.016 & 1145 & 3 & 0.016 \\
\hline 2059 & 3 & 0.036 & 1199 & 3 & 0.037 & 2086 & 3 & 0.040 & 1172 & 3 & 0.040 & 1119 & 4 & 0.015 & 1146 & 3 & 0.016 \\
\hline 2060 & 3 & 0.038 & 1200 & 3 & 0.039 & 2087 & 3 & 0.039 & 1174 & 3 & 0.042 & 1120 & 1 & 0.016 & 1147 & 3 & 0.017 \\
\hline 2061 & 3 & 0.039 & 2035 & 3 & 0.036 & 2088 & 3 & 0.038 & 1175 & 3 & 0.042 & 1121 & 1 & 0.016 & 1168 & 3 & 0.016 \\
\hline 2062 & 3 & 0,036 & 2036 & 3 & 0.037 & 2089 & 3 & 0.039 & 1176 & 3 & 0.040 & 1122 & 3 & 0.016 & 1149 & 3 & 0.016 \\
\hline 2063 & 3 & 0.037 & 2037 & 3 & 0.038 & 2090 & 3 & 0.038 & 1177 & 3 & 0.042 & 1124 & 1 & 0.016 & 1150 & 3 & 0.016 \\
\hline 2065 & 3 & 0.038 & 2038 & 3 & 0.040 & 2091 & 3 & 0.037 & 1178 & 3 & 0.043 & 1125 & 3 & 0.016 & 1151 & 3 & 0.016 \\
\hline 2066 & 3 & 0.037 & 2039 & 3 & 0.039 & 2092 & 3 & 0.038 & 1179 & 3 & 0.040 & 1126 & 4 & 0.016 & 1152 & 3 & 0.016 \\
\hline 2067 & 3 & 0.039 & 2040 & 3 & 0.037 & 2093 & 3 & 0.039 & $1+80$ & 3 & 0.040 & 1127 & 1 & 0.017 & 1154 & 3 & 0.016 \\
\hline 2068 & 3 & 0.038 & 2041 & 3 & 0.039 & 2095 & 3 & 0.039 & 1181 & 3 & 0,040 & 1128 & 3 & 0.016 & 1155 & 3 & 0.016 \\
\hline 2069 & 3 & 0.038 & 2042 & 3 & 0.038 & 2096 & 3. & 0.038 & 1182 & 3 & 0.041 & 1129 & 4 & 0.016 & 1156 & 3 & 0.016 \\
\hline Mean & & 0.038 & & & 0.037 & & & 0.038 & & & 0.041 & & & 0.016 & & & 0.016 \\
\hline std. dev. & & 0.0009 & & & 0.001 & & & 0.001 & & & 0.002 & & & 0.0003 & & & 0.0004 \\
\hline $\begin{array}{l}\text { Calculated } \\
\text { ratio }(b)\end{array}$ & & $\begin{array}{l}0.042 \\
\pm 0 \%\end{array}$ & & & $\begin{array}{l}0.038 \\
\pm 10 \%\end{array}$ & & & $\begin{array}{l}0.039 \\
\pm 10 \%\end{array}$ & & & $\begin{array}{l}0.045 \\
\pm 10 \%\end{array}$ & & & $\begin{array}{l}0.016 \\
\pm 10\end{array}$ & & & $\begin{array}{l}0.016 \\
\pm 10 .\end{array}$ \\
\hline
\end{tabular}

(a) Fission product inventory was decayed back to EOL. The measured isotope ratios were normalized using intrinsic efficiency curve factors and absolute intensities of the isotopes.

(b) Theoretical isotope ratio calculated using the FISS-PROD computer code and attenuation calculations. 
RESULTS OF METALLOGRAPHIC EXAMiNaTion OF FABLLE 4-35 PARTICLES IRRAdIATEd IN CAPSULES P13R AND P13S

\begin{tabular}{|c|c|c|c|c|c|c|c|c|c|c|c|c|c|c|}
\hline \multirow[b]{2}{*}{ Batch No. } & \multirow[b]{2}{*}{$\begin{array}{c}\text { Fuel Rod Data } \\
\text { Retrieval No. } \\
(7161-004-) \\
\end{array}$} & \multirow[b]{2}{*}{ Capsu1e } & \multirow[b]{2}{*}{$\begin{array}{l}\text { Capsule } \\
\text { Position } \\
\end{array}$} & \multirow[b]{2}{*}{$\begin{array}{c}\text { Average } \\
\text { Temp }(a) \\
\left({ }^{\circ} \mathrm{C}\right)\end{array}$} & \multirow[b]{2}{*}{$\begin{array}{c}\text { Fast Fluence } \\
\left(10^{21} \mathrm{n} / \mathrm{cm}^{2}\right) \\
(\mathrm{E}>0.18 \mathrm{MeV})_{\mathrm{HTGR}}\end{array}$} & \multirow[b]{2}{*}{$\begin{array}{c}\text { Burnup } \\
\text { (\% FIMA) } \\
\end{array}$} & \multirow[b]{2}{*}{$\begin{array}{l}\text { Number of } \\
\text { Particles } \\
\text { Examined }\end{array}$} & \multicolumn{2}{|c|}{ OPyc Coating } & \multirow[b]{2}{*}{$\begin{array}{c}\text { IPyC } \\
\text { Coating } \\
\text { Failure } \\
\text { (\%) } \\
\end{array}$} & \multirow[b]{2}{*}{$\begin{array}{c}\text { IPye } \\
\text { Coat ing } \\
\text { Debonded } \\
\text { (\%) } \\
\end{array}$} & \multicolumn{2}{|c|}{ Total Coating (c) } & \multirow[b]{2}{*}{ Remarks } \\
\hline & & & & & & & & $\begin{array}{c}\text { Failure } \\
(\%)\end{array}$ & $\begin{array}{l}95 \% \text { Confidence } \\
\text { Interval, } \mathrm{P}(\mathrm{b}) \\
(\%)\end{array}$ & & & $\begin{array}{c}\text { Failure } \\
(\%)\end{array}$ & $\begin{array}{c}95 \% \text { Conf idence } \\
\text { Interval, } \mathrm{P}(\mathrm{b}) \\
(\%)\end{array}$ & \\
\hline 6151-00-035 & $26-5$ & pi3s & $6 \mathrm{C}$ & 1050 & 4.4 & 48 & 77 & 0 & $0 \leq P \leq 4.9$ & 0 & 13.0 & 0 & $0 \leq p \leq 4.9$ & \\
\hline $6151-00-035$ & $23-5$ & $\mathrm{Pl} 3 \mathrm{R}$ & $6 \mathrm{C}$ & 1005 & 4.5 & 49 & 33 & 0 & $0 \leq P \leq 10.9$ & 0 & 3.0 & 0 & $0 \leq \mathrm{p} \leq 10.9$ & \\
\hline $6151-00-035$ & $18-5$ & P13s & sc & 1335 & 8.4 & 66 & 69 & 2.9 & $0 \leq \mathrm{P} \leq 10.4$ & 0 & 29.0 & 0 & $0 \leq \mathrm{P} \leq 5.2$ & \\
\hline $6151-00-035$ & $03-5$ & P13R & 1C & 1065 & 8.7 & 73 & 30 & 66.7 & $47 \leq \mathrm{P} \leq 83$ & 66.7 & $N D^{(d)}$ & 66.7 & $47 \leq P \leq 83$ & Heated in TRIGA to $1600^{\circ} \mathrm{C}$ \\
\hline $6151-00-035$ & -- & P13S & $4-12$ & 975 & 10.7 & 71 & 84 & 10.7 & $5.0 \leq \mathrm{P} \leq 18.9$ & ND & 9.5 & 10.7 & $5.0 \leq P \leq 18.9$ & \\
\hline $6151-00-035$ & -- & P13R & $3-5$ & 1075 & 12.0 & 74 & 78 & 0 & $0 \leq \mathrm{P} \leq 4.7$ & 0 & 15.4 & 0 & $0 \leq P \leq 4.7$ & \\
\hline $6151-00-035$ & $13-6$ & P13S & $2 \mathrm{C}$ & 975 & 11.9 & 74 & 57 & 0 & $0 \leq P \leq 6.2$ & 0 & 15.8 & 0 & $0 \leq \mathrm{P} \leq 6.2$ & \\
\hline $6151-00-035$ & $07-5$ & P13R & $2 \mathrm{~B}$ & 1055 & 12.0 & 75 & 54 & 0 & $0 \leq P \leq 6.5$ & 0 & 13.0 & 0 & $0 \leq \mathrm{P} \leq 6.5$ & \\
\hline $6151-00-035$ & $08-13$ & P13R & $2 \mathrm{C}$ & 1045 & 12.2 & 75 & .71 & 0 & $0 \leq \mathrm{P} \leq 5.1$ & 0 & 16.9 & 0 & $0 \leq \mathrm{P} \leq 5.1$ & \\
\hline $6151-00-045$ & $01-7$ & P13S & $1 \mathrm{~A}$ & 1030 & 7.0 & 70 & 72 & 15.3 & $8.0 \leq P \leq 26.5$ & 15.3 & 34.3 & 15.3 & $8.0 \leq P \leq 26.5$ & $\begin{array}{l}\text { Faceted coudings, themal } \\
\text { cycled tn-pile }\end{array}$ \\
\hline $6151-00-045$ & $06-5$ & P13R & $2 \mathrm{~A}$ & 1050 & 11.8 & 75 & 16 & 6.3 & $0 \leq \mathrm{P} \leq 30.2$ & 0 & 43.8 & 0 & $0 \leq \mathrm{P} \leq 20.6$ & Faceted coatinus \\
\hline $6151-00-046$ & -- & P13R & $3-10$ & 1025 & 11.8 & 74 & 77 & 5.2 & $1.5 \leq p \leq 11.5$ & 13.0 & 45.5 & 3.9 & $2.0 \leq P \leq 10.6$ & Faceted unatings \\
\hline $615 i-08-015$ & $19-5$ & p13s & 50 & 1325 & 7.9 & 65 & 53 & 17.0 & $8.3 \leq P \leq 29.3$ & 0 & 54.7 & 3.8 & $0 \leq \mathrm{p} \leq 13.3$ & $\begin{array}{l}\text { Low opyc coating density } \\
\text { and coating rate }\end{array}$ \\
\hline $6151-08-015$ & $19-7$ & P13R & 5D & 1225 & 8.2 & 66 & 78 & 5.1 & $1.5 \leq P \leq 11.3$ & 2.2 & 11.1 & 2.6 & $0 \leq P \leq 9.3$ & $\begin{array}{l}\text { Low OPyC coating density } \\
\text { and coating rate }\end{array}$ \\
\hline $6151-08-015$ & $15-5$ & P13s & $2 E$ & 960 & 12.1 & 74 & 19 & 0 & $0 \leq P \leq 17.8$ & 21.1 & 26.3 & 0 & $0 \leq P \leq 17.8$ & $\begin{array}{l}\text { Low oPyC coating density } \\
\text { and coating rate }\end{array}$ \\
\hline $6151-09-015$ & $20-5$ & p13s & $5 \mathrm{E}$ & 1350 & 7.5 & 63 & 62 & 1.6 & $0 \leq P \leq 9.0$ & 4.8 & 40.3 & 1.6 & $0 \leq P \leq 9.0$ & High opyc coating density \\
\hline $6151-09-015$ & $20-6$ & P13R & $5 \mathrm{E}$ & 1280 & 7.7 & 64 & 55 & 0 & $0 \leq P \leq 6.5$ & 0 & ND & 0 & $0 \leq \mathrm{P} \leq 6.5$ & High OPyC coating density \\
\hline $6151-09-015$ & $12-5$ & p13s & $2 \mathrm{~B}$ & 1010 & 11.7 & 74 & 58 & 0 & $0 \leq P \leq 6.1$ & 0 & 25.9 & 0 & $0 \leq \mathrm{P} \leq 6.1$ & High OPyC coating density \\
\hline $6151-09-025$ & -- & $\mathrm{p} 13 \mathrm{R}$ & $4-11$ & 1005 & 10.9 & 73 & 71 & 0 & $0 \leq \mathrm{P} \leq 5.1$ & 12.7 & 52.1 & 0 & $0 \leq \mathrm{P} \leq 5.1$ & High OPyC coating density \\
\hline $6151-04-015$ & -- & $\mathrm{P} 13 \mathrm{R}$ & $3-12$ & 960 & 11.7 & 74 & 71 & 100 & $44.6 \leq P \leq 70.1$ & 1.4 & 4.2 & $95.0^{(\mathrm{e})}$ & $\mathrm{ND}$ & Low OPyC coating rate \\
\hline $5466-37$ & $16-6$ & P13s & $5 \mathrm{~A}$ & 1375 & 9.2 & 36 & 28 & 7.1 & $0.9 \leq P \leq 23.0$ & 0 & 0 & 0 & $0 \leq P \leq 12.9$ & $250-\mu m(T h, U) O_{2}$ \\
\hline $5466-37$ & $16-5$ & P13R & $5 \mathrm{~A}$ & 1285 & 9.4 & 36 & 35 & 5.7 & $0.5 \leq P \leq 19.6$ & 0 & 2.9 & 2.9 & $0 \leq \mathrm{P} \leq 14.8$ & $\begin{array}{l}\text { Heated in TRICA to } 1600^{\circ} \mathrm{C} \text {, } \\
250-\mu \mathrm{m} \text { (Th, U) } \mathrm{O}_{2}\end{array}$ \\
\hline $5466-37$ & -- & P135 & $4-8$ & 1090 & 10.9 & 38 & 57 & 0 & $0 \leq P \leq 6.2$ & 0 & 0 & & $0 \leq P \leq 6.2$ & $250-\mu \mathrm{m} \quad(\mathrm{Th}, \mathrm{U}) \mathrm{O}_{2}$ \\
\hline $6155-01-020$ & -- & P13s & $4-4$ & 1090 & 11.1 & 11 & 34 & 85.3 & $64.2 \leq P \leq 92.9$ & 44.1 & 0 & $50.0^{(e)}$ & ND & $500-\mu \mathrm{m} \quad(8 \mathrm{Th}, 1 \mathrm{~W}) \mathrm{O}_{2}$ \\
\hline
\end{tabular}

(a) Time-averaged fuel centerline temperature.

(b) The $95 \%$ confidence statement means that an infinite population of particles with attributes of the particle batch tested to these conditions will have a failure 1 evel at the $95 \%$ confidence level bounded by this interval.

(c) Total coating failure is defined as the failure of both the OPyC and SiC structural coatings in a fuel particle that would result in the release of gaseous fission products.

(d) ${ }_{N D}=$ not determined.

(e) A large fraction of the cracked SiC coatings observed in these particle batches was an artifact of sample preparation. R/B values yielded failure leve1s of $2.6 \%$ for batch 6155-01-020 and $0.6 \%$ for batch $6151-04-015$. 
TABLE 4-36

RESULTS OF METALLOGRAPHIC EXAMINATION OF FERTILE PARTICLES IRRADIATED IN CAPSULES P13R AND P13S

\begin{tabular}{|c|c|c|c|c|c|c|c|c|c|c|c|}
\hline \multirow[b]{2}{*}{ Batch No. } & \multirow[b]{2}{*}{$\begin{array}{l}\text { Fuel Rod Data } \\
\text { Retrieval No. } \\
(7161-004-)\end{array}$} & \multirow[b]{2}{*}{ Capsule } & \multirow[b]{2}{*}{$\begin{array}{l}\text { Capsule } \\
\text { Position } \\
\end{array}$} & \multirow[b]{2}{*}{$\begin{array}{c}\text { Average } \\
\text { Temp. }(\mathrm{a}) \\
\left({ }^{\circ} \mathrm{C}\right)\end{array}$} & \multirow[b]{2}{*}{ 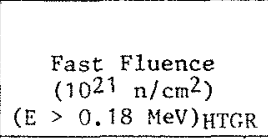 } & \multirow[b]{2}{*}{$\begin{array}{c}\text { Burnup } \\
\text { (\% FIMA) } \\
\end{array}$} & \multirow[b]{2}{*}{$\begin{array}{l}\text { Number of } \\
\text { Particles } \\
\text { Examined }\end{array}$} & \multicolumn{2}{|c|}{ OPyC Coating } & \multirow[b]{2}{*}{$\begin{array}{c}\text { Buffer } \\
\text { Coating } \\
\text { Cracked } \\
(\%)\end{array}$} & \multirow[b]{2}{*}{ Remarks } \\
\hline & & & & & & & & $\begin{array}{c}\text { Failure } \\
(\%)\end{array}$ & $\begin{array}{c}95 \% \text { Confidence } \\
\text { Interval, } \mathrm{P}(\mathrm{b}) \\
(\%)\end{array}$ & & \\
\hline $6542-20-035$ & $01-7$ & 1138 & $1 \mathrm{~A}$ & 1030 & 7.0 & 3.2 & 135 & 51.9 & $43.5 \leq P \leq 60.1$ & 60.0 & $\begin{array}{l}\text { Faceted coatings, thermal } \\
\text { cycled in-pile }\end{array}$ \\
\hline $6542-20-035-2$ & -- & $p 13 k$ & $4-8$ & 1000 & 11.1 & 4.3 & 174 & 2.9 & $1.2 \leq P \leq 6.5$ & 1.7 & Faceted coatings \\
\hline $6542-20-035$ & $06-5$ & $P 13 R$ & $2 \mathrm{~A}$ & 1050 & 11.8 & 5.5 & 57 & 1.8 & $0.2 \leq \mathrm{P} \leq 9.8$ & 1.8 & Faceted coatings \\
\hline $6542-02-020$ & $07-5$ & P13R & $2 \mathrm{~B}$ & 1055 & 12.0 & 5.6 & 161 & 0 & $0 \leq p \leq 2.3$ & 1.9 & \\
\hline 6542-01 010 & $28-13$ & p13S & $6 \mathrm{C}$ & 1050 & 4.4 & 0.8 & 112 & 0 & $0 \leq P \leq 3.3$ & 0 & High OPyC coating rate \\
\hline $6542-01-010$ & $23-5$ & P13R & $6 \mathrm{C}$ & 1005 & 4.5 & 0.8 & 76 & 0 & $0 \leq p \leq 4.8$ & 0 & High OPyC coating rate \\
\hline $6542-01-010$ & $18-5$ & p13s & $5 \mathrm{C}$ & 1335 & 8.4 & 2.4 & 165 & 0 & $0 \leq P \leq 2.3$ & 7.9 & High OPyC coating rate \\
\hline $6542-01-010$ & $03-5$ & P13R & $1 \mathrm{C}$ & 1065 & 8.7 & 4.2 & 69 & 0 & $0 \leq \mathrm{P} \leq 5.2$ & 15.9 & $\begin{array}{l}\text { Heated in TRIGA to } 1600^{\circ} \mathrm{C} \text {, } \\
\text { high OPyC coating rate }\end{array}$ \\
\hline 6542-01-010 & $13-6$ & P13S & $2 \mathrm{C}$ & 975 & 11.9 & 4.7 & 175 & 0 & $0 \leq P \leq 2.1$ & 4.2 & High oPyC coating rate \\
\hline $6542-01-010$ & $08-13$ & P13R & $2 \mathrm{C}$ & 1045 & 12.2 & 5.6 & 144 & 0 & $0 \leq P \leq 2.6$ & 4.2 & High OPyC coating rate \\
\hline $6542-19-015$ & $19-5$ & P13S & $5 \mathrm{D}$ & 1325 & 7.9 & 2.2 & 194 & 5.7 & $3.2 \leq p \leq 9.9$ & 14.9 & Low OPyC coating density \\
\hline $6542-19-015$ & $19-7$ & $P 13 R$ & 5D & 1225 & 8.2 & 2.4 & 206 & 0.5 & $0.1 \leq \mathrm{p} \leq 2.7$ & 0.5 & Low OPyC coating density \\
\hline $6542-19-015$ & $15-5$ & p13s & $2 E$ & 960 & 12.1 & 4.6 & 36 & 0 & $0 \leq P \leq 9.9$ & 8.3 & Low OPyC coating density \\
\hline $6542-09-010$ & $20-5$ & P13S & $5 E$ & 1350 & 7.5 & 2.0 & 247 & 0 & $0 \leq P \leq 1.5$ & 0 & High OPyC coating density \\
\hline $6542-09-010$ & $20-6$ & P13R & $5 E$ & 1280 & 7.7 & 2.1 & 144 & 0.7 & $0.1 \leq \mathrm{P} \leq 3.8$ & 0.7 & High OPyC coating density \\
\hline $6542-09-010$ & $12-5$ & P13S & $2 B$ & 1010 & 11.7 & 4.6 & 129 & 0.8 & $0.1 \leq \mathrm{P} \leq 4.3$ & 0.8 & High OPyC coating density \\
\hline $6542-01-020$ & $16-6$ & P13S & $5 A$ & 1375 & 9.2 & 2.8 & 89 & 5.6 & $2.1 \leq P \leq 12.6$ & $\mathrm{ND}^{(\mathrm{c})}$ & \\
\hline $6542-01-020$ & $16-5$ & $\mathrm{p} 13 \mathrm{R}$ & $5 \mathrm{~A}$ & 1285 & 9.4 & 3.1 & 62 & 16.1 & $7.8 \leq P \leq 27.5$ & ND & Heated in TRIGA to $1600^{\circ} \mathrm{C}$ \\
\hline $6542-18 \sim 015-1$ & -- & P13R & $4-3$ & 1000 & 11.4 & 4.4 & 165 & 0 & $0 \leq \mathrm{P} \leq 2.3$ & 1.2 & No seal coat \\
\hline $6542-25-015-1$ & -- & $P 13 R$ & $4-11$ & 940 & 10.9 & 4.1 & 209 & 1.4 & $0.5 \leq P \leq 4.1$ & 6.2 & Nonround kernels \\
\hline $4252-06-010-1$ & -- & p13s & $3-1$ & 925 & 11.8 & 4.3 & 158 & 2.5 & $1.0 \leq P \leq 6.3$ & 0 & \\
\hline $6542-23-025-1$ & -- & P13S & $3-7$ & 955 & 11.7 & 4.2 & 181 & 0 & $0 \leq \mathrm{R} \leq 2.1$ & 14.4 & Mixed-gas opyc coating \\
\hline $6252-00-025-2$ & -- & $\mathrm{p} 13 \mathrm{~s}$ & $3-12$ & 860 & 11.5 & 4.1 & 37 & 2.7 & $0 \leq p \leq 14.1$ & 10.8 & TRISO coated \\
\hline $6542-12-025-2$ & -- & p13s & $4-12$ & 830 & 10.7 & 3.6 & 177 & 1.1 & $0.3 \leq P \leq 4.0$ & 18.6 & $\mathrm{~N}_{2}$ carrier gas buffer \\
\hline
\end{tabular}

(a) Time-averaged fuel centerline temperature.

(b) The $95 \%$ confidence statement means that an infinite population of particles with attributes of the particle batch tested to these conditions will have a failure level at the $95 \%$ confidence level bounded by this interval.

(c) $\mathrm{ND}=$ not determined. 
TABLE $4-37$

RESULTS OF METALLOGRAPHIC EXAMINATION OF INERT PARTICLES IRRADIATED IN P13R AND P13S FUEL RODS

\begin{tabular}{|c|c|c|c|c|c|c|c|}
\hline $\begin{array}{c}\text { Fuel Rod Data } \\
\text { Retrieval No. } \\
\text { (7161-004-) }\end{array}$ & Capsule & $\begin{array}{l}\text { Capsule } \\
\text { Position } \\
\end{array}$ & $\begin{array}{c}\text { Average } \\
\text { Centerline } \\
\text { Temperature } \\
\left({ }^{\circ} \mathrm{C}\right) \\
\end{array}$ & $\begin{array}{c}\text { Fast Fluence } \\
\left(10^{21} \mathrm{n} / \mathrm{cm}^{2}\right) \\
(\mathrm{E}>0.18 \mathrm{MeV}) \text { HTGR }\end{array}$ & $\begin{array}{l}\text { Number of } \\
\text { Particles } \\
\text { Examined } \\
\end{array}$ & $\begin{array}{c}\text { OPyC } \\
\text { Coating } \\
\text { Failure } \\
(\%) \\
\end{array}$ & $\begin{array}{c}\text { Sic } \\
\text { Coating } \\
\text { Cracked } \\
(\%) \\
\end{array}$ \\
\hline $28-13$ & P13S & $6 \mathrm{C}$ & 1050 & 4.4 & 101 & 1.0 & 3.0 \\
\hline $23-5$ & $\mathrm{P} 13 \mathrm{R}$ & $6 C$ & 1005 & 4.5 & 90 & 0 & 1.1 \\
\hline $20-5$ & P13S & $5 \mathrm{E}$ & 1350 & 7.5 & 260 & 36.9 & 3.1 \\
\hline $20-6$ & P13R & $5 E$ & 1280 & 7.7 & 193 & 14.0 & $\mathrm{ND}^{(\mathrm{a})}$ \\
\hline $19-5$ & P13S & 5D & 1325 & 7.9 & 237 & 22.8 & 0.8 \\
\hline $19-7$ & P13R & 5D & 1225 & 8.2 & 277 & 11.6 & 2.9 \\
\hline $18-5$ & P13S & $5 \mathrm{C}$ & 1335 & 8.4 & 211 & 28.9 & 2.8 \\
\hline $12-5$ & P13S & $2 B$ & 1010 & 11.7 & 259 & 0.7 & 2.4 \\
\hline $06-5$ & P13R & $2 \mathrm{~A}$ & 1050 & 11.8 & 149 & 3.3 & 12.1 \\
\hline $13-6$ & P13S & $2 \mathrm{C}$ & 975 & 11.9 & 123 & 0.8 & 3.3 \\
\hline $15-5$ & P13S & $2 \mathrm{E}$ & 960 & 12.1 & 44 & 2.3 & $\mathrm{ND}$ \\
\hline $07-5$ & P13R & $2 \mathrm{~B}$ & 1055 & 12.0 & 251 & 1.6 & 0.4 \\
\hline $08-13$ & P13R & $2 \mathrm{C}$ & 1045 & 12.2 & 224 & 0 & 0.4 \\
\hline
\end{tabular}

(a) $\mathrm{ND}=$ not determined. 
TABLE $4-38$

IRRADIATION-INDUCED CHANGE IN MACROPOROSTTY OF P13R AND P13S FUEL RODS

\begin{tabular}{|c|c|c|c|c|c|c|c|c|c|c|}
\hline \multirow{2}{*}{$\begin{array}{c}\text { Data } \\
\text { Retrieval } \\
\text { Number } \\
(7161-004-)\end{array}$} & \multirow[b]{2}{*}{ Capsule } & \multirow[b]{2}{*}{$\begin{array}{l}\text { Capsule } \\
\text { Position }\end{array}$} & \multirow{2}{*}{$\begin{array}{l}\text { Average } \\
\text { Centerline } \\
\text { Temp. } \\
\left({ }^{\circ} \mathrm{C}\right)\end{array}$} & \multirow{2}{*}{$\begin{array}{c}\text { Fast Fluence } \\
\left(10^{\left.21 \mathrm{n} / \mathrm{cm}^{2}\right)}\right. \\
(\mathrm{E}>0.18 \mathrm{MeV}) \text { HTGR }\end{array}$} & \multicolumn{3}{|c|}{ Macroporosity $^{(a)}(\%)$} & \multicolumn{3}{|c|}{ Matrix Composition } \\
\hline & & & & & $\begin{array}{l}\text { Pre- } \\
\text { Irrad. (b) }\end{array}$ & $\begin{array}{l}\text { Post- } \\
\text { Irrad. }\end{array}$ & $\Delta \mathrm{x} / \mathrm{x}_{\mathrm{o}}$ & Filler & Additive & $\begin{array}{l}\text { Firing } \\
\text { Conditions (c) }\end{array}$ \\
\hline $28-13$ & P13s & $6 \mathrm{C}$ & 1050 & 4.4 & 38 & 46 & +21 & 6353 & Sc011 & Packed bed \\
\hline $23-5$ & $\mathrm{P} 13 \mathrm{R}$ & $6 \mathrm{C}$ & 1005 & 4.5 & 43 & 43 & 0 & 6353 & $\mathrm{SC0} 27$ & Ref. \\
\hline $01-7$ & P13s & $1 \mathrm{~A}$ & 1030 & 7.0 & 31 & 38 & +23 & 6353 & Sc011 & Ref. \\
\hline $20-5$ & P13S & $5 \mathrm{E}$ & 1350 & 7.5 & 27 & 45 & +67 & 6353 & Sc011 & Ref. \\
\hline $20-6$ & P13R & $5 \mathrm{E}$ & 1280 & 7.7 & 27 & 51 & +89 & 6353 & SC011 & $\operatorname{Ref}$. \\
\hline $19-5$ & P13S & $5 D$ & 1325 & 7.9 & 42 & 44 & +5 & 6353 & $\mathrm{SC} 011$ & $\mathrm{~N}_{2}$ atm \\
\hline $19-7$ & P13R & $5 \mathrm{D}$ & 1225 & 8.2 & 42 & 49 & +17 & 6353 & SC011 & $\mathrm{N}_{2}$ atm \\
\hline $18-5$ & P13S & $5 \mathrm{C}$ & 1335 & 8.4 & 35 & 53 & +51 & 6353 & SC011 & $1500^{\circ} \mathrm{C}$ \\
\hline $03-5$ & P13R & $1 \mathrm{C}$ & 1065 & 8.7 & 42 & 54 & +29 & 6353 & SC011 & Ref. \\
\hline $16-6$ & P13S & $5 \mathrm{~A}$ & 1375 & 9.2 & 37 & 52 & +41 & 6353 & $\mathrm{SC} 011$ & Ref. \\
\hline $16-5$ & P13R & $5 \mathrm{~A}$ & 1285 & 9.4 & 37 & 49 & +32 & 6353 & SC011 & Ref. \\
\hline $12-5$ & P13S & $2 B$ & 1010 & 11.7 & 32 & 58 & +81 & 6353 & sco11 & Ref. \\
\hline $06-5$ & P13R & $2 \mathrm{~A}$ & 1050 & 11.8 & 37 & 48 & +30 & 6353 & Sc011 & $1500^{\circ} \mathrm{C}$ \\
\hline $13-6$ & P13S & $2 C$ & 975 & 11.9 & 35 & 47 & +34 & 6353 & $\mathrm{SC} 027$ & Ref. \\
\hline $15-5$ & P13S & $2 \mathrm{E}$ & 960 & 12.1 & 36 & 55 & +112 & 6353 & Sc011 & Ref. \\
\hline $07-5$ & P13R & $2 \mathrm{~B}$ & 1055 & 12.0 & 28 & 53 & +89 & Lonza & $\mathrm{SC} 011$ & Ref. \\
\hline $08-13$ & P13R & $2 \mathrm{C}$ & 1045 & 12.2 & 37 & 60 & +62 & 6353 & Sc011 & Packed bed \\
\hline
\end{tabular}

(a) Determined by point-count method (Ref. 4-12) on $75 \mathrm{X}$ diametral composite cross section of fuel rod. Macroporosity $=\mathrm{V}_{\text {void }} /\left(\mathrm{V}_{\text {void }}+\mathrm{V}_{\text {matrix }}\right)$.

(b) Measured on companion fuel rods.

(c) Reference firing conditions: in-block (H-451 graphite tubes) to $1800^{\circ} \mathrm{C}$ in argon. Deviations from these conditions are noted. 
TABLE 4-39

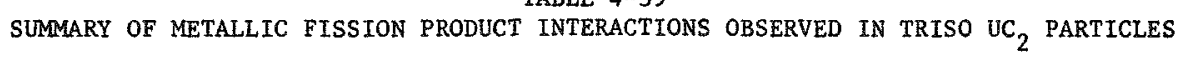

\begin{tabular}{|c|c|c|c|c|c|c|c|c|c|c|c|c|}
\hline \multicolumn{3}{|c|}{ Sample Description } & \multirow{2}{*}{\multicolumn{6}{|c|}{ Irradiation Conditions }} & \multirow{2}{*}{\multicolumn{4}{|c|}{ Results of Metallographic Examination }} \\
\hline \multirow[b]{2}{*}{$\begin{array}{c}\text { Particle Batch } \\
\text { Data Retrieval } \\
\text { Number } \\
\end{array}$} & \multirow{2}{*}{$\begin{array}{c}\text { Mean } \\
\text { Kernel } \\
\text { Diameter } \\
(\mu \mathrm{m})\end{array}$} & \multirow{2}{*}{$\begin{array}{c}\text { Buffer }+ \\
\text { IPyC } \\
\text { Coating } \\
\text { Thickness } \\
(\mu \mathrm{m}) \\
\end{array}$} & & & & & & & & & & \\
\hline & & & Capsule & $\begin{array}{l}\text { Capsule } \\
\text { Position }\end{array}$ & $\begin{array}{c}\text { Average } \\
\text { Temp. }(a) \\
\left({ }^{\circ} \mathrm{C}\right)\end{array}$ & $\begin{array}{l}\text { Maximum } \\
\text { Temp. (b) } \\
\left({ }^{\circ} \mathrm{C}\right)\end{array}$ & $\begin{array}{c}\text { Fast Fluence } \\
\left(10^{21} \mathrm{n} / \mathrm{cm}^{2}\right) \\
(E>0.18 \mathrm{MeV})_{\mathrm{HTGR}}\end{array}$ & $\begin{array}{c}\text { Burnup } \\
(\% \text { FIMA })\end{array}$ & $\begin{array}{l}\text { No. of } \\
\text { Par- } \\
\text { ticles }\end{array}$ & $\begin{array}{c}\text { IPyC } \\
\text { Coating } \\
\text { Reaction }(c) \\
(\%)\end{array}$ & $\begin{array}{c}\text { Sic } \\
\text { Coating } \\
\text { Reaction }(d) \\
(\%)\end{array}$ & $\begin{array}{c}\text { Pressure } \\
\text { Vessel } \\
\text { Failure (e) } \\
(\%)\end{array}$ \\
\hline $6151-00-035$ & 201 & 120 & P13S & $6 \mathrm{C}$ & 1050 & $\$ 215$ & 4.4 & 48 & 77 & 75 & 0 & 0 \\
\hline $6151-00-035$ & 201 & 120 & P13R & $6 \mathrm{C}$ & 1005 & 1160 & 4.5 & 49 & 33 & 39 & 0 & 0 \\
\hline $6151-00-035$ & 201 & 120 & P13S & $5 \mathrm{C}$ & 1335 & 1625 & 8.4 & 66 & 69 & 100 & 88 & 0 \\
\hline $6151-00-035$ & 201 & 120 & P13S & $2 \mathrm{C}$ & 975 & 1090 & 11.9 & 74 & 57 & 100 & 0 & 0 \\
\hline $6154-00-035$ & 201 & 120 & P13R & $2 \mathrm{~B}$ & 1055 & 1220 & 12.0 & 75 & 54 & 100 & 1.9 & 0 \\
\hline $6151-00-035$ & 201 & 120 & P13R & $2 \mathrm{C}$ & 1045 & 1195 & 12.2 & 75 & 71 & 100 & 8.5 & 0 \\
\hline $6151-00-045$ & 196 & 120 & P13S & IA & 1030 & 1545 & 7.0 & 70 & 72 & 100 & 2.9 & 15.3 \\
\hline $6151-00-045$ & 196 & 120 & P13R & $2 \mathrm{~A}$ & 1050 & 1225 & 11.8 & 75 & 16 & 90 & 0 & 6.3 \\
\hline $6151-08-015$ & 202 & 128 & P13S & 5D & 1325 & 1610 & 7.9 & 65 & 53 & 100 & 100 & 3.8 \\
\hline $6151-08-015$ & 202 & 128 & P13R & 5D & 1225 & 1480 & 8.2 & 66 & 78 & 100 & 5.1 & 2.6 \\
\hline $6151-08-015$ & 202 & 128 & P13S & $2 E$ & 960 & 1095 & 12.1 & 74 & 19 & 100 & 0 & 0 \\
\hline $6151-09-015$ & 197 & $\$ 26$ & P135 & $5 E$ & 1350 & 1650 & 7.5 & 63 & 62 & 100 & 86 & 1.6 \\
\hline $6151-09-015$ & 197 & 126 & P13R & $5 \mathrm{E}$ & 1280 & 1590 & 7.7 & 64 & 55 & 100 & 0 & 0 \\
\hline $6151-09-015$ & 197 & 126 & P13S & $2 B$ & 1010 & 1135 & 11.7 & 74 & 58 & 95 & 3.4 & 0 \\
\hline
\end{tabular}

(a) Time-averaged fuel rod centerline irradiation temperature.

(b) Maximum fuel rod centerline temperature during irradiation.

(c) Fraction of particles with large concentrations of metallic fission products in the IPyC coating.

(d) Fraction of particles that exhibited metallic fission product attack of the SiC coating less than one-half the thickness of the SiC coating.

(e) Pailure of the structural opyC and SiC coatings that would result in the release of gaseous fission products. 
TABLE $4-40$

SUMMARY OF FUEL KERNEL MIGRATION DATA

\begin{tabular}{|c|c|c|c|c|c|c|c|c|}
\hline $\begin{array}{c}\text { Particle Batch } \\
\text { Data Retrieval } \\
\text { Number }\end{array}$ & $\begin{array}{c}\text { Kerne1 } \\
\text { Type }\end{array}$ & $\begin{array}{c}\text { Fuel Rod Data } \\
\text { Retrieval No. } \\
\text { (7161-004-) }\end{array}$ & Capsule & $\begin{array}{l}\text { Capsule } \\
\text { Position }\end{array}$ & $\begin{array}{c}\text { Average } \\
\text { Centerline } \\
\text { Temperature } \\
\left({ }^{\circ} \mathrm{C}\right)\end{array}$ & $\begin{array}{l}\text { Part1cle } \\
\text { Ident. } \\
\text { Number }(\alpha) \\
(L 7440-)\end{array}$ & $\begin{array}{c}\text { Particle } \\
\text { Location (b) } \\
(\mathrm{cm})\end{array}$ & $\begin{array}{l}\text { Migration } \\
\text { Distance } \\
\text { (um) }\end{array}$ \\
\hline $6151-08-015$ & $\mathrm{UC}_{2}$ & $19-7$ & P13R & 5D & 1225 & $\begin{array}{l}-381 \\
-383 \\
-385 \\
-393 \\
-397 \\
-401\end{array}$ & $\begin{array}{l}0.5113 \\
0.5808 \\
0.2015 \\
0.1180 \\
0.0085 \\
0.5095\end{array}$ & $\begin{array}{l}\mathrm{ND}(\mathrm{c}) \\
2.0 \\
\mathrm{ND} \\
2.0 \\
6.0 \\
\text { ND }\end{array}$ \\
\hline $6151-00-035$ & $\mathrm{uc}_{2}$ & $18-5$ & P13S & $5 c$ & 1335 & $\begin{array}{l}-403 \\
-428 \\
-431 \\
-434 \\
-437 \\
-444 \\
-601\end{array}$ & $\begin{array}{l}0.2300 \\
0.5915 \\
0.5715 \\
0.1725 \\
0.3730 \\
0.4028 \\
0.1253\end{array}$ & $\begin{array}{r}15.0 \\
2.0 \\
2.0 \\
6.0 \\
3.0 \\
12.0 \\
9.0\end{array}$ \\
\hline $6151-08-015$ & $\mathrm{uc}_{2}$ & $19-5$ & P13S & 5D & 1325 & $\begin{array}{l}-316 \\
-319 \\
-331 \\
-325\end{array}$ & $\begin{array}{l}0.3259 \\
0.2269 \\
0.5961 \\
0.5100\end{array}$ & $\begin{array}{l}4.0 \\
6.0 \\
1.0 \\
\text { ND }\end{array}$ \\
\hline $6151-00-035$ & $\mathrm{uc}_{2}$ & $28-3$ & P13s & $6 C$ & 1050 & $\begin{array}{l}-466 \\
-468 \\
-470 \\
-476 \\
-478 \\
-480 \\
-484 \\
-486 \\
-490\end{array}$ & $\begin{array}{l}0.5000 \\
0.4157 \\
0.4319 \\
0.2748 \\
0.0951 \\
0.4634 \\
0.1541 \\
0.1159 \\
0.5818\end{array}$ & $\begin{array}{l}\text { ND } \\
\text { ND } \\
\text { ND } \\
\text { ND } \\
\text { ND } \\
\text { ND } \\
\text { ND } \\
\text { ND } \\
\text { ND }\end{array}$ \\
\hline $5466-37$ & $(1 \mathrm{Th}, 1 \mathrm{U}) \mathrm{O}_{2}$ & $16-5$ & P13R & SA & 1285 & $\begin{array}{l}-1009 b \\
-1011 \\
-1025 \\
-1027\end{array}$ & $\begin{array}{l}0.2223 \\
0.2110 \\
0.3378 \\
0.5720\end{array}$ & $\begin{array}{l}\text { ND } \\
4.0 \\
1.0 \\
\text { ND }\end{array}$ \\
\hline $5466-37$ & $(1 \mathrm{Th}, 1 \mathrm{v}) \mathrm{O}_{2}$ & $16-6$ & P13S & $5 A$ & 1375 & $\begin{array}{l}-1041 \\
-1043 \\
-1051 \\
-1045 \\
-1055 \\
-1059 \\
-1063 \\
-1061 \\
-1067 \\
-1065 \\
-1070\end{array}$ & $\begin{array}{l}0.5936 \\
0.5395 \\
0.5336 \\
0.3391 \\
0.2795 \\
0.0735 \\
0.4925 \\
0.2606 \\
0.5917 \\
0.4626 \\
0.3022\end{array}$ & $\begin{array}{c}13.0 \\
23.0 \\
15.0 \\
6.0 \\
17.0 \\
8.0 \\
12.0 \\
\mathrm{ND} \\
19.0 \\
7.0 \\
4.0\end{array}$ \\
\hline
\end{tabular}

(a) Identification number of laboratory photograph of fuel particle.

(b) Distance of particle from fuel rod centerline.

(c) $\mathrm{ND}$ = kernel migration was not detectable. 
TABLE 4-41

SUMMARY OF FISSION GAS RELEASE MEASUREMENTS ON UNFAILED BISO Tho ${ }_{2}$ PARTICLE BATCHES

\begin{tabular}{|c|c|c|c|c|c|c|c|c|c|c|c|}
\hline \multirow{2}{*}{$\begin{array}{c}\text { Data } \\
\text { Retrieval } \\
\text { Number } \\
\end{array}$} & \multicolumn{3}{|c|}{ OPyC Coating } & \multirow[b]{2}{*}{ Capsule } & \multirow[b]{2}{*}{$\begin{array}{l}\text { Capsule } \\
\text { Position } \\
\end{array}$} & \multirow{2}{*}{$\begin{array}{c}\text { Fast Fluence } \\
\left(10^{2} 1 \mathrm{n} / \mathrm{cm}^{2}\right) \\
(\mathrm{E}>0.18 \mathrm{MeV})_{\text {HTGR }} \\
\end{array}$} & \multirow[b]{2}{*}{$\begin{array}{c}\text { Burnup } \\
\text { (\% FIMA) } \\
\end{array}$} & \multirow{2}{*}{$\begin{array}{c}\text { No. of } \\
\text { Par- } \\
\text { ticles }\end{array}$} & \multicolumn{2}{|c|}{$\begin{array}{l}\text { OPyC Coating } \\
\text { Failure (b) }(\%)\end{array}$} & \multirow{2}{*}{$\begin{array}{c}\text { Fission Gas } \\
\text { Release (c) } \\
(\mathrm{R} / \mathrm{B} \mathrm{Kr}-85 \mathrm{~m}) \\
\end{array}$} \\
\hline & $\begin{array}{c}\text { Density (a) } \\
\left(\mathrm{g} / \mathrm{cm}^{3}\right)\end{array}$ & $\begin{array}{c}\text { Rate } \\
(\mu \mathrm{m} / \mathrm{min})\end{array}$ & Gas & & & & & & $\frac{\text { Failure }}{\text { Pre-FGR }}$ & $\frac{(\%)}{\text { Post-FGR }}$ & \\
\hline $6542-09-010$ & 1.93 & 5.0 & Propylene & P13R & $3-11,12$ & 11.7 & 4.8 & 998 & 0 & 0.3 & $2.4 \times 10^{-5}$ \\
\hline $6542-02-020$ & 1.91 & 8.5 & Propylene & P13R & $3-7,8$ & 11.9 & 4.9 & 1072 & 0 & 0 & $4.4 \times 10^{-5}$ \\
\hline $6542-18-015$ & 1.84 & 6.5 & Propylene & P13R & $4-3,4$ & 11.4 & 4.5 & 953 & 0 & 0 & $2.2 \times 10^{-6}$ \\
\hline $6542-02-020$ & 1.91 & 8.5 & Propylene & P13R & $4-5,6$ & 11.3 & 4.4 & 1149 & 0 & 0 & $3.3 \times 10^{-5}$ \\
\hline $4252-06-010$ & 1.82 & 5.8 & Propylene & P13S & $3-1,2$ & 11.8 & 4.3 & 872 & 0 & 0 & $6.2 \times 10^{-5}$ \\
\hline $6542-23-025$ & 1.89 & 5.8 & $\mathrm{MG}^{(\mathrm{d})}$ & P13S & $3-7,8$ & 11.7 & 4.2 & 992 & 0 & 0 & $2.8 \times 10^{-6}$ \\
\hline $6542-24-015$ & 1.94 & 6.6 & $M G(d)$ & P13S & $3-9,10$ & 11.6 & 4.1 & 942 & 0 & 0 & $3.9 \times 10^{-6}$ \\
\hline $4252-02-010$ & 1.83 & 4.0 & Propylene & P13S & $4-2,3$ & 11.2 & 3.9 & 925 & 0 & 0 & $1.1 \times 10^{-3}$ \\
\hline $6542-21-016$ & 1.73 & 8.0 & $M G(d)$ & P13S & $4-9$ & 10.9 & 3.7 & 508 & 0 & 0.6 & $2.1 \times 10^{-3}$ \\
\hline $6542-21-015$ & 1.73 & 7.8 & $M^{(d)}$ & P13S & $4-10$ & 10.8 & 3.6 & 513 & 0 & 0 & $2.1 \times 10^{-3}$ \\
\hline
\end{tabular}

(a) Preirradiation density gradient column measurement.

(b) Determined by visual examination before and after fission gas release (FGR) measurements.

(c) Fission gas release measurements made in TRIGA at $1100^{\circ} \mathrm{C}$.

(d) OPyC coating was deposited from mixed gas (MG) (propylene-acetylene). 
TABLE $4-42$

SUMMARY DESCRIPTION OF ThO, PARTICLE SAMPLES SELECTED FOR ACID LEACHING AND FISSION GAS RELEASE MEASUREMENTS

\begin{tabular}{|c|c|c|c|c|c|c|c|c|c|c|}
\hline \multirow[b]{2}{*}{$\begin{array}{l}\text { Data } \\
\text { Retrieval } \\
\text { Number }\end{array}$} & \multicolumn{3}{|c|}{ OPyC Coating } & \multirow[b]{2}{*}{ Capsule } & \multirow[b]{2}{*}{$\begin{array}{l}\text { Capsule } \\
\text { Position } \\
\end{array}$} & \multirow{2}{*}{$\begin{array}{c}\begin{array}{c}\text { Fast Fluence } \\
\left(10^{21} \mathrm{n} / \mathrm{cm}^{2}\right)\end{array} \\
(\mathrm{E}>0.18 \mathrm{MeV})_{\mathrm{HTGR}}\end{array}$} & \multirow[b]{2}{*}{$\begin{array}{c}\text { Burnup } \\
(\% \text { FIMA) }\end{array}$} & \multirow{2}{*}{$\begin{array}{l}\text { No. of } \\
\text { Par- } \\
\text { ticles }\end{array}$} & \multirow{2}{*}{$\begin{array}{c}\text { OPyC } \\
\text { Coating } \\
\text { Failure (b) }\end{array}$} & \multirow{2}{*}{$\begin{array}{l}\text { Fission Gas } \\
\text { Release (c) } \\
\text { (R/B } \mathrm{Kr}-85 \mathrm{~m})\end{array}$} \\
\hline & $\begin{array}{c}\text { Density (a) } \\
\left(\mathrm{g} / \mathrm{cm}^{3}\right)\end{array}$ & $\begin{array}{c}\text { Rate } \\
(\mu \mathrm{m} / \mathrm{min})\end{array}$ & Gas & & & & & & & \\
\hline $6542-21-016-1$ & 1.73 & 8.0 & $M G(d)$ & P13S & $4-9$ & 10.9 & 3.7 & 197 & 1 & $3.0 \times 10^{-3}$ \\
\hline $6542-22-015-1$ & 1.80 & 4.0 & $\mathrm{MG}^{(\mathrm{d})}$ & P13S & $3-3$ & 11.7 & 4.3 & 207 & 0 & $6.7 \times 10^{-4}$ \\
\hline $6542-24-015-1$ & 1.94 & 6.6 & $M G^{(d)}$ & P13S & $3-9$ & 11.6 & $4 \cdot 1$ & 200 & 0 & $1.4 \times 10^{-5}$ \\
\hline $6542-01-020-2$ & 1.82 & 2.7 & Propylene & P13R & $3-4$ & 12.0 & 5.0 & 55 & 0 & $1.6 \times 10^{-3}$ \\
\hline $4252-06-018-1$ & 1.82 & 5.8 & Propylene & P13R & $3-1$ & 12.1 & 5.1 & 196 & $5^{(e)}$ & $3.0 \times 10^{-3}$ \\
\hline $6542-01-010-1$ & 1.80 & 10.0 & Propylene & P13R & $3-5$ & 12.0 & 5.0 & 202 & 0 & $1.5 \times 10^{-3}$ \\
\hline $.6542-02-020-2$ & 1.91 & 8.5 & Propylene & P13R & $3-8$ & 11.9 & 4.9 & 205 & 0 & $5.6 \times 10^{-5}$ \\
\hline $6542-09-010-2$ & 1.93 & 5.0 & Propylene & P13R & $3-12$ & 11.7 & 4.7 & 201 & 0 & $8.5 \times 10^{-6}$ \\
\hline
\end{tabular}

(a) Preirradiation density gradient column measurement.

(b) Number of particles in sample with failed coatings. Failure was detected by visual examination of sample after fission gas release measurements but before acid leaching.

(c) Fission gas release measurements made on particle sample at $1100^{\circ} \mathrm{C}$ prior to acid leaching.

(d) OPyC coating was deposited from mixed gas (MG) (propylene-acetylene).

(e) Particles contained very fine hair-line cracks. 
TABLE $4-43$
RESULTS OF SPECTROPHOTOMETRIC ANALYSIS OF LEACH SOLUTIONS

\begin{tabular}{|c|c|c|c|c|c|c|c|c|c|c|c|c|c|c|c|c|c|c|c|c|c|}
\hline \multirow[b]{3}{*}{ Aliquot } & \multicolumn{3}{|c|}{$6542-21-016-1$} & \multicolumn{3}{|c|}{$6542-22-015-1$} & \multicolumn{3}{|c|}{$6542-24-015-1$} & \multicolumn{3}{|c|}{$6542-01-020-2$} & \multicolumn{3}{|c|}{$4252-06-018-9$} & \multicolumn{3}{|c|}{$6542-02-020-2$} & \multicolumn{3}{|c|}{$6542-09-010-2$} \\
\hline & \multicolumn{2}{|c|}{$\begin{array}{l}\text { Leach } \\
\text { Time(a) } \\
\text { (hr) }\end{array}$} & \multirow{2}{*}{$\begin{array}{l}\text { Thorium } \\
\text { Leached } \\
\text { (ug) }\end{array}$} & \multicolumn{2}{|c|}{$\begin{array}{l}\text { Leach } \\
\text { Time } \\
\text { (hr) }\end{array}$} & \multirow{2}{*}{$\begin{array}{l}\text { Thorium } \\
\text { Leached } \\
\text { (ig) }\end{array}$} & \multicolumn{2}{|c|}{$\begin{array}{l}\text { Leach } \\
\text { Tine } \\
\text { (hr) }\end{array}$} & \multirow{2}{*}{$\begin{array}{l}\text { Thorium } \\
\text { Leached } \\
(\mu \mathrm{g})\end{array}$} & \multicolumn{2}{|c|}{$\begin{array}{l}\text { Leach } \\
\text { Time } \\
\text { (hr) }\end{array}$} & \multirow{2}{*}{$\begin{array}{l}\text { Thor lum } \\
\text { Leached } \\
\text { ( } \mathrm{gg})\end{array}$} & \multicolumn{2}{|c|}{$\begin{array}{l}\text { Leach } \\
\text { Time } \\
\text { (hr) } \\
\end{array}$} & \multirow{2}{*}{ 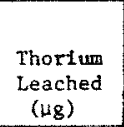 } & \multicolumn{2}{|c|}{$\begin{array}{l}\text { Leach } \\
\text { Time } \\
\text { (hr) }\end{array}$} & \multirow{2}{*}{$\begin{array}{l}\text { Thorium } \\
\text { Leached } \\
(\mu g)\end{array}$} & \multicolumn{2}{|c|}{$\begin{array}{l}\text { Leach } \\
\text { Time } \\
\text { (hr) }\end{array}$} & \multirow{2}{*}{$\begin{array}{l}\text { Thorium } \\
\text { Leached } \\
\text { (Hg) }\end{array}$} \\
\hline & Hot & Cold & & Hot & Cold & & Hot & $\operatorname{cold}$ & & Hot & $\cos d$ & & Hot & cold & & Hot & Cold & & Hot & Cold & \\
\hline 1 & 4 & 15.5 & $1.0 \times 10^{4}$ & 6 & -- & $2.5 \times 10^{3}$ & 5.5 & -- & $2.7 \times 10^{2}$ & 3.5 & -- & $6.3 \times 10^{2}$ & 5.5 & -- & $2.2 \times 10^{4}$ & 2.5 & -- & $2.5 \times 10^{2}$ & 2.5 & -- & $2.4 \times 10^{2}$ \\
\hline 2 & 11 & 31 & $1.1 \times 10^{5}$ & 9 & 16 & $1.0 \times 10^{4}$ & 7.0 & 16.0 & $2.5 \times 10^{2}$ & 5.5 & 16.0 & $4.1 \times 10^{3}$ & 7.0 & 16.0 & $3.6 \times 10^{4}$ & 5.5 & 16.0 & $2.4 \times 10^{2}$ & 5.5 & 16.0 & $2.4 \times 10^{2}$ \\
\hline 3 & 17 & 31 & $1.1 \times 10^{5}$ & 13 & 16 & $4.5 \times 10^{4}$ & 12.5 & 16.0 & $3.7 \times 10^{2}$ & 13.0 & 16.5 & $2.2 \times 10^{4}$ & 12.5 & 16.0 & $1.2 \times 10^{5}$ & 9.0 & 16.0 & $8.7 \times 10^{2}$ & 9.0 & 16.0 & $4.8 \times 10^{2}$ \\
\hline 4 & 21 & 46 & $1.1 \times 10^{5}$ & 17.5 & 31.5 & $1.1 \times 10^{5}$ & 15.0 & 32.5 & $6.5 \times 10^{2}$ & 16.5 & 32.0 & $2.9 \times 10^{4}$ & 15.0 & 32.5 & $1.2 \times 10^{5}$ & 12.0 & 79.5 & $3.6 \times 10^{3}$ & 12.0 & 79.5 & $2.9 \times 10^{3}$ \\
\hline 5 & -- & - & -- & 22.5 & 31.5 & $\mathrm{NA}(\mathrm{b})$ & -- & 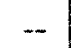 & - & 20.5 & 47.5 & $3.3 \times 10^{4}$ & -- & $\ldots$ & $\ldots$ & 18.0 & 79.5 & $1.8 \times 10^{4}$ & 18.0 & 79.5 & NA \\
\hline 6 & -- & - & -- & 26.0 & 47.0 & $\mathrm{NA}$ & -- & -- & -- & 24.5 & 63.0 & $\mathrm{NA}$ & -- & -- & -- & 18.0 & 85.0 & $2.6 \times 10^{4}$ &.- & -- & - \\
\hline
\end{tabular}

(a) All leach times are cumulative. Hot is the boiling time and cold is with the hot plate turned off at night.

(b) Leach solution was not analyzed. 
TABLE $4-44$

RESULTS OF VISUAL AND METALLOGRAPHIC EXAMINATIONS OF BISO COATED PARTICLES AFTER ACID LEACHING

\begin{tabular}{|c|c|c|c|c|c|c|c|}
\hline \multirow[b]{2}{*}{$\begin{array}{l}\text { Data } \\
\text { Retrieval } \\
\text { Number }\end{array}$} & \multirow[b]{2}{*}{ Capsule } & \multirow[b]{2}{*}{$\begin{array}{l}\text { Capsule } \\
\text { Position }\end{array}$} & \multicolumn{2}{|c|}{ Visual Examination } & \multicolumn{2}{|c|}{$\begin{array}{l}\text { Metallographic } \\
\text { Examination }\end{array}$} & \multirow[b]{2}{*}{ Remarks } \\
\hline & & & $\begin{array}{l}\text { Number of } \\
\text { Particles } \\
\text { Recovered }\end{array}$ & $\begin{array}{c}\text { Number of } \\
\text { Particles } \\
\text { with Failed } \\
\text { OPyC Coatings }\end{array}$ & $\begin{array}{l}\text { Number of } \\
\text { Particles } \\
\text { Examined }\end{array}$ & $\begin{array}{c}\text { Number of } \\
\text { Particles } \\
\text { with Failed } \\
\text { opyo Coatings }\end{array}$ & \\
\hline $6542-21-016-1$ & P13S & $4-9$ & 190 & $0^{(a)}$ & 148 & 2 & All kernels were heavily leached \\
\hline $6542-22-015-1$ & P13S & $3-3$ & 202 & 43 & 144 & 68 & $\begin{array}{l}\text { Severe degradation of } O P y C \text { coating and } \\
\text { all kernels were heavily leached }\end{array}$ \\
\hline $6542-24-015-1$ & P13S & $3-9$ & 195 & 1 & 157 & 2 & $\begin{array}{l}\text { Mild degradation of oPyC coating; } 290 \% \\
\text { of kernels were unleached }\end{array}$ \\
\hline $6542-01-020-2$ & P13R & $3-4$ & 44 & 9 & 27 & 16 & $\begin{array}{l}\text { OPyC coating appeared pitted and porous; } \\
\text { all kernels were heavily leached }\end{array}$ \\
\hline $4252-06-018-1$ & P13R & $3-1$ & 187 & 6 & 151 & 21 & $\begin{array}{l}\text { OPyC coating appeared porous; all kernels } \\
\text { were heavily leached }\end{array}$ \\
\hline $6542-01-010-1$ & P13R & $3-5$ & 155 & 5 & 143 & 2 & $\begin{array}{l}\text { OPyC coating appeared porous; all kernels } \\
\text { were heavily leached }\end{array}$ \\
\hline $6542-02-020-2$ & P13R & $3-8$ & 204 & 22 & 158 & 31 & $\begin{array}{l}\text { Mild degradation of OPyC coating; } \sim 90 \% \\
\text { of kernels were unleached }\end{array}$ \\
\hline $6542-09-010-2$ & P13R & $3-12$ & 200 & 0 & 161 & 0 & $\begin{array}{l}\text { Mild degradation of OPyC coating; } 290 \% \\
\text { of kernels were unleached }\end{array}$ \\
\hline
\end{tabular}

(a) The surface of all particles was crazed; however, during the metallographic examination it was observed that the microcracks did not penetrate the thickness of the OPyC coating. 
TABLE 4-45

CHEMICAL ANALYSIS OF LEACH SOLUTIONS

\begin{tabular}{c|c|c|c}
\hline Aliquot & $\begin{array}{c}\text { Time } \\
\text { (hr) }\end{array}$ & $\begin{array}{c}\text { Volume of } \\
\text { Aliquot } \\
(\mathrm{m} \ell)\end{array}$ & $\begin{array}{c}\text { Tota1 U } \\
\text { (mg/ml) }\end{array}$ \\
\hline 1 & 3.5 & 5.9 & $247 \pm 4.5 \%$ \\
2 & 7.0 & 4.5 & $255 \pm 0.2 \%$ \\
3 & 13.0 & 4.7 & $245 \pm 0.5 \%$ \\
4 & 15.0 & 6.8 & $259 \pm 2.7 \%$ \\
\hline
\end{tabular}

TABLE $4-46$

URANIUM ISOTOPIC COMPOSITION OF THE LEACH SOLUTION

\begin{tabular}{c|c|l}
\hline Isotope & \multicolumn{1}{|c|}{$\begin{array}{c}\text { Weight } \\
(\mathrm{mg})\end{array}$} & \multicolumn{1}{|c}{$\begin{array}{c}\text { Weight } \\
(\%)\end{array}$} \\
\hline $\mathrm{U}-233$ & $17.92 \pm 0.025$ & $71.930 \pm 0.08$ \\
$\mathrm{U}-234$ & $5.367 \pm 0.007$ & $21.739 \pm 0.004$ \\
$\mathrm{U}-235$ & $1.110 \pm 0.0015$ & $4.496 \pm 0.02$ \\
$\mathrm{U}-236$ & $0.385 \pm 0.007$ & $1.558 \pm 0.01$ \\
$\mathrm{U}-238$ & $0.069 \pm 0.002$ & $0.279 \pm 0.002$ \\
\hline
\end{tabular}


TABLE 4-47

\begin{tabular}{c|c|c|c}
\multirow{2}{*}{ URANIUM ISOTOPIC COMPOSITION OF THE FUEL ROD } \\
\hline & \multicolumn{3}{|c}{ Weight (mg) } \\
\cline { 2 - 4 } Isotope & Fissile & Fertile & Total \\
\hline U-233 & 0 & 16.72 & 16.72 \\
U-234 & 0.10 & 5.97 & 6.07 \\
U-235 & 1.61 & 1.29 & 2.90 \\
U-236 & 4.97 & 0.23 & 5.20 \\
U-238 & 1.67 & 0 & 1.67 \\
\hline
\end{tabular}

(a) Composition at EOL +120 days calculated using FISSIN computer program. 
TABLE $4-48$

POSTIRRADIATIUN PARTICLE DIMENSIONS DETERMINED BY CONTACT MICRORADIOGRAPHY

\begin{tabular}{|c|c|c|c|c|c|c|c|c|c|c|c|c|c|c|c|c|c|c|c|}
\hline \multirow[b]{2}{*}{$\begin{array}{c}\text { Data } \\
\text { Retrieval } \\
\text { Number }\end{array}$} & \multirow[b]{2}{*}{ Capsule } & \multirow[b]{2}{*}{$\begin{array}{l}\text { Capsule } \\
\text { Position }\end{array}$} & \multirow[b]{2}{*}{$\begin{array}{l}\text { Radio- } \\
\text { graph } \\
\text { Plate } \\
\text { No. }\end{array}$} & \multirow[b]{2}{*}{$\begin{array}{l}\text { Number } \\
\text { of } \\
\text { Parti- } \\
\text { cles }\end{array}$} & \multicolumn{3}{|c|}{ Kernel } & \multicolumn{3}{|c|}{ Buffer } & \multicolumn{3}{|c|}{$\mathrm{SiC}$} & \multicolumn{3}{|c|}{$O P Y C$} & \multicolumn{3}{|c|}{ Total Particle } \\
\hline & & & & & $\begin{array}{l}\text { Diam } \\
(\mathrm{m})\end{array}$ & $\begin{array}{l}\text { Std. } \\
\text { De: } \\
\text { (.m) }\end{array}$ & $\begin{array}{l}\text { Range } \\
(\llcorner\mathrm{n})\end{array}$ & $\begin{array}{c}\text { Thick- } \\
\text { ness } \\
(\mathrm{um})\end{array}$ & $\begin{array}{l}\text { Std. } \\
\text { Dev. } \\
\text { (in) }\end{array}$ & $\begin{array}{l}\text { Range } \\
(\text { um) }\end{array}$ & $\begin{array}{c}\text { Thick- } \\
\text { ness } \\
(\text { nm) }\end{array}$ & $\begin{array}{l}\text { Std. } \\
\text { Dev: } \\
\text { (ari) }\end{array}$ & $\begin{array}{c}\text { Kange } \\
(\ldots)\end{array}$ & $\begin{array}{l}\text { Thick- } \\
\text { ness } \\
\text { (um) }\end{array}$ & $\begin{array}{l}\text { Std. } \\
\text { Dev. } \\
(\ldots \pi)\end{array}$ & $\begin{array}{l}\text { Range } \\
(\mu-1)\end{array}$ & $\begin{array}{l}\text { Diax } \\
(\ldots \mathrm{m})\end{array}$ & $\begin{array}{l}\text { Std. } \\
\text { Jev. } \\
\text { (-m) }\end{array}$ & $\begin{array}{l}\text { Range } \\
\text { (un) }\end{array}$ \\
\hline $6151-00-035-2$ & P13R & $3-5$ & $\begin{array}{l}\text { HA370, } \\
377\end{array}$ & 57 &. $\mathrm{x}^{(\mathrm{a})}$ & $\mathrm{ND}$ & $\mathrm{D}$ & $\mathrm{SD}$ & $\mathrm{ND}$ & ND & 26 & 2.6 & $20-37$ & 35 & 5.5 & $25-60$ & 580 & 31.0 & $490-660$ \\
\hline $4252-06-018-1$ & P13R & $3-1$ & $\begin{array}{c}\text { HA349, } \\
352, \\
354\end{array}$ & 69 & 529 & 12.8 & $508-576$ & 69 & 6.5 & $55-91$ & - & - & -- & 73 & 6.0 & $58-87$ & 810 & 25.6 & $730-877$ \\
\hline $4252-06-018-2$ & $\mathrm{P} 13 \mathrm{R}$ & $3-2$ & $\begin{array}{c}\text { HA } 353, \\
355, \\
358\end{array}$ & 59 & 532 & 20.9 & $510-65=$ & 67 & 6.3 & $49-82$ & -- & - & - & 73 & 5.5 & $59-81$ & 812 & 26.1 & $728-872$ \\
\hline $6542-19-016-1$ & PIJS & $4-8$ & $\begin{array}{r}\text { HA } 359, \\
363, \\
369\end{array}$ & 87 & 506 & $1^{-} .8$ & $+62-542$ & 71 & 6.7 & $54-89$ & -- & -. & -. & 72 & 6.5 & $55-88$ & $79 k$ & 27.6 & $722-84 i$ \\
\hline $6542-21-016-1$ & P13S & $4-9$ & $\begin{array}{l}\text { HA364, } \\
368\end{array}$ & 75 & 505 & 17.9 & $463-538$ & 66 & 6.2 & $53-85$ & -- & -- & -- & 78 & 7.1 & $60-97$ & 796 & 29.8 & $730-855$ \\
\hline
\end{tabular}

(a) $x D=$ not determined. 
TABLE 4-49

IRRADIATION-INDUCED PARTICLE DIMENSIONAL CHANGE DETERMINED BY MICRORADIOGRAPHY

\begin{tabular}{|c|c|c|c|c|c|c|c|c|c|c|c|c|c|}
\hline \multirow{2}{*}{$\begin{array}{c}\text { Data } \\
\text { Retrieval } \\
\text { Number (a) } \\
\end{array}$} & \multirow[b]{2}{*}{ Capsule } & \multirow[b]{2}{*}{$\begin{array}{l}\text { Capsule } \\
\text { Position }\end{array}$} & \multirow{2}{*}{$\begin{array}{c}\text { Fast Fluence } \\
\left(10^{21} \mathrm{n} / \mathrm{cm}^{2}\right) \\
(\mathrm{E}>0.18 \mathrm{MeV}) \text { HTGR }\end{array}$} & \multirow[b]{2}{*}{$\begin{array}{c}\text { Burnup } \\
(\% \text { FIMA })\end{array}$} & \multirow[b]{2}{*}{$\begin{array}{c}\text { Particle } \\
\text { Type }\end{array}$} & \multicolumn{2}{|c|}{$\begin{array}{c}\text { Kernel } \\
\text { Diameter }\end{array}$} & \multicolumn{2}{|c|}{$\begin{array}{l}\text { Buffer } \\
\text { Thickness }\end{array}$} & \multicolumn{2}{|c|}{$\begin{array}{c}\text { OPyC } \\
\text { Thickness }\end{array}$} & \multicolumn{2}{|c|}{$\begin{array}{l}\text { Total } \\
\text { Particle } \\
\text { Diameter }\end{array}$} \\
\hline & & & & & & $\begin{array}{c}\Delta D \\
(\mu \mathrm{m})\end{array}$ & $\begin{array}{c}\Delta \mathrm{D} / \mathrm{D}_{\mathrm{O}} \\
(\%)\end{array}$ & $\begin{array}{c}\Delta \mathrm{T} \\
(\mu \mathrm{m})\end{array}$ & $\begin{array}{c}\Delta \mathrm{T} / \mathrm{T}_{\mathrm{O}} \\
(\%)\end{array}$ & $\begin{array}{c}\Delta \mathrm{T} \\
(\mu \mathrm{m})\end{array}$ & $\begin{array}{c}\Delta \mathrm{T} / \mathrm{T}_{\mathrm{O}} \\
(\%)\end{array}$ & $\begin{array}{c}\Delta D \\
(\mu \mathrm{m})\end{array}$ & $\begin{array}{c}\Delta \mathrm{D} / \mathrm{D}_{\mathrm{O}} \\
(\%)\end{array}$ \\
\hline $6151-00-035-2$ & P13R & $3-5$ & 12.0 & 74 & $\mathrm{UC}_{2}$ TRISO & $N D^{(b)}$ & ND & ND & ND & -1 & -2.8 & 0 & 0 \\
\hline $4252-06-018-1$ & P13R & $3-1$ & 12.1 & 5.1 & $\mathrm{ThO}_{2}$ BISO & +8 & +1.5 & -10 & -12.7 & -9 & -11.0 & -30 & -3.6 \\
\hline $4252-06-018-2$ & P13R & $3-2$ & 12.1 & 5.1 & $\mathrm{ThO}_{2} \mathrm{BISO}$ & +11 & +2.1 & -13 & -16.3 & -8 & -9.9 & -30 & -3.6 \\
\hline $6542-19-016-1$ & P13S & $4-8$ & 10.9 & 3.7 & $\mathrm{ThO}_{2}$ BISO & +9 & +1.8 & -11 & -13.4 & -10 & -12.2 & -31 & -3.7 \\
\hline $6542-21-016-1$ & P13s & $4-9$ & 10.9 & 3.7 & $\mathrm{ThO}_{2} \mathrm{BISO}$ & +8 & +1.6 & -14 & -17.5 & -11 & -12.4 & -41 & -4.9 \\
\hline
\end{tabular}

(a) Preirradiation dimensions of the actual sample tested, not the parent batch, were used to calculate dimensional change.

(b) $\mathrm{ND}=$ not determined. 
TABLE 4-50

RESULTS OF OPYC DENSITY MEASUREMENTS OF UNBONDED FISSILE PARTICLE SAMPLES IRRADTATED IN CAPSULES P13R AND P13S

\begin{tabular}{|c|c|c|c|c|c|c|c|c|c|c|c|c|}
\hline \multicolumn{6}{|c|}{ Sample Description } & \multicolumn{7}{|c|}{ Postirradiation Density Measurements (a) } \\
\hline \multirow{2}{*}{$\begin{array}{c}\text { Data } \\
\text { Retrieval } \\
\text { Number } \\
\end{array}$} & \multirow{2}{*}{$\begin{array}{c}\text { Preirradiation } \\
\text { Density }(\mathrm{a}) \\
\left(\mathrm{g} / \mathrm{cm}^{3}\right)\end{array}$} & \multirow[b]{2}{*}{ Capsule } & \multirow[b]{2}{*}{$\begin{array}{l}\text { Capsule } \\
\text { Position } \\
\end{array}$} & \multirow{2}{*}{$\begin{array}{c}\text { Fast Fluence } \\
\left(10^{21} \mathrm{n} / \mathrm{cm}^{2}\right) \\
(\mathrm{E}>0.18 \mathrm{MeV}) \mathrm{HTGR}\end{array}$} & \multirow{2}{*}{$\begin{array}{l}\text { Average } \\
\text { Irrad. } \\
\text { Temp. } \\
\left({ }^{\circ} \mathrm{C}\right)\end{array}$} & \multicolumn{2}{|c|}{$\begin{array}{c}\text { Fragments } \\
\text { Obtained From }\end{array}$} & \multirow[b]{2}{*}{$\begin{array}{l}\text { Number of } \\
\text { Fragments }\end{array}$} & \multirow{2}{*}{$\begin{array}{l}\text { Mean } \\
\text { Density } \\
\left(\mathrm{g} / \mathrm{cm}^{3}\right) \\
\end{array}$} & \multirow{2}{*}{$\begin{array}{c}\text { Density } \\
\text { Range } \\
\left(\mathrm{g} / \mathrm{cm}^{3}\right) \\
\end{array}$} & \multirow[b]{2}{*}{$\begin{array}{l}\text { Standard } \\
\text { Deviation } \\
\left(\mathrm{g} / \mathrm{cm}^{3}\right)\end{array}$} & \multirow[b]{2}{*}{$\begin{array}{c}\text { Densification } \\
(\%)\end{array}$} \\
\hline & & & & & & $\begin{array}{c}\text { Failed } \\
\text { Particles }\end{array}$ & $\begin{array}{c}\text { Intact } \\
\text { Particles }\end{array}$ & & & & & \\
\hline $6151-00-010-10$ & 1.80 & P13R & $3-1$ & 12.1 & 1015 & $\mathrm{x}$ & $\mathrm{x}$ & 13 & 2.07 & $2.01-2.09$ & 0.021 & +15.3 \\
\hline $6151-00-035-1$ & 1.85 & P13R & $3-4$ & 12.0 & 1075 & & $\mathrm{x}$ & 5 & 2.01 & $2.01-2.02$ & 0.007 & +8.6 \\
\hline $6151-04-015-1$ & 1.82 & P13R & $3-11$ & 11.7 & 960 & $\mathrm{x}$ & & 16 & 2.06 & $1.97-2.09$ & 0.025 & +13.2 \\
\hline $6151-04-015-1$ & 1.82 & P13R & $3-11$ & 11.7 & 960 & $\mathrm{x}$ & & 9 & 2.08 & $2.07-2.08$ & 0.003 & +14.3 \\
\hline $6151-01-015-2$ & 1.90 & P13R & $4-2$ & 11.5 & 1070 & $\mathrm{x}$ & & 31 & 2.01 & $1.92-2.03$ & 0.019 & +5.8 \\
\hline $6151-00-025-2$ & 1.88 & P13R & $4-4$ & 11.4 & 1070 & $\mathrm{x}$ & & 19 & 2.02 & $1.97 \cdot-2.09$ & 0.038 & +7.4 \\
\hline $6151-09-015-1$ & 1.94 & P13R & $4-7$ & 11.2 & 1070 & & $\mathrm{x}$ & 17 & 2.07 & $2.03-2.10$ & 0.019 & +6.7 \\
\hline $6151-08-015-1$ & 1.76 & P13s & $3-7$ & 11.7 & 1020 & & $\mathrm{x}$ & 10 & 2.01 & $2.00-2.02$ & 0.007 & +14.2 \\
\hline $6151-02-025-1$ & 1.50 & P13S & $3-10$ & 11.6 & 980 & $\mathrm{x}$ & & 20 & 2.12 & $1.84-2.16$ & 0.070 & +41.3 \\
\hline $6155-01-020-10$ & 1.81 & P13S & $4-4$ & 11.1 & 1090 & $\mathrm{x}$ & & 15 & 2.00 & $1.97-2.03$ & 0.002 & +10.5 \\
\hline
\end{tabular}

(a) Measured using a density gradient column. 
TABLE $4-51$

RESULTS OF DENSITY MEASUREMENTS OF UNBONDED FERTILE PARTICLE SAMPLES IRRADIATED IN CAPSULES P13R AND P13S

\begin{tabular}{|c|c|c|c|c|c|c|c|c|c|c|c|c|c|}
\hline \multicolumn{5}{|c|}{ Sample Description } & \multicolumn{4}{|c|}{ Preirradiation $^{(a)}$} & \multicolumn{5}{|c|}{ Postirradiation (a) } \\
\hline $\begin{array}{l}\text { Data } \\
\text { Retrieval } \\
\text { Number }\end{array}$ & Capsule & $\begin{array}{l}\text { Capsule } \\
\text { Position }\end{array}$ & 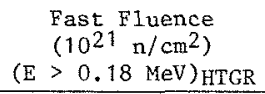 & $\begin{array}{c}\text { Burnup } \\
\text { (\% FTMA) }\end{array}$ & $\begin{array}{c}\text { No. of } \\
\text { Par- } \\
\text { ticles }\end{array}$ & $\begin{array}{l}\text { Mean } \\
\text { Density } \\
\left(\mathrm{g} / \mathrm{cm}^{3}\right)\end{array}$ & $\begin{array}{l}\text { Density } \\
\text { Range } \\
\left(\mathrm{g} / \mathrm{cm}^{3}\right)\end{array}$ & $\begin{array}{l}\text { Standard } \\
\text { Deviation } \\
\left(\mathrm{g} / \mathrm{cm}^{3}\right)\end{array}$ & $\begin{array}{c}\text { No. of } \\
\text { Par- } \\
\text { ticles }\end{array}$ & $\begin{array}{l}\text { Mean } \\
\text { Density } \\
\left(\mathrm{g} / \mathrm{cm}^{3}\right)\end{array}$ & $\begin{array}{l}\text { Density } \\
\text { Range } \\
\left(\mathrm{g} / \mathrm{cm}^{3}\right) \\
\end{array}$ & $\begin{array}{l}\text { Standard } \\
\text { Deviation } \\
\left(\mathrm{g} / \mathrm{cm}^{3}\right)\end{array}$ & $\begin{array}{c}\text { Densification } \\
(\%)\end{array}$ \\
\hline $4252-06-018-1$ & P13R & $3-1$ & 12.1 & 5.1 & 25 & 3.72 & $3.69-3.97$ & 0.053 & 30 & 3.98 & $3.96-4.01$ & 0.010 & +7.0 \\
\hline $4252-06-018-2$ & P13R & $3-2$ & 12.1 & 5.1 & 25 & 3.72 & $3.69-3.97$ & 0.053 & 30 & 4.00 & $3.99-4.19$ & 0.050 & +7.5 \\
\hline $6542-19-016-1$ & P13S & $4-8$ & 10.9 & 3.7 & $\mathrm{ND}^{(\mathrm{b})}$ & 3.45 & ND & ND & 33 & 3.98 & $3.96-3.98$ & 0.005 & +15.4 \\
\hline $6542-21-016-1$ & P13S & $4-9$ & 10.9 & 3.7 & $\mathrm{ND}$ & 3.41 & $\mathrm{ND}$ & ND & 11 & 3.94 & $3.85-3.96$ & 0.034 & +15.5 \\
\hline
\end{tabular}

(a) Measured using a density gradient column.

(b) $\mathrm{ND}=$ not determined. 


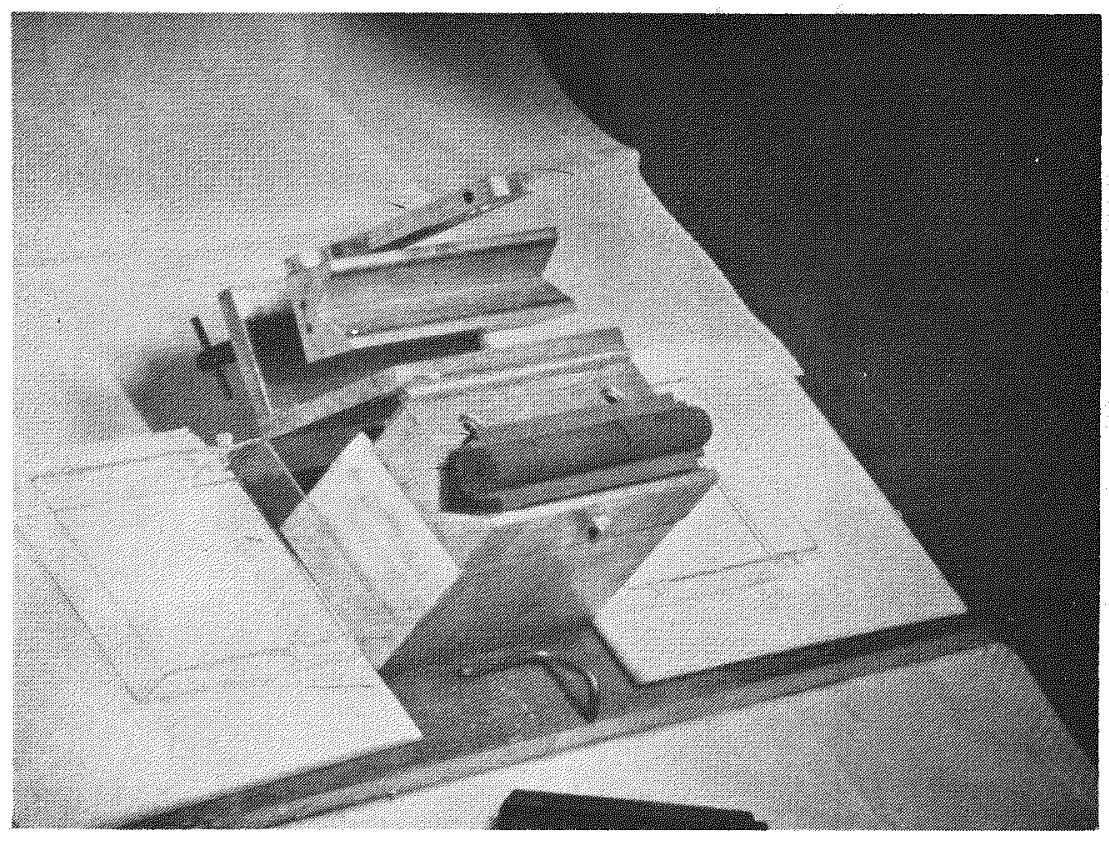

K7440-80b

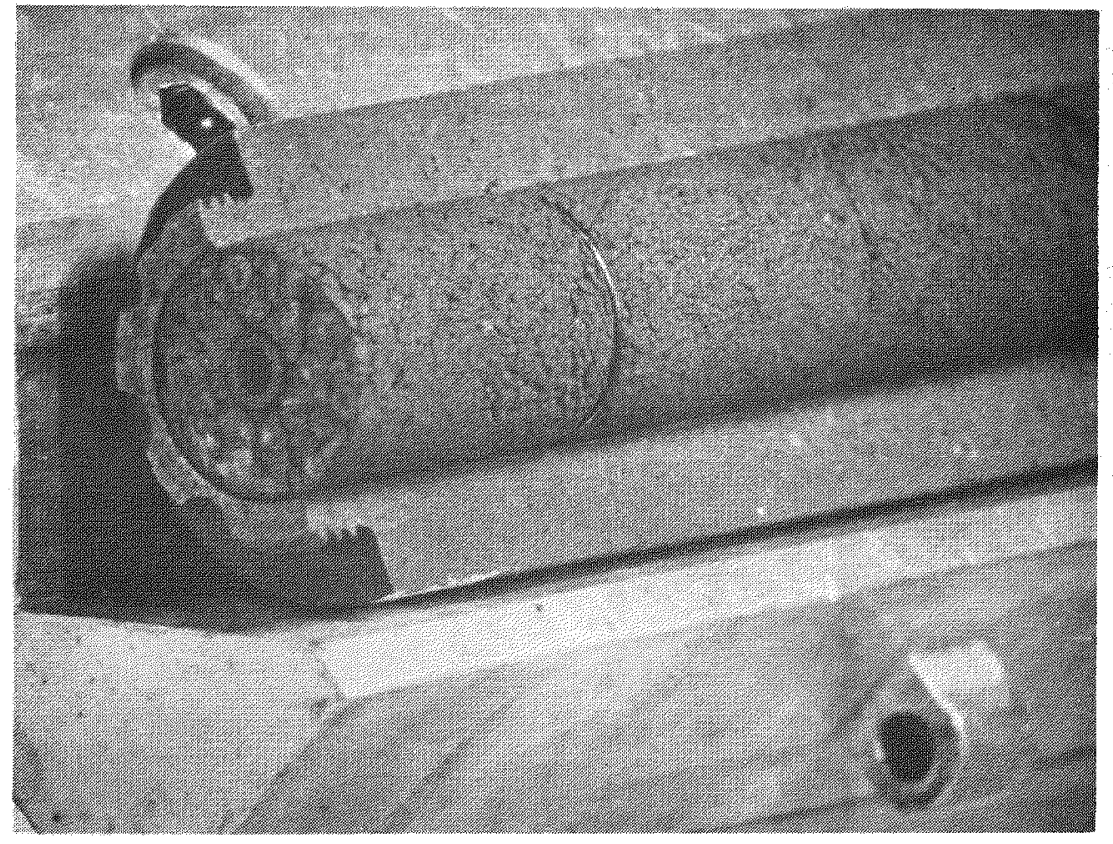

$\mathrm{K} 7440-81$

Fig. 4-1. Photomicrographs showing P13S cel1 No. 2 fuel rods during removal from split graphite crucible 


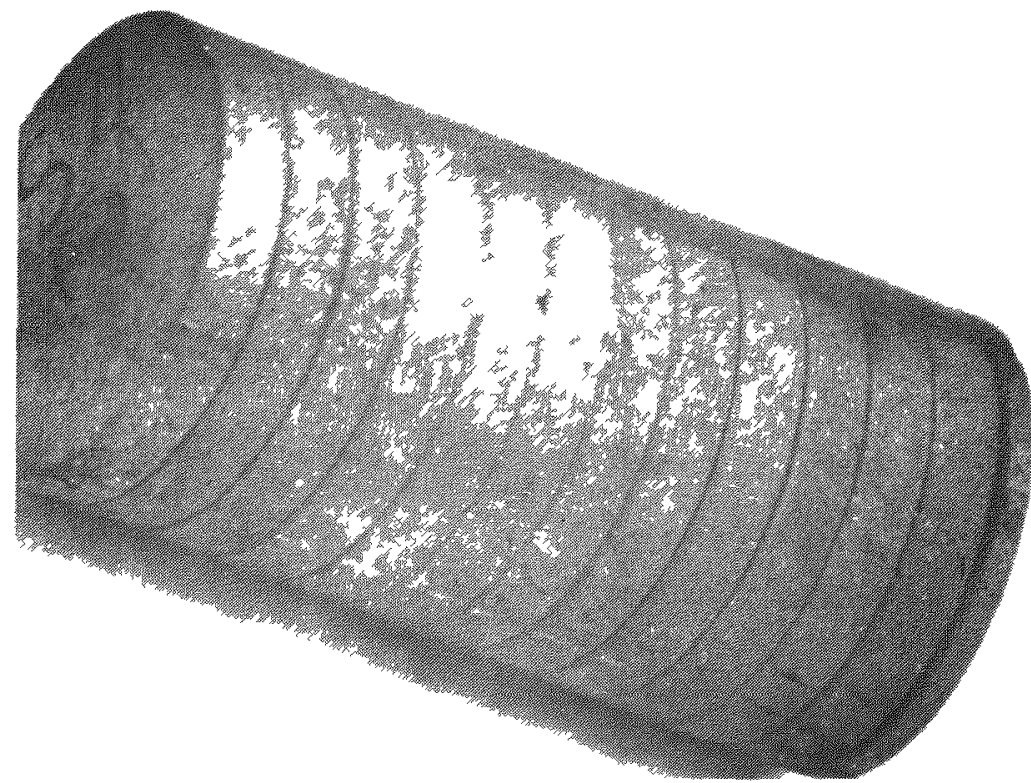

K.7425-95

(a)

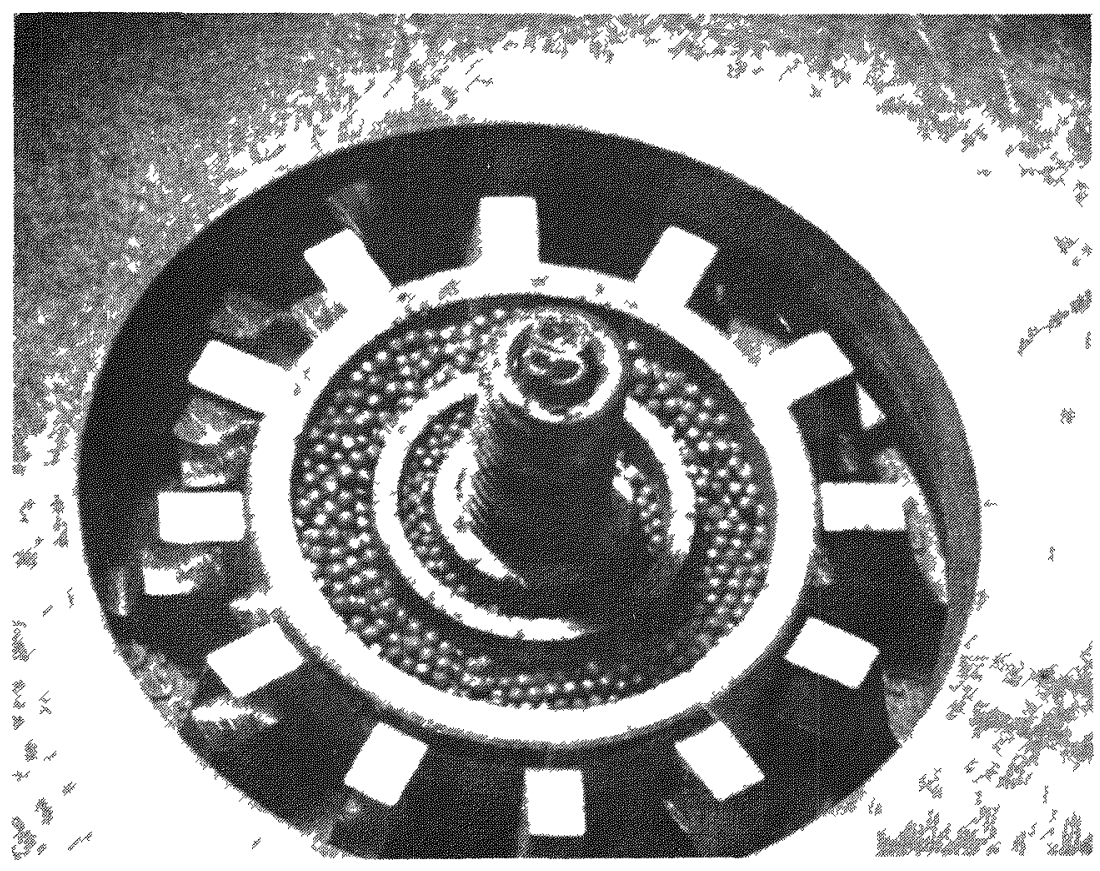

K7425-102

(b)

Fig. 4-2. Representative photomicrographs showing (a) unbonded particle crucible assembly after removal from primary containment, and (b) unloading of unbonded particle samples from crucible 


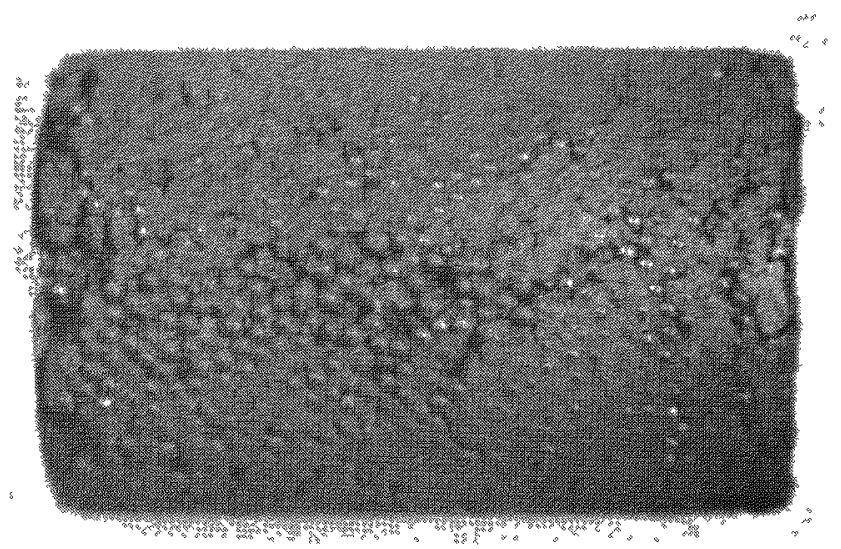

$57440-11$

$0.5 \mathrm{~cm}$

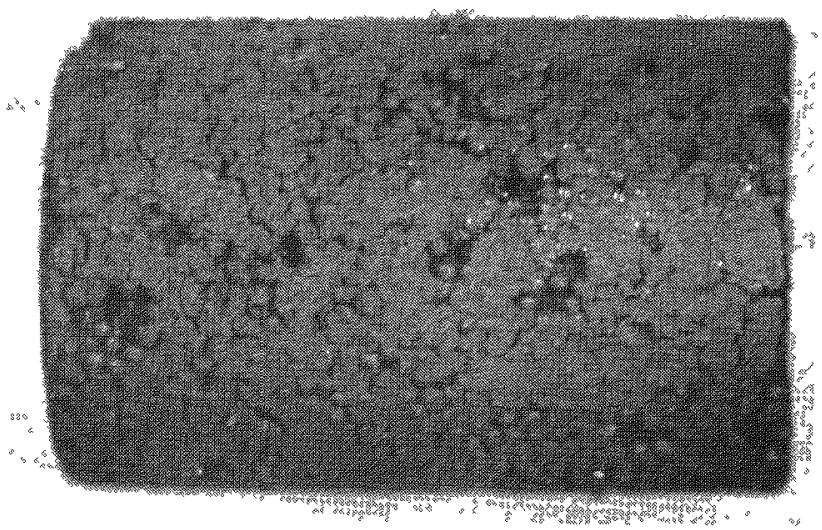

$57440-101$

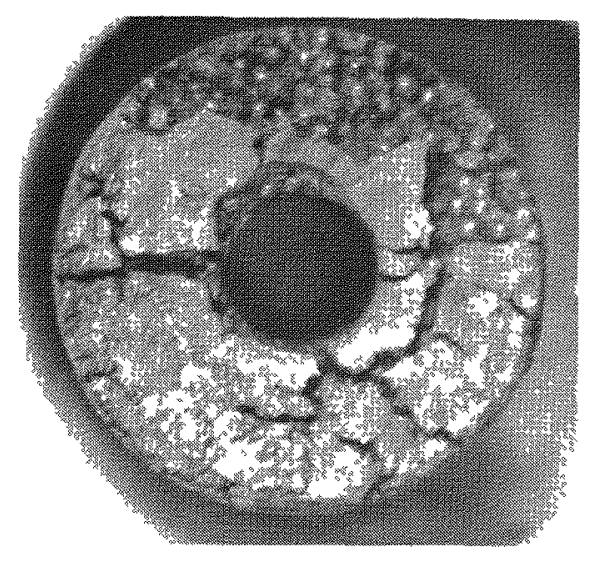

$57440-9$

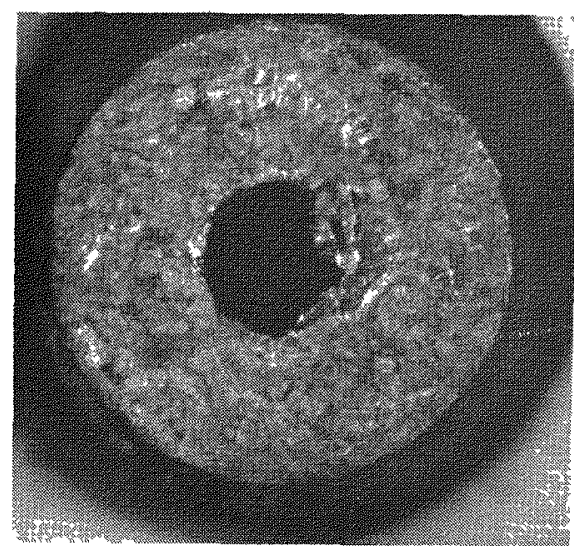

$57440-98$

Fig. 4-3. Photomicrographs of fuel rod 7161-004-01-5 (top) after irradiation in capsule P13R (position 1A) to a fast neutron fluence of

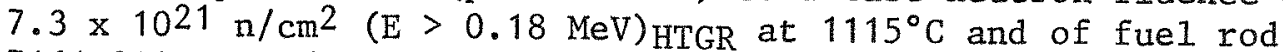
7161-004-01-7 (bottom) after irradiation in capsule P13S (position 1A) to a fast neutron fluence of $7.0 \times 1021 \mathrm{n} / \mathrm{cm}^{2}$ (E $>0.18 \mathrm{MeV})_{\text {HTGR }}$. Cell No. 1 of capsule P13S was therma1 cycled 24 times during irradiation. 


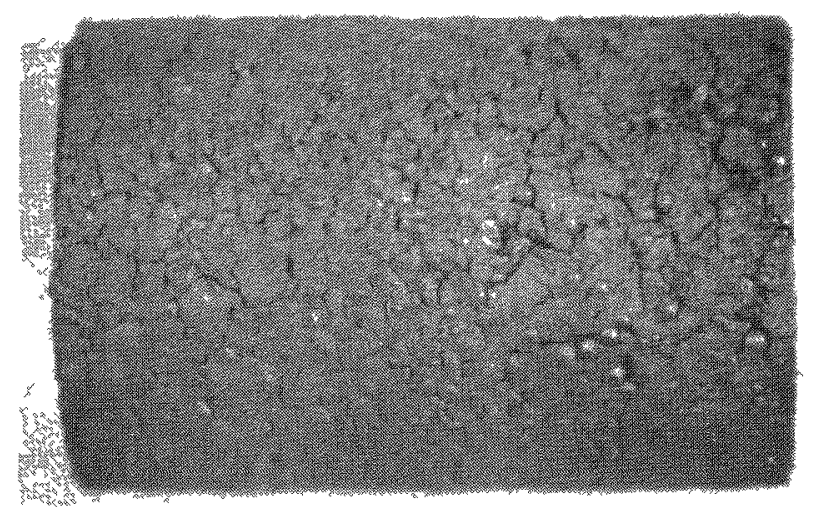

$57440-7$

\section{$0.5 \mathrm{~cm}$}

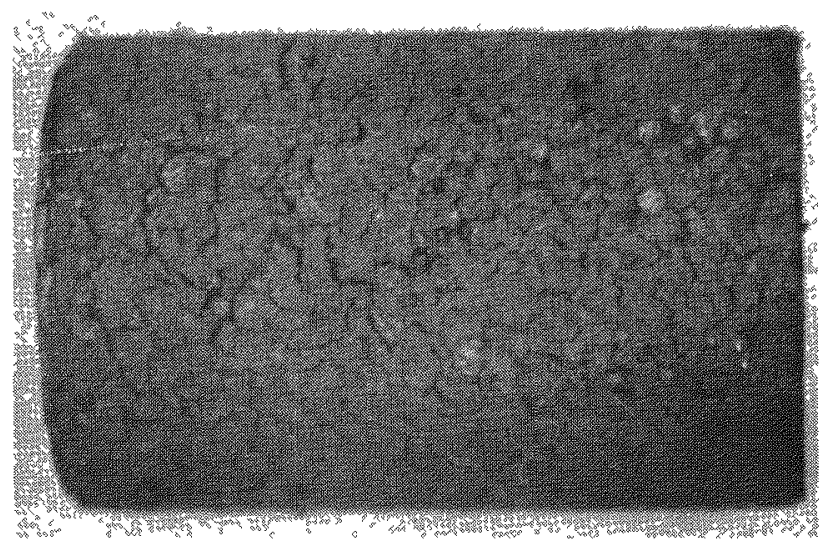

$57440-104$

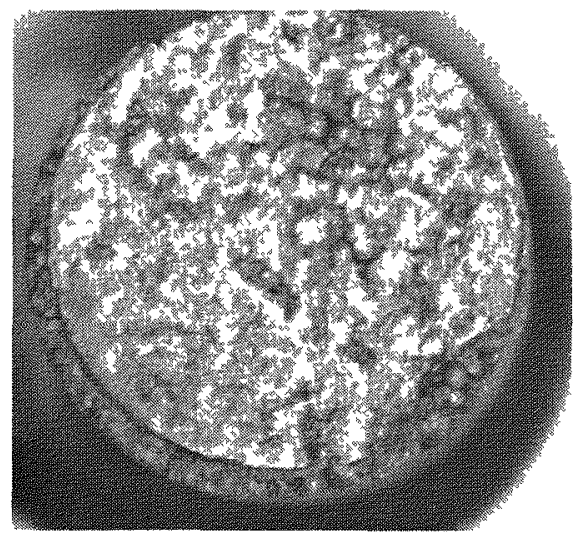

$57440-5$

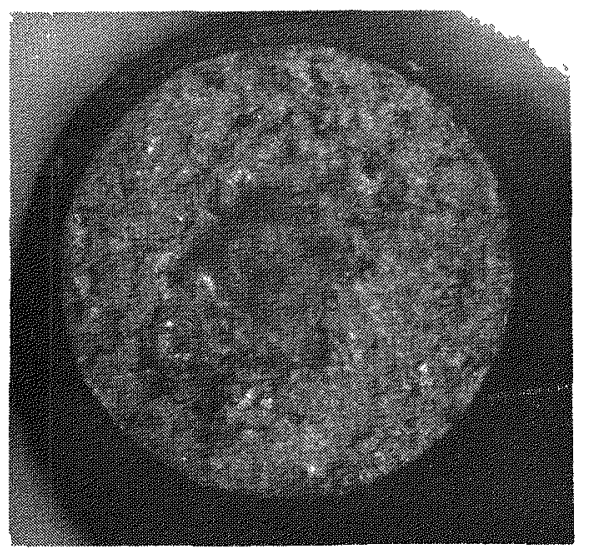

S7440-103

Fig. 4-4. Photomicrographs of fuel rod 7161-004-02-6 (top) after irradiation in capsule P13R (position 1B) to a fast neutron fluence of $8.1 \times 1021 \mathrm{n} / \mathrm{cm}^{2}(\mathrm{E}>0.18 \mathrm{MeV})_{\mathrm{HTGR}}$ at $1050^{\circ} \mathrm{C}$ and of fuel rod 7161-004-02-5 (bottom) after irradiation in capsule P13S (position $1 \mathrm{~B}$ ) to a fast neutron fluence of $7.7 \times 10^{21} \mathrm{n} / \mathrm{cm}^{2}$ $(E>0.18 \mathrm{MeV})_{\mathrm{HTGR}}$. Cell No. 1 of capsule P13S was thermal cycled 24 times during irradiation. 


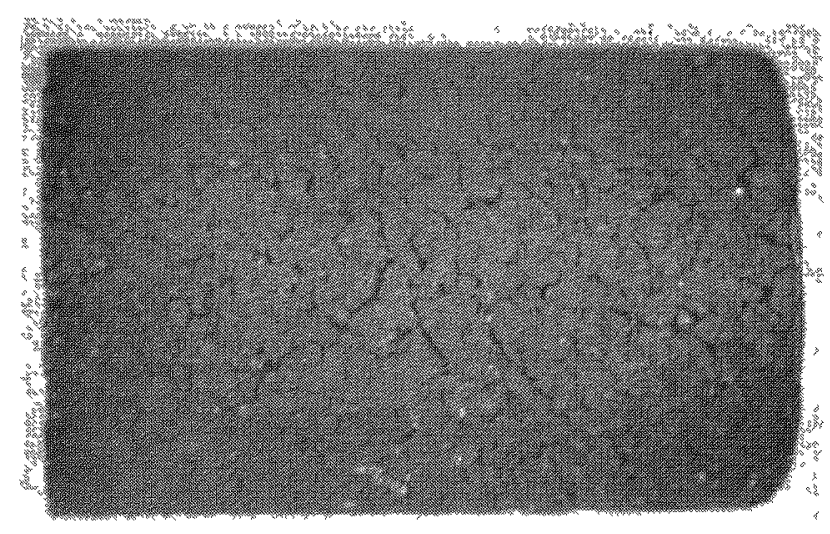

$57440-20$

$$
0.5 \mathrm{~cm}
$$

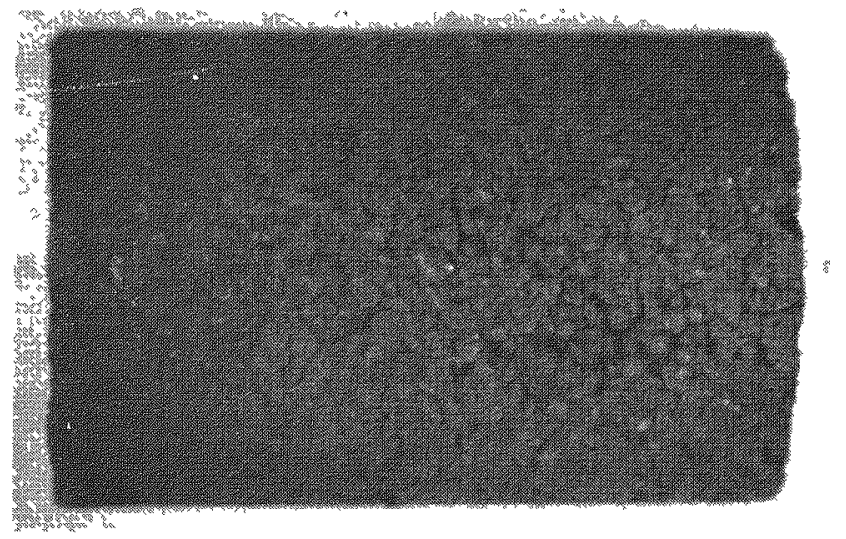

$57440-108$

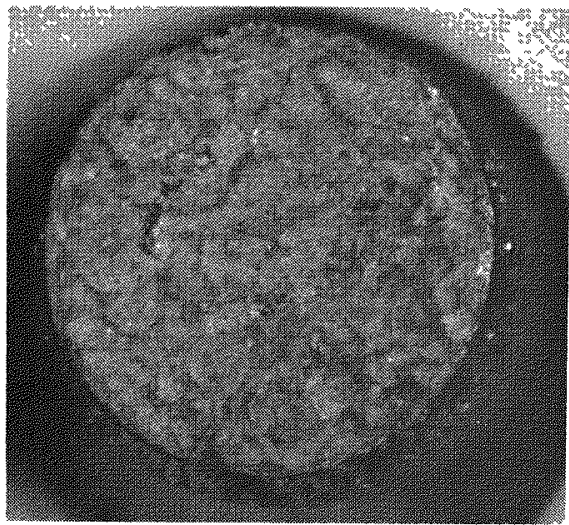

$57440-19$

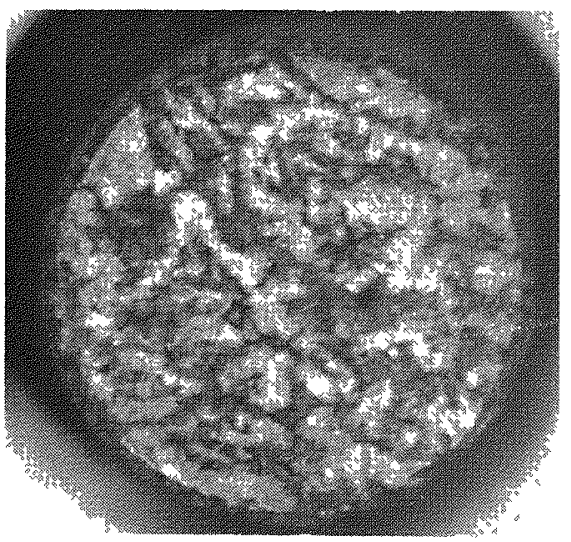

S7440-107

Fig. 4-5. Photomicrographs of fuel rod 7161-004-03-5 (top) after irradiation in capsule P13R (position 1C) to a fast neutron fluence of $8.7 \times 10^{21} \mathrm{n} / \mathrm{cm}^{2}$ (E $>0.18 \mathrm{MeV}$ ) HTGR at $1065^{\circ} \mathrm{C}$ and of fuel rod 7161-004-03-6 (bottom) after irradiation in capsule P13S (position 1C) to a fast neutron fluence of $8.5 \times 10^{21} \mathrm{n} / \mathrm{cm}^{2}$ $(\mathrm{E}>0.18 \mathrm{MeV})_{\text {HTGR }}$. Cell No. 1 of capsule P13S was thermal cycled 24 times during irradiation. 


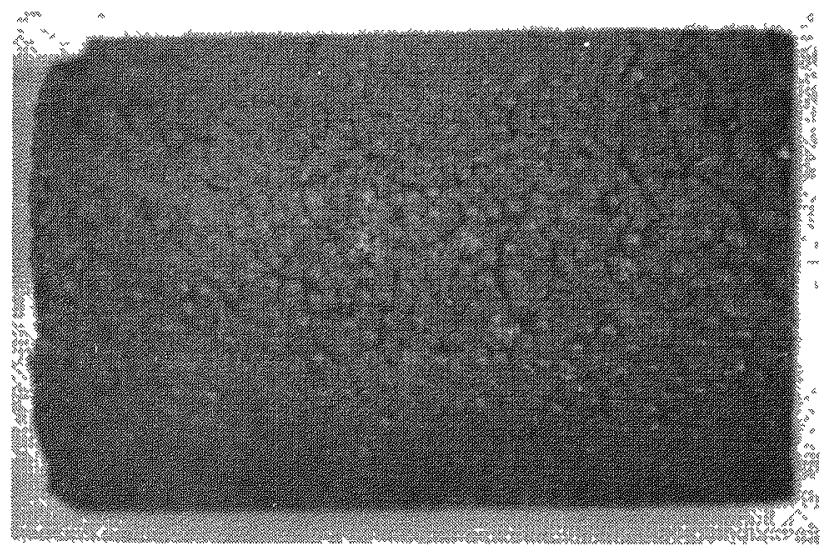

$57440-15$
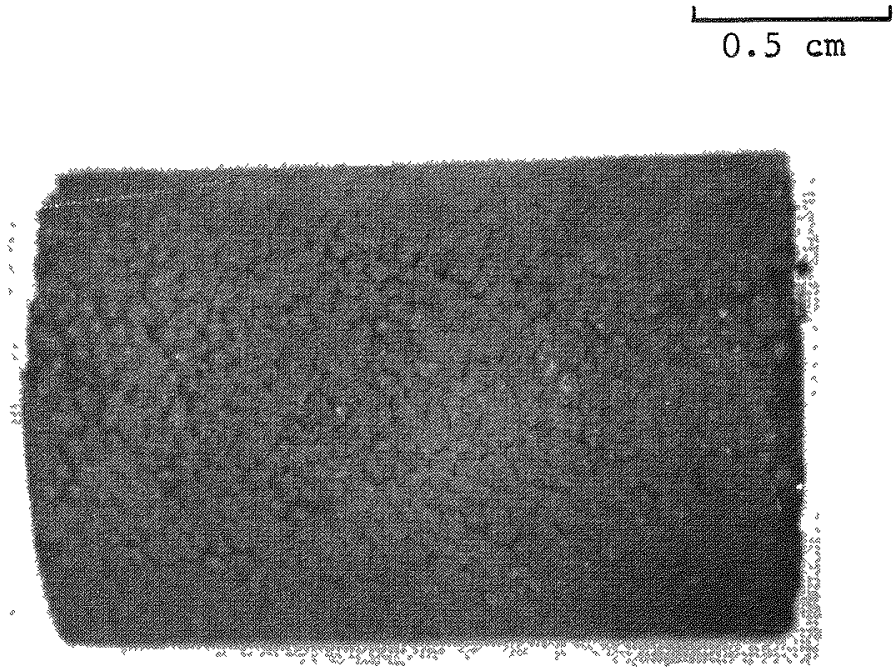

$57440-114$

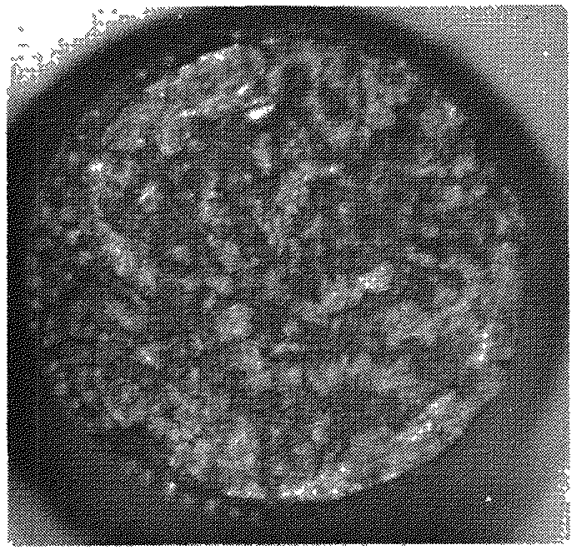

$57440-13$

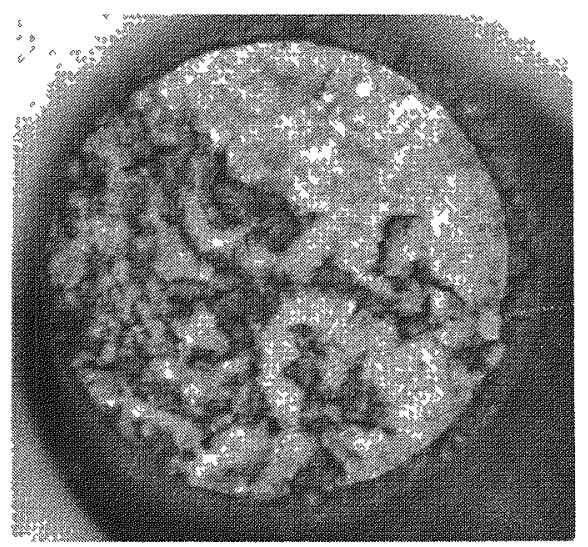

$57440-111$

Fig. 4-6. Photomicrographs of fuel rod 7161-004-04-6 (top) after irradiation in capsule P13R (position 1D) to a fast neutron fluence of $9.3 \times 10^{21} \mathrm{n} / \mathrm{cm}^{2}(\mathrm{E}>0.18 \mathrm{MeV})_{\mathrm{HTGR}}$ at $1115^{\circ} \mathrm{C}$ and of fuel rod 7161-004-04-5 (bottom) after irradiation in capsule P13S (position 1D) to a fast neutron fluence of $9.1 \times 10^{21} \mathrm{n} / \mathrm{cm}^{2}$ $(E>0.18 \mathrm{MeV})_{\text {HTGR }}$. Cell No. 1 of capsule P13S was therma1 cycled 24 times during irradiation. 


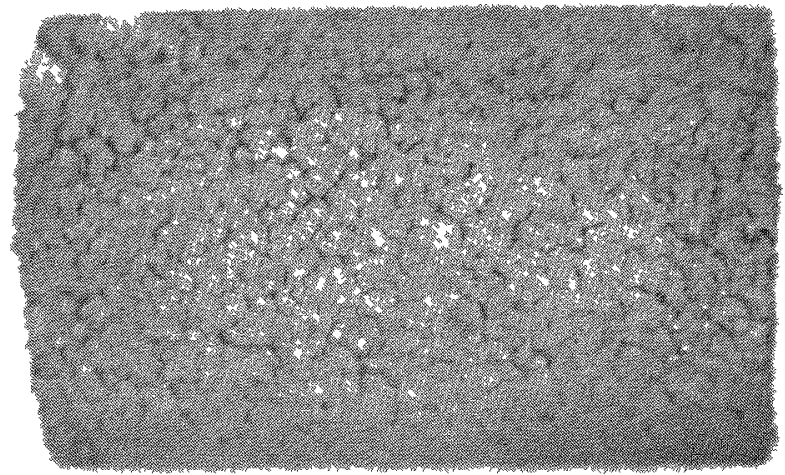

S7440-1

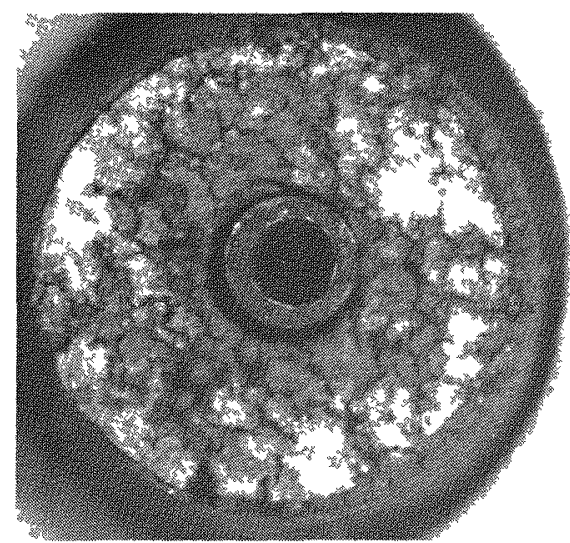

$57440-3$

$$
0.5 \mathrm{~cm}
$$

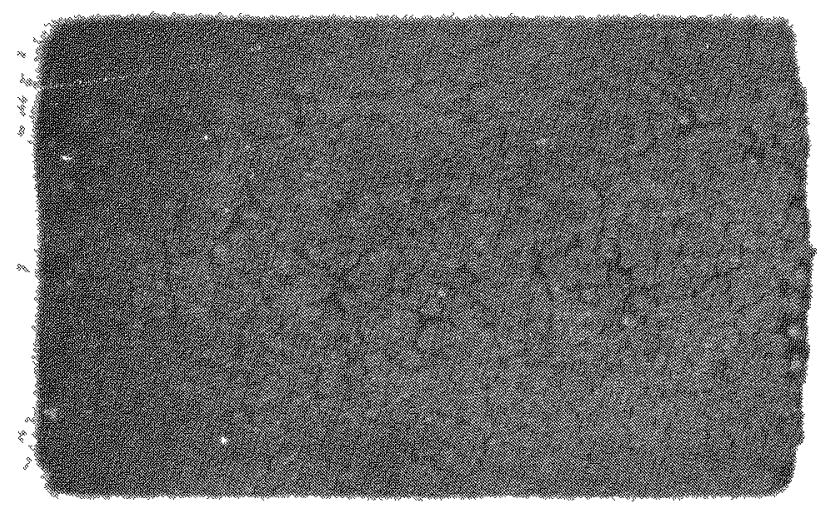

$57440-117$

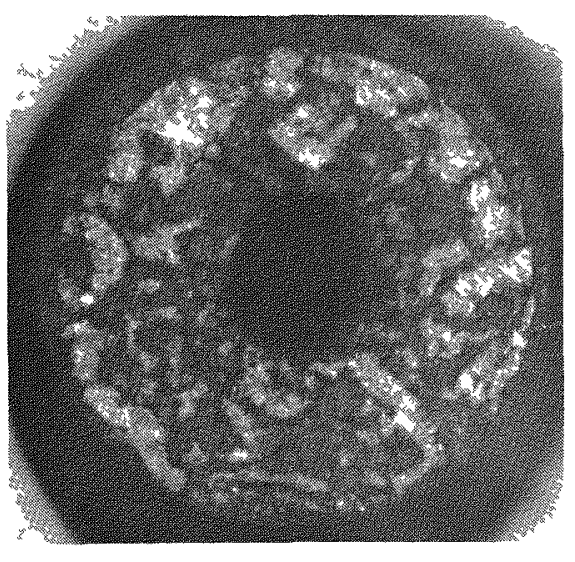

$57440-116$

Fig. 4-7. Photomicrographs of fuel rod 7161-004-05-6 (top) after irradiation in capsule P13R (position $1 \mathrm{E}$ ) to a fast neutron fluence of $9.9 \times 10^{21} \mathrm{n} / \mathrm{cm}^{2}(\mathrm{E}>0.18 \mathrm{MeV})$ HTGR at $1125^{\circ} \mathrm{C}$ and of fuel rod 7161-004-05-5 (bottom) after irradiation in capsule P13S (position 1E) to a fast neutron fluence of $9.6 \times 10^{21} \mathrm{n} / \mathrm{cm}^{2}$ $(\mathrm{E}>0.18 \mathrm{MeV})_{\mathrm{HTGR}}$. Cell No. 1 of capsule P13S was thermal cycled 24 times during irradiation. 


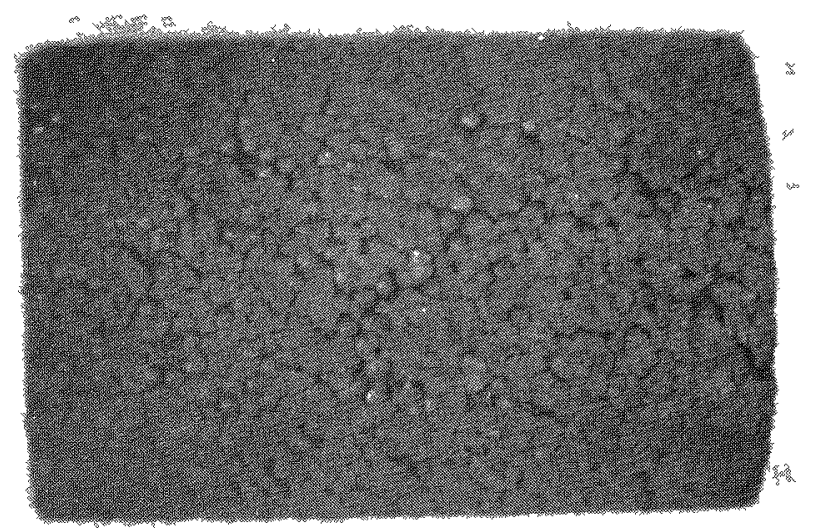

$57440-26$

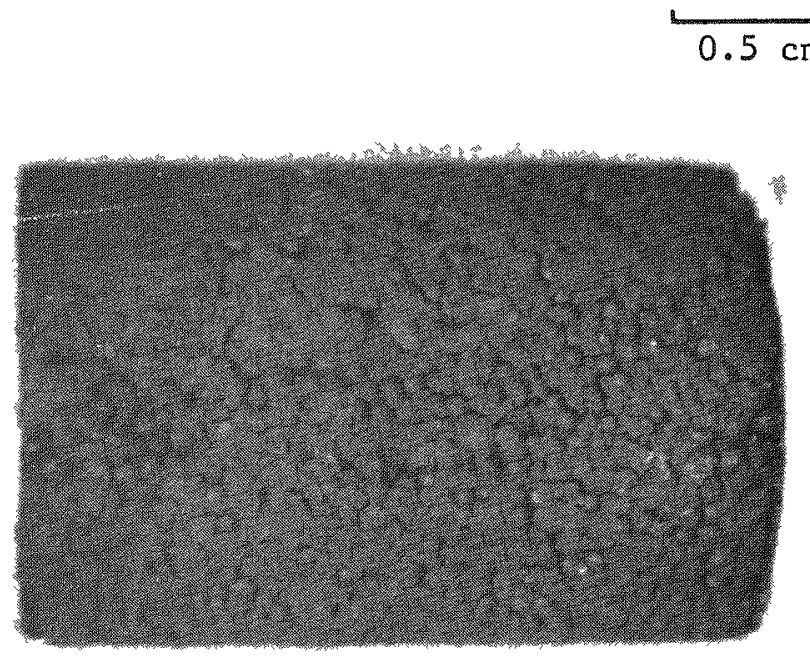

$57440-122$

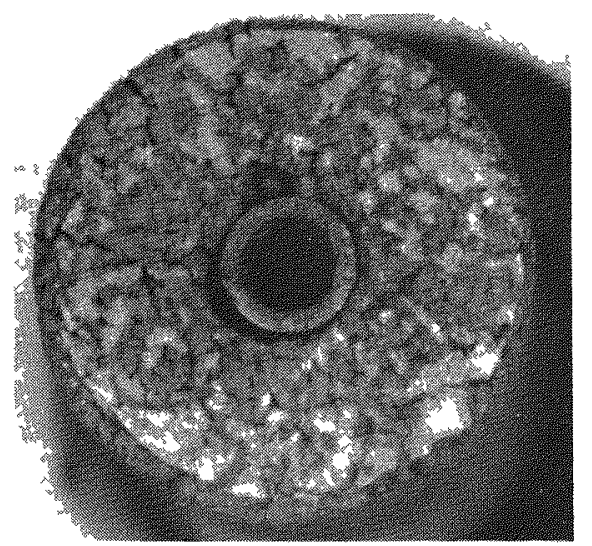

$57440-24$

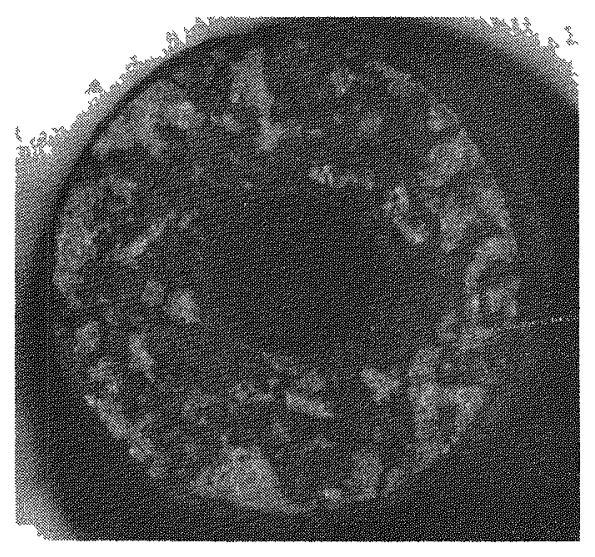

$57440-121$

Fig. 4-8. Photomicrographs of fuel rod 7161-004-06-5 (top) after irradiation in capsule $\mathrm{P} 13 \mathrm{R}$ (position 2A) to a fast neutron fluence of $11.8 \times 10^{21} \mathrm{n} / \mathrm{cm}^{2}$ (E $>0.18 \mathrm{MeV}$ ) HTGR at $1050^{\circ} \mathrm{C}$ and of fuel rod 7161-004-11-6 (bottom) after irradiation in capsule P13S (position 2A) to a fast neutron fluence of $11.5 \times 1021 \mathrm{n} / \mathrm{cm}^{2}$ $(\mathrm{E}>0.18 \mathrm{MeV})_{\mathrm{HTGR}}$ at $1040^{\circ} \mathrm{C}$ 


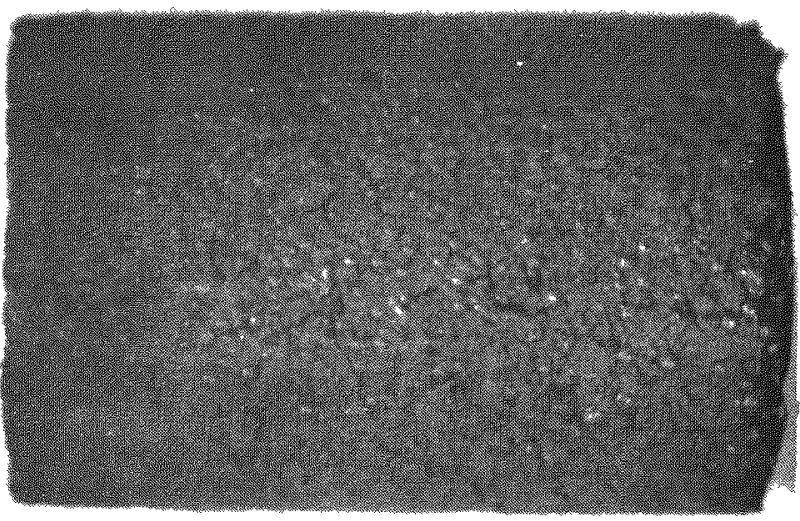

$57440-53$

$$
0.5 \mathrm{~cm}
$$

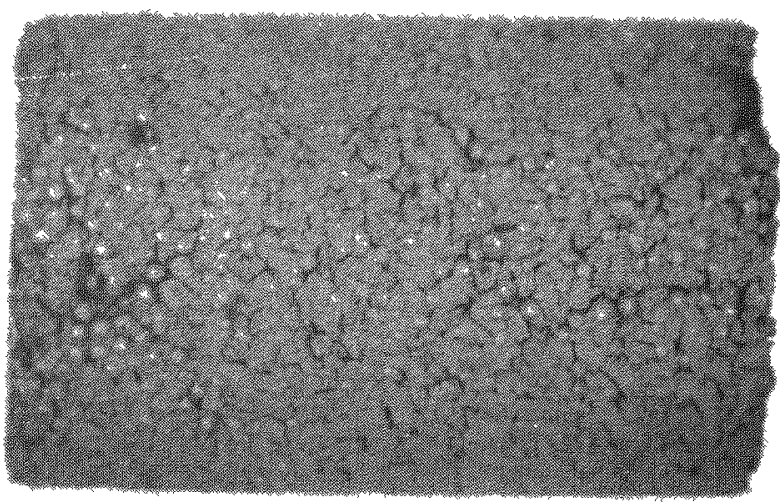

$57440-127$

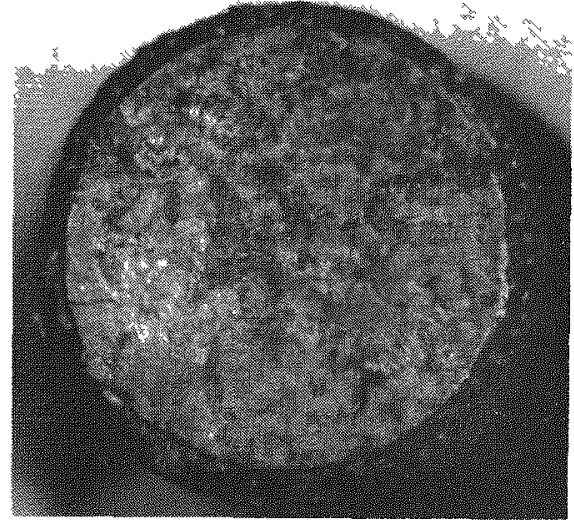

$57440-52$

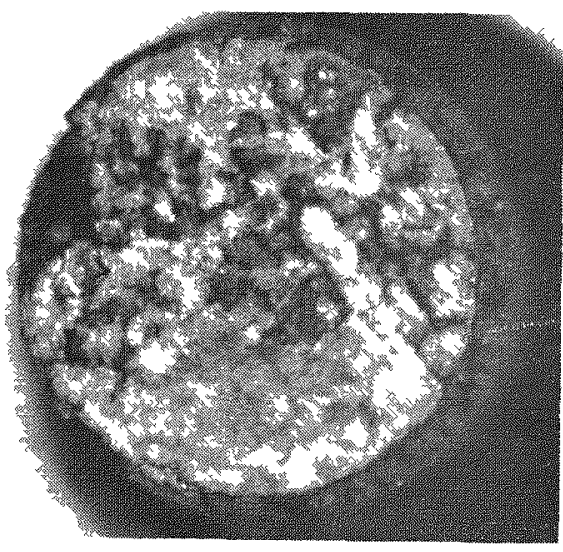

$57440-125$

Fig. 4-9. Photomicrographs of fuel rod 7161-004-07-5 (top) after irradiation in capsule P13R (position $2 \mathrm{~B}$ ) to a fast neutron fluence of $12.0 \times 10^{21} \mathrm{n} / \mathrm{cm}^{2}(\mathrm{E}>0.18 \mathrm{MeV})_{\mathrm{HTGR}}$ at $1055^{\circ} \mathrm{C}$ and of fuel rod 7161-004-12-5 (bottom) after irradiation in capsule P13S (position 2B) to a fast neutron fluence of $11.7 \times 10^{21} \mathrm{n} / \mathrm{cm}^{2}$ $(\mathrm{E}>0.18 \mathrm{MeV})_{\mathrm{HTGR}}$ at $1010^{\circ} \mathrm{C}$ 


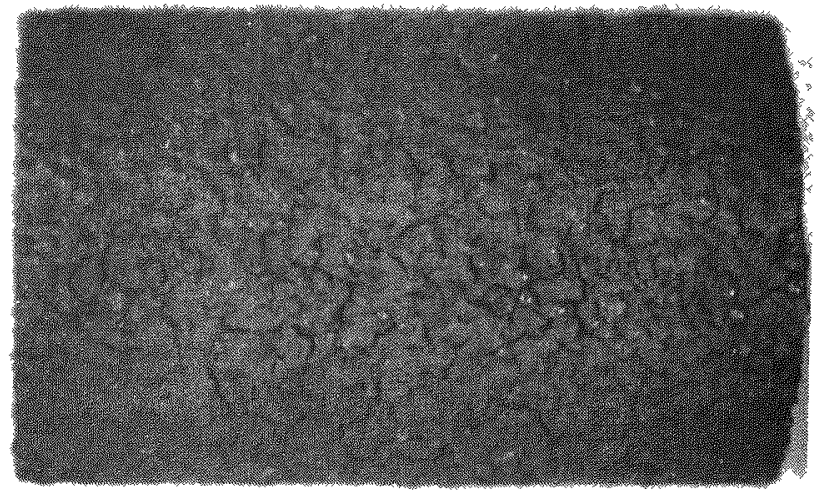

$57440-58$

\section{$0.5 \mathrm{~cm}$}

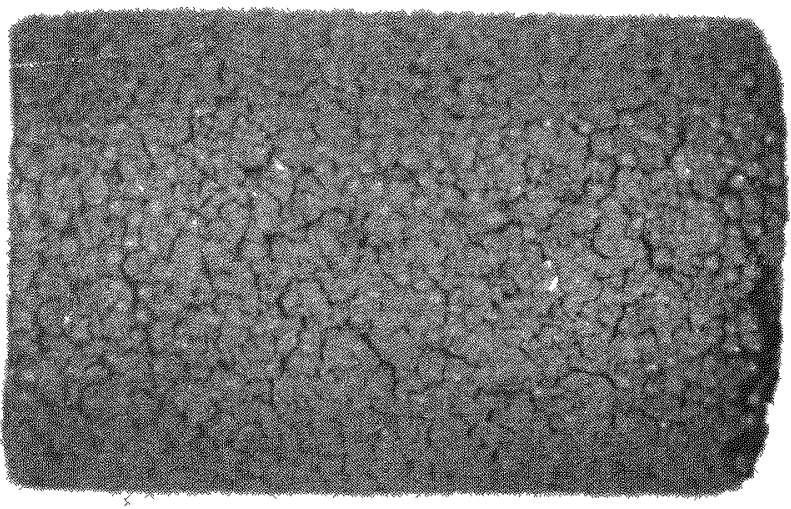

$57440-130$

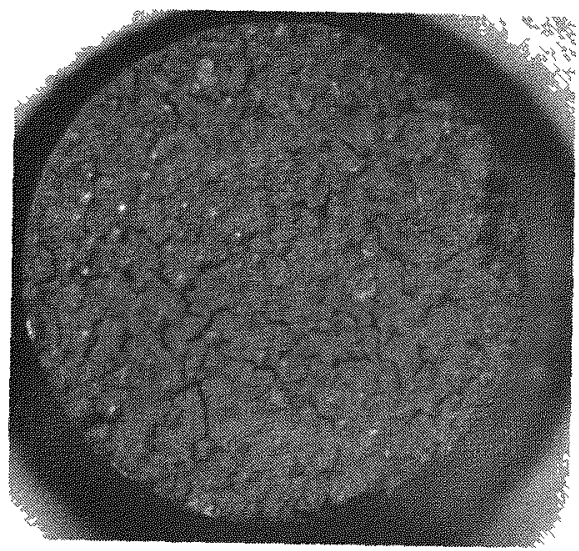

$57440-56$

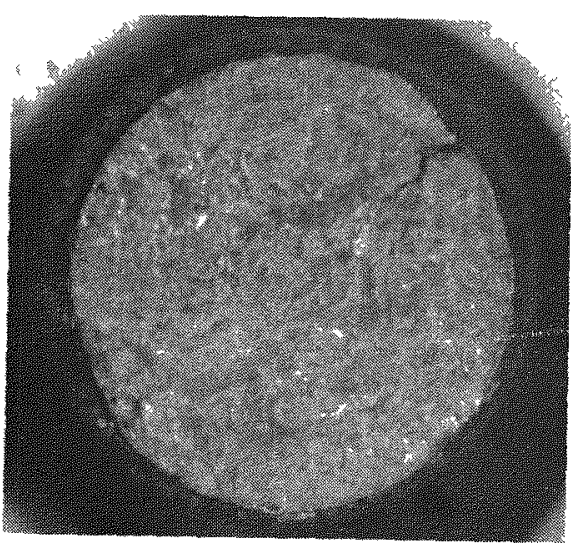

S7440-129

Fig. 4-10. Photomicrographs of fuel rod 7161-004-08-013 (top) after irradiation in capsule P13R (position 2C) to a fast neutron fluence of

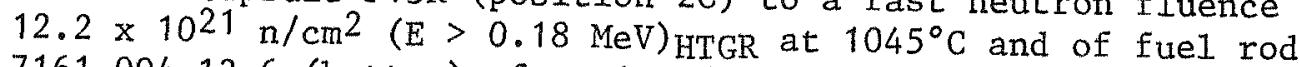
7161-004-13-6 (bottom) after irradiation in capsule P13S

(position 2C) to a fast neutron fluence of $11.9 \times 10^{21} \mathrm{n} / \mathrm{cm}^{2}$ $(E>0.18 \mathrm{MeV})_{\mathrm{HTGR}}$ at $975^{\circ} \mathrm{C}$ 


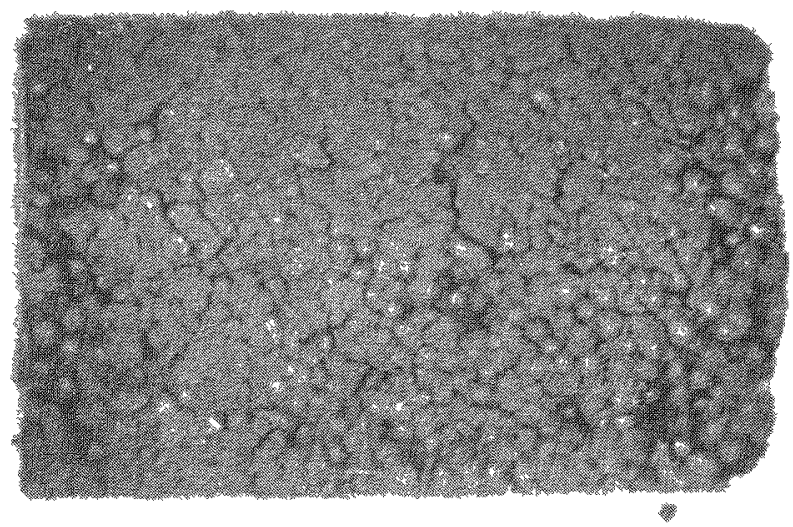

$57440-63$

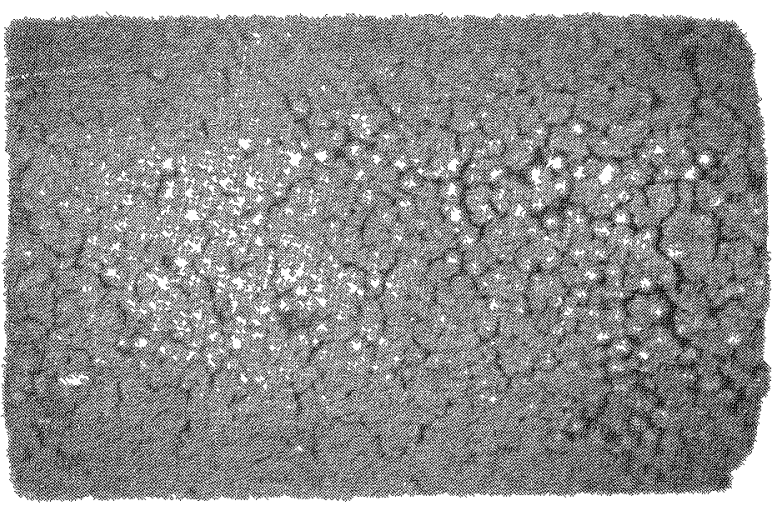

$57440-134$

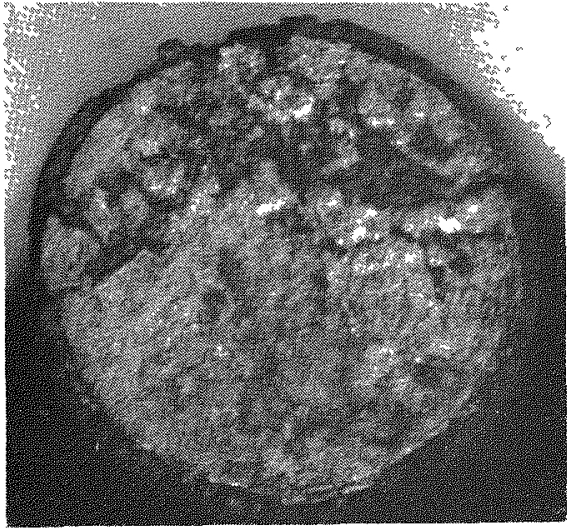

$57440-60$

\section{$0.5 \mathrm{~cm}$}

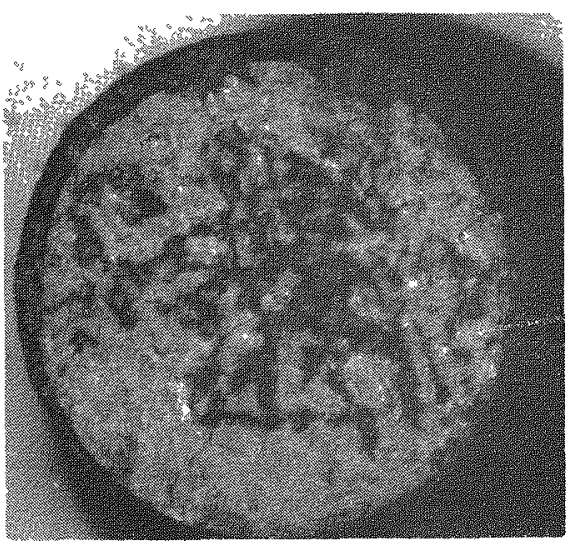

$\$ 7440-132$

Fig. 4-11. Photomicrographs of fuel rod 7161-004-09-5 (top) after irradiation in capsule P13R (position 2D) to a fast neutron fluence of $12.3 \times 1021 \mathrm{n} / \mathrm{cm}^{2}$ (E $\left.>0.18 \mathrm{MeV}\right)_{\mathrm{HTGR}}$ at $1035^{\circ} \mathrm{C}$ and of fuel rod 7161-004-14-5 (bottom) after irradiation in capsule P13S (position 2D) to a fast neutron fluence of $12.0 \times 10^{21} \mathrm{n} / \mathrm{cm}^{2}$ (E $>0.18 \mathrm{MeV})_{\mathrm{HTGR}}$ at $985^{\circ} \mathrm{C}$ 


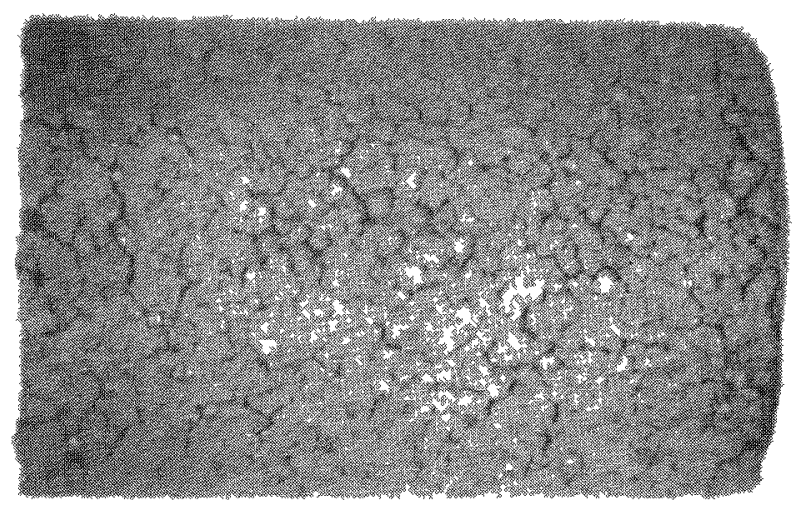

$57440-66$

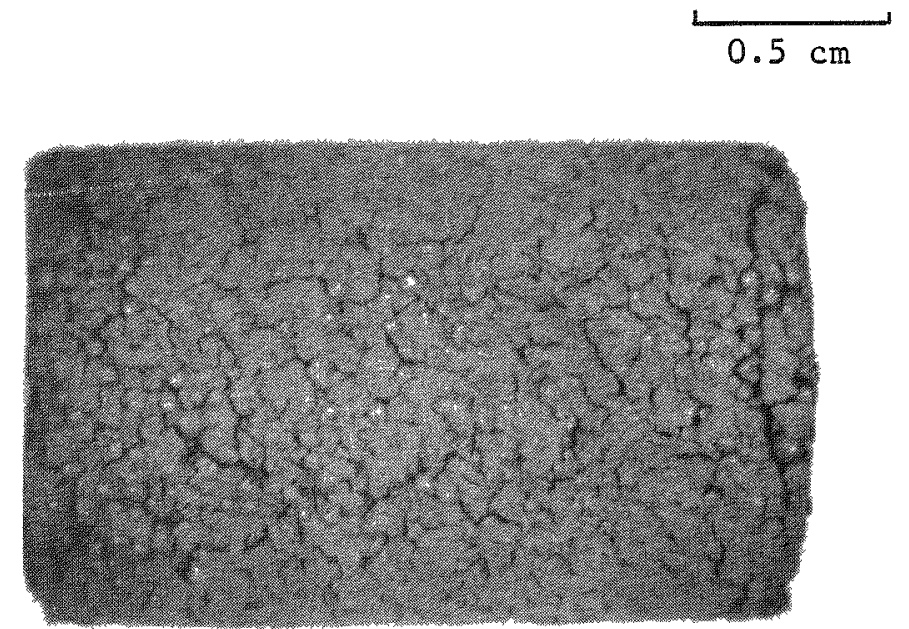

$57440-138$

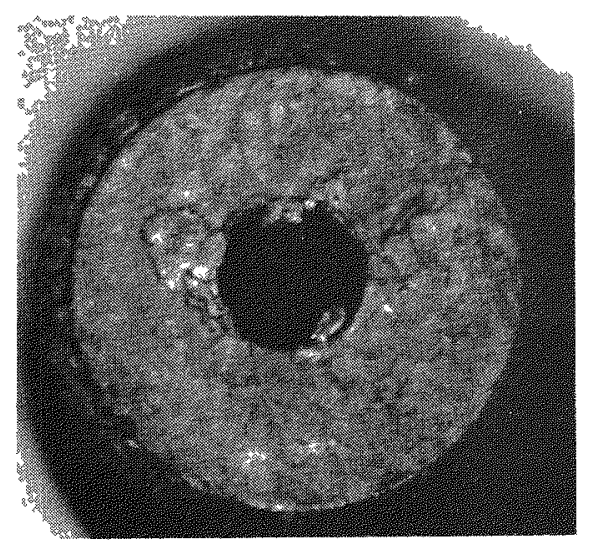

$57440-64$

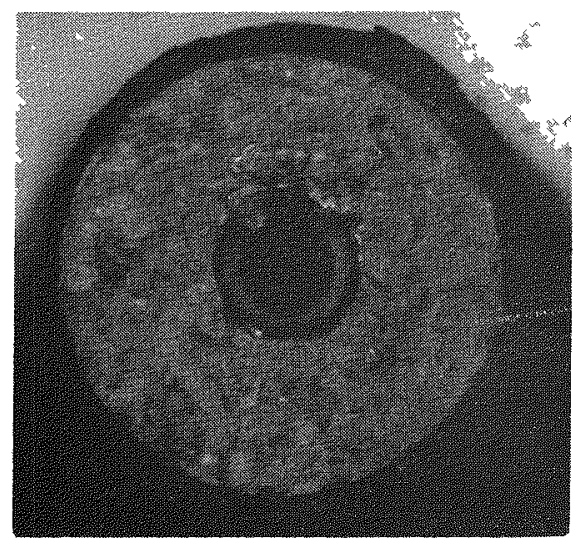

$57440-136$

Fig. 4-12. Photomicrographs of fuel rod 7161-004-10-5 (top) after irradiation in capsule P13R (position 2E) to a fast neutron fluence of $12.4 \times 10^{21} \mathrm{n} / \mathrm{cm}^{2}$ ( $\mathrm{E}>0.18 \mathrm{MeV}$ ) HTGR at $965^{\circ} \mathrm{C}$ and of fuel rod 7161-004-15-5 (bottom) after irradiation in capsule P13S (position 2E) to a fast neutron fluence of $12.1 \times 10^{21} \mathrm{n} / \mathrm{cm}^{2}$ $(\mathrm{E}>0.18 \mathrm{MeV})_{\mathrm{HTGR}}$ at $960^{\circ} \mathrm{C}$ 


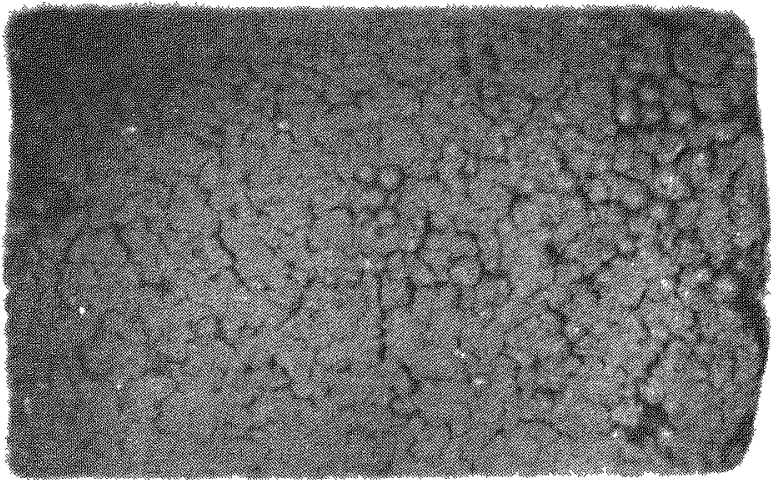

$57440-73$

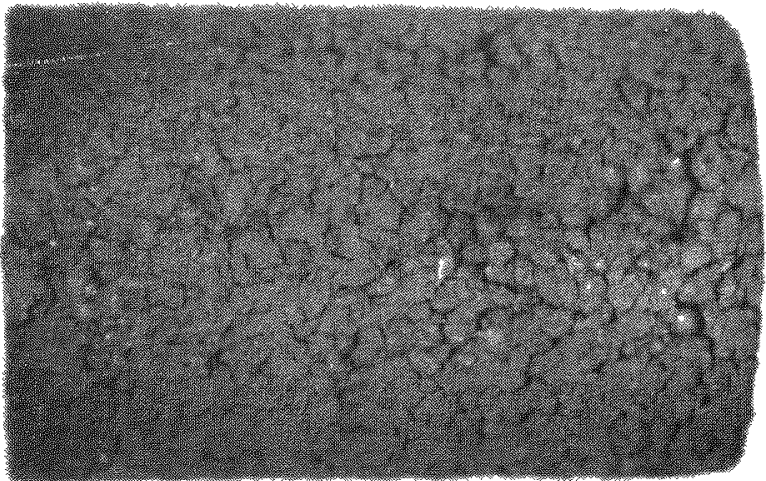

S7440-145

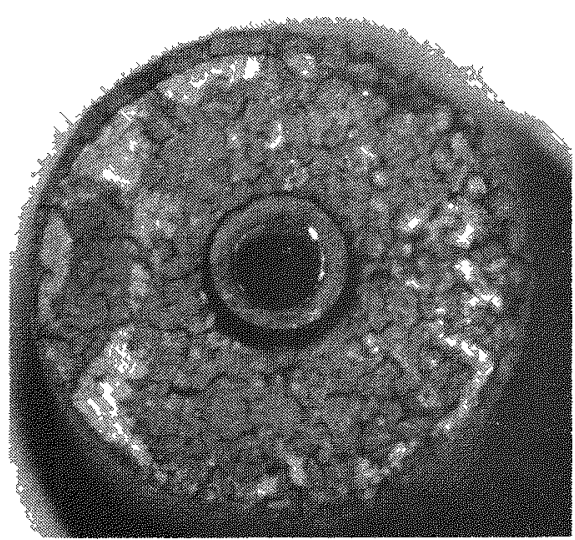

$57440-68$

\section{$0.5 \mathrm{~cm}$}

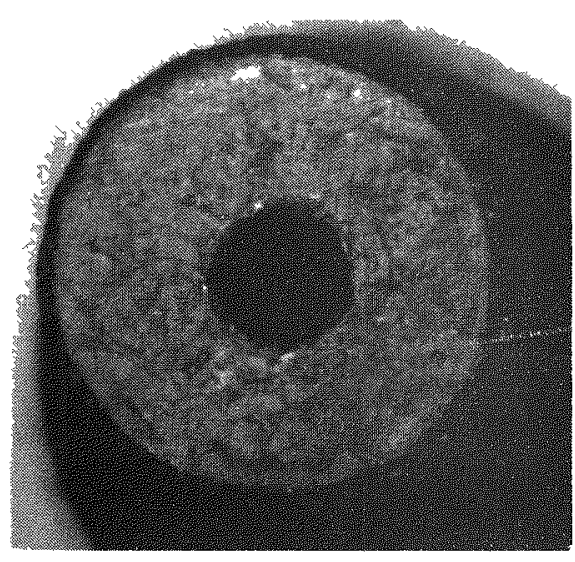

S7440-142

Fig. 4-13. Photomicrographs of fuel rod 7161-004-16-5 (top) after irradiation in capsule P13R (position 5A) to a fast neutron fluence of $9.4 \times 10^{21} \mathrm{n} / \mathrm{cm}^{2}\left(\mathrm{E}>0.18 \mathrm{MeV}\right.$ ) HTGR at $1285^{\circ} \mathrm{C}$ and of fuel rod 7161-004-16-6 (bottom) after irradiation in capsule P13S (position $5 \mathrm{~A}$ ) to a fast neutron fluence of $9.2 \times 10^{21} \mathrm{n} / \mathrm{cm}^{2}$ $(\mathrm{E}>0.18 \mathrm{MeV})_{\mathrm{HTGR}}$ at $1375^{\circ} \mathrm{C}$ 


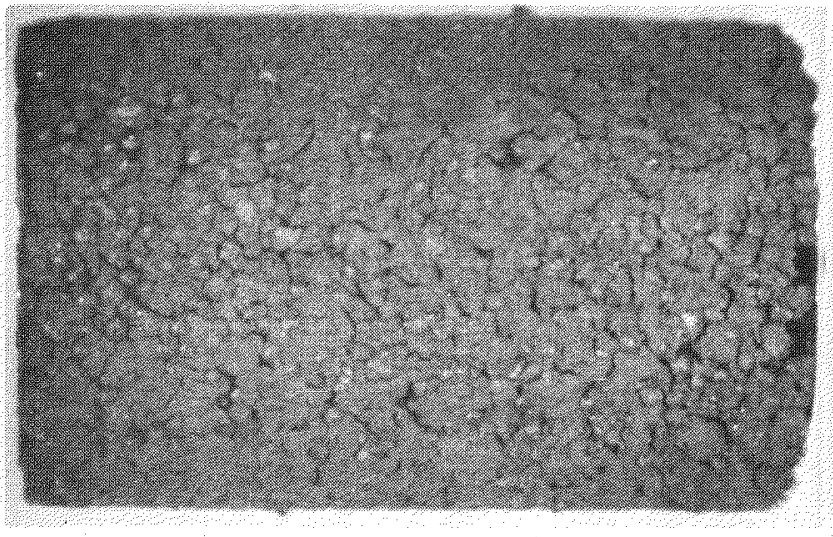

$57440-80$

\section{$0.5 \mathrm{~cm}$}

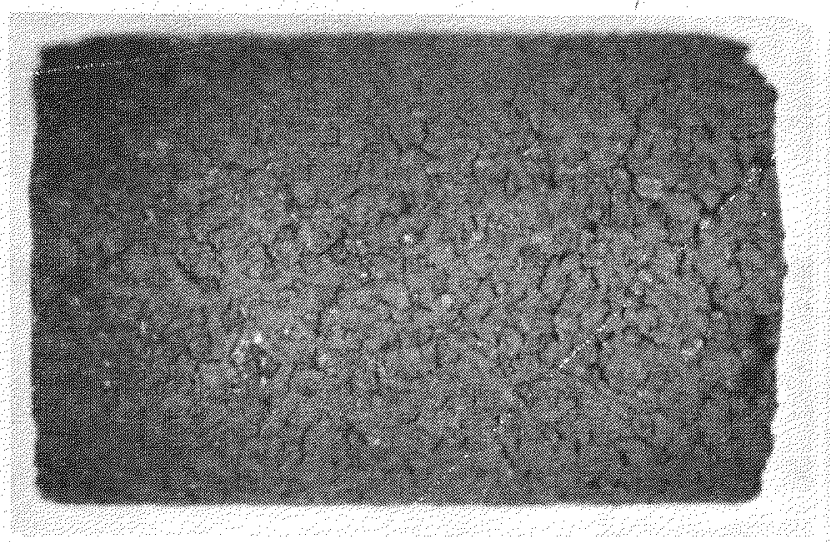

$57440-150$

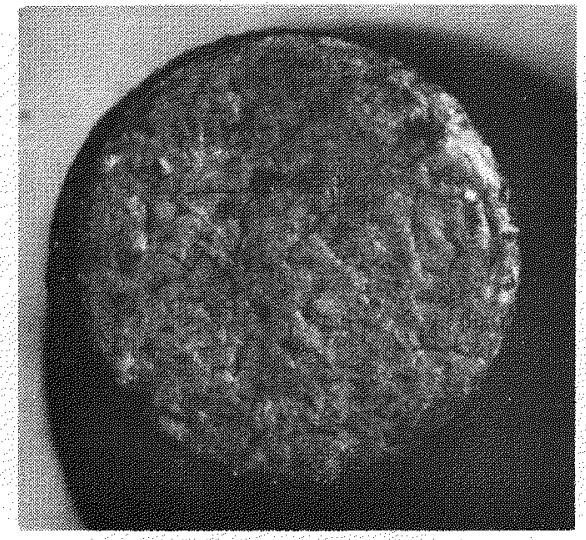

$57440-76$

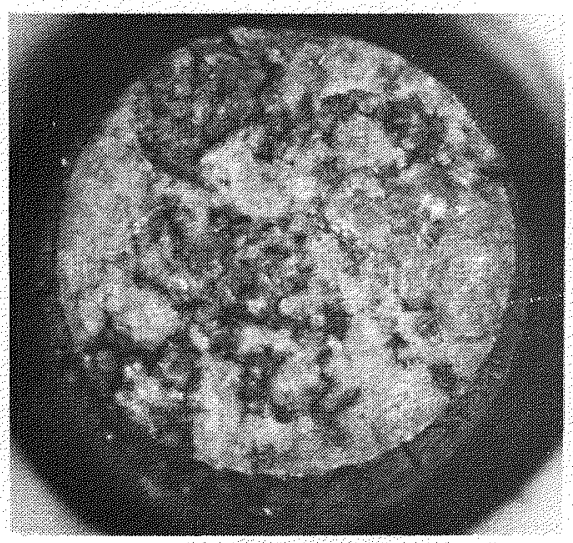

S7440-147

Fig. 4-14. Photomicrographs of fuel rod 7161-004-17-5 (top) after irradiation in capsule P13R (position 5B) to a fast neutron fluence of $9.0 \times 10^{21} \mathrm{n} / \mathrm{cm}^{2}(\mathrm{E}>0.18 \mathrm{MeV})$ HTGR at $1225^{\circ} \mathrm{C}$ and of fuel rod 7161-004-17-6 (bottom) after irradiation in capsule P13S (position 5B) to a fast neutron fluence of $8.7 \times 10^{21} \mathrm{n} / \mathrm{cm}^{2}$ $(\mathrm{E}>0.18 \mathrm{MeV})_{\mathrm{HTGR}}$ at $1330^{\circ} \mathrm{C}$ 


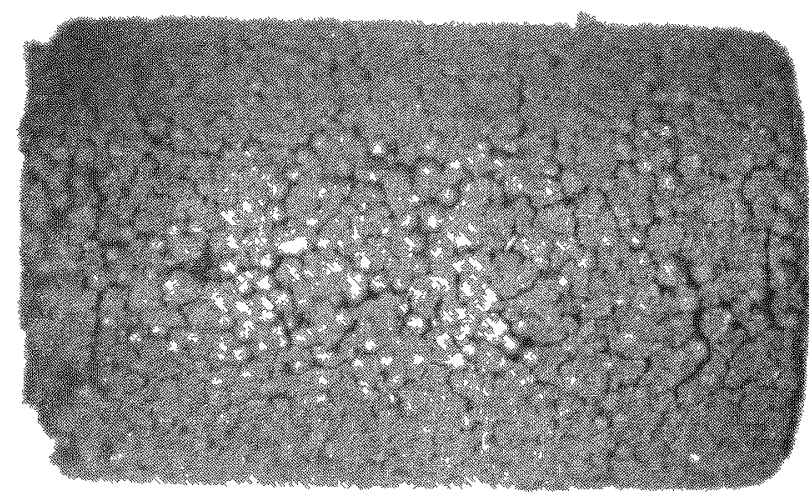

S7440-84

$$
0.5 \mathrm{~cm}
$$

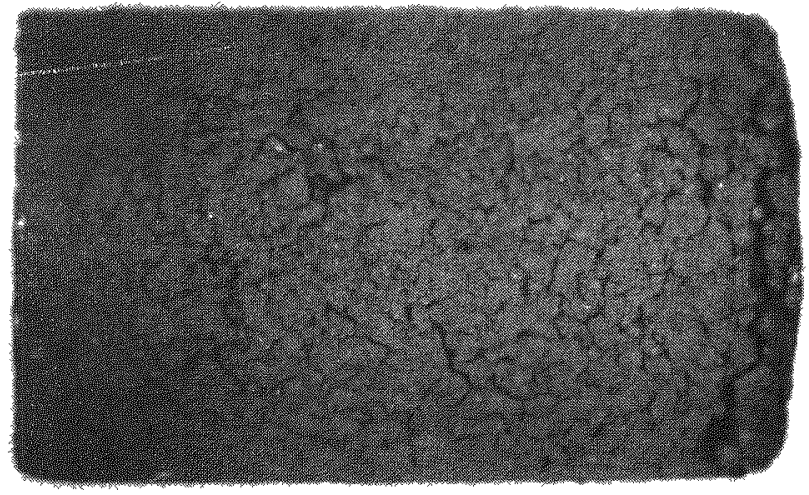

S7440-153

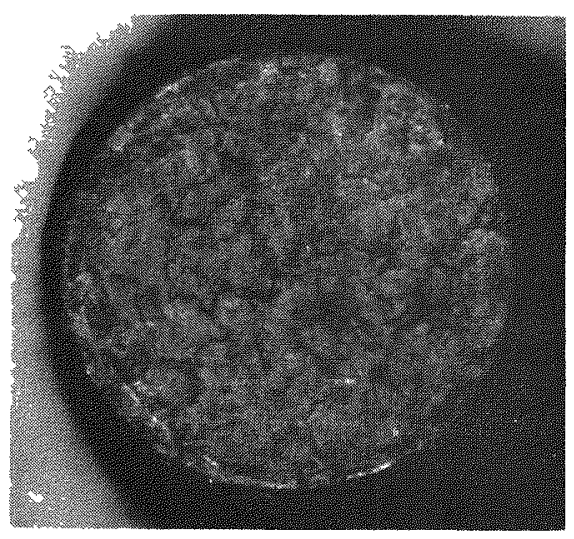

$57440-83$

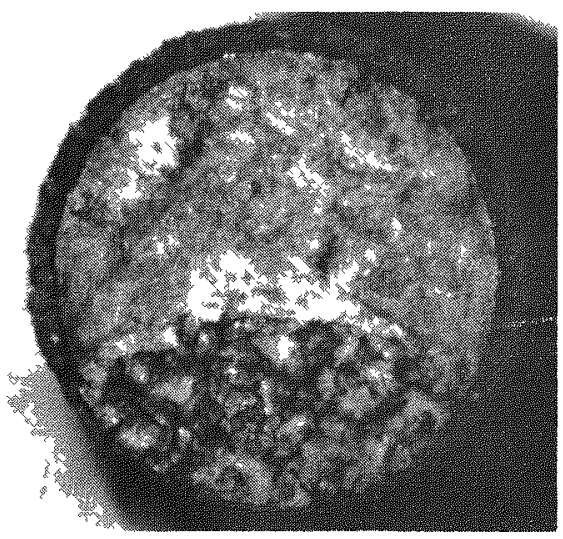

$57440-152$

Fig. 4-15. Photomicrographs of fuel rod 7161-004-18-6 (top) after irradiation in capsule P13R (position 5C) to a fast neutron fluence of $8.5 \times 10^{21} \mathrm{n} / \mathrm{cm}^{2}\left(\mathrm{E}>0.18 \mathrm{MeV}\right.$ ) $\mathrm{HTGR}$ at $1235^{\circ} \mathrm{C}$ and of fuel rod 7161-004-18-5 (bottom) after irradiation in capsule P13S (position $5 \mathrm{C}$ ) to a fast neutron fluence of $8.4 \times 10^{21} \mathrm{n} / \mathrm{cm}^{2}$ $(\mathrm{E}>0.18 \mathrm{MeV})_{\text {HTGR }}$ at $1335^{\circ} \mathrm{C}$ 


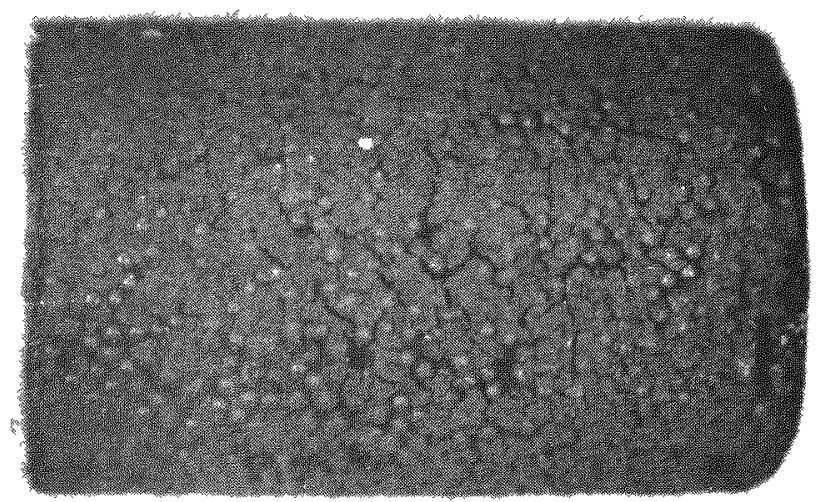

$57440-89$

$$
0.5 \mathrm{~cm}
$$

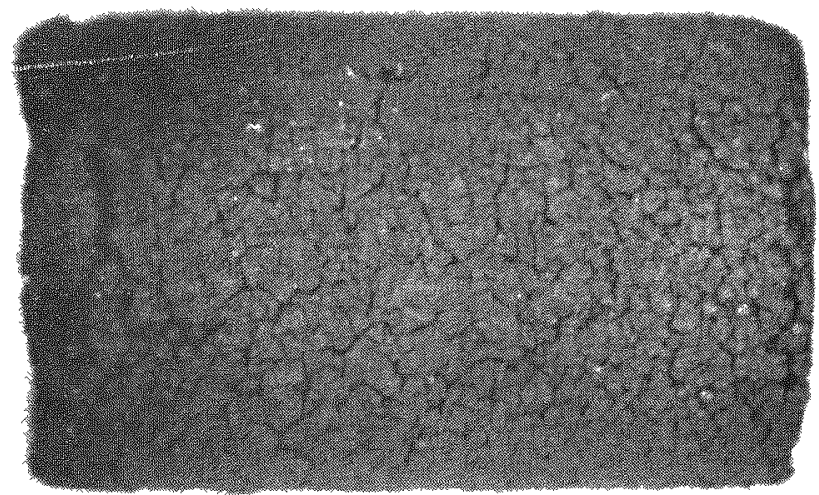

$57440-160$

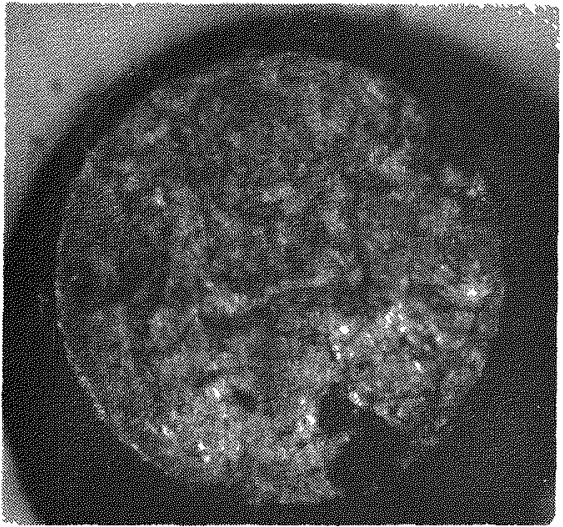

57440-88

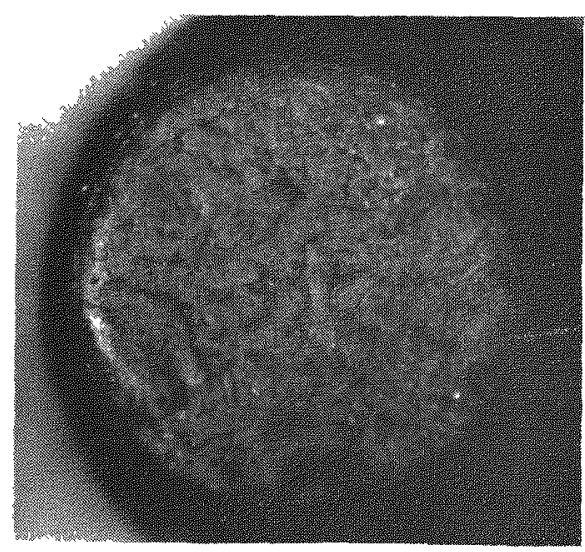

S7440-157

Fig. 4-16. Photomicrographs of fuel rod 7161-004-19-7 (top) after irradiation in capsule P13R (position 5D) to a fast neutron fluence of $8.2 \times 10^{21} \mathrm{n} / \mathrm{cm}^{2}(\mathrm{E}>0.18 \mathrm{MeV})_{\mathrm{HTGR}}$ at $1225^{\circ} \mathrm{C}$ and of fuel rod 7161-004-19-5 (bottom) after irradiation in capsule P13S (position 5D) to a fast neutron fluence of $7.9 \times 10^{21} \mathrm{n} / \mathrm{cm}^{2}$ $(\mathrm{E}>0.18 \mathrm{MeV})_{\mathrm{HTGR}}$ at $1325^{\circ} \mathrm{C}$ 


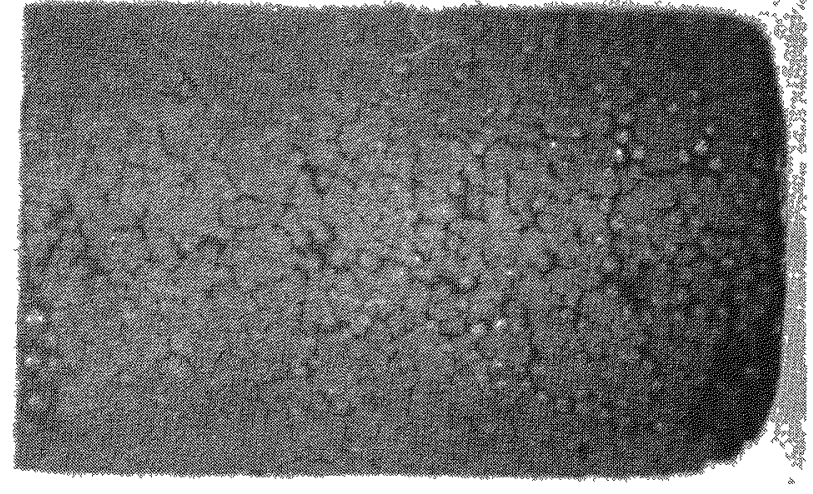

$57440-94$
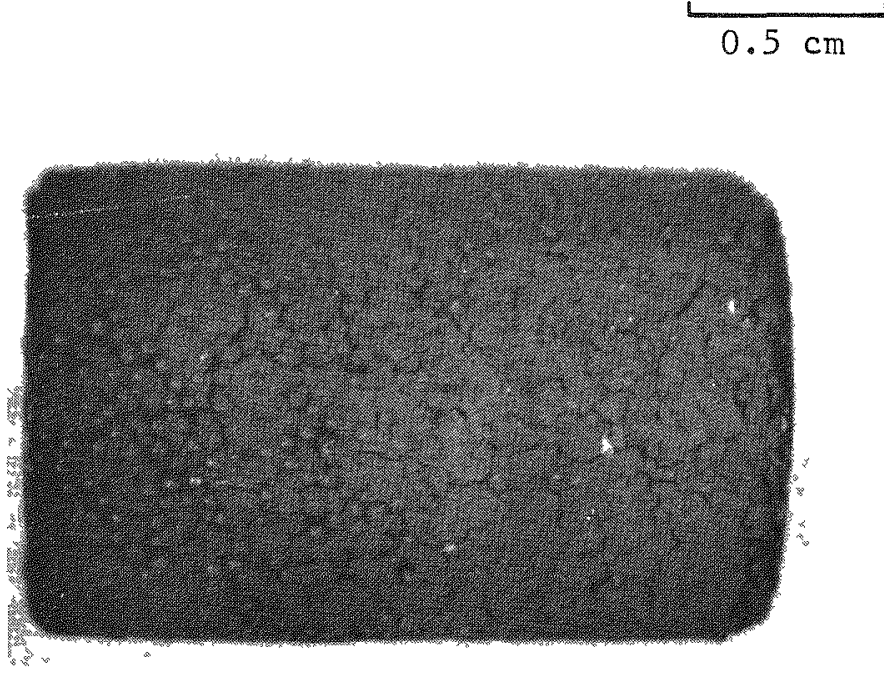

S7440-163

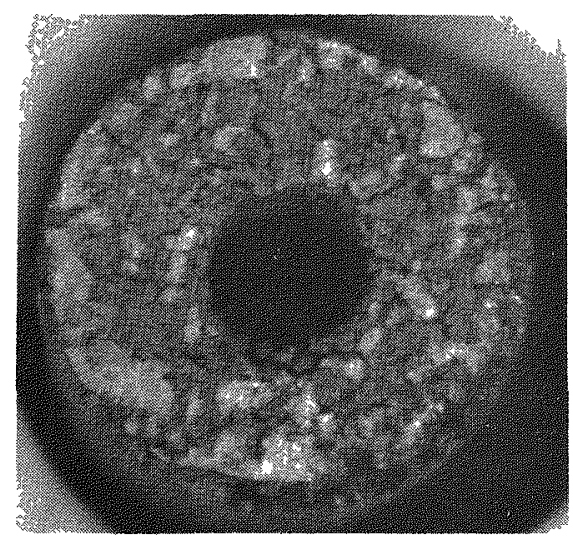

S7440-92

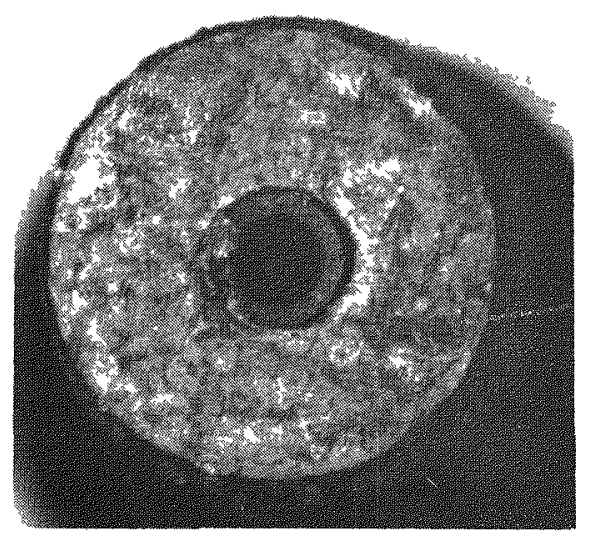

$57440-161$

Fig. 4-17. Photomicrographs of fuel rod 7161-004-20-6 (top) after irradiation in capsule P13R (position 5E) to a fast neutron fluence of $7.7 \times 10^{21} \mathrm{n} / \mathrm{cm}^{2}\left(\mathrm{E}>0.18 \mathrm{MeV}\right.$ ) HTGR at $1280^{\circ} \mathrm{C}$ and of fuel rod 7161-004-20-5 (bottom) after irradiation in capsule P13S (position 5E) to a fast neutron fluence of $7.5 \times 10^{21} \mathrm{n} / \mathrm{cm}^{2}$ $(\mathrm{E}>0.18 \mathrm{MeV})_{\mathrm{HTGR}}$ at $1350^{\circ} \mathrm{C}$ 


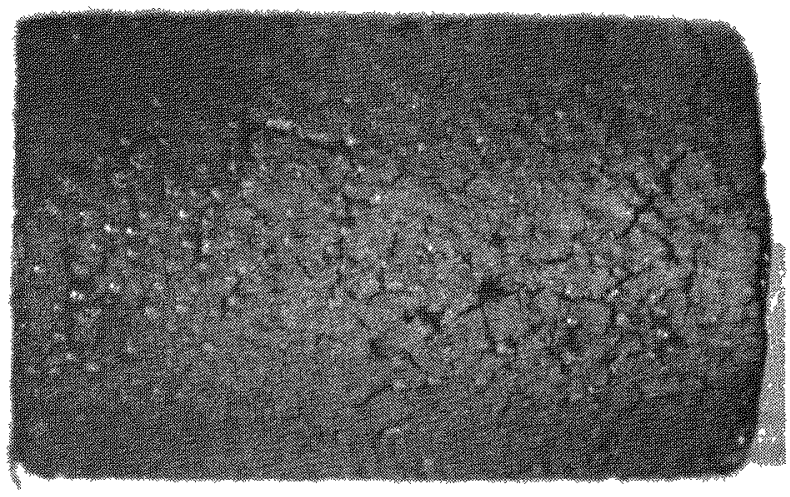

$57440-30$

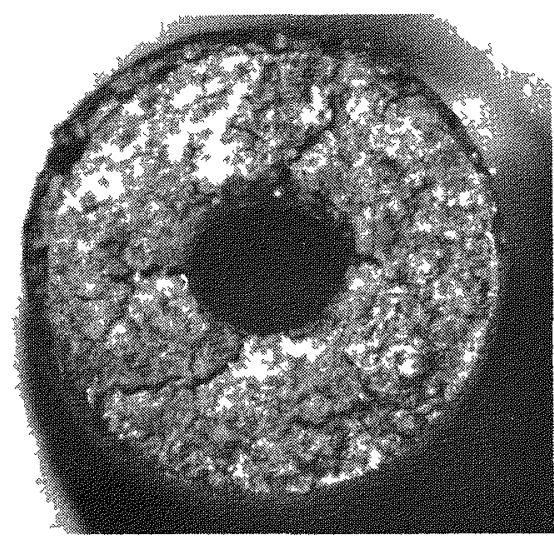

$57440-29$

\section{$0.5 \mathrm{~cm}$}

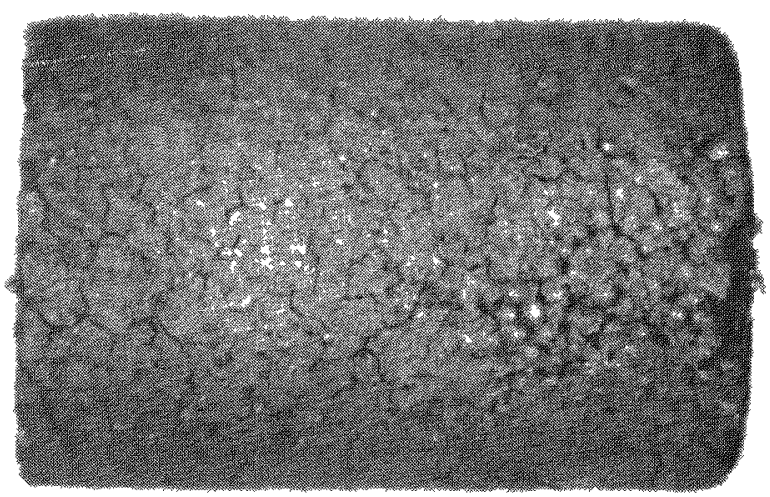

$57440-169$

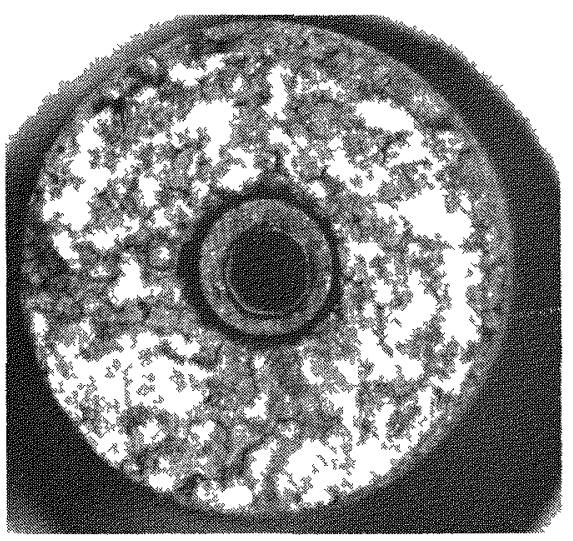

\$7440-166

Fig. 4-18. Photomicrographs of fuel rod 7161-004-21-5 (top) after irradiation in capsule P13R (position 6A) to a fast neutron fluence of $5.4 \times 10^{21} \mathrm{n} / \mathrm{cm}^{2}(\mathrm{E}>0.18 \mathrm{MeV})_{\mathrm{HTGR}}$ at $1065^{\circ} \mathrm{C}$ and of fuel rod 7161-004-26-5 (bottom) after irradiation in capsule P13S (position 6A) to a fast neutron fluence of $5.3 \times 10^{21} \mathrm{n} / \mathrm{cm}^{2}$ $(\mathrm{E}>0.18 \mathrm{MeV})_{\mathrm{HTGR}}$ at $1085^{\circ} \mathrm{C}$ 


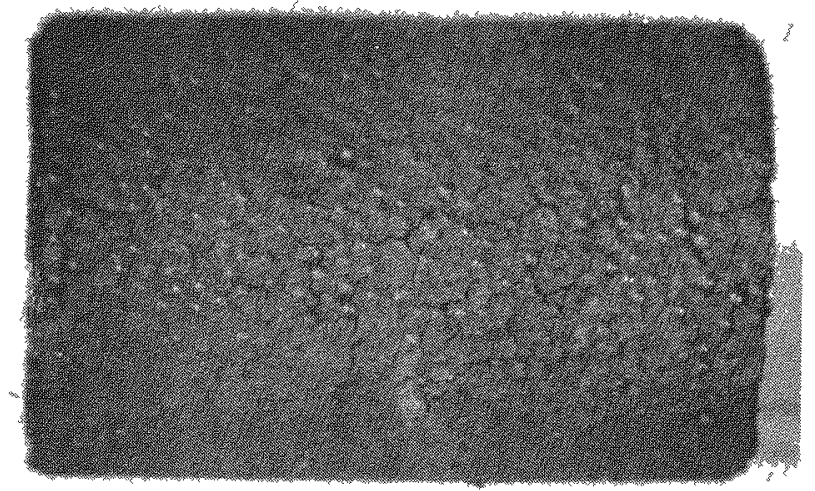

$57440-36$

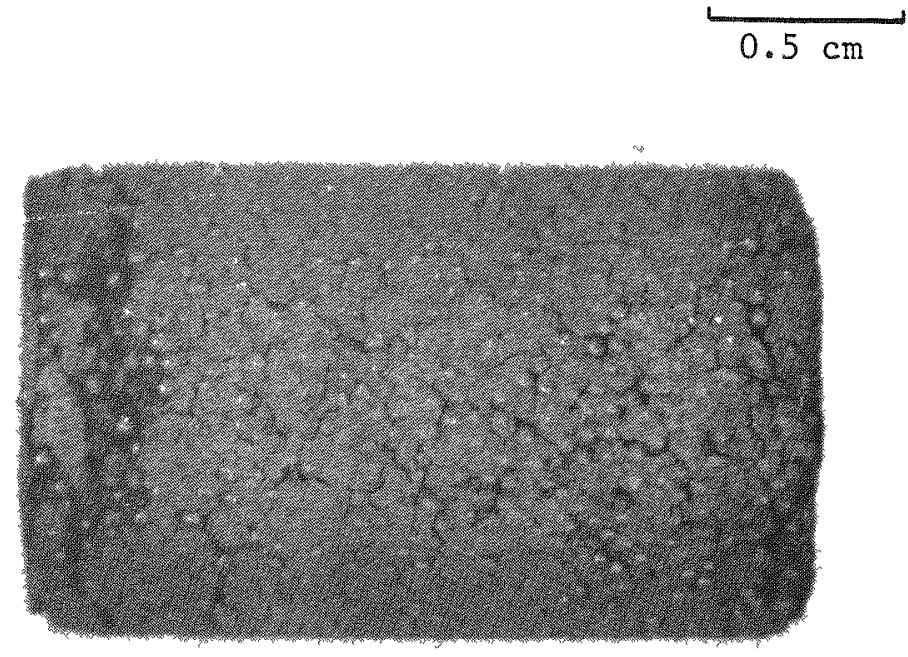

S7440-173

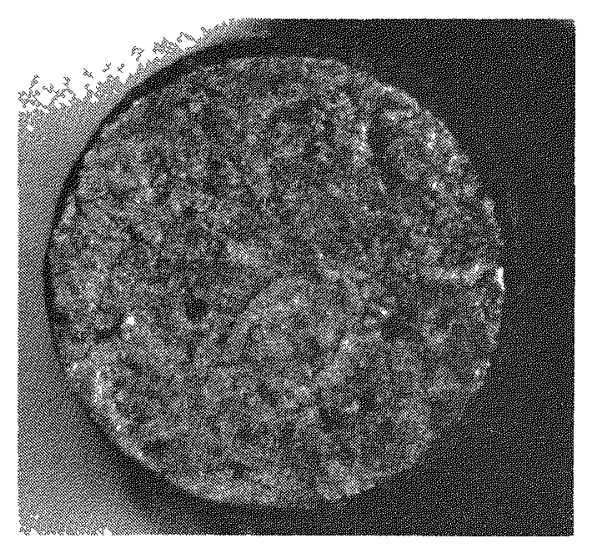

$57440-34$

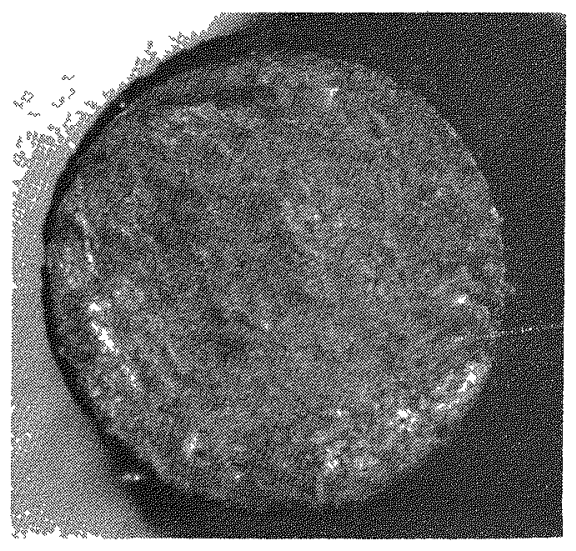

$57440-171$

Fig. 4-19. Photomicrographs of fuel rod 7161-004-22-5 (top) after irradiation in capsule P13R (position $6 \mathrm{~B}$ ) to a fast neutron fluence of $5.0 \times 10^{21} \mathrm{n} / \mathrm{cm}^{2}\left(\mathrm{E}>0.18 \mathrm{MeV}\right.$ ) HTGR at $1055^{\circ} \mathrm{C}$ and of fuel rod 7161-004-27-5 (bottom) after irradiation in capsule P13S (position $6 \mathrm{~B}$ ) to a fast neutron fluence of $4.8 \times 10^{21} \mathrm{n} / \mathrm{cm}^{2}$ $(\mathrm{E}>0.18 \mathrm{MeV})_{\mathrm{HTGR}}$ at $1045^{\circ} \mathrm{C}$ 


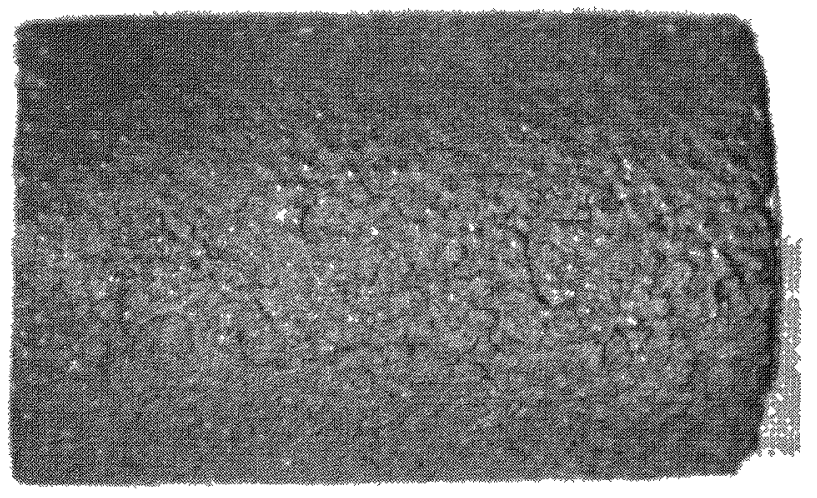

$57440-40$

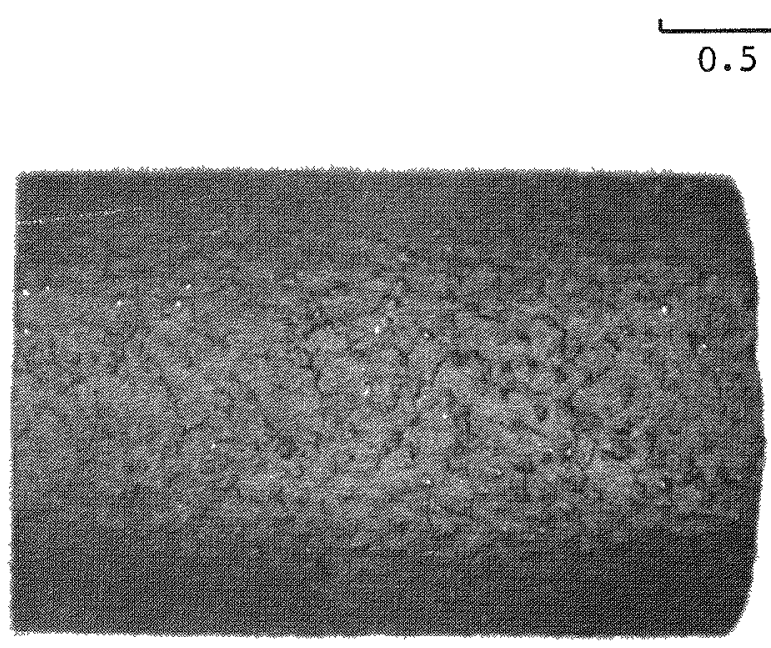

S7440-176

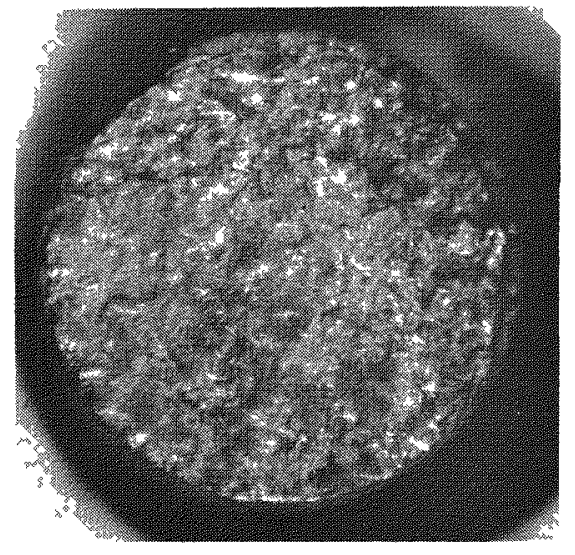

$57440-38$

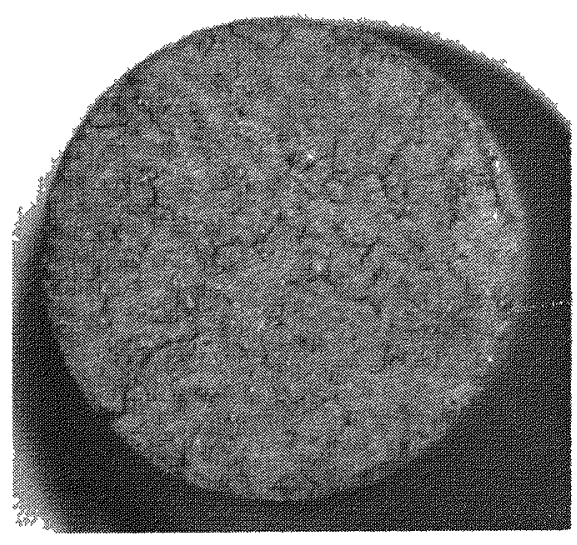

$57440-174$

Fig. 4-20. Photomicrographs of fuel rod 7161-004-23-5 (top) after irradiation in capsule P13R (position 6C) to a fast neutron fluence of $4.5 \times 10^{21} \mathrm{n} / \mathrm{cm}^{2}(\mathrm{E}>0.18 \mathrm{MeV})$ HTGR at $1005^{\circ} \mathrm{C}$ and of fuel rod 7161-004-28-13 (bottom) after irradiation in capsule P13S (position $6 \mathrm{C}$ ) to a fast neutron fluence of $4.4 \times 10^{21} \mathrm{n} / \mathrm{cm}^{2}$ $(\mathrm{E}>0.18 \mathrm{MeV})_{\mathrm{HTGR}}$ at $1050^{\circ} \mathrm{C}$ 


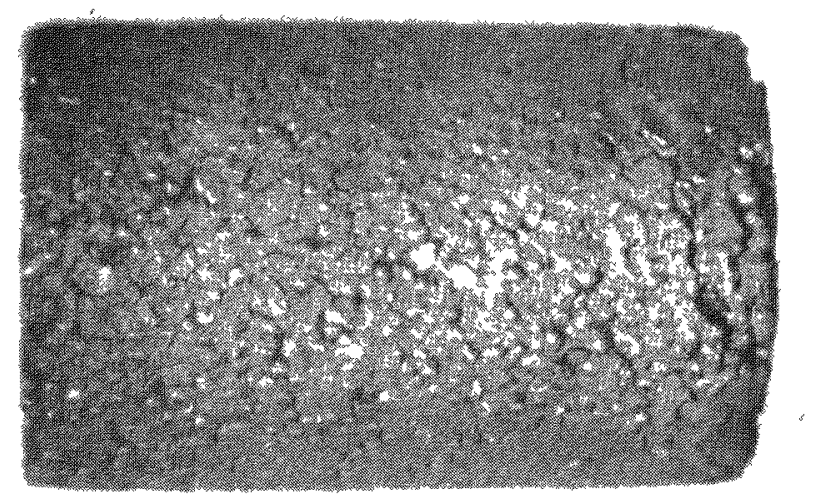

$57440-43$

\section{$0.5 \mathrm{~cm}$}

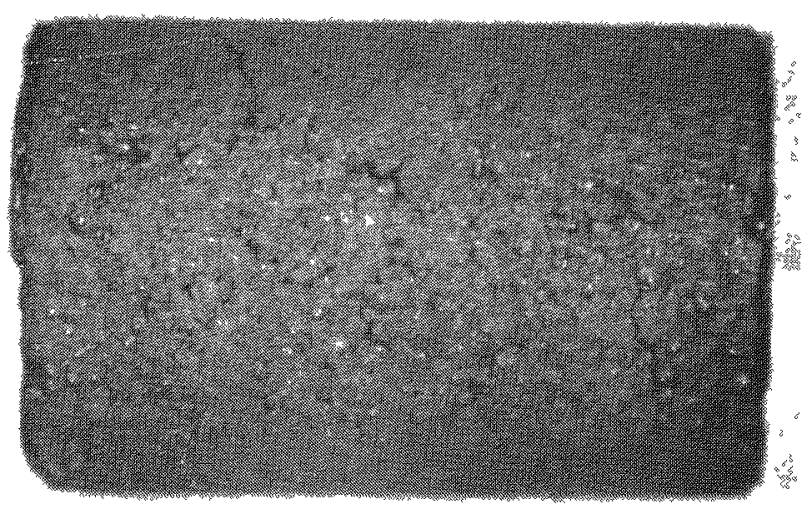

$57440-180$

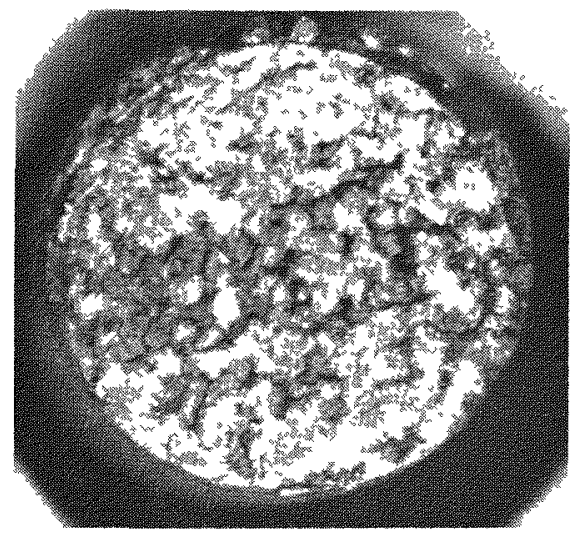

$57440-41$

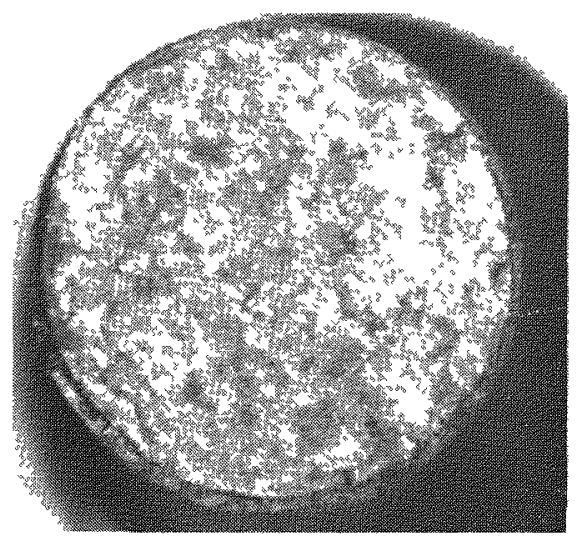

$57440-179$

Fig. 4-21, Photomicrographs of fuel rod 7161-004-24-5 (top) after irradiation in capsule P13R (position 6D) to a fast neutron fluence of $4.1 \times 1021 \mathrm{n} / \mathrm{cm}^{2}$ (E $>0.18 \mathrm{MeV}$ ) $\mathrm{HTGR}$ at $1020^{\circ} \mathrm{C}$ and of fuel rod 7161-004-29-5 (bottom) after irradiation in capsule P13S (position 6D) to a fast neutron fluence of $3.9 \times 10^{21} \mathrm{n} / \mathrm{cm}^{2}$ $(\mathrm{E}>0.18 \mathrm{MeV})_{\mathrm{HTGR}}$ at $1015^{\circ} \mathrm{C}$ 


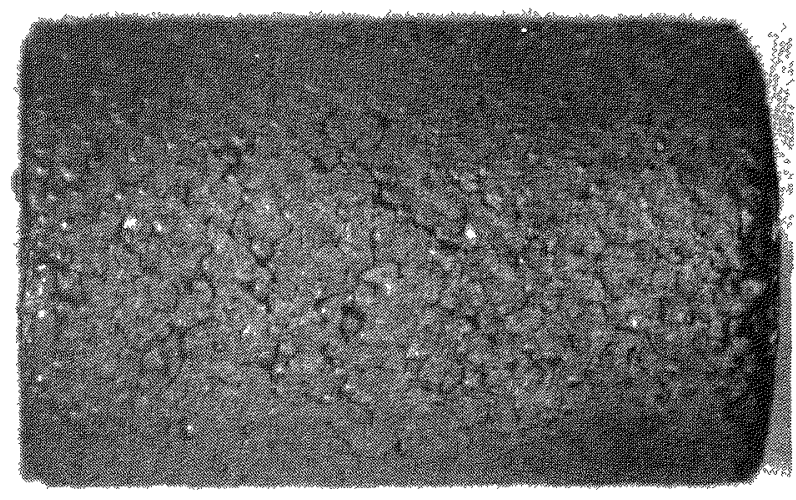

$57440-50$

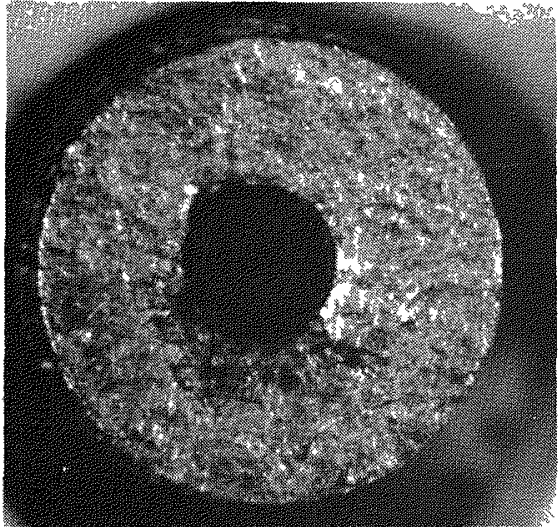

$57440-47$

\section{$0.5 \mathrm{~cm}$}

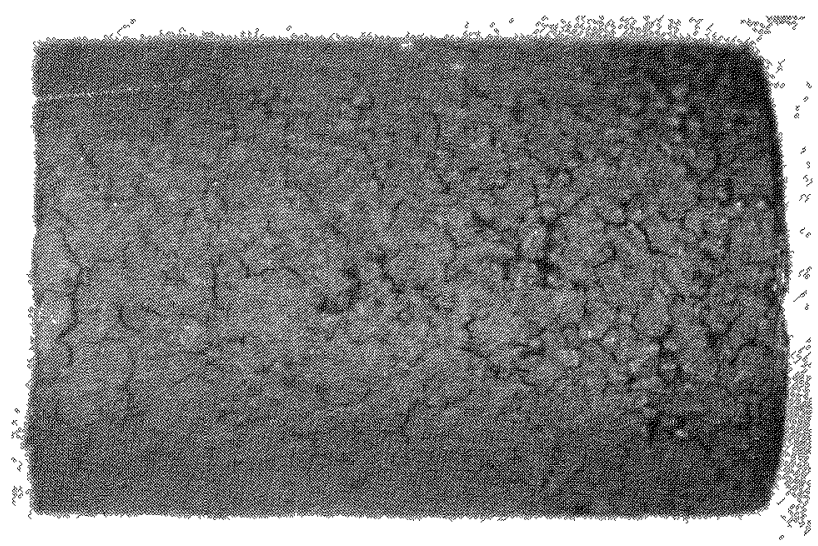

$57440-185$

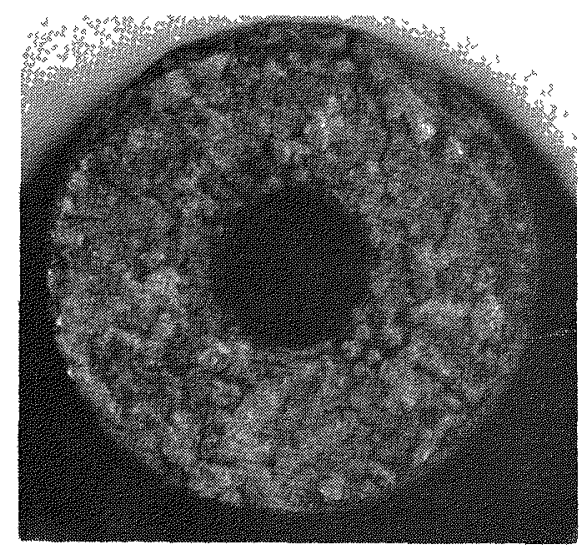

S7440-182

Fig. 4-22. Photomicrographs of fuel rod 7161-004-25-5 (top) after irradiation in capsile P13R (position 6E) to a fast neutron fluence of $3.6 \times 1021 \mathrm{n} / \mathrm{cm}^{2}(\mathrm{E}>0.18 \mathrm{MeV})_{\mathrm{HTGR}}$ at $1000^{\circ} \mathrm{C}$ and of fuel rod 7161-004-30-5 (bottom) after irradiation in capsule P13S (position 6E) to a fast neutron fluence of $3.5 \times 10^{21} \mathrm{n} / \mathrm{cm}^{2}$ $(\mathrm{E}>0.18 \mathrm{MeV})_{\mathrm{HTGR}}$ at $995^{\circ} \mathrm{C}$ 


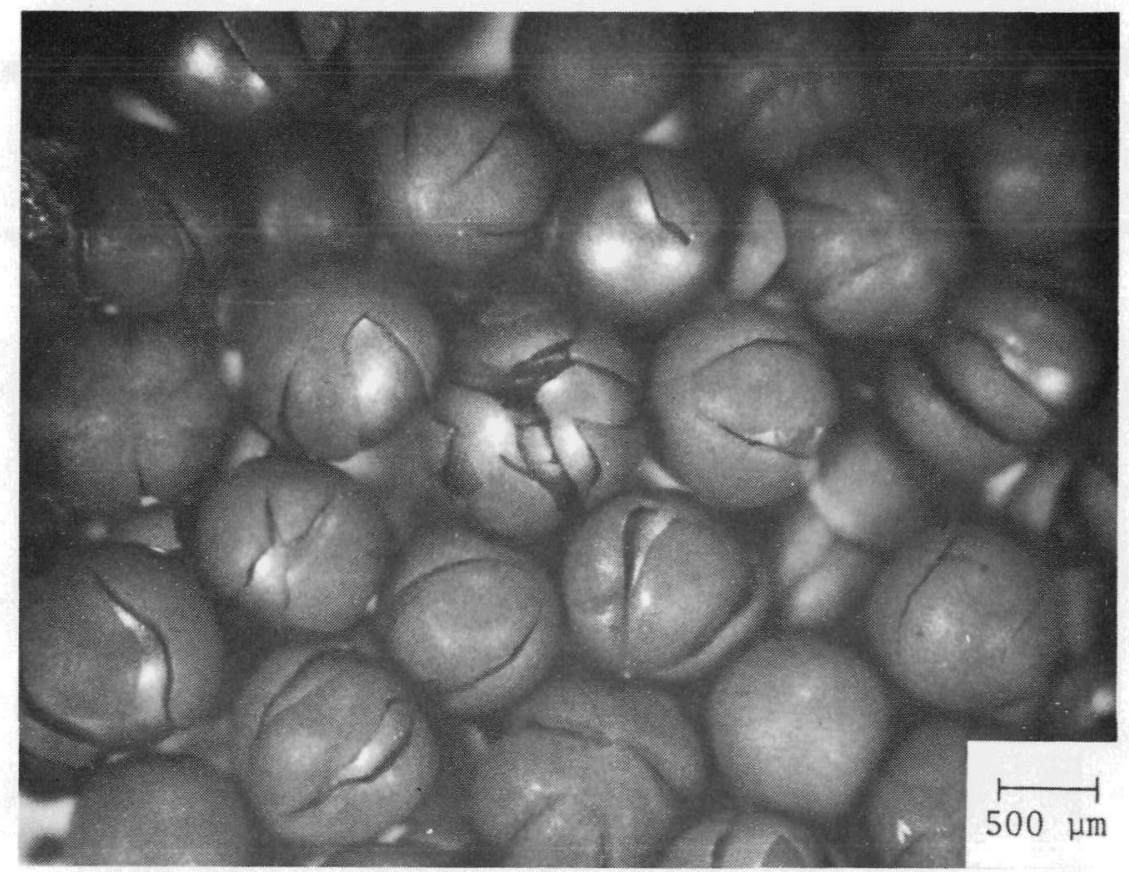

S7440-372

Fig. 4-23. Photograph of TRISO (8Th,1U) $0_{2}$ particles (6155-01-020) after irradiation in position 4-4 of capsule P13S to a fast neutron fluence of $11.1 \times 10^{21} \mathrm{n} / \mathrm{cm}^{2}(\mathrm{E}>0.18 \mathrm{MeV})_{\mathrm{HTGR}}$. In some TRISO coated particle batches, the fragments of failed OPyC coatings remained attached to the SiC coating substrate, which made an accurate assessment of the total coating failure level impossible by visual examination. 


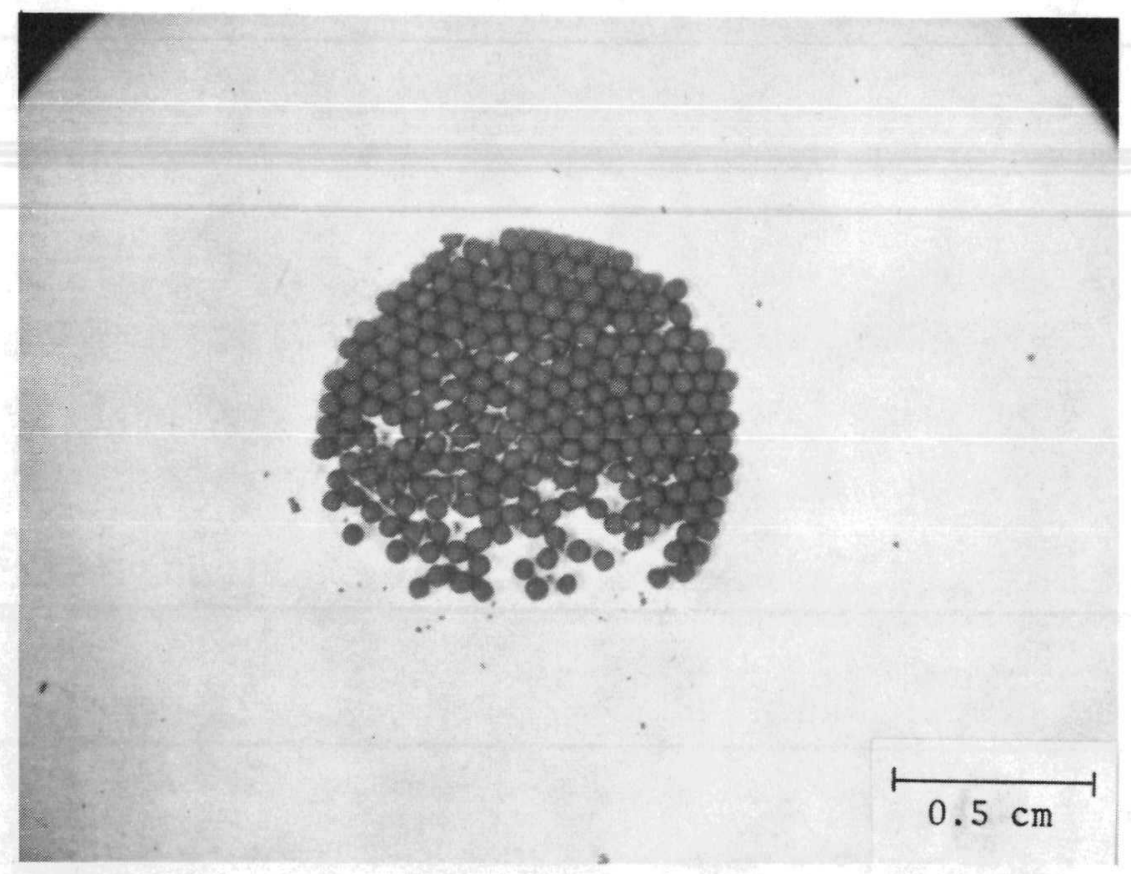

S7440-273

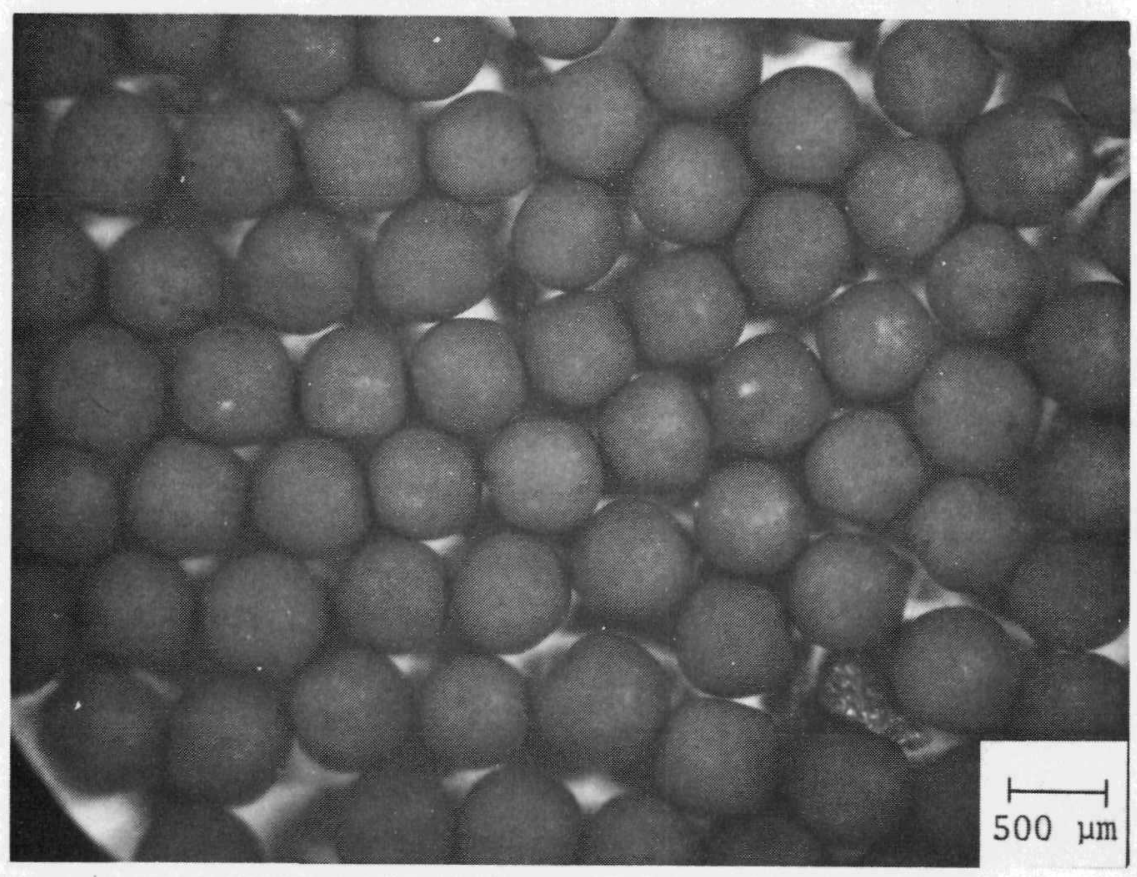

S7440-274

Fig. 4-24. Representative photographs of LHTGR reference design type fissile particles. The particle batch (6151-00-035) exhibited $0 \%$ $(0 \leq \mathrm{P} \leq 0.8)$ failure after irradiation in positions $3-4$ and $3-5$ of capsüle P13R to a fast neutron fluence of $12.0 \times 10^{21} \mathrm{n} / \mathrm{cm}^{2}$ and burnup of $74 \%$ FIMA at $1075^{\circ} \mathrm{C}$. 


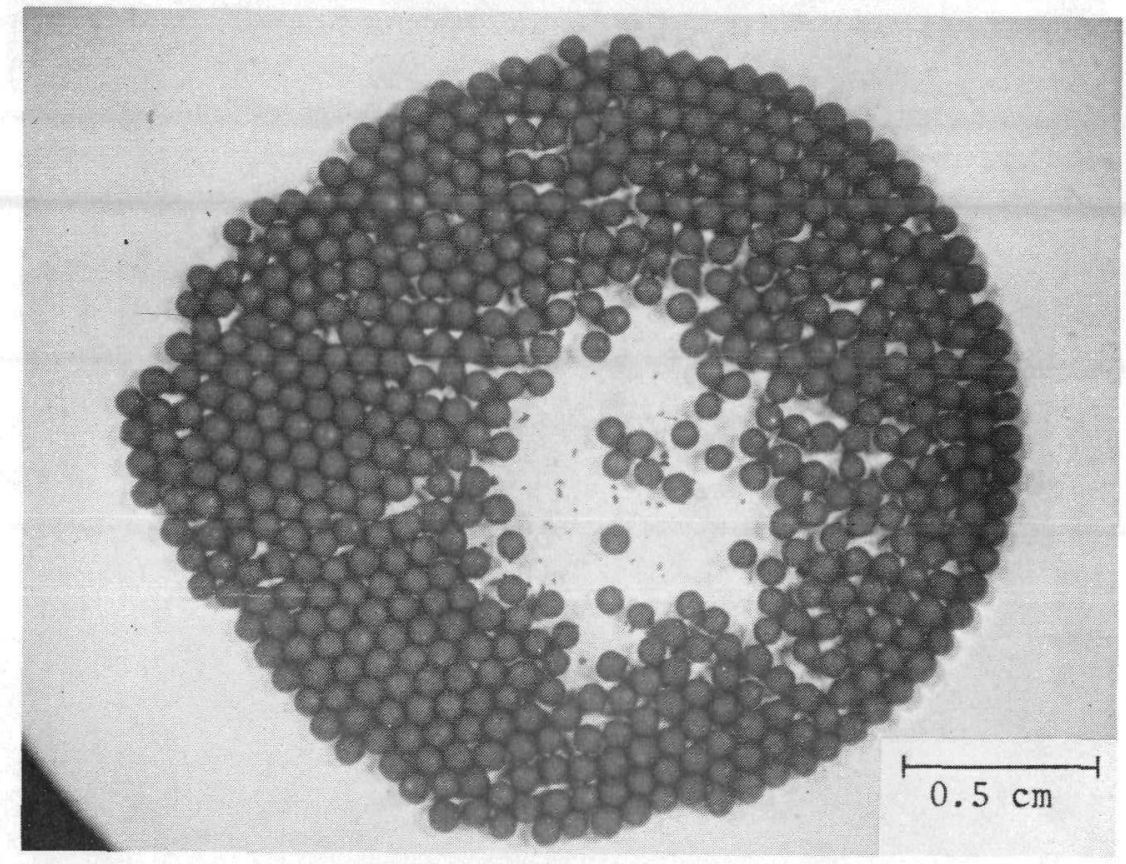

S7440-266

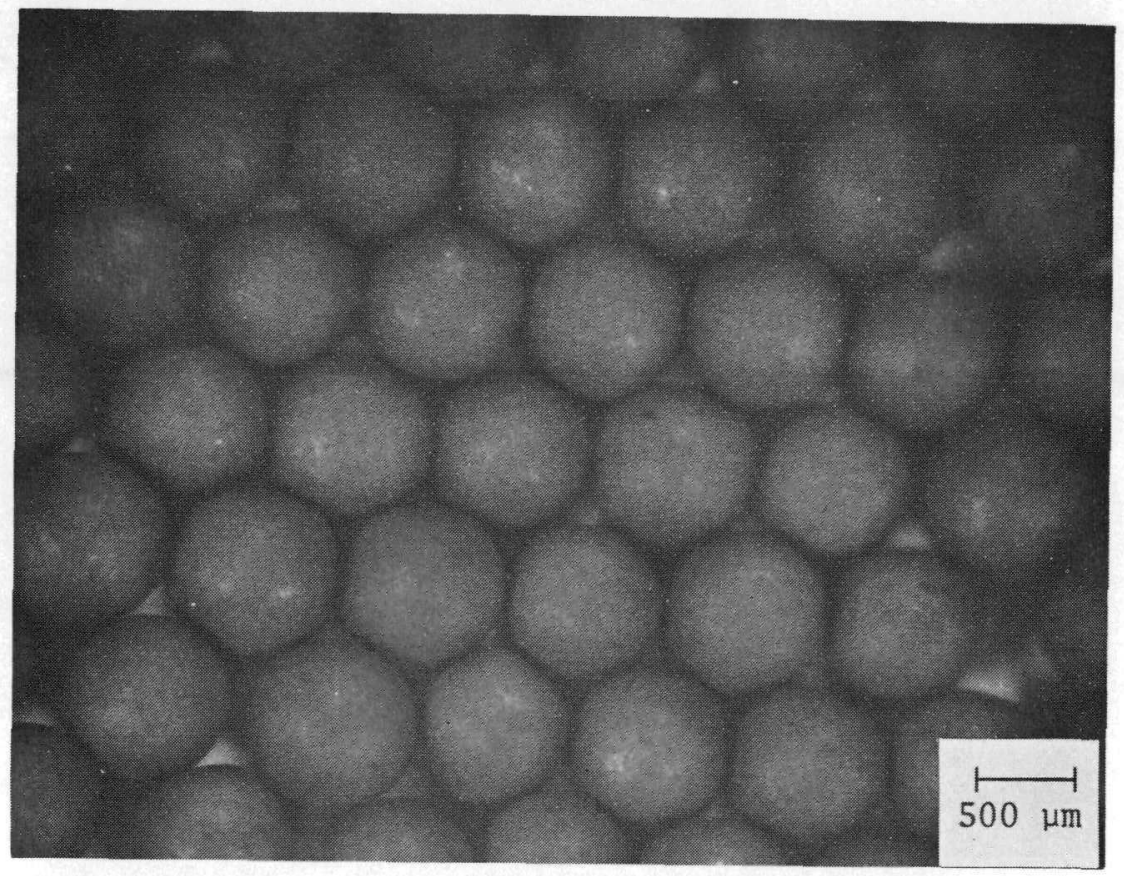

S7440-267

Fig. 4-25. Representative photographs of LHTGR reference design type fertile particles. The particle batch (6542-02-020) exhibited 0\% $(0 \leq \mathrm{P} \leq 0.5)$ failure after irradiation in positions $3-7$ and $3-8$ of capsüle P13R to a fast neutron fluence of $11.9 \times 10^{21} \mathrm{n} / \mathrm{cm}^{2}$ $(\mathrm{E}>0.18 \mathrm{MeV})_{\mathrm{HGTR}}$ and burnup of $4.9 \%$ FIMA at $1010^{\circ} \mathrm{C}$. 


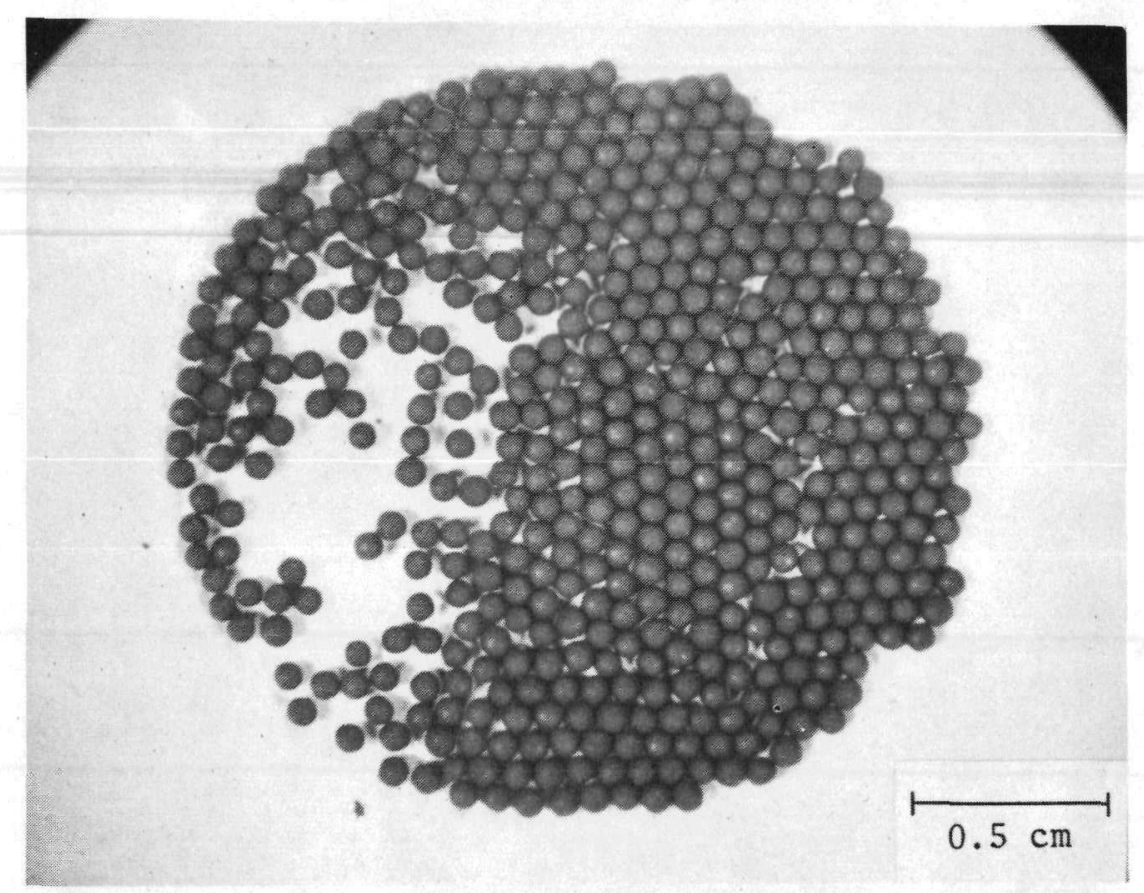

S7440-311

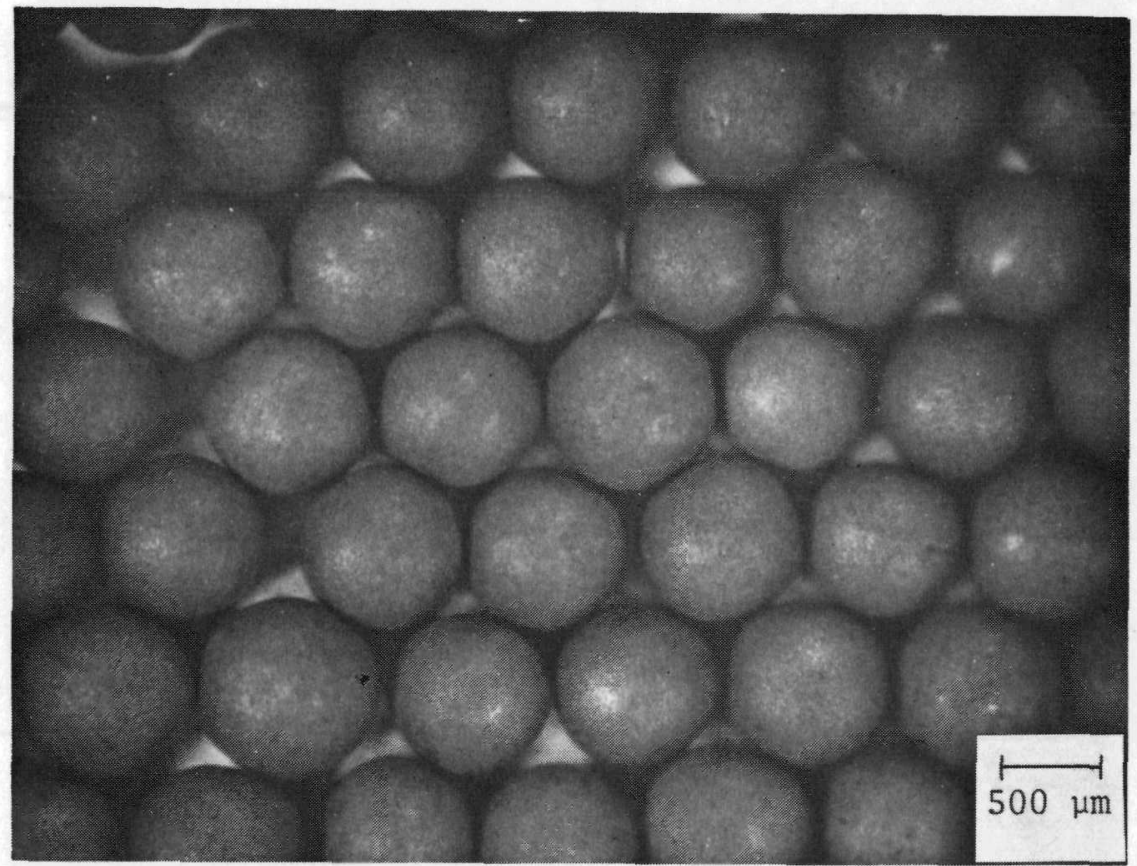

S7440-312

Fig. 4-26. Representative photographs of LHTGR reference design type fertile particles with the OPyC coating deposited from mixed gas. The particle batch (6542-23-025) exhibited $0 \%(0<P<0.5)$ failure after irradiation in positions $3-7$ and $3-8$ of capsule P13S to a fast neutron fluence of $11.7 \times 10^{21} \mathrm{n} / \mathrm{cm}^{2}(\mathrm{E}>0.18 \mathrm{MeV}) \mathrm{HTGR}$ and burnup of $4.2 \%$ FIMA at $955^{\circ} \mathrm{C}$. 


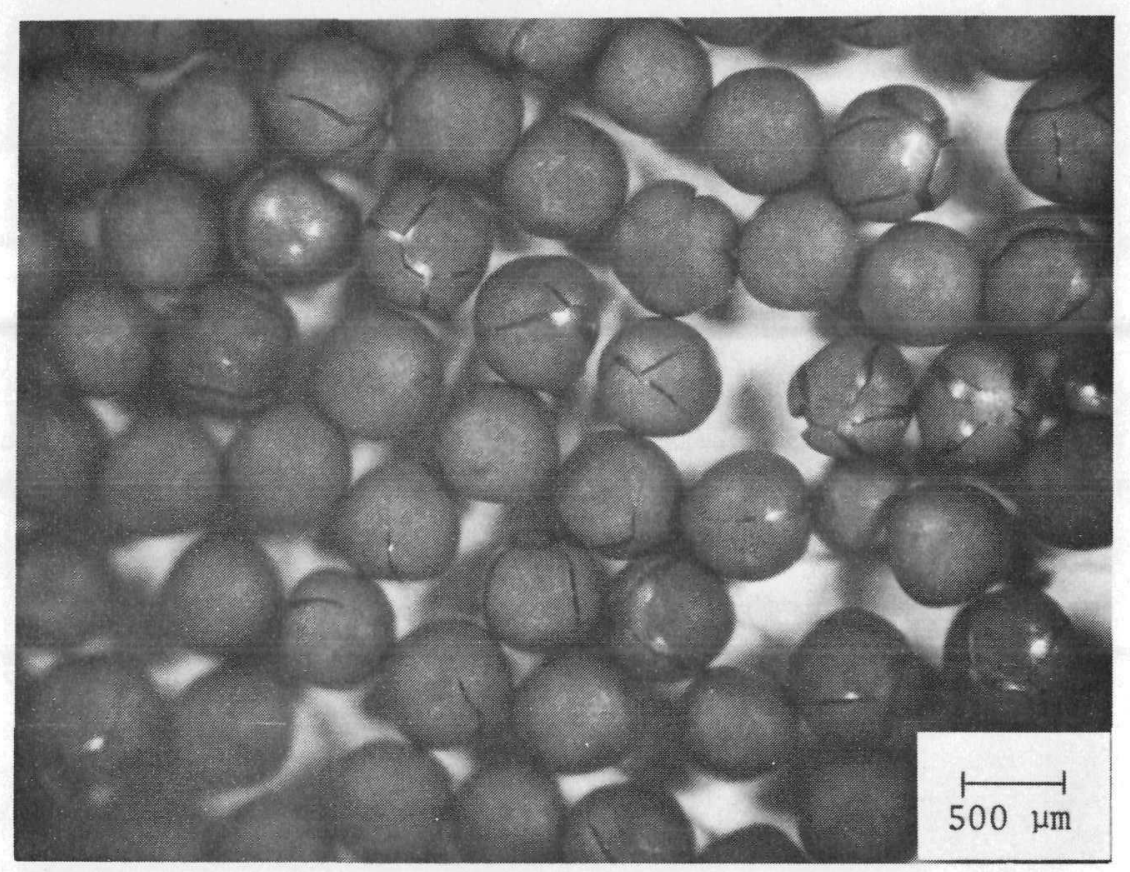

S7440-244

(a)

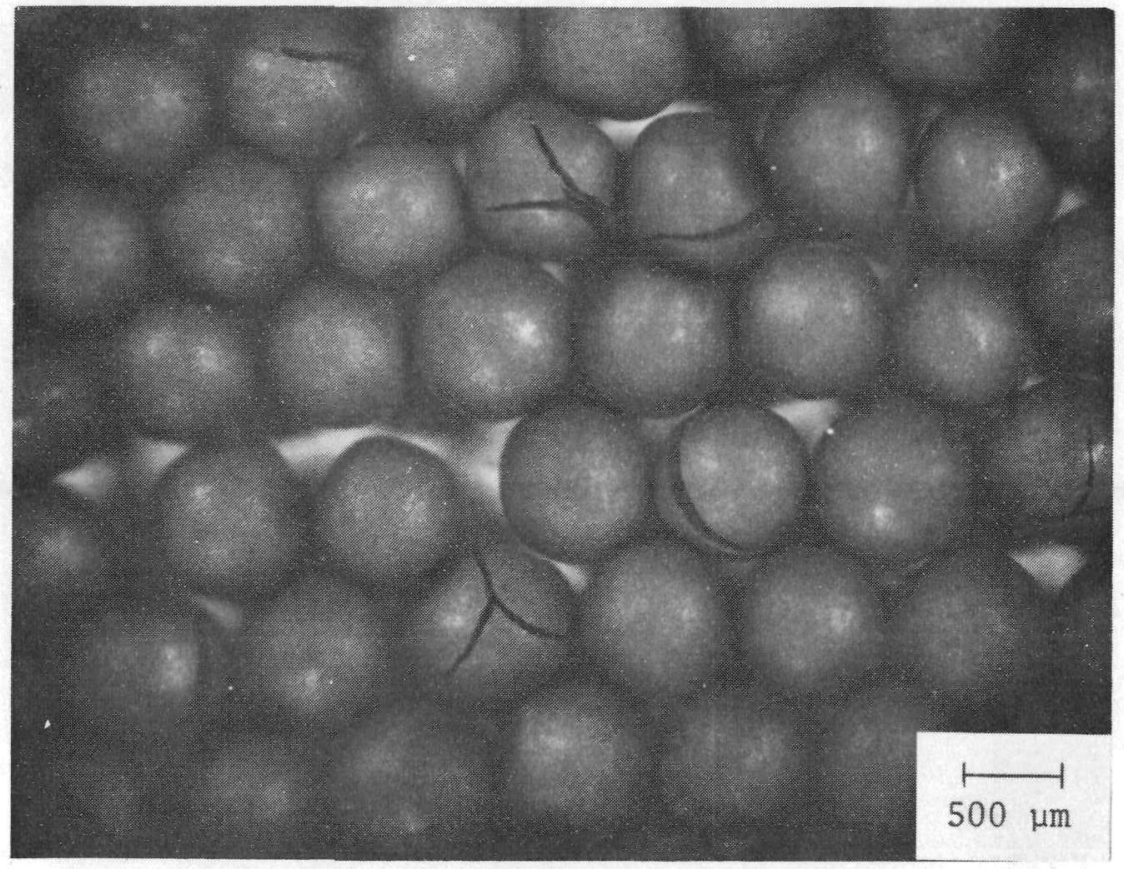

S7440-256

(b)

Fig. 4-27. Photographs of TRISO $\mathrm{UC}_{2}$ and $\mathrm{BISO} \mathrm{ThO}_{2}$ particles after irradiation in capsule P13R. The high OPyC coating failure levels of 49.0 and $10.2 \%$ for the fissile and fertile particles, respectively, were due to the poor irradiation stability of the OPyC coatings, which were deposited at relatively low coating rates and therefore had high $\mathrm{BAF}_{\mathrm{o}}$ values. (a) TRISO $\mathrm{UC}_{2}$ particles (6151-04-015) irradiated to $11.7 \times 10^{21} \mathrm{n} / \mathrm{cm}^{2}(\mathrm{E}>0.18 \mathrm{MeV})_{\mathrm{HTGR}}$ and (b) BISO $\mathrm{ThO}_{2}$ particles (6542-02-030) irradiated to $11.8 \times 10^{21} \mathrm{n} / \mathrm{cm}^{2}(\mathrm{E}>0.18 \mathrm{MeV})_{\mathrm{HTGR}}$. 


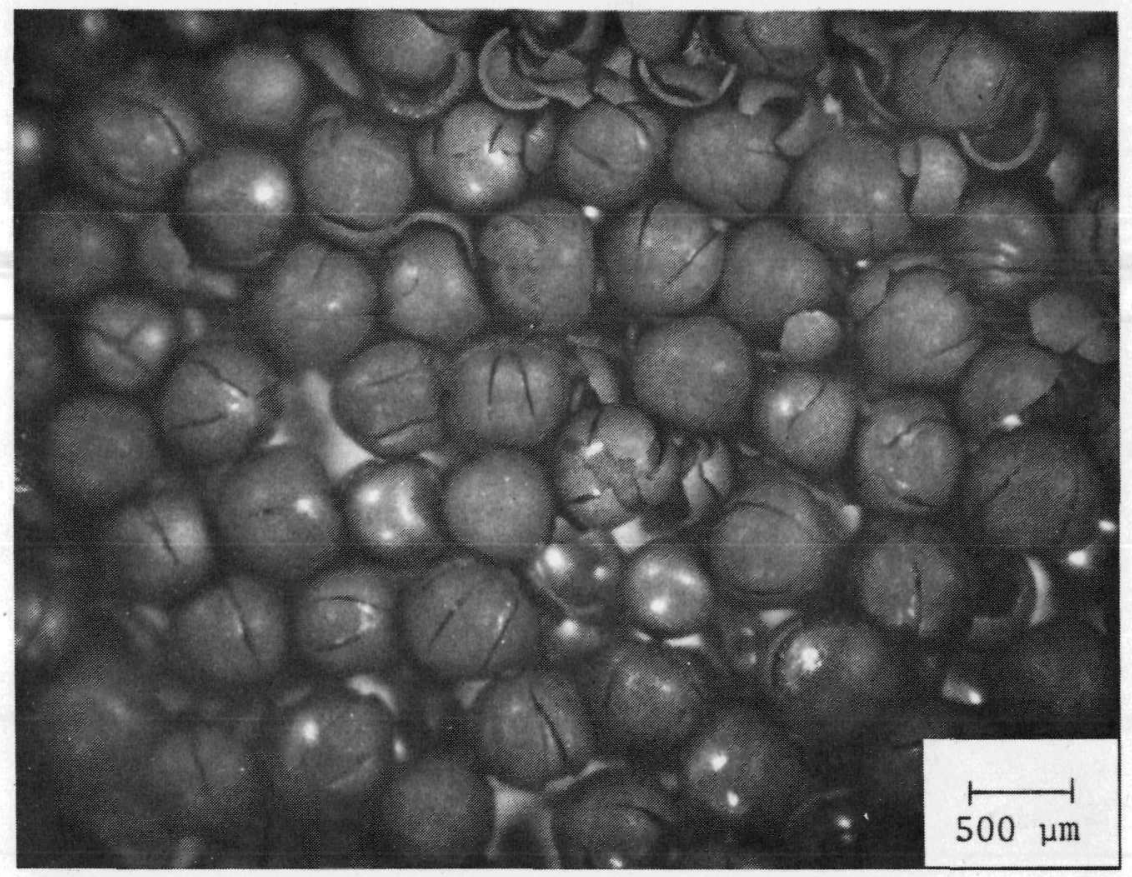

S7440-224

(a)

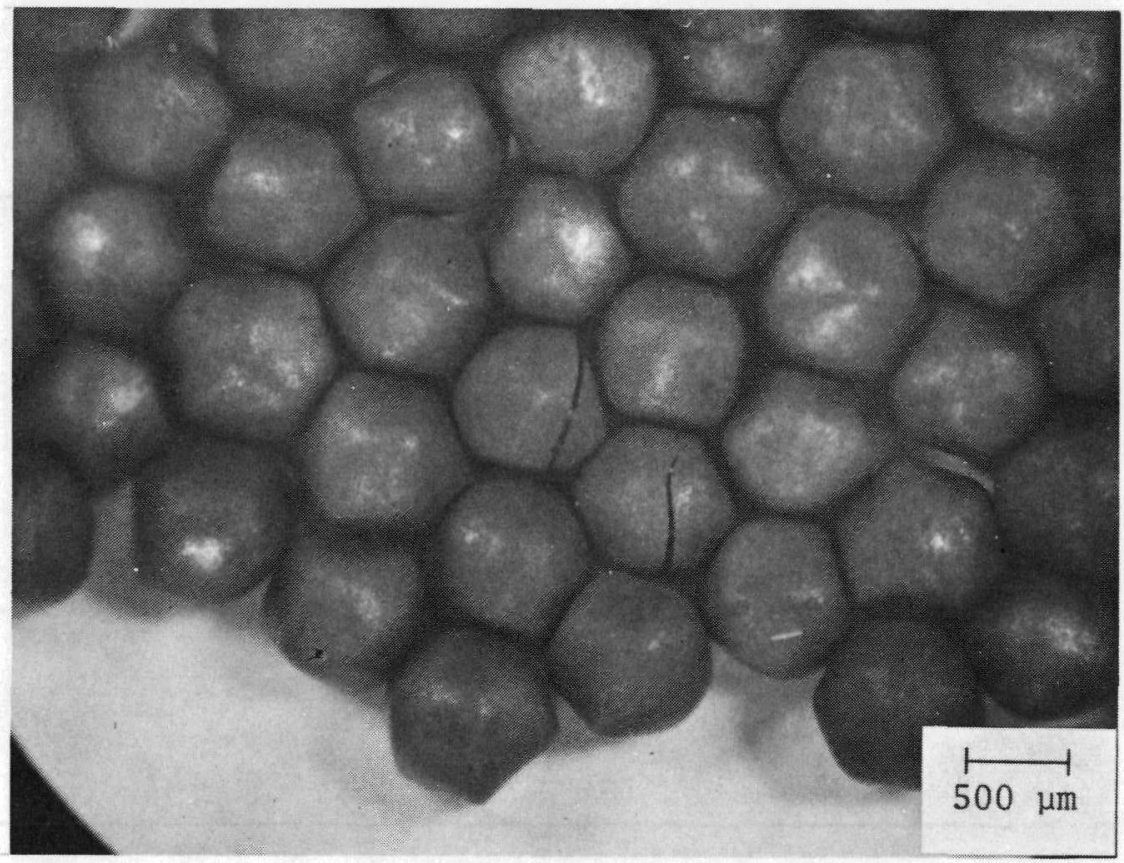

S7440-204

(b)

Fig. 4-28. Photographs of TRISO $\mathrm{UC}_{2}$ and $\mathrm{BISO} \mathrm{ThO}_{2}$ particles after irradiation in capsule P13R. Coating failure of both particle types was due to the poor irradiation stability of the severely faceted coatings. (a) TRISO UC2 particles (6151-00-025) irradiated to $11.3 \times 10^{21} \mathrm{n} / \mathrm{cm}^{2}(\mathrm{E}>0.18 \mathrm{MeV})_{\mathrm{HTGR}}$ and (b) $\mathrm{BISO}^{\mathrm{ThO}} 2$ particles (6542-20-025) irradiated to $11.1 \times 1021 \mathrm{n} / \mathrm{cm}^{2}$ $(\mathrm{E}>0.18 \mathrm{MeV})_{\mathrm{HTGR}}$. 


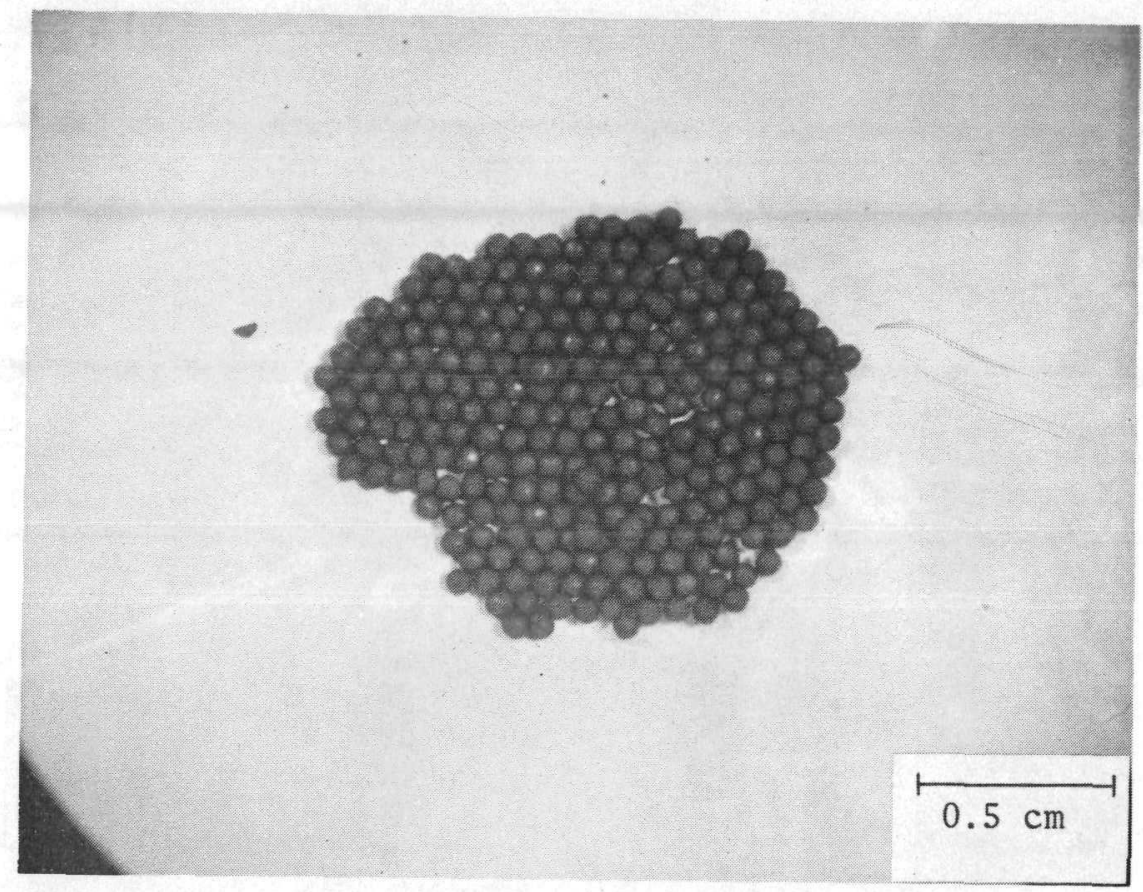

S7440-383

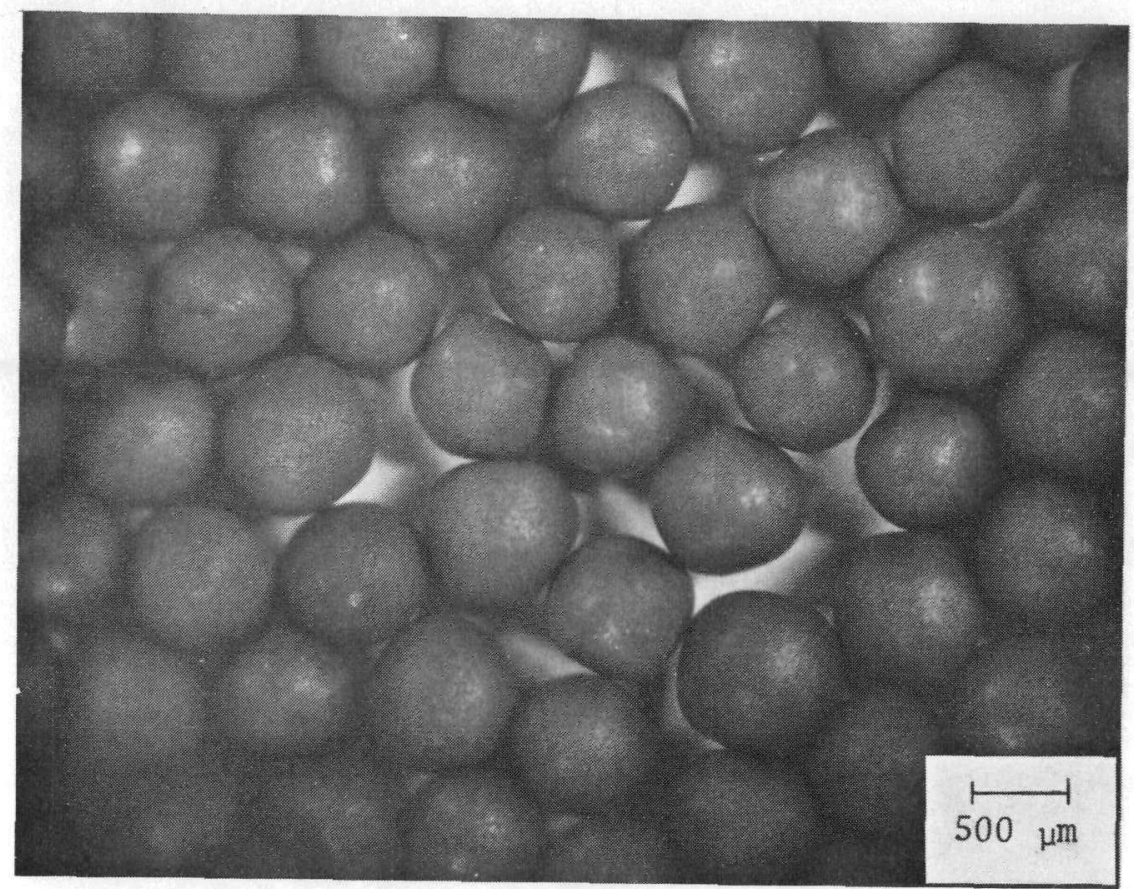

S7440-384

Fig. 4-29. Representative photographs of TRISO (Th,U) $0_{2}$ fissile particles. The particle batch (4163-00-011) exhibited $0 \%(0 \leq \mathrm{P} \leq 0.5)$ failure after irradiation in positions $4-1,4-2$, and $\overline{4}-3$ of capsule P13S to a fast neutron fluence of $11.2 \times 10^{21} \mathrm{n} / \mathrm{cm}^{2}$ $(\mathrm{E}>0.18 \mathrm{MeV})_{\mathrm{HTGR}}$ and burnup of $27 \%$ FIMA at $1045^{\circ} \mathrm{C}$. 


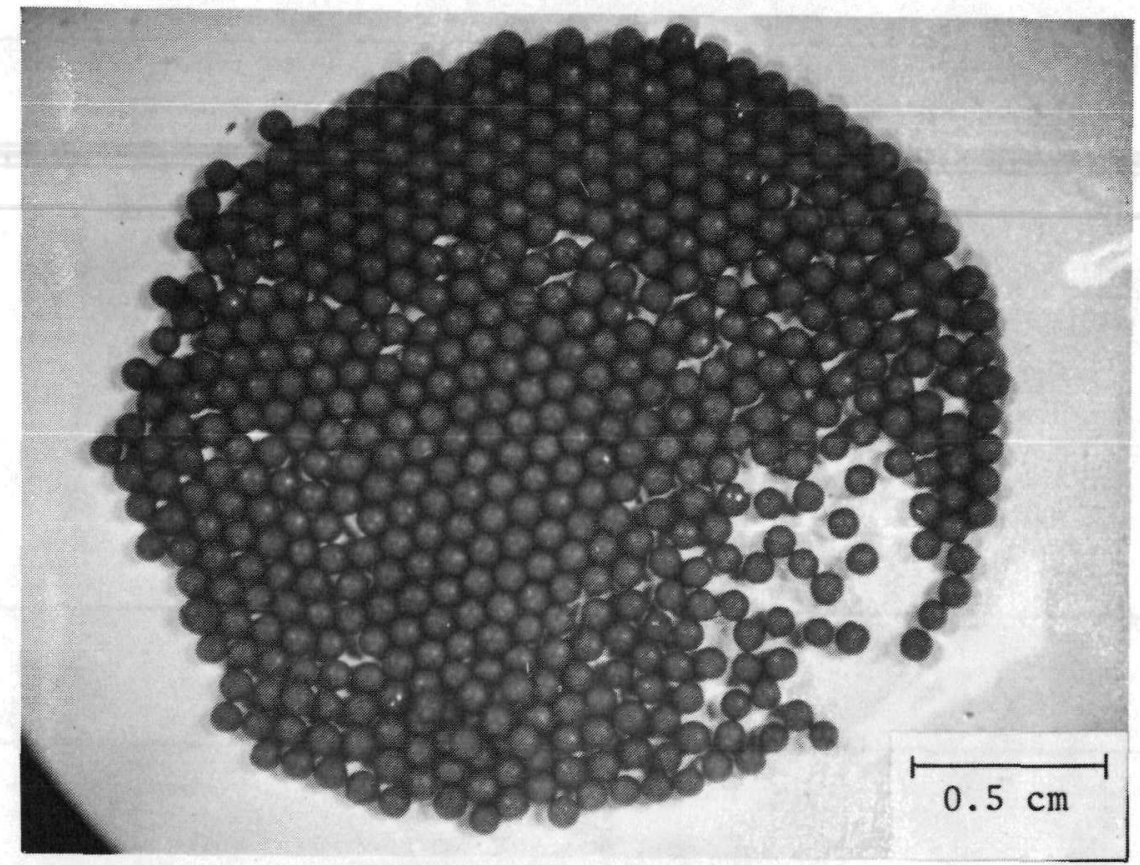

S7440-294

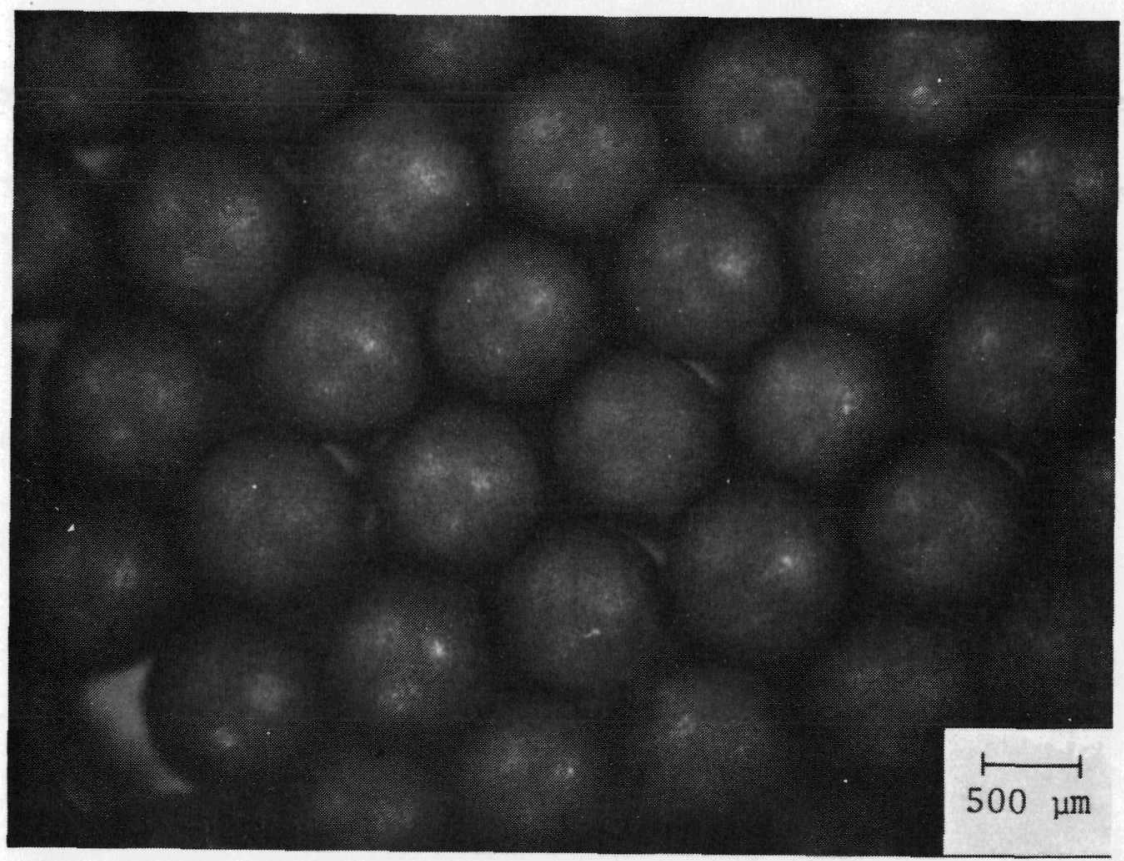

S7440-296

Fig. 4-30. Representative photographs of TRISO $\mathrm{ThO}_{2}$ fertile particles. The particle batch (6252-00-025) exhibited $0.4 \%(0.1<\mathrm{P}<1.4)$ OPyC coating failure after irradiation in position $3-\overline{12}$ of capsule P13S to a fast neutron fluence of $11.5 \times 10^{21} \mathrm{n} / \mathrm{cm}^{2}$ $(\mathrm{E}>0.18 \mathrm{MeV})_{\mathrm{HTGR}}$ and burnup of $4.1 \% \mathrm{FIMA}$ at $860^{\circ} \mathrm{C}$. 


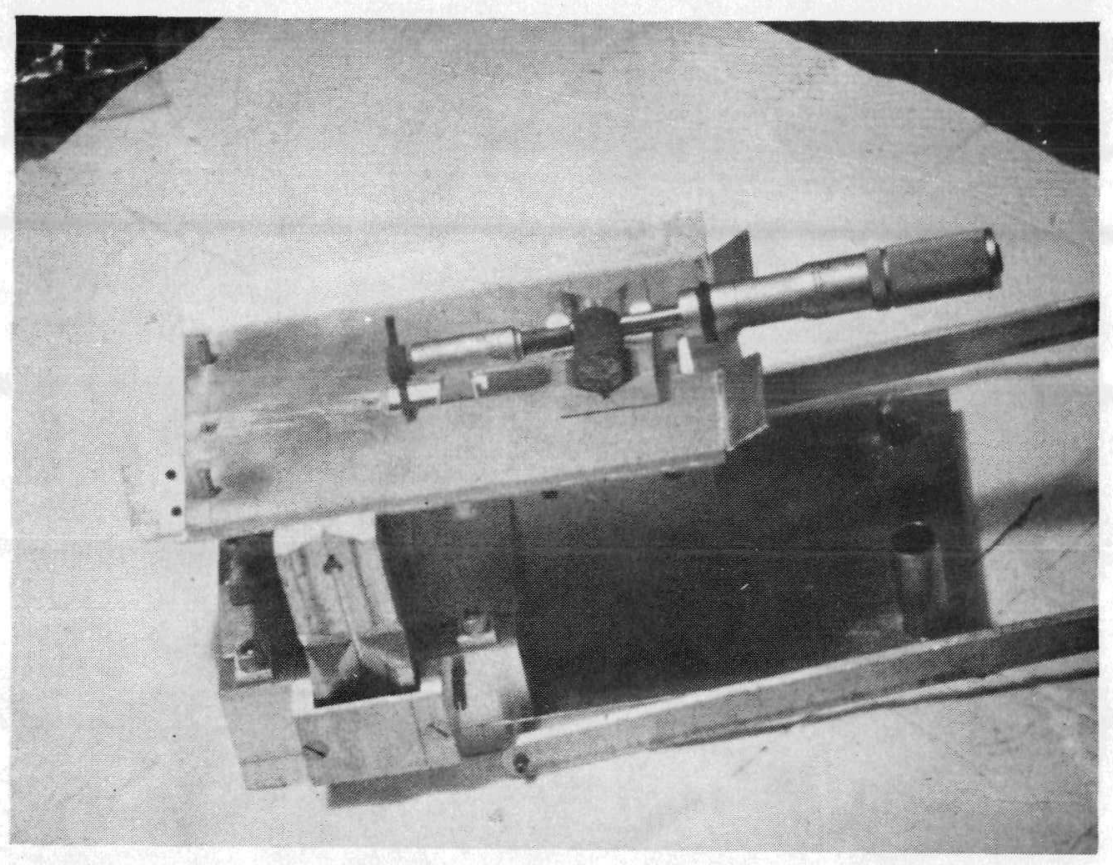

K7440-31

(a)

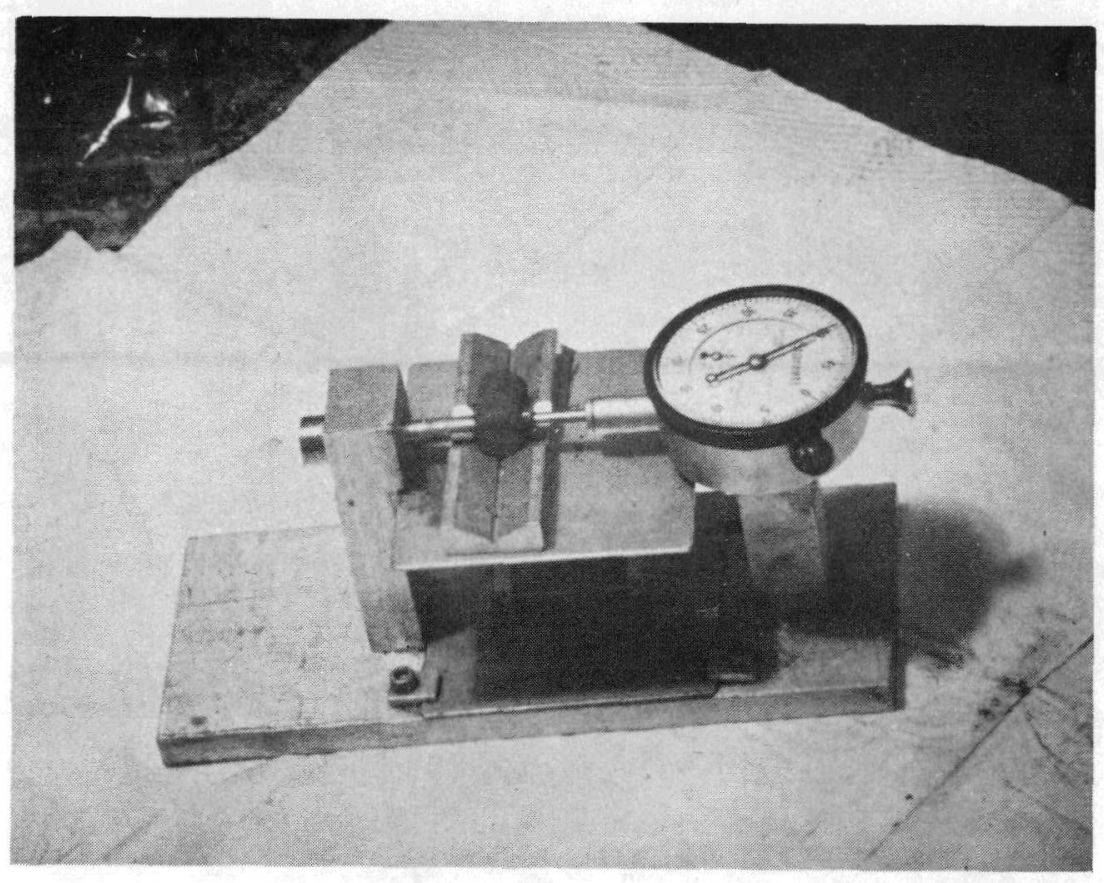

K7440-32

(b)

Fig. 4-31. Photographs showing fuel rod dimensional measurements being made using (a) a barrel micrometer and (b) a dial gauge micrometer 

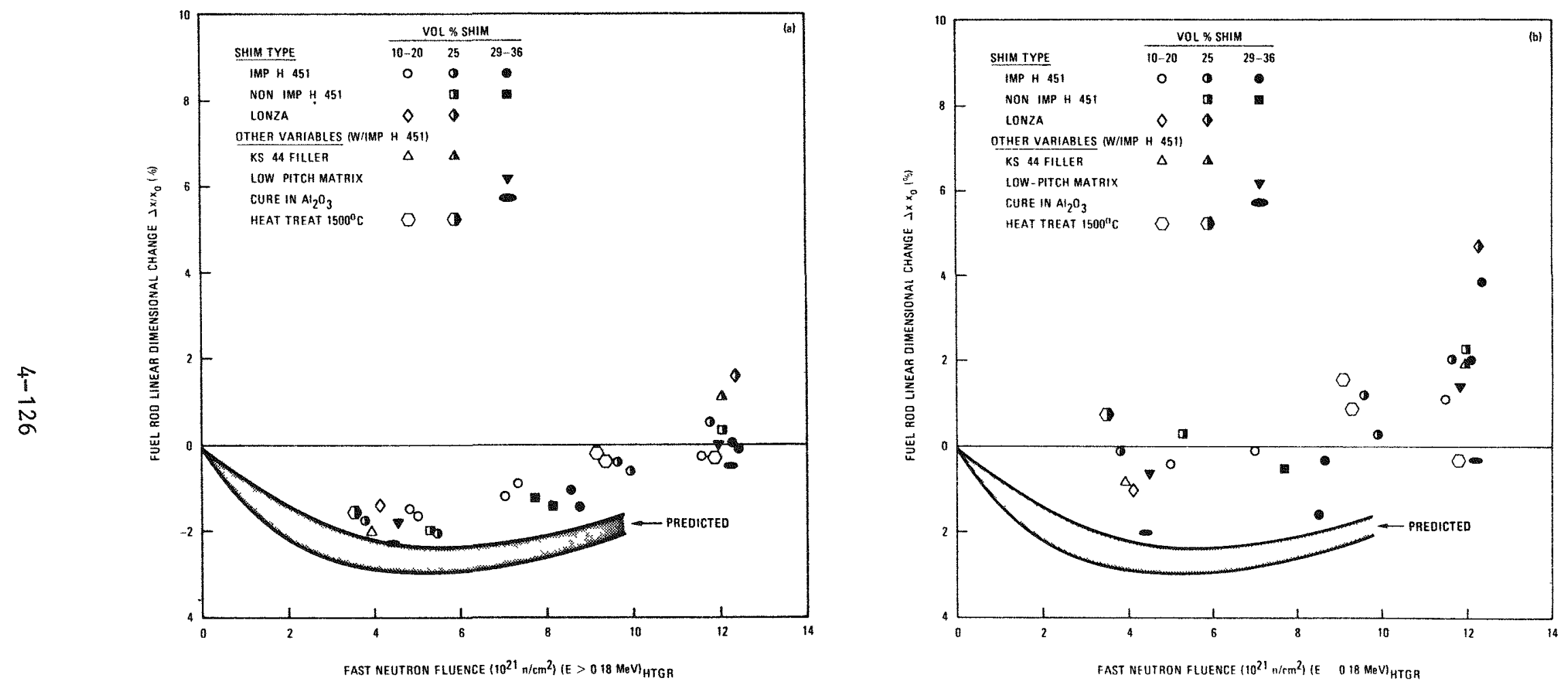

Fig. 4-32. Measured and predicted linear dimensional change data for P13R and P13S fuel rods irradiated at $1100^{\circ} \mathrm{C}$ (centerline temperature): (a) $\Delta \mathrm{D} / \mathrm{D}_{\mathrm{O}}$ and (b) $\Delta \mathrm{L} / \mathrm{L}_{\mathrm{O}}$ 


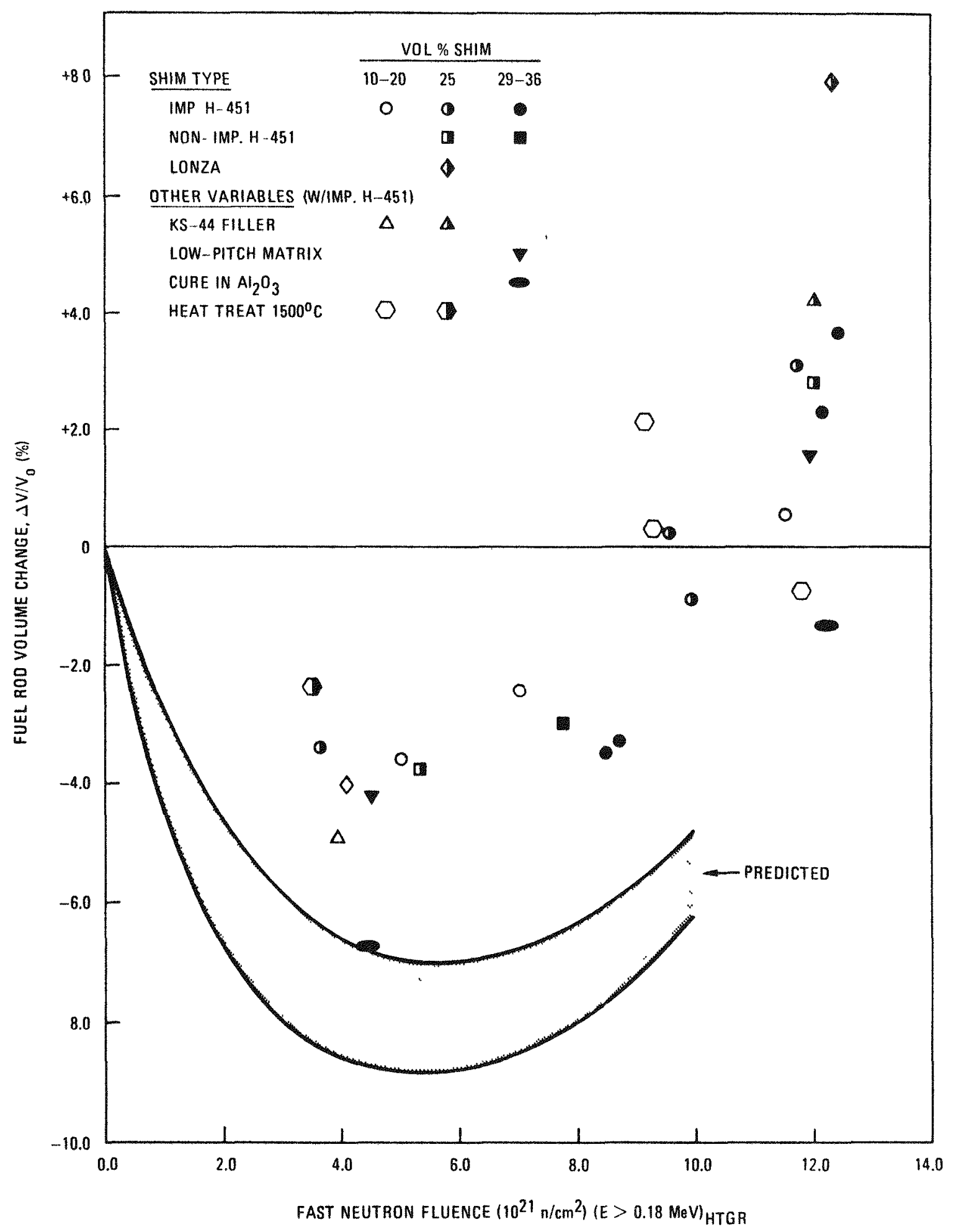

Fig. 4-33. Measured and predicted volume change data for P13R and P13S fuel rods irradiated at $1100^{\circ} \mathrm{C}$ (centerline temperature) 


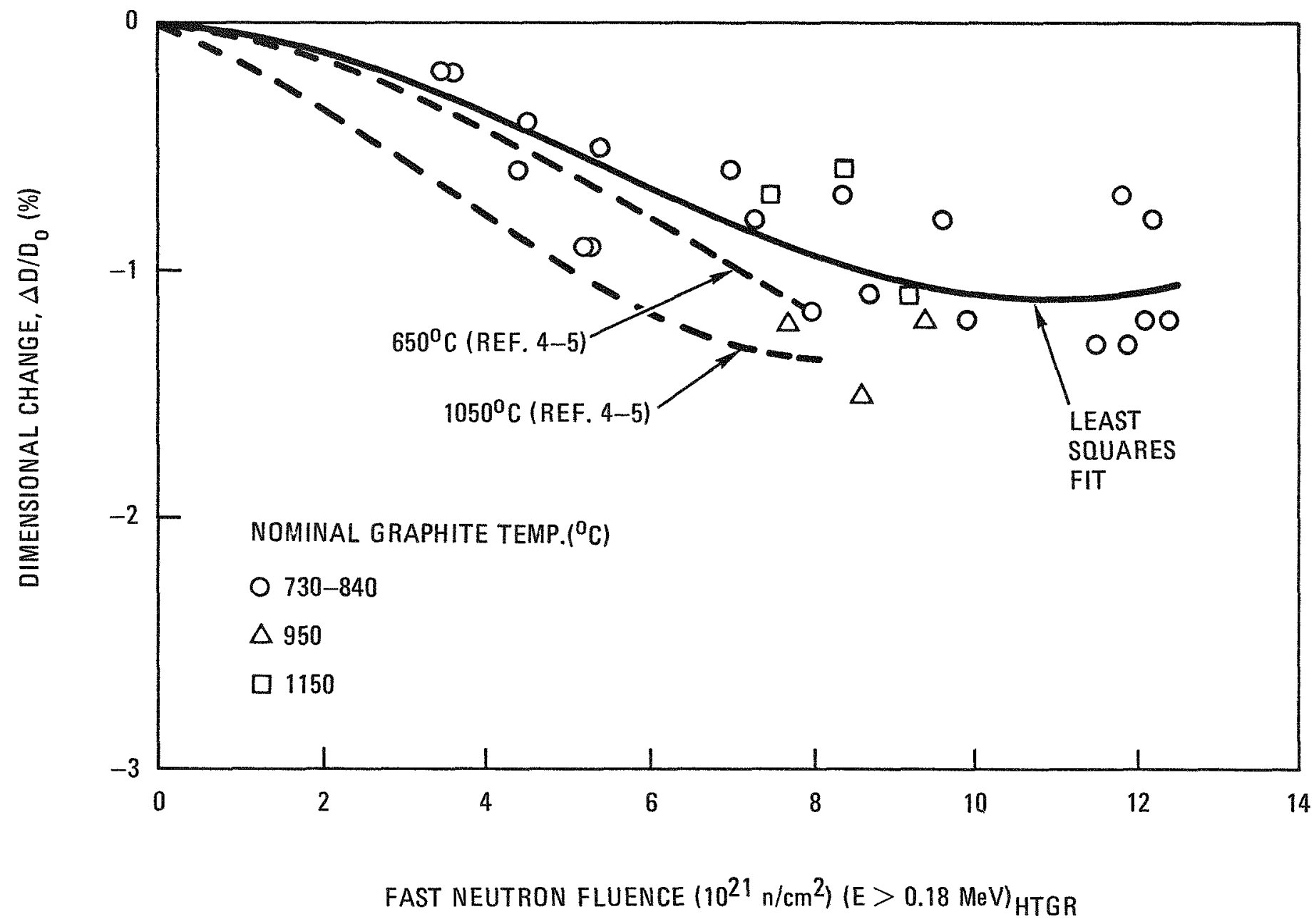

Fig. 4-34. Irradiation-induced diametral change of graphite crucibles as a function of fast neutron fluence 


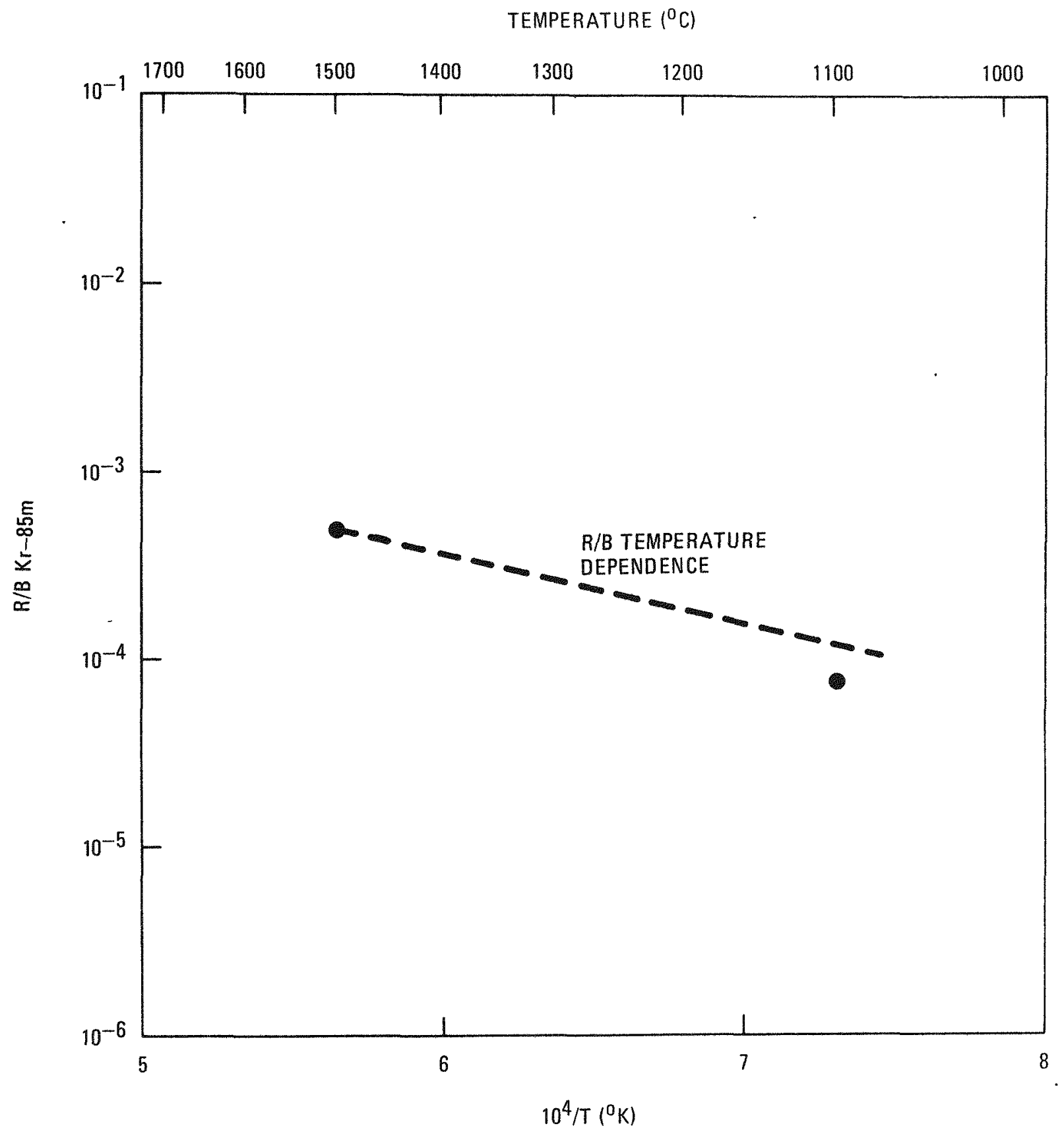

Fig. 4-35. Fission gas release versus reciprocal temperature for fuel rod 7161-004-19-5 irradiated in capsule P13S (position 5D) 


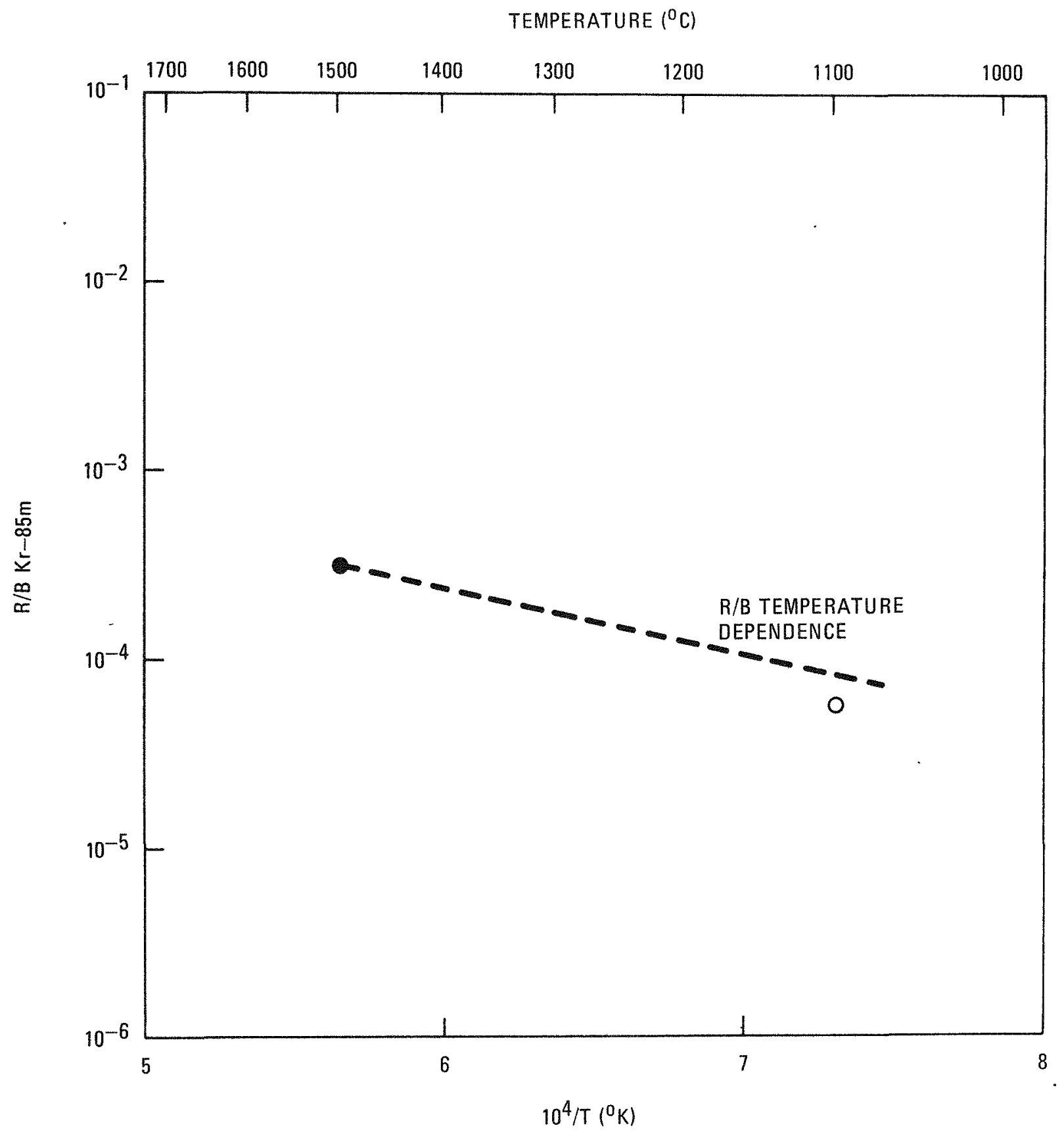

Fig. 4-36. Fission gas release versus reciprocal temperature for fuel rod 7161-004-17-6 irradiated in capsule P13S (position 5B) 


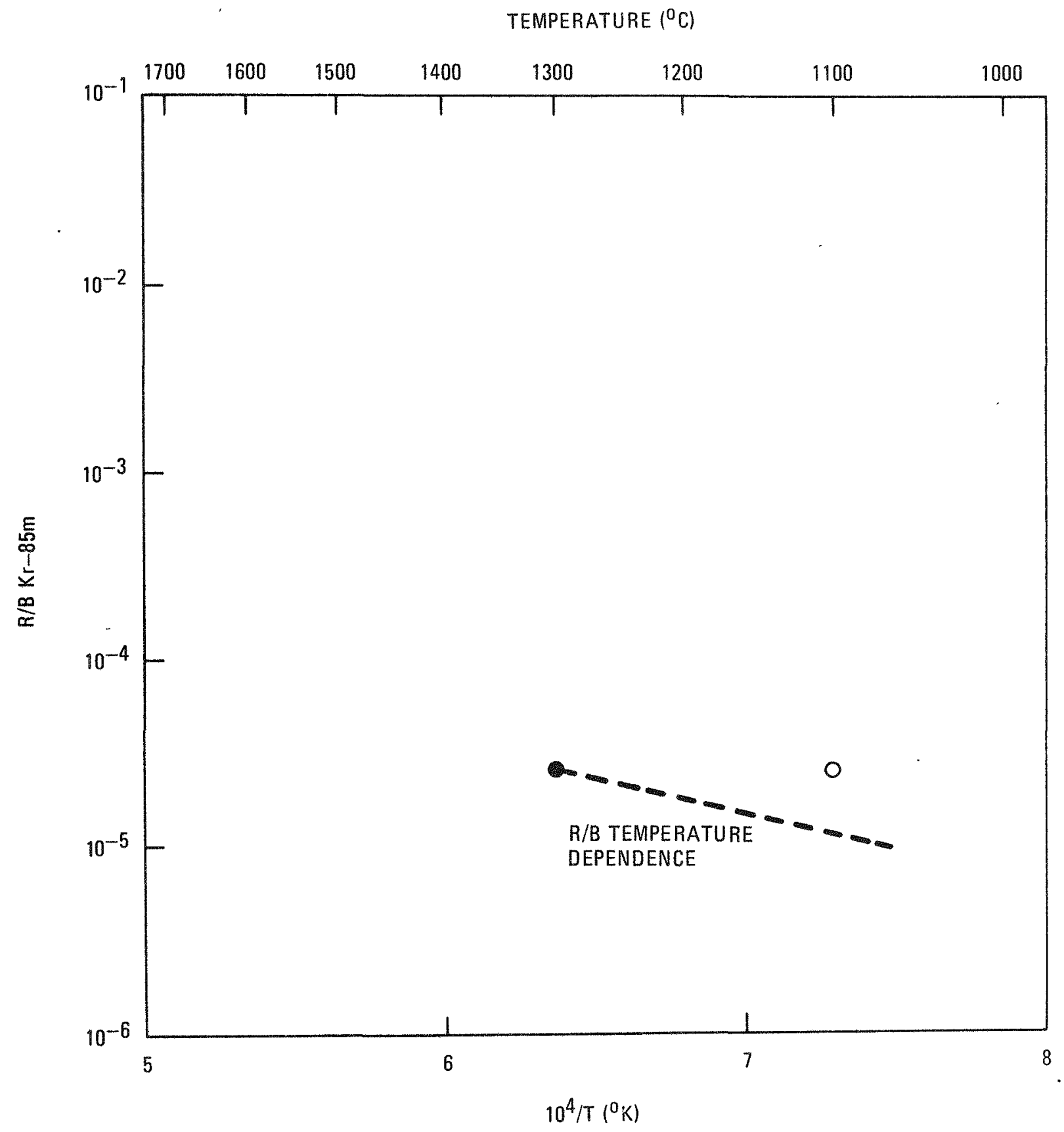

Fig. 4-37. Fission gas release versus reciprocal temperature for fuel rod 7161-004-19-7 irradiated in capsule P13R (position 5D) 


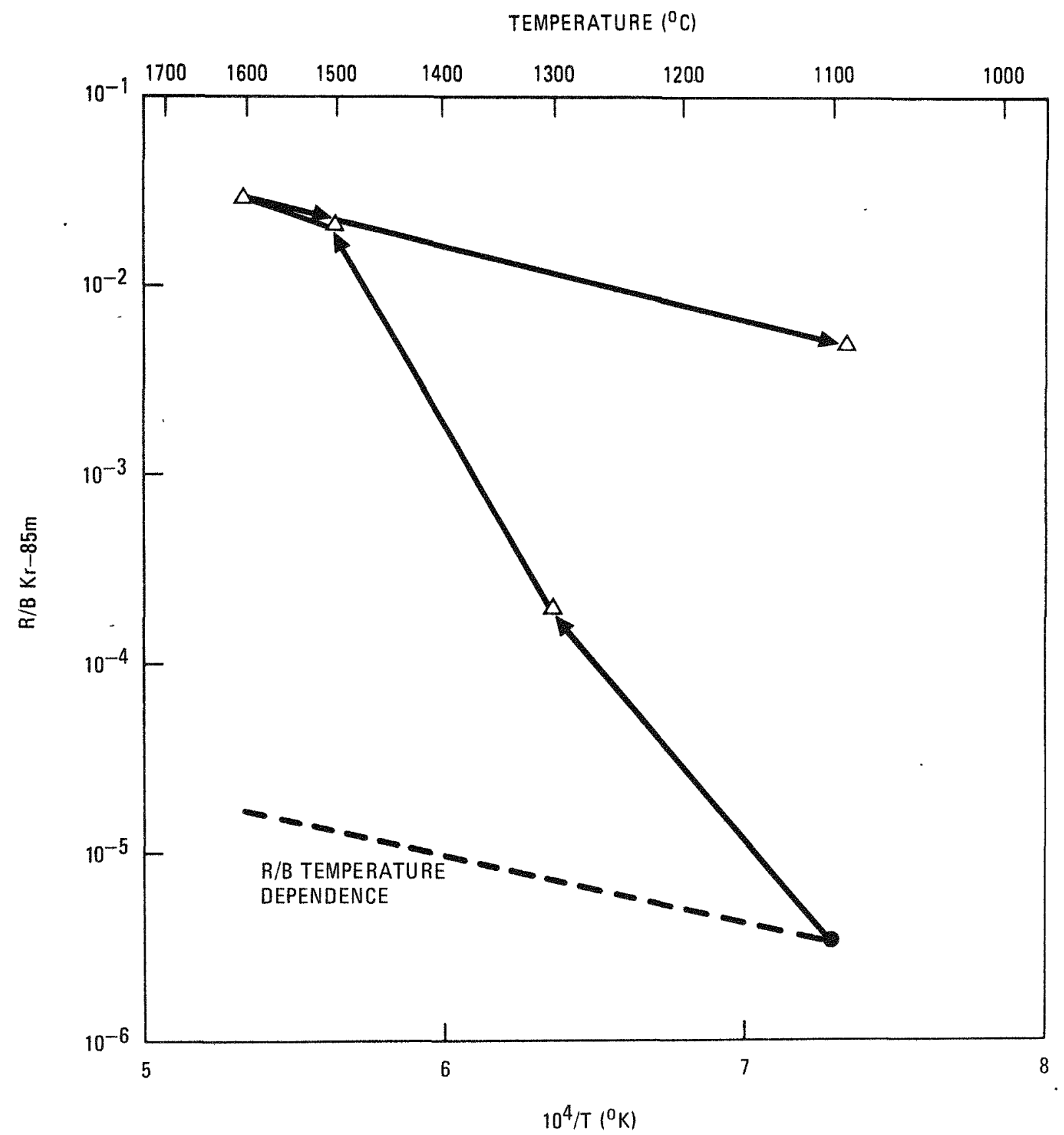

Fig. 4-38. Fission gas release versus reciprocal temperature for fuel rod 7161-004-03-5 irradiated in capsule P13R (position 1C) 


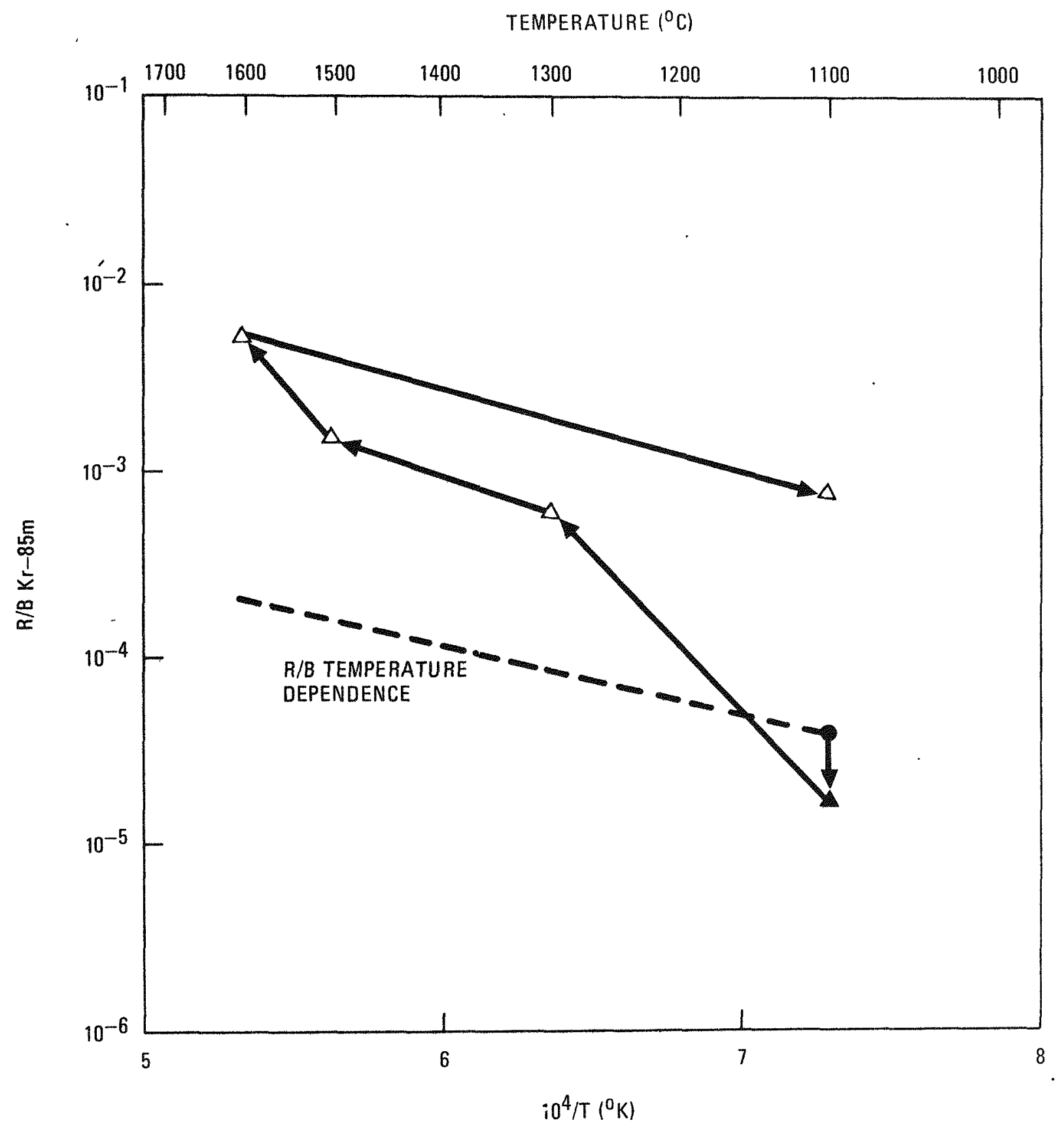

Fig, 4-39. Fission gas release versus reciprocal temperature for fuel rod 7161-004-16-5 irradiated in capsule P13R (position 5A) 


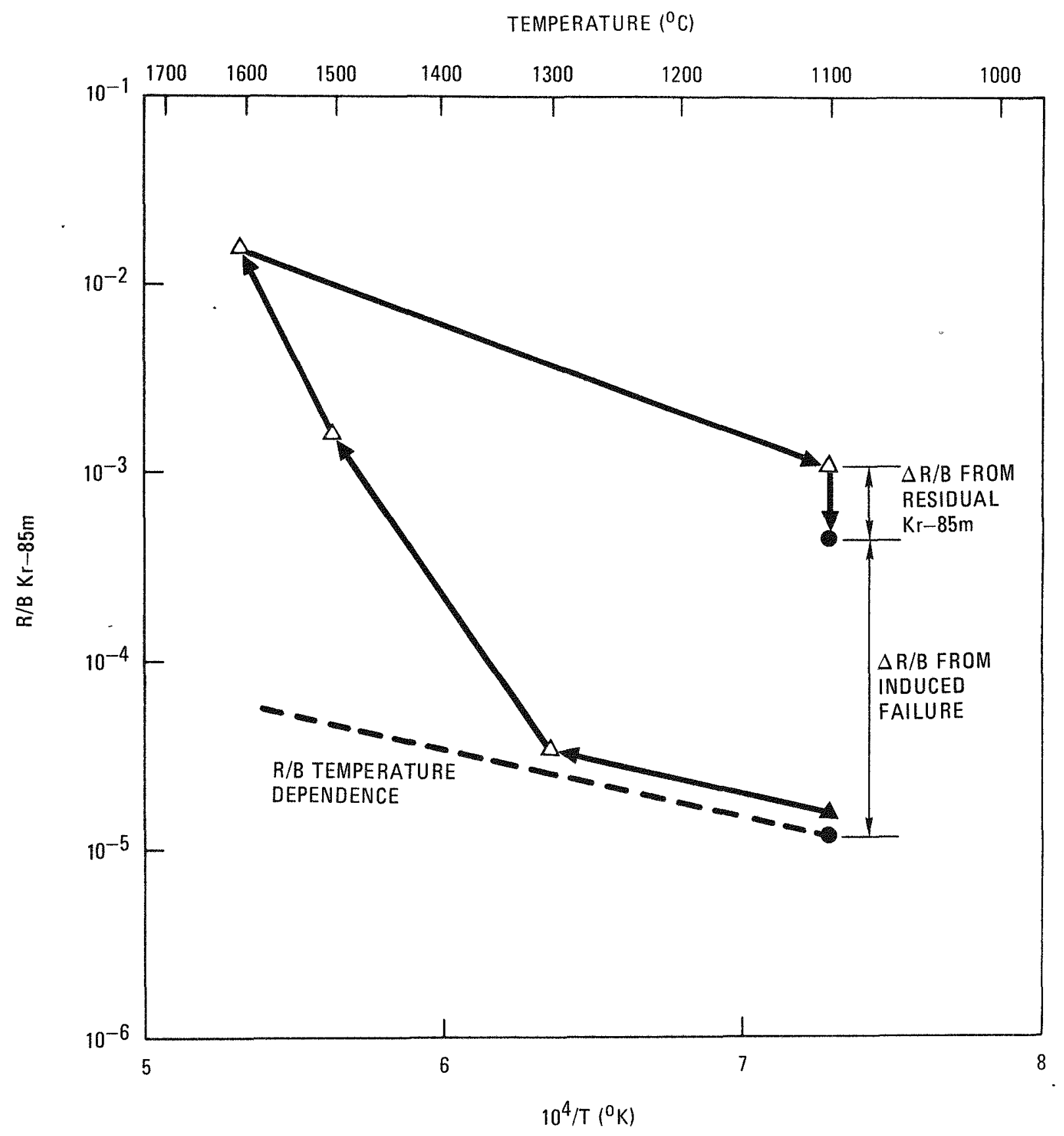

Fig. 4-40. Fission gas release versus reciprocal temperature for fue1 rod 7161-004-14-5 irradiated in capsule P13S (position 2D) 


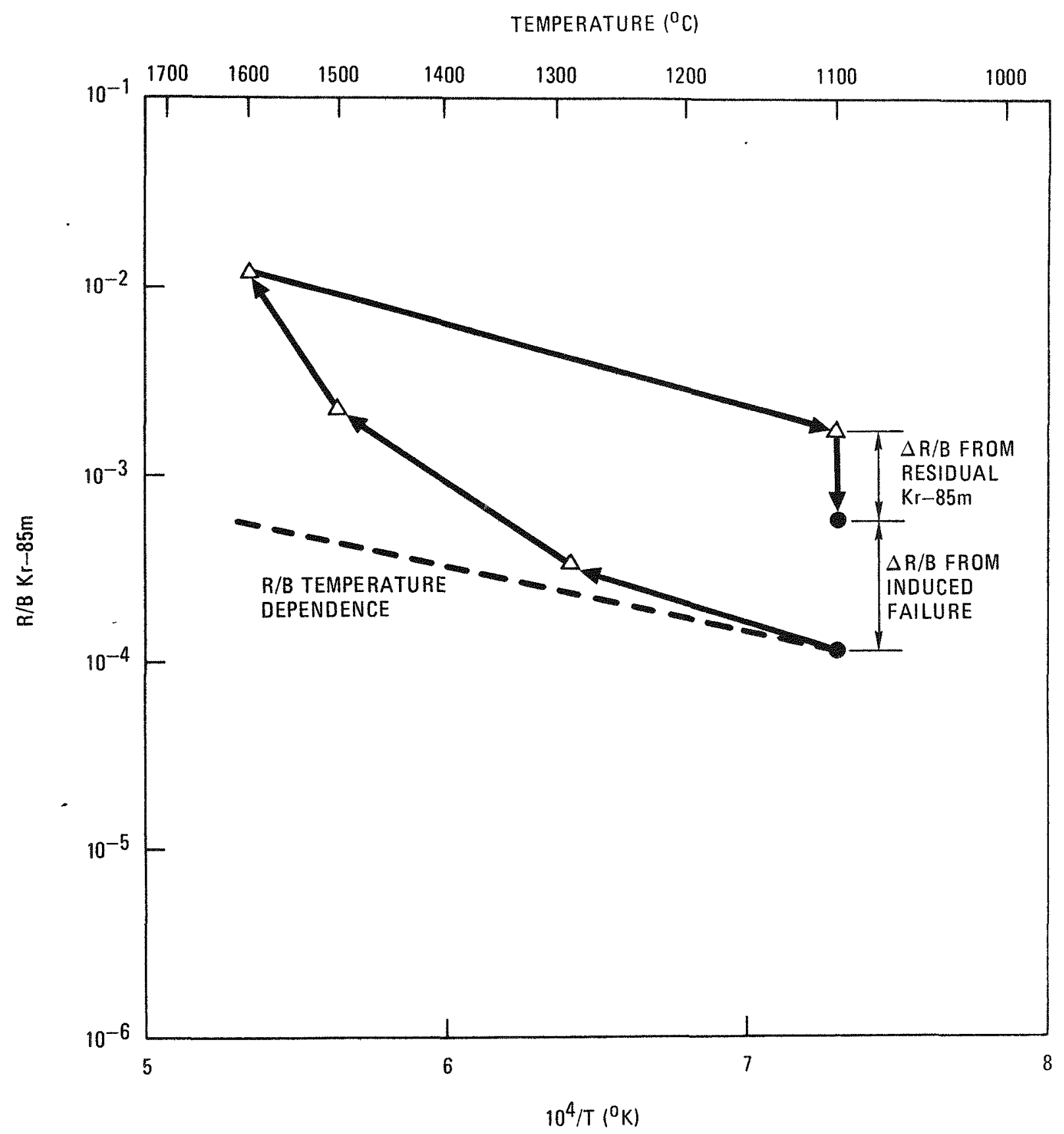

Fig. 4-41. Fission gas release versus reciprocal temperature for fuel rod 7161-004-03-6 irradiated in capsule P13S (position 1C) 


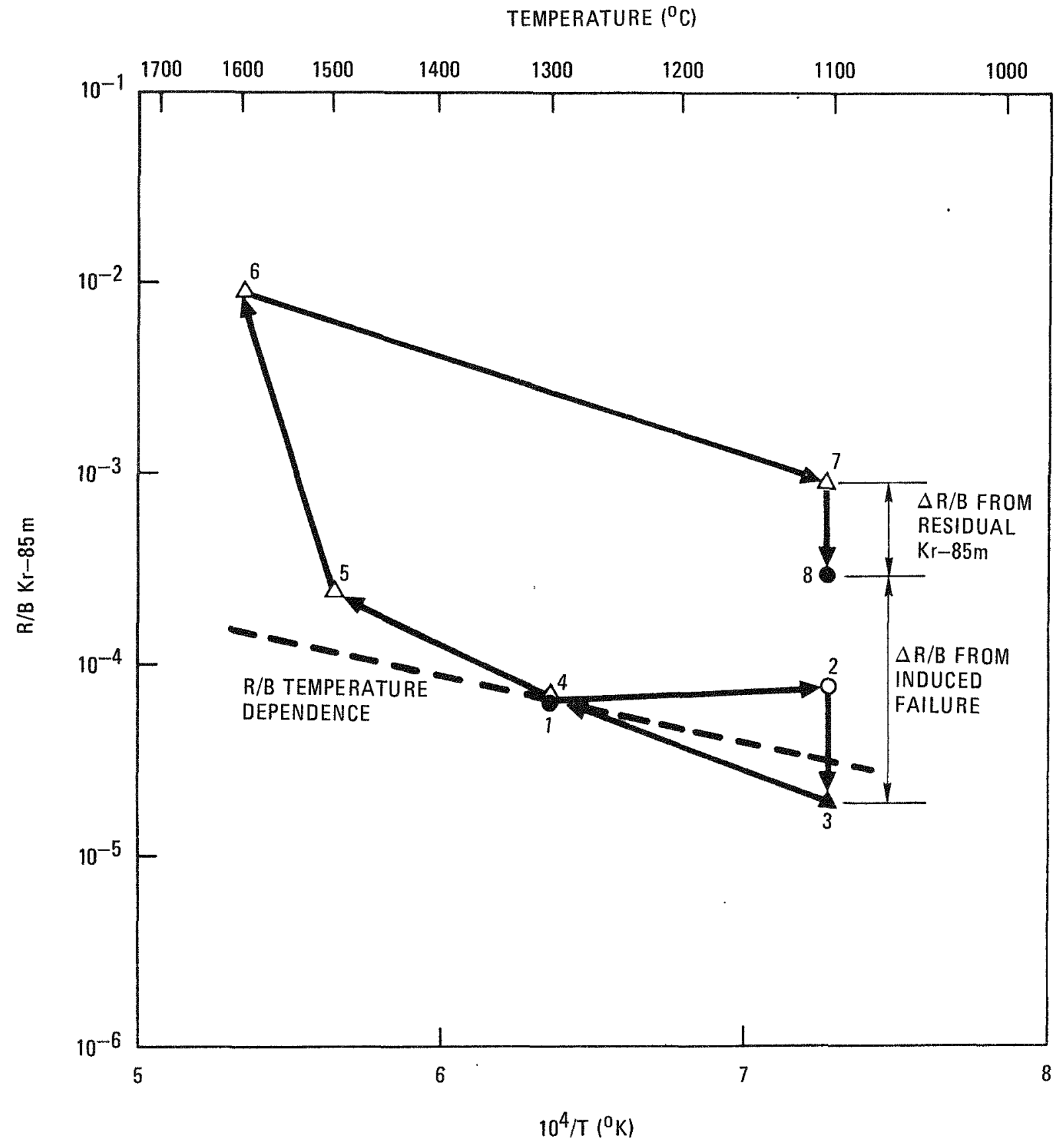

Fig. 4-42. Fission gas release versus reciprocal temperature for fuel rod 7161-004-17-5 irradiated in capsule P13R (position 5B) 

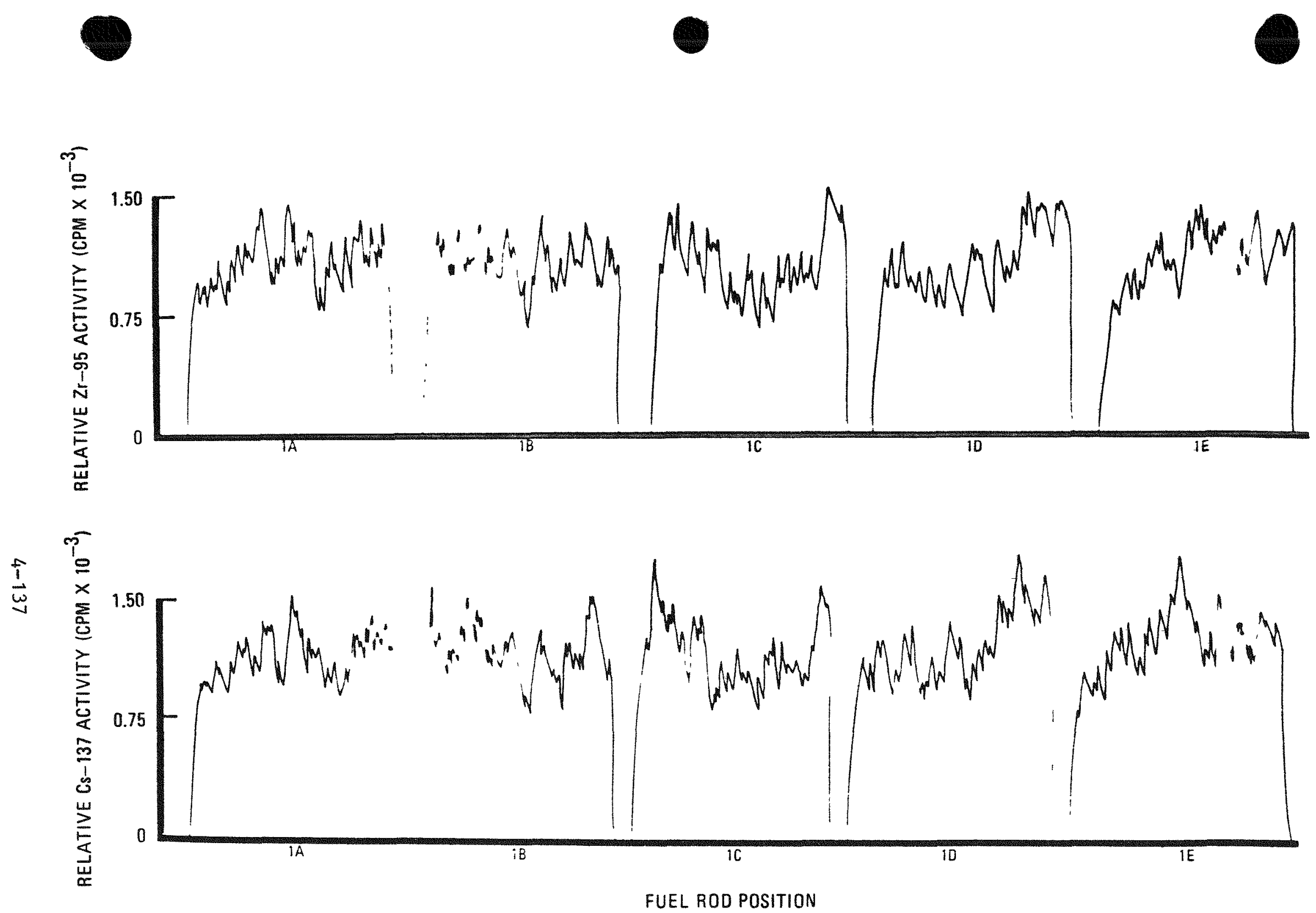

Fig. 4-43. Single-channe1 Cs-137 and Zr-95 gamma-ray scans of fuel rods irradiated in cell 1 of capsule P13R 


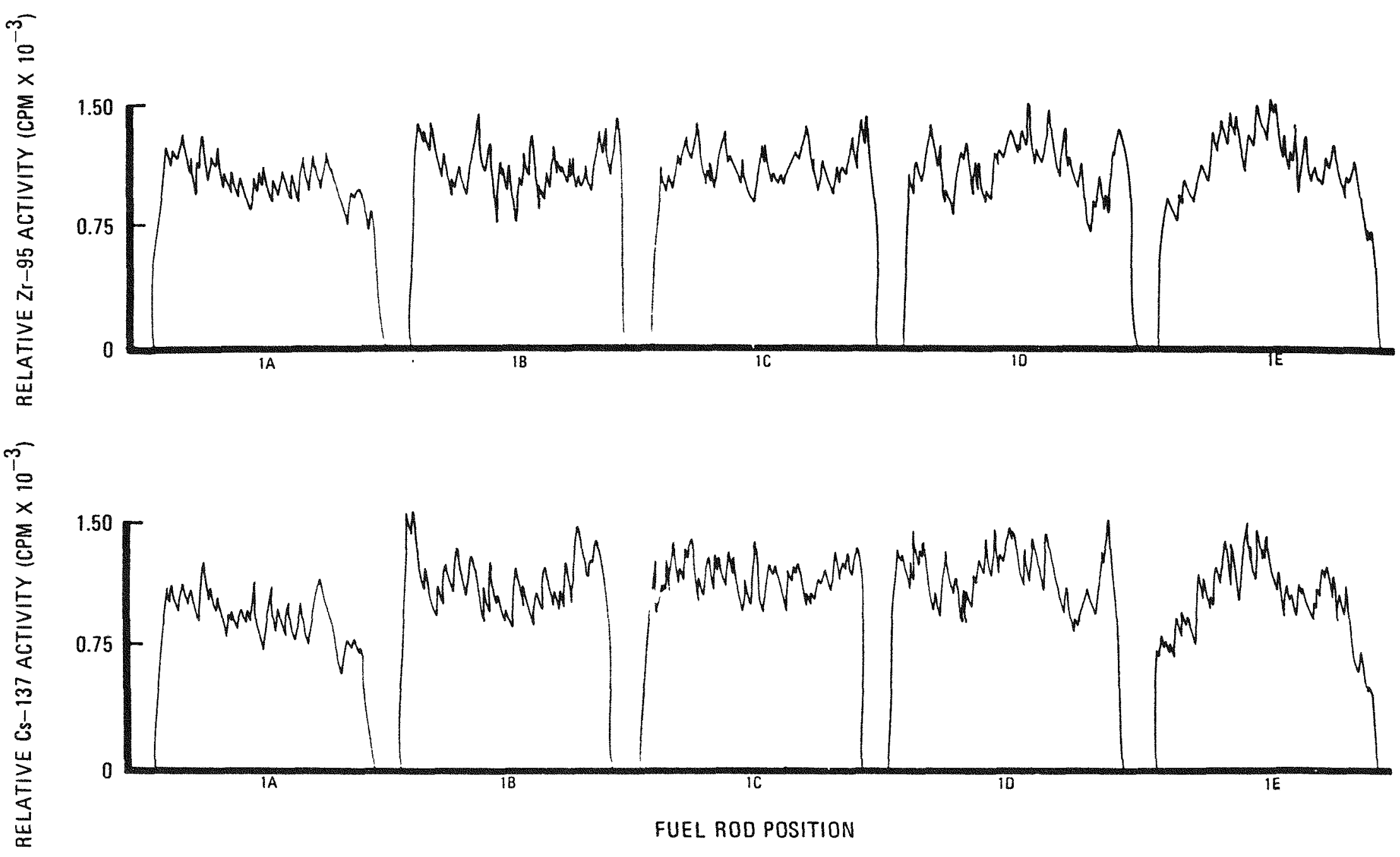

Fig. 4-44. Single-channel $\mathrm{Cs}-137$ and $\mathrm{Zr}-95$ gamma-ray scans of fuel rods irradiated in cel1 1 of capsule P13S 

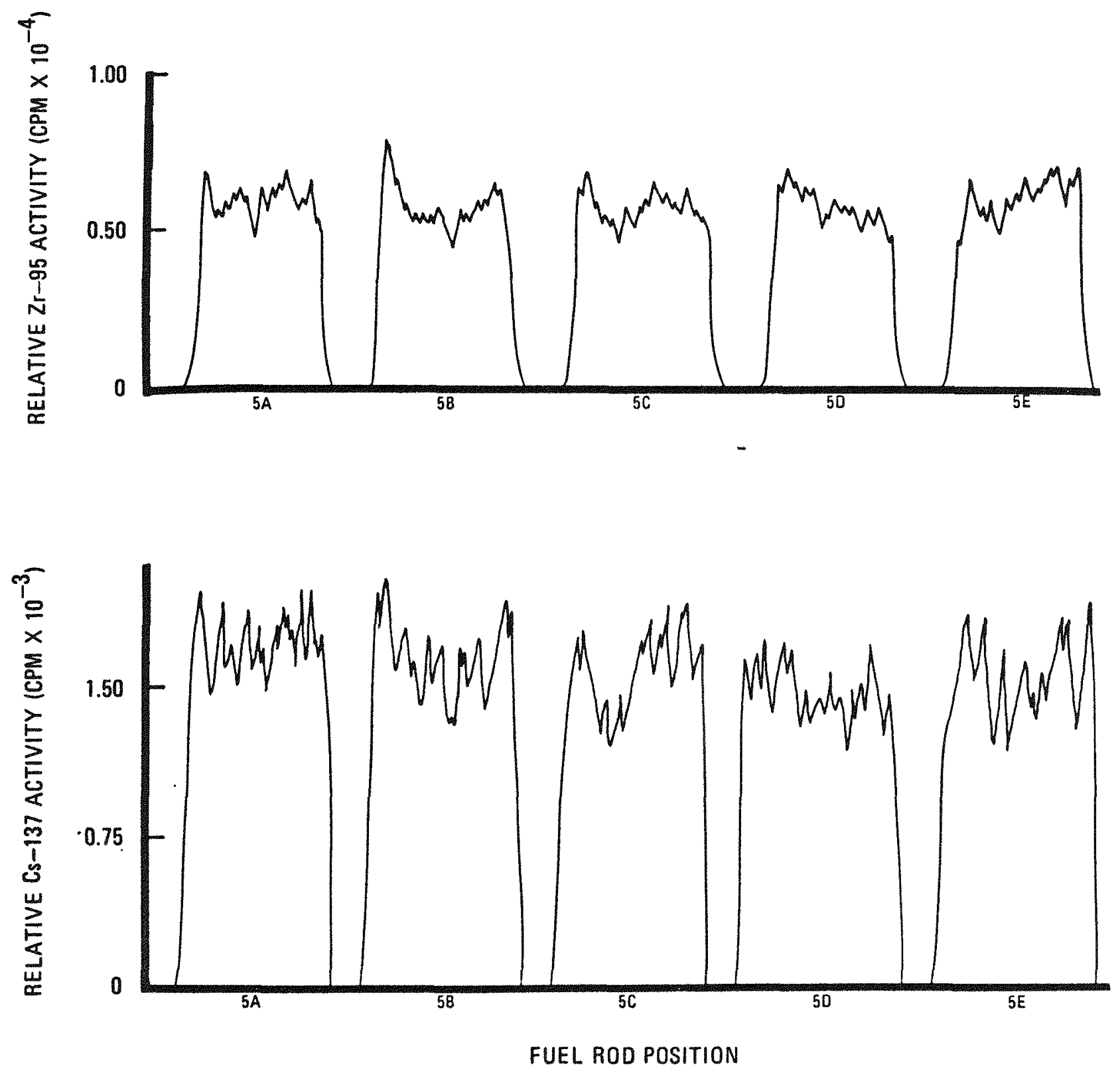

Fig. 4-45. Single-channel Cs-137 and Zr-95 gamma-ray scans of fue1 rods irradiated in cel1 5 of capsule P13R 

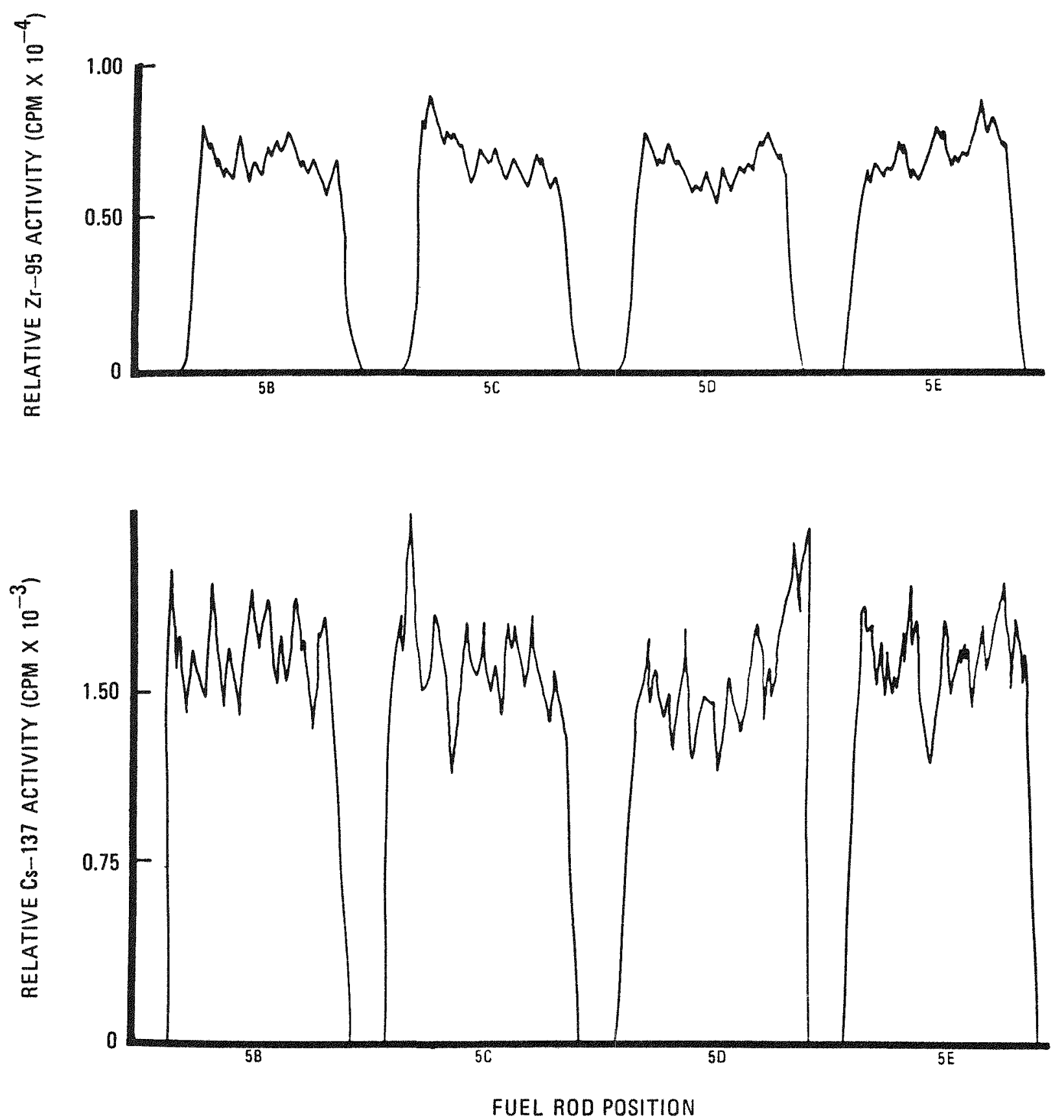

Fig. 4-46. Single-channel $\mathrm{Cs}-137$ and $\mathrm{Zr}-95$ gamma-ray scans of fuel rods irradiated in cel1 5 of capsule P13S 
SPECTRUM IDENTIFICATION

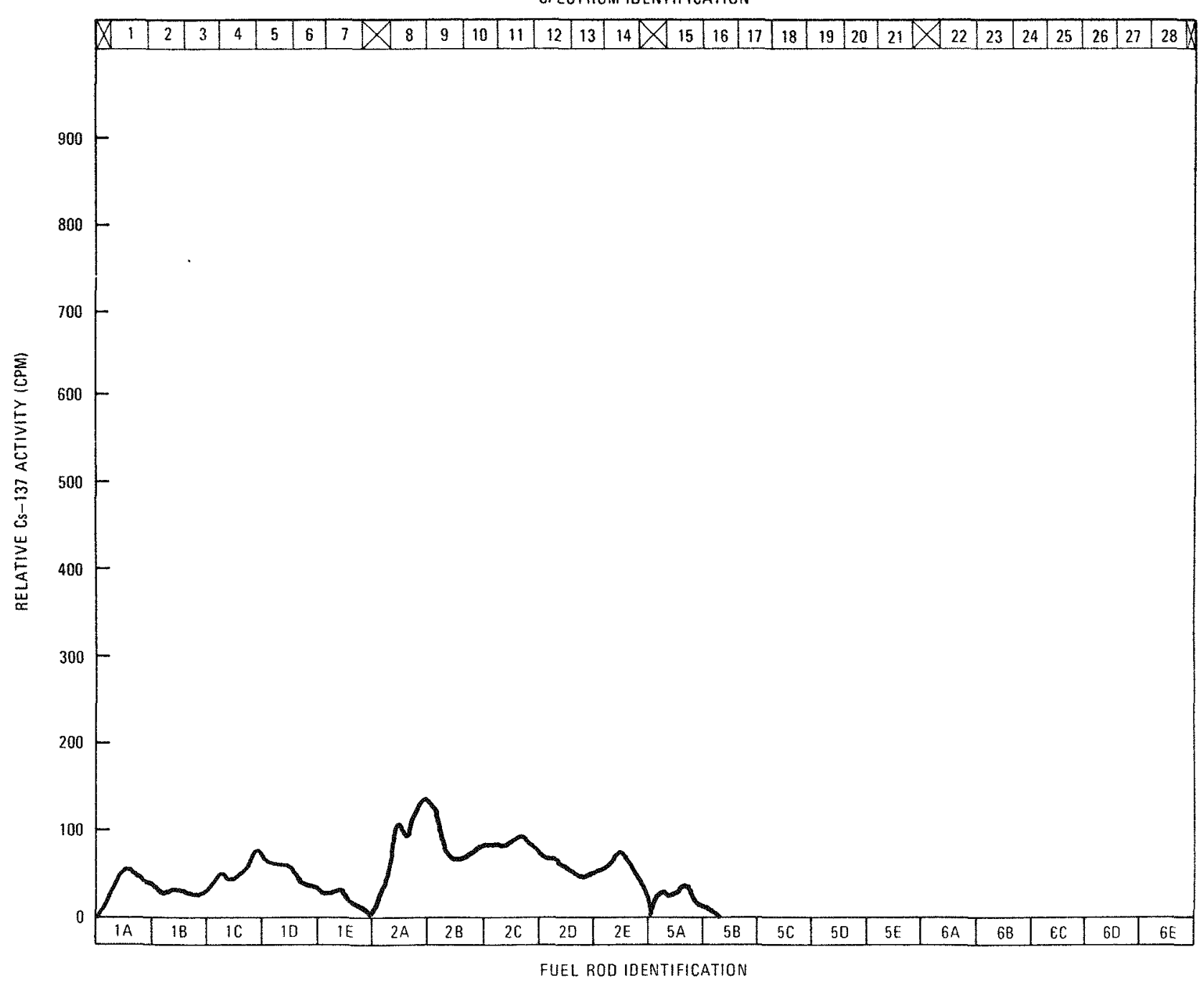

Fig. 4-47. Single-channel gamma scan of Cs-137 (661 keV) and location of multichannel gamma scan spectra for P13R graphite fuel rod crucibles 
SPECTRUM IDENTIFICATION

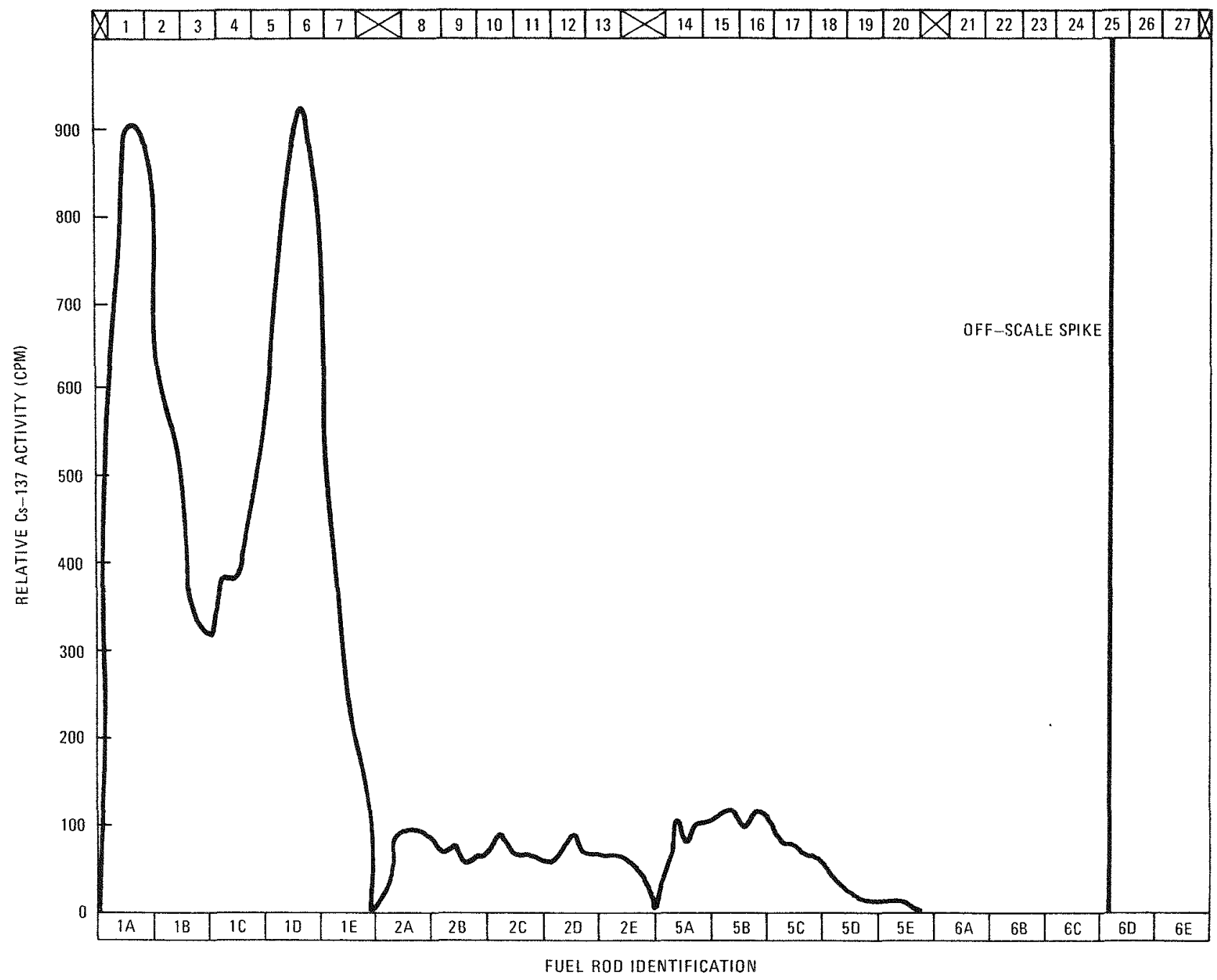

Fig. 4-48. Single-channel gamma scan of $\mathrm{Cs}^{-137}$ (661 keV) and location of multichannel gamma scan spectra for P13S graphite fuel rod crucibles 


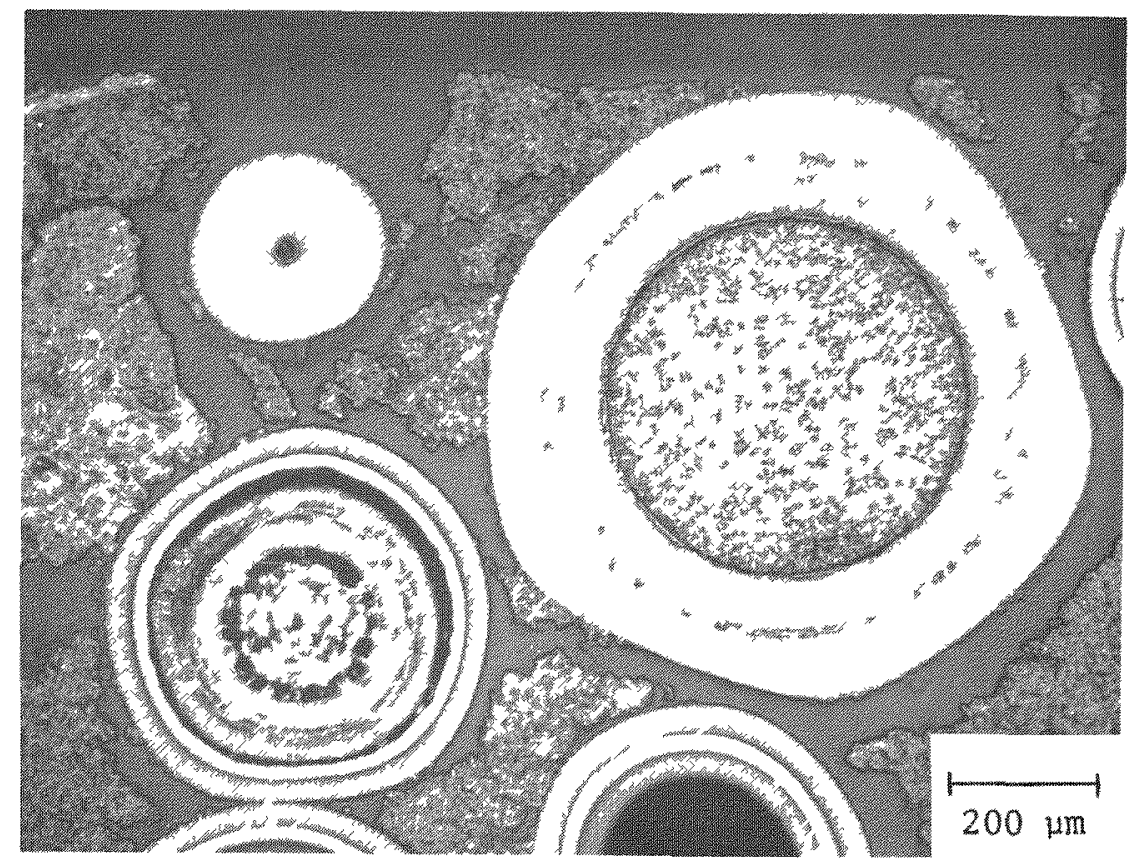

$27440-41$

(a)

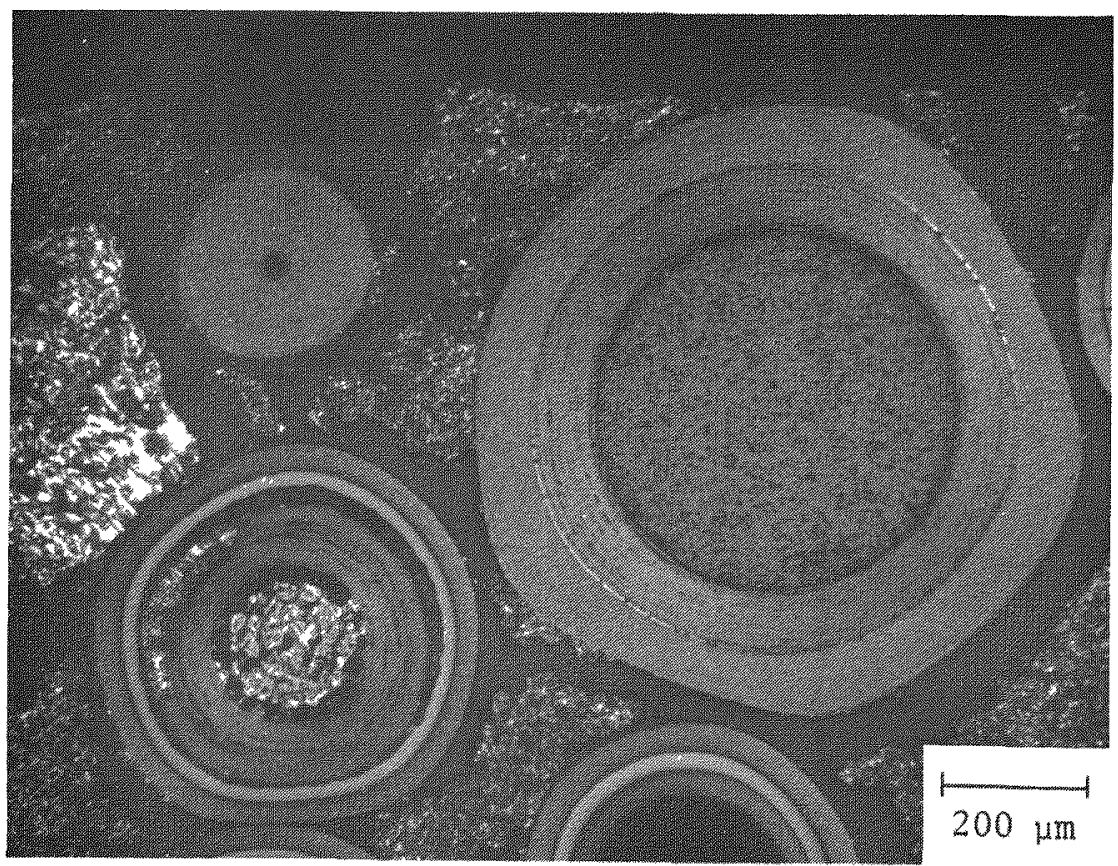

$17440-42$

(b)

Fig. 4-49. Photomicrographs of representative particles from fuel rod 7161-004-06-5 irradiated in capsule $\mathrm{P} 13 \mathrm{R}$ (position 2A) to a fast neutron fluence of $11.8 \times 1021 \mathrm{n} / \mathrm{cm}^{2}$ (E $\left.>0.18 \mathrm{MeV}\right)_{\text {HTGR }}$ at $1050^{\circ} \mathrm{C}$. (a) Bright field and (b) polarized light. 


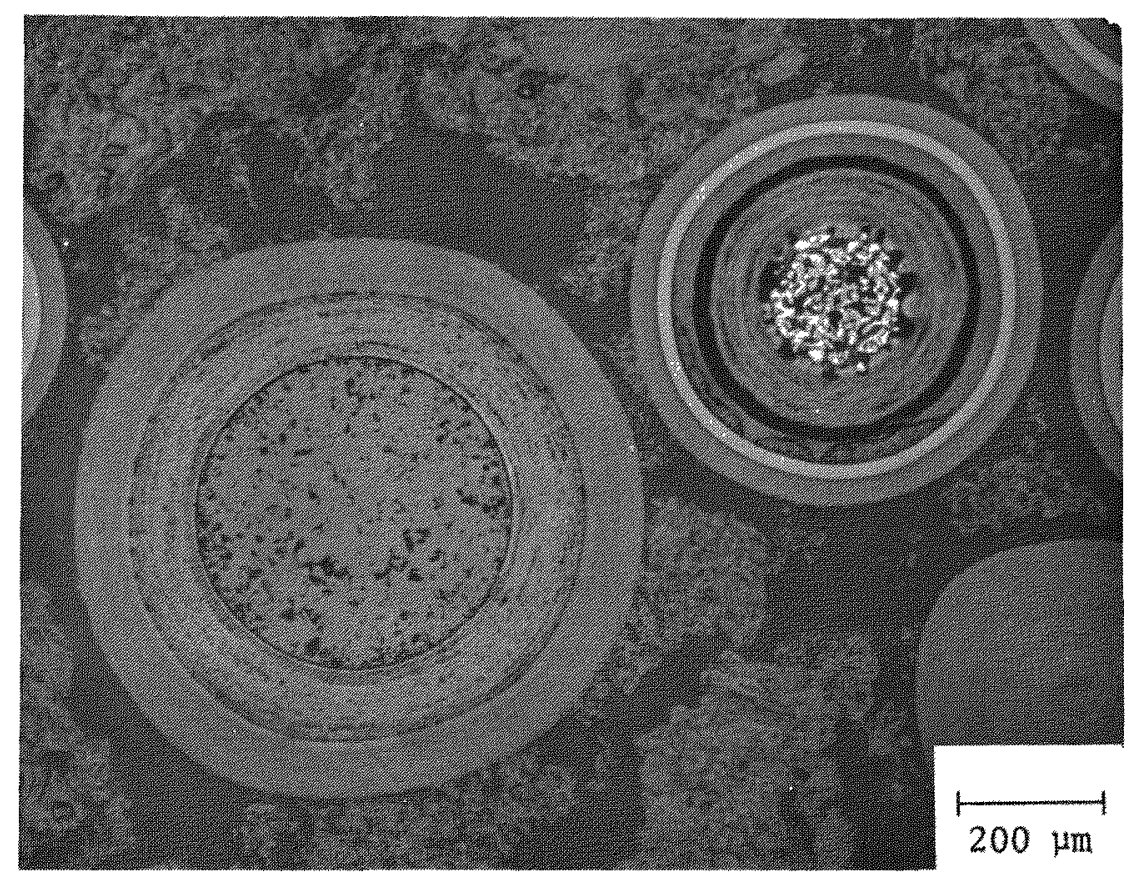

(a)

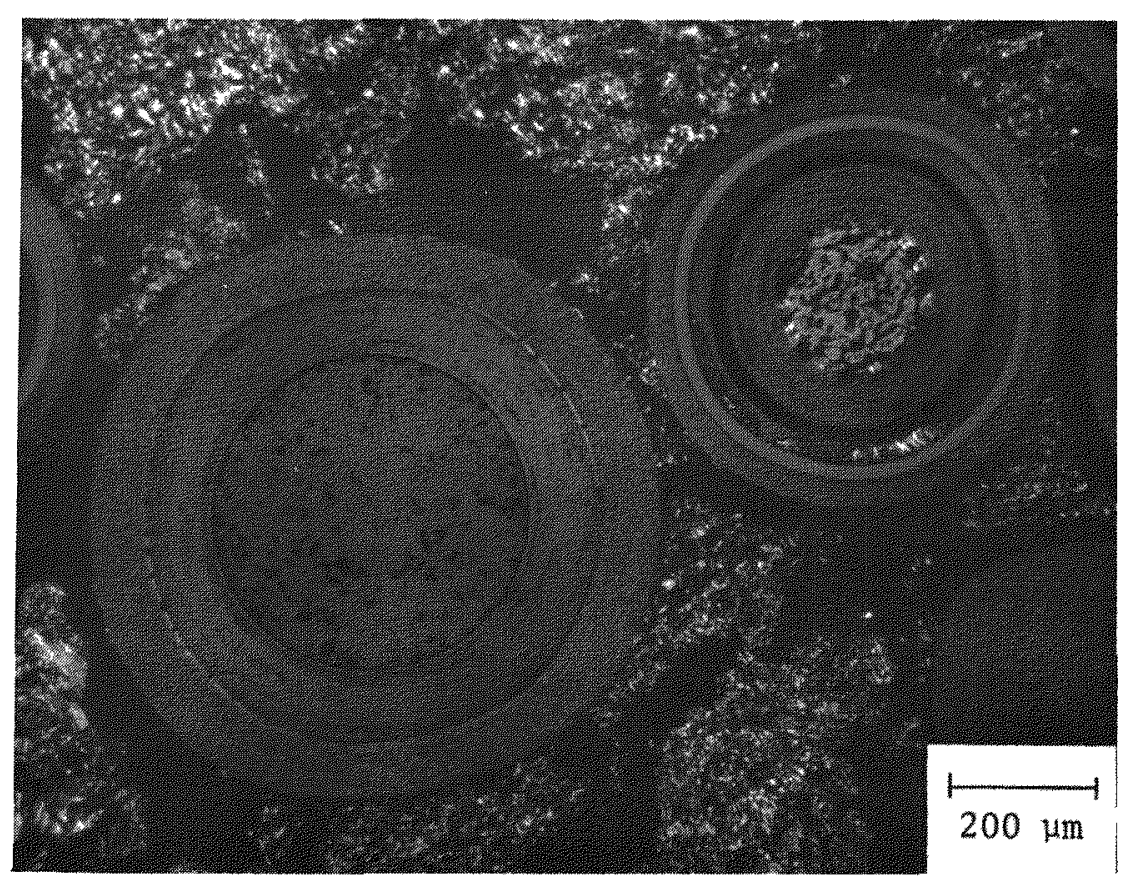

L7440-290

(b)

Fig. 4-50. Photomicrographs of representative particles from fuel rod 7161-004-07-5 irradiated in capsule P13R (position 2B) to a fast neutron fluence of $12.0 \times 10^{21} \mathrm{n} / \mathrm{cm}^{2}(\mathrm{E}>0.18 \mathrm{MeV})_{\text {HTGR }}$ at $1055^{\circ} \mathrm{C}$. (a) Bright field and (b) polarized light. 


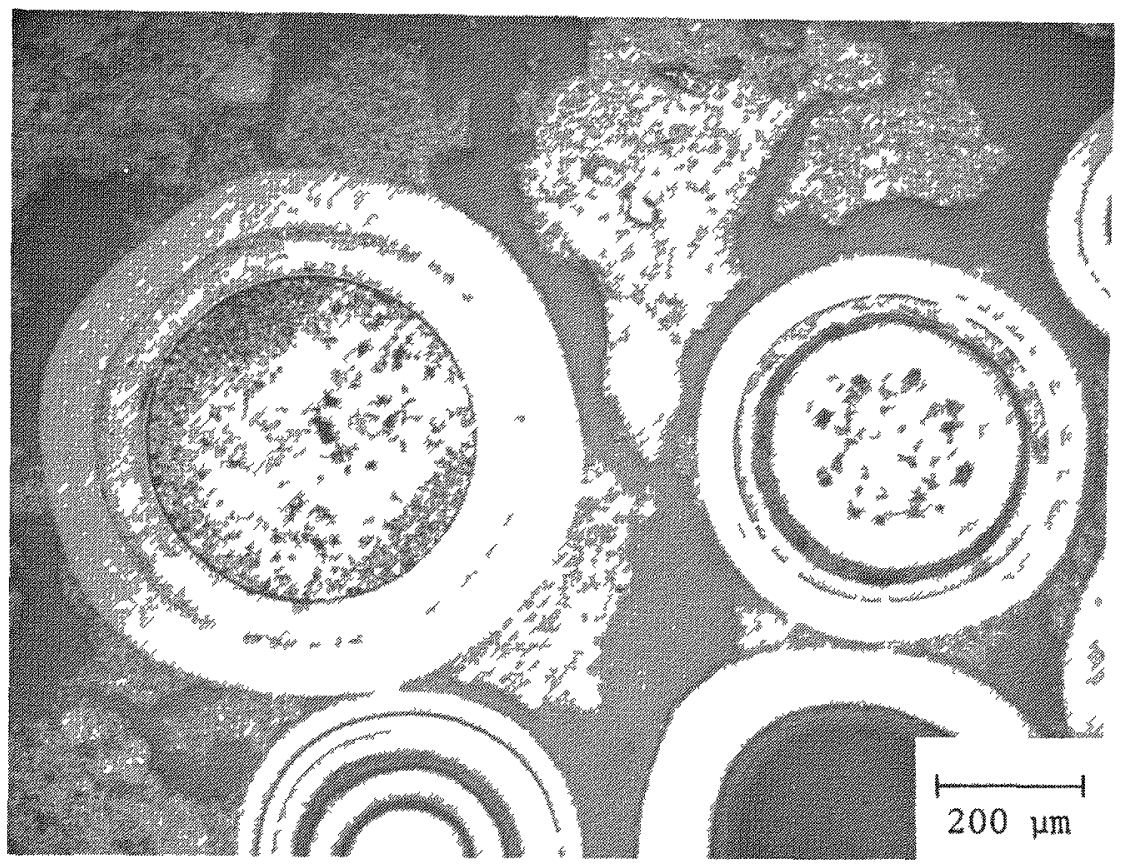

L 7440-254

(a)

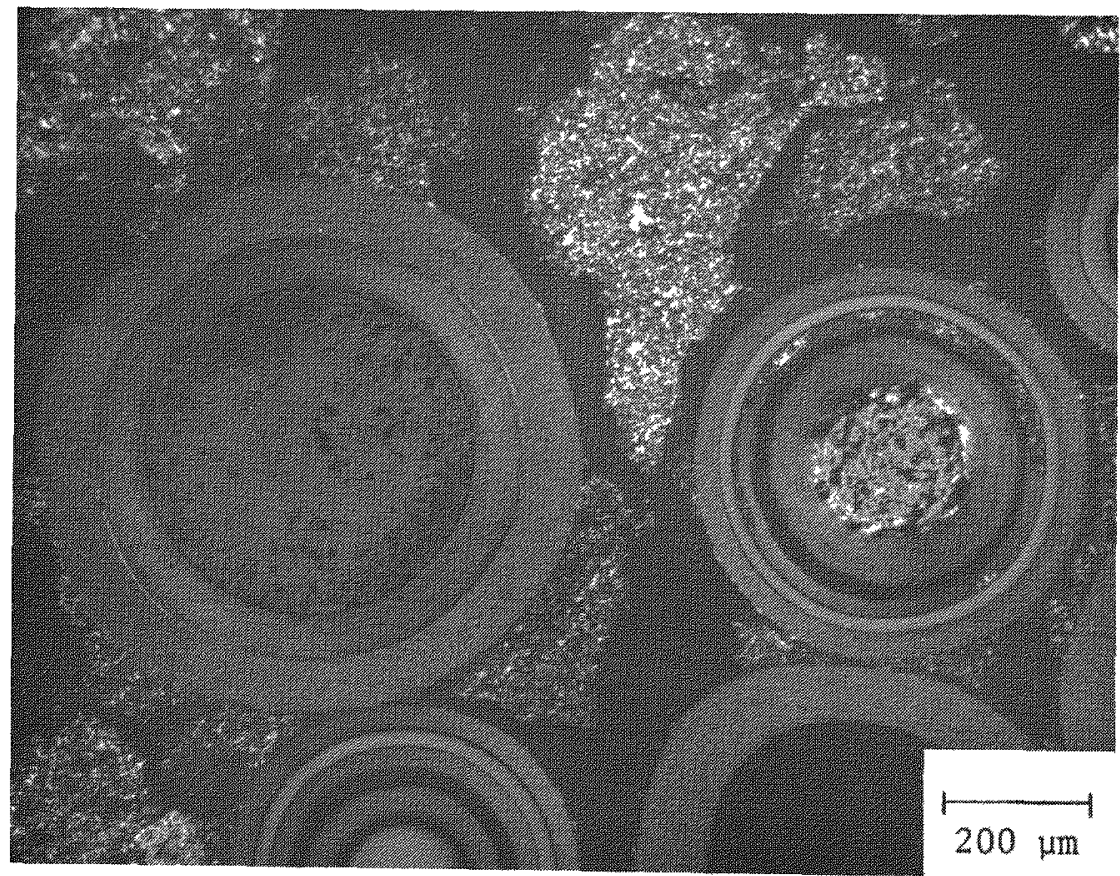

L7440-255

(b)

Fig. 4-51. Photomicrographs of representative particles from fuel rod 7161-004-08-13 irradiated in capsule P13R (position 2C) to a fast neutron fluence of $12.2 \times 1021 \mathrm{n} / \mathrm{cm}^{2}$ (E>0.18 MeV) HTGR at $1045^{\circ} \mathrm{C}$. (a) Bright field and (b) polarized light. 


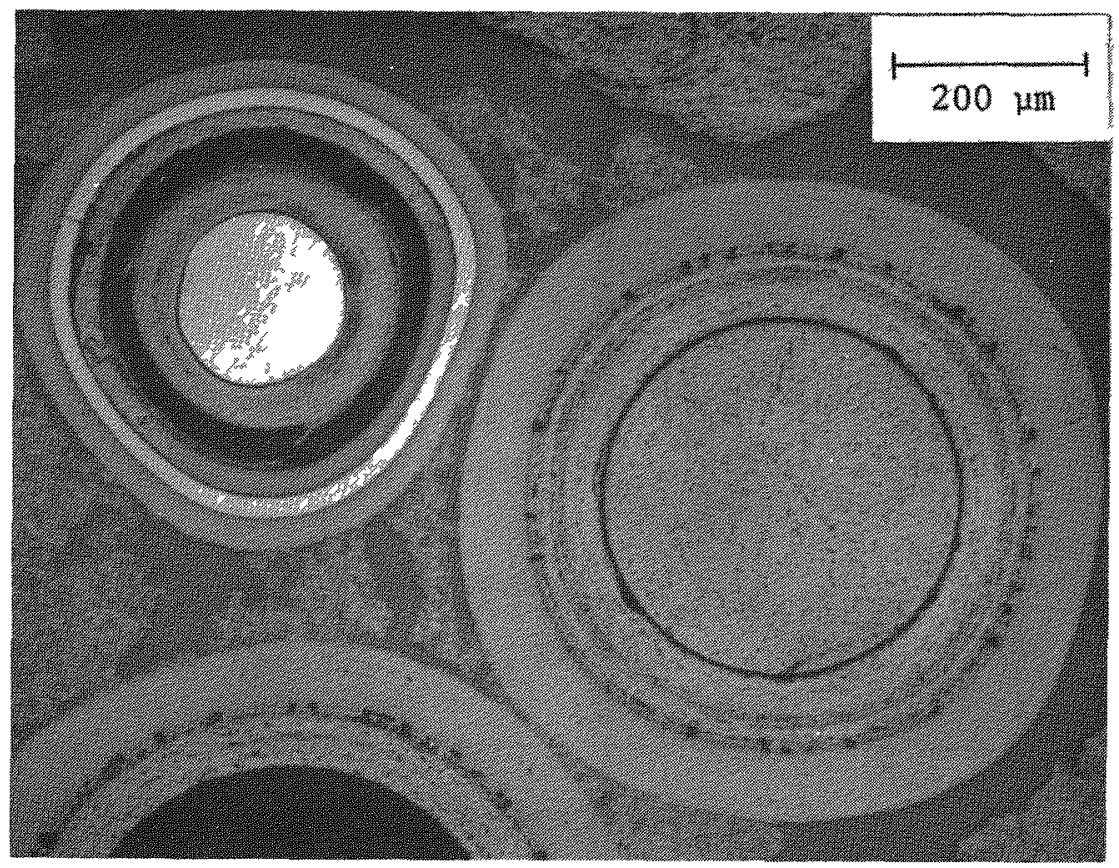

L7440-113

(a)

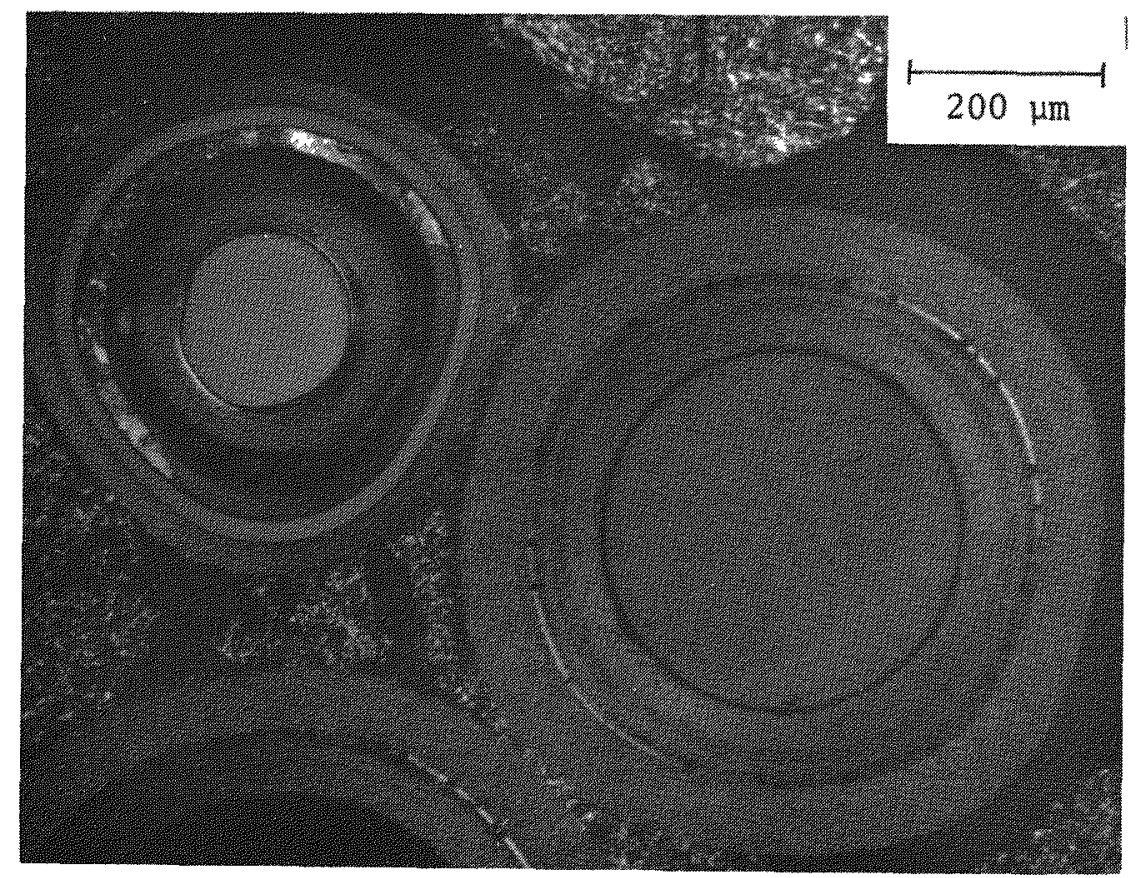

L7440-114

(b)

Fig. 4-52. Photomicrographs of representative particles from fuel rod 7161-004-19-7 irradiated in capsule P13R (position 5D) to a fast neutron fluence of $8.2 \times 10^{21} \mathrm{n} / \mathrm{cm}^{2}(\mathrm{E}>0.18 \mathrm{MeV})$ HTGR at $1225^{\circ} \mathrm{C}$. (a) Bright field and (b) polarized light. 


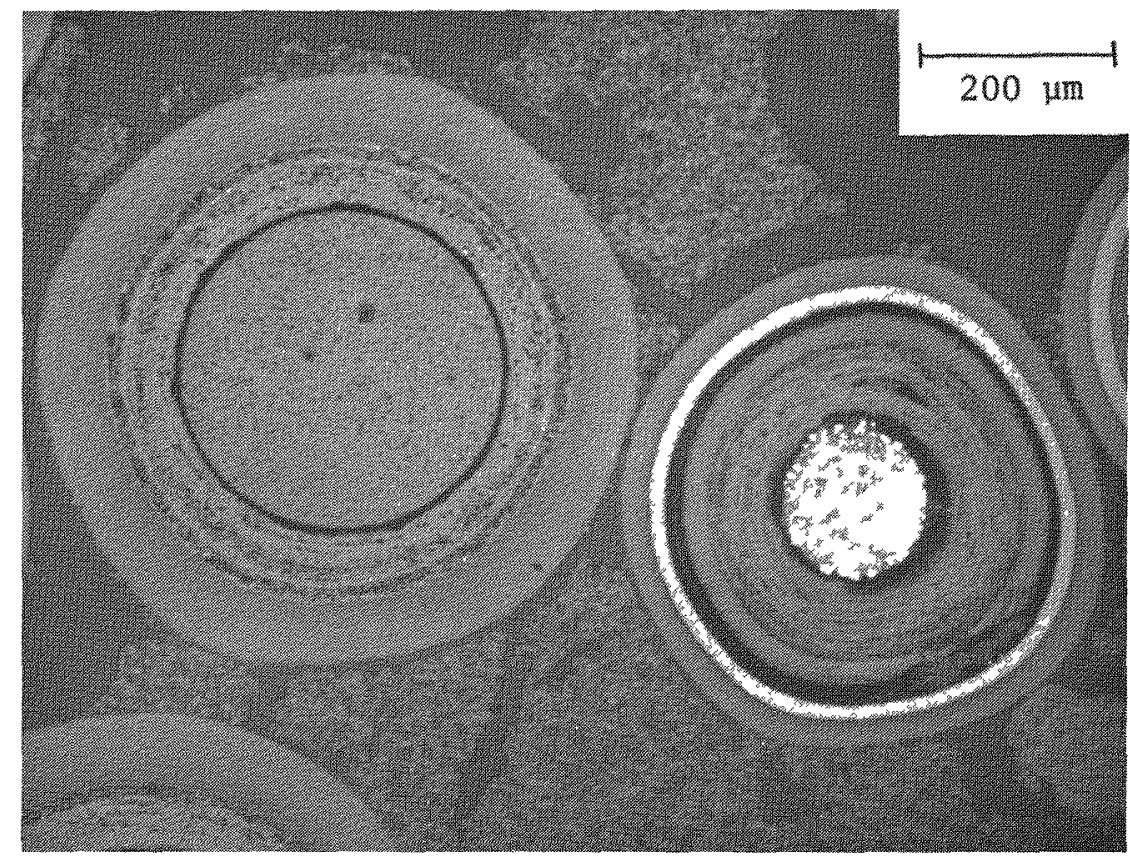

(a)

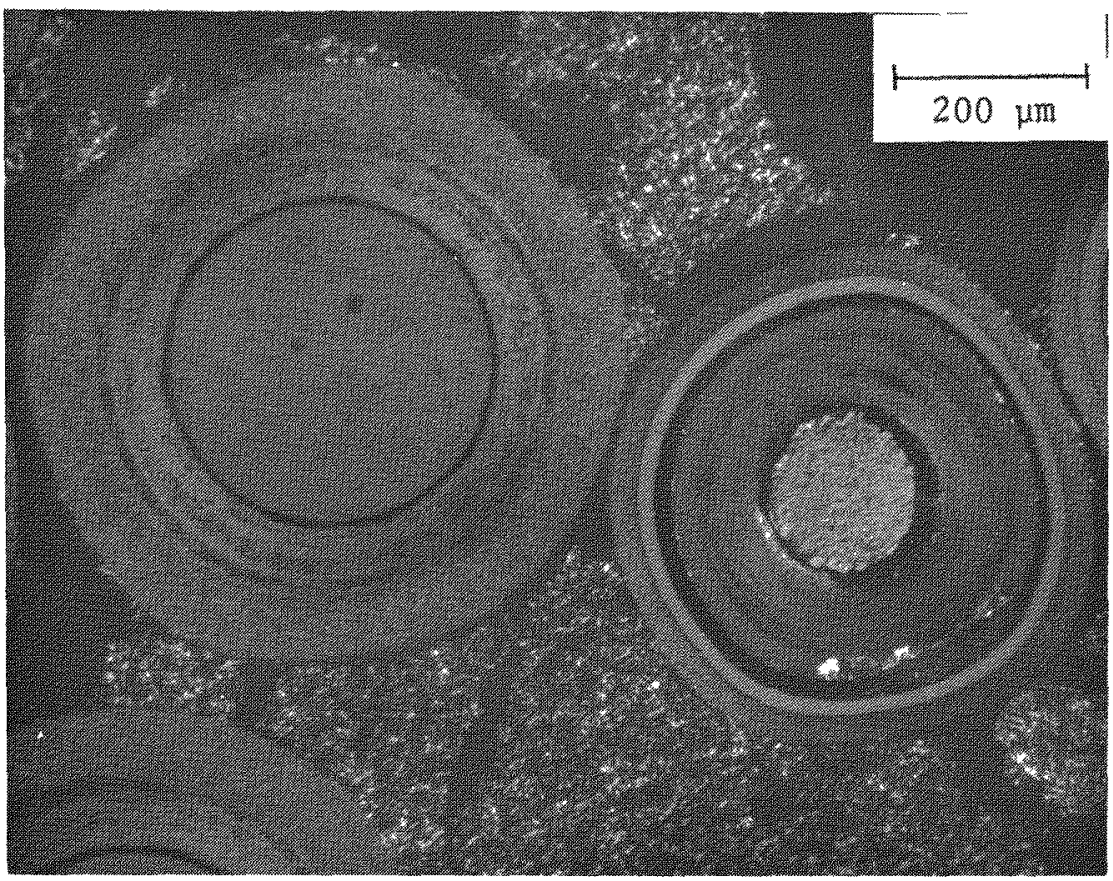

L7440-80

(b)

Fig. 4-53. Photomicrographs of representative particles from fuel rod 7161-004-20-6 irradiated in capsule P13R (position 5E) to a fast neutron fluence of $7.7 \times 10^{21} \mathrm{n} / \mathrm{cm}^{2}$ (E > $0.18 \mathrm{MeV}$ ) HTGR at $1280^{\circ} \mathrm{C}$. (a) Bright field and (b) polarized light. 


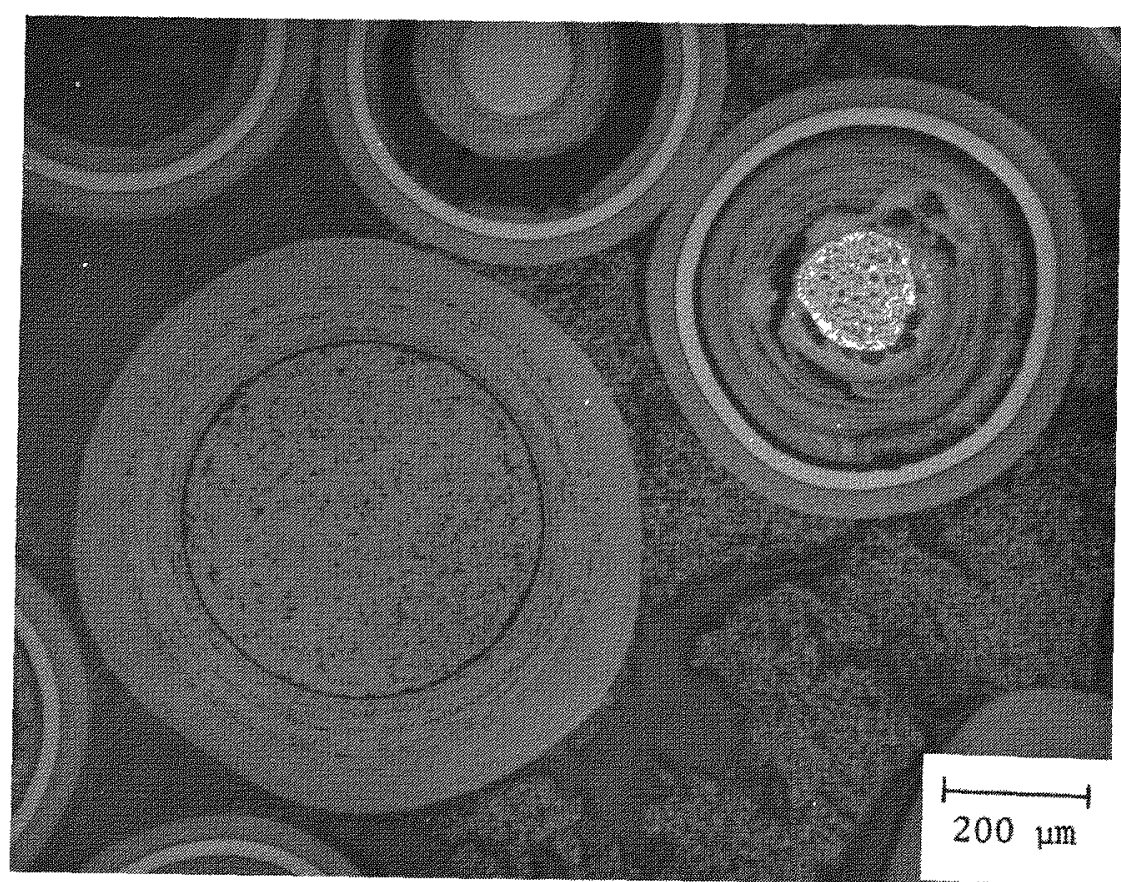

(a)

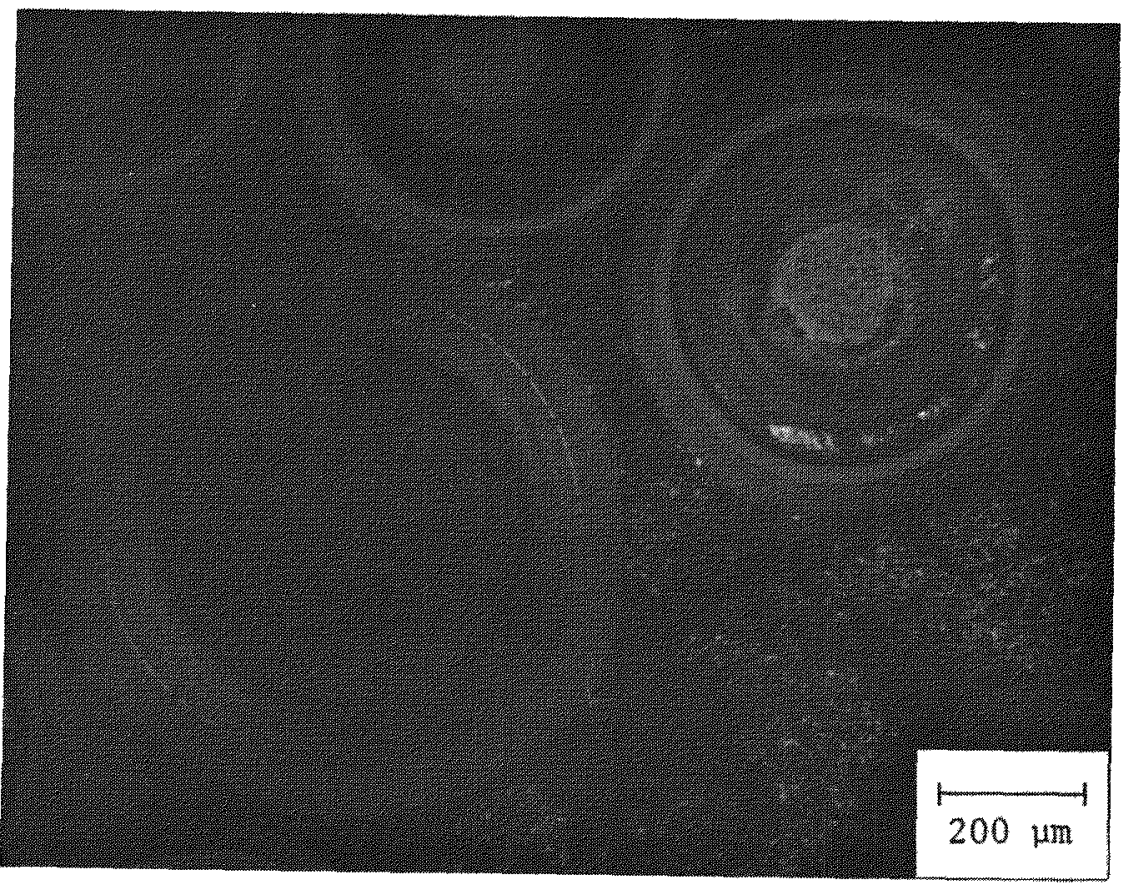

L7440-880

(b)

Fig. 4-54. Photomicrographs of representative particles from fuel rod 7161-004-01-7 irradiated in capsule P13S (position 1A) to a fast neutron fluence of $7.0 \times 10^{21} \mathrm{n} / \mathrm{cm}^{2}(\mathrm{E}>0.18 \mathrm{MeV})_{\mathrm{HTGR}}$. This rod was thermal cycled to high temperatures twenty-four times during irradiation. (a) Bright field and (b) polarized light. 


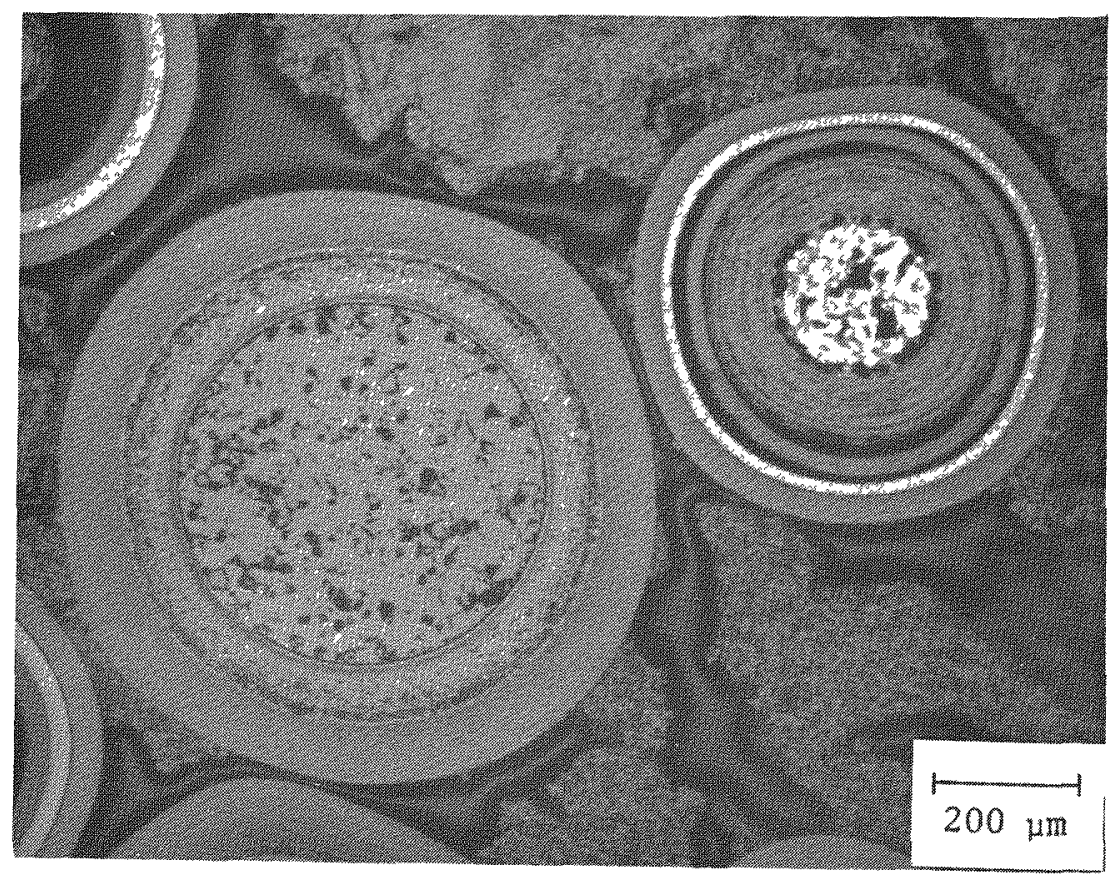

L7440-620

(a)

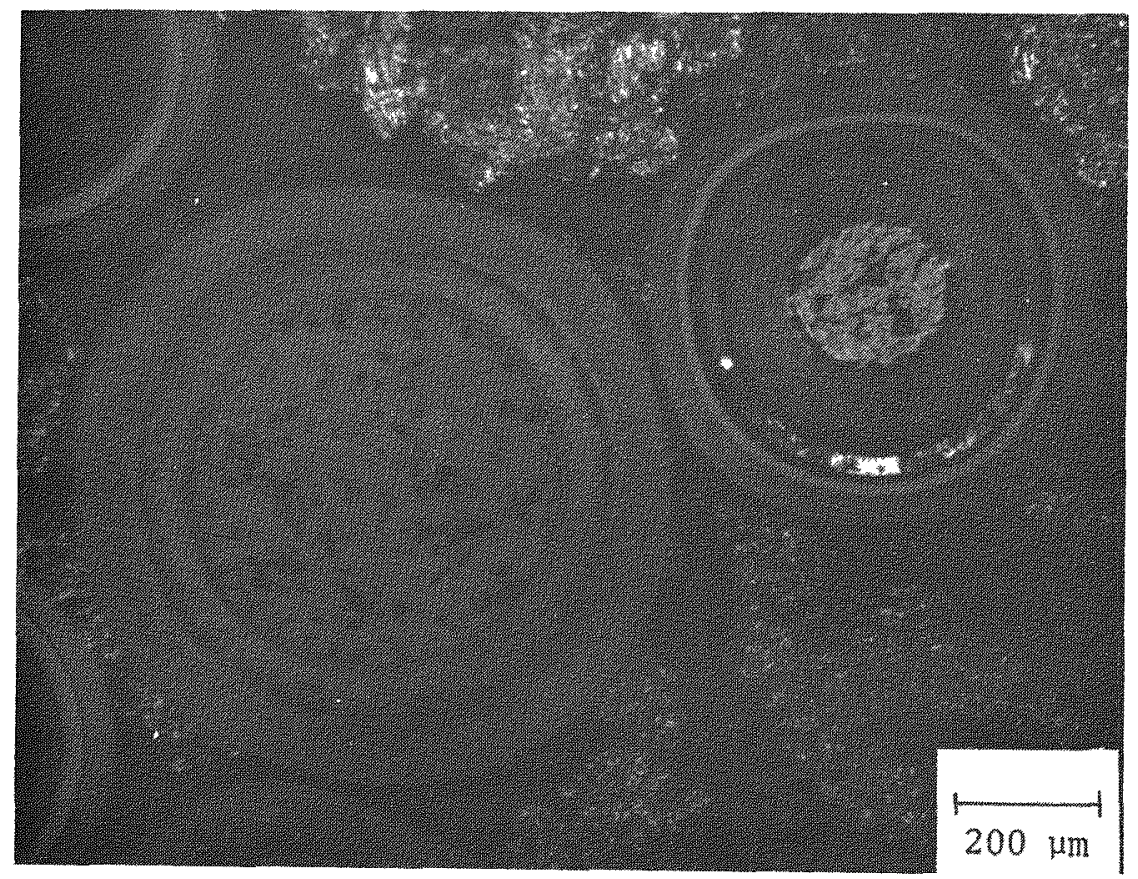

$\mathbf{L} 7440-621$

(b)

Fig. 4-55. Photomicrographs of representative particles from fuel rod 7161-004-12-5 irradiated in capsule P13S (position 2B) to a fast neutron fluence of $11.7 \times 10^{21} \mathrm{n} / \mathrm{cm}^{2}$ (E $\left.>0.18 \mathrm{MeV}\right)_{\text {HTGR }}$ at $1010^{\circ} \mathrm{C}$. (a) Bright field and (b) polarized light. 


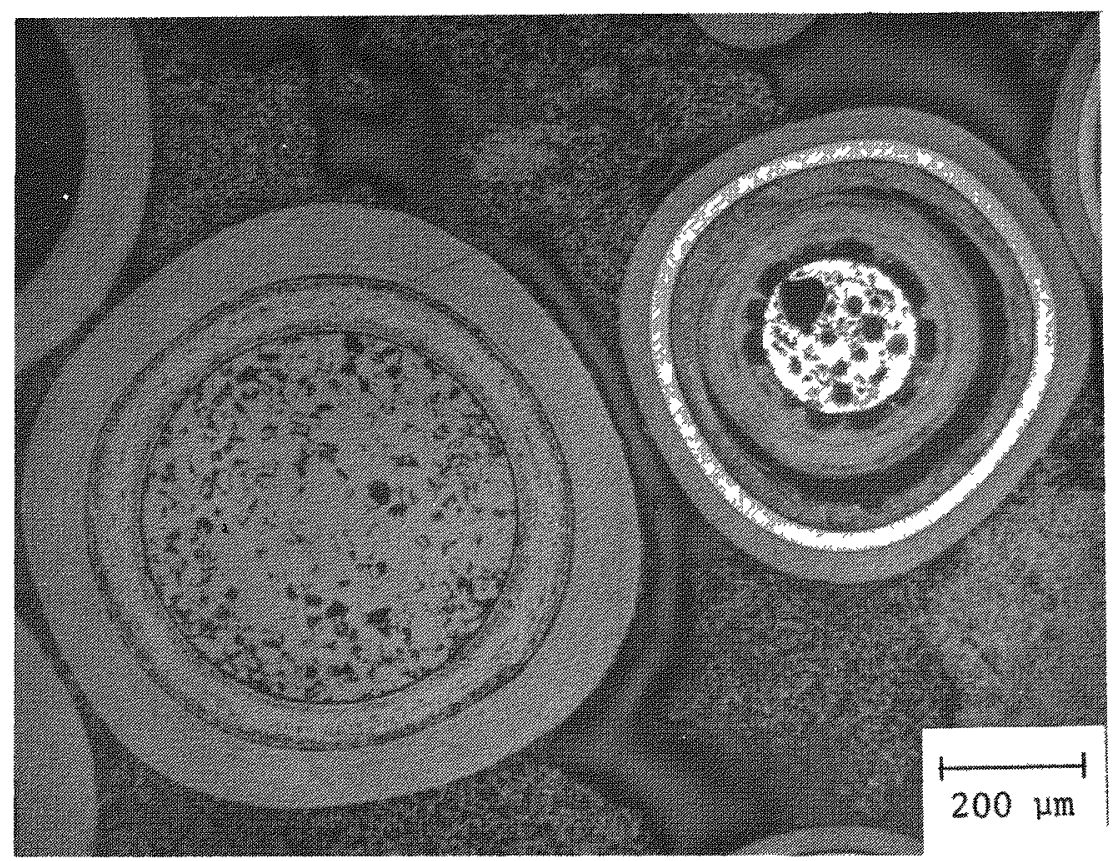

L7440-536

(a)

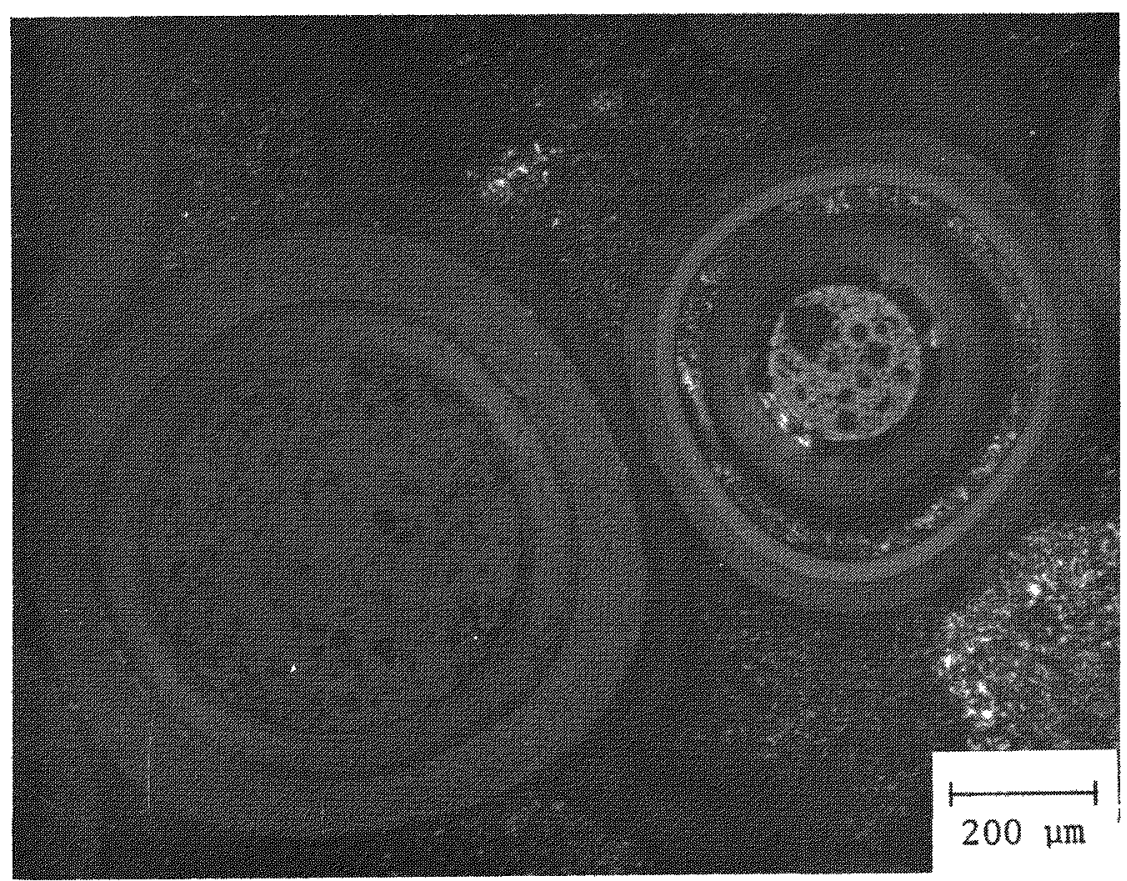

L7440-537

(b)

Fig. 4-56. Photomicrographs of representative particles from fuel rod 7161-004-13-6 irradiated in capsule P13S (position 2C) to a fast neutron fluence of $11.9 \times 10^{21} \mathrm{n} / \mathrm{cm}^{2}$ (E $\left.>0.18 \mathrm{MeV}\right)_{\text {HTGR }}$ at $975^{\circ} \mathrm{C}$. (a) Bright field and (b) polarized light. 


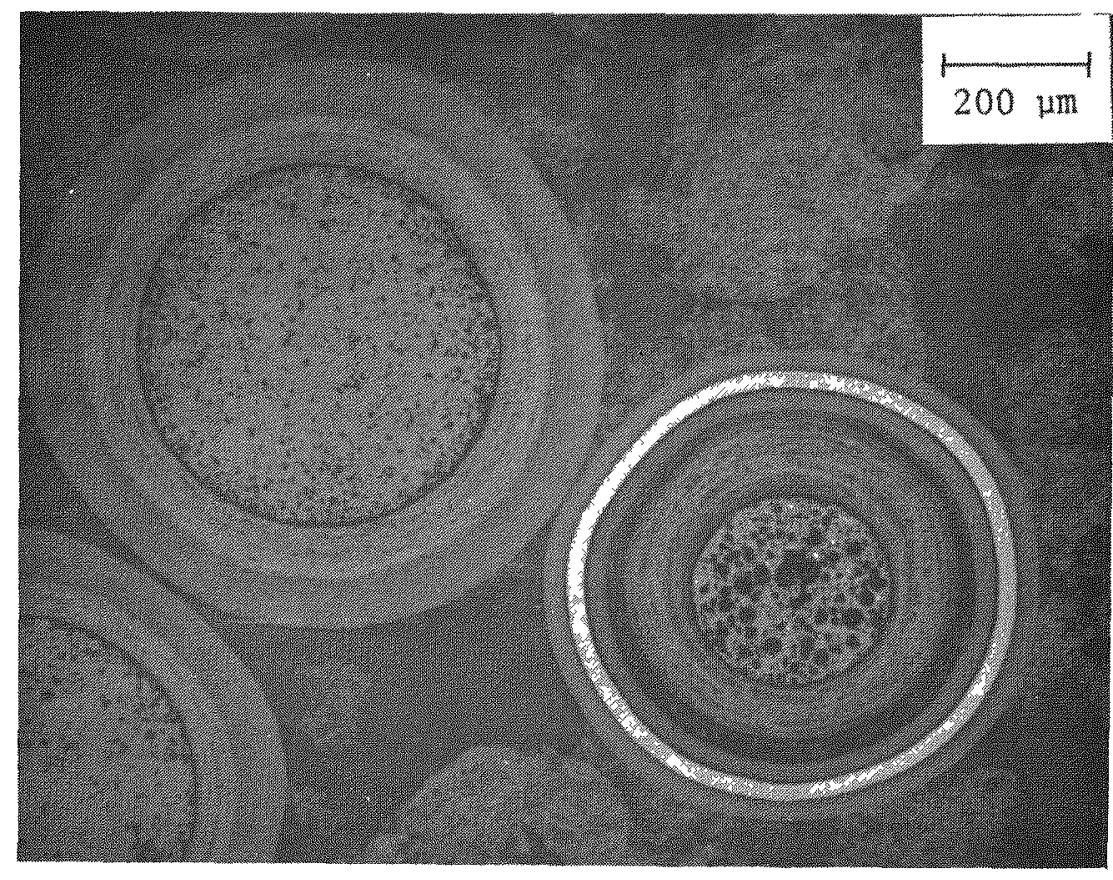

L7440-1068

(a)

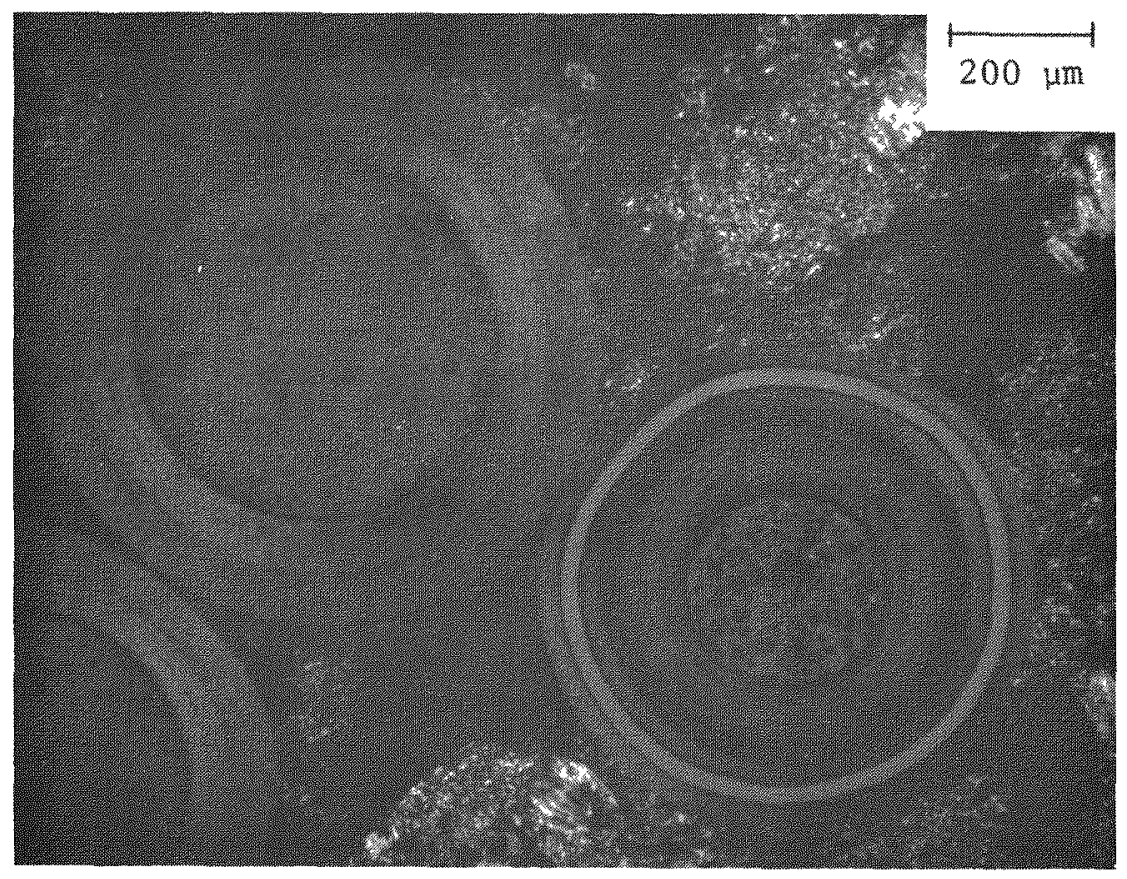

L7440-1069

(b)

Fig. 4-57. Photomicrographs of representative particles from fuel rod 7161-004-16-6 irradiated in capsule P13S (position 5A) to a fast neutron fluence of $9.2 \times 10^{21} \mathrm{n} / \mathrm{cm}^{2}$ (E $\left.>0.18 \mathrm{MeV}\right)$ HTGR at $1375^{\circ} \mathrm{C}$. (a) Bright field and (b) polarized light. 


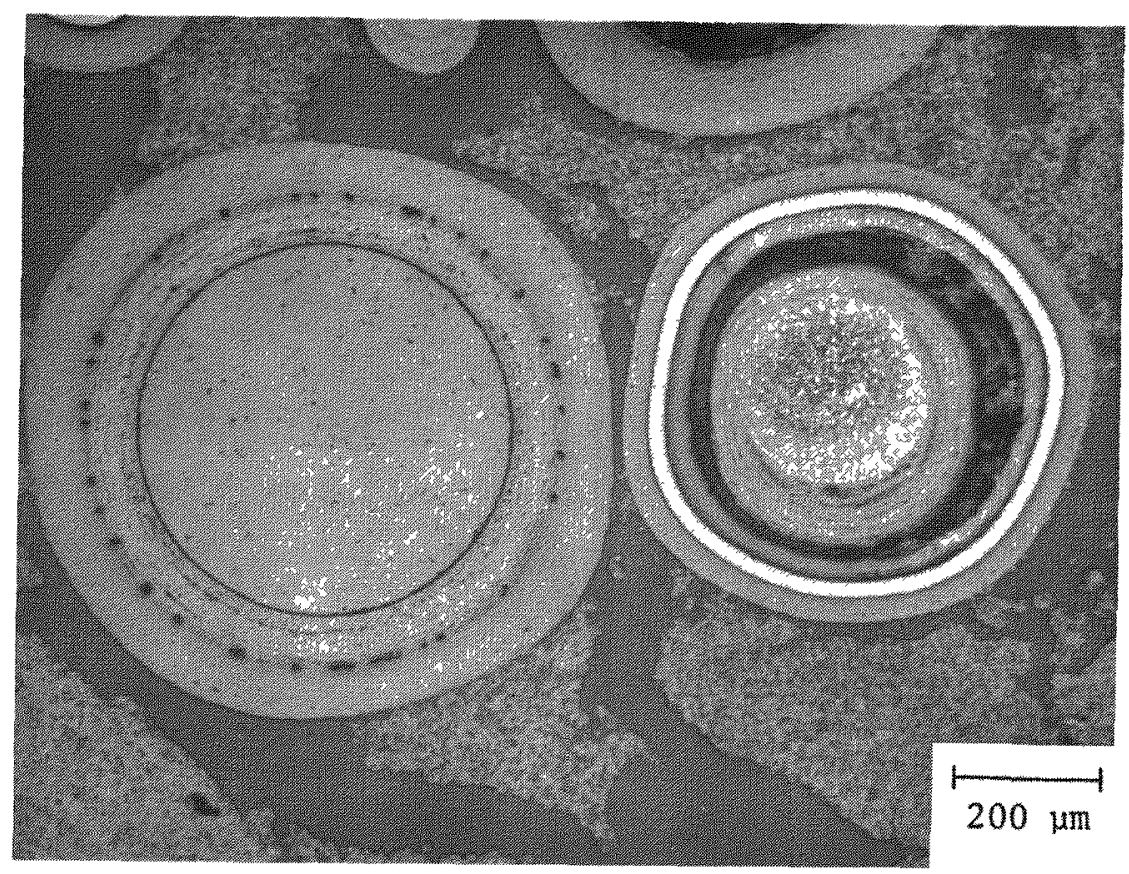

27440-209

(a)

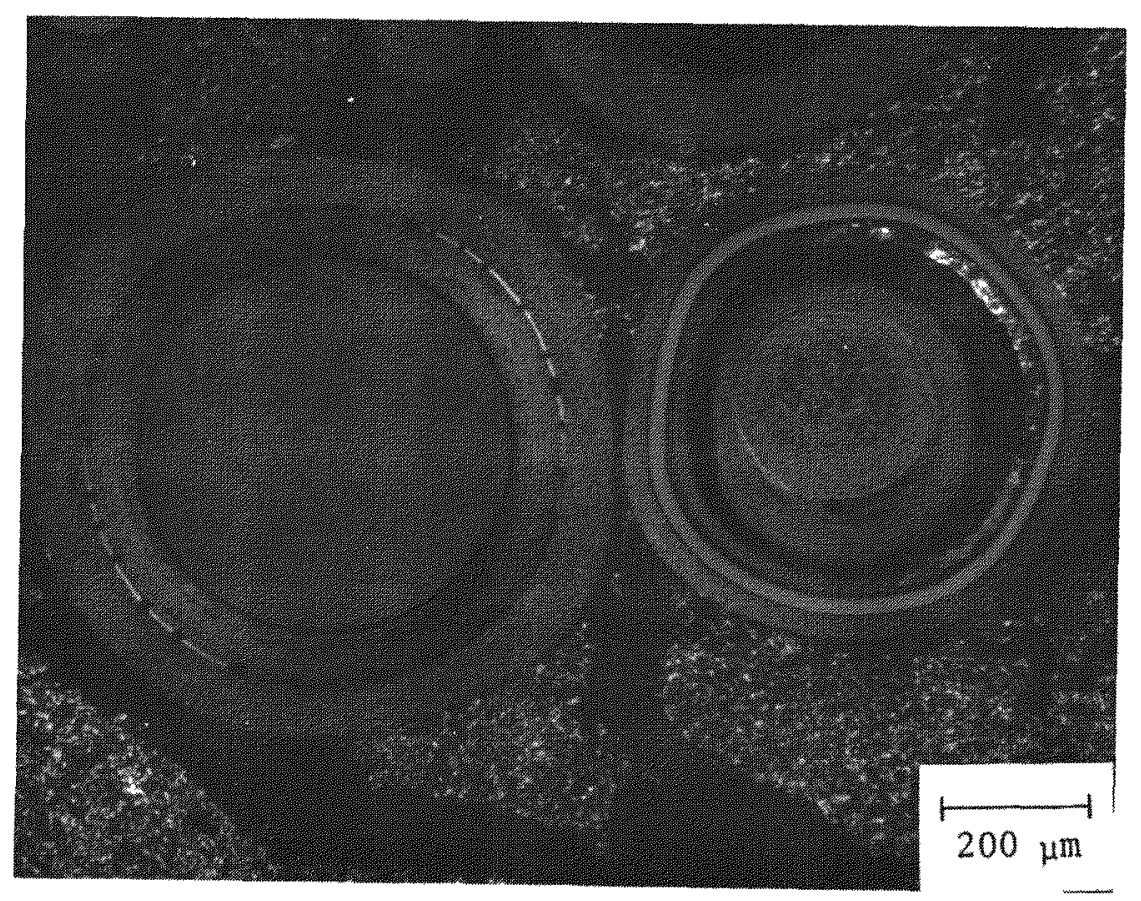

L7440-210

(b)

Fig. 4-58. Photomicrographs of representative particles from fuel rod 7161-004-19-5 irradiated in capsule P13S (position 5D) to a fast neutron fluence of $7.9 \times 10^{21} \mathrm{n} / \mathrm{cm}^{2}(\mathrm{E}>0.18 \mathrm{MeV})_{\mathrm{HTGR}}$ at $1325^{\circ} \mathrm{C}$. (a) Bright field and (b) polarized light. 

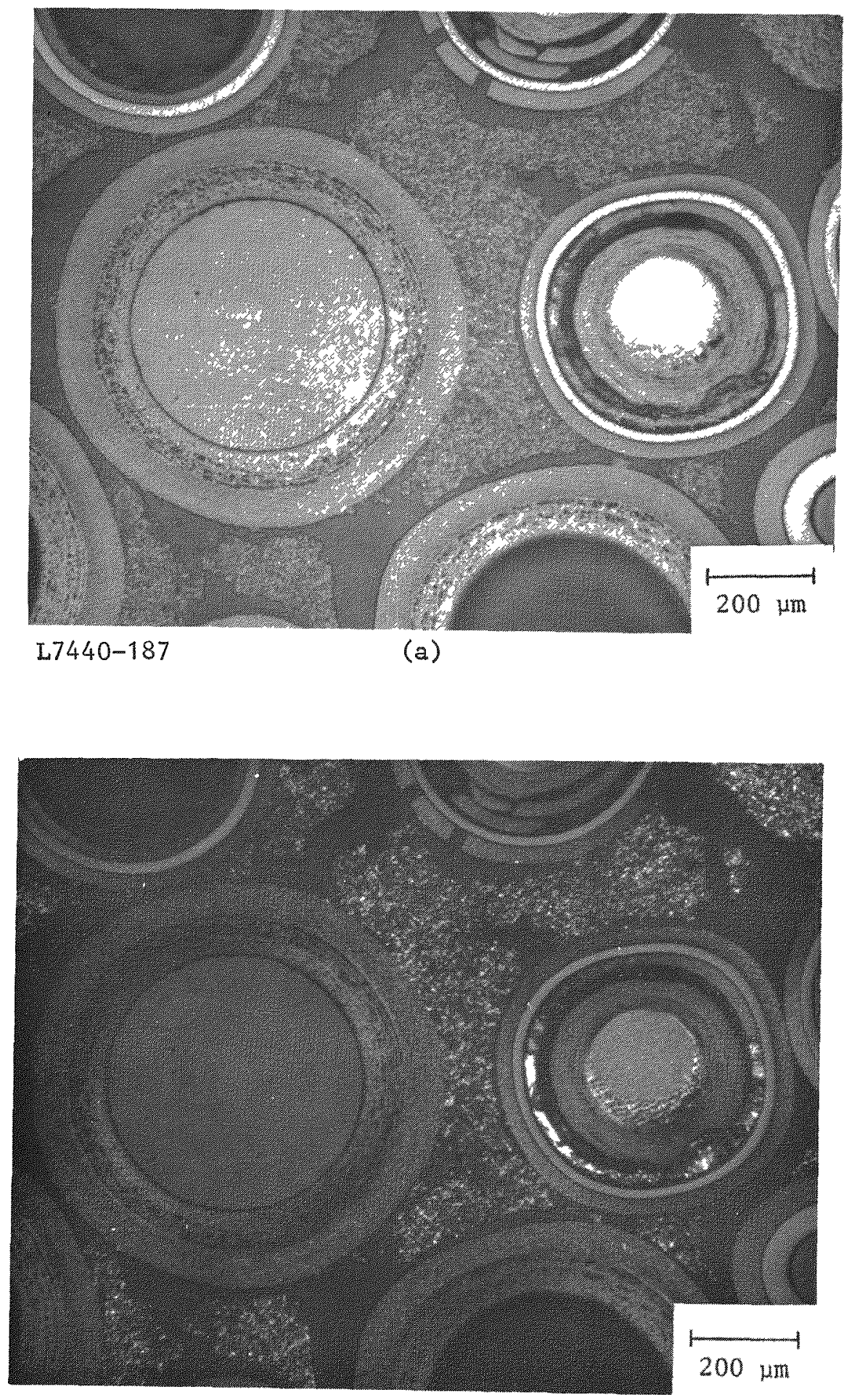

L7440-188

(b)

Fig. 4-59. Photomicrographs of representative particles from fuel rod 7161-004-20-5 irradiated in capsule P13S (position 5E) to a fast neutron fluence of $7.5 \times 10^{21} \mathrm{n} / \mathrm{cm}^{2}$ (E $\left.>0.18 \mathrm{MeV}\right)_{\mathrm{HTGR}}$ at $1350^{\circ} \mathrm{C}$. (a) Bright field and (b) polarized light. 


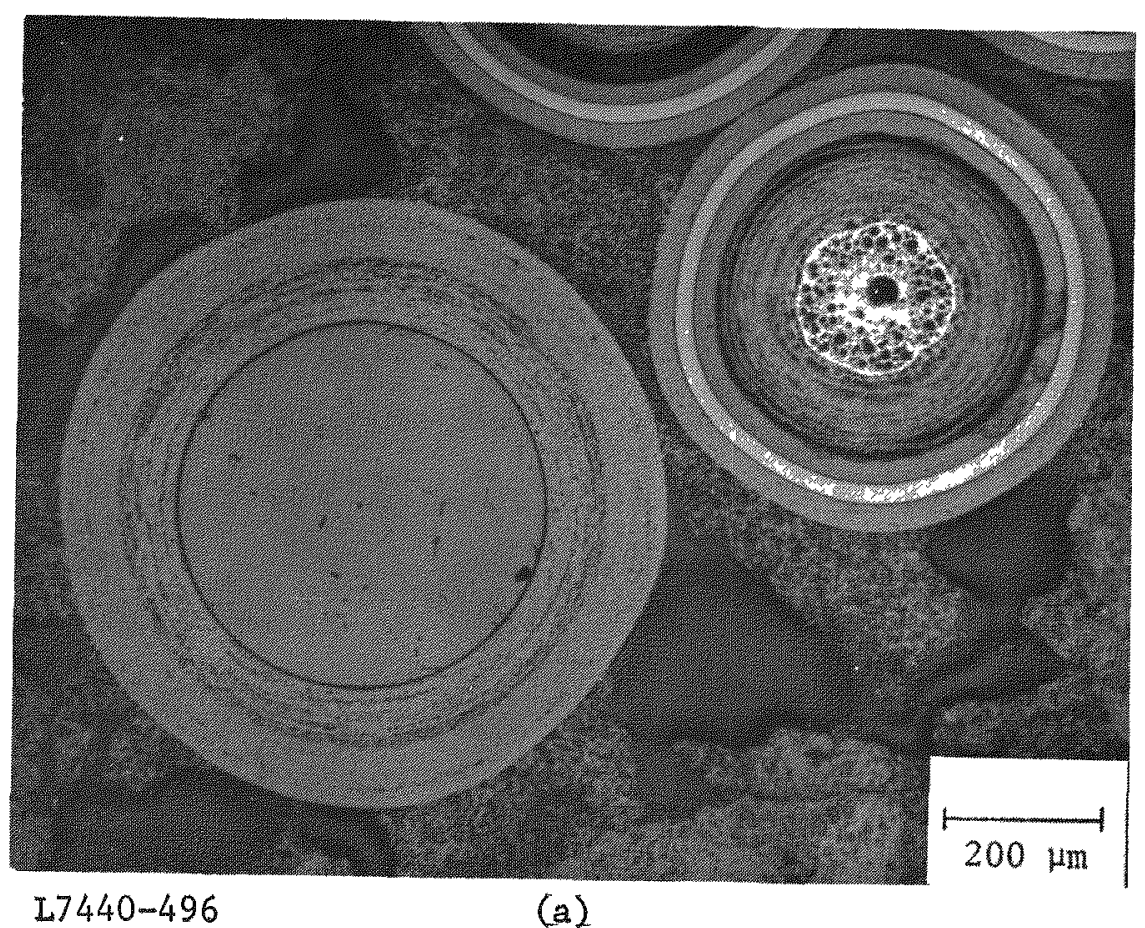

L7 $440-496$

(a)

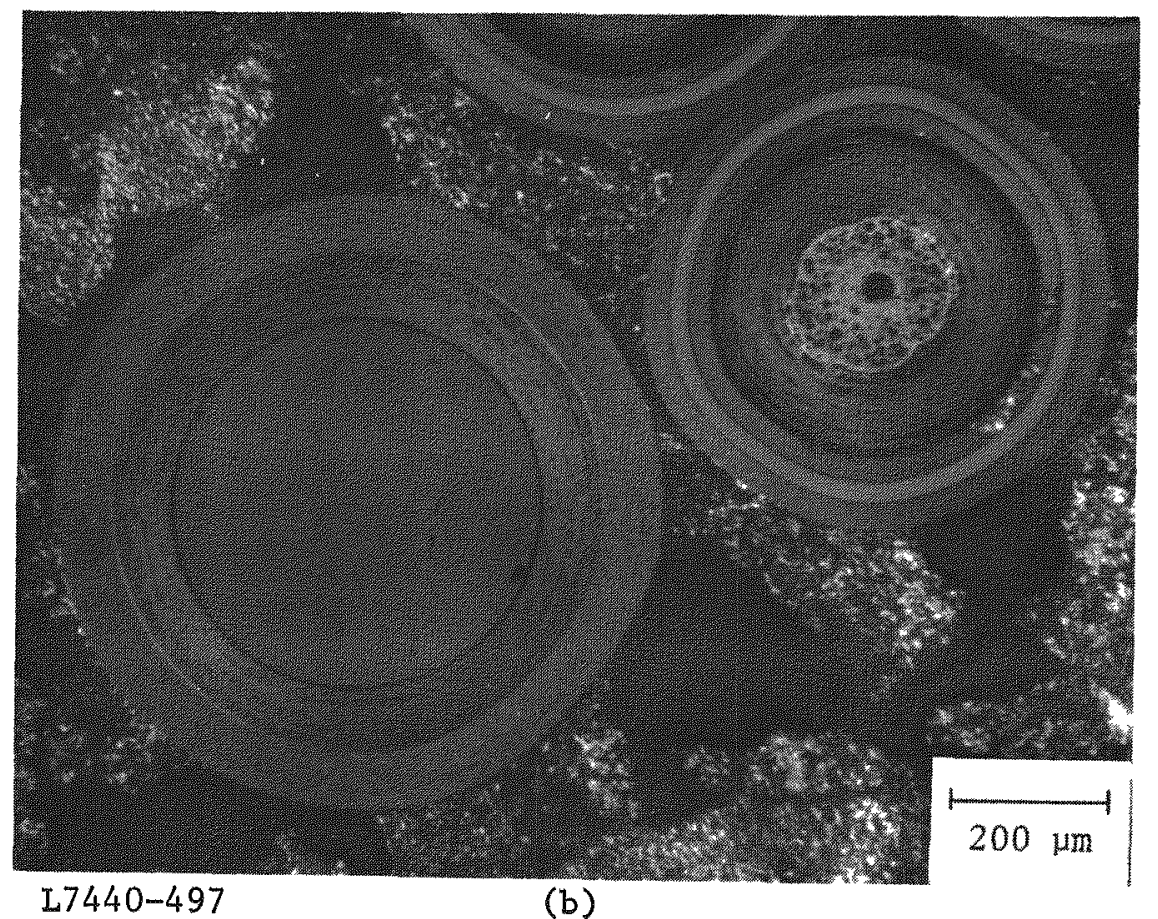

Fig. 4-60. Photomicrographs of representative particles from fuel rod 7161-004-28-13 irradiated in capsule P13S (position 6C) to a fast neutron fluence of $4.4 \times 10^{21} \mathrm{n} / \mathrm{cm}^{2}(\mathrm{E}>0.18 \mathrm{MeV})_{\text {HTGR }}$ at $1050^{\circ} \mathrm{C}$. (a) Bright field and (b) polarized light. 


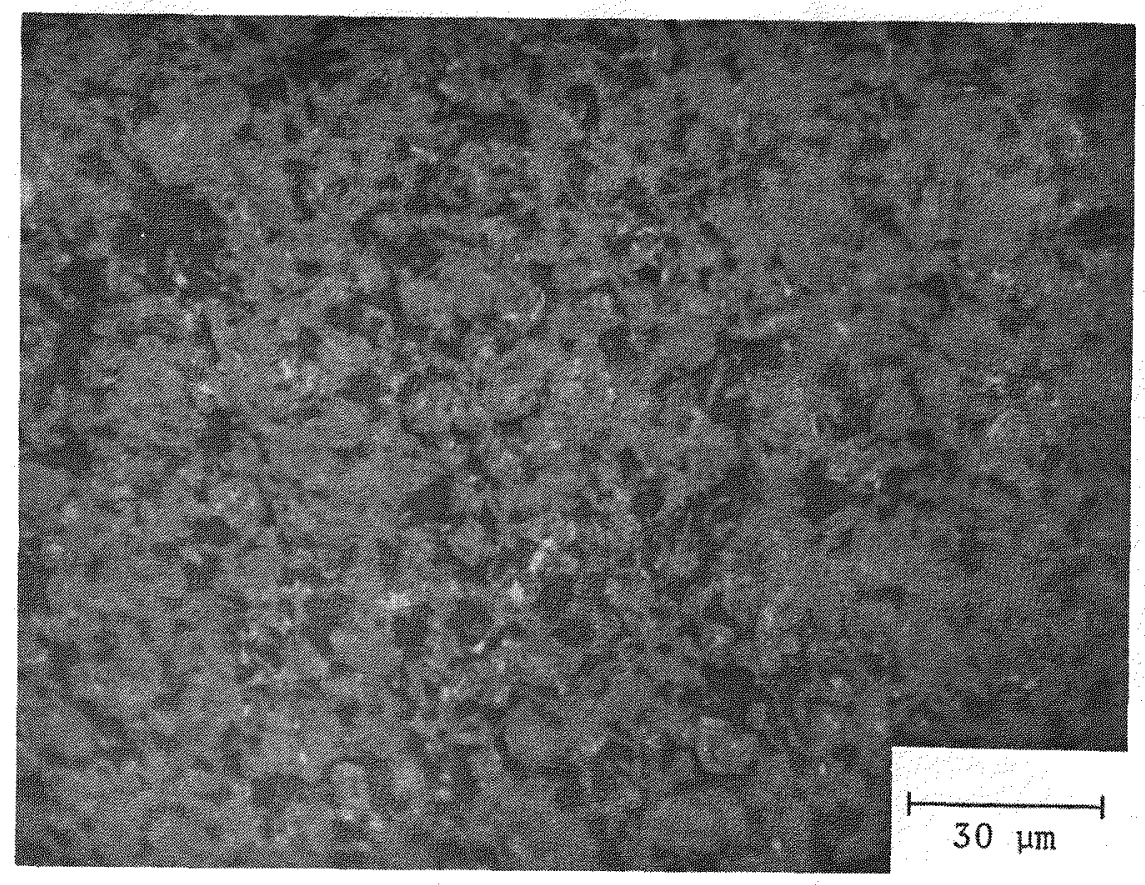

L $7440-553$

(a)

FUEL ROD

FUEL ROD

SURFACE

CENTERLINE

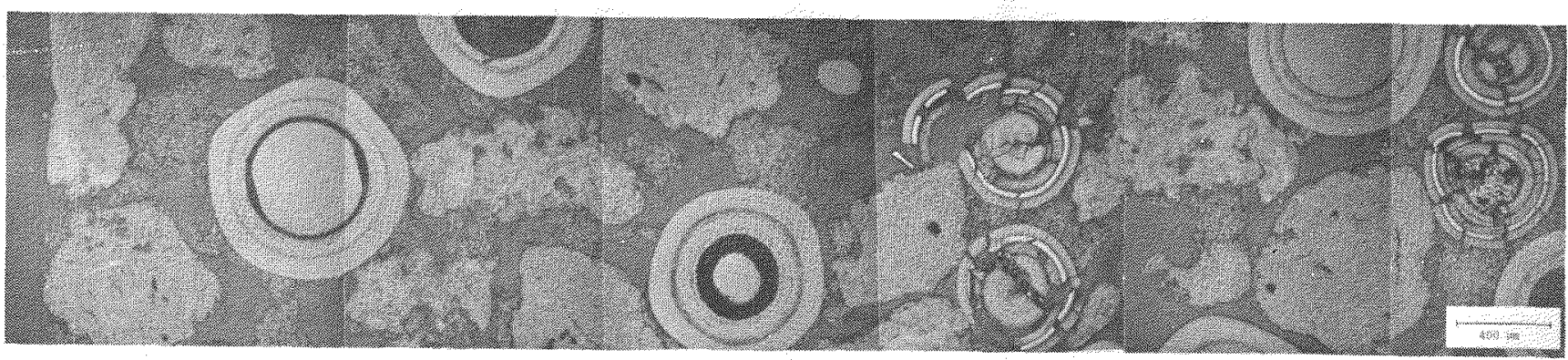

$75 \mathrm{~K} 3400-3$

(b)

Fig. 4-61. Representative photomicrographs of fuel rod 7161-004-03-5 after irradiation in capsule P13R (position $1 \mathrm{C}$ ) to a fast neutron. fluence of $8.7 \times 10^{21} \mathrm{n} / \mathrm{cm}^{2}(\mathrm{E}>0.18 \mathrm{MeV})_{\text {HTGR }}$. After irradiation this rod was heated to $1600^{\circ} \mathrm{C}$ in TRIGA for fission gas release measurements. (a) Typical appearance of matrix and (b) composite showing radial cross section. 


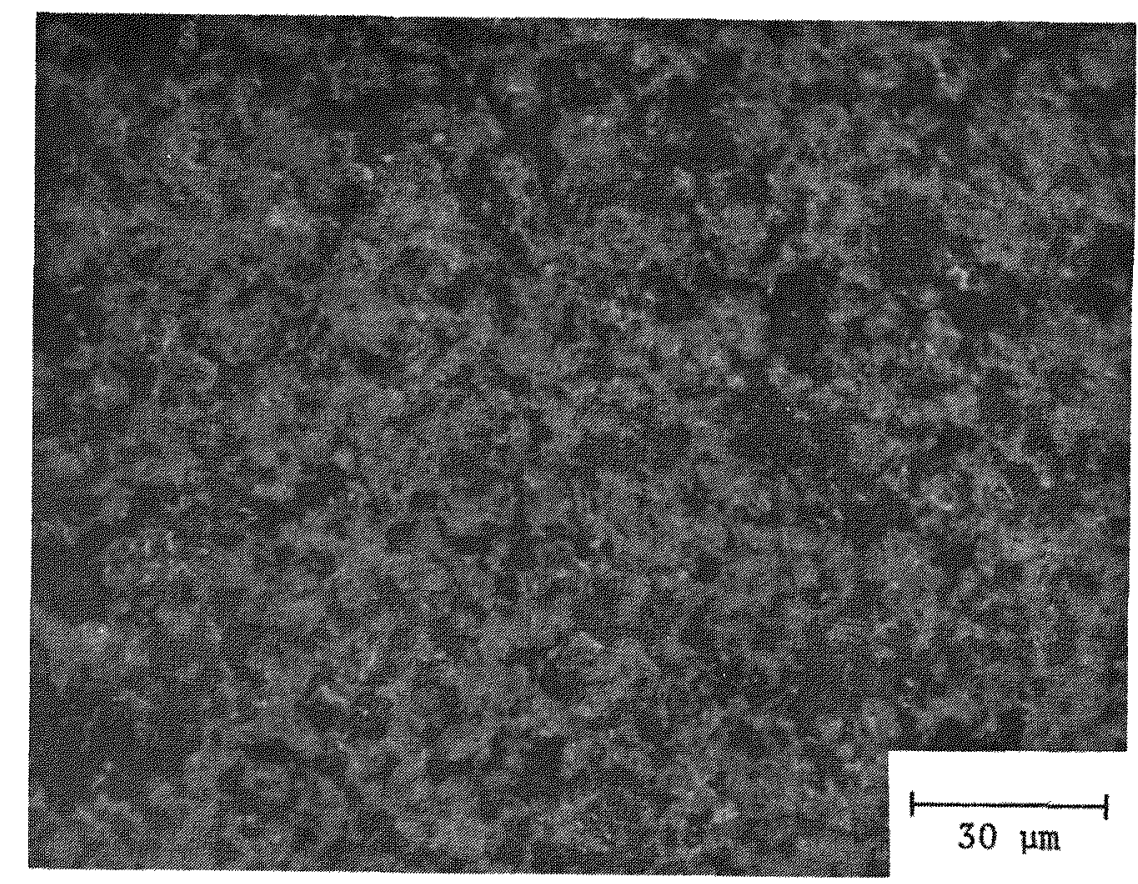

L7440-52

(a)

FUEL ROD

FUEL ROD

SURFACE

CENTERLINE

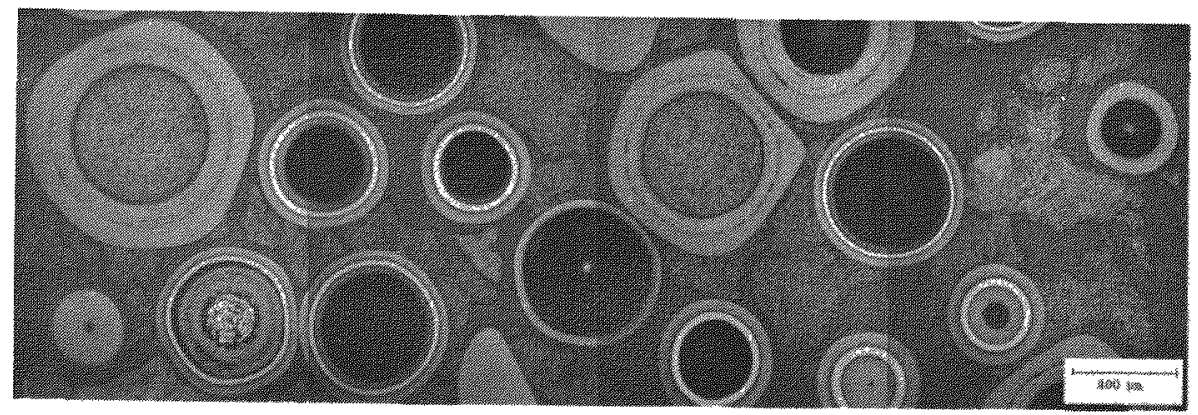

$75 \mathrm{~K} 3398-2$

(b)

Fig. 4-62. Representative photomicrographs of fuel rod 7161-004-06-5 after irradiation in capsule P13R (position 2A) to a fast neutron fluence of $11.8 \times 10^{21} \mathrm{n} / \mathrm{cm}^{2}$ ( $(\mathrm{E}>0.18 \mathrm{MeV})$ HTGR at $1050^{\circ} \mathrm{C}$. (a) Typical appearance of matrix and (b) composite showing radial cross section. 


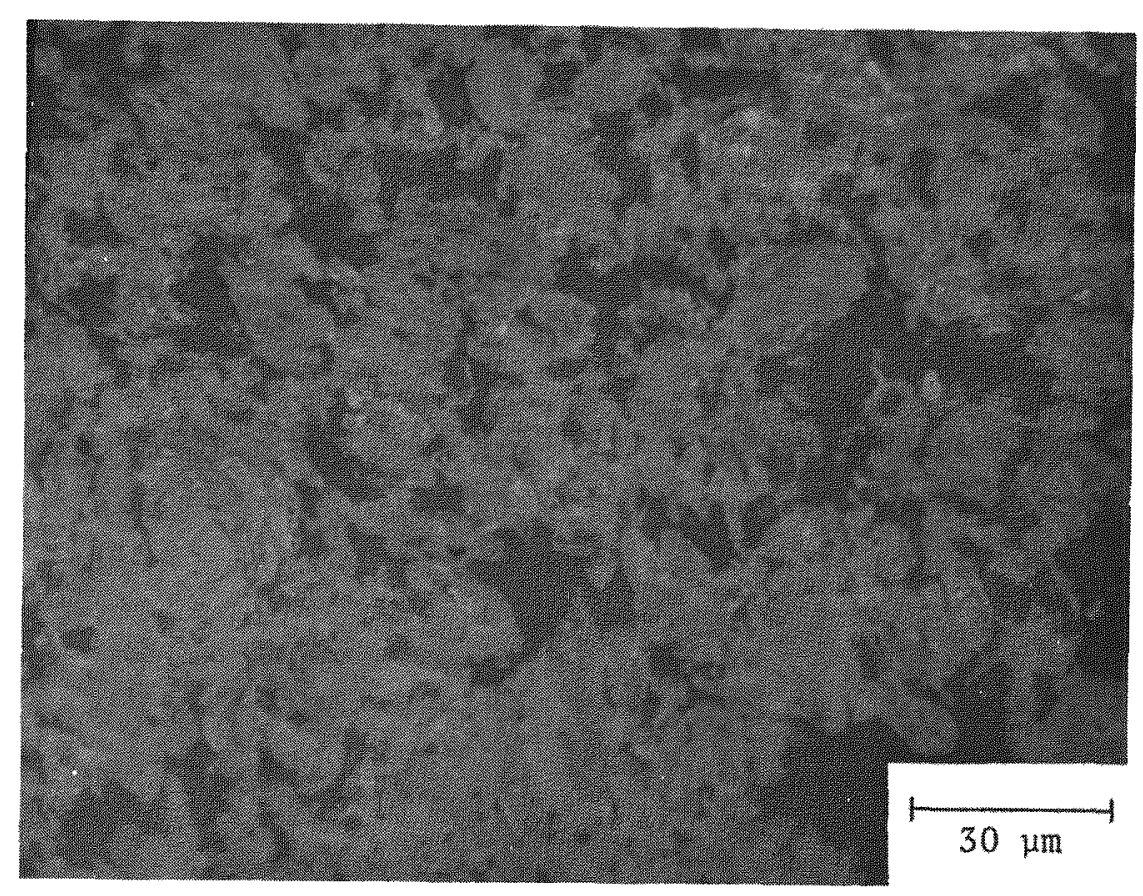

L7440-300

(a)

FUEL ROD

FUEL ROD

SURFACE

CENTERLINE

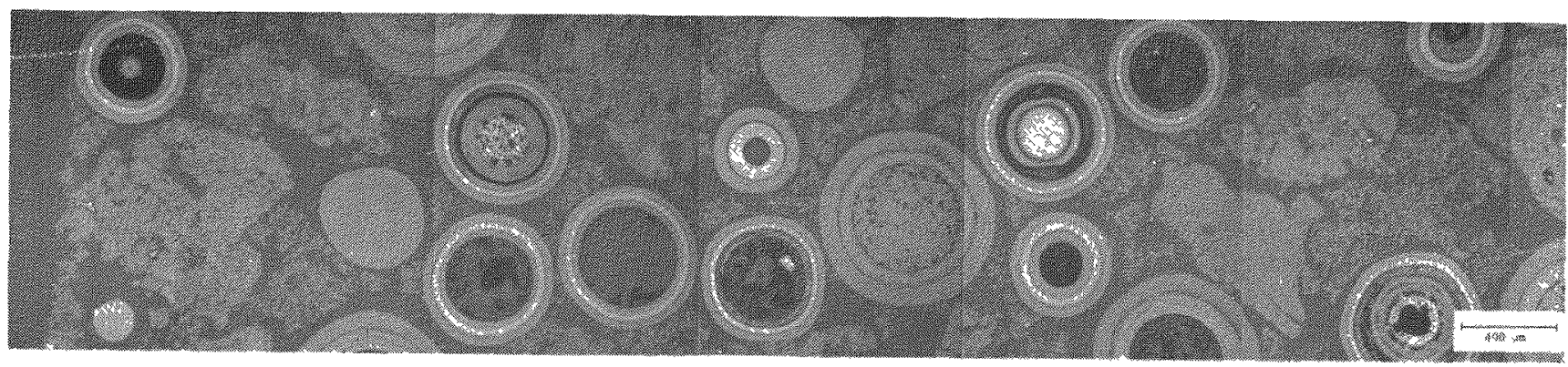

$75 \mathrm{~K} 3400-4$

(b)

Fig. 4-63. Representative photomicrographs of fuel rod 7161-004-07-5 after irradiation in capsule $\mathrm{P} 13 \mathrm{R}$ (position 2B) to a fast neutron fluence of $12.0 \times 10^{21} \mathrm{n} / \mathrm{cm}^{2}(\mathrm{E}>0.18 \mathrm{MeV})_{\mathrm{HTGR}}$ at $1055^{\circ} \mathrm{C}$. (a) Typical appearance of matrix and (b) composite showing radial cross section. 


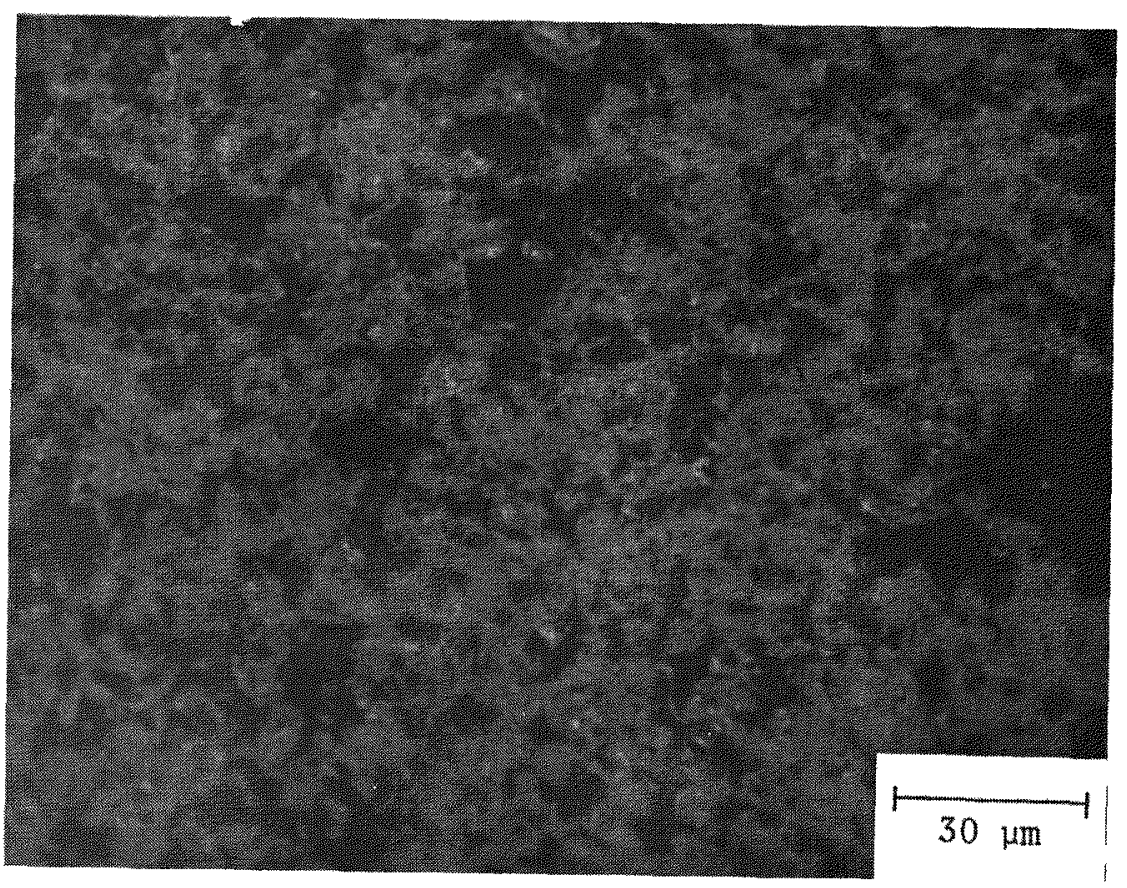

L7440-272

(a)

FUEL ROD

SURFACE

FUEL ROD

CENTERLINE

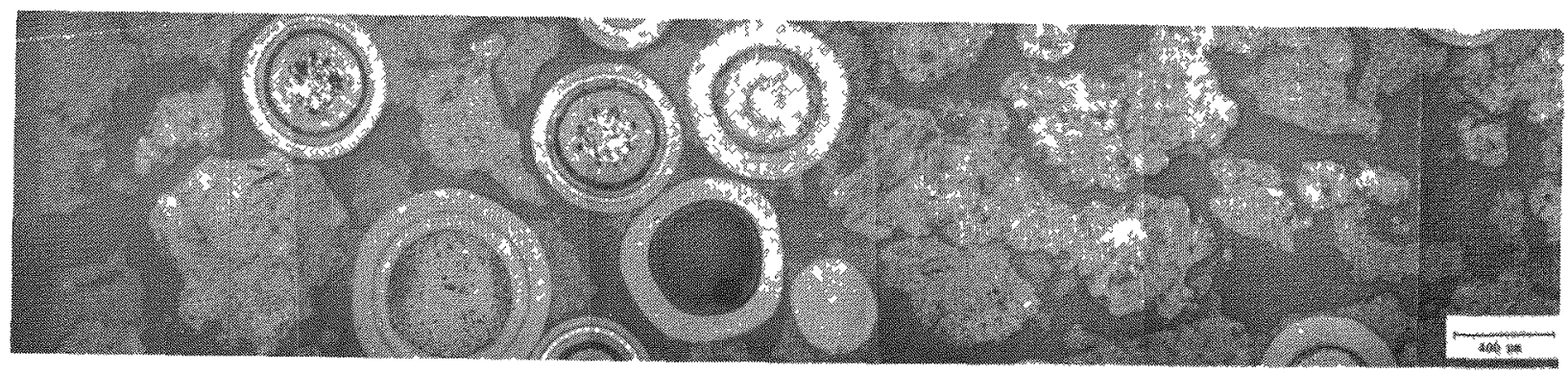

$75 \times 3399-4$

(b)

Fig. 4-64. Representative photomicrographs of fuel rod 7161-004-08-13 after irradiation in capsule $\mathrm{P} 13 \mathrm{R}$ (position $2 \mathrm{C}$ ) to a fast neutron fluence of $12.2 \times 10^{21} \mathrm{n} / \mathrm{cm}^{2}(\mathrm{E}>0.18 \mathrm{MeV})_{\mathrm{HTGR}}$ at $1045^{\circ} \mathrm{C}$. (a) Typical appearance of matrix and (b) composite showing radial cross section. 


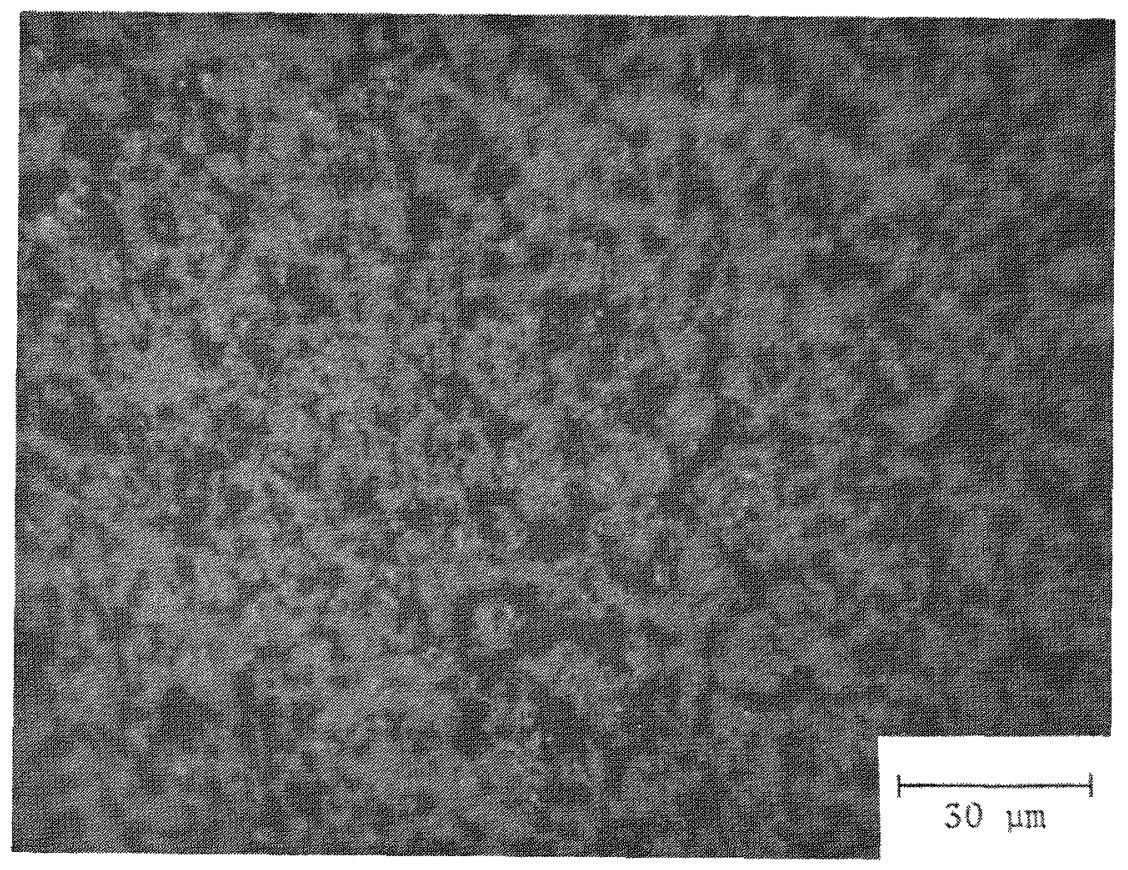

L7440-1032

(a)

FUEL ROD

SURFACE

FUEL ROD

CENTERLINE

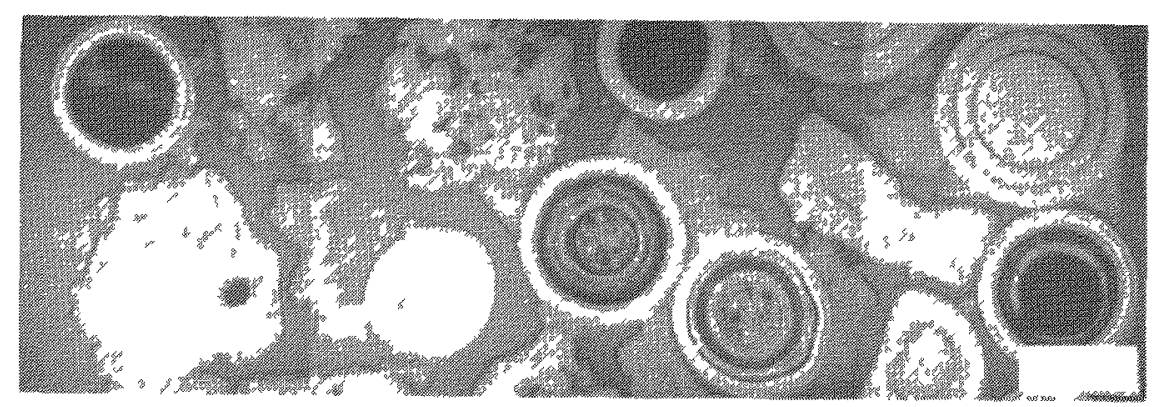

75K3401-1

(b)

Fig. 4-65. Representative photomicrographs of fuel rod 7161-004-16-5 after irradiation in capsule $\mathrm{P} 13 \mathrm{R}$ (position $5 \mathrm{~A}$ ) to a fast neutron fluence of $9.4 \times 10^{21} \mathrm{n} / \mathrm{cm}^{2}(\mathrm{E}>0.18 \mathrm{MeV})$ HTGR. After irradiation this rod was heated to $1600^{\circ} \mathrm{C}$ in TRIGA for fission gas release measurements. (a) Typical appearance of matrix and (b) composite showing radial cross section. 


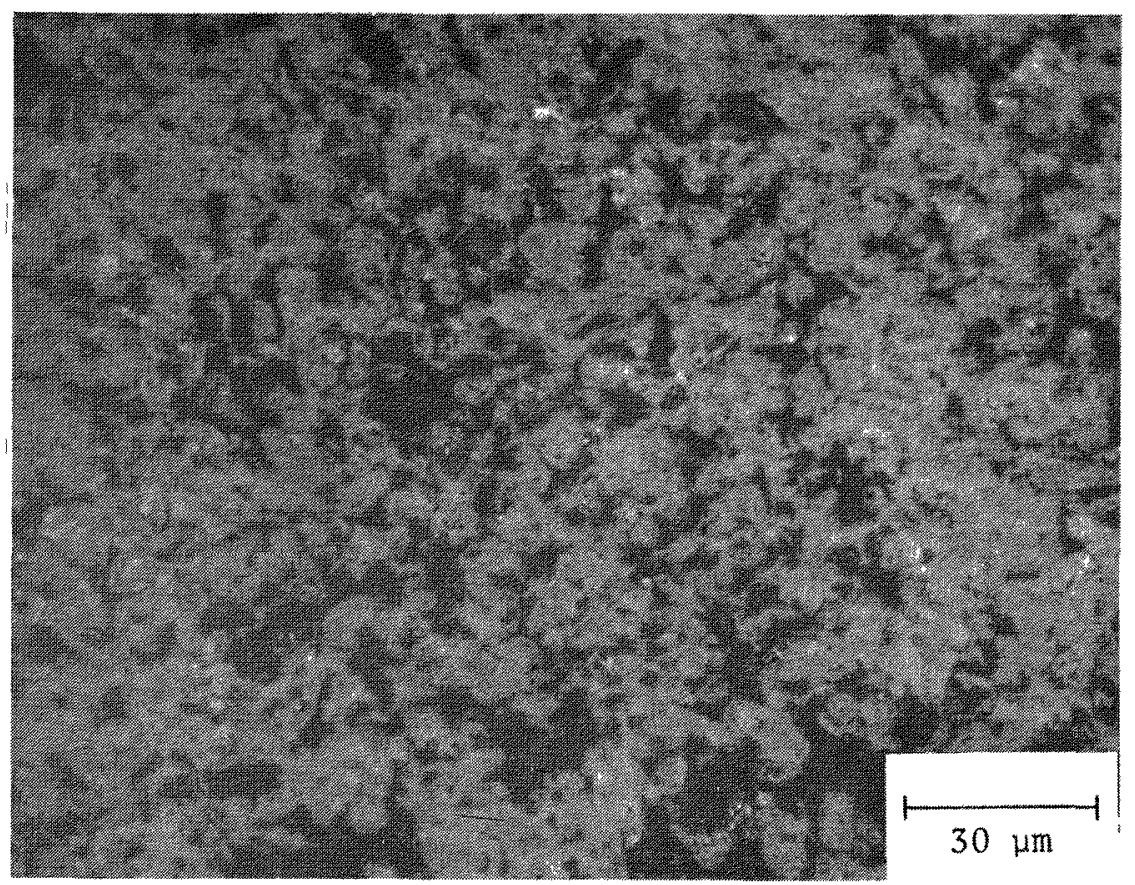

L7440-135

(a)

FUEL ROD

FUEL ROD

SURFACE

CENTERLINE

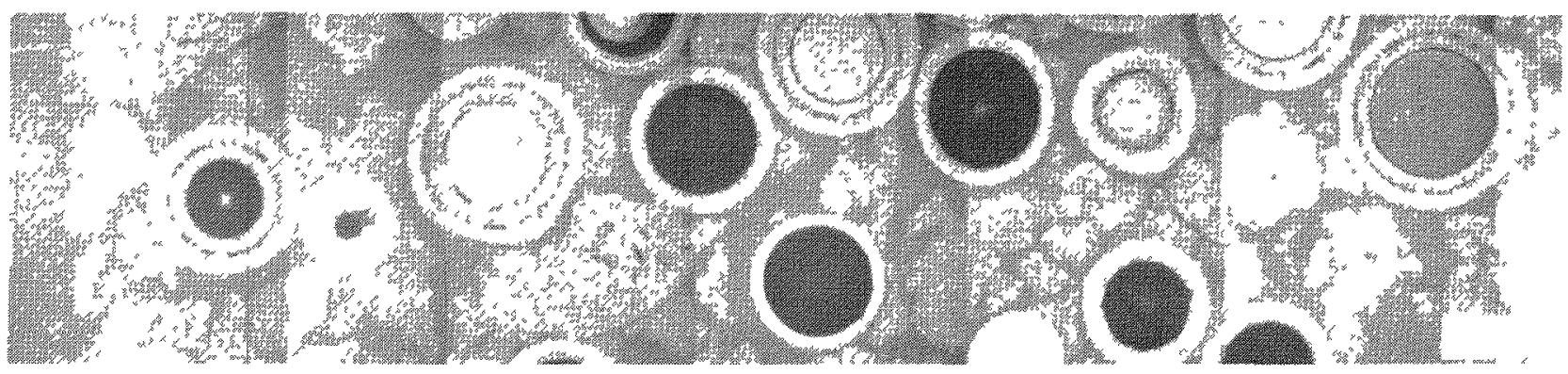

$75 \mathrm{~K} 3399-5$

(b)

Fig. 4-66. Representative photomicrographs of fuel rod 7161-004-19-7 after irradiation in capsule $\mathrm{P} 13 \mathrm{R}$ (position 5D) to a fast neutron fluence of $8.2 \times 10^{21} \mathrm{n} / \mathrm{cm}^{2}$ (E $>0.18 \mathrm{MeV}$ ) $\mathrm{HTGR}$ at $1225^{\circ} \mathrm{C}$.

(a) Typical appearance of matrix and (b) composite showing radial cross section. 


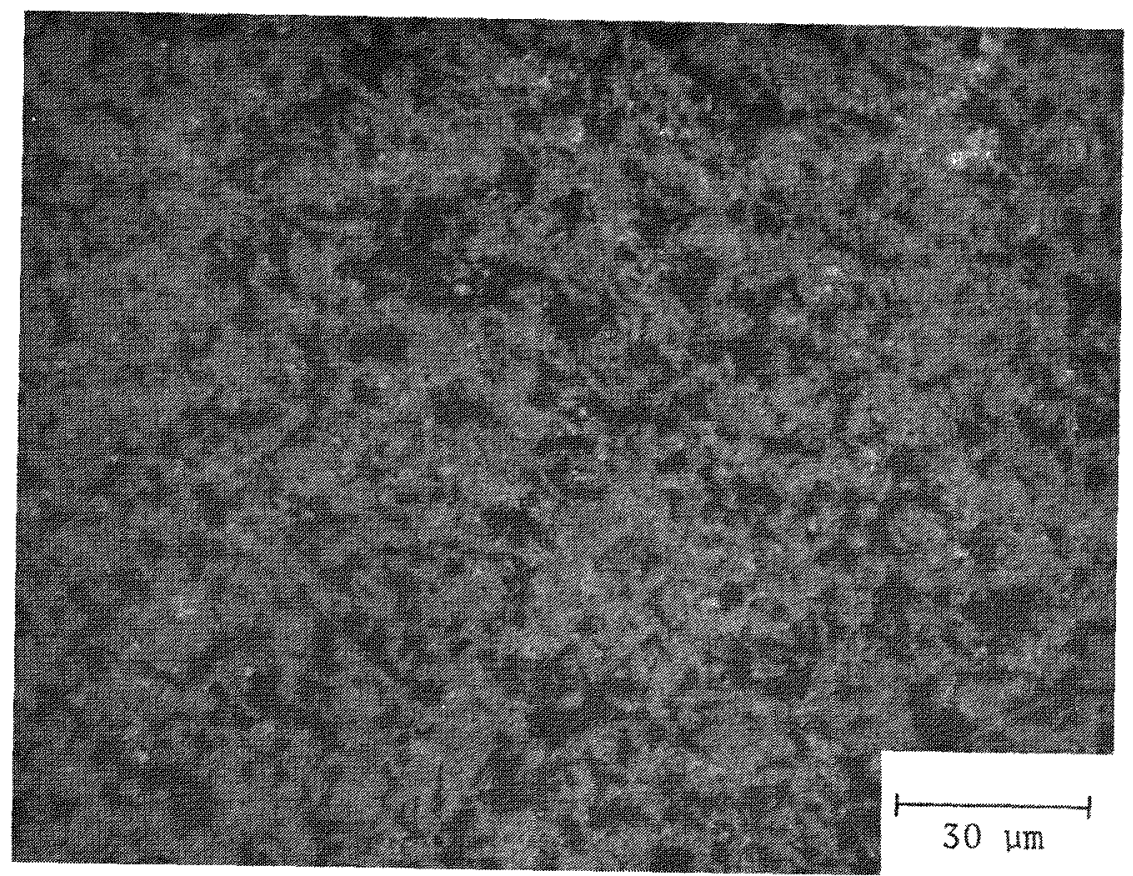

L7440-20

FUEL ROD

SURFACE

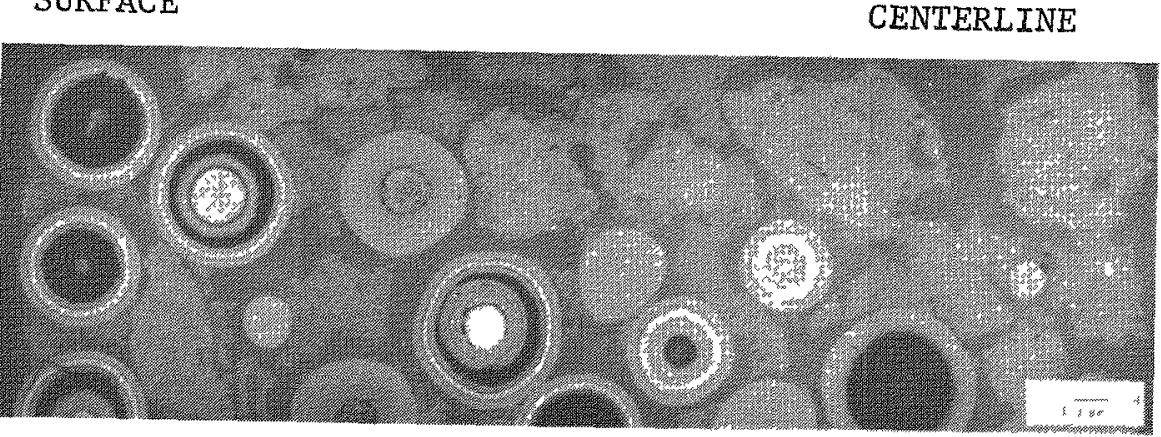

75K3398-5 (a) 


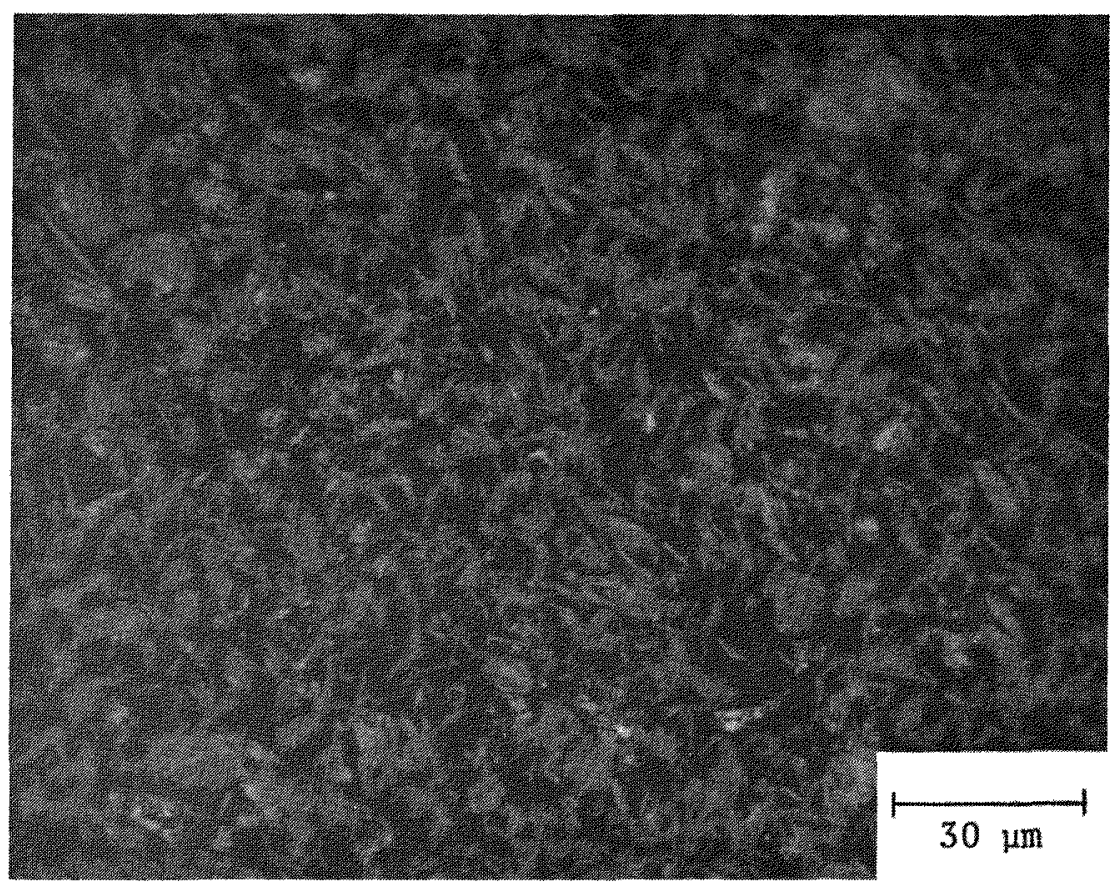

L.7440-161

(a)

FUEL ROD

SURFACE

FUEL ROD

CENTERLINE

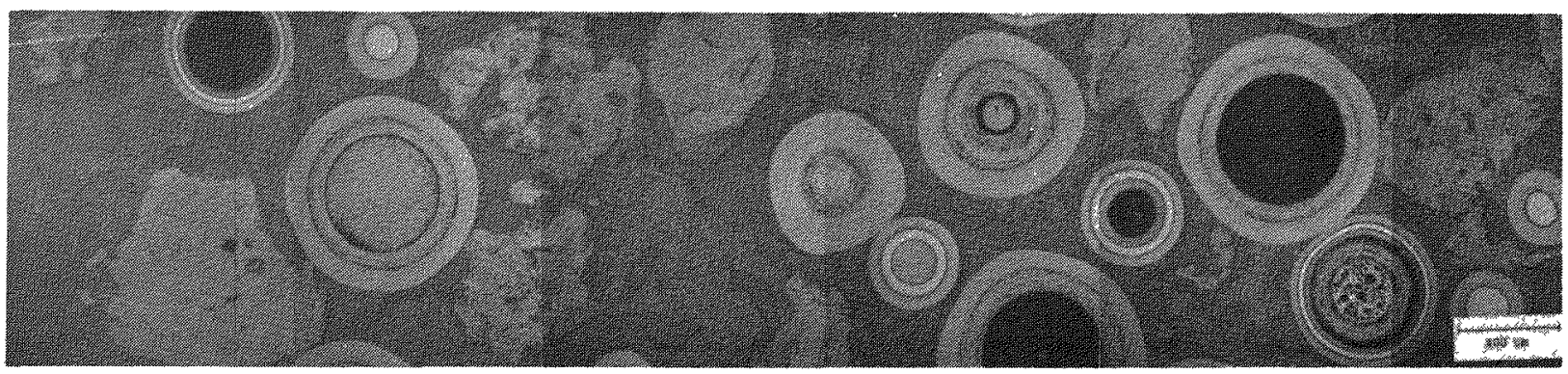

$75 \mathrm{~K} 3398-3$

(b)

Fig. 4-68. Representative photomicrographs of fuel rod 7161-004-23-5 after irradiation in capsule $\mathrm{P} 13 \mathrm{R}$ (position $6 \mathrm{C}$ ) to a fast neutron fluence of $4.5 \times 10^{21} \mathrm{n} / \mathrm{cm}^{2}$ (E > $0.18 \mathrm{MeV}$ ) HTGR at $1005^{\circ} \mathrm{C}$. (a) Typical appearance of matrix and (b) composite showing radial cross section. 


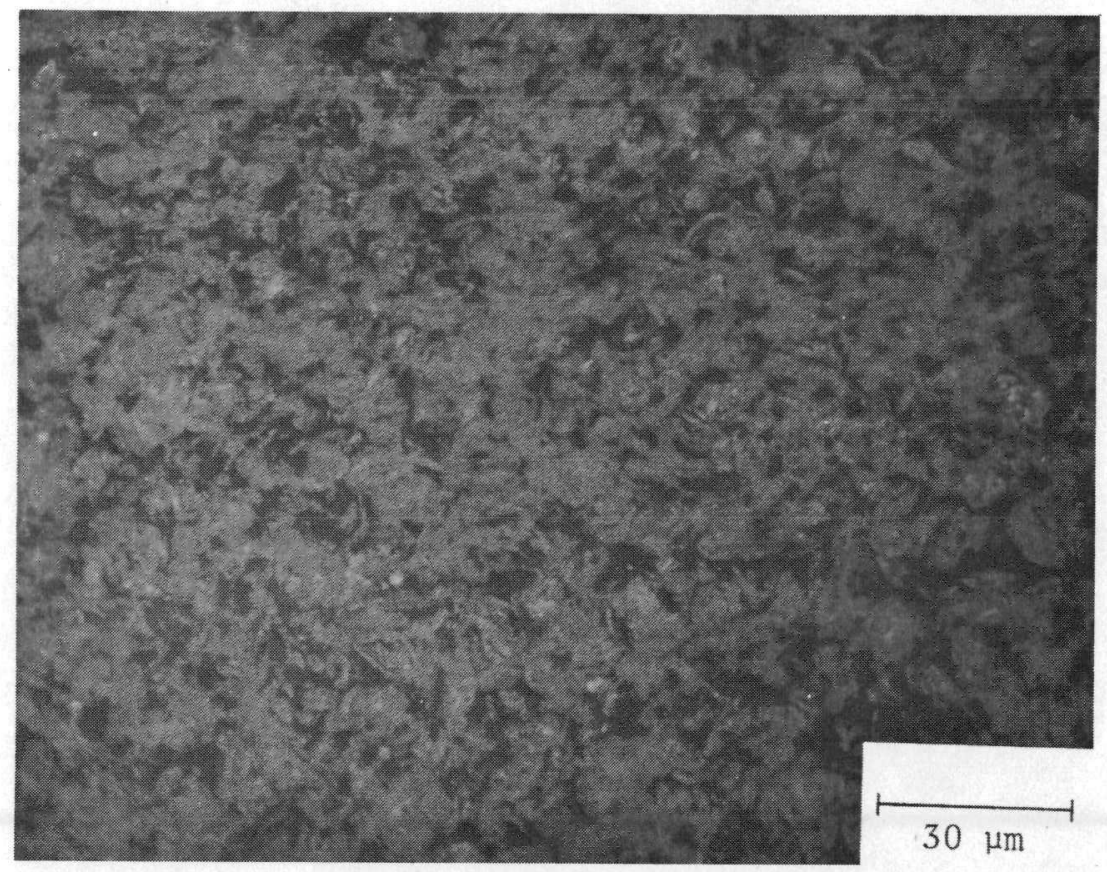

L7440-843

(a)

FUEL ROD

FUEL ROD

SURFACE

CENTERLINE

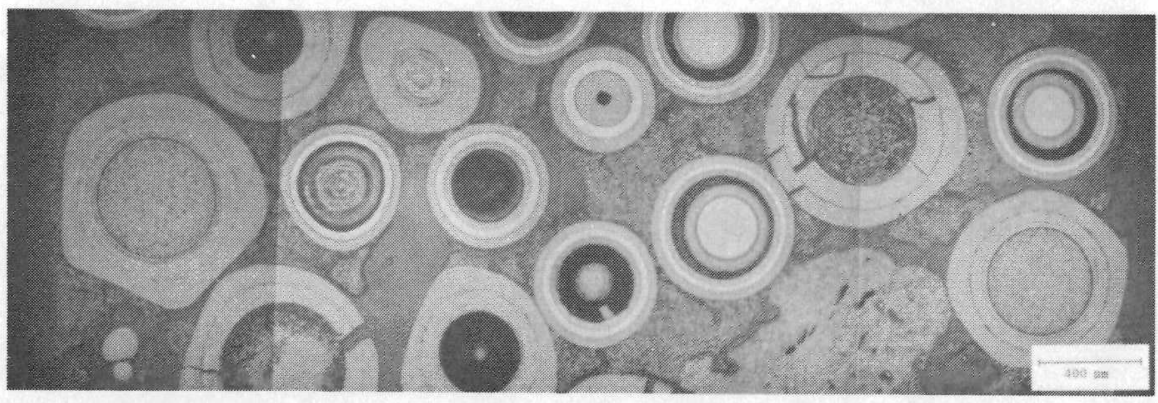

$75 \mathrm{~K} 3401-2$

(b)

Fig. 4-69. Representative photomicrographs of fuel rod 7161-004-01-7 after irradiation in capsule P13S (position 1A) to a fast neutron fluence of $7.1 \times 10^{21} \mathrm{n} / \mathrm{cm}^{2}(\mathrm{E}>0.18 \mathrm{MeV})_{\mathrm{HTGR}}$. This rod was thermal cycled to high temperatures 24 times during irradiation. (a) Typical appearance of matrix and (b) composite showing radial cross section. 


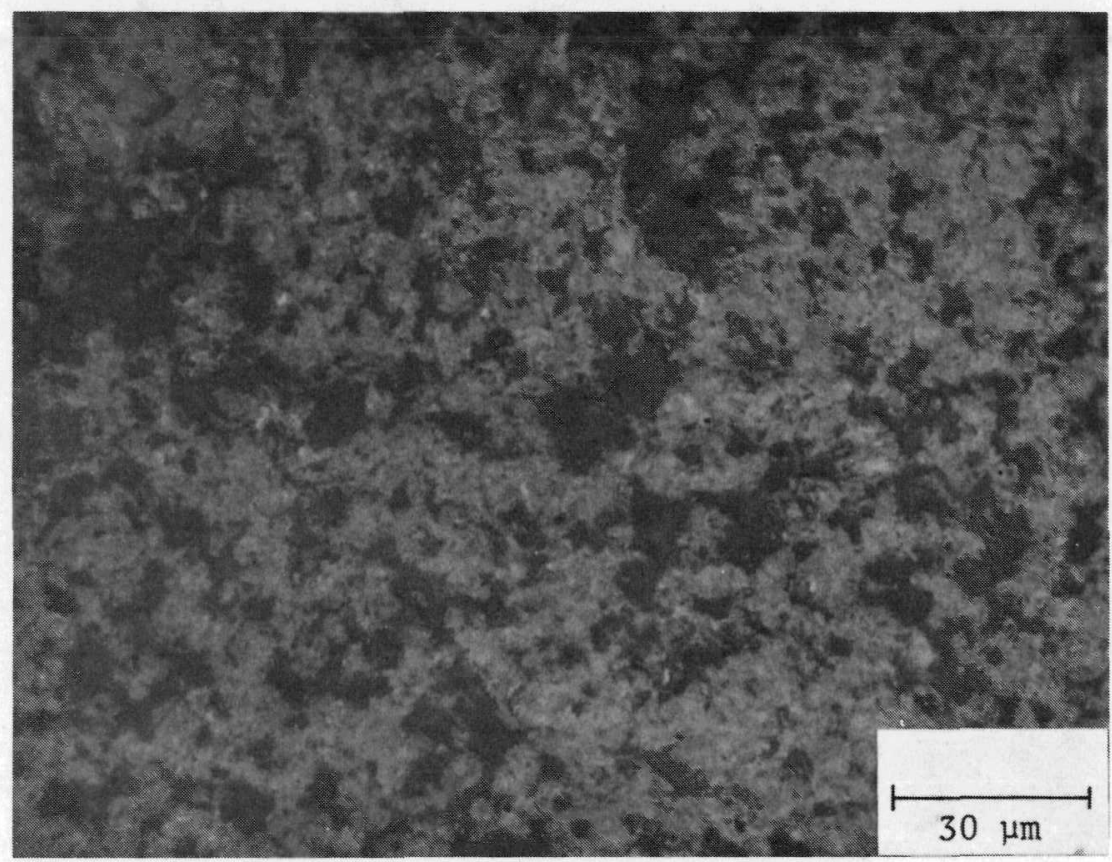

L7440-581

(a)

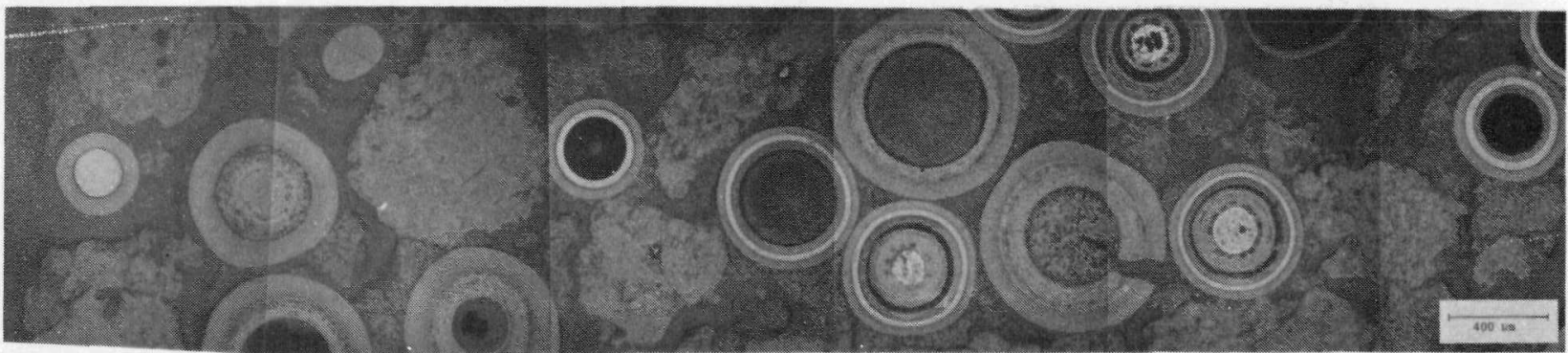

$75 \mathrm{~K} 3400-2$

(b)

Fig. 4-70. Representative photomicrographs of fuel rod 7161-004-12-5 after irradiation in capsule P13S (position 2B) to a fast neutron fluence of $11.7 \times 10^{21} \mathrm{n} / \mathrm{cm}^{2}(\mathrm{E}>0.18 \mathrm{MeV})$ HTGR at $1010^{\circ} \mathrm{C}$. (a) Typical appearance of matrix and (b) composite showing radial cross section. 


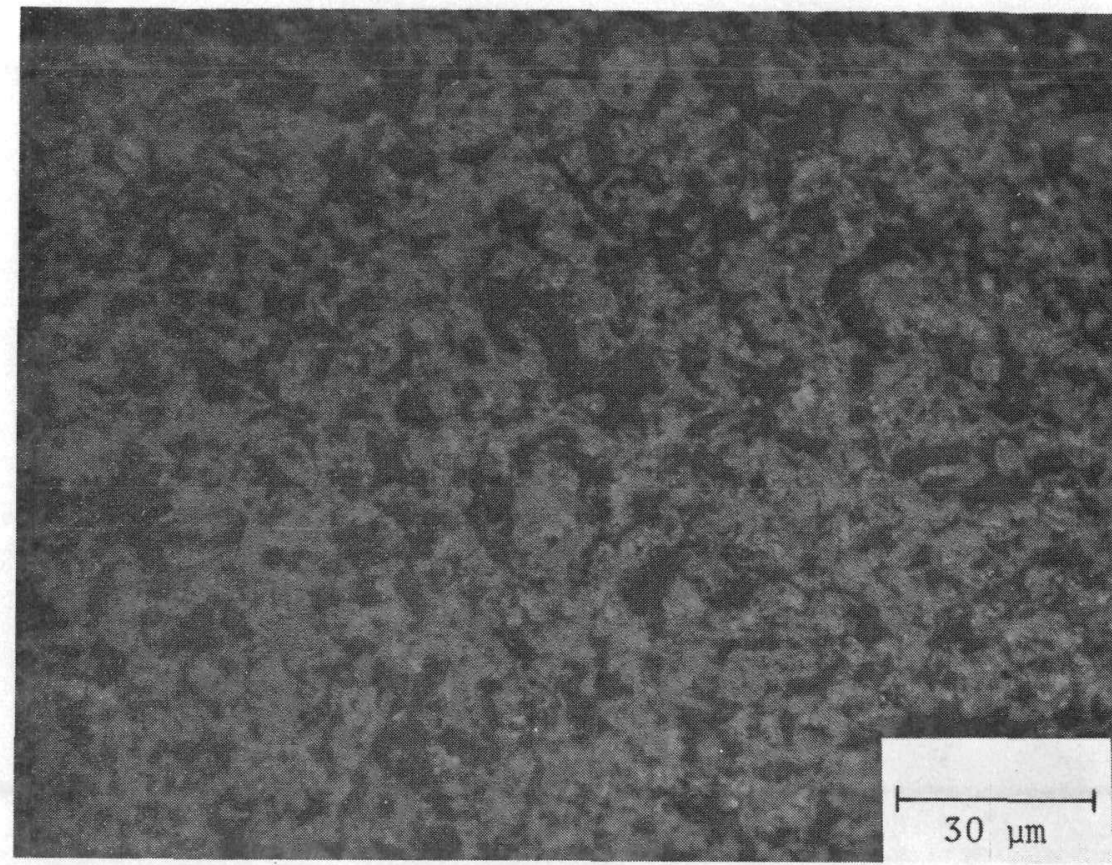

L7440-523

(a)

FUEL ROD

FUEL ROD

SURFACE

CENTERLINE

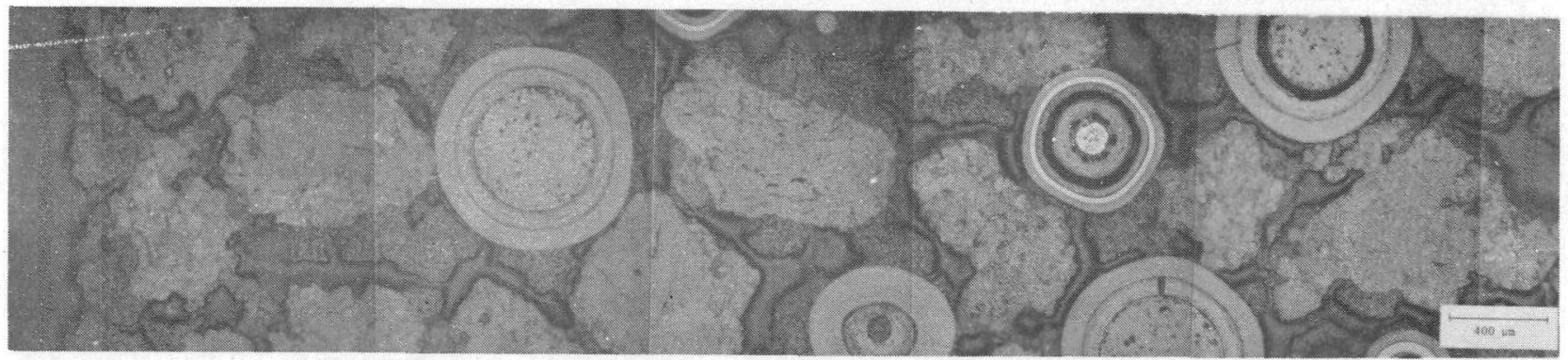

$75 \mathrm{~K} 3400-5$

(b)

Fig. 4-71. Representative photomicrographs of fuel rod 7161-004-13-6 after irradiation in capsule P13S (position 2C) to a fast neutron fluence of $11.9 \times 10^{21} \mathrm{n} / \mathrm{cm}^{2}$ ( $\mathrm{E}>0.18 \mathrm{MeV}$ ) HTGR at $975^{\circ} \mathrm{C}$. (a) Typical appearance of matrix and (b) composite showing radial cross section. 


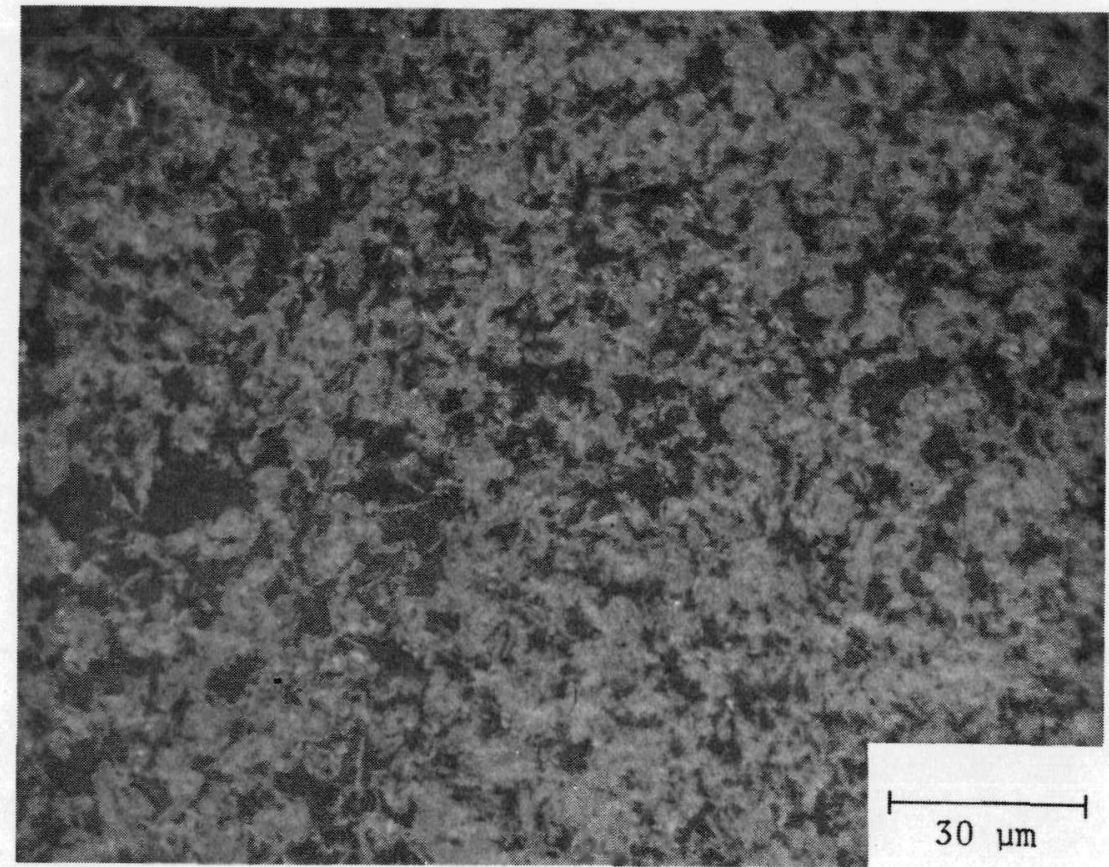

L7440-94

(a)

FUEL ROD

SURFACE

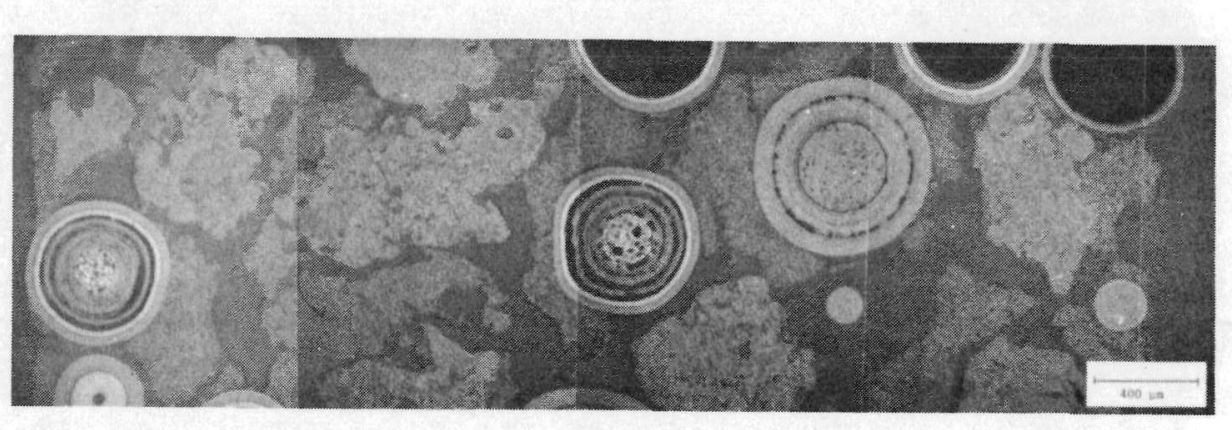

75K3398-4
FUEL ROD

CENTERLINE (b)

Fig. 4-72. Representative photomicrographs of fuel rod 7161-004-15-5 after irradiation in capsule $\mathrm{P} 13 \mathrm{~S}$ (position $2 \mathrm{E}$ ) to a fast neutron fluence of $12.1 \times 10^{21} \mathrm{n} / \mathrm{cm}^{2}$ (E $>0.18 \mathrm{MeV}$ ) HTGR at $960^{\circ} \mathrm{C}$. (a) Typical appearance of matrix and (b) composite showing radial cross section. 


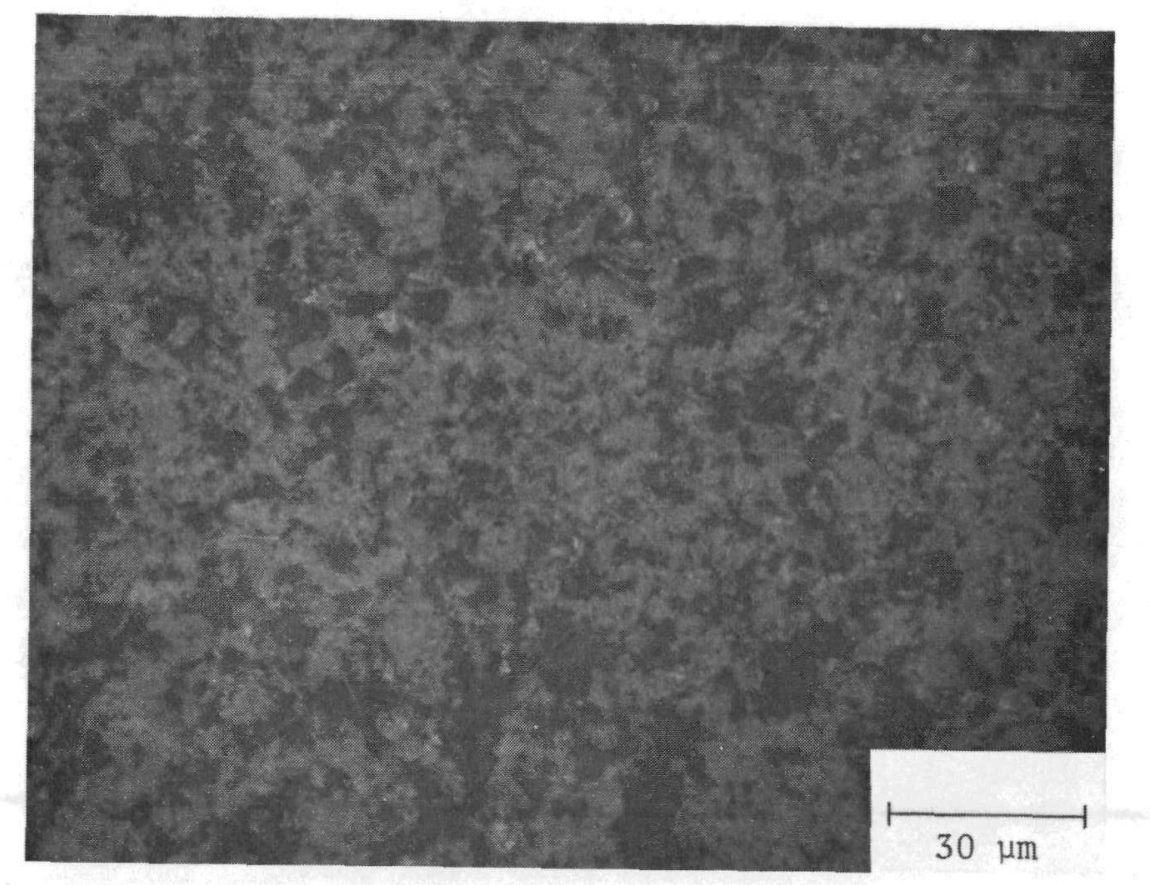

L7440-1072

(a)

FUEL ROD

FUEL ROD

SURFACE

CENTERLINE

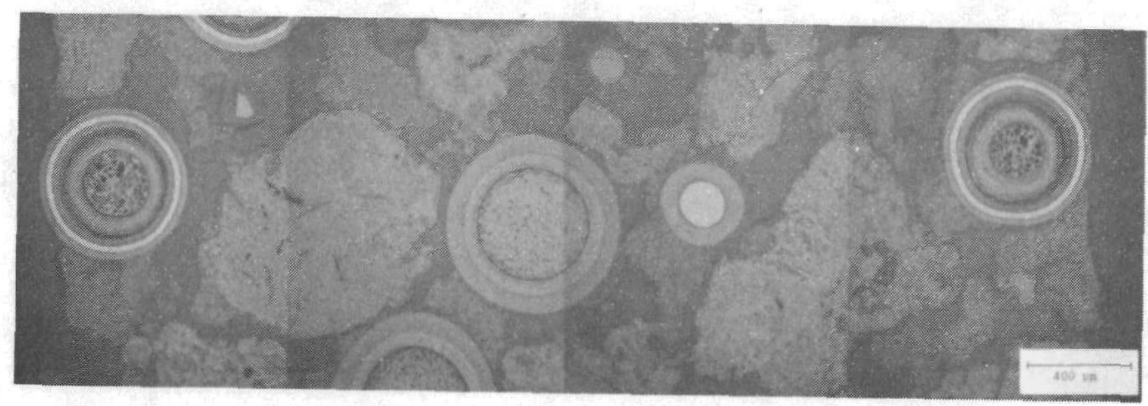

75K3398-1

(b)

Fig. 4-73. Representative photomicrographs of fuel rod 7161-004-16-6 after irradiation in capsule P13S (position 5A) to a fast neutron fluence of $9.2 \times 10^{21} \mathrm{n} / \mathrm{cm}^{2}(\mathrm{E}>0.18 \mathrm{MeV}) \mathrm{HTGR}$ at $1375^{\circ} \mathrm{C}$. (a) Typical appearance of matrix and (b) composite showing radial cross section. 


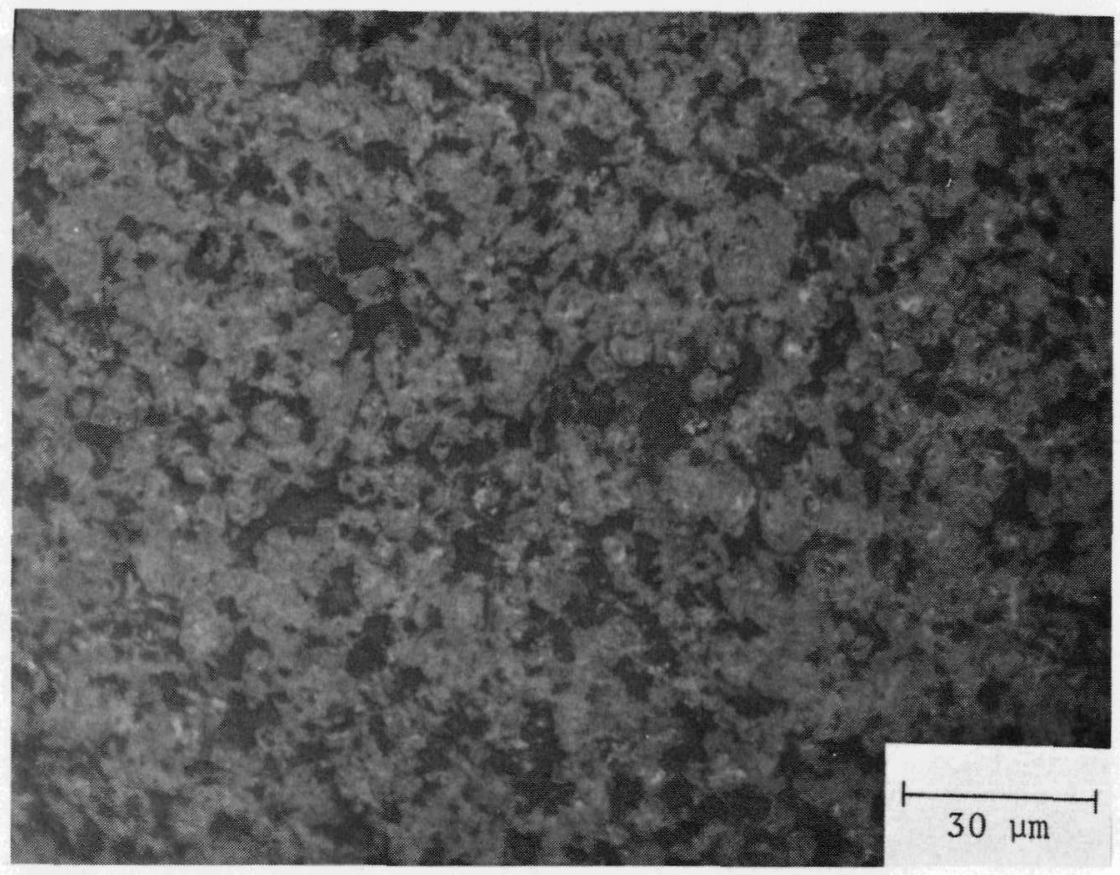

L7440-463

(a)

\section{FUEL ROD}

SURFACE

FUEL ROD

CENTERLINE

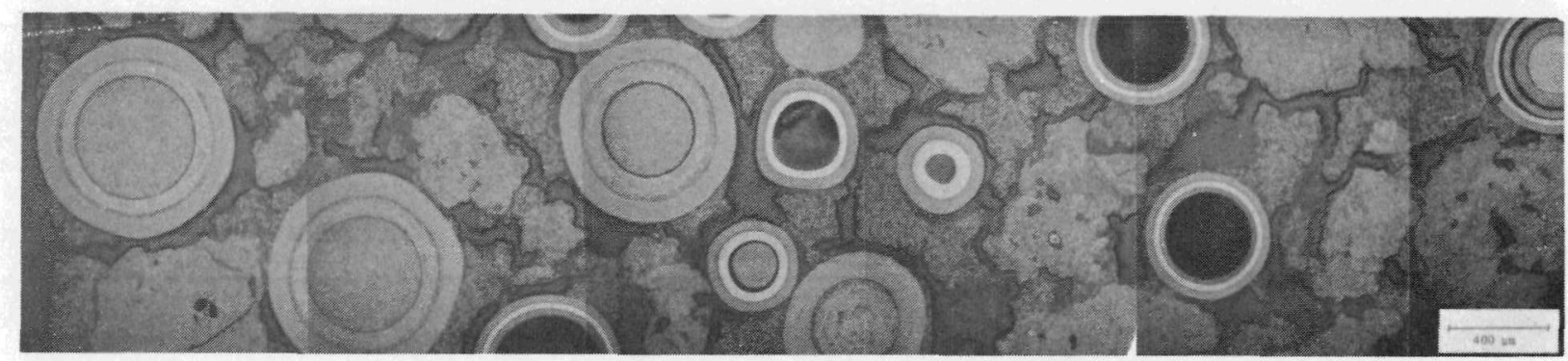

$75 \mathrm{~K} 3399-3$

(b)

Fig. 4-74. Representative photomicrographs of fuel rod 7161-004-18-5 after irradiation in capsule P13S (position 5C) to a fast neutron fluence of $8.4 \times 10^{21} \mathrm{n} / \mathrm{cm}^{2}\left(\mathrm{E}>0.18 \mathrm{MeV}\right.$ ) $\mathrm{HTGR}$ at $1335^{\circ} \mathrm{C}$. (a) Typical appearance of matrix and (b) composite showing radial cross section. 


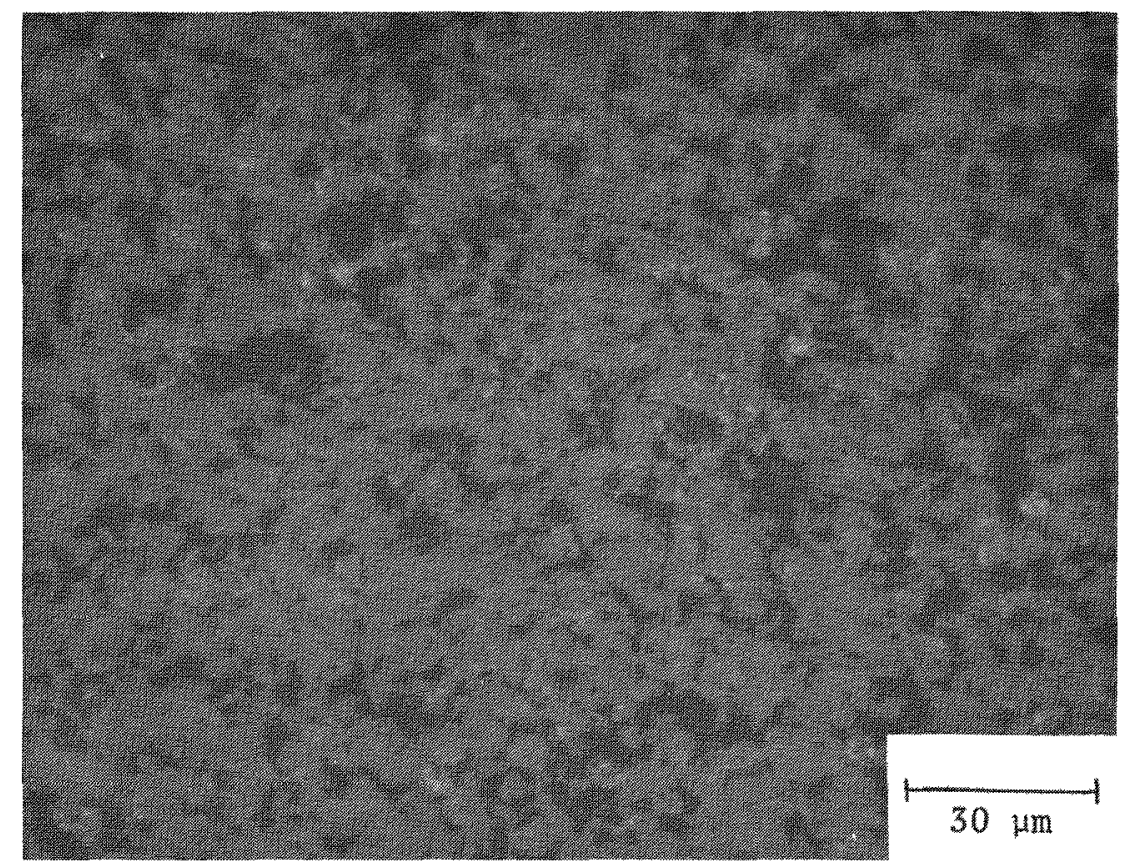

L7440-218

(a)

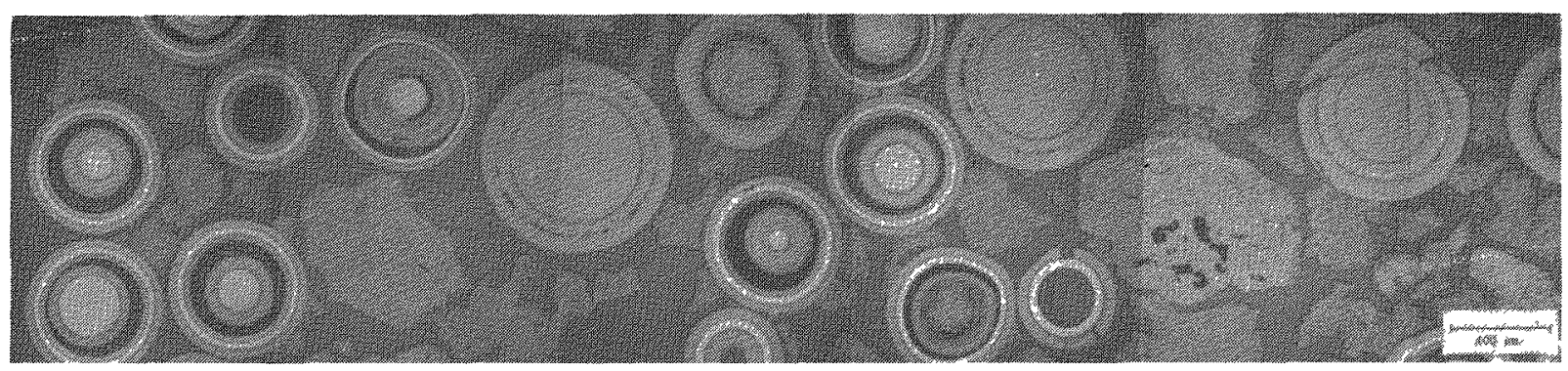

$75 \mathrm{~K} 3399-1$

(b)

Fig. 4-75. Representative photomicrographs of fuel rod 7161-004-19-5 after irradiation in capsule $\mathrm{P} 13 \mathrm{~S}$ (position 5D) to a fast neutron fluence of $7.9 \times 10^{21} \mathrm{n} / \mathrm{cm}^{2}$ ( $\mathrm{E}>0.18 \mathrm{MeV}$ ) HTGR. After irradiation this rod was heated to $1500^{\circ} \mathrm{C}$ in TRIGA for fission gas release measurements. (a) Typical appearance of matrix and (b) composite showing radial cross section. 


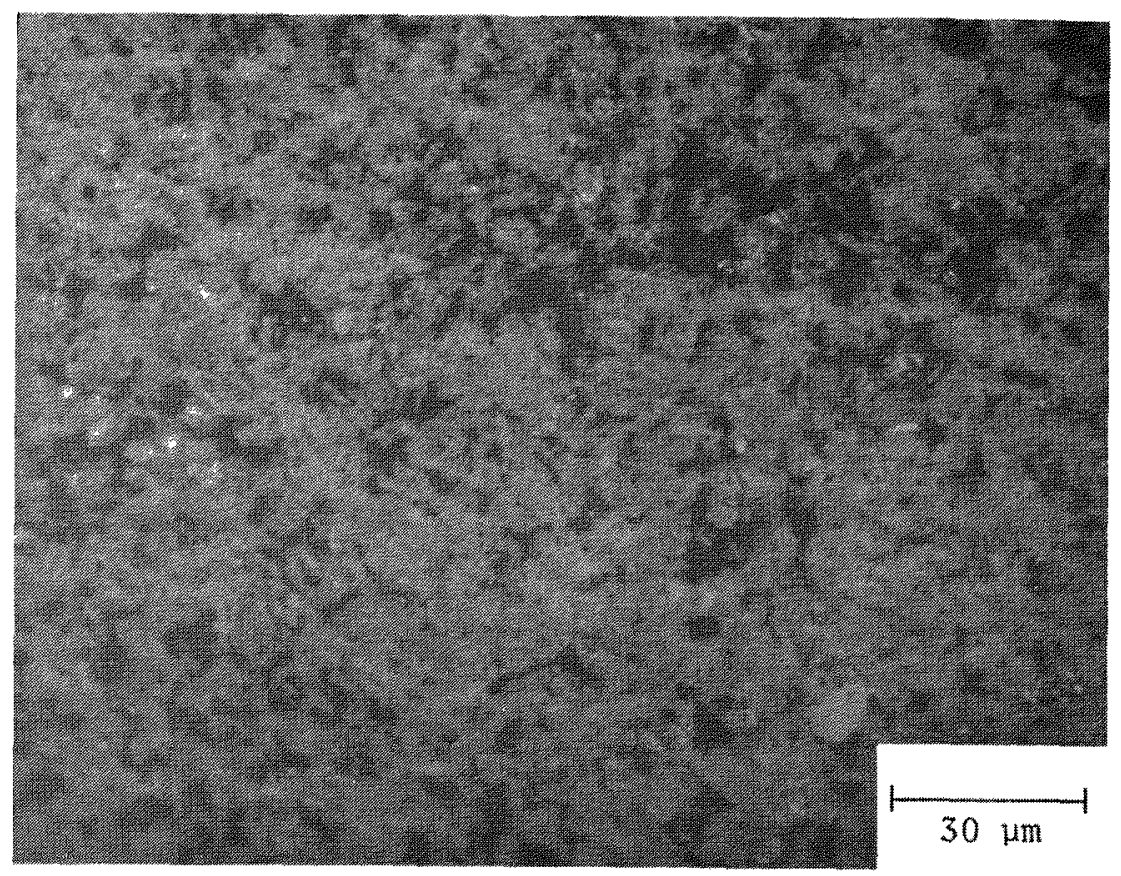

L7440-202

(a)

FUEL ROD

FUEL ROD

SURFACE

CENTERLINE

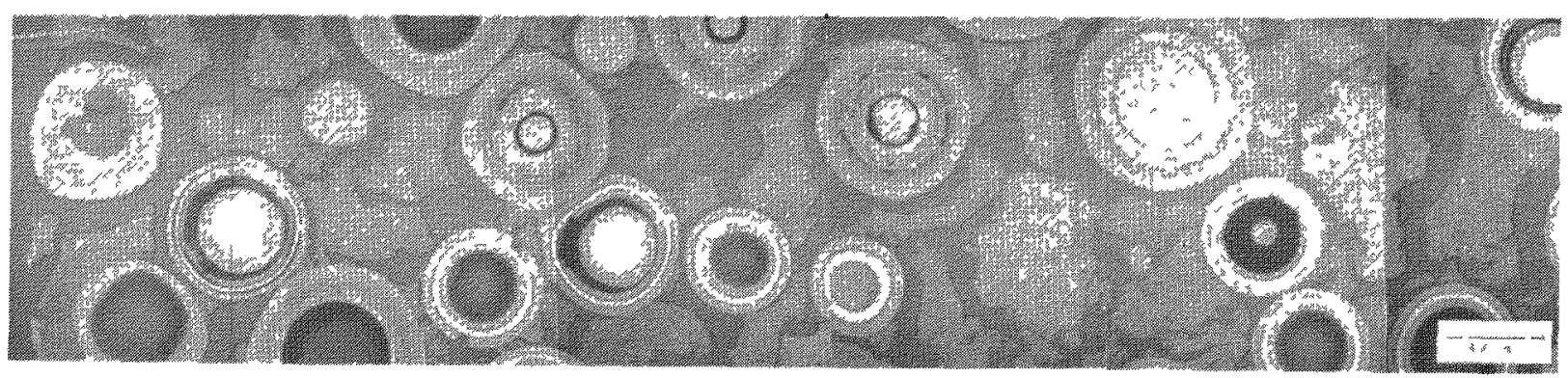

75K3399-2

(b)

Fig. 4-76. Representative photomicrographs of fuel rod 7161-004-20-5 after irradiation in capsule P13S (position $5 \mathrm{E}$ ) to a fast neutron fluence of $7.5 \times 10^{21} \mathrm{n} / \mathrm{cm}^{2}(\mathrm{E}>0.18 \mathrm{MeV})_{\mathrm{HTGR}}$ at $1350^{\circ} \mathrm{C}$. (a) Typical appearance of matrix and (b) composite showing radial cross section. 


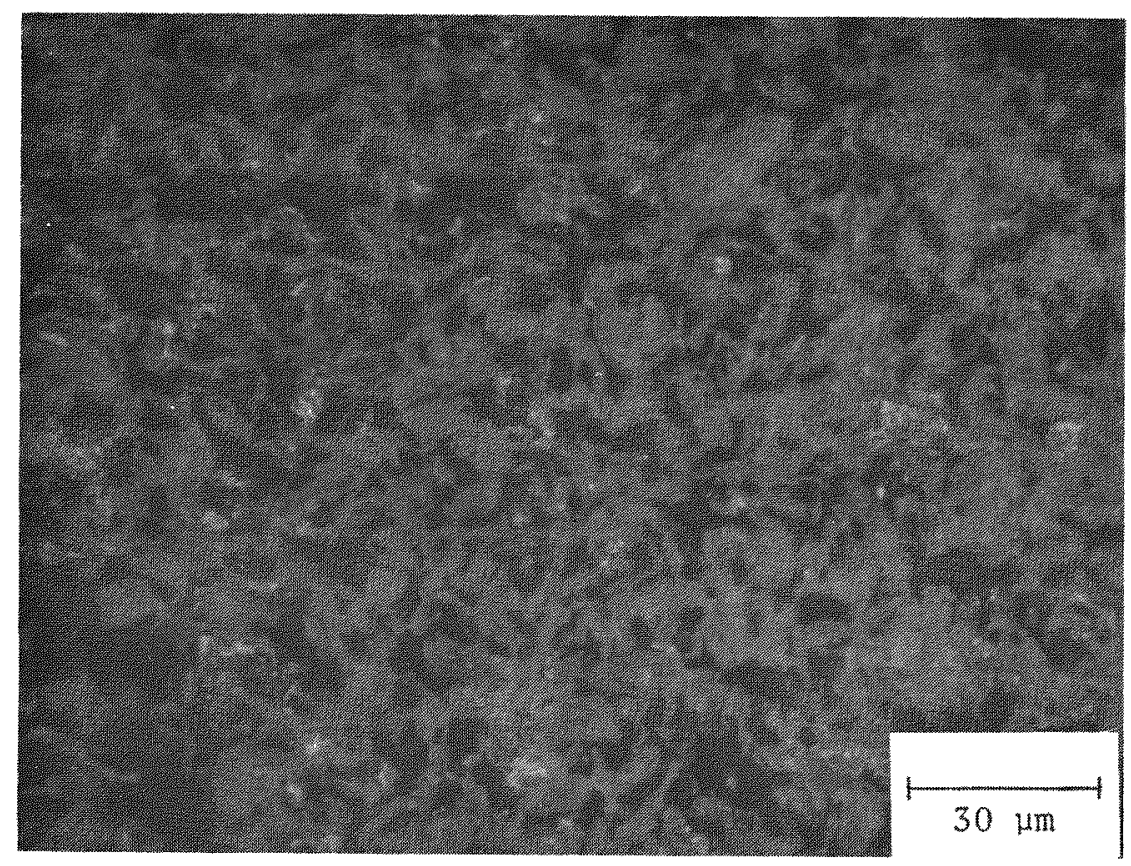

L7440-499

(a)

FUEL ROD SURFACE

FUEL ROD CENTERLINE

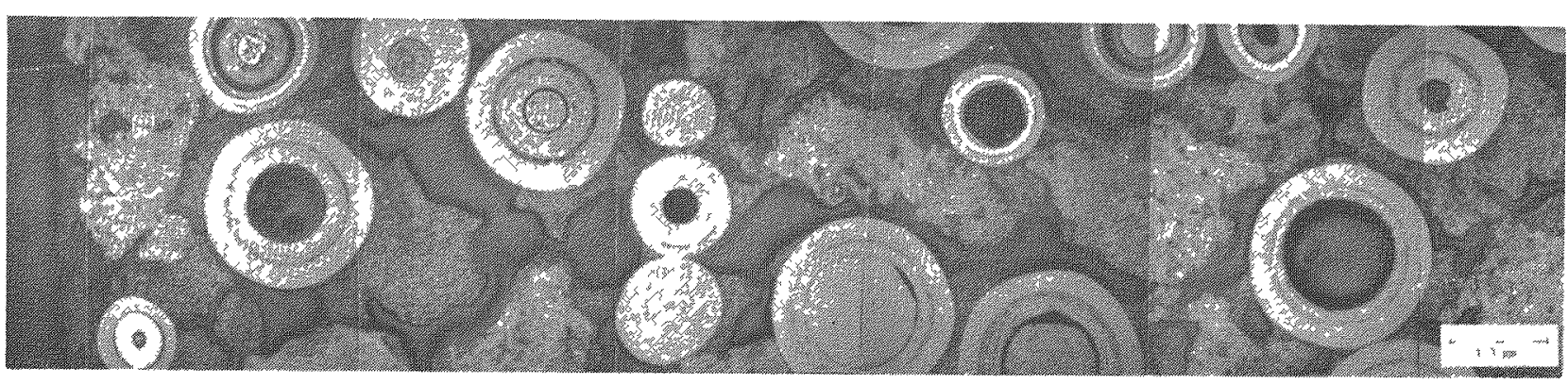

$75 \times 3400-1$

(b)

Fig. 4-77. Representative photomicrographs of fuel rod 7161-004-28-13 after irradiation in capsule P13S (position 6C) to a fast neutron fluence of $4.4 \times 10^{21} \mathrm{n} / \mathrm{cm}^{2}(\mathrm{E}>0.18 \mathrm{MeV})_{\mathrm{HTGR}}$ at $1050^{\circ} \mathrm{C}$. (a) Typical appearance of matrix and (b) composite showing radial cross section. 


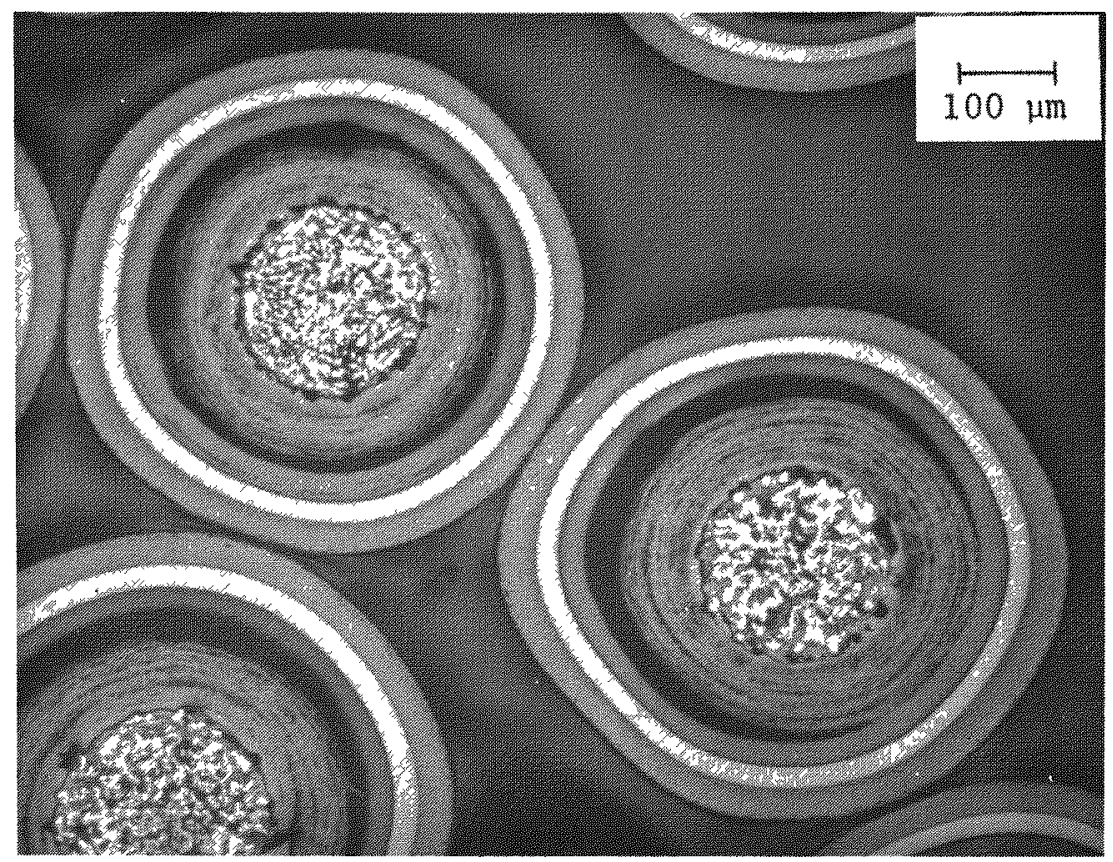

L7440-833

(a)

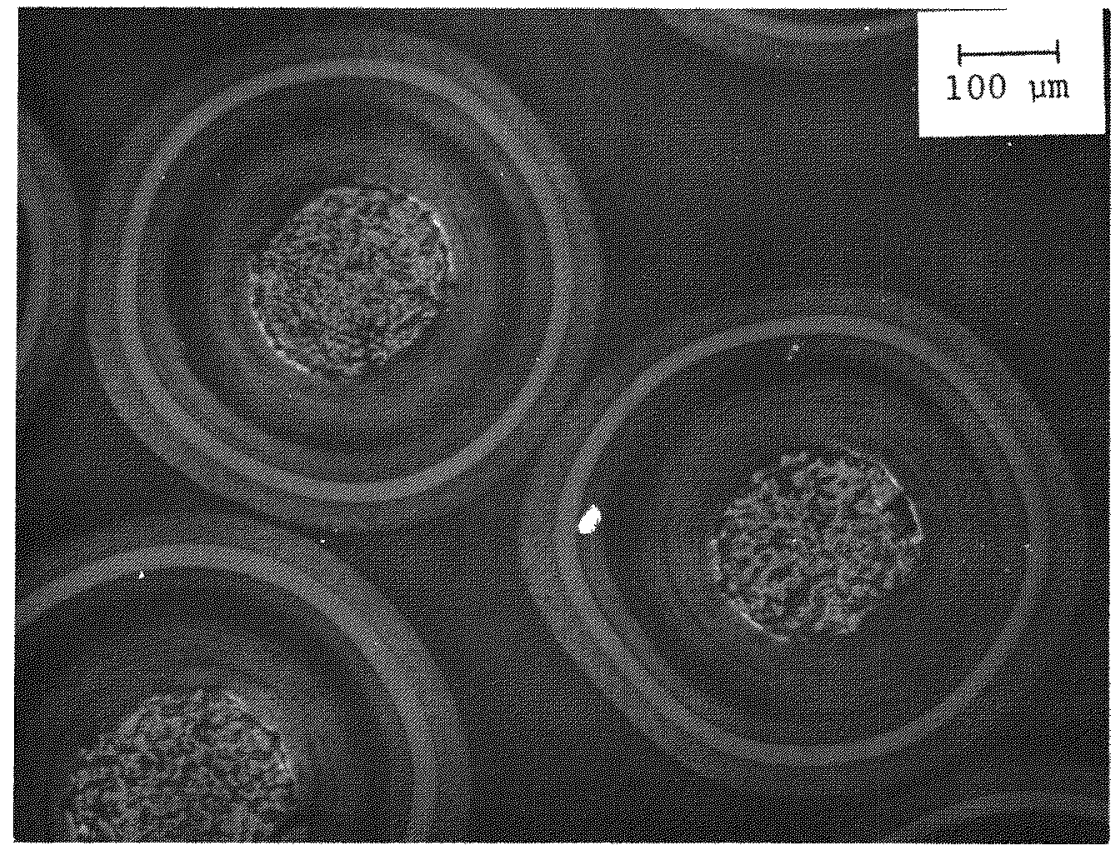

L7440-834

(b)

Fig. 4-78. Representative photomicrographs of TRISO UC 2 particles (6151-00-035) irradiated in capsule P13S (position 4-12) to a fast neutron fluence of $10.7 \times 10^{2} \mathrm{n} / \mathrm{cm}^{2}(\mathrm{E}>0.18$ $\mathrm{MeV})_{\mathrm{HTGR}}$ and burnup of $71 \%$ FIMA at $975^{\circ} \mathrm{C}$. (a) Bright field and (b) polarized 1ight. 


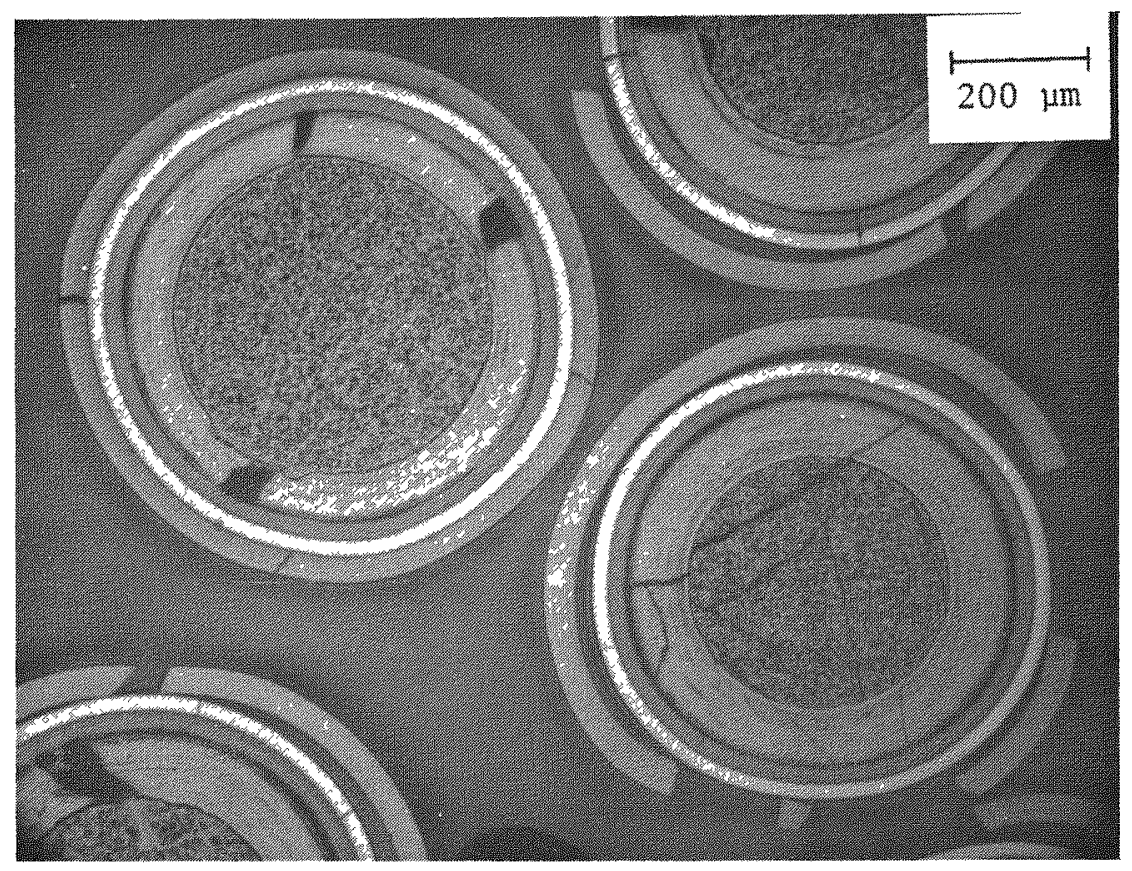

L7440-800

(a)

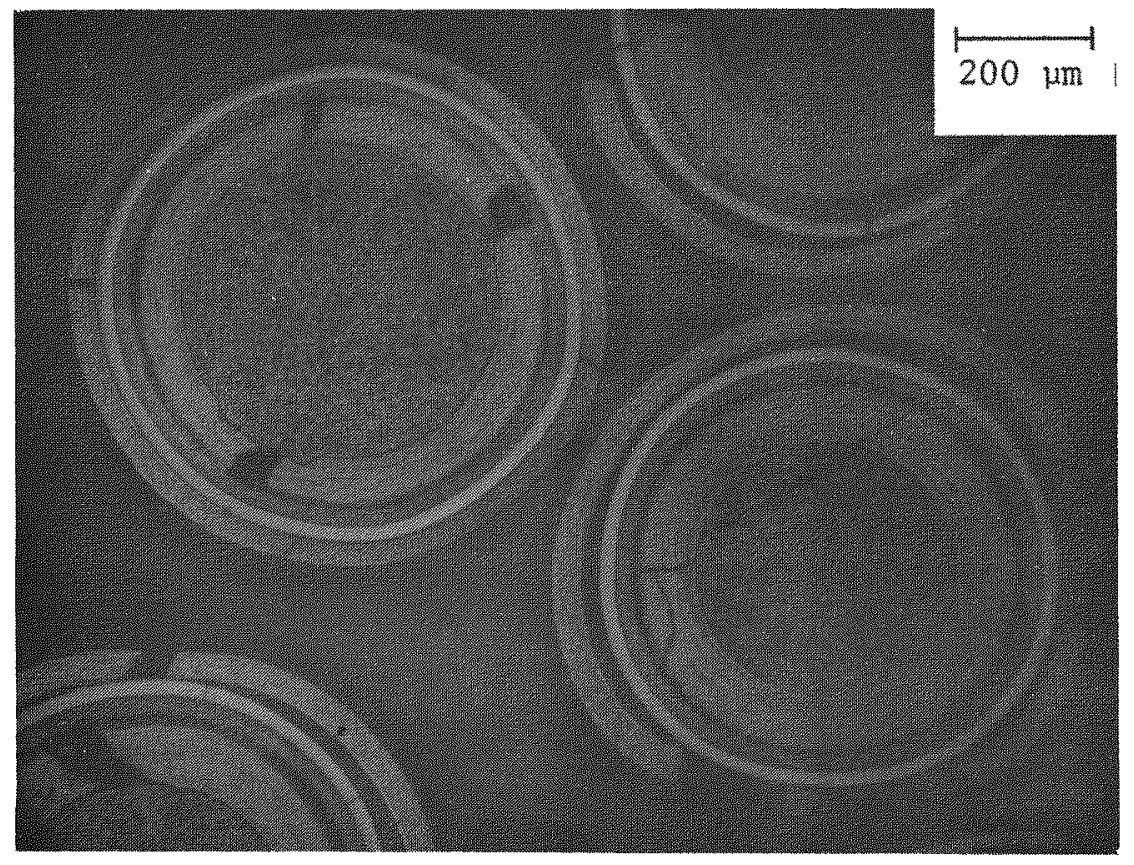

L7440-801

(b)

Fig. 4-79. Representative photomicrographs of TRISO (8Th, 1U) $\mathrm{O}_{2}$ particles (6155-01-020) irradiated in capsule P13S (position 4-4) to a fast neutron fluence of $11.1 \mathrm{x}$

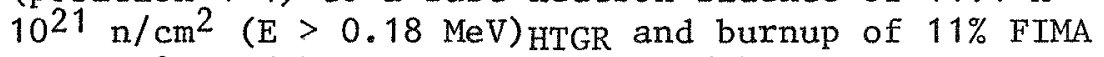
at $1090^{\circ} \mathrm{C}$. (a) Bright field and (b) polarized light. 


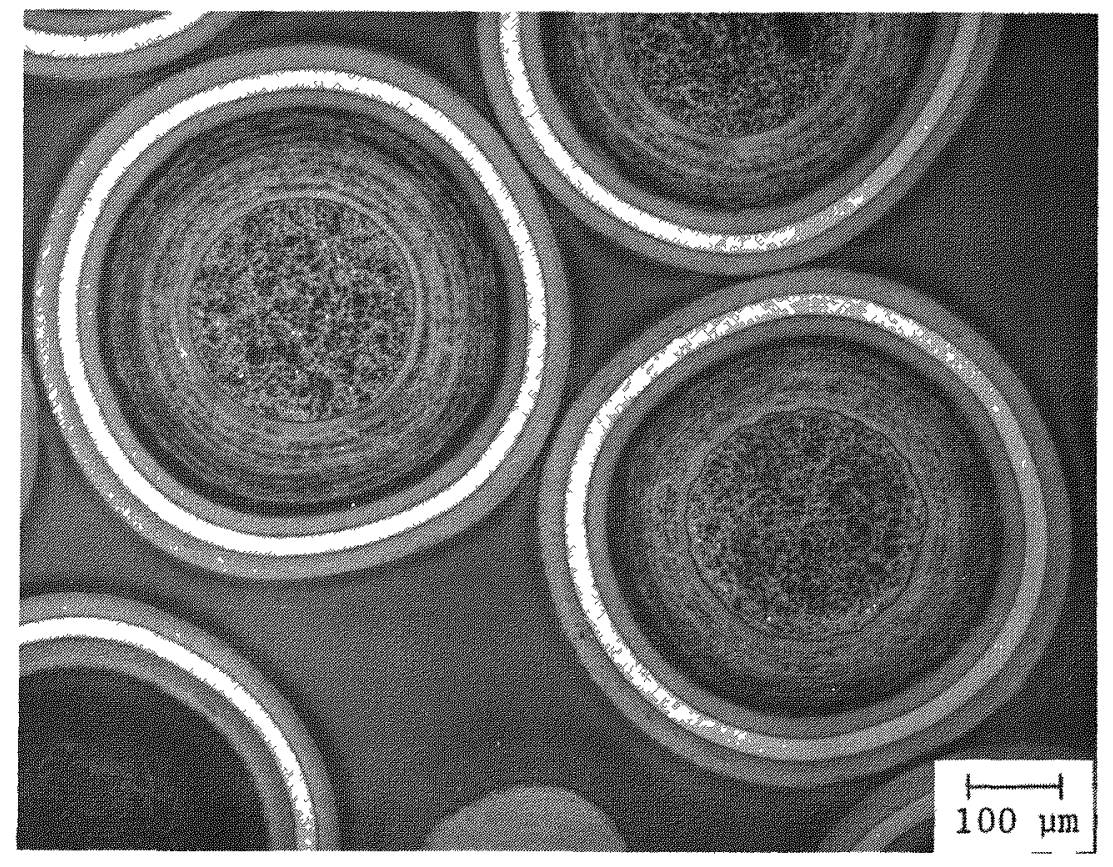

L7440-816

(a)

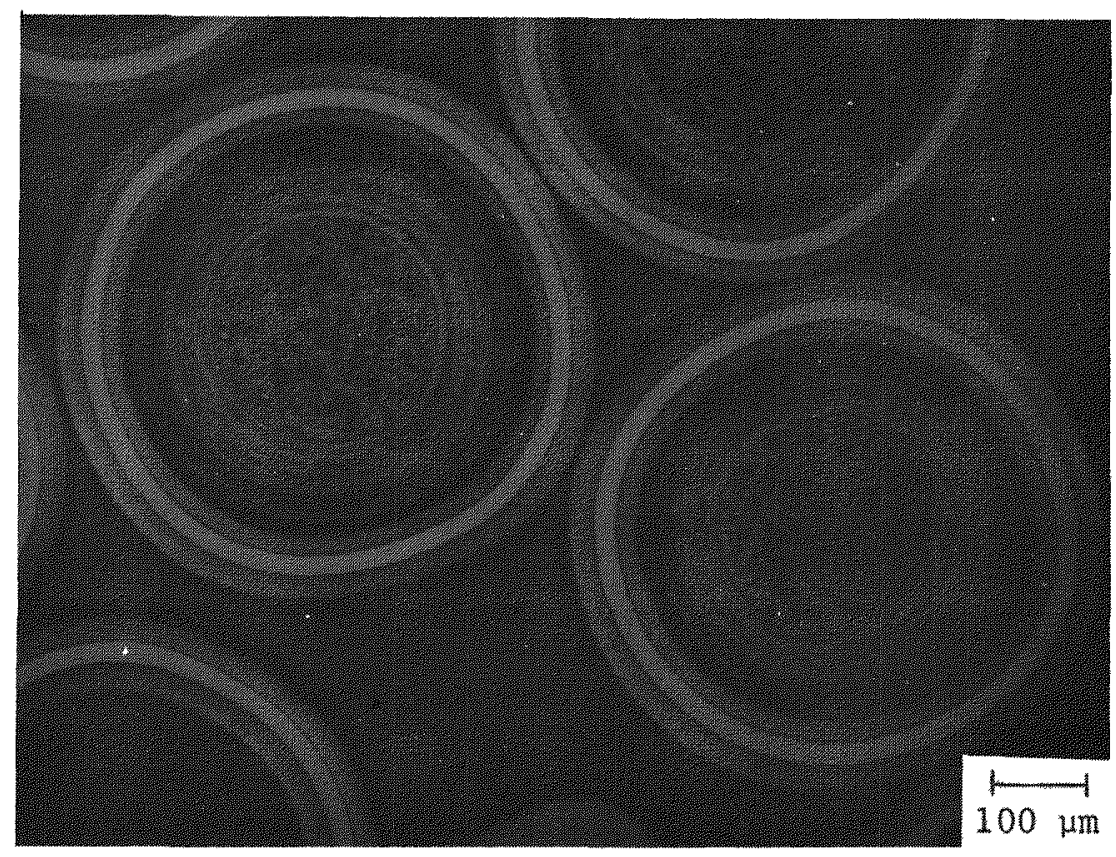

L7440-817

(b)

Fig. 4-80. Representative photomicrographs of TRISO (Th, U) $\mathrm{O}_{2}$ particles (5466-37) irradiated in capsule P13S (position 4-8) to a fast neutron fluence of $10.9 \times 10^{21} \mathrm{n} / \mathrm{cm}^{2}(\mathrm{E}>0.18 \mathrm{MeV})_{\mathrm{HTGR}}$ and burnup of $38 \%$ FIMA at $1090^{\circ} \mathrm{C}$. (a) Bright field and (b) polarized light. 


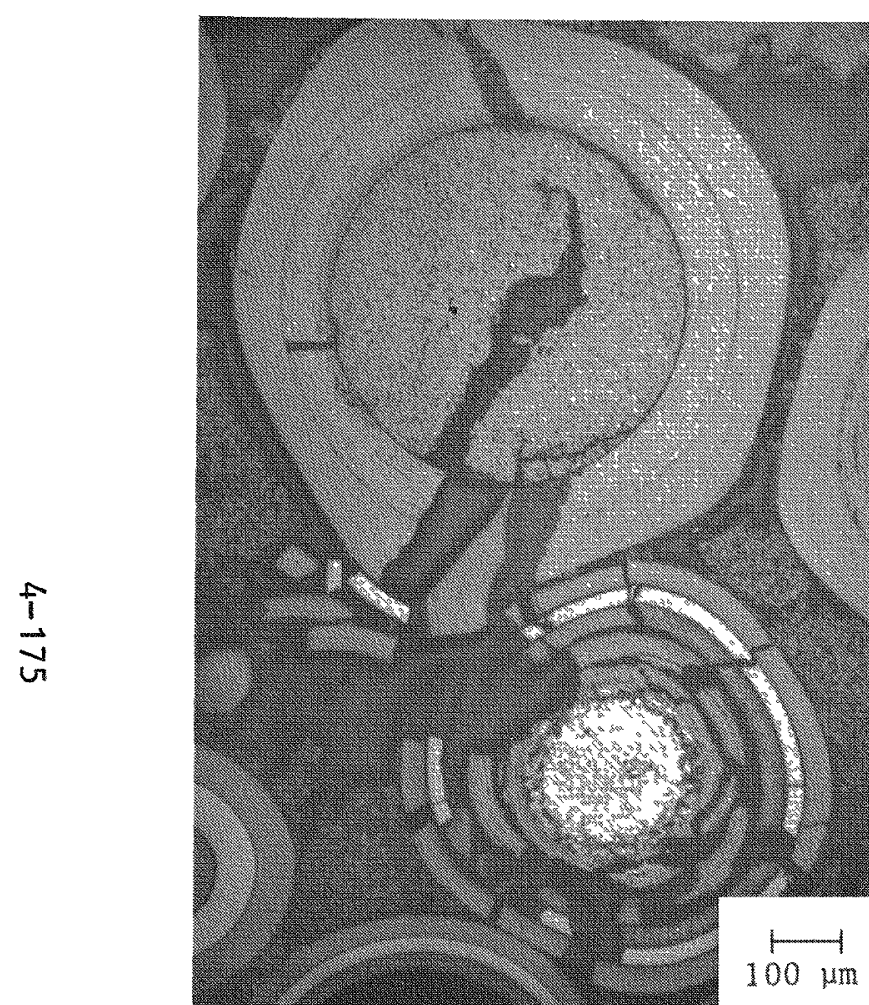

L7440-881

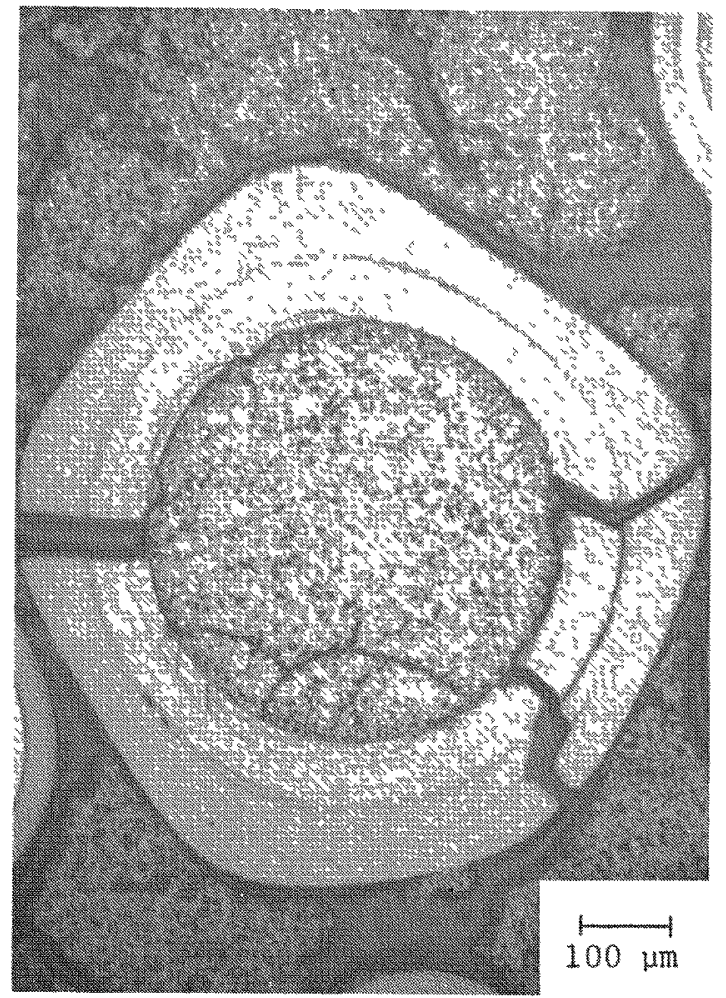

L7440-885

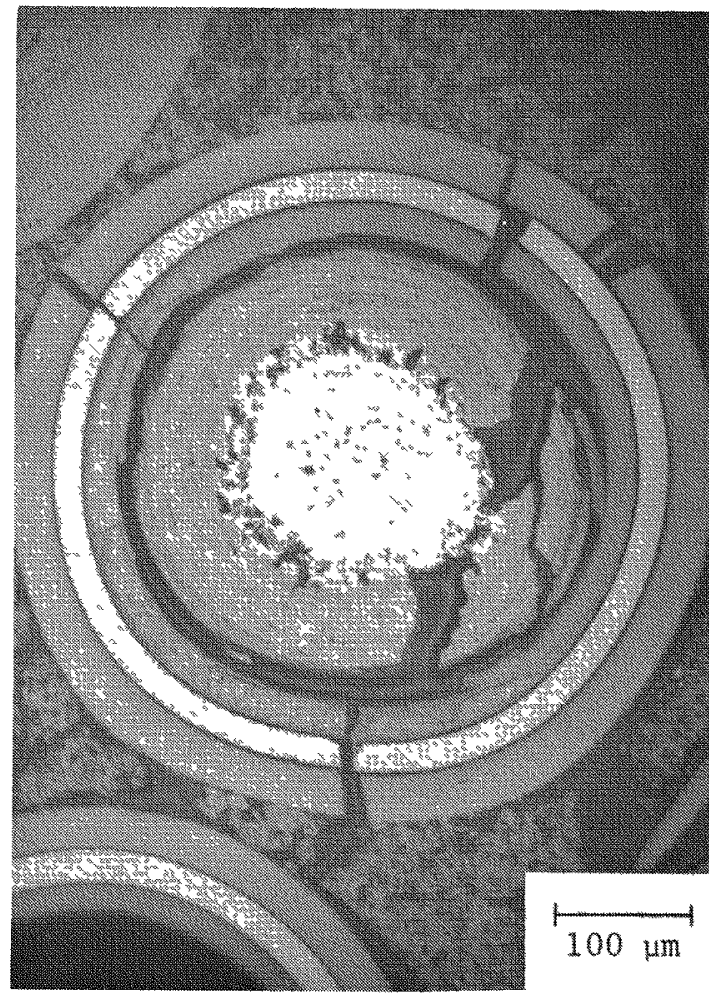

L7440-857

Fig. 4-81. Photomicrographs of fissile and fertile particles from fuel rod 7161-004-01-7 that failed during irradiation in capsule P13S (position 1A), which was thermal cycled to high temperatures 24 times during irradiation. Both the fissile (6151-00-045) and fertile (6542-20035) particles had severely faceted coatings. 


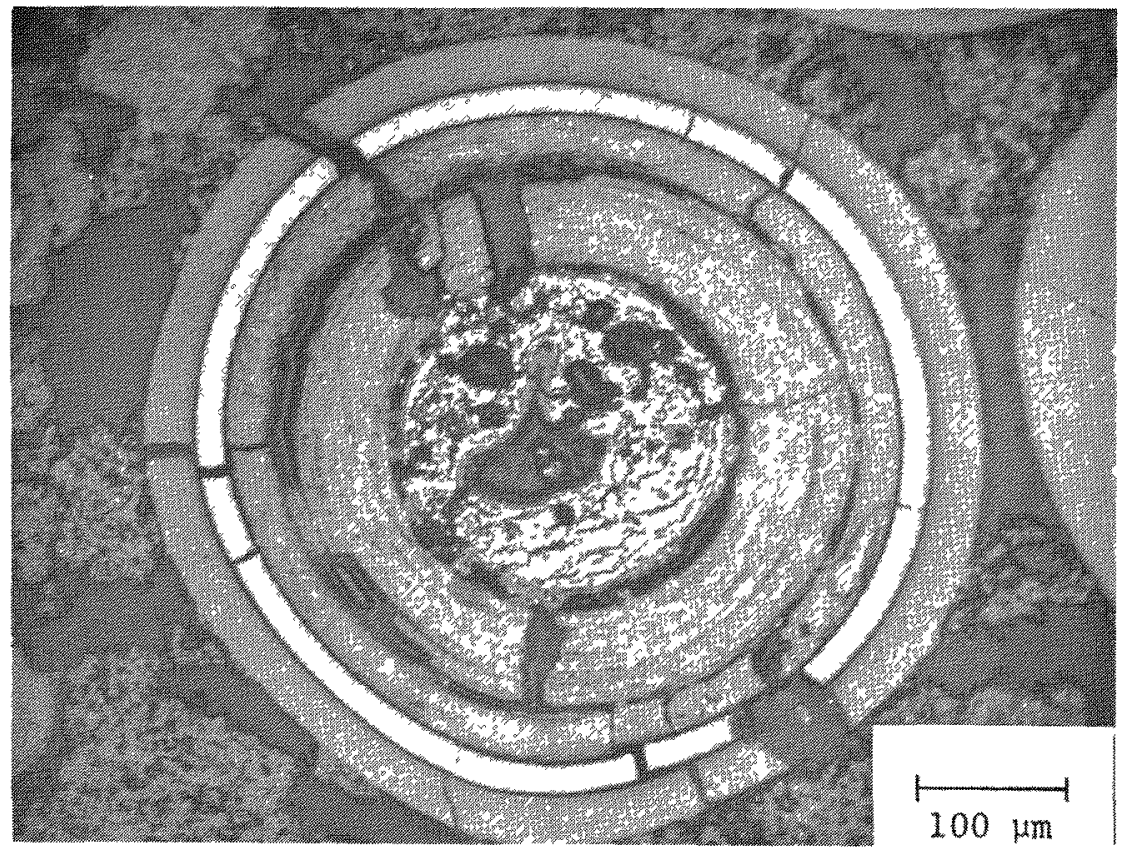

L7440-538

(a)

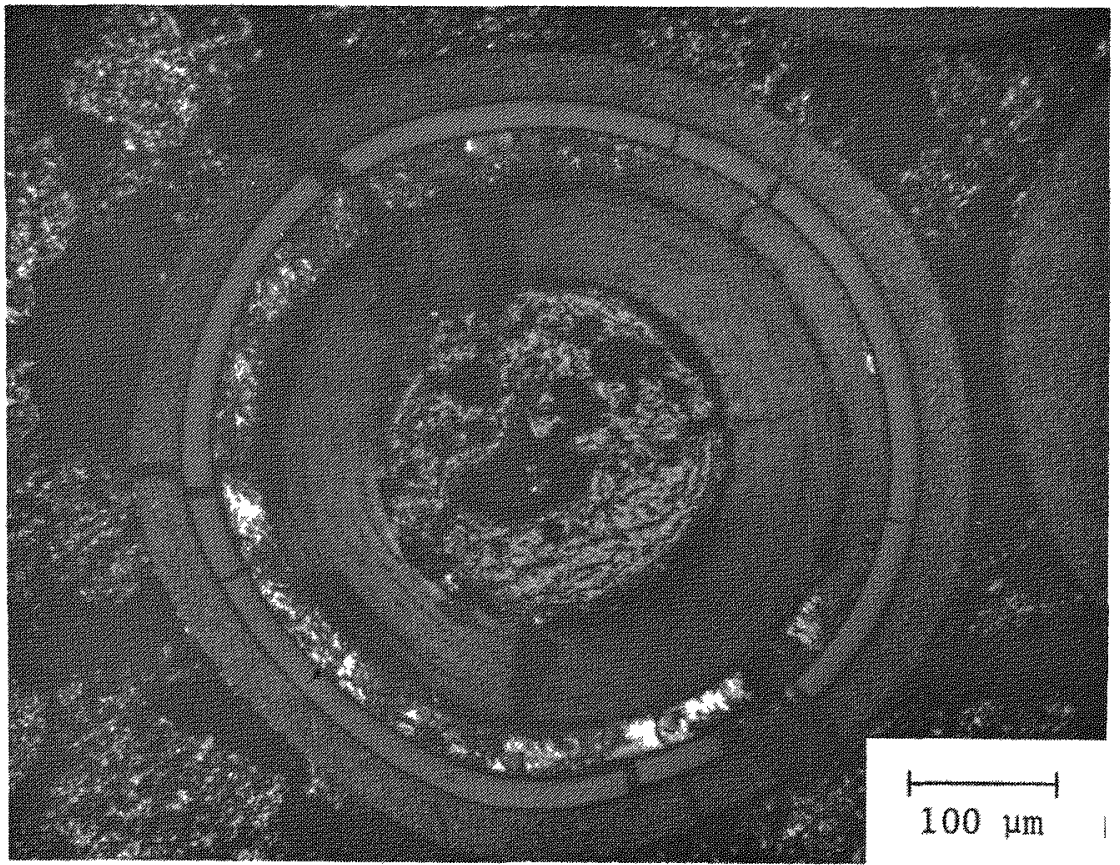

$17440-539$

(b)

Fig. 4-82. Photomicrographs showing a failed fissile particle (6151-00-035) from fuel rod 7161-004-035 irradiated in capsule P13R (position 1C) to a fast neutron fluence of $8.7 \times 10^{21} \mathrm{n} / \mathrm{cm}^{2}(\mathrm{E}>0.18$ $\mathrm{MeV})_{\mathrm{HTGR}}$ and burnup of $73 \%$ FIMA. After irradiation the rod was heated in the TRIGA facility to $1600^{\circ} \mathrm{C}$. (a) Bright field and (b) polarized light. 


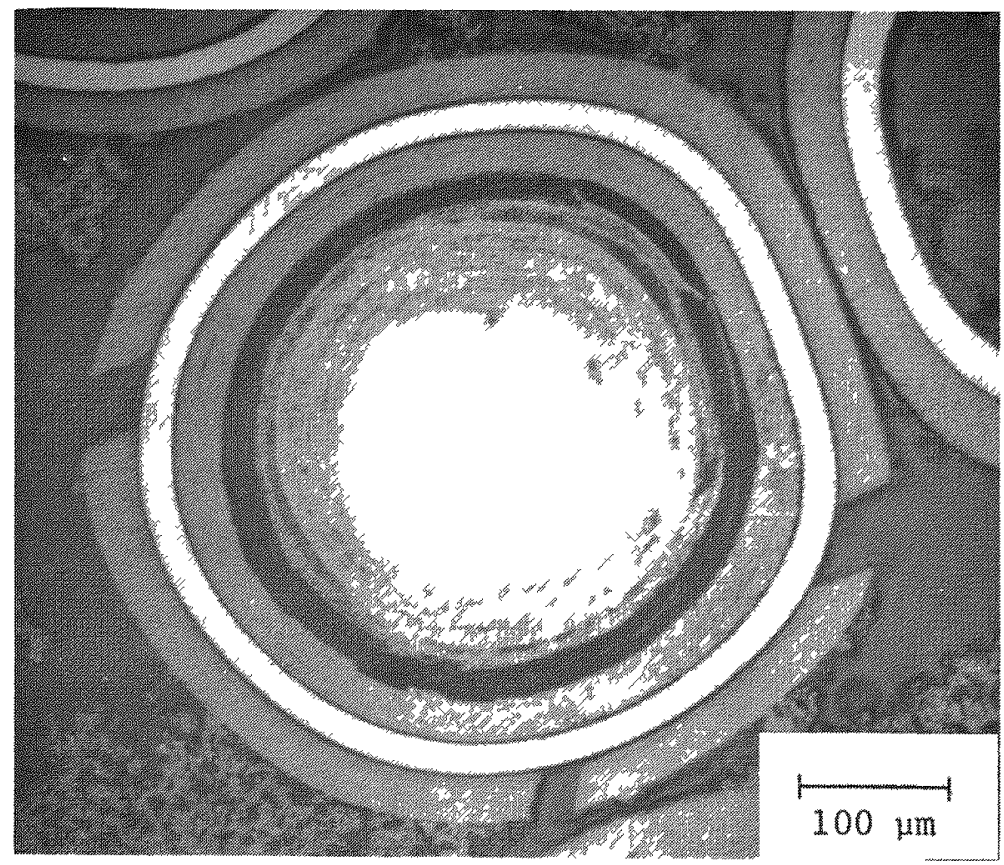

L7440-599

(a)

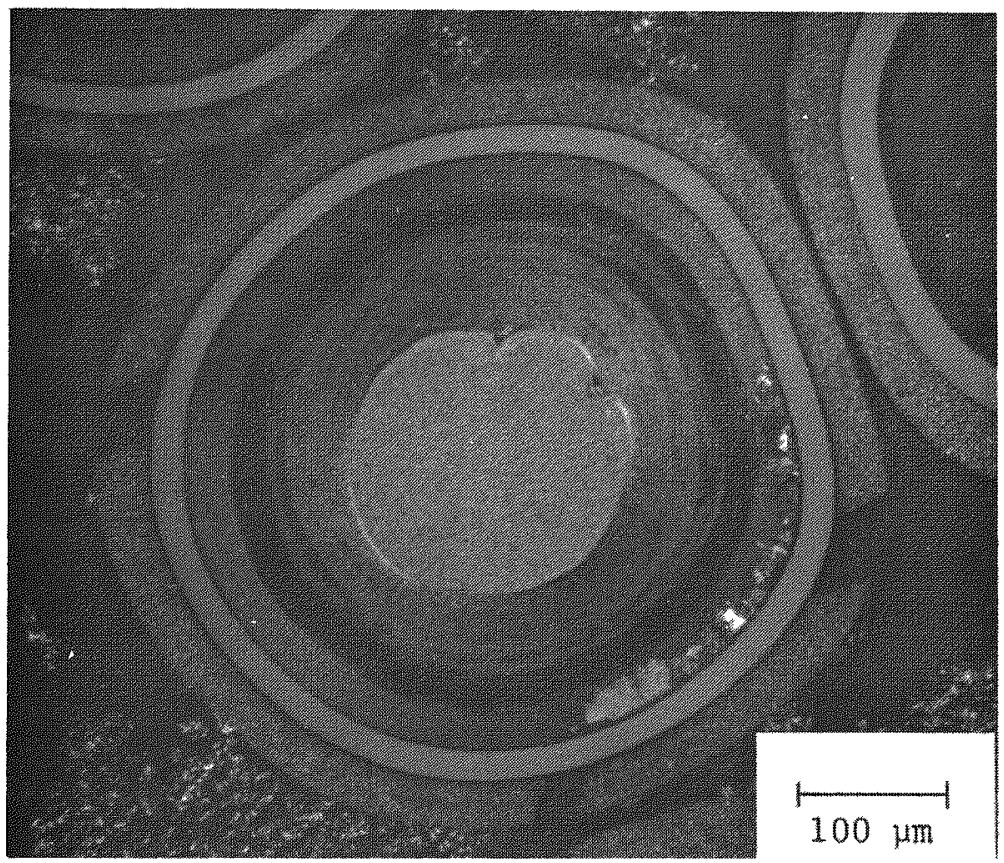

L7440-600

(b)

Fig. 4-83. Photomicrographs showing OPyC coating failure of fissile particle in fuel rod 7161-004-18-5 that was irradiated in capsule P13S (position 5C) to a fast neutron fluence of $8.4 \times 10^{21} \mathrm{n} / \mathrm{cm}^{2}$ $(\mathrm{E}>0.18 \mathrm{MeV})_{\mathrm{HTGR}}$ at $1335^{\circ} \mathrm{C}$. (a) Bright field and (b) polarized light. 


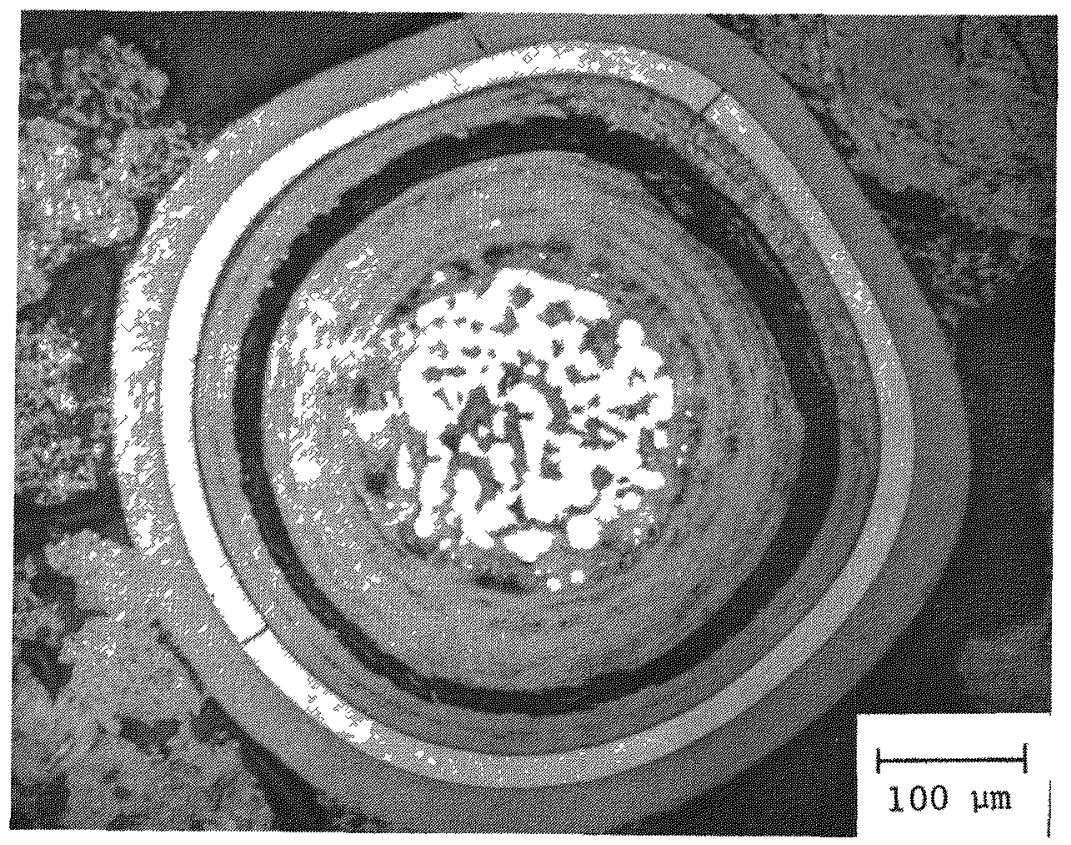

L7440-356

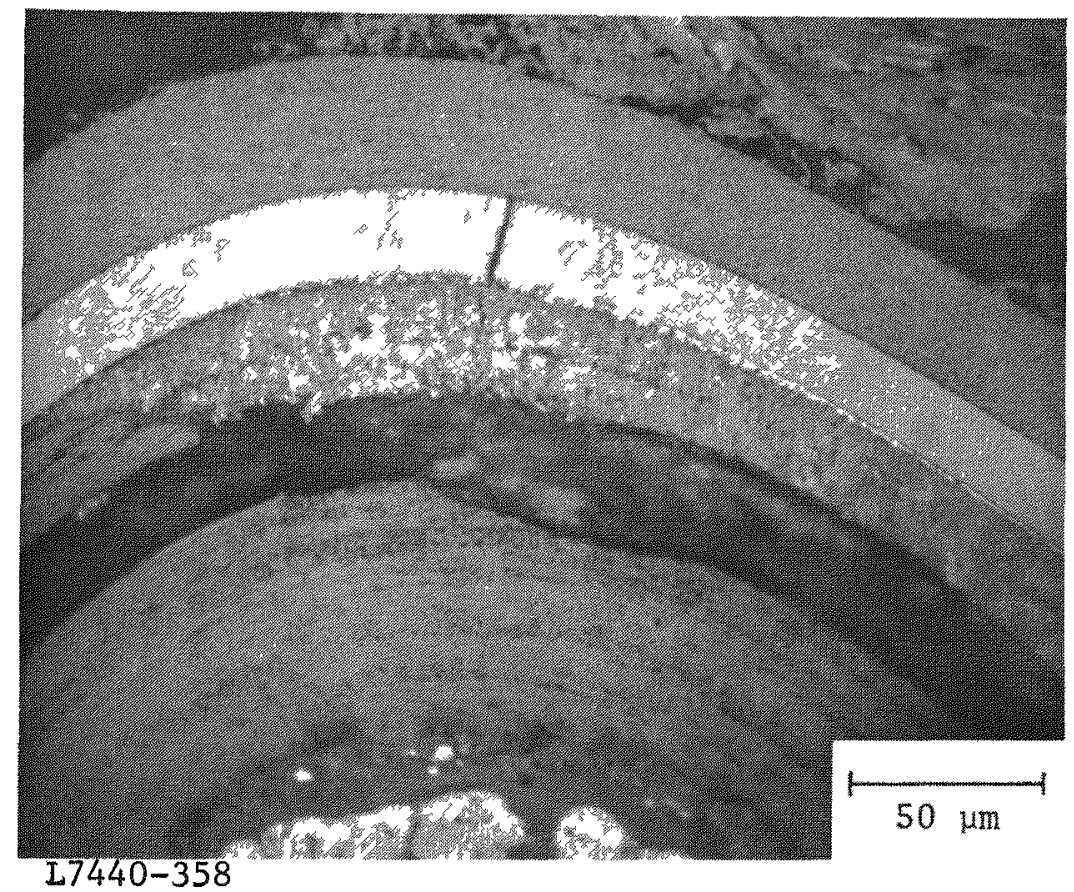

Fig. 4-84. Photomicrographs showing the appearance of a typical cracked SiC coating in a TRISO coated particle. A significant fraction of the cracked SiC coatings observed was an artifact of sample preparation. 


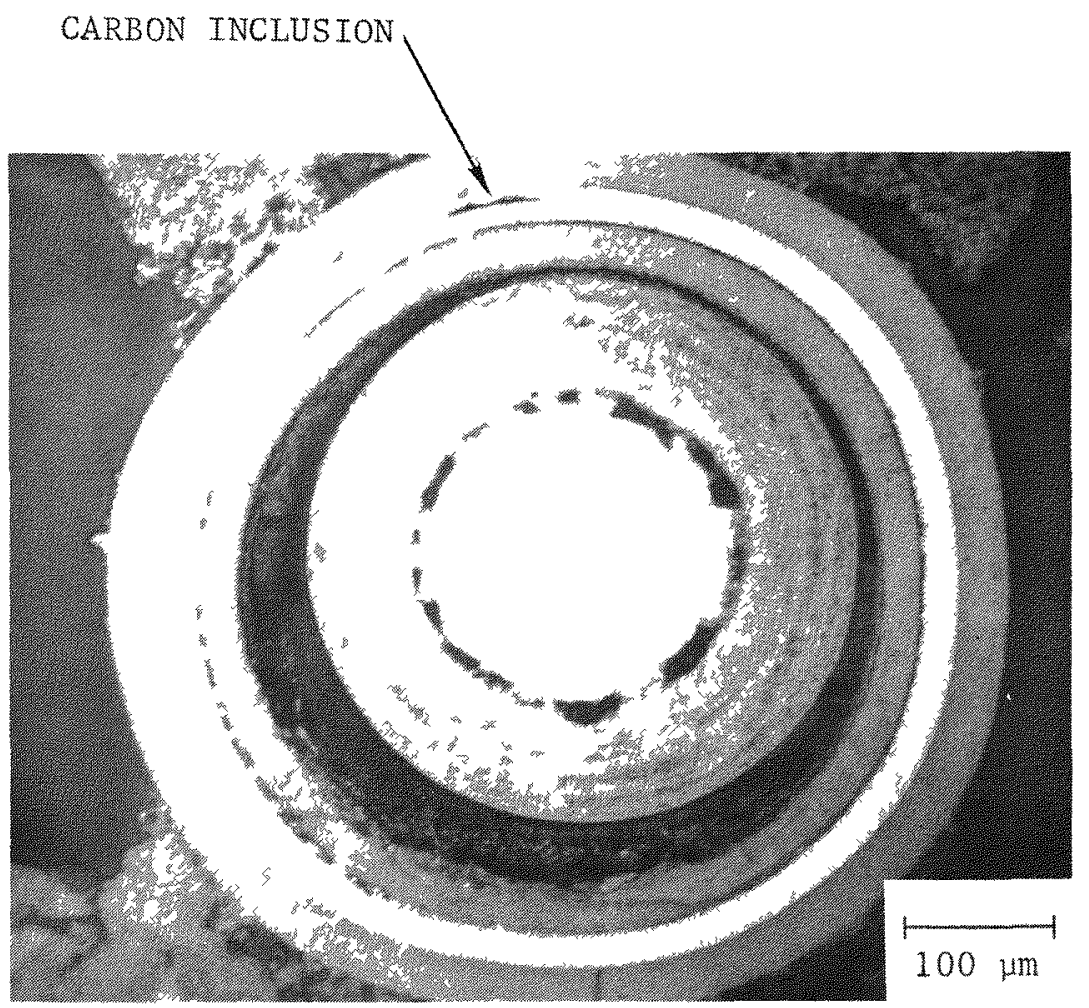

$27440-385$

Fig. 4-85. Photomicrograph showing a small laminar carbon inclusion in SiC coating of a TRISO UC2 particle irradiated in fuel rod 7161-004-19-7 in capsule P13R (position 5D) 

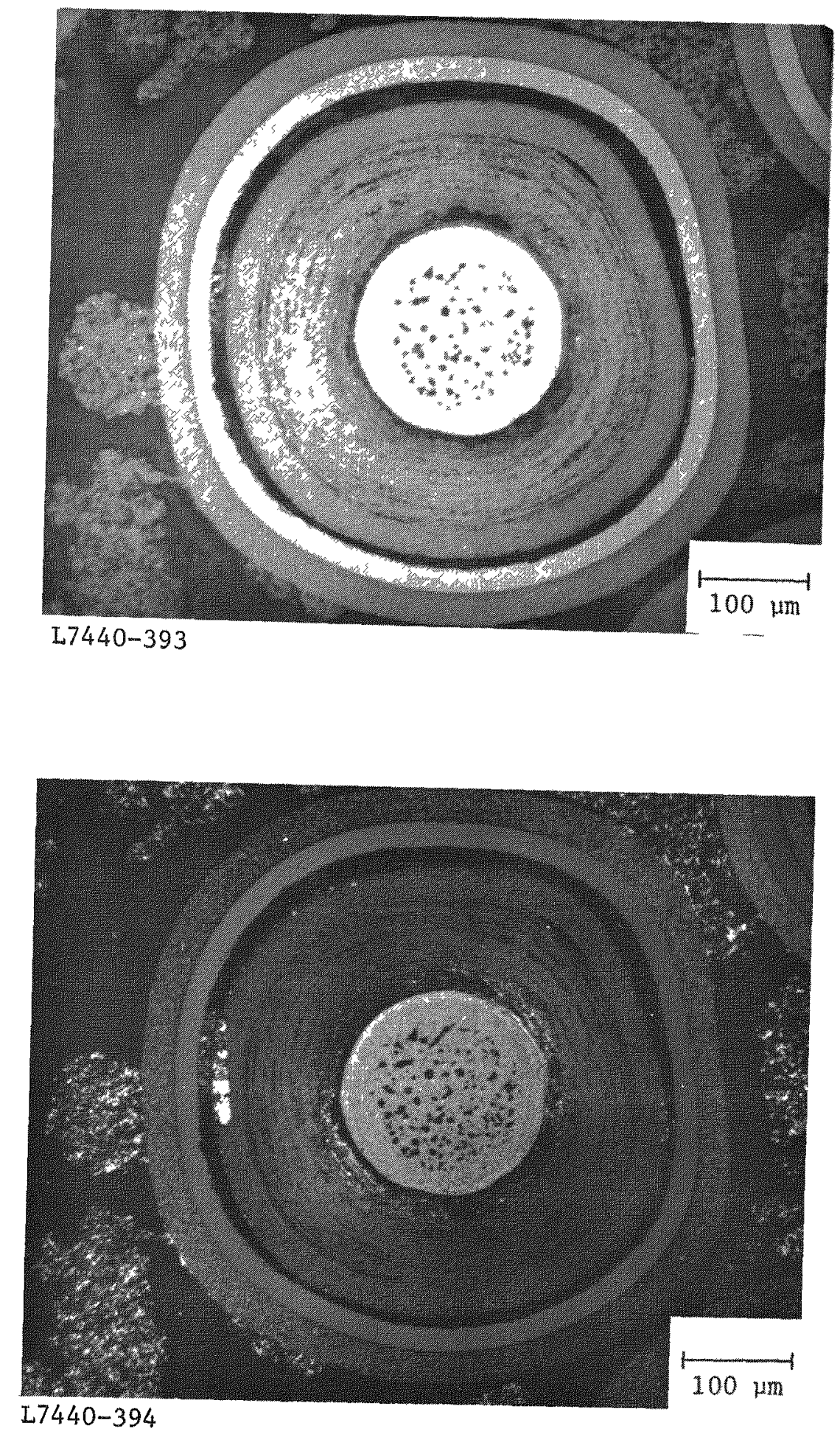

Fig. 4-86. Photomicrographs showing an example of IPyC coating debonding that occurred in a TRISO $\mathrm{UC}_{2}$ particle during irradiation to a fast neutron fluence of $8.2 \times 10^{21} \mathrm{n} / \mathrm{cm}^{2}(\mathrm{E}>0.18 \mathrm{MeV}$ ) HTGR 


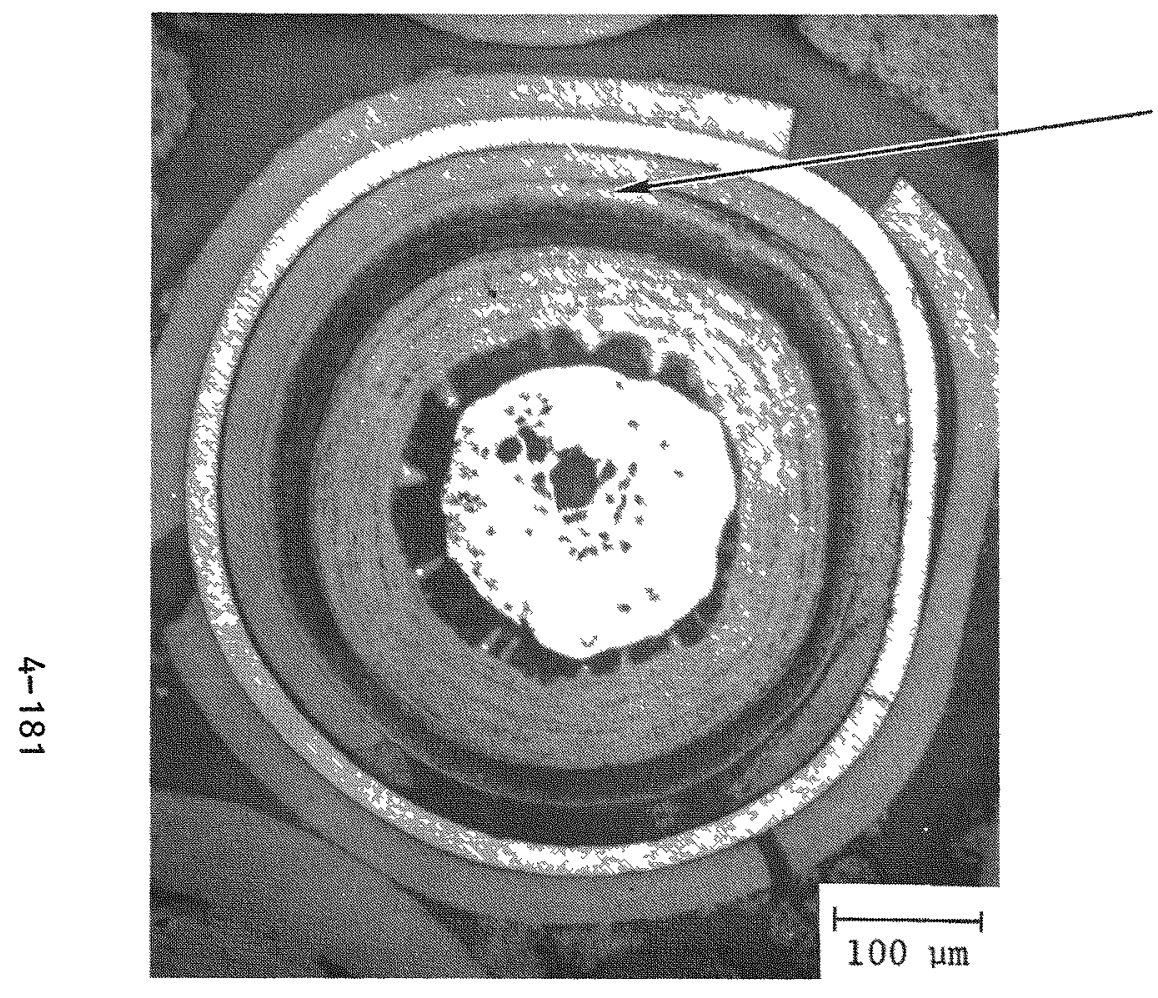

L7440-395

(a)

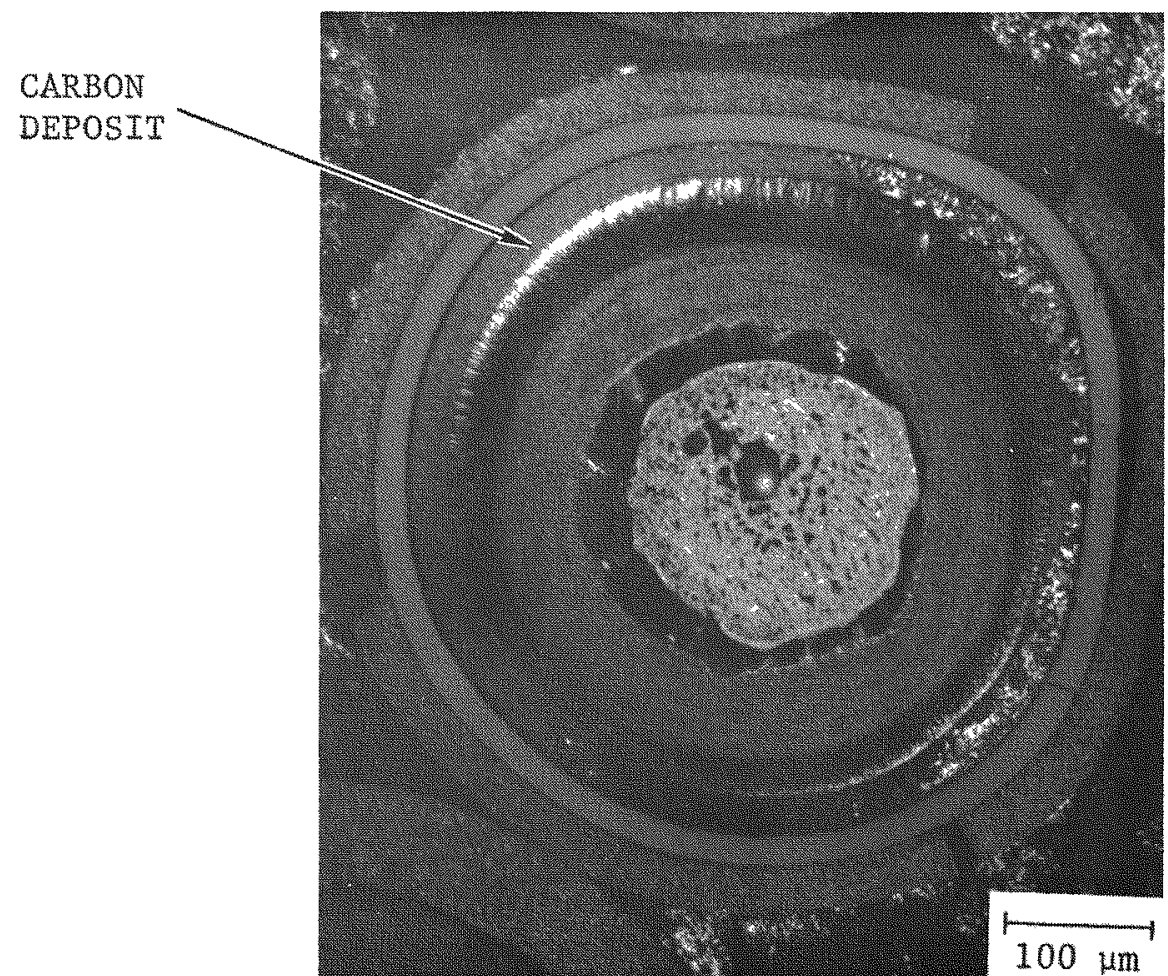

L7440-396

(b)

Fig. 4-87. Photomicrographs showing a carbon deposit in the gap between the buffer and IPyC coating of a TRISO $\mathrm{UC}_{2}$ particle from fuel rod 7161-004-19-7 irradiated in capsule P13R (position 5D) at $1225^{\circ} \mathrm{C}$. (a) Bright field and (b) polarized light. 


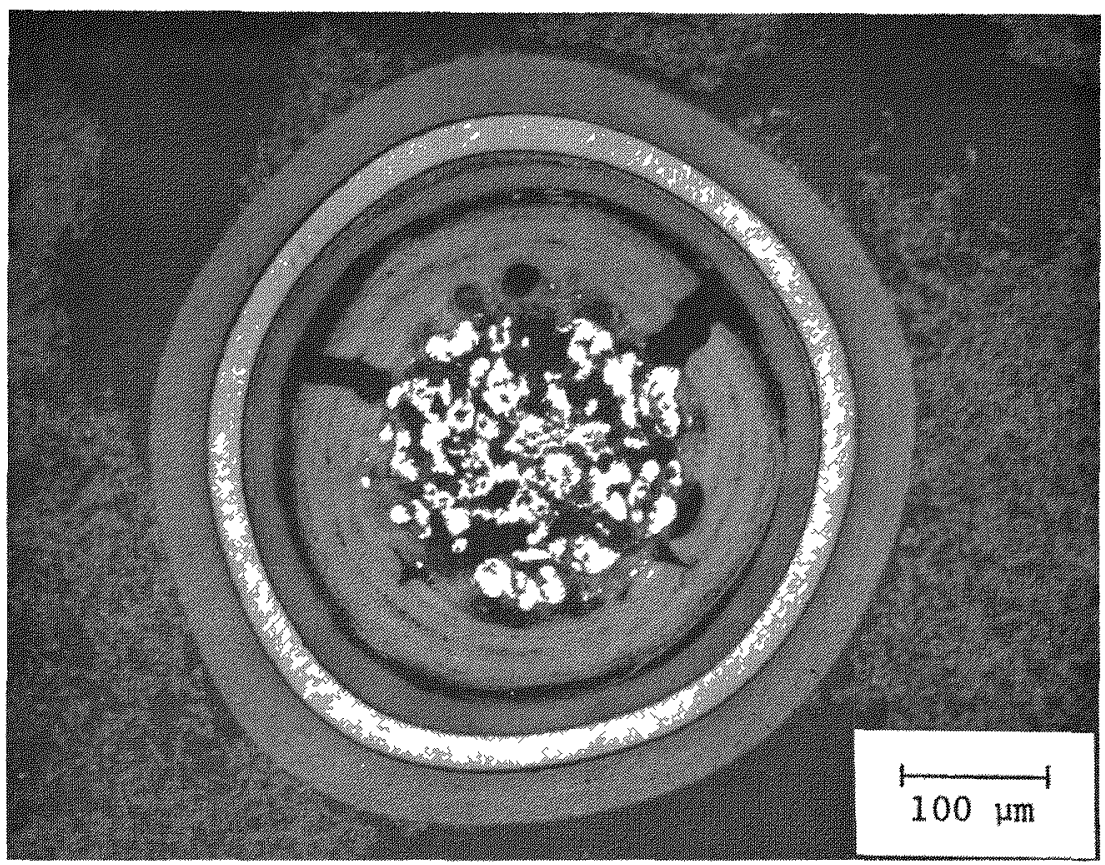

$L 7440-31$

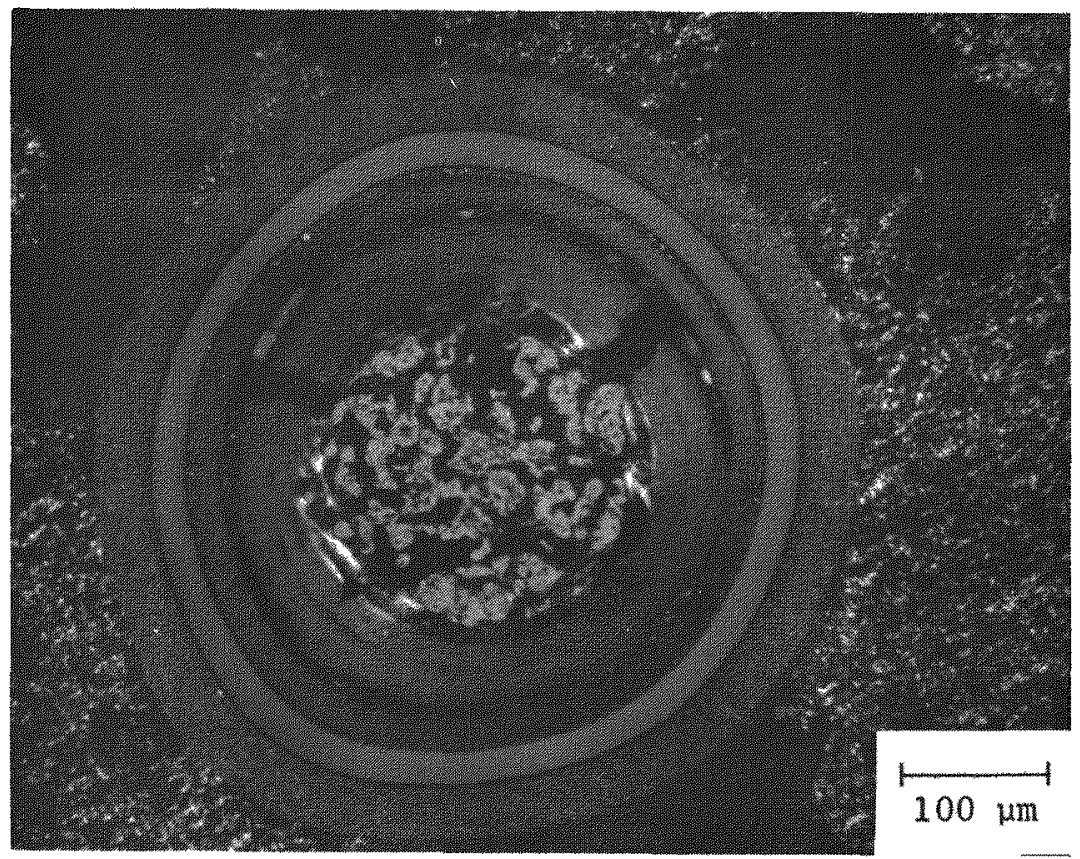

L7440-32

Fig. 4-88. Photomicrographs showing an example of a cracked buffer coating that occurred in a TRISO $\mathrm{UC}_{2}$ particle during irradiation to a fast neutron fluence of $11.8 \times 10^{21} \mathrm{n} / \mathrm{cm}^{2}(\mathrm{E}>0.18 \mathrm{MeV})_{\mathrm{HTGR}}$ 


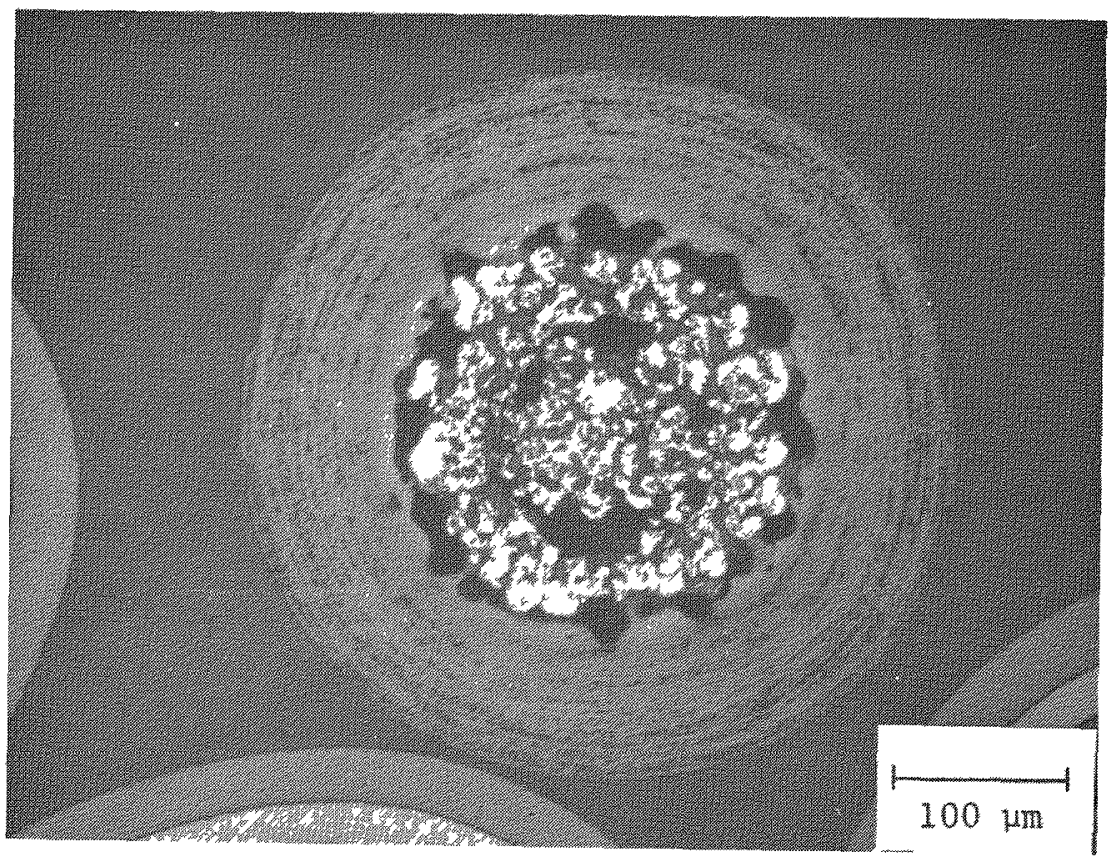

L7440-719

(a)

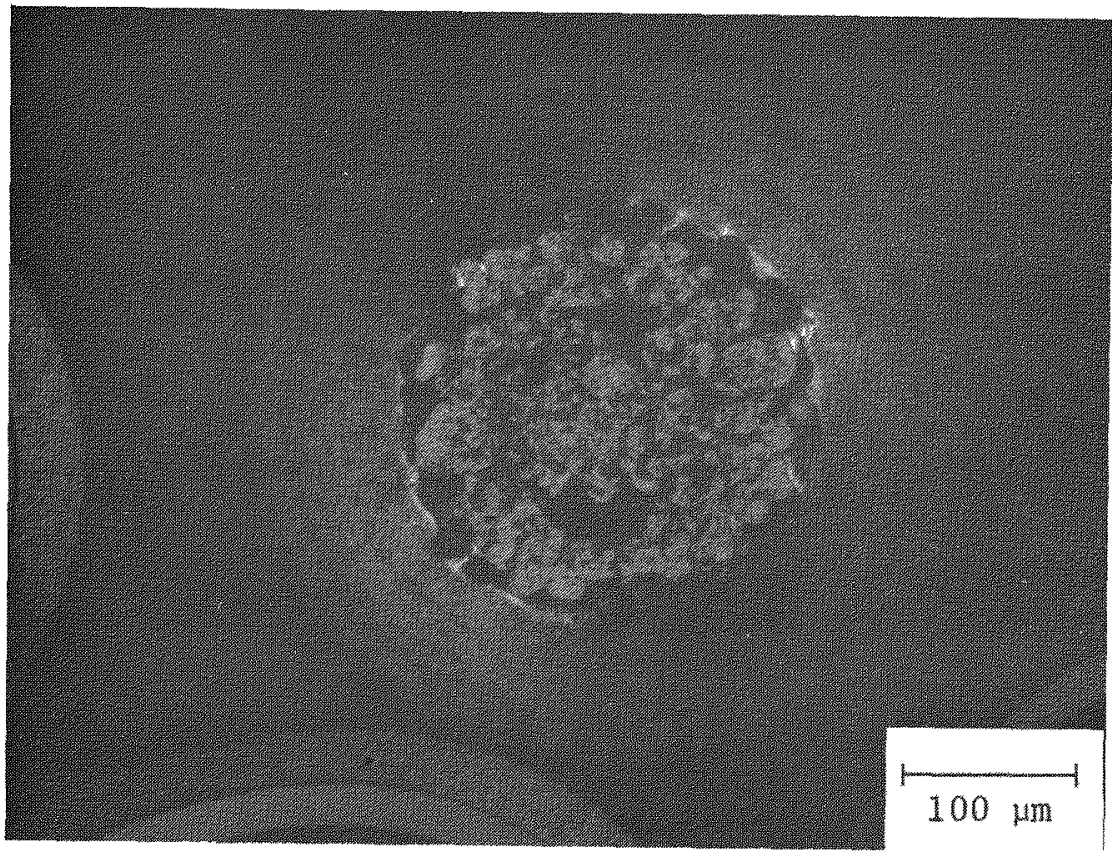

L7440-720

(b)

Fig. 4-89. Photomicrographs showing a TRISO $\mathrm{UC}_{2}$ particle from batch 6151-04-015-2 in which the structural coatings failed during irradiation leaving the buffer coating intact on the fuel kernel. (a) Bright field and (b) polarized light. 


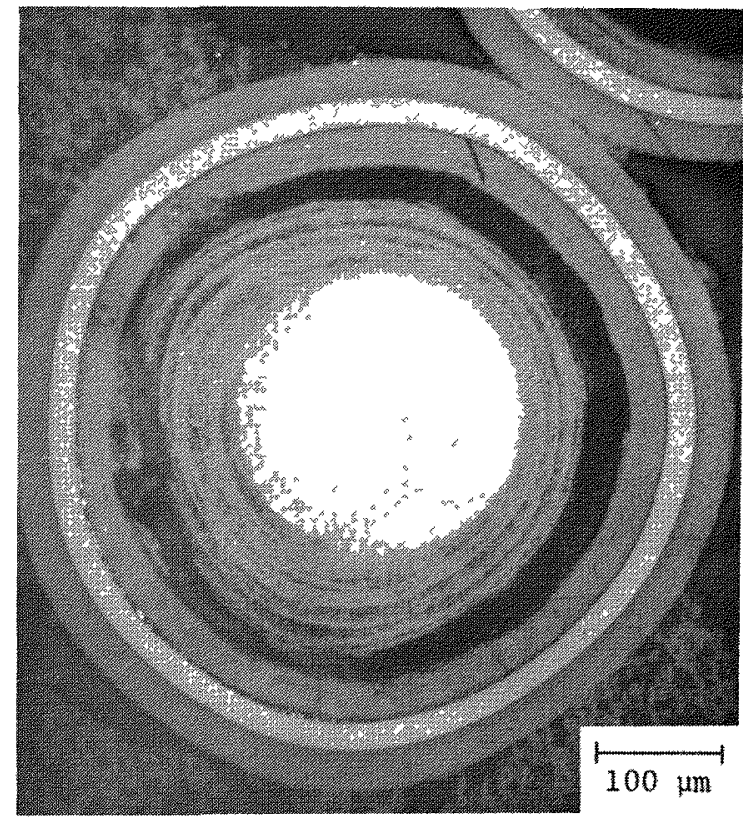

L7440-444

(a)

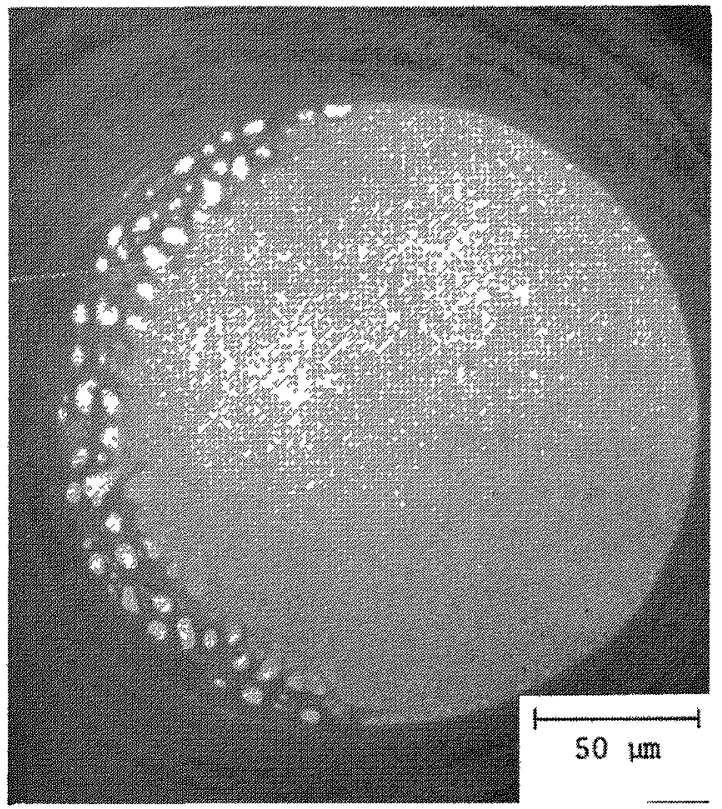

$27440-446$

(c)

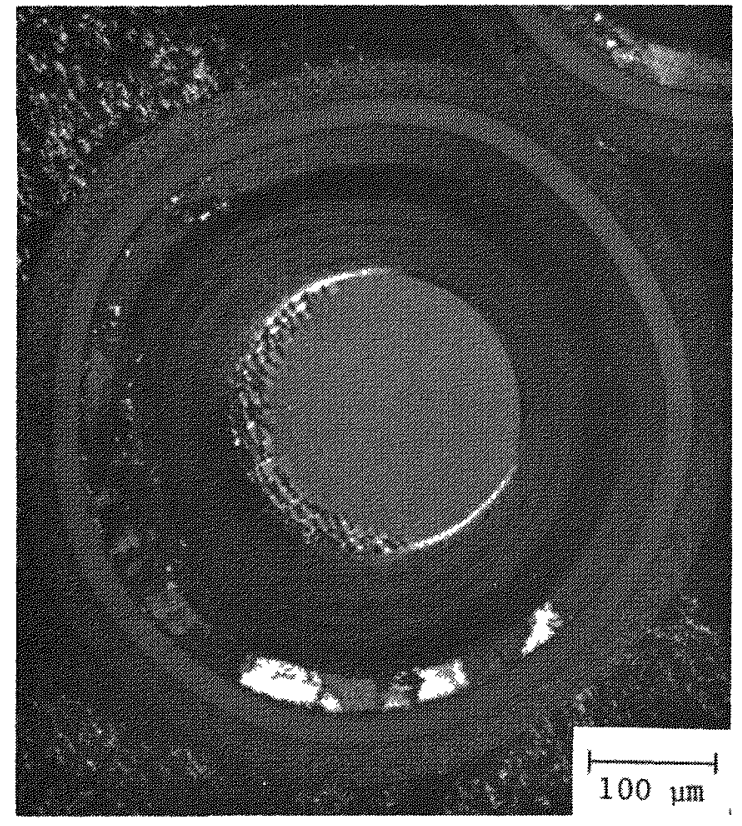

L7440-445

(b)

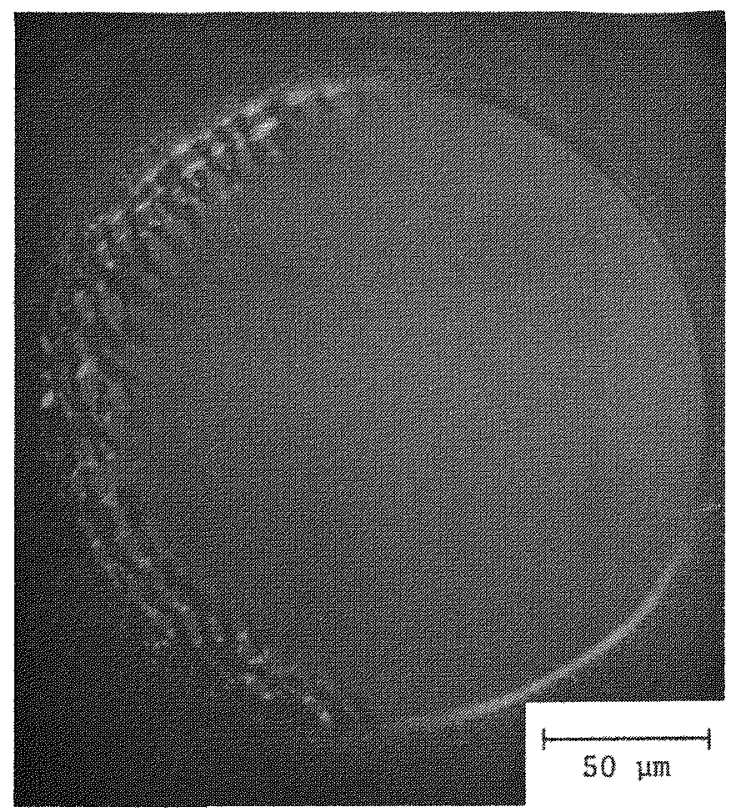

$27440-447$

Fig. 4-90. Photomicrographs showing an example of $\mathrm{UC}_{2}$ fuel kernel migration that was observed in fuel rod 7161-004-18-5 irradiated in capsule $\mathrm{P} 13 \mathrm{~S}$ (position $5 \mathrm{C}$ ) at $1335^{\circ} \mathrm{C}$. The kernel microstructure appeared homogeneous except for small flakes of light-colored material dispersed evenly throughout. (a,c) Bright field and $(b, d)$ polarized light. 


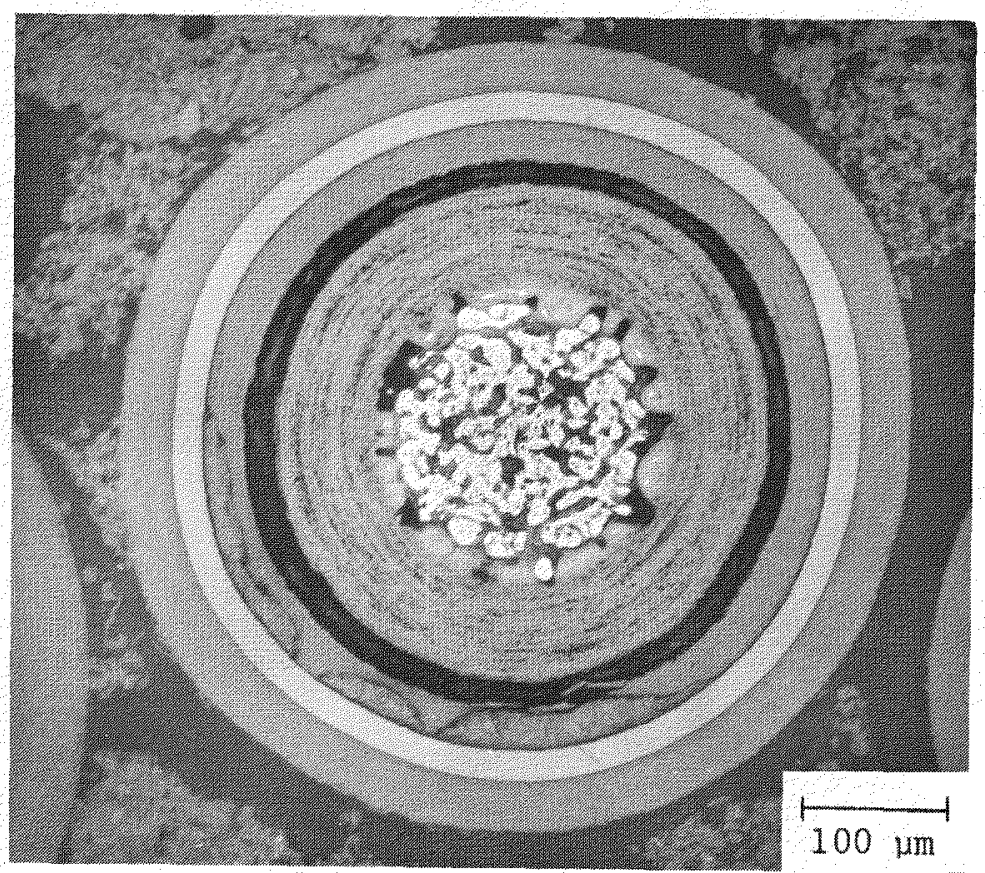

L7440-291

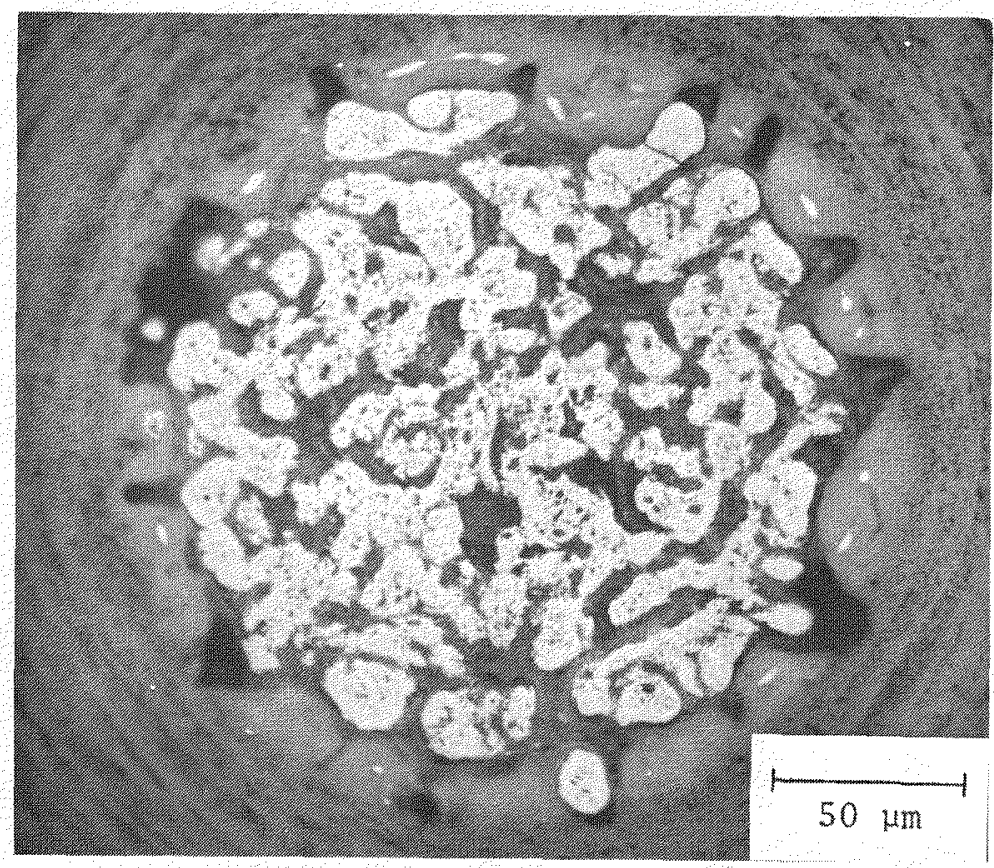

L7440-293

Fig. 4-91. Photomicrographs showing the microstructure of a $\mathrm{UC}_{2}$ fuel kernel irradiated in fuel rod $7161-004-07-5$ at $1055^{\circ} \mathrm{C}$. The kernel apparently segregated into a fuel phase and regions of rejected carbon. 


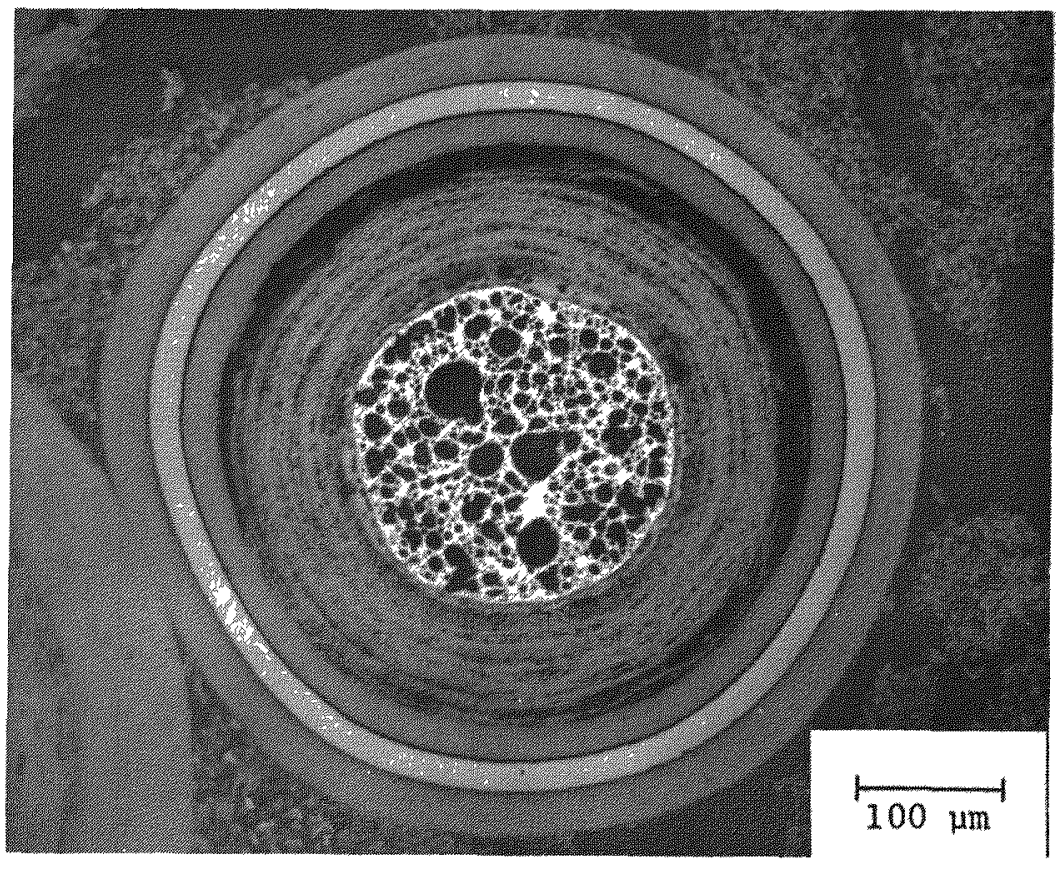

L7440-147

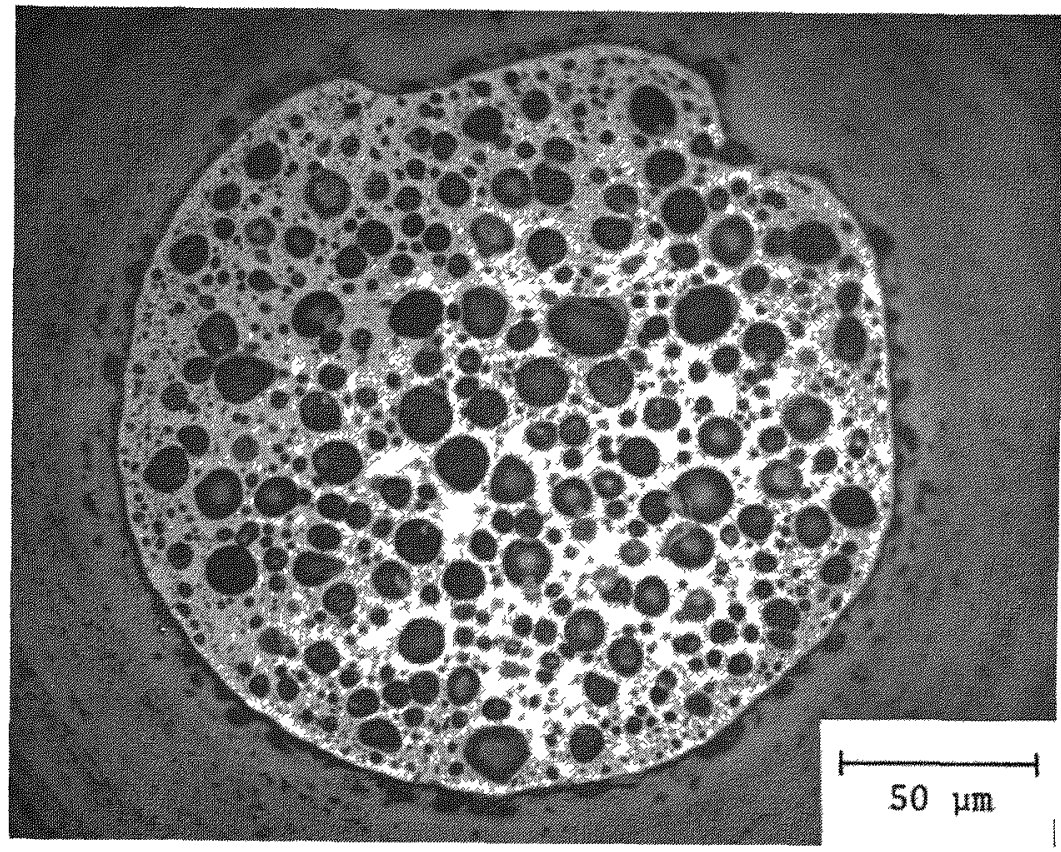

L7440-158

Fig. 4-92. Photomicrographs showing the microstructure of a $\mathrm{UC}_{2}$ fuel kernel irradiated in fuel rod $7161-004-23-5$ at $1005^{\circ} \mathrm{C}$. The kernel was relatively spherical and contained large fission gas bubbles. 


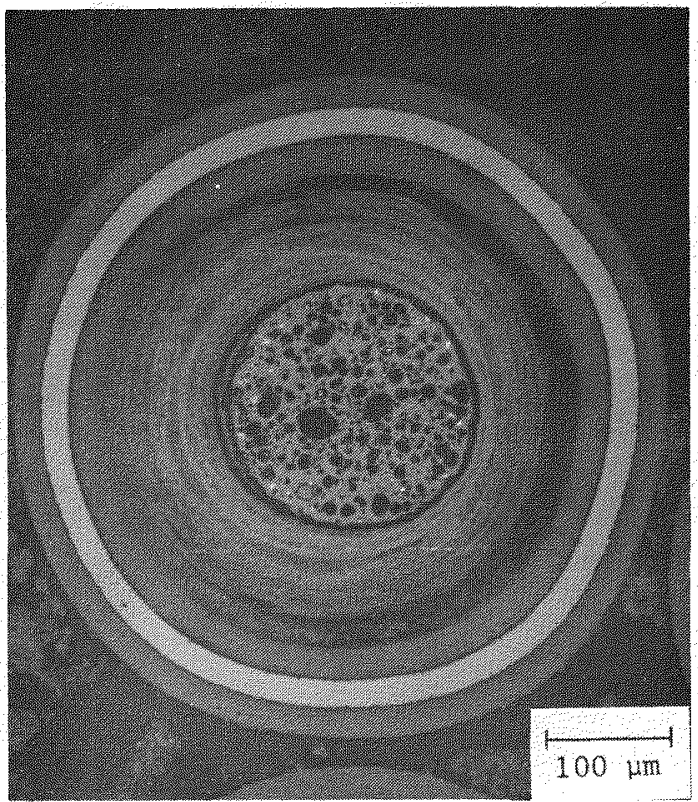

L7440-1063

(a)

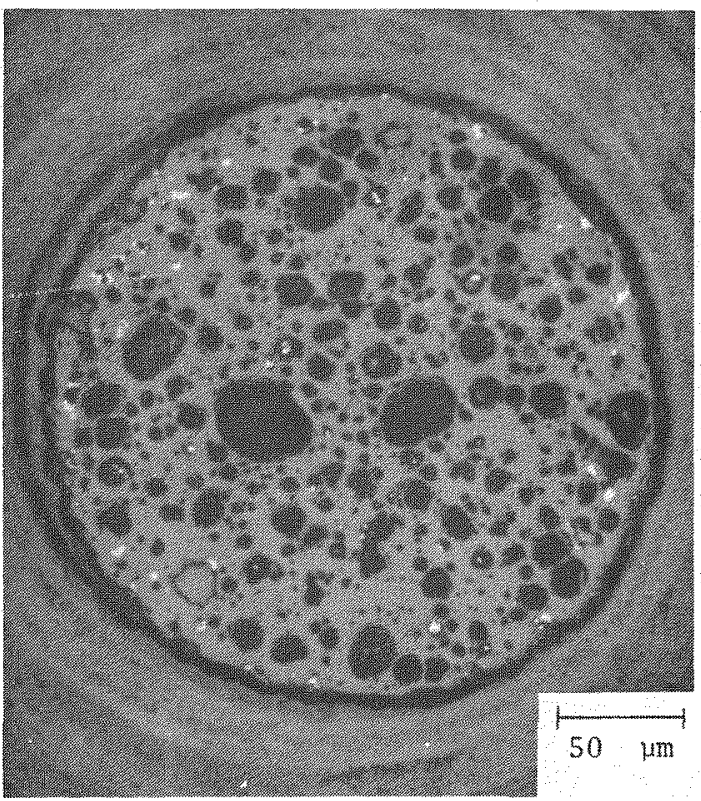

L7440-1073 (c)

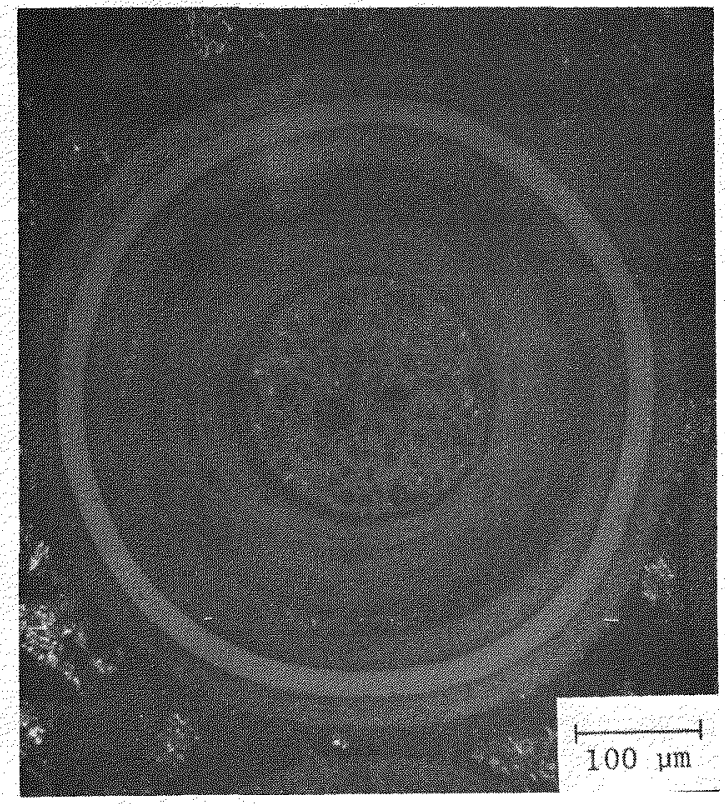

L7440-1064

(b)

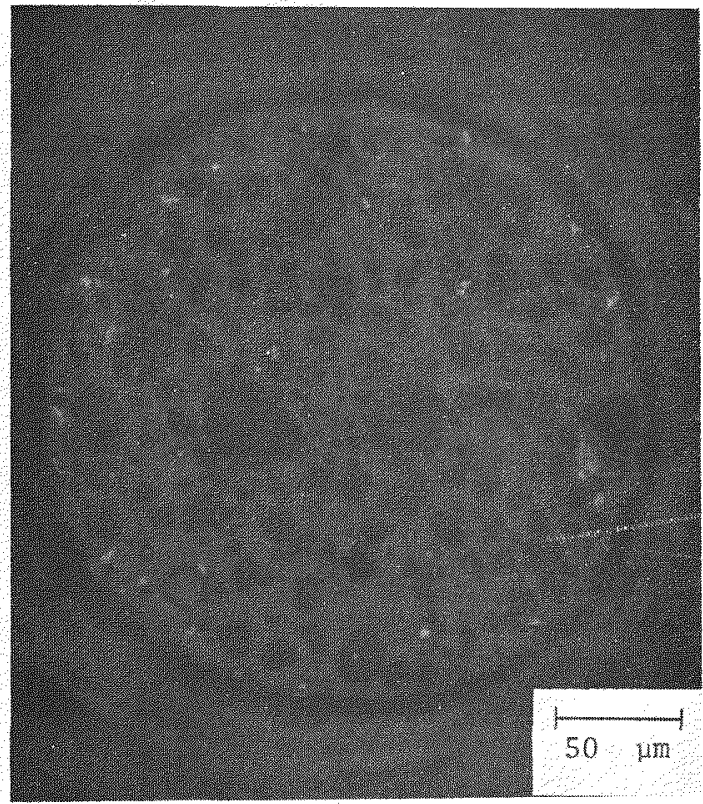

L7440-1074

(d)

Fig. 4-93. Photomicrographs showing an example of (1Th, $1 \mathrm{U}) \mathrm{O}_{2}$ kernel migration that was observed in fuel rod 7161-004-16-6 irradiated in capsule $\mathrm{P} 13 \mathrm{~S}$ (position $5 \mathrm{~A}$ ) at $1375^{\circ} \mathrm{C}$. The kernel was relatively spherical and contained large fission gas bubbles. $(a, c)$ Bright field and $(b, d)$ polarized light. 


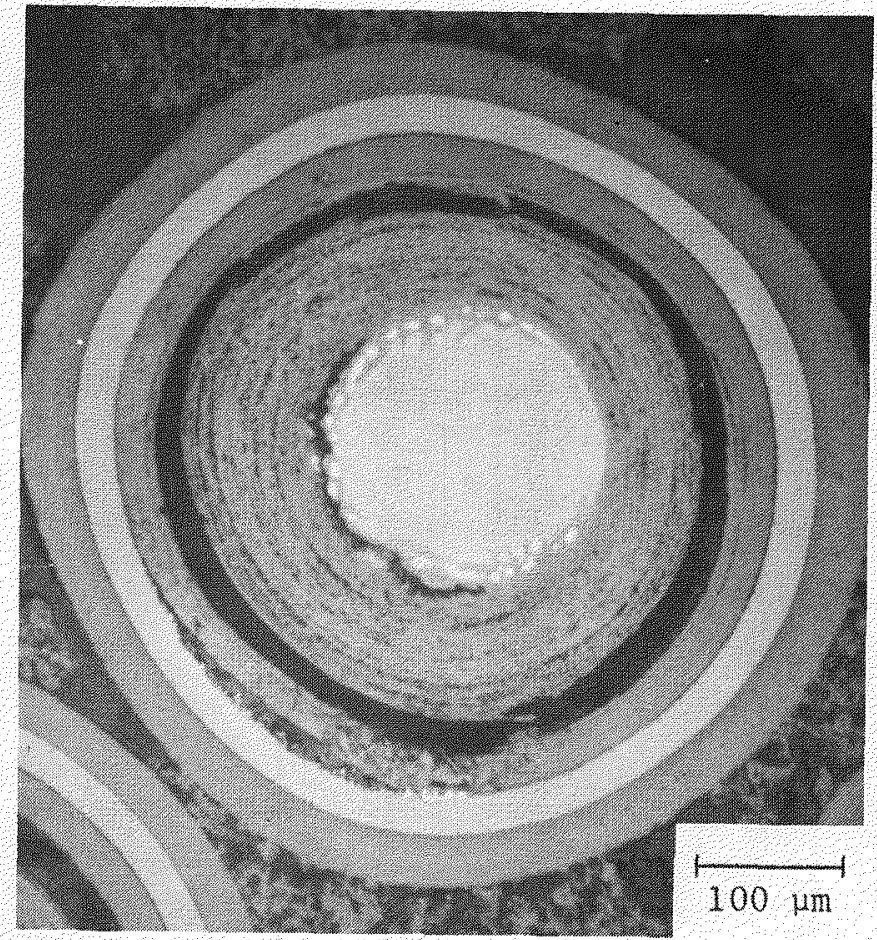

L7440-416 (a)

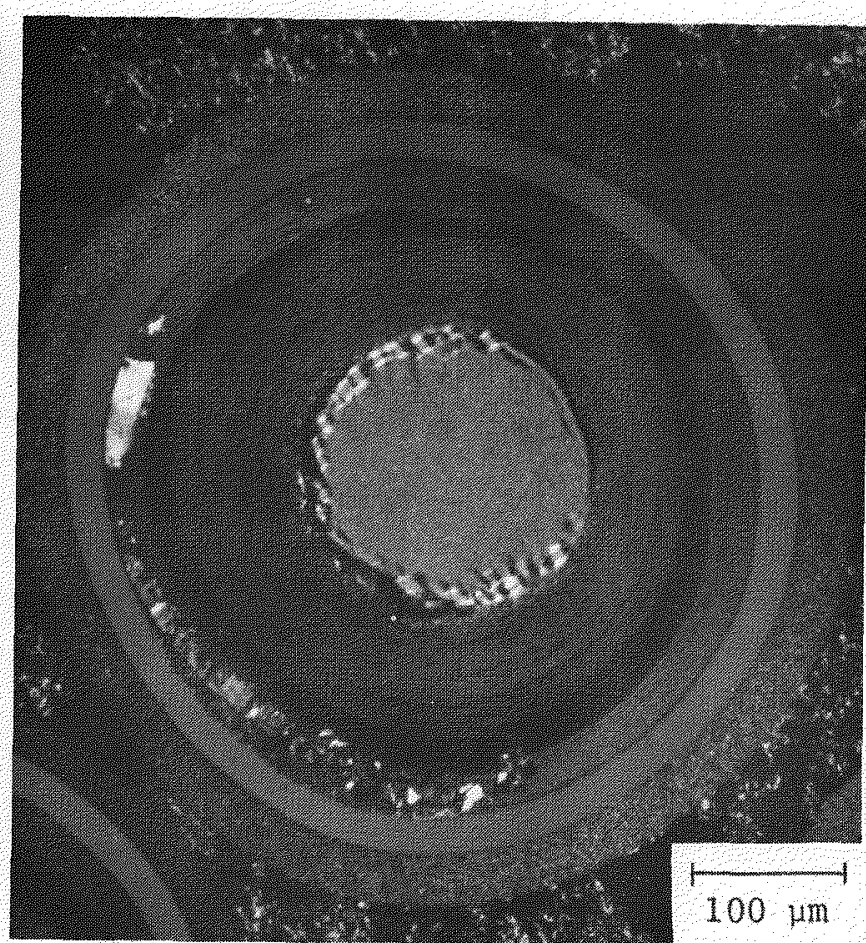

(b)
L7440-417

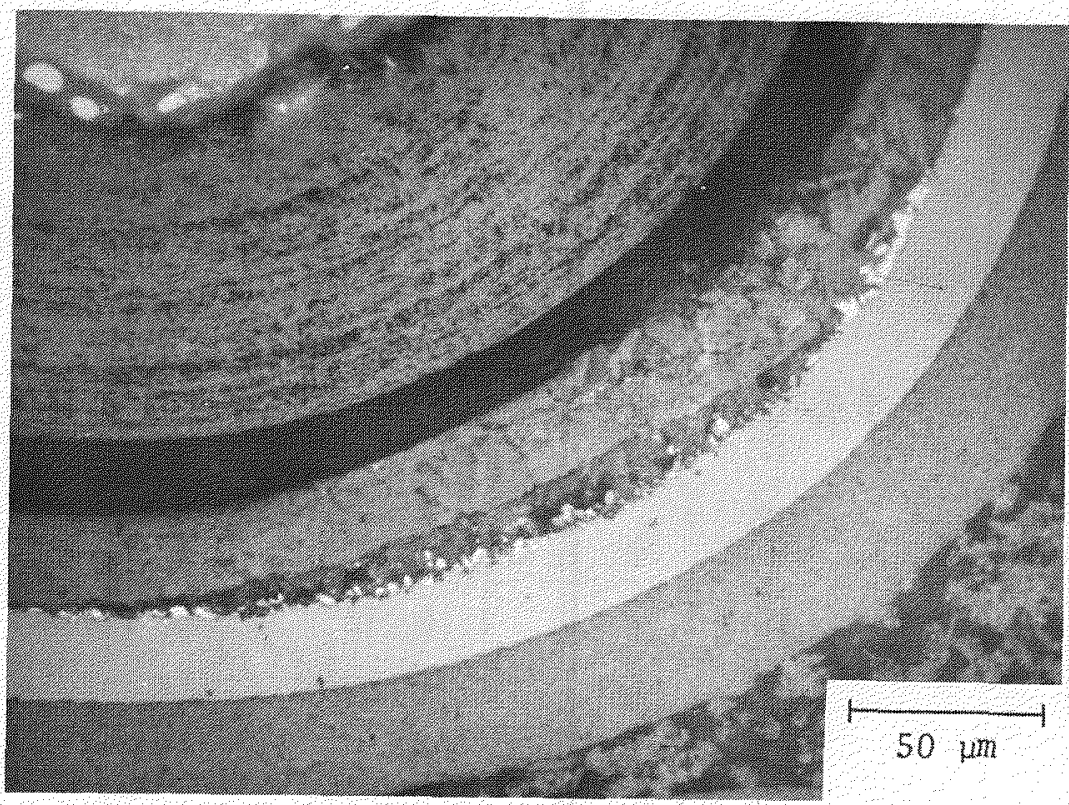

L7440-418

(c)

Fig. 4-94. Photomicrographs showing metallic fission product attack of the SiC coating in a TRISO $\mathrm{UC}_{2}$ particle irradiated in fuel rod $7161-004-18-5$ at an average centerline temperature of $1335^{\circ} \mathrm{C}$. (a,c) Bright field and (b) polarized light. 


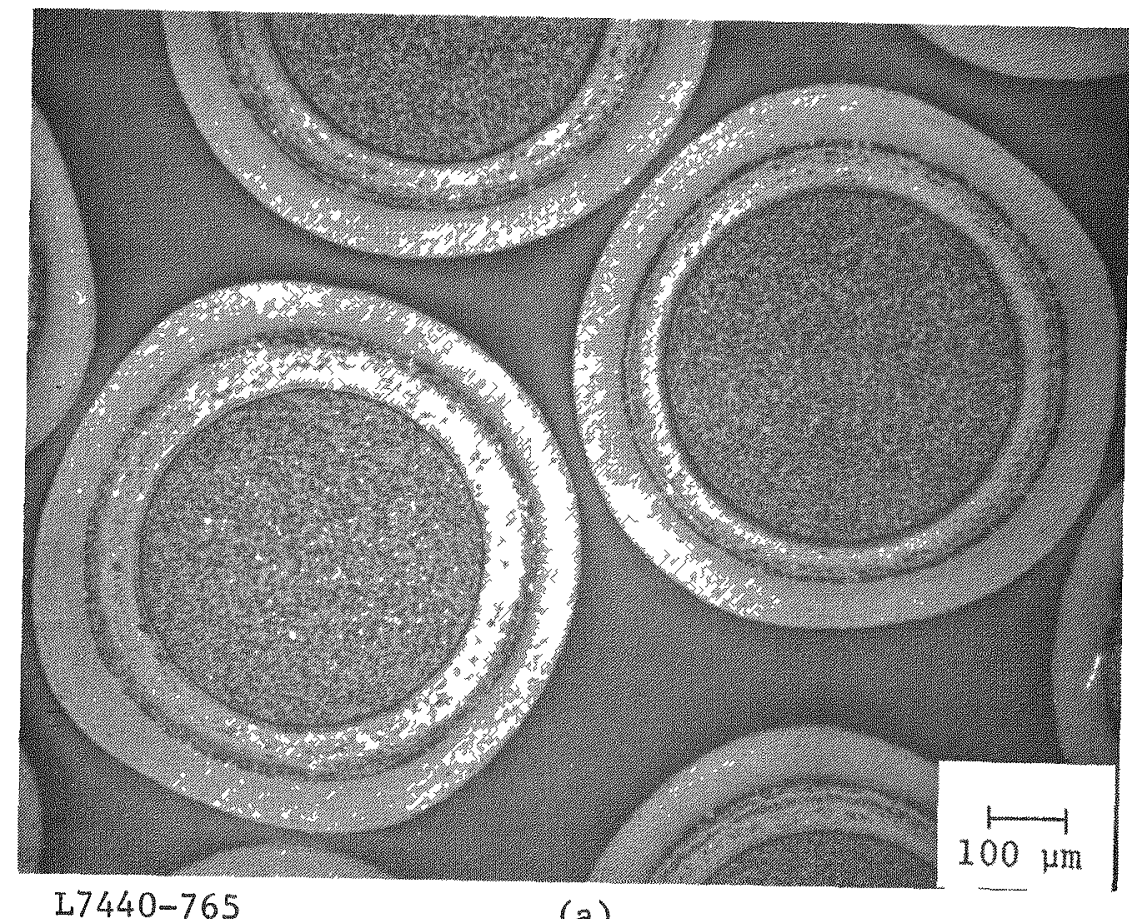

(a)

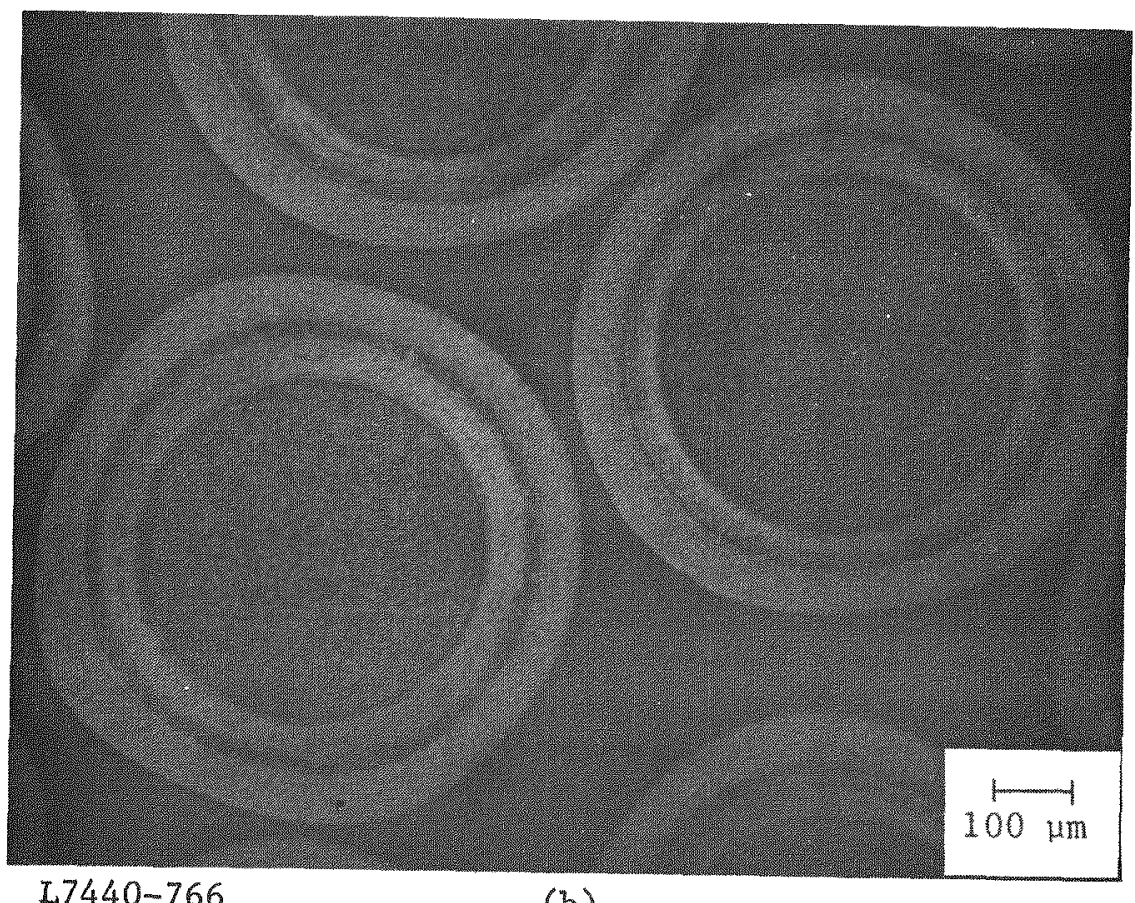

L7440-766

(b)

Fig. 4-95. Photomicrographs of BISO $\mathrm{ThO}_{2}$ particles (4252-06-010-1) with an OPyC coating deposited from propylene. The particle batch was irradiated in capsule P13S (position 3-1) to a fast neutron fluence of $11.8 \times 10^{21} \mathrm{n} / \mathrm{cm}^{2}$ ( $\mathrm{E}>0.18 \mathrm{MeV}$ ) HTGR and burnup of $4.3 \%$ FIMA at $925^{\circ} \mathrm{C}$. (a) Bright field and (b) polarized light. 


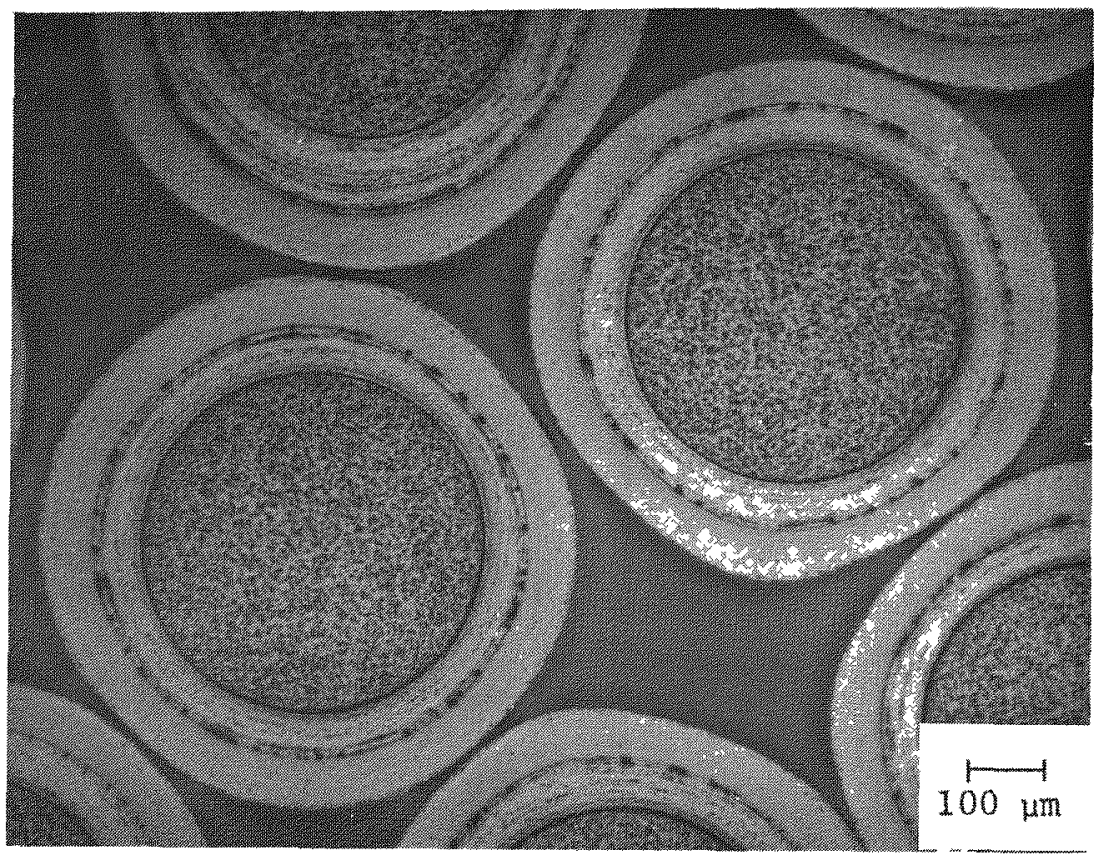

L7440-769

(a)

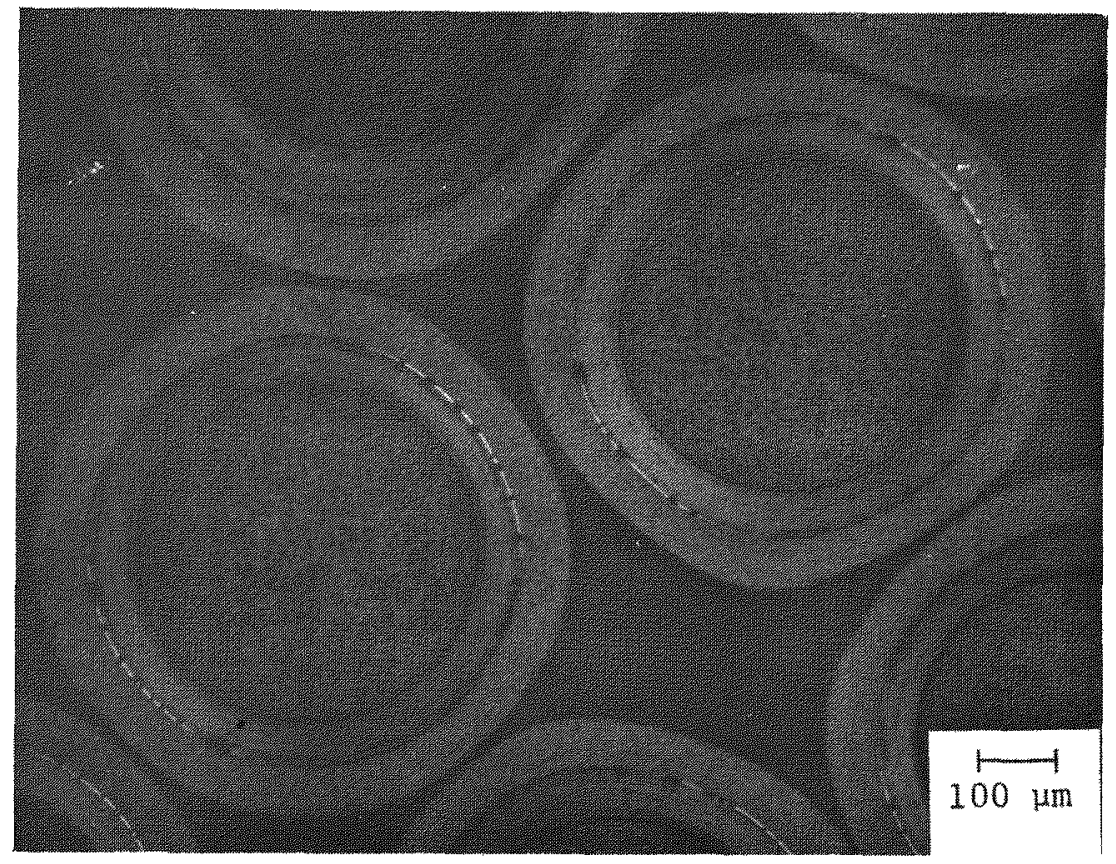

$27440-770$

(b)

Fig. 4-96. Photomicrographs of BISO $\mathrm{ThO}_{2}$ particles (6542-23-025-1) with an OPyC coating deposited from mixed gas. The particle batch was irradiated in capsule P13S (position 3-7) to a fast neutron fluence of $11.7 \times 10^{21} \mathrm{n} / \mathrm{cm}^{2}$ (E $>0.18 \mathrm{MeV}$ ) HTGR and burnup of $4.2 \%$ FIMA at $955^{\circ} \mathrm{C}$. (a) Bright field and (b) polarized light. $4-190$ 


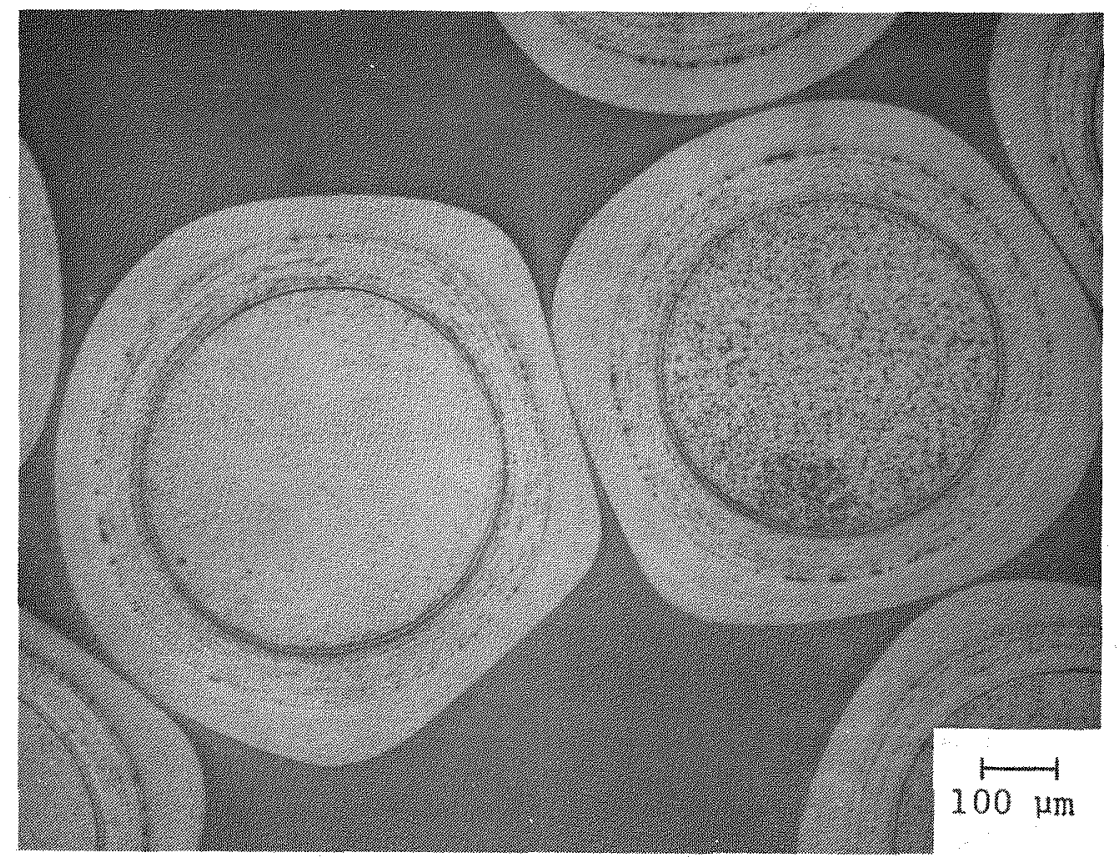

L7440-697

(a)

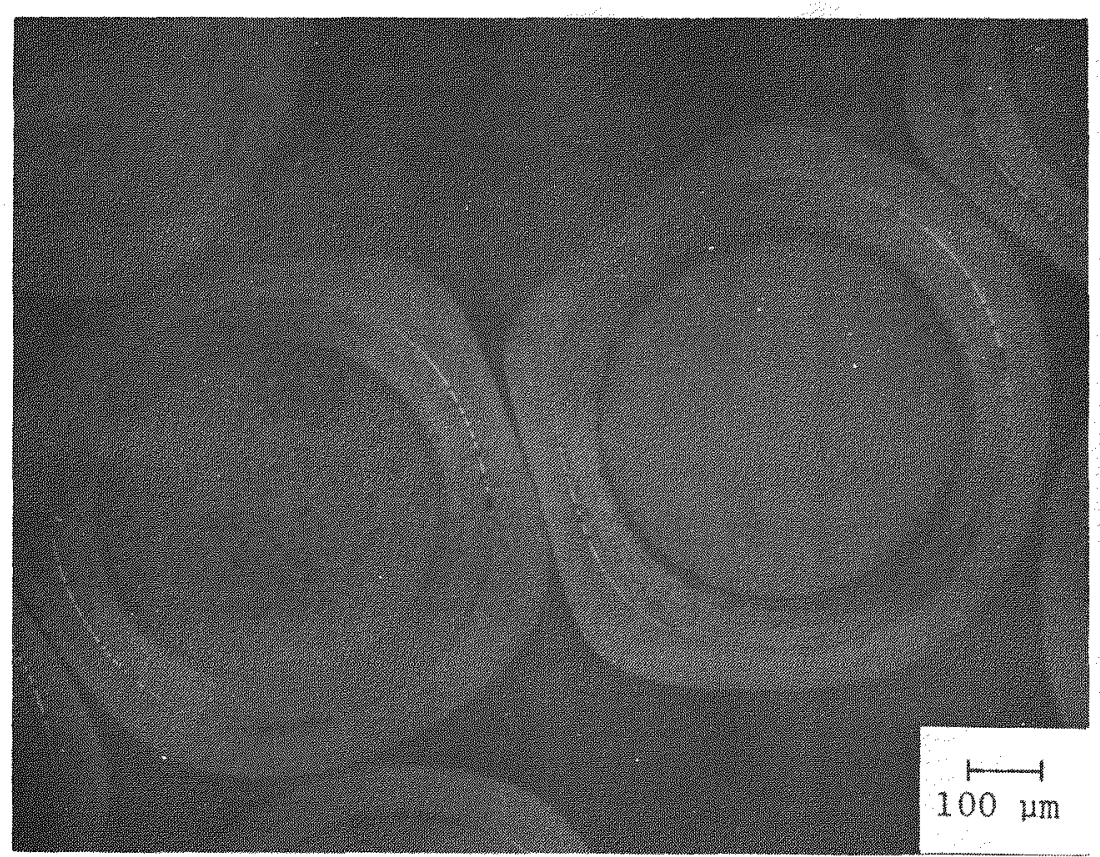

$17440-698$

(b)

Fig. 4-97. Photomicrographs of severely faceted BISO $\mathrm{ThO}_{2}$ particles (6542-20-035-2) after irradiation in capsule $\mathrm{P} 13 \mathrm{R}$ (position $4-8)$ to a fast neutron fluence of $11.1 \times 10^{21} \mathrm{n} / \mathrm{cm}^{2}$ $(\mathrm{E}>0.18 \mathrm{MeV})_{\mathrm{HTGR}}$ and burnup of $4.3 \%$ FIMA at $1000^{\circ} \mathrm{C}$. (a) Bright field and (b) polarized light. 


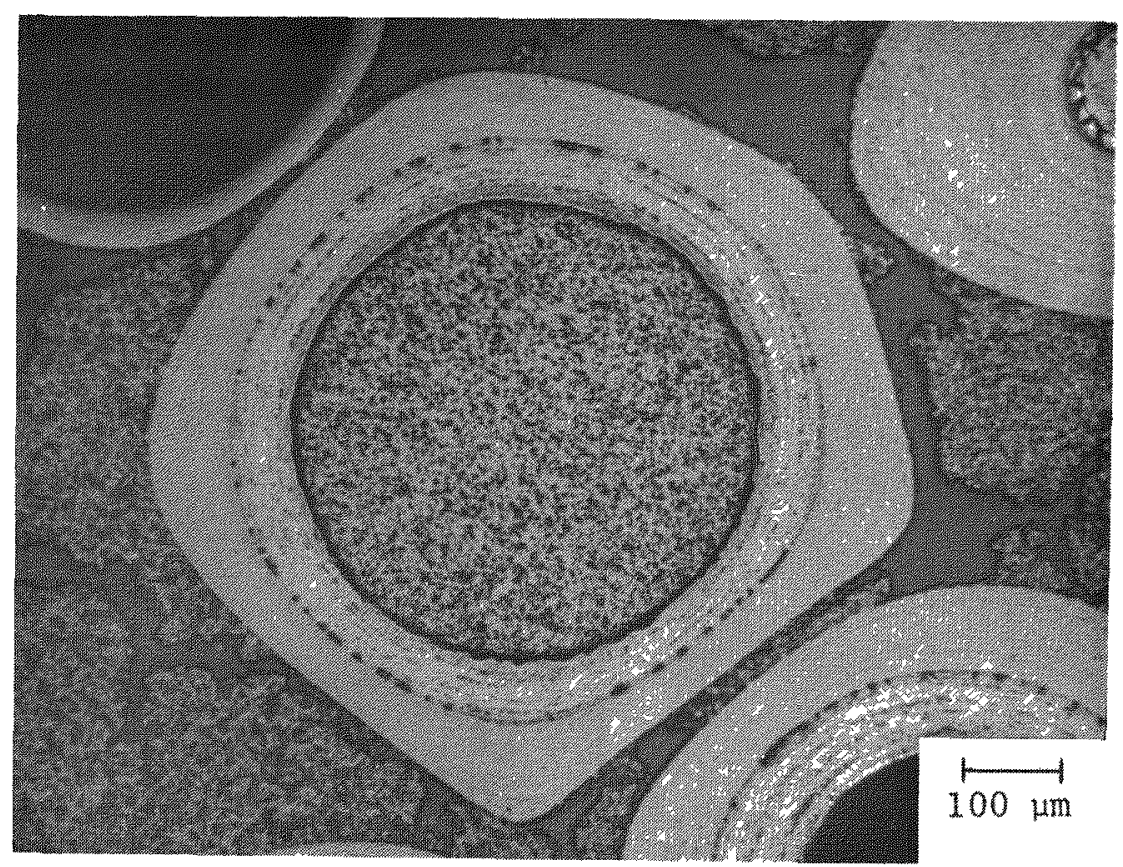

(a)

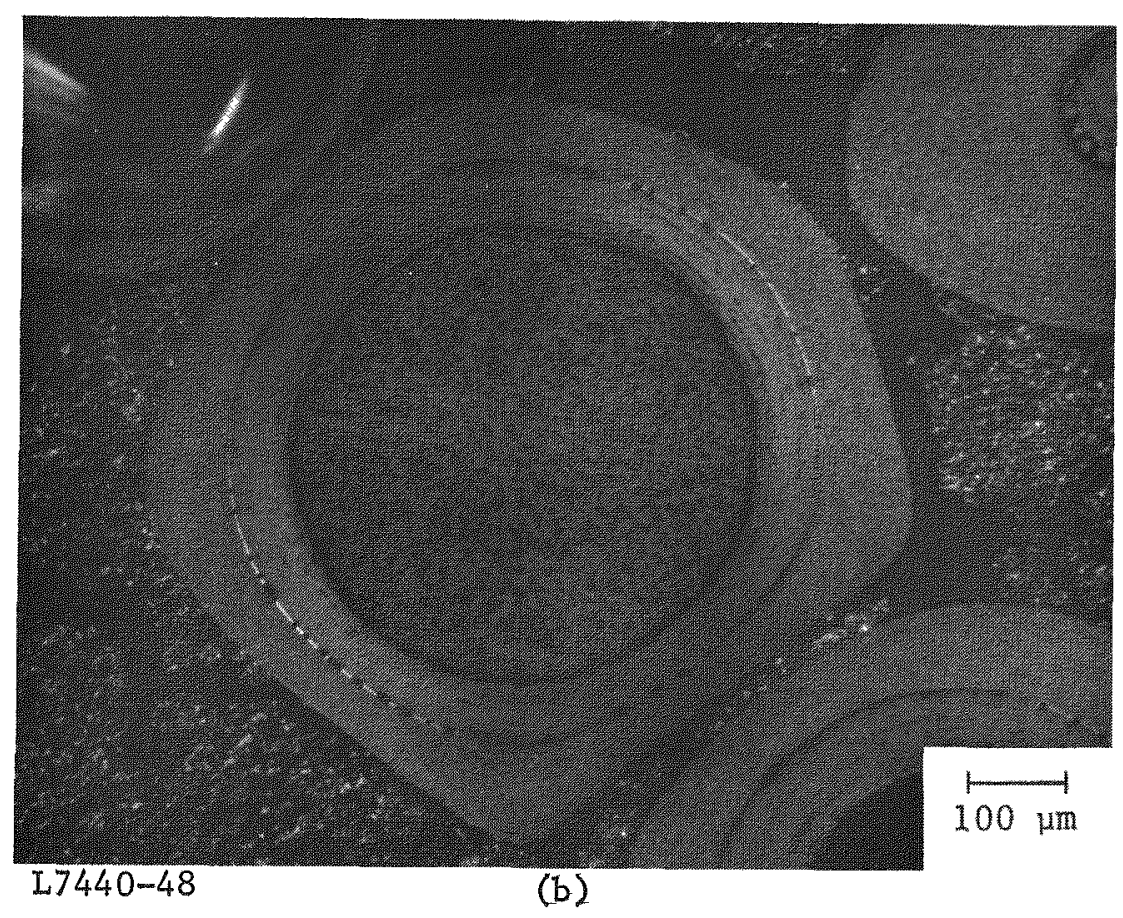

Fig. 4-98. Photomicrographs of a severely faceted $\mathrm{BISO} \mathrm{ThO}_{2}$ particle (6542-20-035) from fuel rod 7161-004-06-5 that survived irradiation in capsule P13R (position 2A) to a fast neutron exposure of $11.8 \times 10^{21} \mathrm{n} / \mathrm{cm}^{2}(\mathrm{E}>0.18 \mathrm{MeV})_{\mathrm{HTGR}}$ and burnup of $5.5 \%$ FTMA at $1050^{\circ} \mathrm{C}$. (a) Bright field and (b) polarized 1ight. 


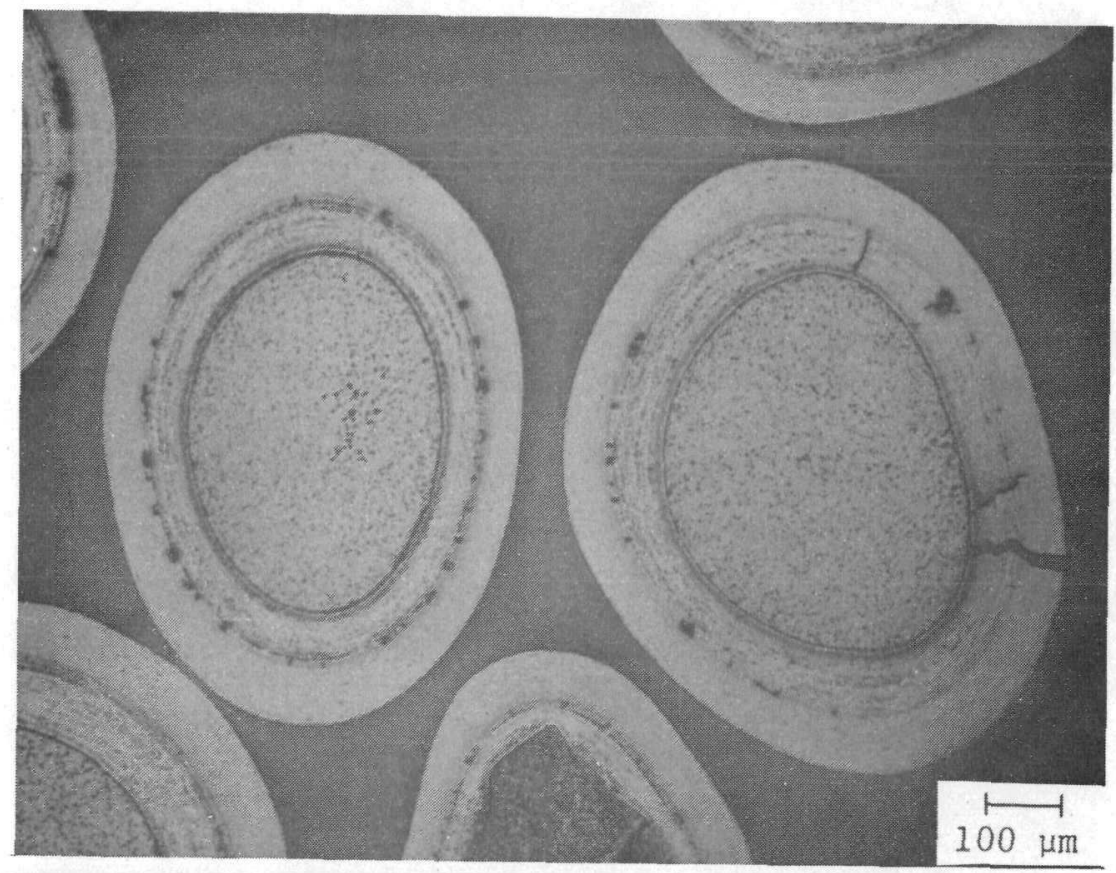

(a)

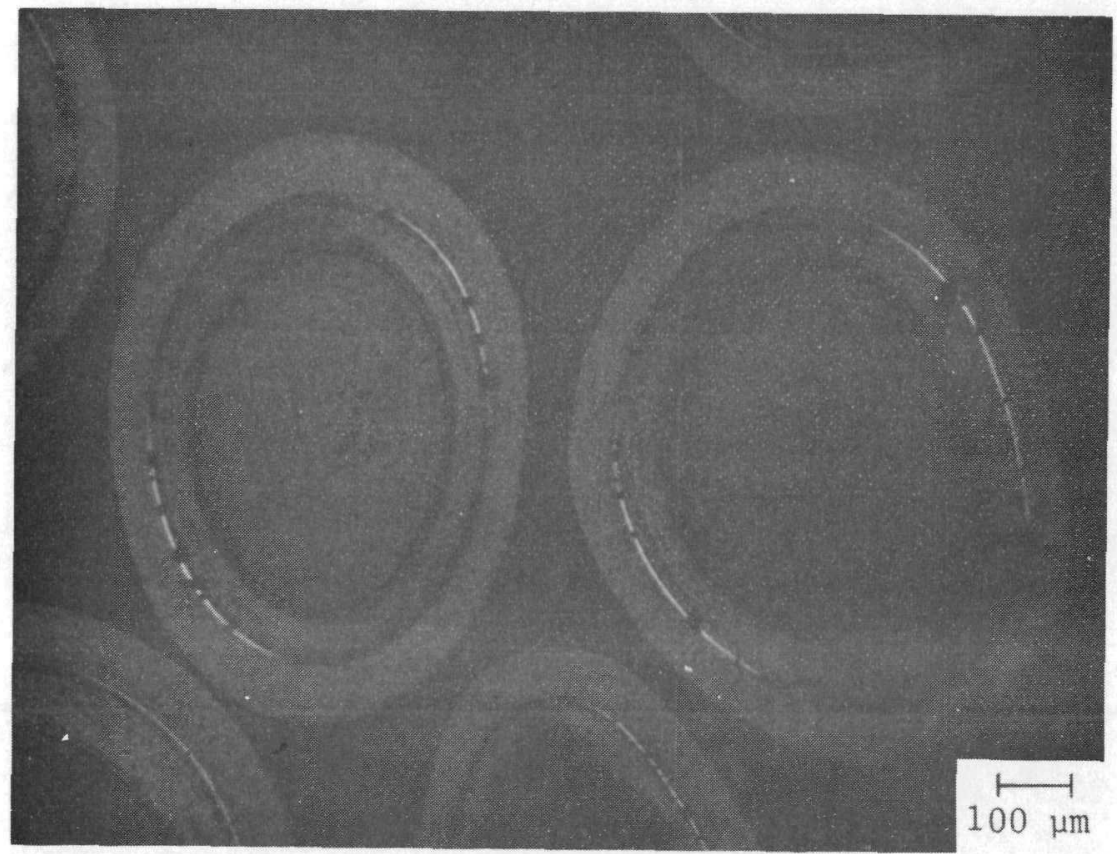

L7440-736

(b)

Fig. 4-99. Photomicrographs of $\mathrm{BISO} \mathrm{ThO}_{2}$ particles with nonround fuel kernels (6542-25-015-1) after irradiation in capsule P13R (position 4-11) to a fast neutron fluence of $10.9 \times 10^{21}$ $\mathrm{n} / \mathrm{cm}^{2}(\mathrm{E}>0.18 \mathrm{MeV})_{\mathrm{HTGR}}$ and burnup of $4.1 \%$ FIMA at $940^{\circ} \mathrm{C}$. (a) Bright field and (b) polarized light. 


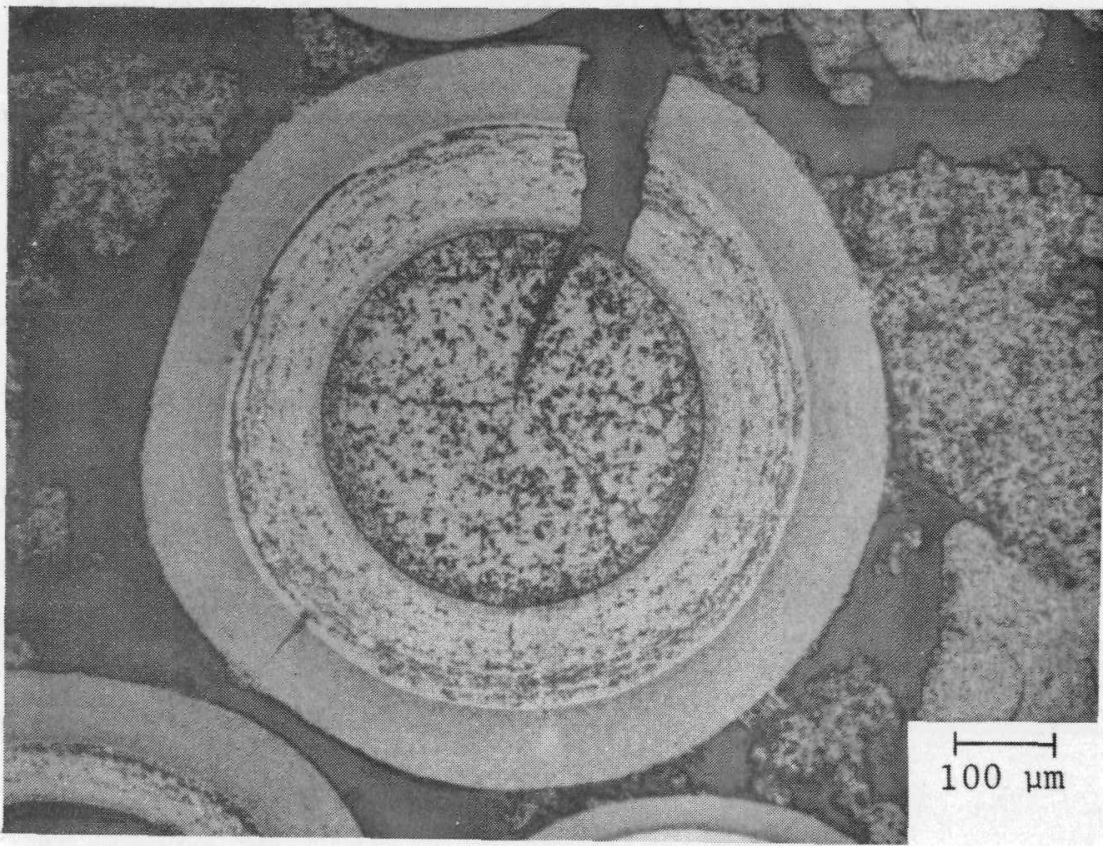

L7440-574

(a).

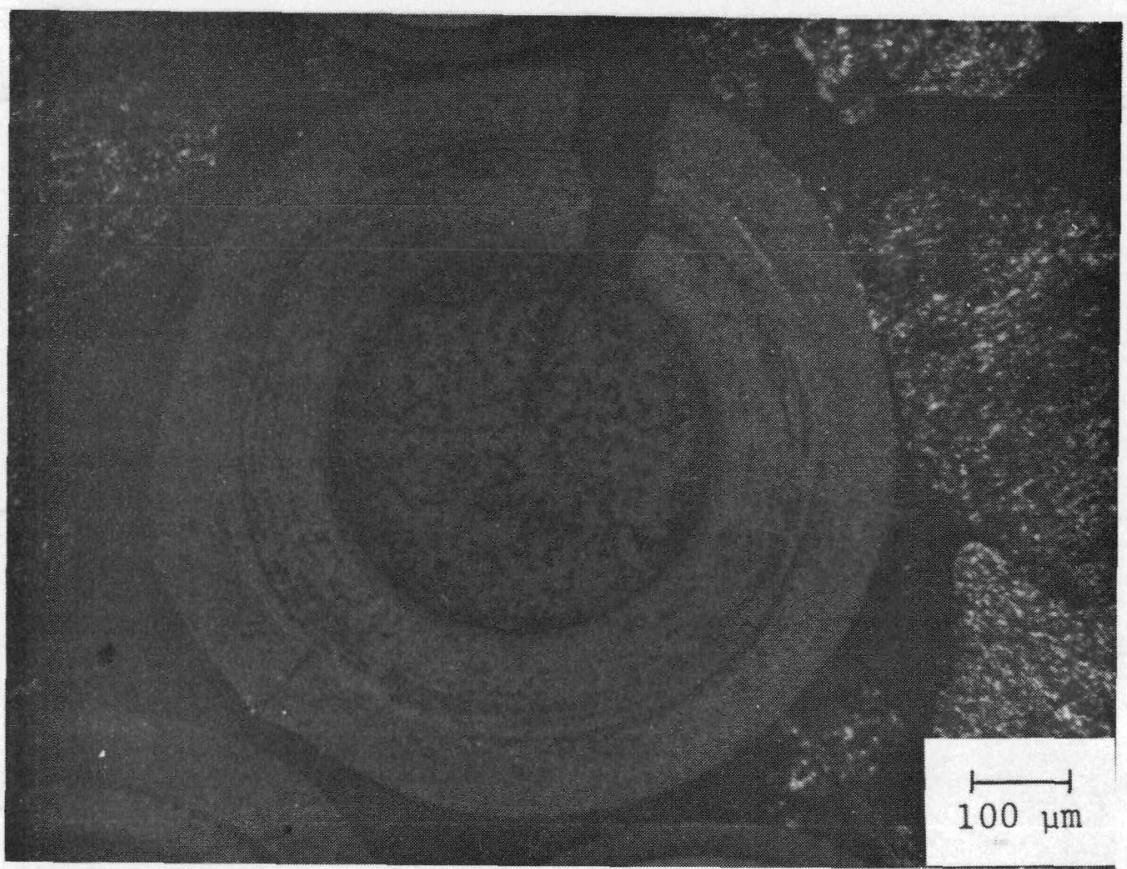

Fig. 4-100. Photomicrographs showing irradiation-induced failure in a BISO $\mathrm{ThO}_{2}$ particle (6542-09-010) with a high-density $\left(1.93 \mathrm{~g} / \mathrm{cm}^{3}\right)$ OPyC coating. The particle was irradiated in fuel rod 7161004-12-5 of capsule P13S (position 2B) to a fast neutron fluence of $11.7 \times 10^{21} \mathrm{n} / \mathrm{cm}^{2}$ ( $\mathrm{E}>0.18 \mathrm{MeV}$ ) HTGR and burnup of $4.6 \%$ FIMA at $1010^{\circ} \mathrm{C}$. (a) Bright field and (b) polarized light. 


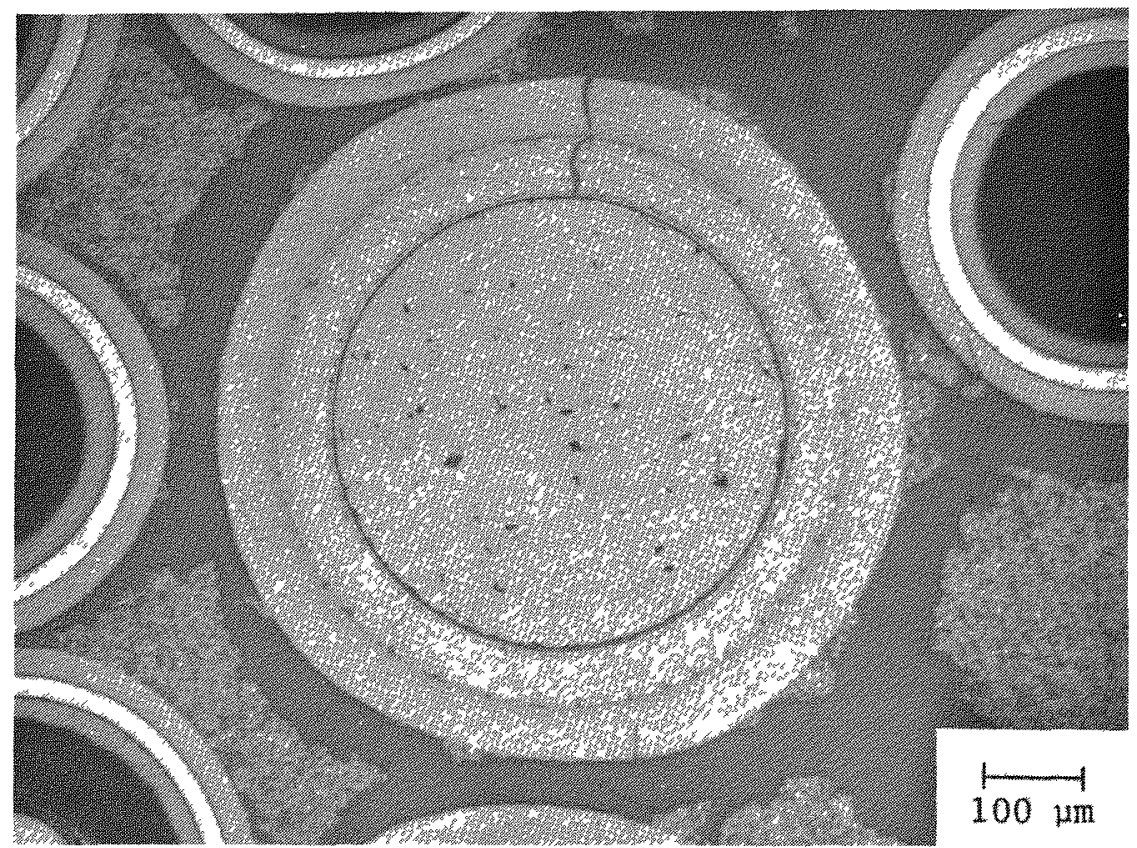

L7440-119

(a)

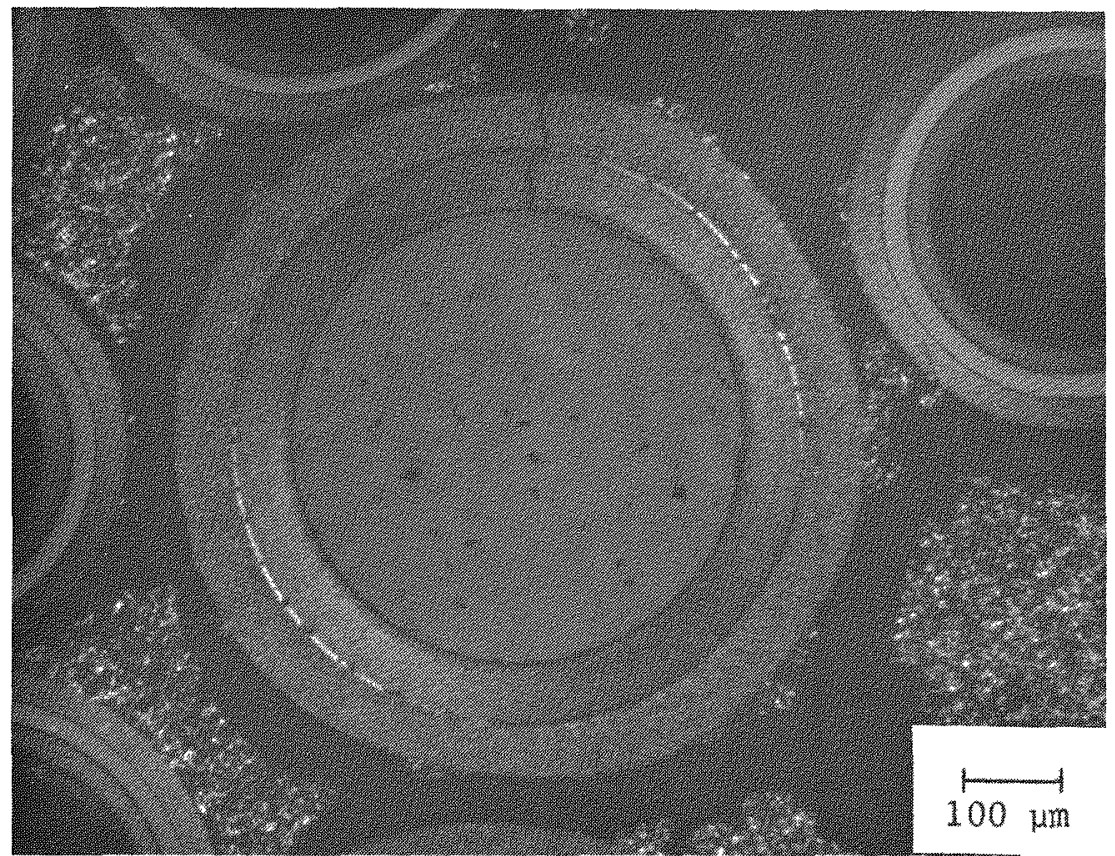

L7 $440-120$

(b)

Fig. 4-101. Photomicrographs showing irradiation-induced failure in a BISO $\mathrm{ThO}_{2}$ particle $(6542-19-015)$ with a $10 \mathrm{w}$-density $\left(1.78 \mathrm{~g} / \mathrm{cm}^{3}\right)$ OPyC coating. The particle was irradiated in fuel rod 7161004-19-7 of capsule P13R (position 5D) to a fast neutron fluence of $8.2 \times 1021 \mathrm{n} / \mathrm{cm}^{2}(\mathrm{E}>0.18 \mathrm{MeV}$ ) HTGR and burnup of $2.4 \%$ FIMA at $1225^{\circ} \mathrm{C}$. (a) Bright field and (b) polarized 1ight. 


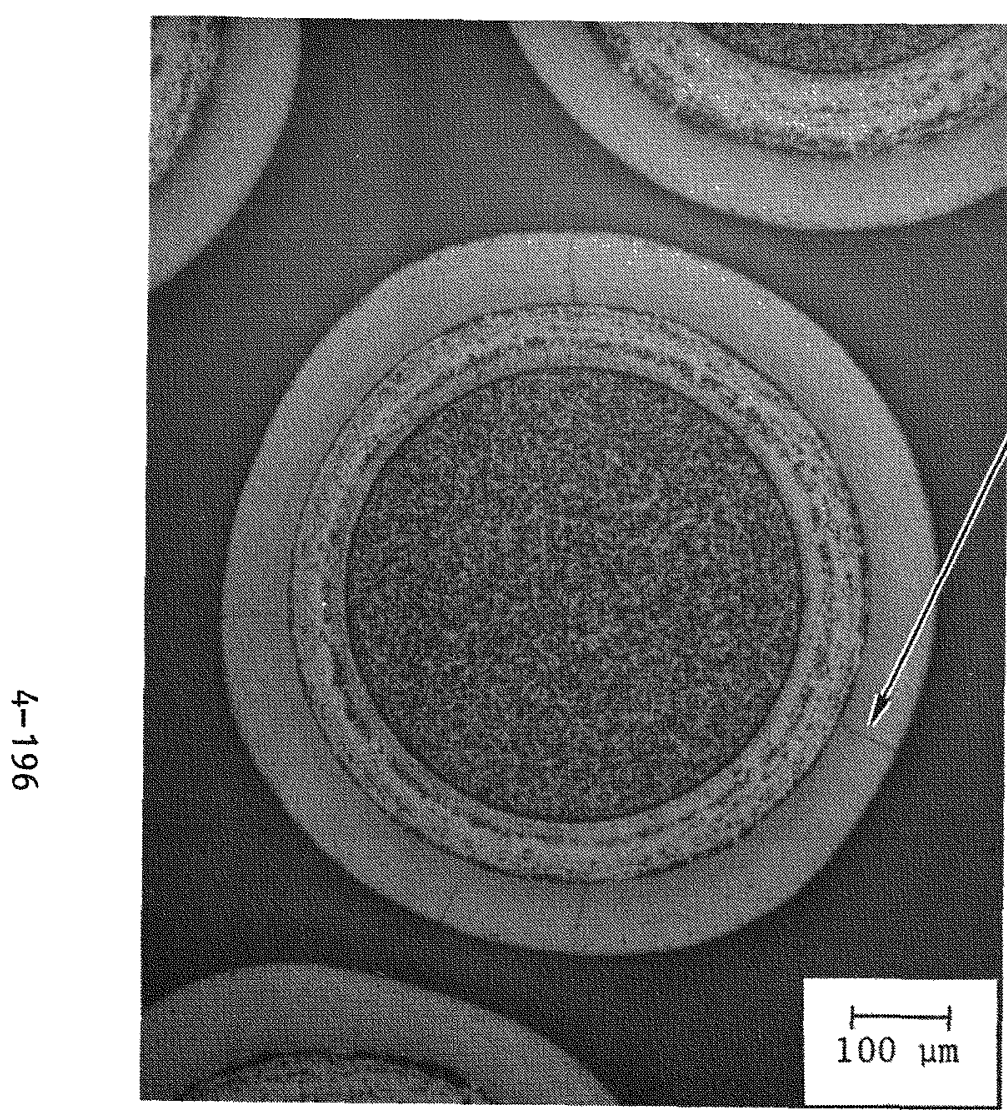

L7440-760

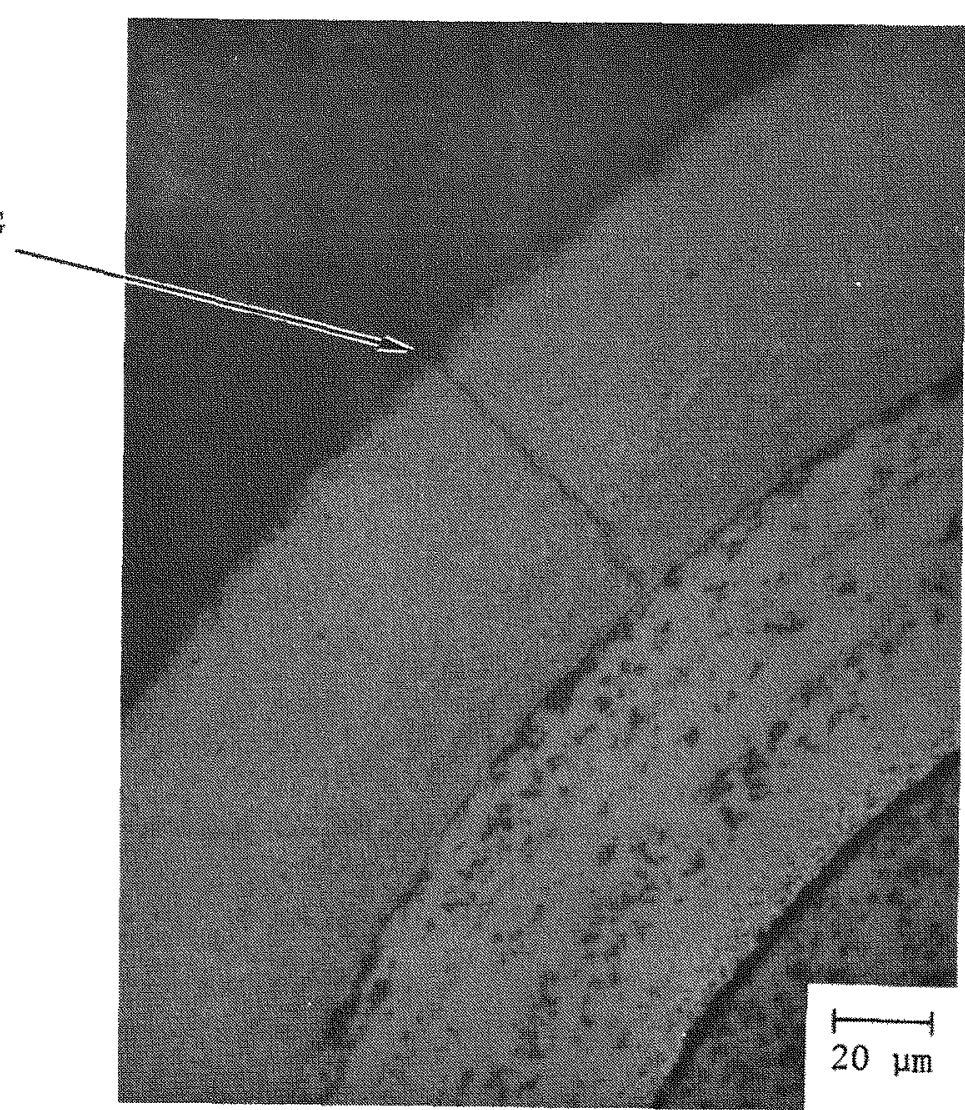

L7440-762 Fig. 4-102. Photomicrographs showing microcracks in OPyC coating of BISO $\mathrm{ThO}_{2}$ particle (4252-06-010-1)
irradiated in capsule P13S (position 3-1) 


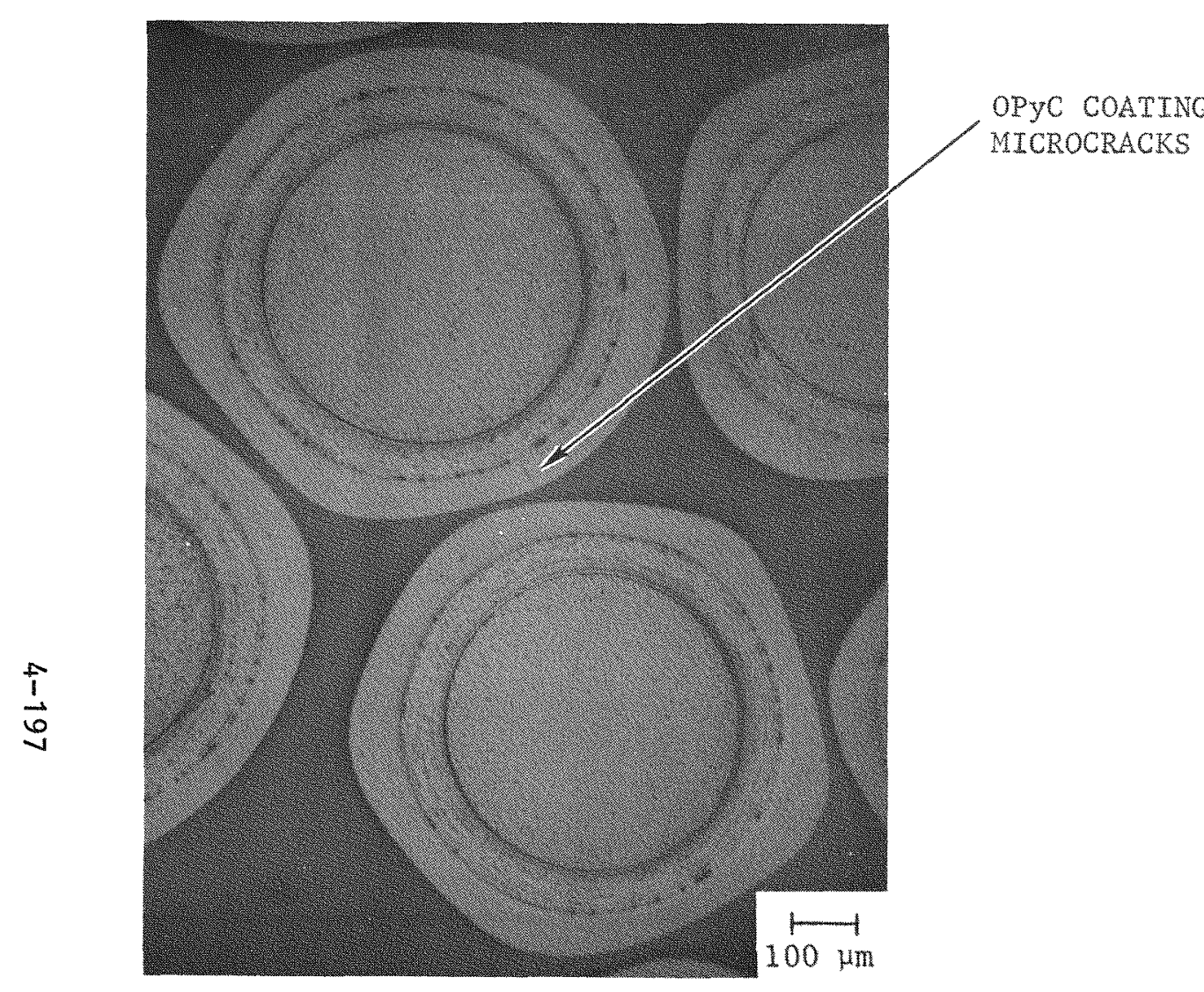

L7440-705

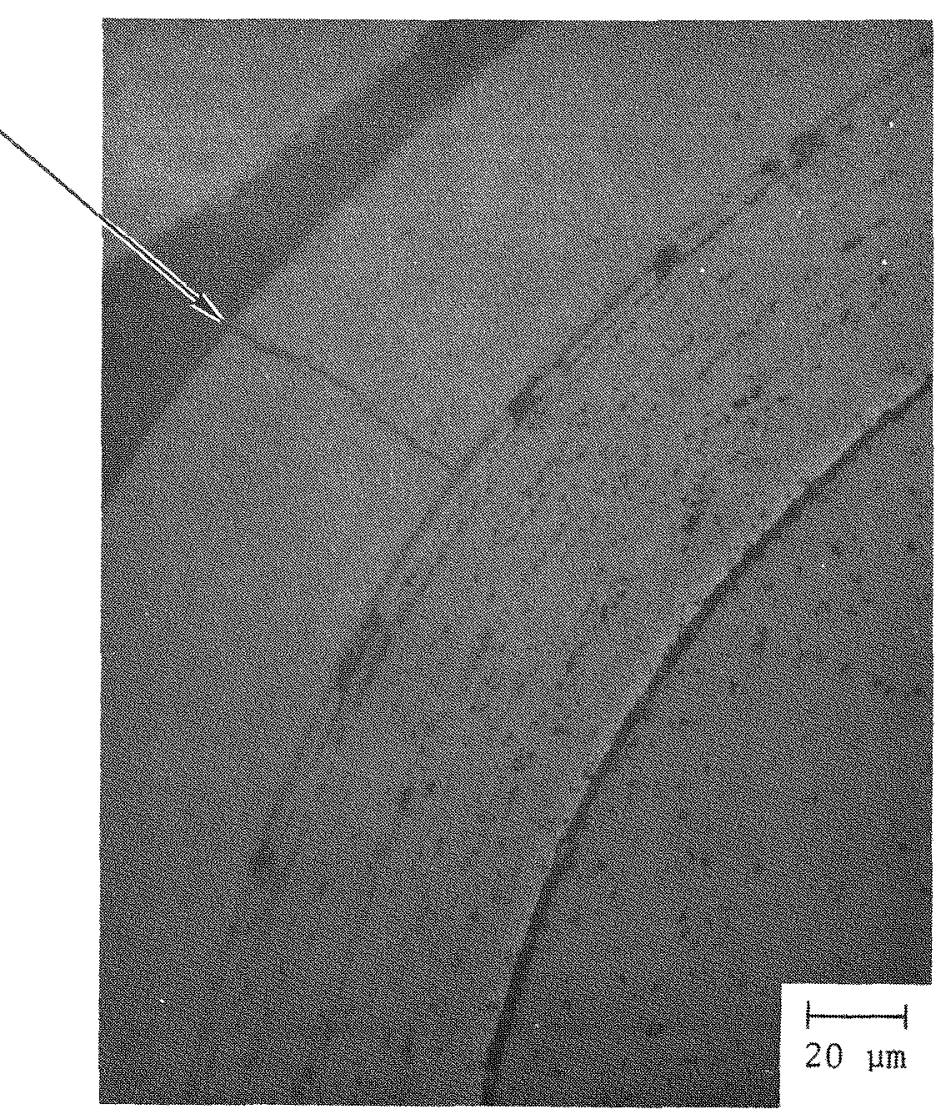

$17440-707$

Fig. 4-103. Photomicrographs showing microcracks in OPyC coating of BISO $\mathrm{ThO}_{2}$ particle (6542-20-035-2) irradiated in capsule P13R (position 4-8) 


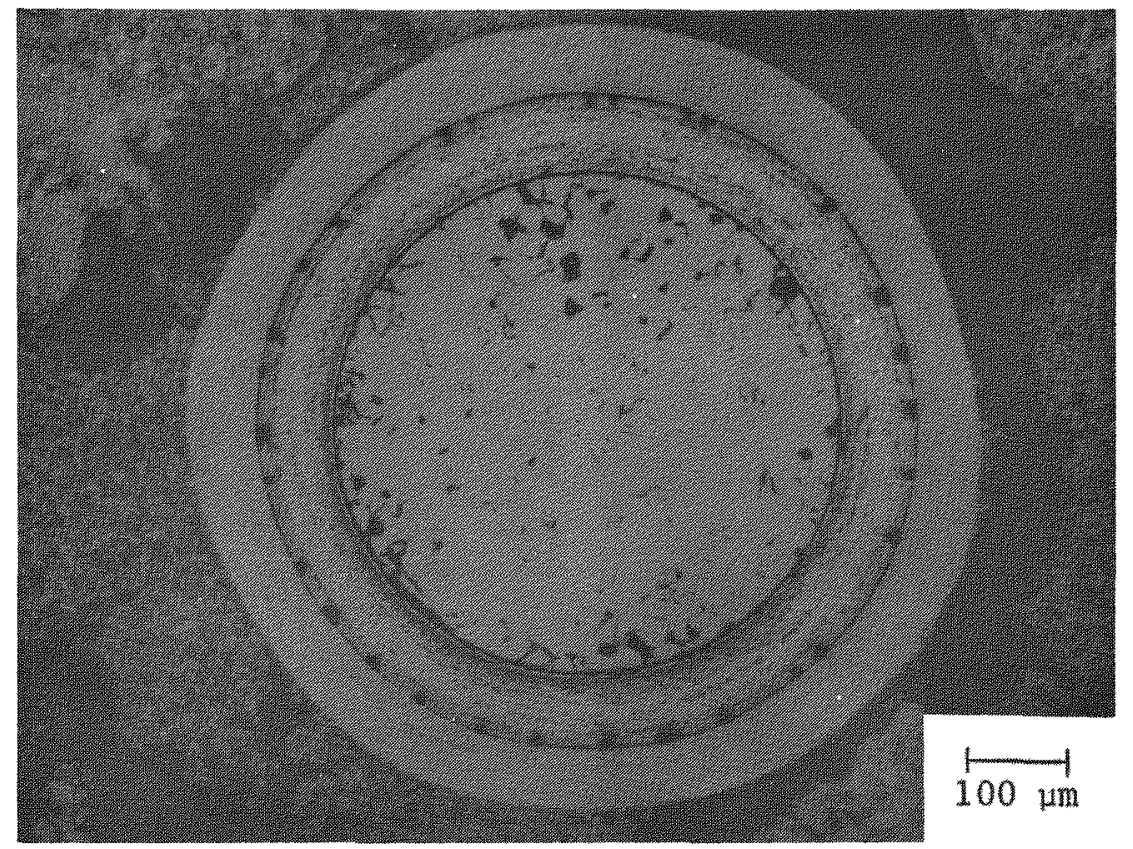

(a)

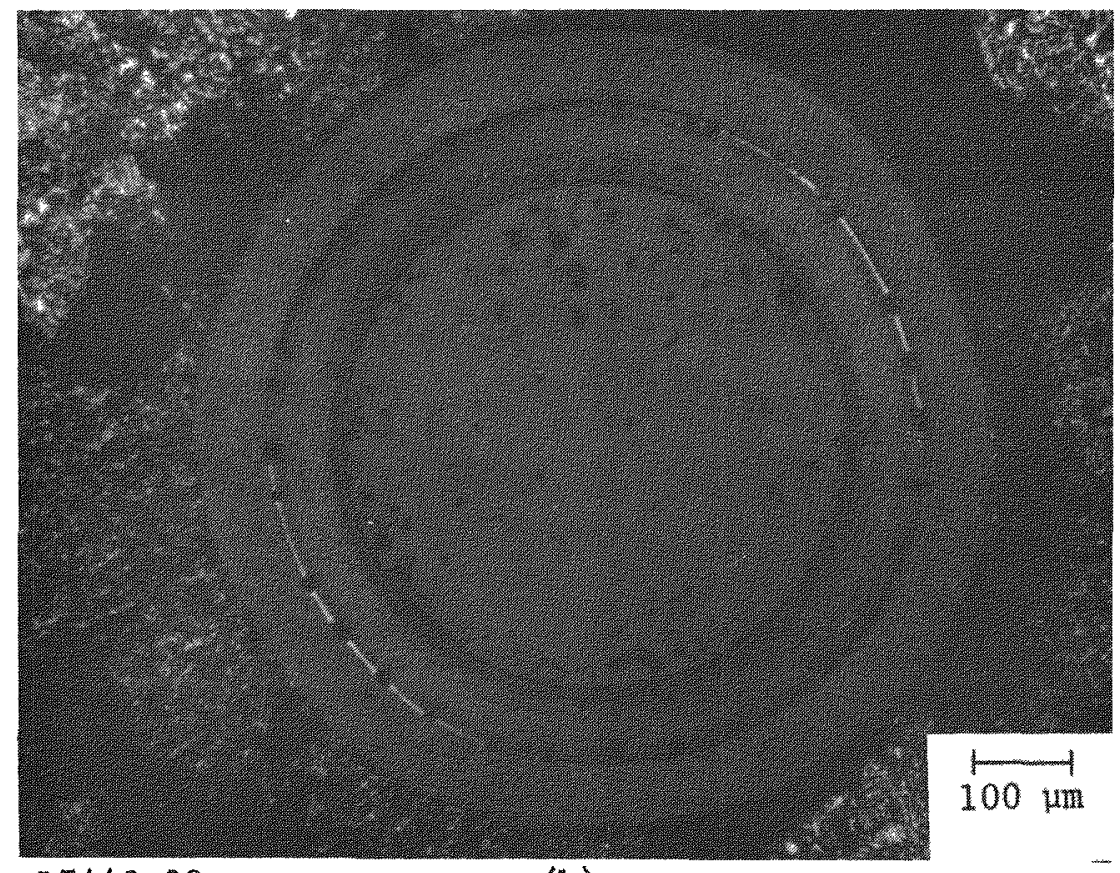

L7440-90

(b)

Fig. 4-104. Photomicrographs of a $\mathrm{BISO} \mathrm{ThO}_{2}$ particle showing numerous small gaps formed at the buffer/OPyC coating interface where the seal coat cracked and densified during irradiation. (a) Bright field and (b) polarized light. 


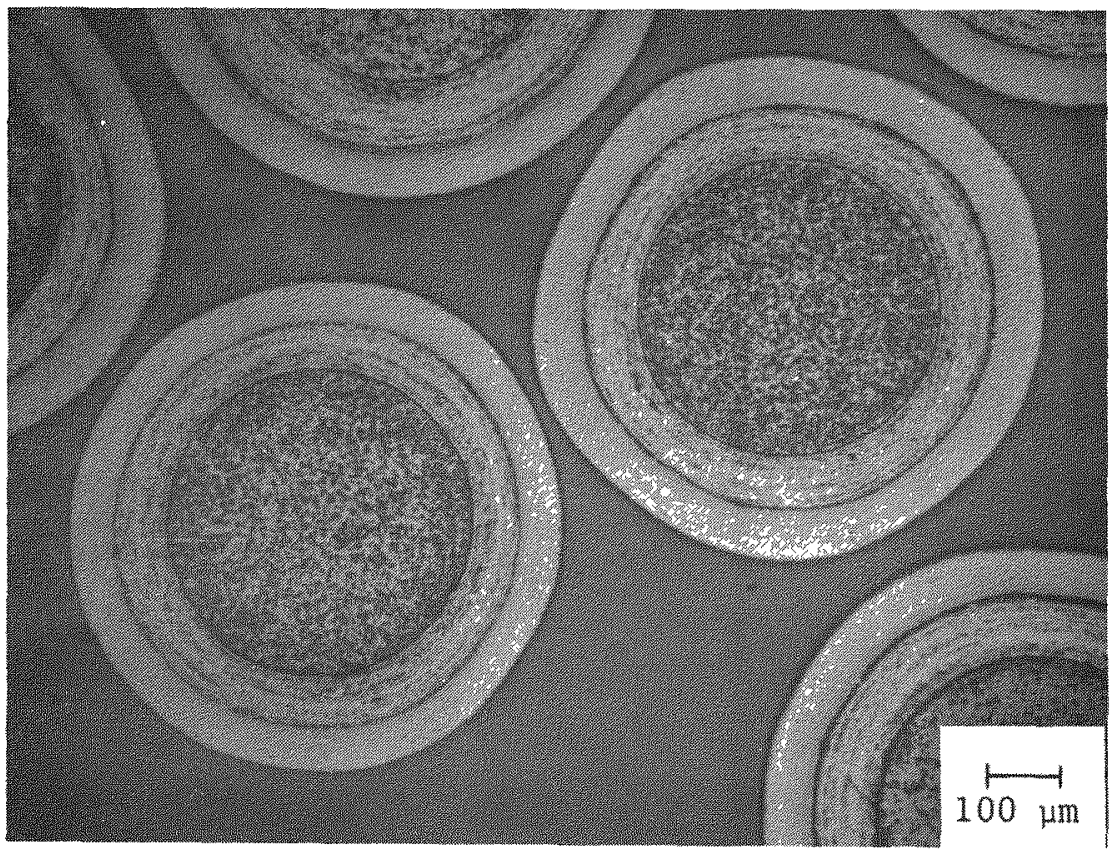

L7440-654

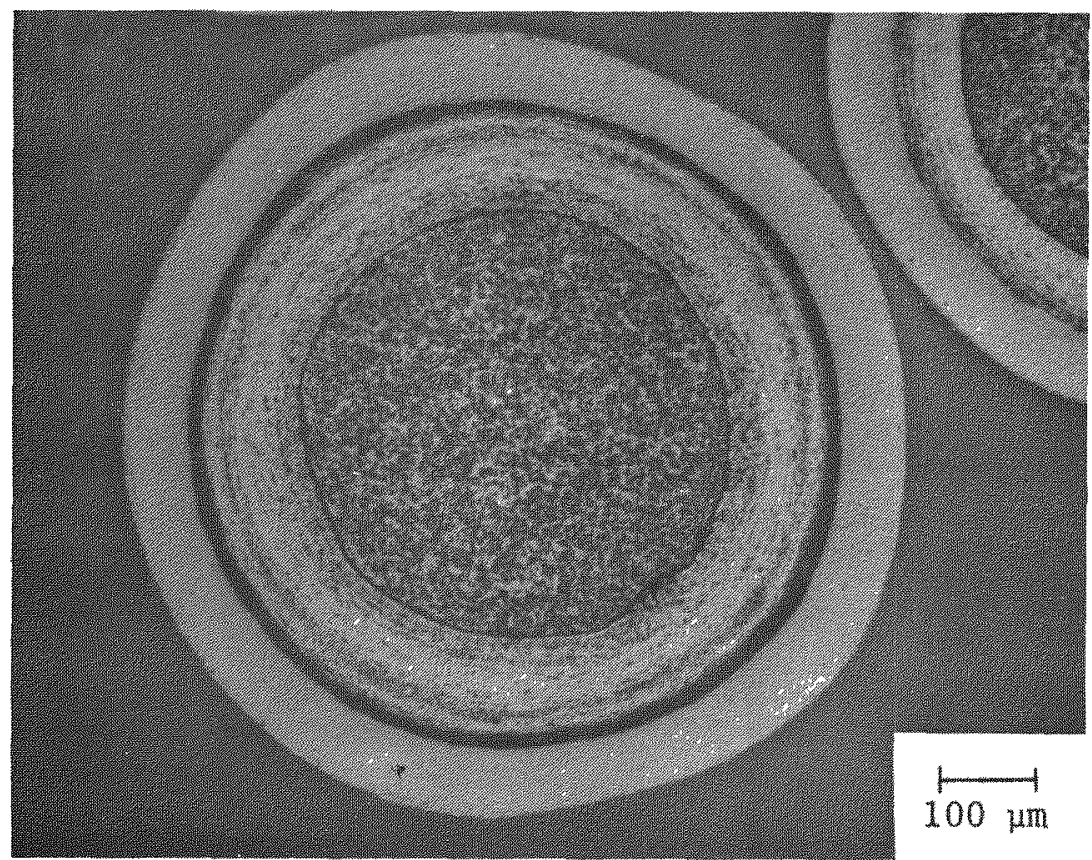

L7440-650

Fig. 4-105. Photomicrographs of BISO Th0 2 particles (6542-18-015-1) with no seal coat between the buffer and OPyC coatings. The particle batch was irradiated in capsule P13R (position 4-3) to a fast neutron fluence of $11.4 \times 10^{21} \mathrm{n} / \mathrm{cm}^{2}$ (E>0.18 MeV) HTGR and burnup of $4.4 \%$ FIMA at $1000^{\circ} \mathrm{C}$. 


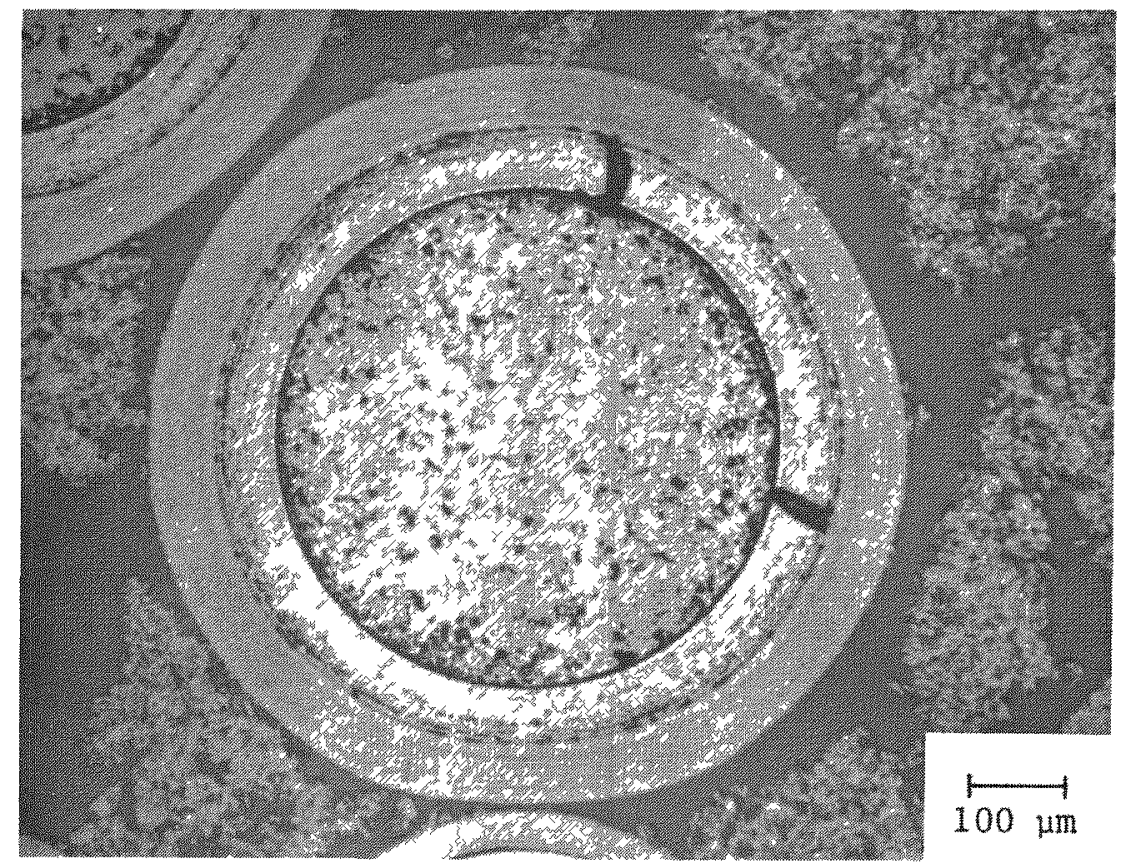

L7440-281

(a)

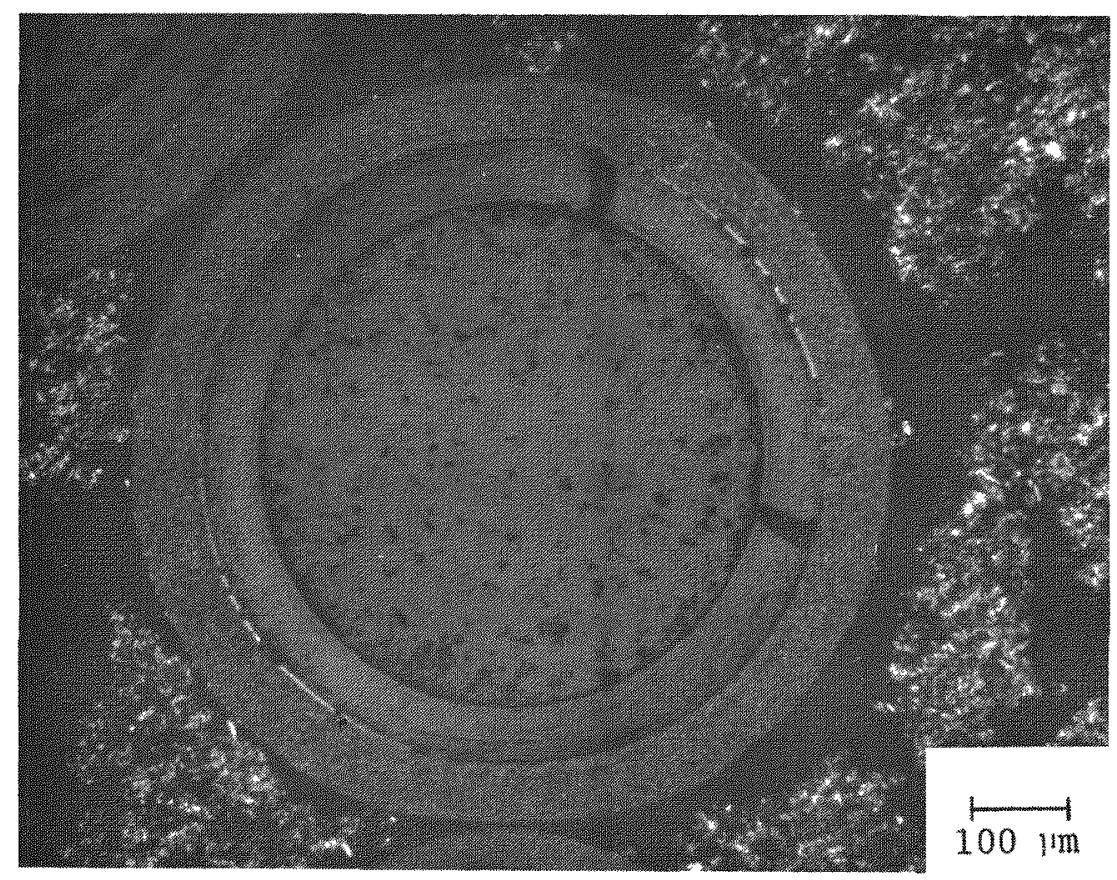

L7440-282

(b)

Fig. 4-106. Photomicrographs of a BISO $\mathrm{ThO}_{2}$ particle where the buffer coating cracked and densified while the OPyC coating remained intact. (a) Bright field and (b) polarized light. 
PARTIAL

CONVERSION

OF $\mathrm{ThO}_{2}$ TO $\mathrm{ThC}_{2}$

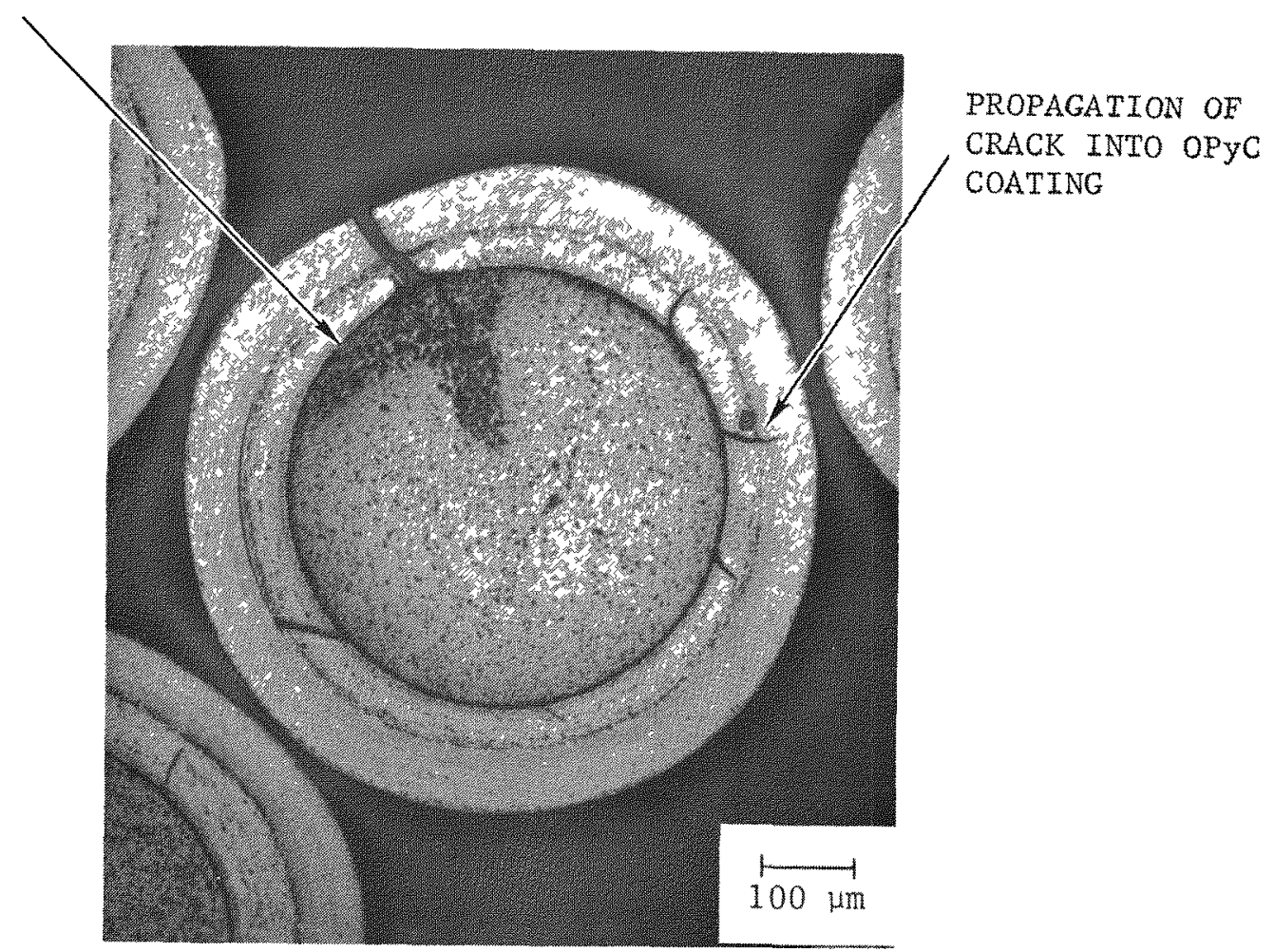

L7440-788

Fig. 4-107. Photomicrograph of a BISO $\mathrm{ThO}_{2}$ particle (6542-12-025)

which shows the propagation of a buffer coating crack into the adjacent OPyC coating and the partial conversion of a localized region of the fuel kernel to carbide. The presence of a seal coat on this particle batch did not prevent propagation of the crack from the buffer coating into the OPyC coating. 

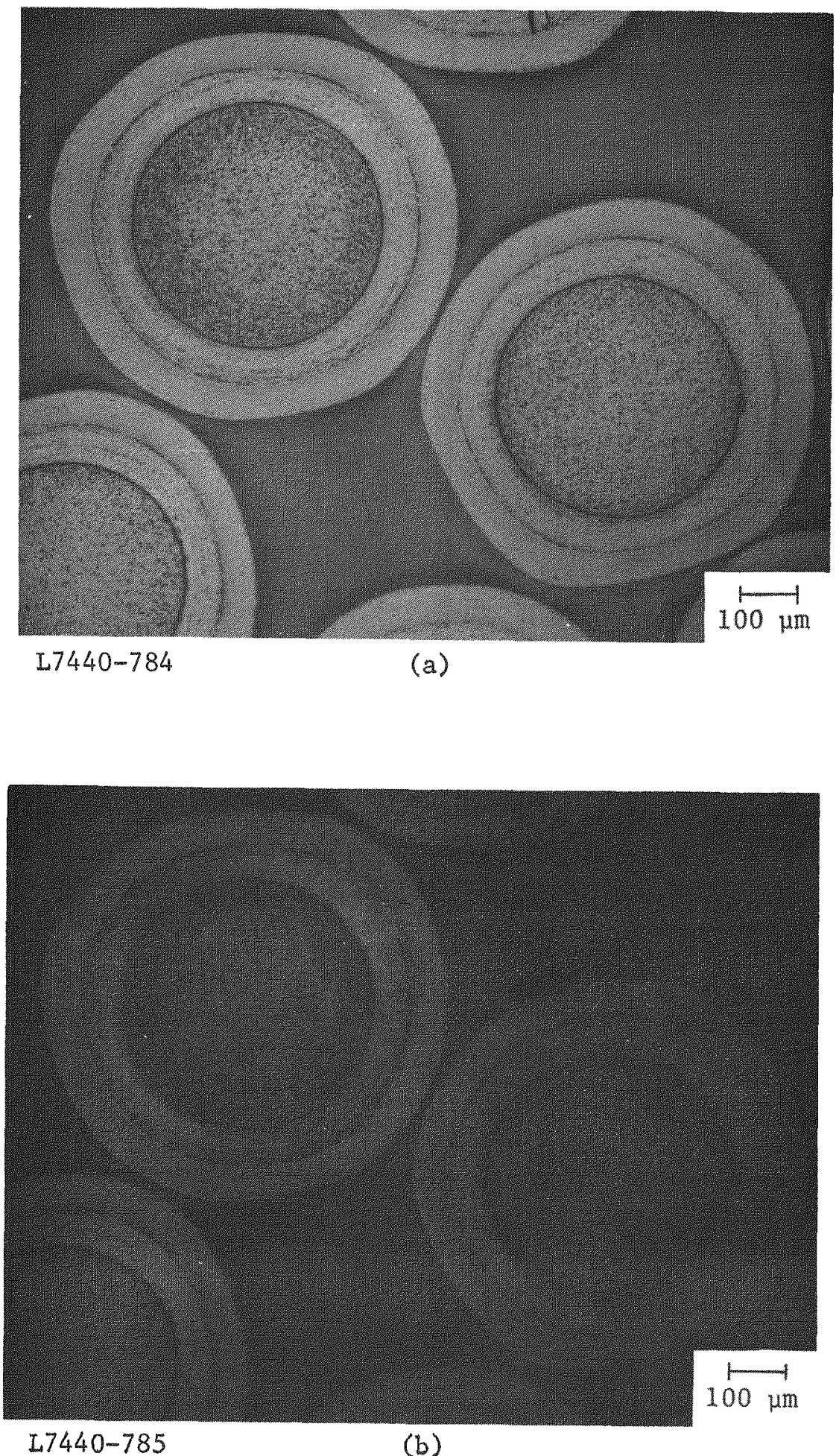

Fig. 4-108. Photomicrographs of BISO $\mathrm{ThO}_{2}$ particles (6542-12-025-2) with the buffer coating applied with $\mathrm{N}_{2}$ carrier gas. The sample was irradiated in capsule P13S (position 4-12) to a fast neutron fluence of $10.7 \times 10^{21} \mathrm{n} / \mathrm{cm}^{2}$ ( $\mathrm{E}>0.18 \mathrm{MeV}$ ) HTGR and burnup of $3.6 \%$ FIMA at $830^{\circ} \mathrm{C}$. (a) Bright field and (b) polarized light. 

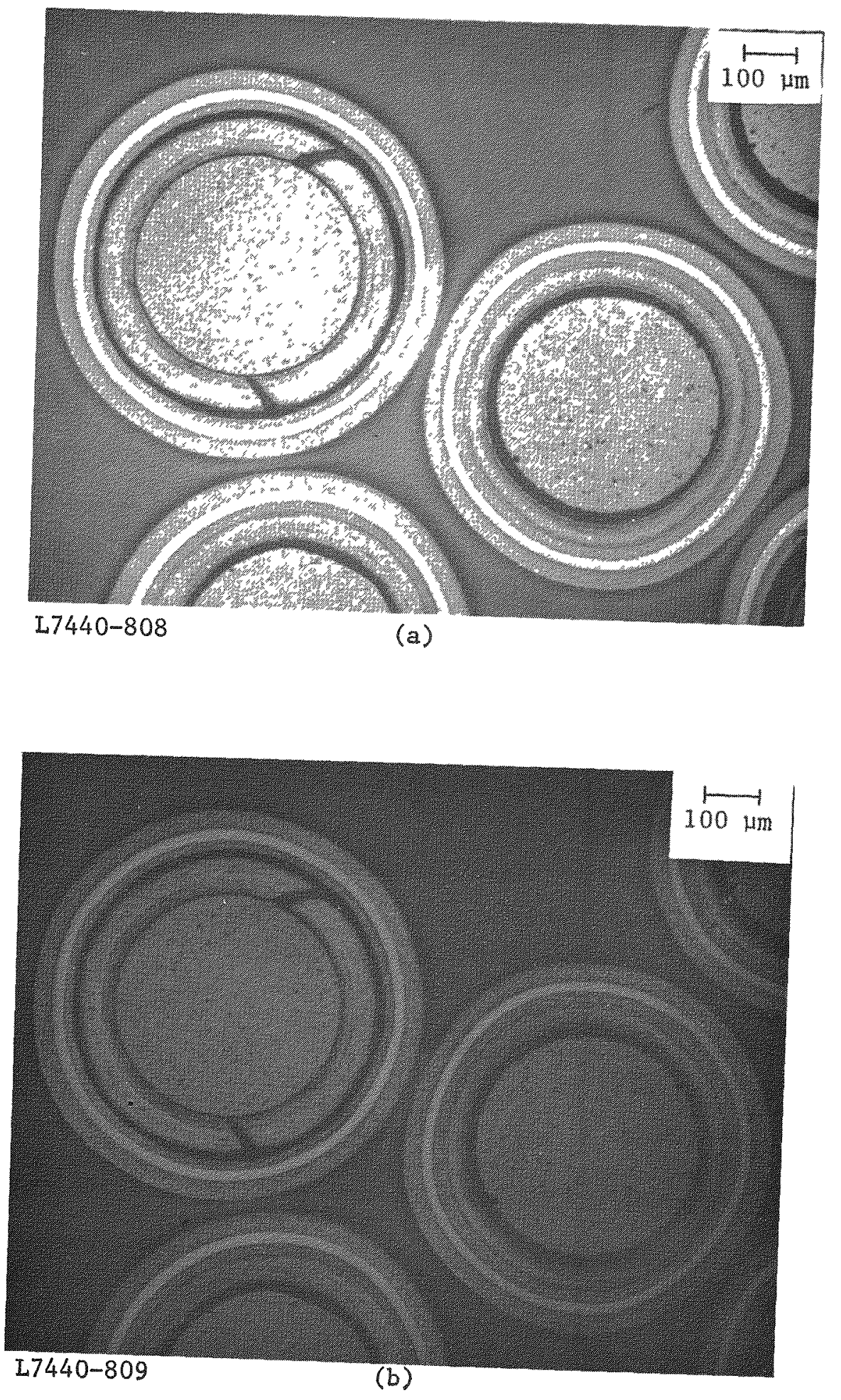

Fig. 4-109. Photomicrographs of TRISO $\mathrm{ThO}_{2}$ particles (6252-00-025-2) irradiated in capsule P13S (position 3-12) to a fast neutron fluence of $11.5 \times 10^{21} \mathrm{n} / \mathrm{cm}^{2}$ (E $>0.18 \mathrm{MeV}$ ) $\mathrm{HTGR}$ and burnup of $4.1 \%$ FIMA at $860^{\circ} \mathrm{C}$. (a) Bright field and (b) polarized light. 


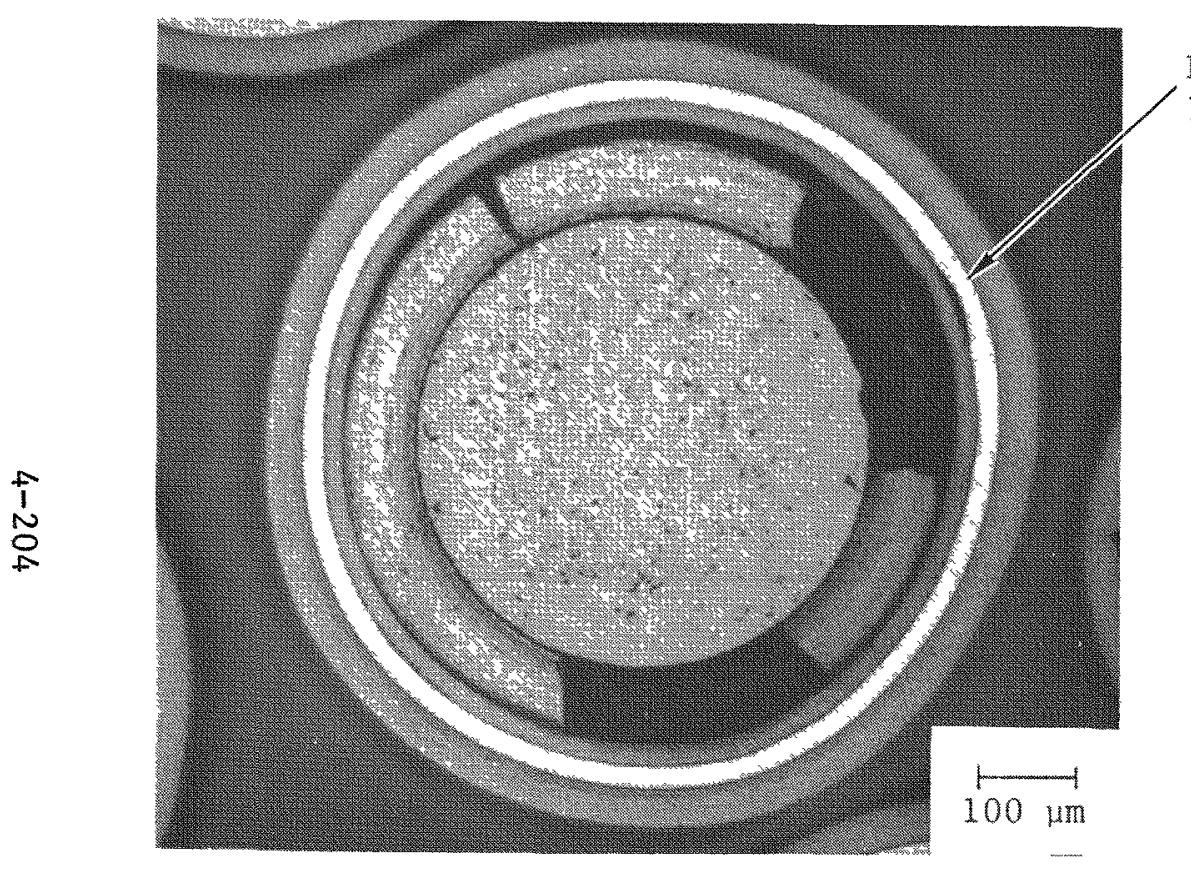

$27440-822$
REACTION ZONE IN SIC COATINO

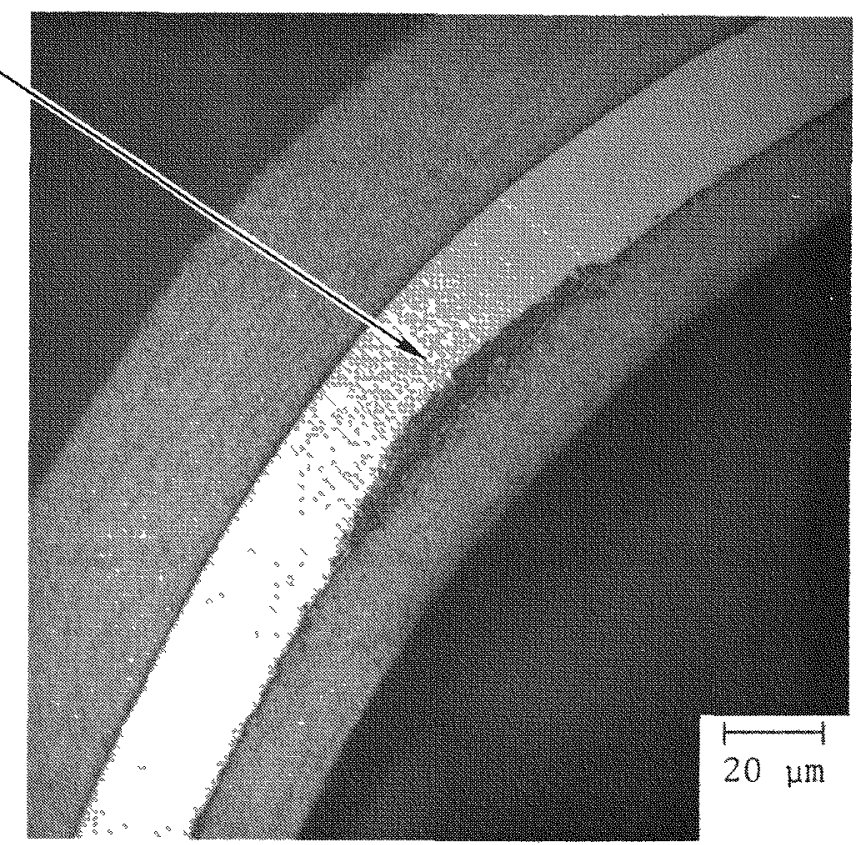

L7 7440-824

Fig. 4-110. Photomicrographs showing reaction zone observed in TRISO $\mathrm{ThO}_{2}$ particle (6252-00-025-2) irradiated in capsule P13S (position 3-12) to a fast neutron fluence of $11.5 \times 10^{21}$ $\mathrm{n} / \mathrm{cm}^{2}(\mathrm{E}>0.18 \mathrm{MeV})_{\mathrm{HTGR}}$ and burnup of $4.1 \%$ FIMA at $860^{\circ} \mathrm{C}$ 


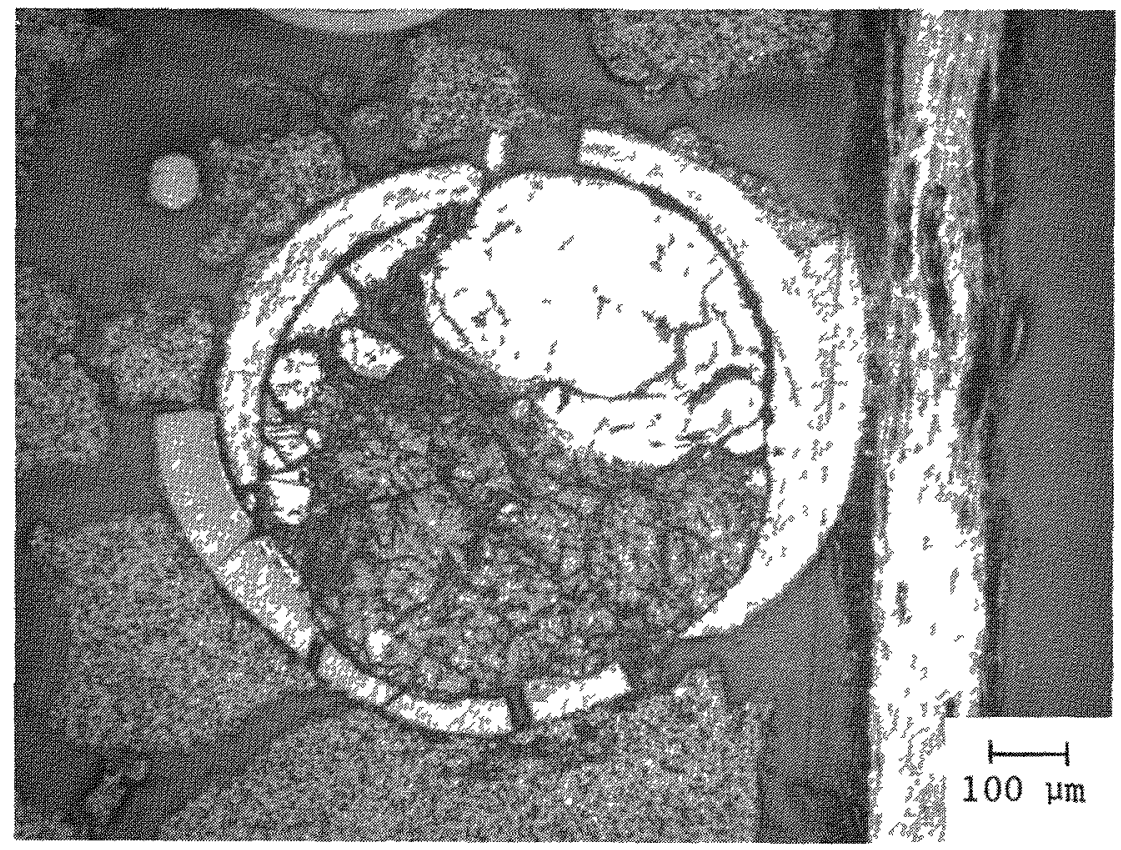

L7440-176

Fig. 4-111. Photomicrograph of a defective BISO $\mathrm{ThO}_{2}$ particle in fuel rod 7161-004-20-5. Matrix material is bonded to the buffer coating in the region where the OPyC coating is missing. During irradiation the coating broke and the fuel kernel converted to carbide and subsequently hydrolyzed. 


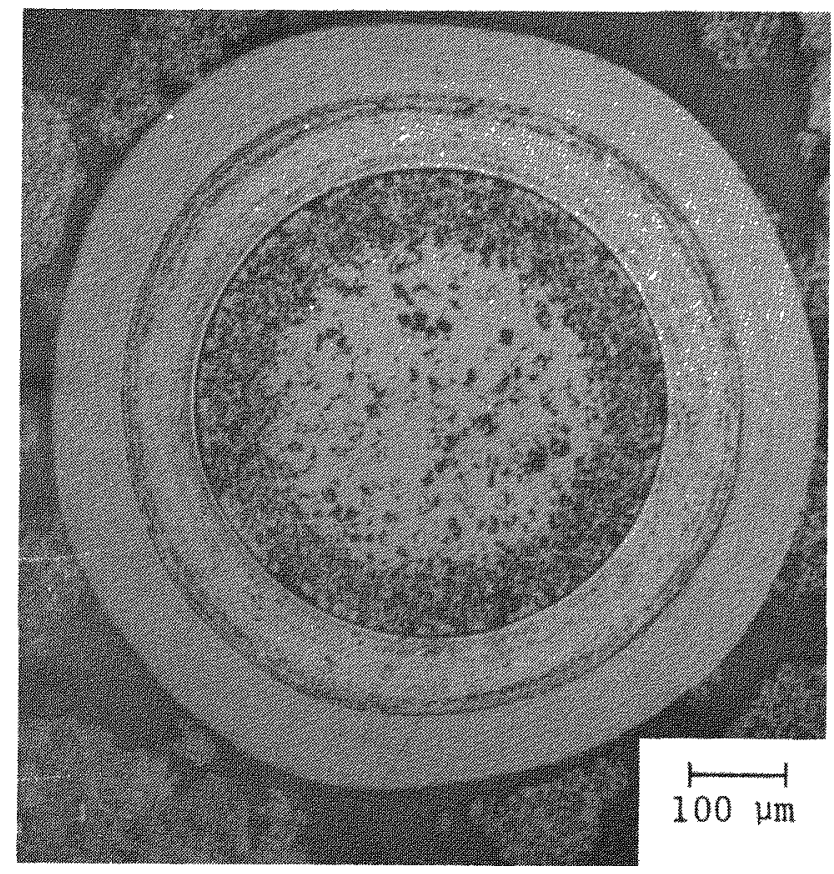

L7440-374 (a)

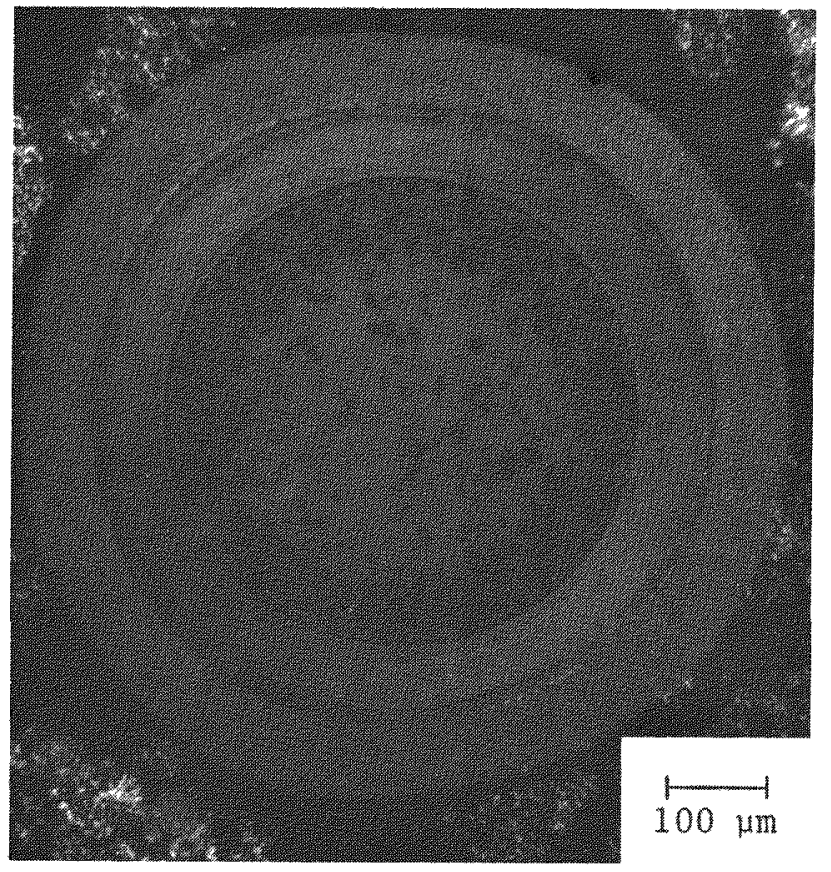

L7440-375

(b)

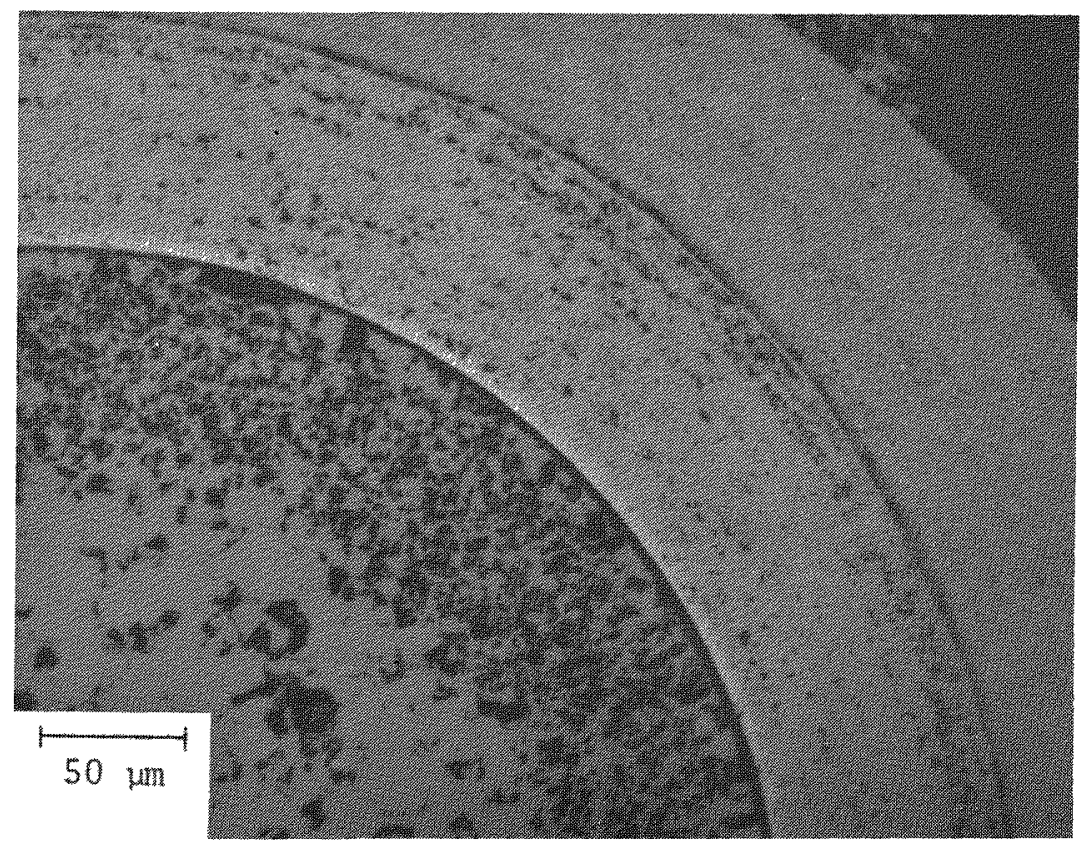

L7440-376

(c)

Fig. 4-112. Photomicrographs showing the microstructure of a $\mathrm{ThO}_{2}$ kernel from particle batch 6542-01-010 irradiated in fuel rod 7161-004-08-13 of capsule P13R (position 2C) to a fast neutron fluence of $12.2 \times 10^{21} \mathrm{n} / \mathrm{cm}^{2}$ (E $>0.18 \mathrm{MeV}$ ) HTGR and burnup of $5.6 \%$ FIMA at $1045^{\circ} \mathrm{C}$. The formation of pores has occurred in the outer region of the fuel kernel. $(a, c)$ Bright field and (b) polarized light. 


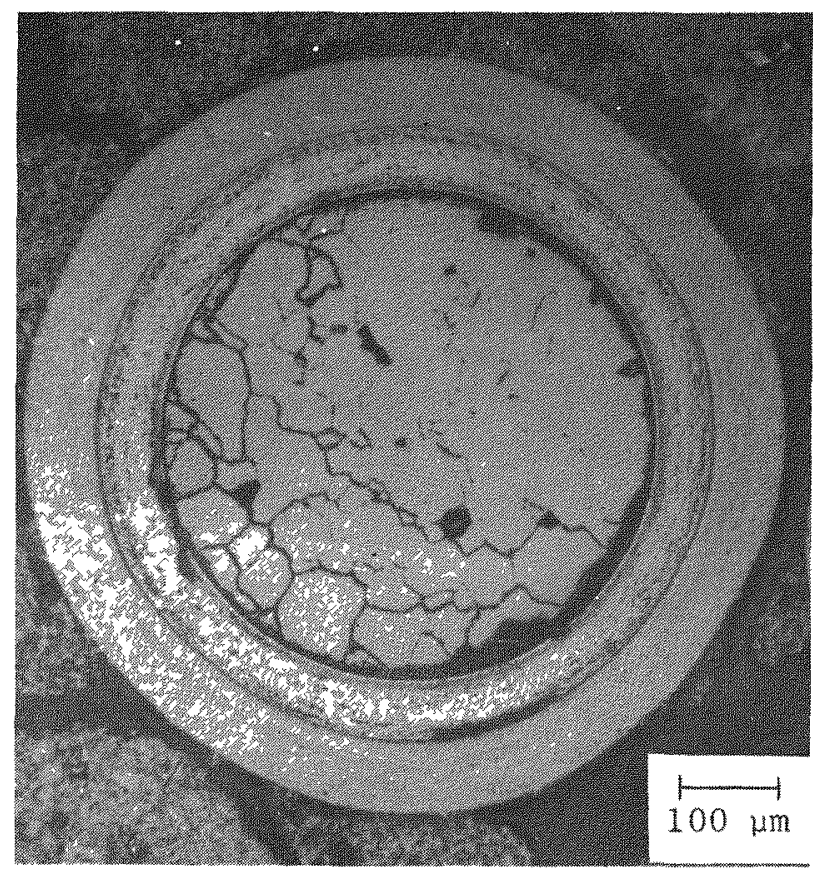

$17440-378$

(a)

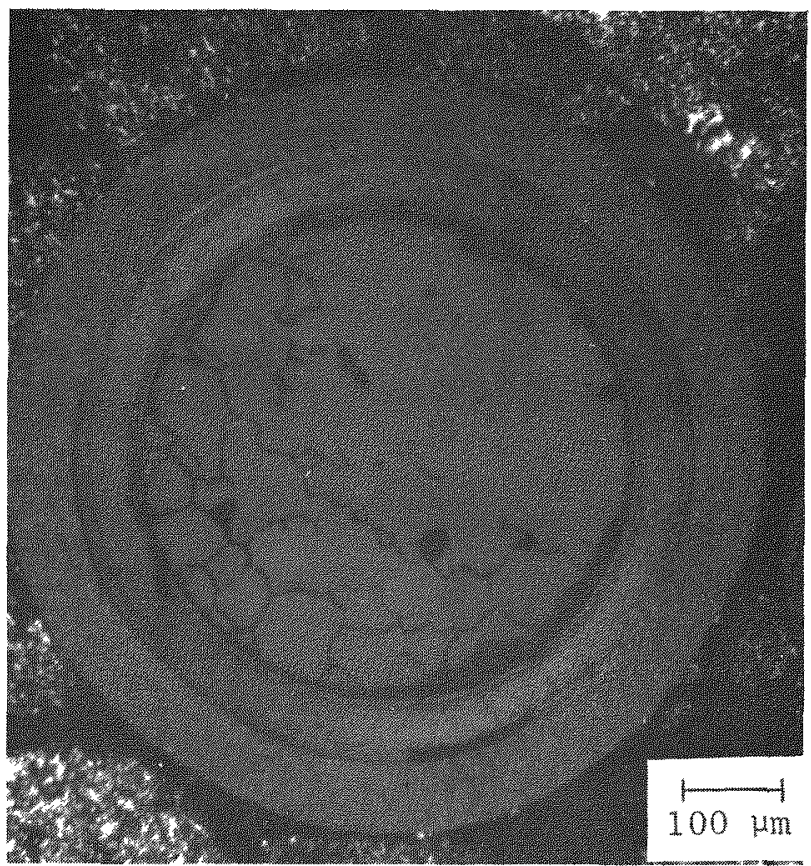

L7440-379

(b)

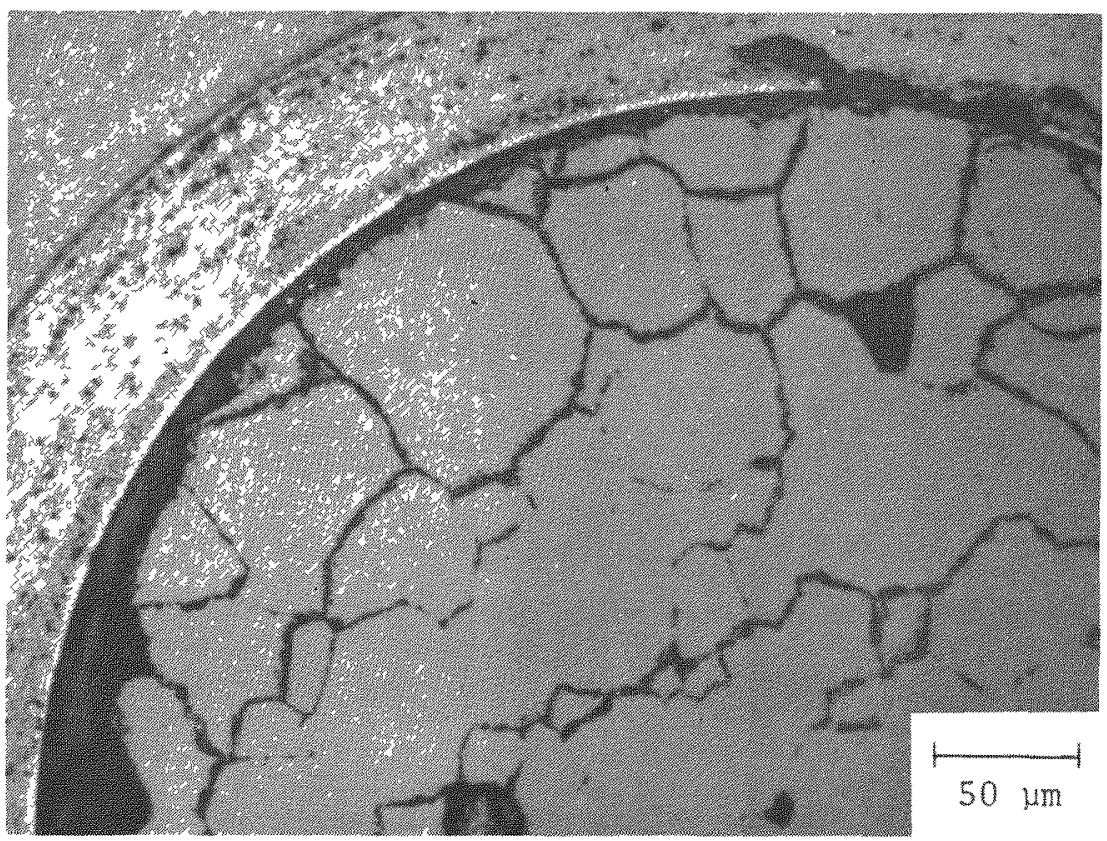

L7440-380

(c)

Fig. 4-113. Photomicrographs showing the microstructure of a $\mathrm{ThO}_{2}$ kernel from particle batch 6542-01-010 irradiated in fuel rod 7161-004-08-13 of capsule P13R (position 2C). Grain boundary thickening occurred on the hot side of the fuel kernel. ( $a, c)$ Bright field and (b) polarized light. 


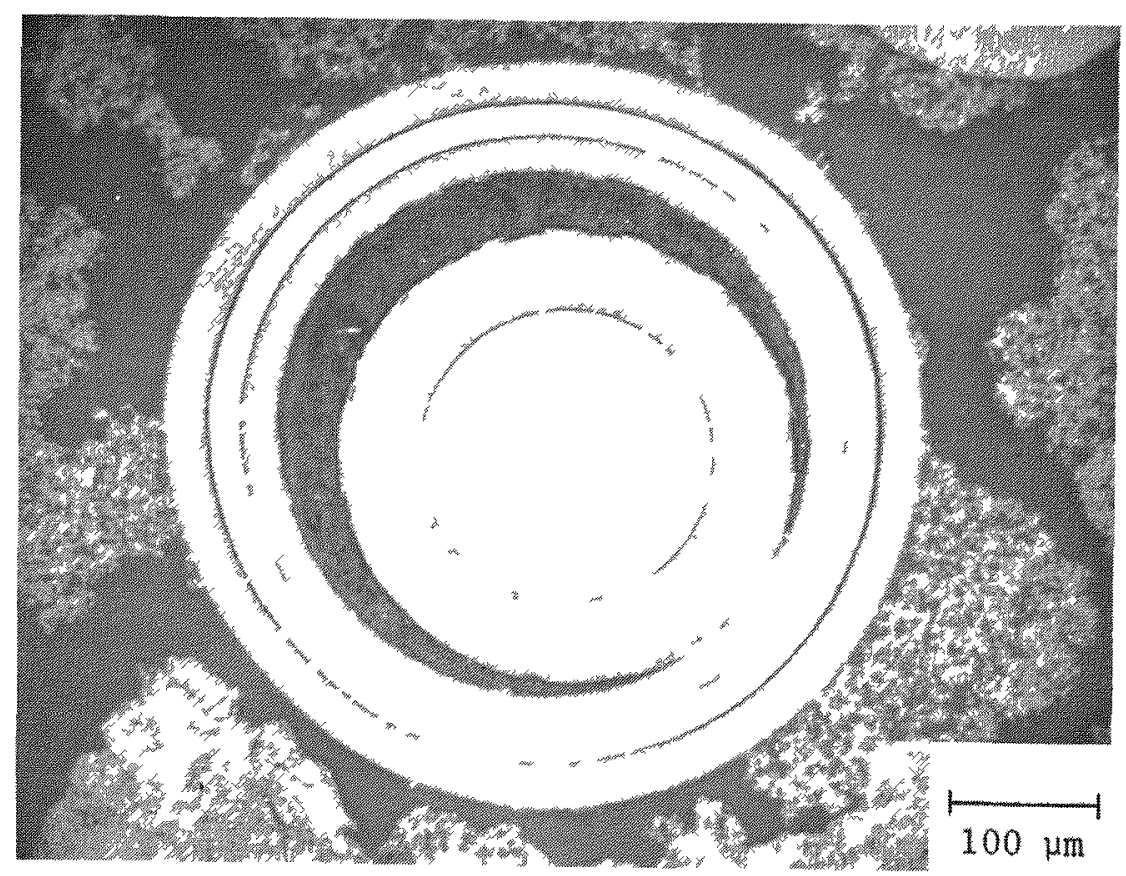

L7440-269

(a)

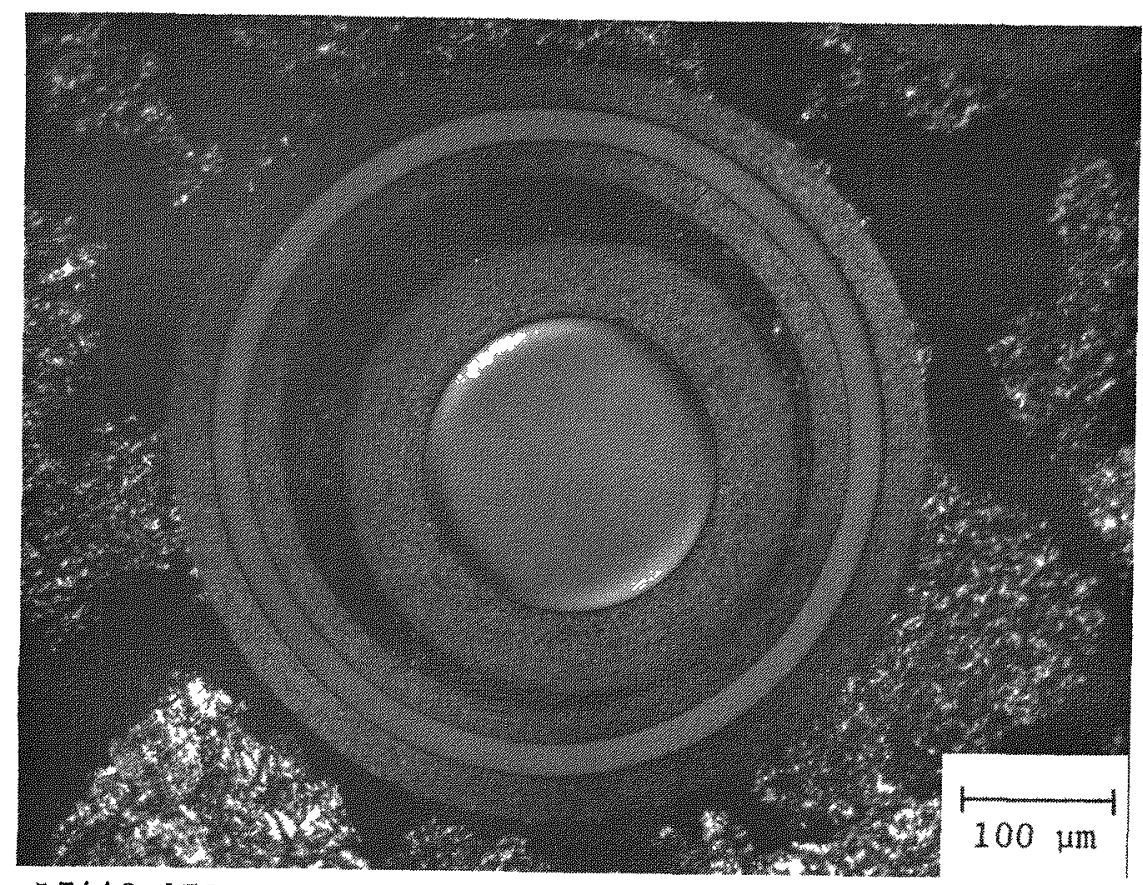

L7440-270

(b)

Fig. 4-114. Photomicrographs of TRISO inert particle (6351-01-020) irradiated in fuel rod 7161-004-08-13 of capsule P13R (position 2C) to a fast neutron fluence of $12.2 \times 10^{21}$ $\mathrm{n} / \mathrm{cm}^{2}(\mathrm{E}>0.18 \mathrm{MeV})_{\mathrm{HTGR}}$ at $1045^{\circ} \mathrm{C}$. (a) Bright field and (b) polarized 1ight. 


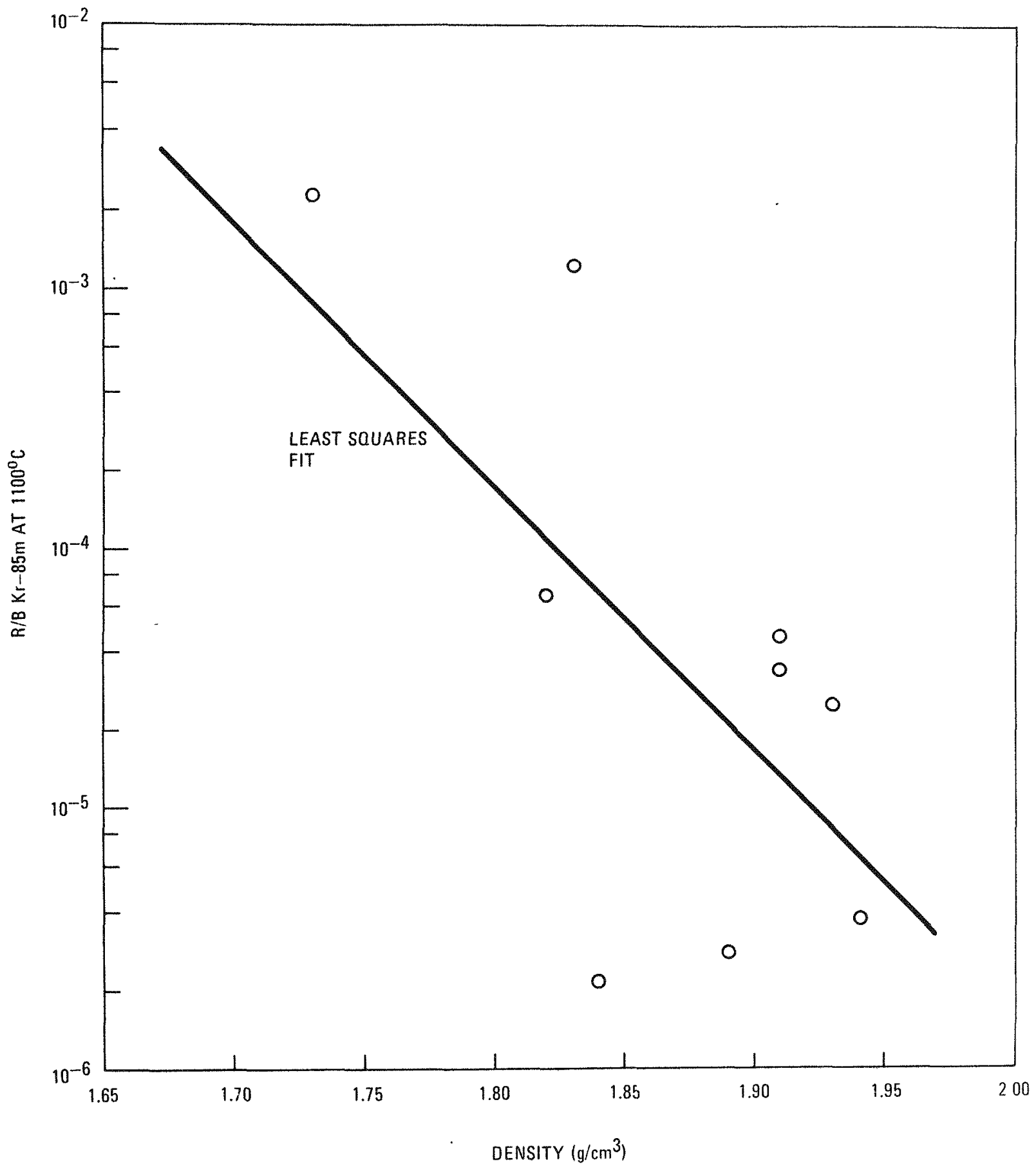

Fig. 4-115. Postirradiation fission gas release versus unirradiated oPyC coating density (density gradient column) for intact BISO $\mathrm{ThO}_{2}$ particles 


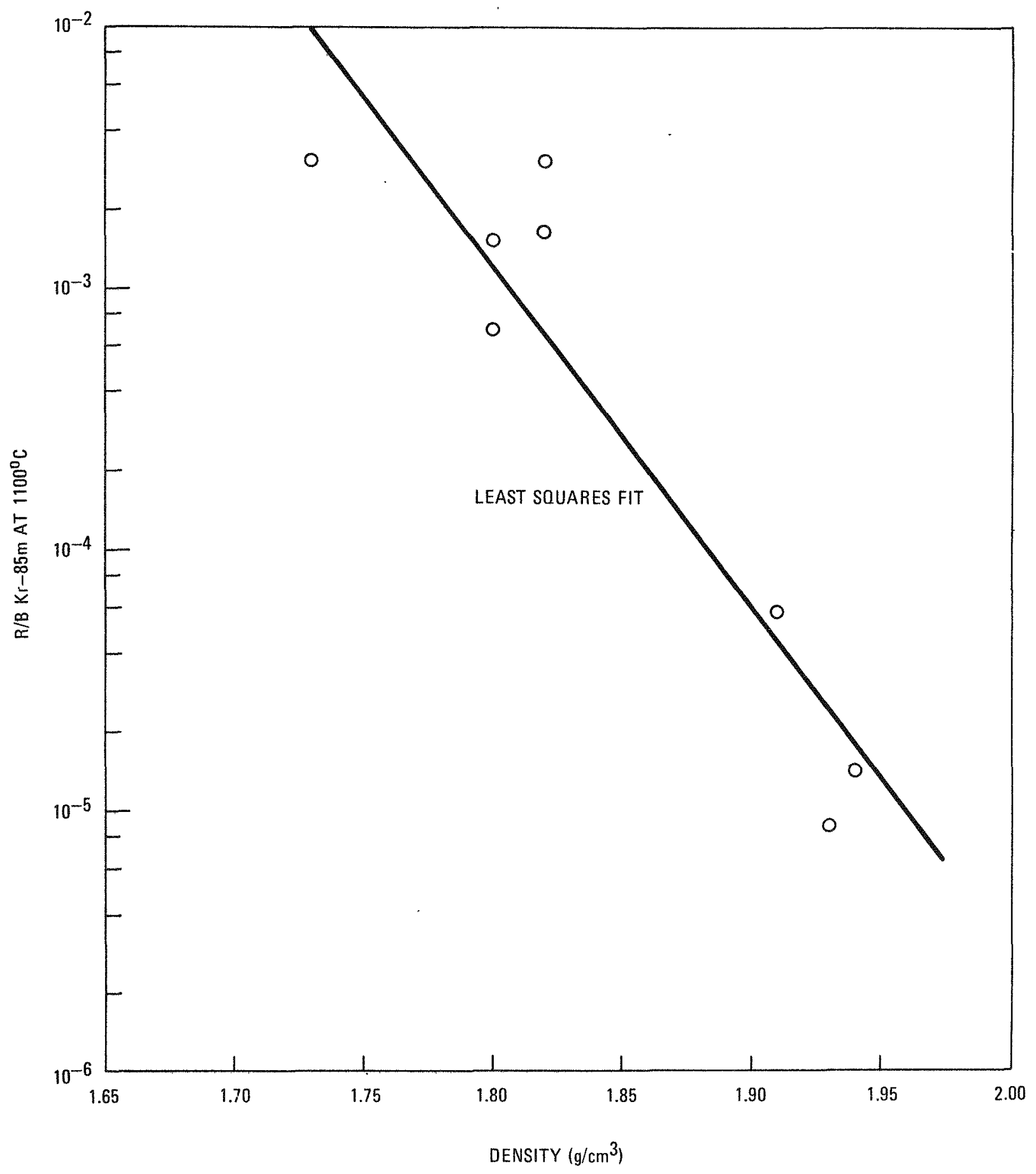

Fig. 4-116. Postirradiation fission gas release versus unirradiated OPyC coating density (density gradient column) for intact BISO $\mathrm{ThO}_{2}$ particles. The particles were cleaned with diluted nitric acid and examined visually prior to fission gas release measurements. 


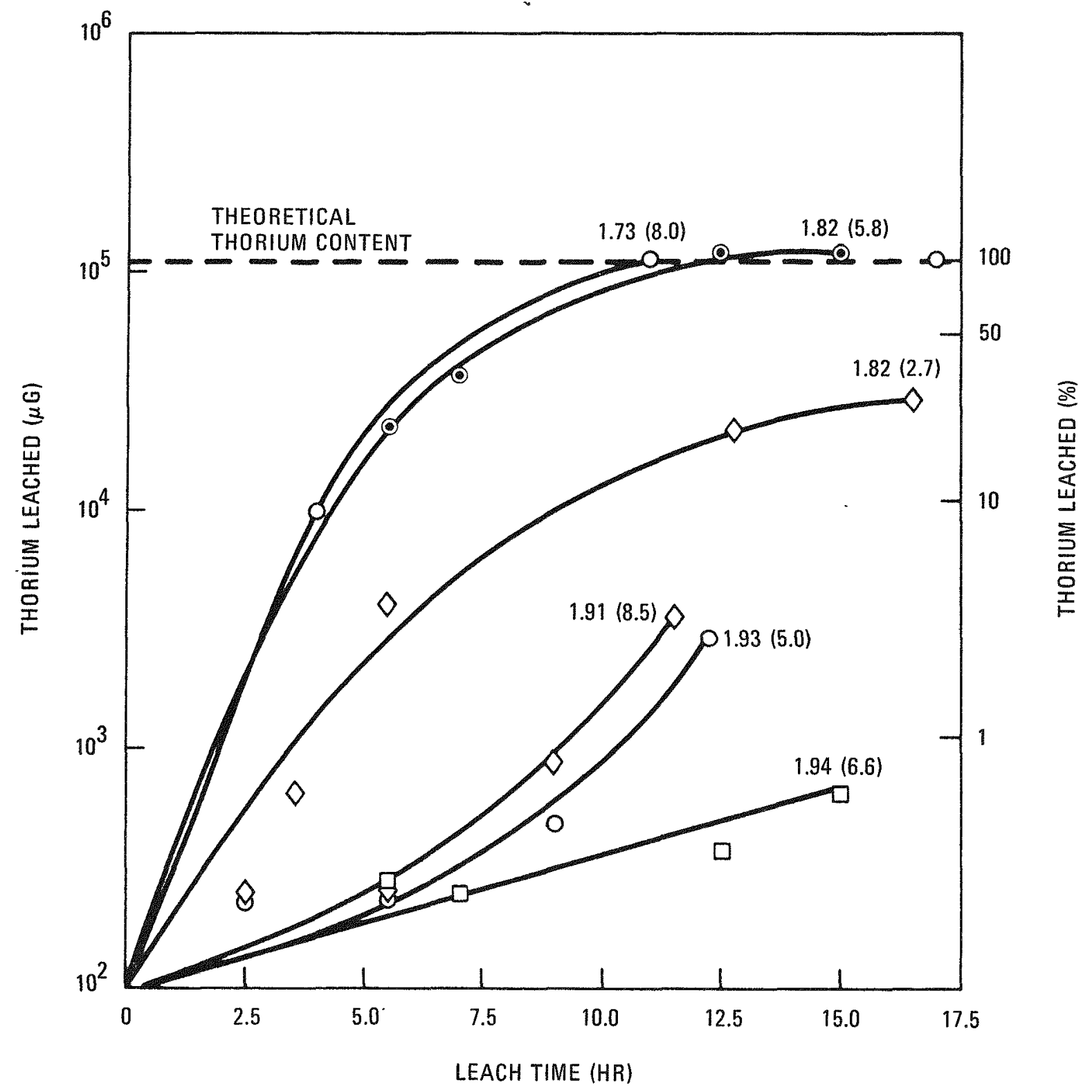

Fig. 4-117. Amount of thorium leached from irradiated $\mathrm{BISO} \mathrm{ThO}_{2}$ particle samples as a function of hot leaching time. The OPyC coating density in $\mathrm{g} / \mathrm{cm}^{3}$ and $\mathrm{OPyC}$ coating rate in $\mu \mathrm{m} / \mathrm{min}$ (numbers in parentheses) are also shown for each batch. 

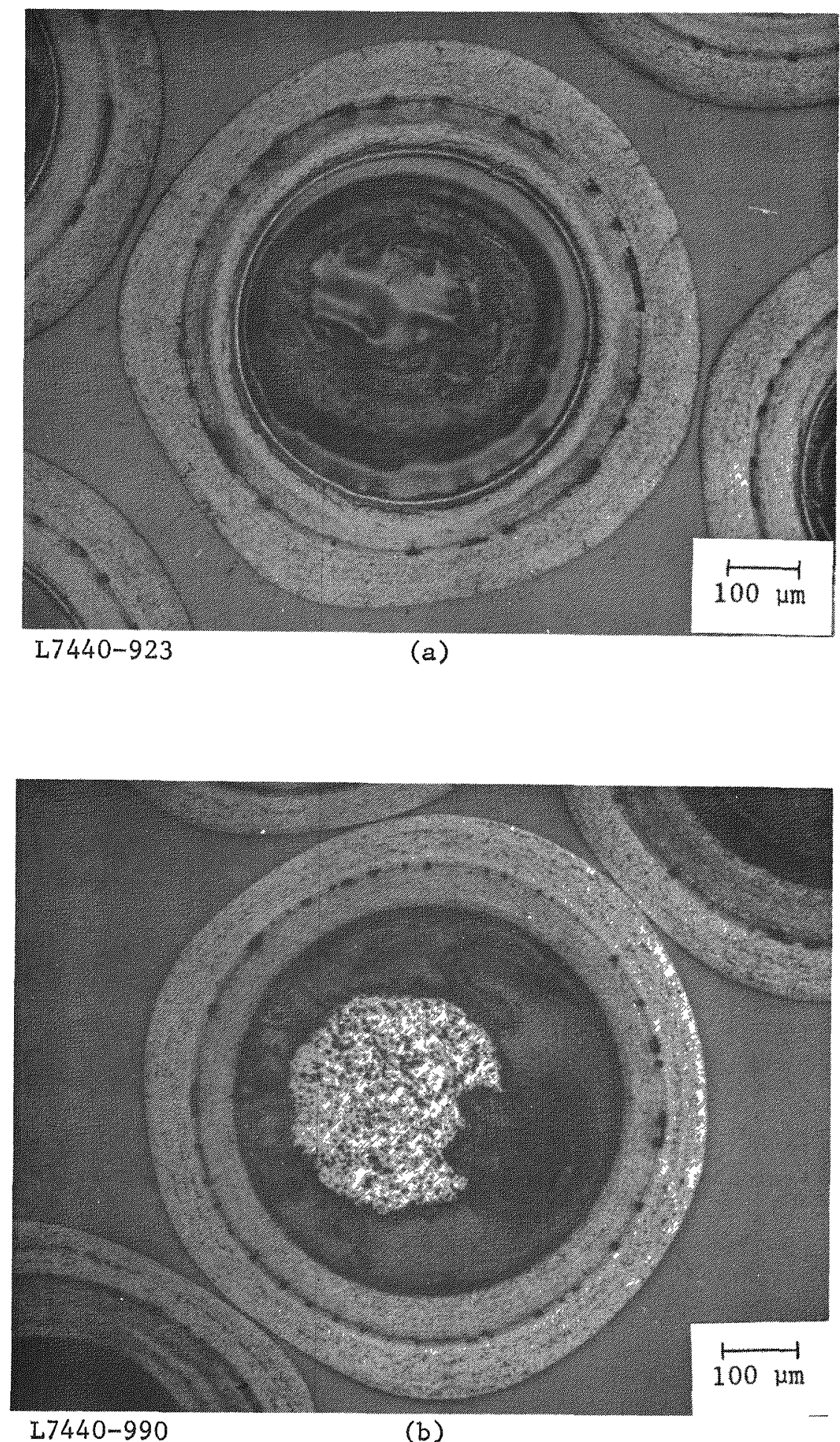

L7440-990

(b)

Fig. 4-118. Photomicrographs showing the condition of irradiated $\mathrm{BISO} \mathrm{ThO}_{2}$ particles after acid leaching. (a) Batch 6542-21-016-1 with an $\mathrm{OPYC}$ coating density of $1.73 \mathrm{~g} / \mathrm{cm}^{3}$ and a coating rate of $8.0 \mu \mathrm{m} / \mathrm{min}$ and (b) batch 6542-22-015-1 with an OPyC coating density of $1.80 \mathrm{~g} / \mathrm{cm}^{3}$ and a coating rate of $4.0 \mu \mathrm{m} / \mathrm{min}$. 


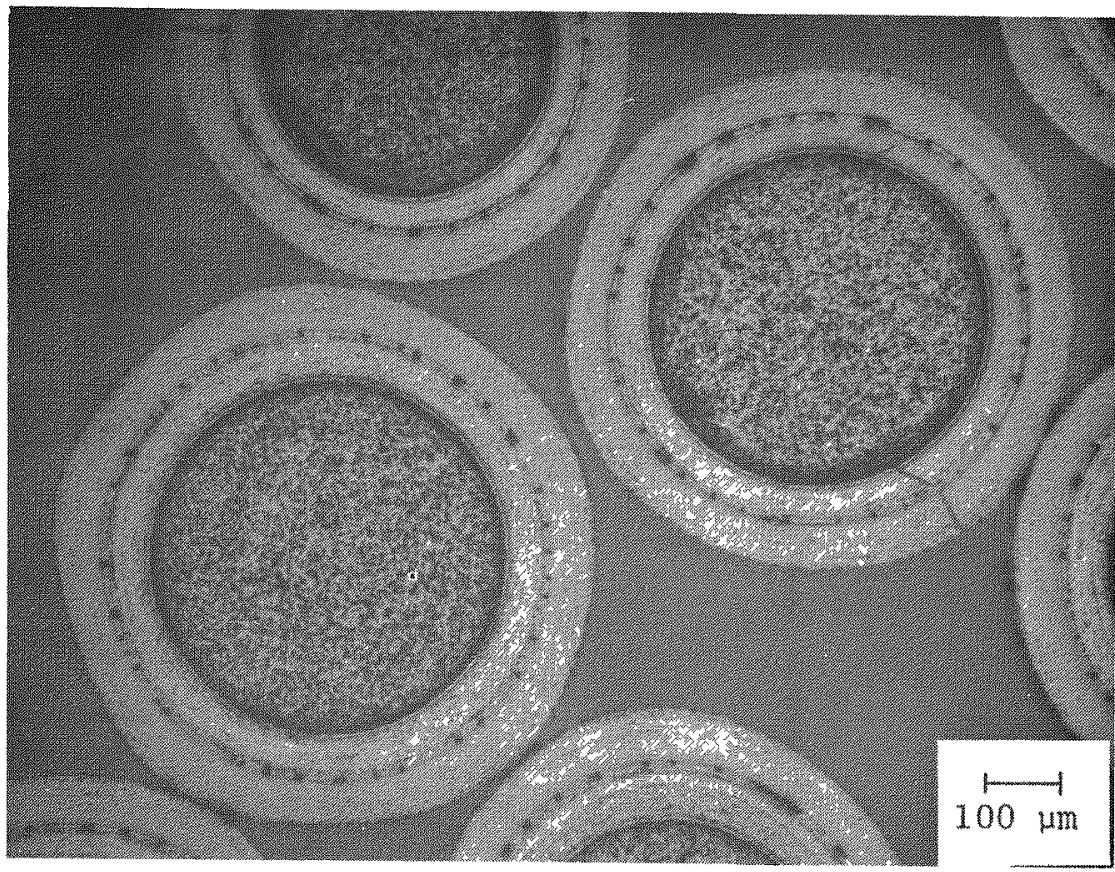

(a)

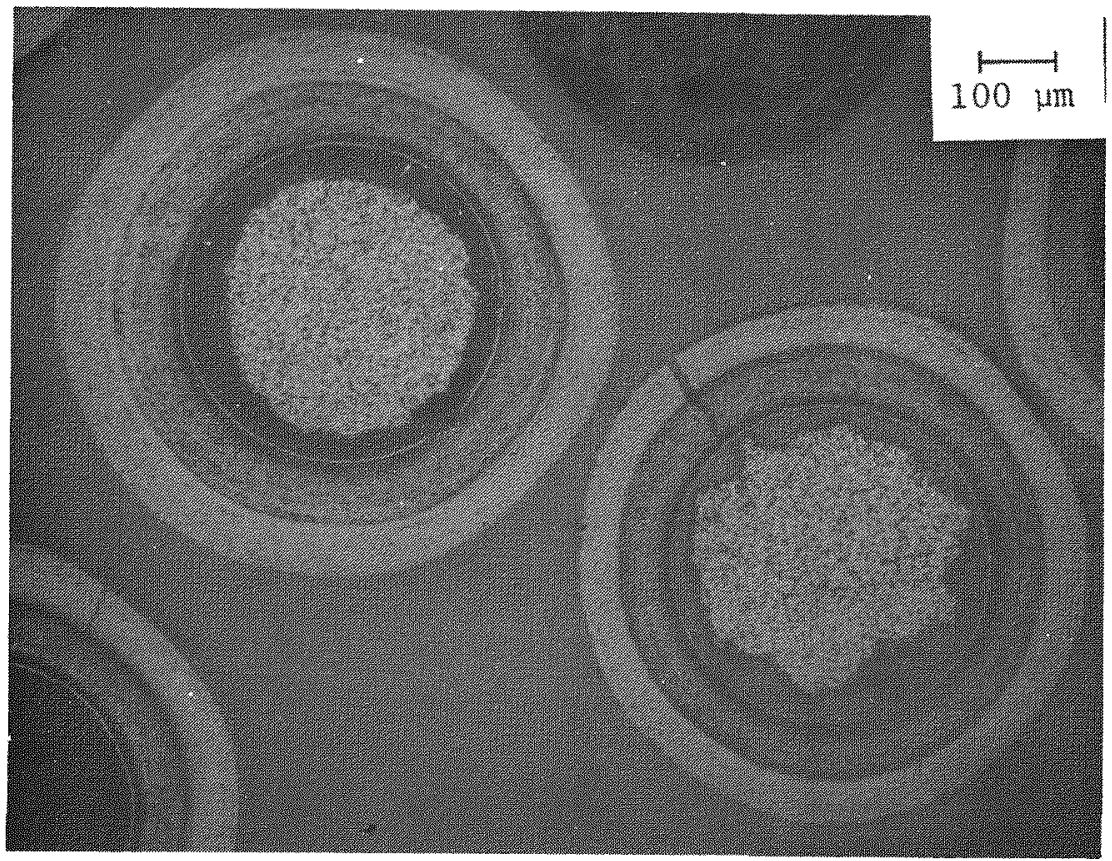

Fig. 4-119. Photomicrographs showing the condition of irradiated BISO $\mathrm{ThO}_{2}$ particles after acid leaching. (a) Batch 6542-24-015-1 with an OPyC coating density of $1.94 \mathrm{~g} / \mathrm{cm}^{3}$ and a coating rate of $6.6 \mu \mathrm{m} / \mathrm{min}$ and (b) batch 6542-02-020-2 with an OPyC coating density of $1.91 \mathrm{~g} / \mathrm{cm}^{3}$ and a coating rate of $8.5 \mu \mathrm{m} / \mathrm{min}$. 


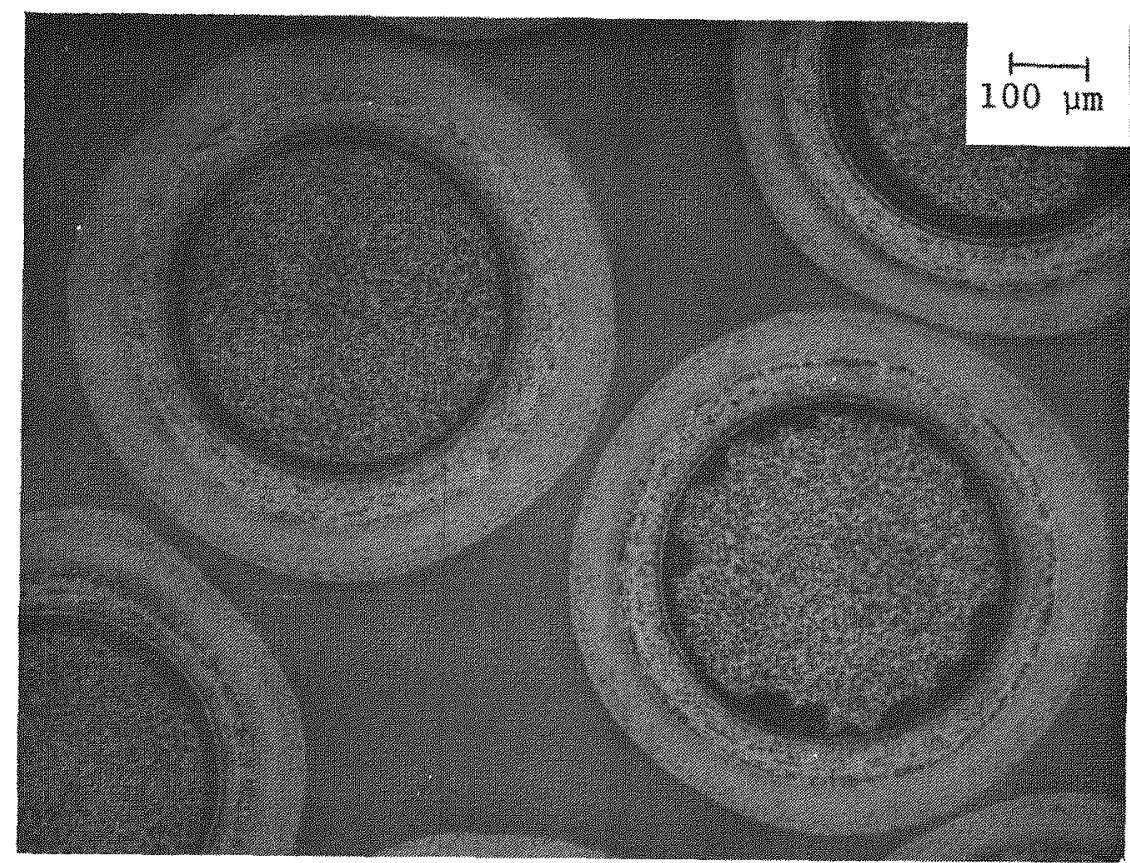

L7440-951

(a)

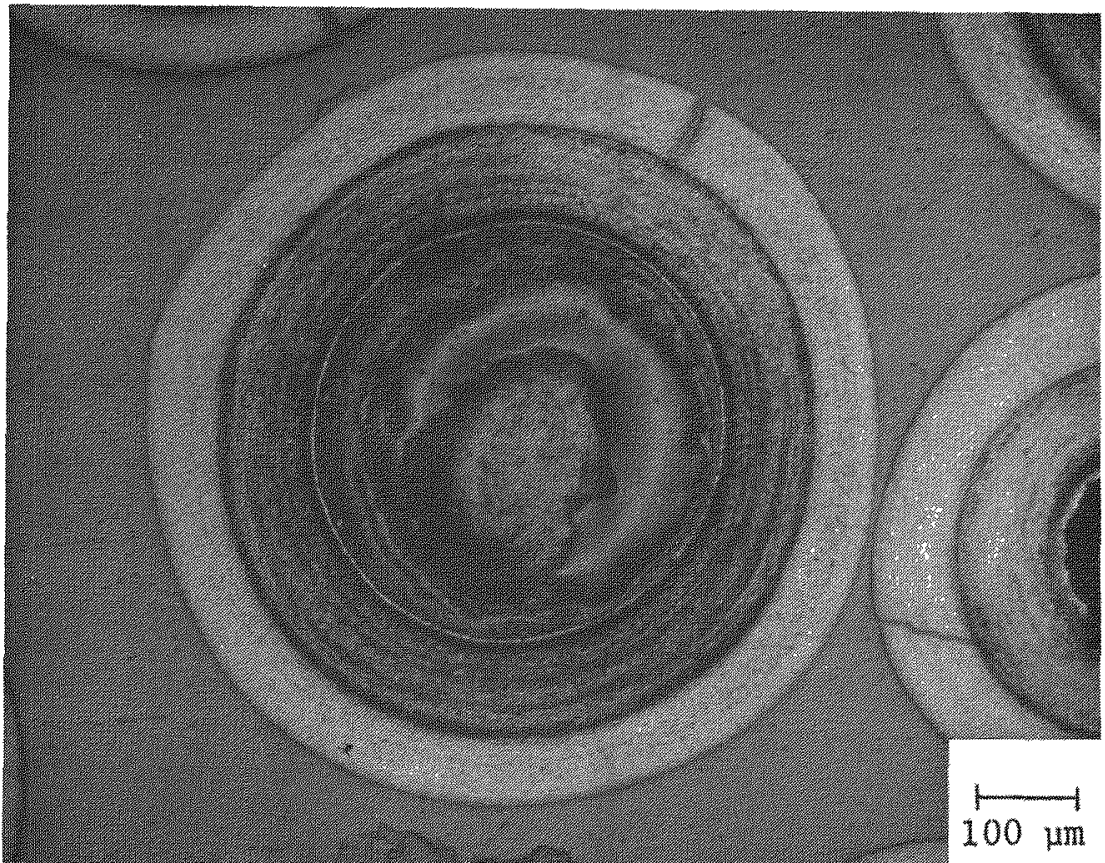

(b)

Fig. 4-120. Photomicrographs showing condition of irradiated $\mathrm{BISO} \mathrm{ThO}_{2}$ particles after acid leaching. (a) Batch 6542-09-010-2 with an OPyC coating density of $1.93 \mathrm{~g} / \mathrm{cm}^{3}$ and a coating rate of $5.0 \mathrm{\mu m} / \mathrm{min}$ and (b) batch 6542-01-020-2 with an OPyC coating density of $1.82 \mathrm{~g} / \mathrm{cm}^{3}$ and a coating rate of $2.7 \mu \mathrm{m} / \mathrm{min}$. 


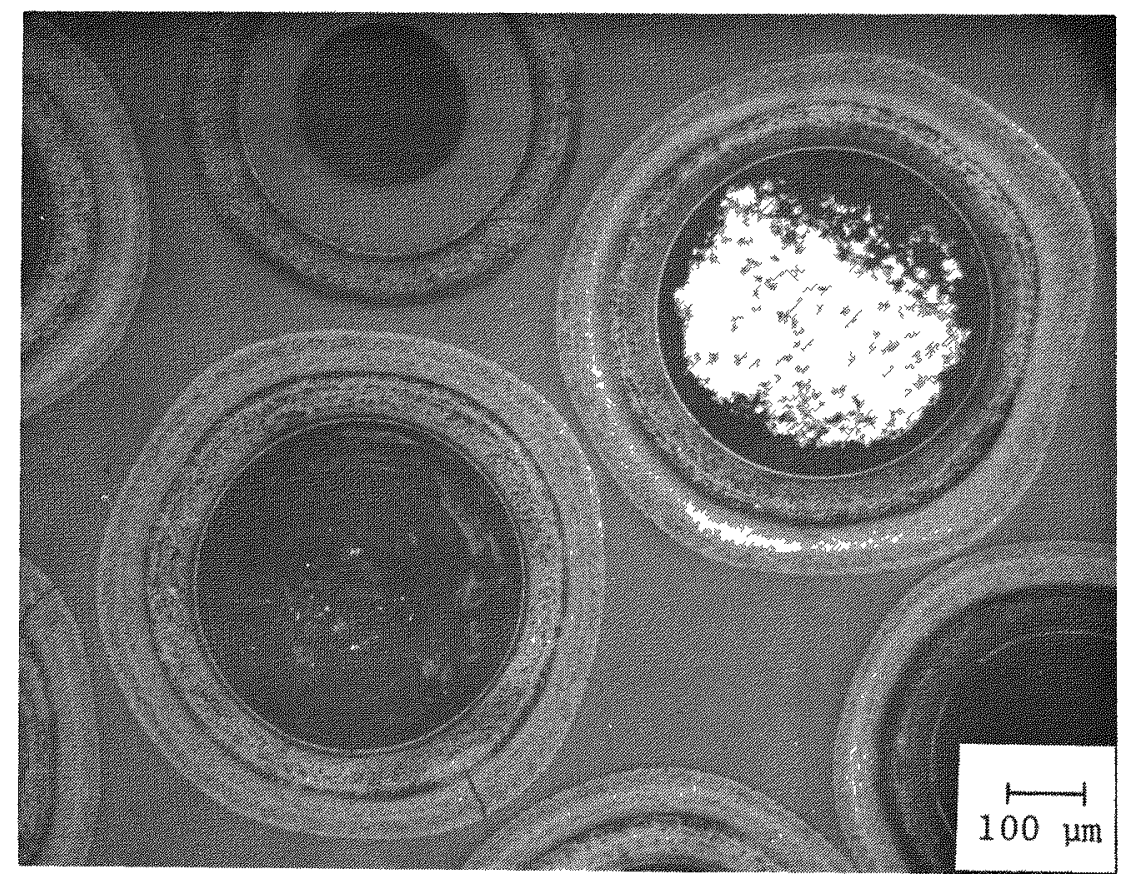

L7440-965

(a)

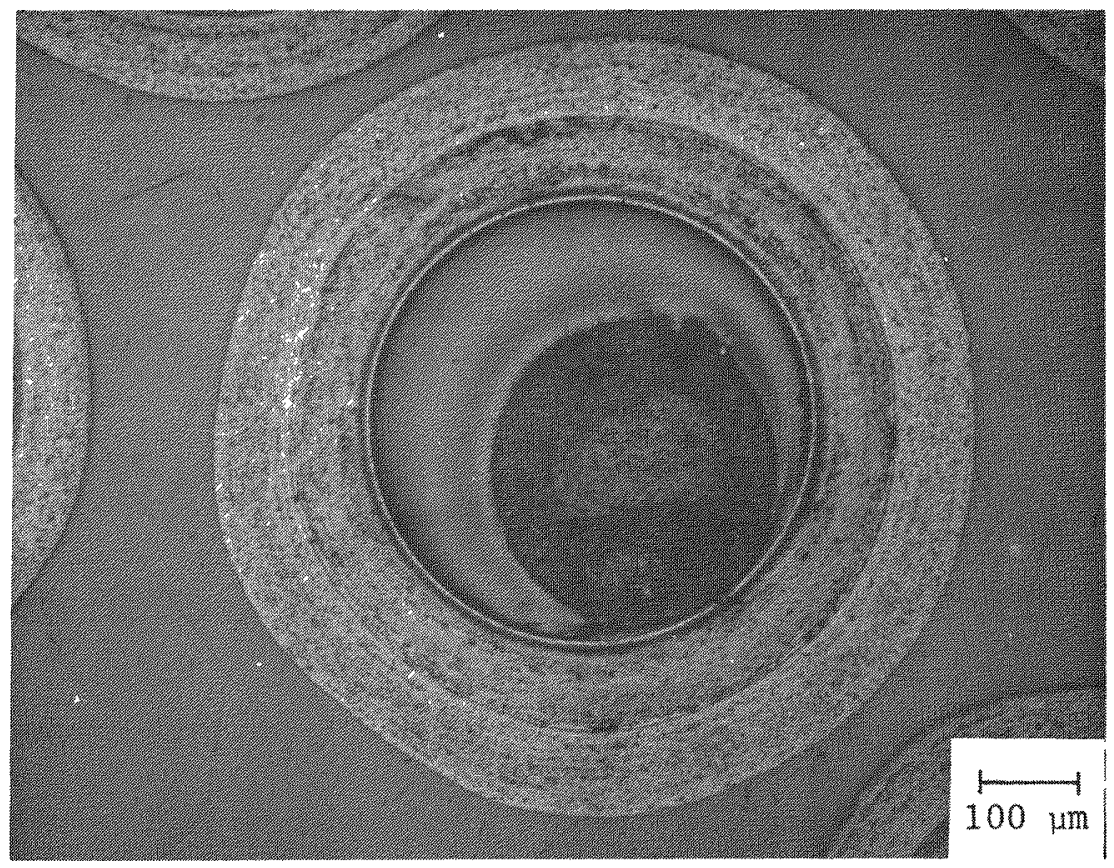

L7440-937

(b)

Fig. 4-121. Photomicrographs showing condition of irradiated $\mathrm{BISO} \mathrm{ThO}_{2}$ particles after acid leaching. (a) Batch 4252-06-018-1 with an OPyC coating density of $1.82 \mathrm{~g} / \mathrm{cm}^{3}$ and a coating rate of $5.8 \mu \mathrm{m} / \mathrm{min}$ and (b) batch 6542-01-010-1 with an OPyC coating density of $1.80 \mathrm{~g} / \mathrm{cm}^{3}$ and a coating rate of $10.0 \mu \mathrm{m} / \mathrm{min}$. 


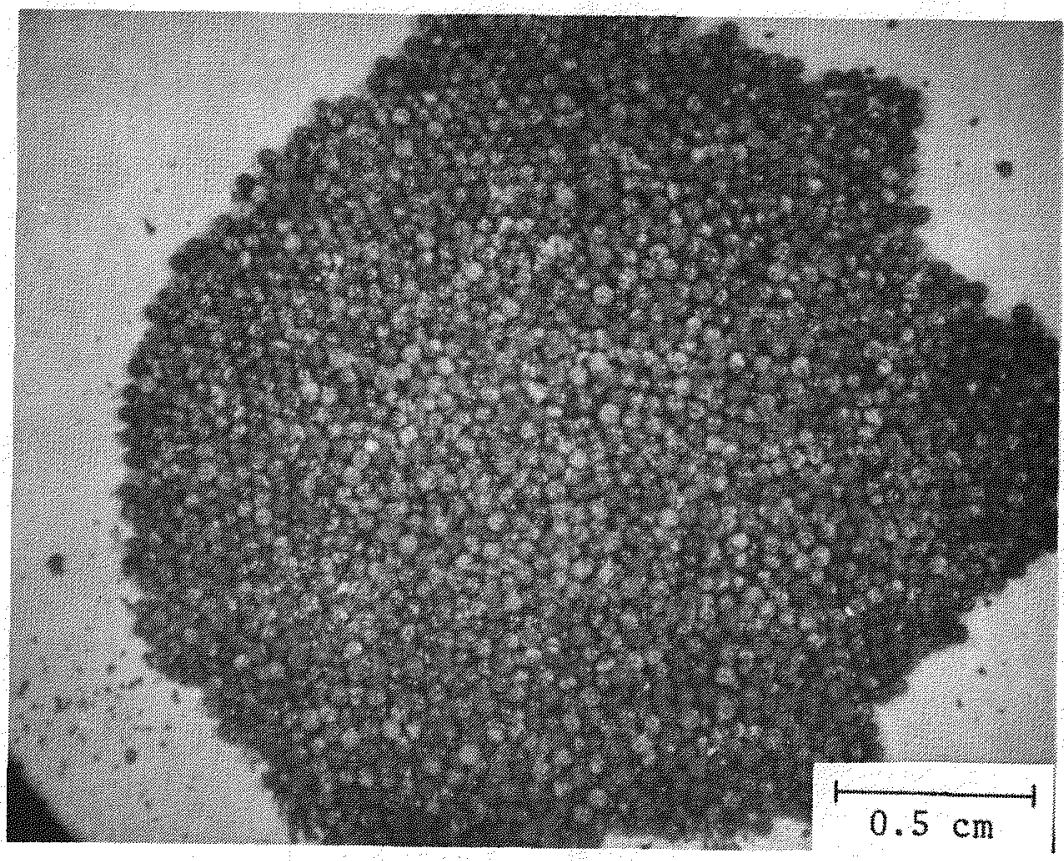

$57440-457$

(a)

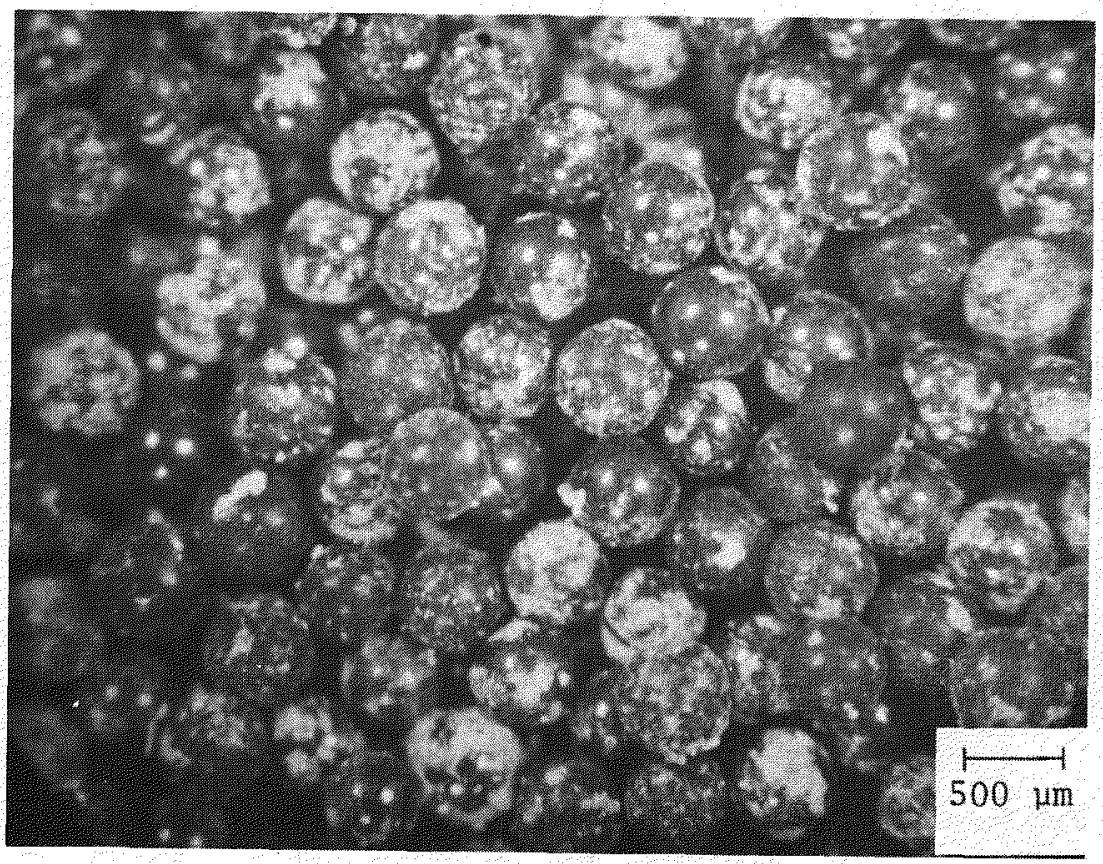

$57440-458$

(b)

Fig. 4-122. Photographs of fuel particles recovered from rod 7161-004-02-6 (P13R, 1B) after it was burned in air at $950^{\circ} \mathrm{C}$ for $6 \mathrm{hr}$ 


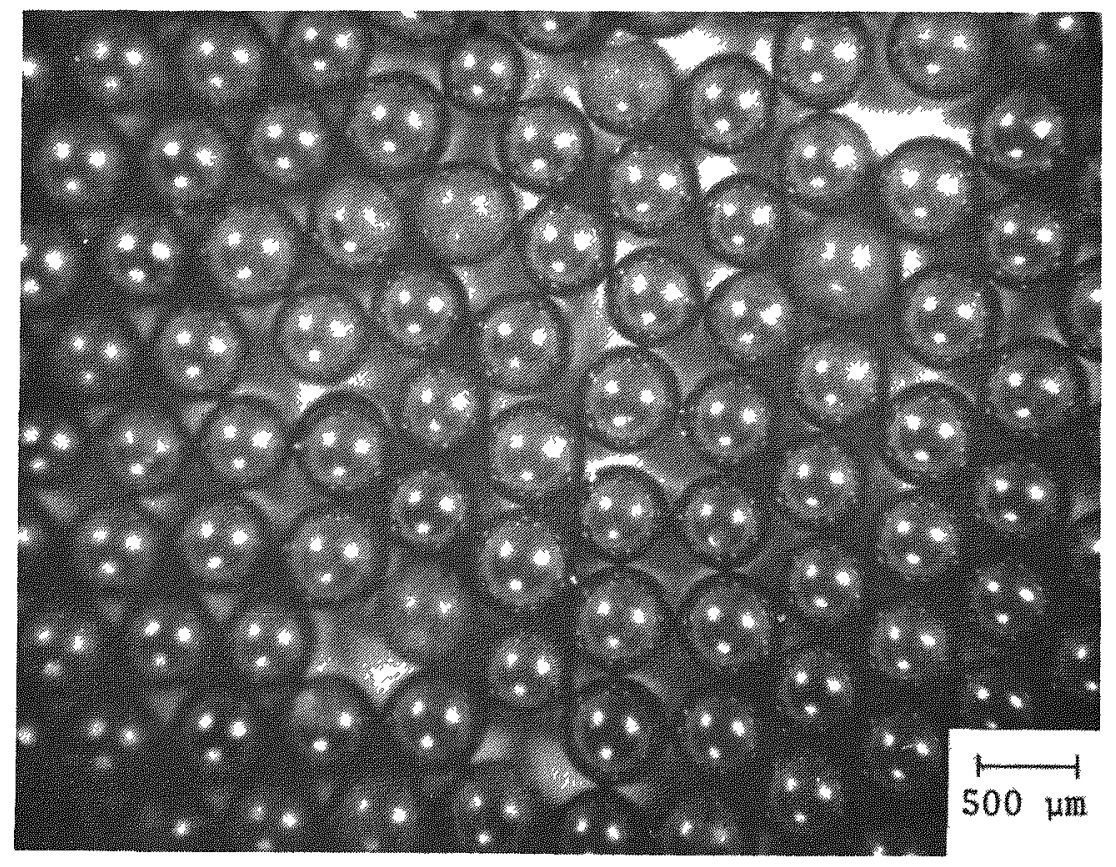

$57440-463$

(a)

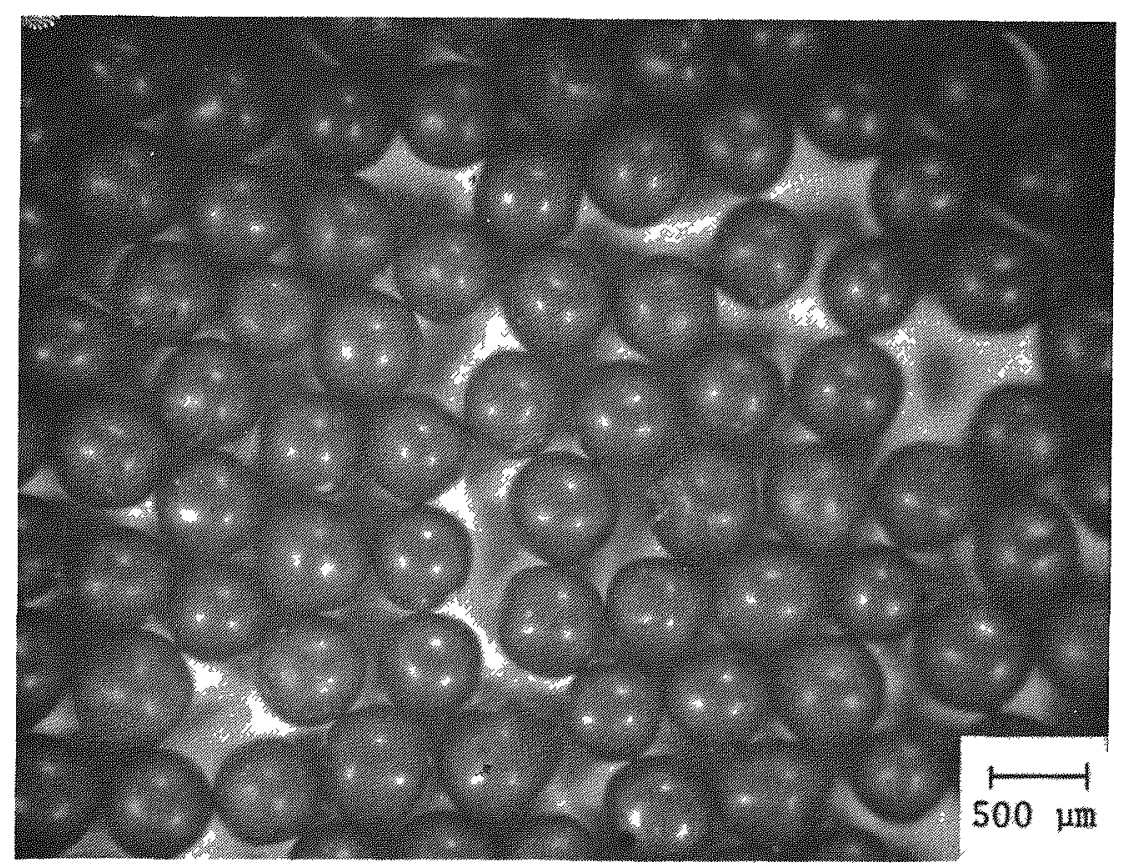

$57440-465$

(b)

Fig. 4-123. Photographs showing $\mathrm{ThO}_{2}$ kernels and $\mathrm{SiC}$ coated fissile particles recovered from fuel rod 7161-004-02-6 (P13R, 1B). The two particle types were segregated using a separatory funnel. (a) $\mathrm{ThO}_{2}$ kernels and (b) SiC coated fissile particles. 


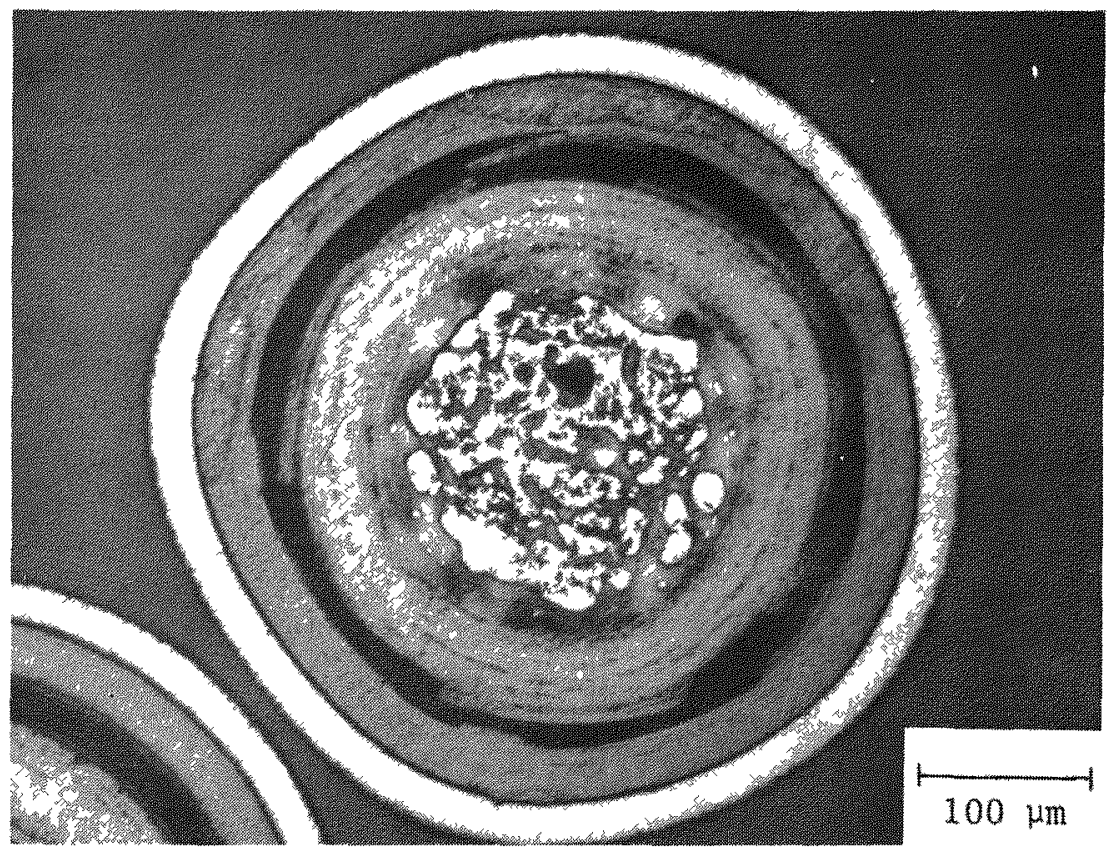

L7440-1002

Fig. 4-124. Photomicrograph showing condition of TRISO coated fissile particle from fuel rod 7161-004-11-6 (P13S, 2A). After the fuel rod was burned, the remnants were boiled in an acid solution for $15 \mathrm{hr}$. No SiC coating attack or failure due to the acid leaching was observed. 


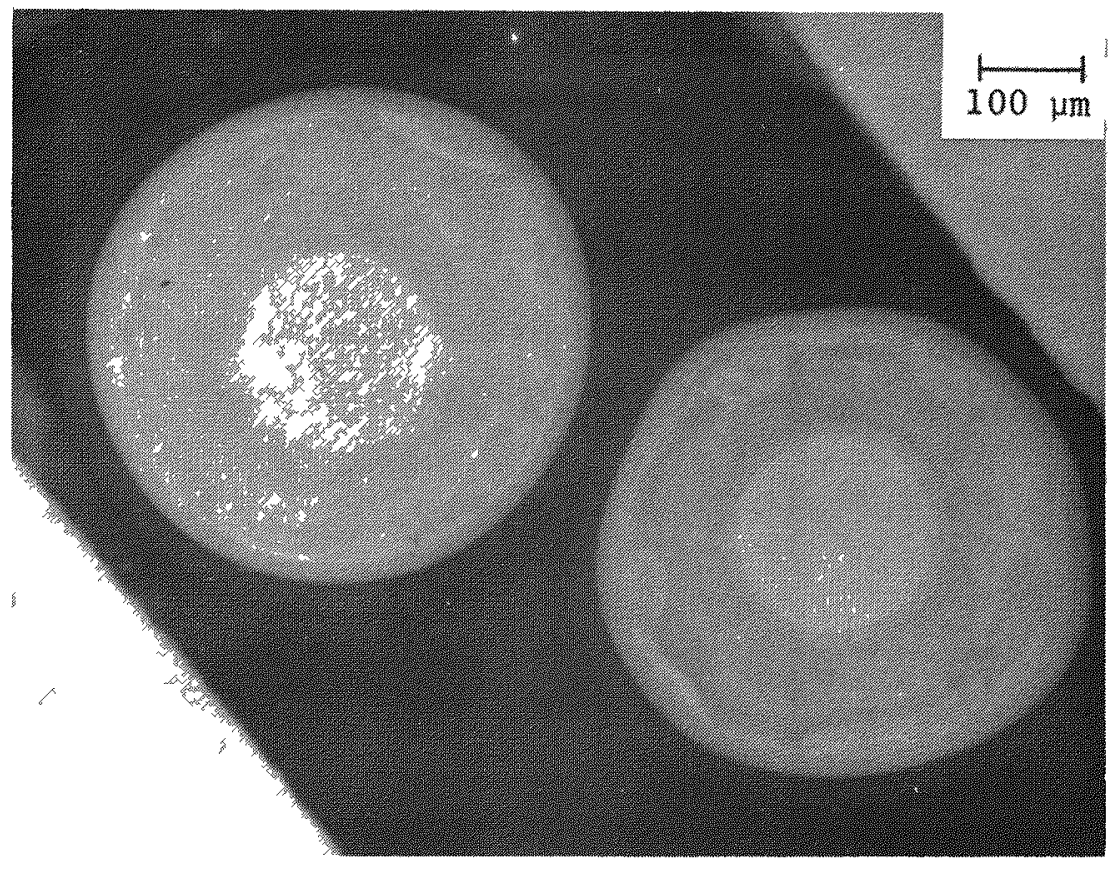

$\mathrm{HA}-372$

(a)

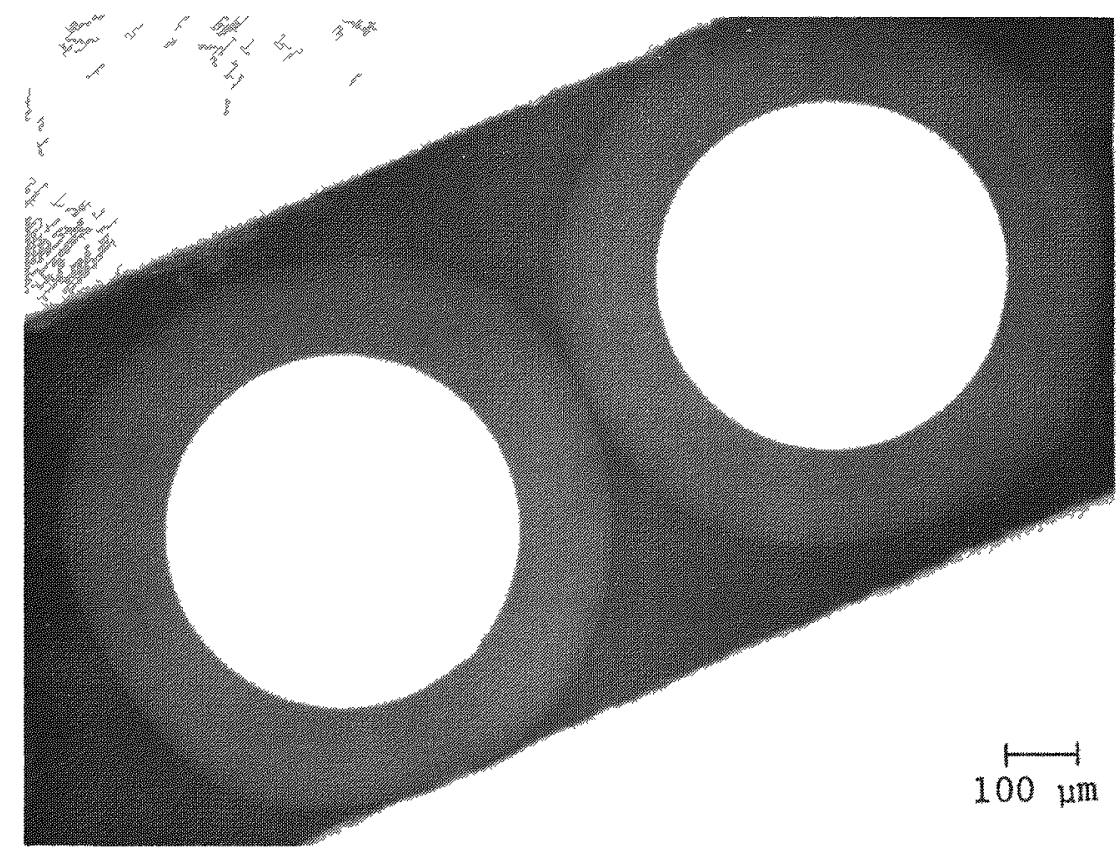

$\mathrm{HA}-364$

(b)

Fig. 4-125. Photographs taken of microradiographs of fissile and fertile particles. (a) Fissile particles irradiated in capsule P13R (position 3-5) and (b) fertile particles irradiated in capsule P13S (position 4-9). 
5. DISCUSSION

5.1. COATED PARTICLE IRRADIATION PERFORMANCE

5.1.1. Fissile Particles

5.1.1.1. Failure Analyses. Quantitative failure levels of the unbonded fissile particle samples were ascertained by visual examination (see

Section 4.2.2) and by fission gas release measurements (see Section 4.4.2). The results of these examinations are summarized in Table 5-1. The agreement between the pressure vessel failure levels observed during the visual examination and those calculated from the fission gas release data was excellent for 11 of the particle batches. Three batches (6151-00-010, 6151-02-025, and 6151-00-025) exhibited a high fraction of OPyC coating failure. An accurate assessment of pressure vessel failure (failure of the OPyC and SiC coating) could not be made during the visual examination because fragments of the failed OPyC coatings remained attached to the SiC substrate. Fission gas release measurements revealed pressure vessel failure levels of $4.0,6.5$, and $4.3 \%$ for these particle batches. Low levels of OPyC coating and pressure vessel failure were observed in the other three particle batches (6151-00-045, 6151-00-046, and 4161-01-21); however, the samples exhibited high fission gas release. The samples were reexamined visually after the TRIGA measurements and no significant additional particle failure had occurred. The reason for the large discrepancy between the observed and calculated failure levels for these three particle batches is not apparent from the results of these examinations.

Fissile particle failure levels determined during the metallographic examination are reported in Table 4-35. The irradiation performance of fissile particles tested in the fuel rods was similar to the performance 
of the unbonded particle samples. Failure levels determined by metallographic examination of the fuel rods were similar to the failure levels determined for the unbonded particle samples by visual examination and fission gas release measurements. There is a larger uncertainty in the metallography failure data, however, because only a small portion of the sample population was examined. Fission gas release measurements were made on each fuel rod. However, accurate fissile particle failure fractions could not be determined from the data because only a small fraction of the total fissions occurred in the fissile particles at end-of-life.

\subsubsection{Influence of Product and Process Variables}

5.1.1.2.1. OPyC Coating Rate, Anisotropy, and Density

The OPyC layers were deposited on the TRISO fissile particles using coating rates varying between 0.7 and $4.0 \mu \mathrm{m} / \mathrm{min}$. This produced coatings having a range of anisotropy values. This series also included particles having different coating densities. The objective of these tests was to determine the influence of these properties on OPYC coating performance at peak HTGR fluences. This section is concerned primarily with coating rate and anisotropy; however, the interaction of density with these variables is mentioned briefly.

At the time the P13R and P13S fissile TRISO samples were fabricated, coating rate was considered the primary process variable controlling coating anisotropy. Since previous irradiation results had shown a good correlation between coating performance and coating rate (Refs. 5-1 and 5-2), this parameter was a key factor in selecting samples for this test series.

Data for the nine different $\mathrm{UC}_{2}$ TRISO particle batches included in this study are given in Table 4-3 along with the results of the postirradiation examination. Each of these samples was irradiated in unbonded particle trays, and typically particles from each batch were tested in two or 
three trays. The OPyC failure levels are plotted in Fig. 5-1 as a function of the batch mean coating rate. The data show that the failures occurred for coatings having apparent deposition rates of 1 ess than $2 \mu \mathrm{m} / \mathrm{min}$. Also, onset of failure is shifted toward lower coating rates for the lower-density samples. These results are very consistent with data from earlier irradiation tests of fissile TRISO particles (Ref. 5-2), and indicate a dependence of OPyC failure on both coating rate and coating density. However, when care is taken to precisely measure PyC coating anisotropy values, there is a noticeable effect of density as well as coating rate (Fig. 5-2). Data presented in Fig. 5-2 were obtained using an optical anisotropy measuring apparatus developed by OSGAE (Osterreichischen Studien Gesellschaft für Atomenergie), Seibersdorf, Austria (Ref. 5-3). This instrument has greater accuracy and precision than most alternate methods used to characterize the particle coatings during their manufacture. It can be seen from Fig. 5-2 that at a given coating rate, the lower-density OPyC coatings have lower anisotropies. Plotting OPyC coating failure against BAF optical anisotropy values reveals that the initiation of failure occurs in batches having mean anisotropies around 1.02 and that failures increase as the batch mean $\mathrm{BAF}_{\mathrm{O}}$ increases (Fig. 5-3). There also appears to be a trend for the 1ow-density coatings to have higher failure levels at a given anisotropy. These two observations are in agreement with results from characterization studies of PyC irradiation behavior (Refs. 5-4 and 5-5) which show (1) coating stresses increase with increasing coating anisotropy and (2) higher-density pyrocarbons are stronger than lower-density ones.

These trends become even clearer when the particle performance data from the fuel rod tests are evaluated. These tests contained UC 2 TRISO particles having OPYC coatings with low to medium anisotropies and densities ranging between 1.76 and $1.95 \mathrm{~g} / \mathrm{cm}^{3}$. In addition, most of the rods contained fissile size (600- $\mu$ m diameter) TRISO inert particles which had lowdensity coatings that were moderately anisotropic (BAF $\sim 1.03$ ). OPyC failure levels were determined from metallographic examination (see Tables 4-35 and 4-37). The data have been plotted in Fig. 5-4. These plots show 
the importance of temperature as well as fluence on the coating irradiation performance. These results also magnify the effects of anisotropy and density on TRISO OPYC coating survival. Comparing Figs. 5-4a and 5-4b illustrates the influence of anisotropy for coatings having nearly identical densities. The coatings represented in Fig. 5-4a had relatively high anisotropies for their low density. These coatings behaved well at low temperature; however, as the irradiation temperature increased to $1250^{\circ} \mathrm{C}$ and above, their performance was poor. A fissile TRISO sample having an OPyC coating with nearly identical density but lower anjsotropy had better performance (Fig. 5-4b), although the extent of the OPyC failure is still considered excessive at the higher temperatures. The influence of density on performance is seen when Fig. 5-4c is compared with Figs. 5-4a and b. Particles having OPYC coating densities between 1.85 and $1.95 \mathrm{~g} / \mathrm{cm}^{3}$ had no failures in samples irradiated to full fluence at $1250^{\circ} \mathrm{C}$ and approximately $2 \%$ failure at $1350^{\circ} \mathrm{C}$. Since the samples irradiated at an average temperature of $1350^{\circ} \mathrm{C}$ had operated as high as $1600^{\circ} \mathrm{C}$ for a period of time, their performance was judged to be excellent.

In summary, the results from this series of unbonded particle and fuel rod tests have provided an excellent basis for setting the specification limit for the TRISO OPYC coating. Results from the unbonded particles irradiated at $1000^{\circ} \mathrm{C}$ illustrate the need to maintain low anisotropy values. Batch mean anisotropies of $<1.03 \mathrm{BAF}_{\mathrm{o}}$ values are required. These results are very consistent with tests conducted on fuel rods at similar temperatures. However, once the lowest practical anisotropy values have been achieved (i.e., the plateau in the coating rate - optical anisotropy curve, Fig. 5-2), it becomes necessary to restrict the range of allowable coating density to achieve satisfactory performance at higher temperatures. This conclusion is drawn from the high-temperature fuel rod tests, which show coatings having densities between 1.85 and $1.95 \mathrm{~g} / \mathrm{cm}^{3}$ exhibited the most satisfactory performance. Coatings having densities below approximately $1.75 \mathrm{~g} / \mathrm{cm}^{3}$ had excessive failure, even when the optical anisotropy values were very low ( $1.02 \mathrm{BAF}_{0}$ units). 


\subsection{OPyC Coating Irradiation-Induced Densification}

Postirradiation density measurements were made on OPyC coating fragments from nine fissile particle batches, as discussed in Section 4.10.1, and the data are sumarized in Table 4-50. The postirradiation densities of OPyC coatings with initial densities in the range of 1.76 to $1.82 \mathrm{~g} / \mathrm{cm}^{3}$ and 1.85 to $1.94 \mathrm{~g} / \mathrm{cm}^{3}$ are plotted in Fig. 5-5 as a function of fast neutron fluence. Also shown in the figure are the densification curves obtained from previous measurements on OPyC coatings irradiated to lower fast neutron fluences (Ref. 5-2). The degree of irradiation-induced densification of OPyC coatings with initial densities in the range of 1.76 to $1.82 \mathrm{~g} / \mathrm{cm}^{3}$ was in good agreement with the lower-fluence data. Postirradiation densities of the OPyC coatings having an initial density in the range of 1.85 to 1.94 $\mathrm{g} / \mathrm{cm}^{3}$ were consistent with studies by Kaae (Ref. 5-6) which indicate that high-density, unrestrained PyC densifies a small amount initially and then expands during subsequent irradiation.

\subsection{SiC Coating Performance}

The SiC coating is the primary load-bearing member of the TRISO coating as well as an absolute barrier to certain metallic fission products. Lowdensity $\left(<3.18 \mathrm{~g} / \mathrm{cm}^{3}\right) \mathrm{SiC}$ is undesirable as a coating material because it can contain free silicon, which has a high vapor pressure and forms lowmelting eutectic compounds. Also, low-density SiC shows a decrease in strength at temperatures above $1000^{\circ} \mathrm{C}$, whereas higher-density $\left(>3.18 \mathrm{~g} / \mathrm{cm}^{3}\right)$ SiC exhibits an increase in strength at these temperatures (Ref. 5-7). For these reasons, $\mathrm{SiC}$ coatings with densities $\geq 3.18 \mathrm{~g} / \mathrm{cm}^{3}(>99 \%$ theoretical density) are specified for TRISO particles.

The SiC coating density of fissile particles tested in capsules P13R and P13S was $23.18 \mathrm{~g} / \mathrm{cm}^{3}$. The irradiation performance of these coatings was good. Failure of the SiC coating in particles which suffered pressure vessel failure was attributed to other variables, such as faceted coatings, rather than to properties of the SiC coating. 
Tensile stresses in the OPyC coating generated by irradiation-induced densification help maintain the SiC coating in compression. Failure of the OPyC coating during irradiation would therefore be expected to increase the probability of SiC coating failure. To evaluate the effect of OPyC coating failure on SiC coating performance, the failure levels in several TRISO coated $U_{2}$ particle batches with coating parameters (except the OPyC coating) representative of LHTGR design requirements were analyzed. Particle batches that had a specific attribute which would be expected to increase the probability of pressure vessel failure, i.e., nonround kernels, faceted coatings, or interrupted SiC coatings, were not considered. The irradiation performance of these particle batches is summarized in Table 5-2. The same SiC substrate number for more than one particle indicates that one batch was split after deposition of the SiC coating and different OPyC coatings were applied. One batch that was split from each of the parent batches had the OPYC coating deposited at a very low coating rate in order to establish the specification limit for this parameter. These batches, 6151-02-025, 6151-01-015, and 6151-00-010, experienced OPyC coating failure levels of $46.3 \%, 100 \%$, and $32.8 \%$, respectively. Even though high levels of OPyC coating failure occurred, the total coating failure levels for these batches only ranged from 1.4 to $4.0 \%$. These results indicate the conservative nature of the LHTGR reference fissile particle design.

During fabrication, deposition of the SiC may be inadvertently interrupted. To determine whether it was acceptable to interrupt the SiC coating process if the final product met specification, two batches (6151-03-015 and 4161-01-021) of TRISO $\mathrm{UC}_{2}$ particles with interrupted SiC coatings were tested in capsule P13S. Results of the visual examination and fission gas release measurements performed on these two particle batches are summarized in Table 5-1. Batches 6151-03-015 and 4161-01-021 exhibited pressure vessel failure levels of 0.1 and $3.3 \%$, respectively, as determined by visual examination after irradiation to fast neutron fluences of 11.8 and $11.7 \times 10^{21} \mathrm{n} / \mathrm{cm}^{2}(\mathrm{E}>0.18 \mathrm{MeV})_{\mathrm{HTGR}}$. The equivalent failure leve1 of $26.9 \%$ determined from the fission gas release data for batch 
4161-01-021 was anomalous. The high release could have resulted from contamination pickup in the hot cell during handling, from microcracks in the particles that were not observed during the visual examination, or from failure induced during the TRIGA measurement. Visual examination of the batch after the TRIGA measurement indicated significantly higher failure levels than were originally observed. Batch 4161-01-021 had a high metal density (thin buffer coating) and severely faceted coatings. These two variables probably contributed more to the high failure observed in this batch than the presence of an interrupted SiC coating. The low failure level exhibited by batch 6151-03-015 indicated that an interruption in the SiC coating deposition may not be detrimental to coated particle irradiation performance. However, these limited results do not justify use of interrupted SiC coatings.

\subsection{IPyC Coating Performance}

The function of the IPyC layer of the $\mathrm{UC}_{2}$ TRISO particle is to protect the SiC layer from detrimental chemical reactions during manufacturing and irradiation. For manufacturing purposes a dense, impermeable coating is most desirable in order to prevent infiltration of gaseous by-products into the buffer during the SiC deposition step. Process studies have shown that the presence of C1-compounds in the buffer can cause extensive fuel dispersion. Although quantitative data relating the effect of fuel dispersion on particle performance are not available, the occurrence of such dispersion is specified to be kept to a minimum during fuel production (Ref. 5-8).

The IPyC layer also appears to act as a sink for some of the more mobile fission products during irradiation (see Section 5.1.1.2). Its presence may slow down the migration of these fission products (primarily the rare earths) or deter them from reacting with the SiC layer. However, under the more severe irradiation conditions these fission products do alter the structure of the IPyC layer (for example, see Fig. 4-94), and in 
some cases cause the IPyC layer to fail. Failure of the IPyC layer under these circumstances presumably should be independent of the physical properties of the PyC.

Results of metallographic examination of a large number of fissile particles tested in capsules P13R and P13S revealed that the majority of the IPyC layers survived. Only six of the 24 batches examined meta11ographically exhibited a significant fraction of cracked IPyC coatings (see Table 4-35). In no case was failure of the IPyC layer found to be detrimental to particle performance; i.e., no deleterious chemical reactions occurred as a result of the failure of this layer. A correlation of the failures with coating properties (density and anisotropy) indicated that the batches in which failure occurred had the most anisotropic coatings. These samples had optical anisotropy values (measurements made after the $\mathrm{SiC}$ and OPyC layers were deposited) of $>1.08 \mathrm{BAF}_{\mathrm{O}} \cdot$ Thus, if other tests show it is necessary to have the IPyC layer retain its integrity during irradiation, this anisotropy value (measured after the SiC deposition) should be a reasonable limit. Results from these tests do not dictate a need for this layer to remain intact; therefore, manufacturing considerations probably should influence property specification limits for this coating.

\subsection{Buffer Coating Performance}

The purpose of a low-density buffer coating immediately adjacent to the fuel kernel is to attenuate fission recoils and to provide a void volume to accommodate fission gases and fuel kernel swelling. The thickness and density of buffer coatings required for TRISO particles irradiated to peak conditions are interrelated and are a function of the fuel density in the particle and the coating design.

\footnotetext{
TRISO coated $\mathrm{UC}_{2}$ particles with buffer coating thicknesses ranging from 82 to $105 \mu \mathrm{m}$ and densities ranging from 0.87 to $1.31 \mathrm{~g} / \mathrm{cm}^{3}$ were tested
} 
in capsules P13R and P13S. During irradiation the buffer coatings decoupled from the IPyC coatings and densified around the fuel kernels. From 0 to $2.0 \%$ of the buffer coatings were cracked in the particles examined metallographically. The cracking was not a function of the buffer coating thickness or density. It occurred only in particles irradiated in the temperature range of $1000^{\circ}$ to $1100^{\circ} \mathrm{C}$ where the fuel kernel had segregated into a twophase structure as shown in Fig. 4-88. In some particles irradiated as unbonded particle samples, the structural coatings failed and the buffer coating remained on the fuel kernel as shown in Fig. 4-89. Results from capsules $\mathrm{P} 13 \mathrm{R}$ and $\mathrm{P} 13 \mathrm{~S}$ and from previous irradiation experiments will be analyzed using a TRISO particle stress model to establish the buffer coating thickness and density limits for the LHTGR fuel specifications.

Less than $1.0 \%$ of the buffer coatings were cracked in the TRISO $(1 \mathrm{Th}, 1 \mathrm{U}) \mathrm{O}_{2}$ particles (5466-37) with 250- $\mu \mathrm{m}$-diameter kernels. However, $88.0 \%$ of the buffer coatings were cracked in the TRISO ( $8 \mathrm{Th}, 1 \mathrm{U}) \mathrm{O}_{2}$ particles (6155-01-020) with larger (500 $\mu \mathrm{m})$ diameter kernels. Batch 6155-01-020 had a 5- $\mu \mathrm{m}$-thick seal coat which allowed the buffer coating to decouple from the IPyC coating during irradiation and shrink onto the fuel kernels. Cracking of the buffer coating occurred as a result of buffer densification and fuel kernel swelling. In contrast, the TRISO $\mathrm{ThO}_{2}$ particles with 500- $\mu \mathrm{m}$-diameter kernels (batch 6252-00-025) exhibited buffer cracking in only $11.0 \%$ of the particles. This batch did not have a seal coat, and the buffer coatings remained bonded to the IPyC coating. Buffer cracking occurred only in the particles that debonded from the IPyC coating and shrank onto the fuel kernel. Buffer coating density may also have had an effect on the levels of cracking observed. Batch 6155-01-020 had a buffer density of $1.24 \mathrm{~g} / \mathrm{cm}^{3}$, whereas batches 5466-37 and 6252-00-025 had buffer densities of 1.12 and $1.16 \mathrm{~g} / \mathrm{cm}^{3}$, respectively. Higher-density buffer coatings may be more susceptible to irradiation-induced densification and cracking. 


\subsection{Faceted Particles}

Severely faceted particles would be expected to have a higher probability of failure than more spherical particles because the smaller radius of curvature would enhance the stresses in the coating. Two batches (6151-00045 and 6151-00-025) of fissile particles were tested to evaluate the effect of faceting on TRISO particle performance. In addition, one sample (6151-00-046) of severely misshaped particles segregated from batch 6151-00-045 was also irradiated. Particles from each of these three samples were tested as unbonded particle samples, and particles from batch 6151-00045 also were tested in six fuel rods. Results from metallographic evaluation (Table 4-35) and from visual and fission gas release evaluations (Table 5-1) all show that these particles performed poorly.

Fission gas release measurements revealed that batches 6151-00-045, 6151-00-046, and 6151-00-025, tested as unbonded particle samples, experienced failure levels of $19.1,14.0$, and $6.5 \%$, respectively. No failure was observed in batch 6151-00-045 when it was irradiated in fuel rod 7161-00406-5 at $1050^{\circ} \mathrm{C}$; however, this batch experienced $15.3 \%$ failure in rod $7161-$ 004-01-7, which was thermal cycled up to $1650^{\circ} \mathrm{C}$ in-pile. These results indicate that severely faceted TRISO particles are more susceptible to pressure vessel failure during irradiation than similarly designed, nonfaceted fuel particles (e.g., compare with results of batches 6151-09-025 and 6151-00-035). However, the effect of faceting on the irradiation performance of these particle batches could not be evaluated quantitatively because both particle batches had other attributes that would have been expected to contribute to the failure levels observed. Batch 6151-00-045 was non-conservatively designed since it had a buffer coating thickness of $85 \mu \mathrm{m}$, and batch 6151-00-025 had an anisotropic OPyC coating (deposited at a coating rate of $1.4 \mathrm{\mu m} / \mathrm{min}$ ) which resulted in a high level of OPyC coating failure, independent of the pressure vessel failure observed in the particle batch. 


\subsubsection{Thermochemical Stability}

\subsection{Meta11ic Fission Product Interactions}

Metallic fission products diffuse from $\mathrm{UC}_{2}$ kernels during irradiation at high temperatures. In TRISO particles concentrations of metallic fission products build up in the IPyC coating on the cooler side of the fuel particles and subsequently react with the SiC coating at the SiC/IPyC coating interface. Reactions of this type have been observed in $\mathrm{UC}_{2}$ TRISO particles irradiated in capsules P13L, P13M, P13N, and P13P. Electron microprobe analysis revealed the only heavy metals preferentially diffusing out of the fuel kernel to the SiC coating were the rare earth fission products cerium, neodymium, lanthanum, samarium, praseodymium, and europium (Ref. 5-2).

Slight degradation of the SiC coating by metallic fission products was observed in TRISO $\mathrm{UC}_{2}$ particles irradiated in capsule P13R and P13S fuel rods at average temperatures above $1050^{\circ} \mathrm{C}$. The extent of attack increased with increasing temperature. A photomicrograph of fission product attack in a $\mathrm{UC}_{2}$ TRISO particle irradiated in fuel rod 7161-004-18-5 at an average centerline temperature of $1335^{\circ} \mathrm{C}$ is shown in Fig. 4-94. In this particle the buildup of metallic fission products at the SiC/IPyC coating interface was large enough to mechanically force the IPyC coating away from the SiC coating. A summary of the metallic fission product interactions observed in $\mathrm{UC}_{2}$ TRISO particles during the metallographic examination (see Section 4.6.2) is given in Table 4-39. In particles where metallic fission products reacted with the SiC coating, maximum depths of penetration into the SiC coating generally ranged from 2 to $5 \mu \mathrm{m}$. No coating failure was observed as a result of metallic fission product interaction. Although concentrations of metallic fission products were observed in the IPyC coatings, no SiC coating reaction was observed in the $\mathrm{UC}_{2}$ TRISO unbonded particle samples which were irradiated at lower temperatures than the fuel rods. No metallic fission product interactions were observed in the TRISO (Th, U) $\mathrm{O}_{2}$ particles. This observation is consistent with previous results which indicated that in oxide fuel particles, 
the rare earth metallic fission products are retained in the fuel kernel in the form of stable oxides.

Metallic fission product attack in TRISO $\mathrm{UC}_{2}$ particles is dependent on the concentration of fission products in the particle or fission density, time, and temperature. The kinetics of this reaction are not well known. Consequently, extensive out-of-pile annealing studies are being conducted to evaluate the degradation of TRISO coated fissile particles by metallic fission products. In order to provide irradiation data that can be used in conjunction with out-of-pile postirradiation heating data to determine the kinetics of metallic fission product attack, a more quantitative assessment has been made of reactions observed in some of the particles (see Table 5-3). The depth of fission product penetration in the SiC coating of these particles was measured from high-magnification photomicrographs taken of localized regions of SiC attack. The location of each particle with respect to the fuel rod centerline was established. An average irradiation temperature and temperature gradient for each particle were calculated using the following equations:

$$
\begin{aligned}
T & =T_{C \cdot L \cdot}-b x^{2}, \\
\frac{\Delta T}{\Delta x} & =-2 b x, \\
b & =+\frac{\Delta T(F \cdot R \cdot)}{r^{2}},
\end{aligned}
$$

where

$$
\begin{aligned}
\mathrm{T}= & \text { particle irradiation temperature }\left({ }^{\circ} \mathrm{C}\right) \\
\mathrm{T}_{\mathrm{C} . \mathrm{L} .}= & \text { average fuel rod centerline irradiation temperature }\left({ }^{\circ} \mathrm{C}\right) \\
\mathrm{x}= & \text { distance of particle from fuel rod centerline }(\mathrm{cm}) \\
\Delta \mathrm{T} / \Delta \mathrm{x}= & \text { temperature gradient in fuel rod at location of particle } \\
& \left({ }^{\circ} \mathrm{C} / \mathrm{cm}\right) \\
\mathrm{r}= & \text { radius of fuel rod }(\mathrm{cm}) \\
\Delta \mathrm{T}\left(\mathrm{F} \cdot \mathrm{R}_{0}\right)= & \text { time-averaged temperature drop from fuel rod centerline to } \\
& \text { surface }
\end{aligned}
$$


These temperatures and temperature gradients were calculated using timeaveraged irradiation conditions and therefore only approximate the temperature history of the fuel particles.

\subsection{Fuel Kernel Migration}

During operation at high temperatures, carbide fuel kernels migrate into the surrounding $\mathrm{PyC}$ coatings under the influence of a temperature gradient. Kernel migration up the temperature gradient results from carbon transport in the fuel phase and is characterized by a rejected graphite layer on the cool side of the kernel. The temperature and thermal gradient dependence of carbide fuel kernel migration and its impact on HTGR core thermal design have been discussed in Ref. 5-9.

Fuel kernel migration was observed in TRISO $\mathrm{UC}_{2}$ and TRISO (1Th, $\left.1 \mathrm{U}\right) \mathrm{O}_{2}$ particles irradiated in the high-temperature (cell 5) fuel rods of capsules P13R and P13S. A quantitative evaluation of kernel migration in six fue1 rods was made, as discussed in Section 4.6.2, and the data are presented in Table 4-40.

Time-averaged particle temperatures and temperature gradients were calculated using Eqs. 5-1, 5-2, and 5-3 for the particles noted in Table 4-40 that exhibited kernel migration. Kernel migration coefficients calculated for the $\mathrm{UC}_{2}$ TRISO particles were in good agreement with migration data obtained for $\mathrm{UC}_{2}$ TRISO particles irradiated in capsule P13N (Ref. 5-9). The use of time-averaged irradiation temperatures provides only approximate kernel migration coefficients because the rate of migration follows an Arrhenius type of dependence rather than a linear temperature dependence. It is concluded from a comparison of the in-pile data with kernel migration coefficients obtained from out-of-pile annealing studies on unirradiated and irradiated fuel particles that the rate of kernel migration is not significantly enhanced during irradiation. 
To compare fuel kernel migration rates observed in capsules P13R and P13S with rates predicted for LHTGR operation, fuel performance subroutines which are similar to those contained in the SURVEY code were incorporated into the thermal analysis CAPTEM code. The SURVEY code is used in LHTGR core thermal design to predict the extent of kernel migration in an LHTGR core at any time during operation. Using the $\mathrm{UC}_{2}$ kernel migration coefficient design curve, the CAPTEM code predicted the fuel kernel migration distance in 10 equally divided annular rings of a fuel rod for the 5,50 (nomina1), and $95 \%$ confidence levels on a daily basis over the operating life of the capsules. The total predicted migration distance at the end of irradiation as a function of the distance from the fuel rod centerline was obtained for each rod. The predicted migration distances for kernels in fuel rods 7161-004-18-5, 7161-004-19-7, and 7161-004-19-5 are plotted in Figs. 5-6, 5-7, and 5-8, respectively, as a function of radial location in the fuel rod. The solid line represents the predicted migration distance using the mean of the $90 \%$ confidence level, and the dashed lines represent the predicted migration distance using the range at the $90 \%$ confidence 1 evel of the kernel migration coefficient design curve. Also plotted are the measured migration distances from Table 4-40.

There was good agreement between the measured and predicted kernel migration distances. The scatter in the observed migration distances results from a number of factors, as discussed in Ref. 5-9. The migration distance of $6.0 \mathrm{\mu m}$ measured for one particle in fuel rod 7161-004-19-7 (Fig. 5-7) is significantly greater than the predicted value. This occurred because the particle was located at the end of the fuel rod on the centerline. The direction of migration was up the fuel rod centerline, which indicates a steep axial thermal gradient in the rod. The CAPTEM code used in thermal analysis takes into account two-dimensional (radial and axial) effects in calculating the radial temperature distribution in the fuel rod; however, axial temperature distributions are not calculated. Consequently, migration distances based on a radial temperature distribution were calculated and the severe axial temperature gradient was not taken into account. Grafoil discs were placed between the fuel rods; 
consequently, the end thermal effects were more severe than would be expected for fuel rods in the LHTGR. Fuel kernel migration was not detected in fuel rod 7161-004-28-3 (Table 4-40). This observation is consistent with the maximum migration distance of $0.03 \mu \mathrm{m}$ predicted by the CAPTEM code.

Kernel migration was also observed in TRISO (Th, U) $\mathrm{O}_{2}$ particles, and the data are reported in Table 4-40. Design kernel migration coefficient curves were not available for $(\mathrm{Th}, \mathrm{U}) \mathrm{O}_{2}$ particles. Therefore, in-pile migration distances were not calculated. However, the observed ( $\mathrm{Th}, \mathrm{U}) \mathrm{O}_{2}$ kernel migration rates were greater than the $\mathrm{UC}_{2}$ migration rates. This result was expected based on available migration data.

\subsubsection{Fertile Particles}

5.1.2.1. Failure Analyses. Quantitative failure levels of the unbonded fertile particle samples were ascertained by visual examination (see Section 4.2.2) and by fission gas release measurements (see Section 4.4.2). The results of these examinations are summarized in Table 5-4. The inservice failure levels determined from the fission gas release data were generally higher than the total coating failure levels determined during the visual examination. This discrepancy was attributed to the partial permeability of lower-density OPyC coatings to gaseous fission products, as discussed in Sections 4.7 and 5.1.2.2.3. As a result of this phenomenon, the failure analysis performed by visual examination provided the more accurate measure of pressure vessel failure in the unbonded fertile particle samples.

Failure levels of fertile particles tested in fuel rods were determined by metallography and from fission gas release data. The results of these analyses are presented in Table 5-5. There is a large uncertainty in the metallography failure data because only a small sample population was examined. Metallography data on fuel rods 7161-004-03-5 and 7161-004-16-5 are not presented in Table 5-5 because the examination was performed after the fuel rods were heated to $1600^{\circ} \mathrm{C}$ in the TRIGA facility. Evaluation of fuel failure levels in these rods is discussed in Section 5.1.3. Maximum 
fertile particle failure levels were calculated using fission gas release data by assuming all the fission gas released during the TRIGA measurement came from failed fertile particles. This is a good approximation since more than $80 \%$ of the total fissions occurred in the fertile particles during the TRIGA measurement, with the exception of fuel specimens from cel1 6 , and metallographic examination revealed low fissile particle failure levels (Table 4-36).

There was good agreement between the failure levels observed during the metallographic examination and the levels calculated from the fission gas release data. In 11 of the 15 rods examined metallographically, the failure level determined from the R/B data was within the $95 \%$ confidence interval for failure observed metallographically. Fuel rod 7161-004-20-5 was the only rod for which the maximum failure level calculated from the $\mathrm{R} / \mathrm{B}$ data was greater than the upper $95 \%$ confidence level calculated on the basis of metallographic observations.

Comparison of failure levels revealed that the irradiation performance of fertile particles tested in fuel rods was similar to the performance of the unbonded particle samples. However, there was a discrepancy in the failure levels determined from the R/B data for two particle batches. Failure levels of 6.0 and $12.0 \%$ were determined from $R / B$ data for batches 6542-01-010 and 6542-19-015, respectively, tested as unbonded particle samples. However, in 12 fuel rods maximum failure levels ranging from only 0 to $2.1 \%$ were determined for these batches based on $R / B$ data. The high fission gas release measured for the unbonded particle samples was attributed to the permeability of the OPYC coatings since (1) the coatings were of relatively low density, (2) they had high coating rates, and (3) low failure levels were observed during the visual examination. The permeability of OPYC coatings is discussed further in Section 5.1.2.2.2.

Based on these results the fission gas release measurements gave a fairly accurate measure of pressure vessel failure that occurred in the fertile particles tested in the bonded fuel rods. However, there is a 
level of uncertainty of at least a factor of two in the reported failure fractions because of the assumptions made in calculating the $R / B$ failure levels and the effects of OPyC coating permeability on gaseous fission product release.

\subsubsection{Influence of Property and Process Variables}

\subsection{OPyC Coating Rate, Anisotropy, and Density}

An analysis similar to that presented in Section 5.1.1.2.1 for the behavior of fissile particle OPyC coatings was performed for the $\mathrm{ThO}_{2} \mathrm{BISO}$ fertile particles. Thirteen different batches were included in this evaluation (see Tables 4-4 and 4-5). All of these batches had kernel diameters and buffer and OPyC coating thicknesses within the limits of the current HTGR Fuel Product Specification (Ref. 5-8). OPyC coating rates ranged between 2.2 and $10.0 \mu \mathrm{m} / \mathrm{min}$ and densities ranged between 1.73 and 1.94 $\mathrm{g} / \mathrm{cm}^{3}$. Batch mean $B A F$ values varied from about 1.02 to 1.04 .

A plot of OPyC optical anisotropy as a function of coating rate (Fig. 5-9) did not reveal as good a correlation as was found for fissile particle outer coatings (see Fig. 5-2). It should be noted that within some batches, significant variation in BAF。 values was obtained. For example, in batch 6542-09-010 the maximum and minimum values were 1.06 and 1.02, respectively; the batch mean $B A F$ was 1.04 . Since this batch apparent1y had a moderately high coating rate, $5.0 \mu \mathrm{m} / \mathrm{min}$, one would expect the $\mathrm{BAF}_{\mathrm{o}}$ values to be much lower and have an insignificant amount of scatter.

The reason for the poor correlation between anisotropy and coating rate is not known at this time. Since these coatings are much thicker than the OPyC layers on the TRISO fissile particles, one might suspect gradients in density and/or anisotropy to be the cause for part of the scatter. Also, a contributing factor could be related to the fact that these measurements were not made at the same time as those for the fissile particles. Since 
these measurements require care and precision, part of the scatter could be introduced by different techniques employed in gathering the data. Both of the above possibilities will be investigated further.

Plotting OPyC failure levels as a function of apparent coating rate and optical anisotropy $\left(\mathrm{BAF}_{\mathrm{o}}\right.$ ) reveals correlations much like those for TRISO fissile particle OPyC coatings. In the case of coating rate (Fig. 5-10a), onset of failure appears to begin around $4 \mu \mathrm{m} / \mathrm{min}$, at least for the low-density samples. The correlation between anisotropy and failure relies heavily upon samples 6542-01-020 and 6542-02-030, since these were the only two batches which exhibited any significant amounts of failure (Fig. 5-10b). Although the trends parallel those observed for fissile particles, care must be taken not to overemphasize these results in view of the apparent scatter observed in the optical anisotropy measurements. It is concluded from these unbonded particle tests, however, that the 1.85 to $1.95 \mathrm{~g} / \mathrm{cm}^{3}$ OPyC coatings should perform better than low-density ones, for much the same reasons given earlier in connection with fissile particle coatings.

Fertile particle failure levels in fuel rods calculated from TRIGA fission gas measurements (Table 5-5) are plotted as a function of temperature and fluence in Fig. 5-11. Fuel burnup is not considered here, although the fertile kernel burnup generally increases exponentially with fluence. Several trends can be noted from these plots. First, OPyC coating failure increased with both fluence (burnup) and temperature. At the higher temperatures $\left(>1250^{\circ} \mathrm{C}\right)$, there is not a significant difference in coating performance for particles with different densities and different coating rates (anisotropy). However, at $1000^{\circ} \mathrm{C}$ there is a notable difference in OPyC coatings. That is, higher-density coatings perform better than 1ower-density ones (compare Figs. 5-11a and 5-11b), and for a given density coatings having higher coating rates (and presumably lower anisotropies) perform better than those having lower coating rates (compare Figs. 5-11b and 5-11c). As can be seen, the OPyC performance results from 
these fuel rods yield conclusions similar to those drawn from the constanttemperature unbonded particle tests. However, in reviewing these data it should become obvious that this simplistic, straightforward treatment of OPyC coating performance on BISO particles will not yield results as consistent as those obtained for TRISO particles. This is because stress levels in OPYC coatings on TRISO particles depend primarily on the irradiation temperature and fluence. For TRISO particles, burnup need not be considered a variable because the rigid SiC layer shields the OPyC from any stresses resulting from fission gas pressure. BISO OPyC coating failure, on the other hand, depends on fuel burnup, buffer coating properties, and OPyC thickness, as well as the irradiation temperature and fluence. Rigorous interpretation of coating anisotropy and density requirements can be performed only with the aid of particle stress models which account for effects of these coating properties. Such models are currently being developed. However, in the absence of these analytical tools a recommendation of appropriate property limits based on this analysis alone must be made. To reiterate previous statements, OPyC coatings on BISO particles exhibiting optimum performance are those having densities between 1.85 and $1.95 \mathrm{~g} / \mathrm{cm}^{3}$ and anisotropies below $1.04 \mathrm{BAF}$ units (i.e., coating rates $>4 \mu / \min )$.

\subsection{OPyC Permeability}

In addition to maintaining structural integrity during irradiation to peak LHTGR fast neutron exposures, BISO OPyC coatings must remain impermeable to gaseous fission products. A number of BISO fertile particle batches were irradiated to fast neutron exposures up to $12.1 \times 10^{21} \mathrm{n} / \mathrm{cm}^{2}$ (E > $0.18 \mathrm{MeV})_{\text {HTGR }}$ without any apparent loss of structural integrity of the OPyC coatings. However, these particle batches exhibited varying levels of fission gas release and loss of thorium when leached in acid solutions (see Section 4.7). Fission gas release levels and thorium leachability appeared to increase with decreasing OPyC coating density (unirradiated). These results indicate that the OPyC coatings were permeable to gases and 
liquid solutions and that the degree of permeability was not altered significantly by irradiation-induced densification of the OPyC coatings.

Pyrolytic carbons contain several density defects, including large inter-growth feature pores, intercrystalline microporosity, and low-density carbon inclusions (Ref. 5-10). Any surface-connected porosity available to gases or liquids will undoubtedly be related to the fraction of large inter-growth feature pores rather than the smaller intercrystalline pores. The amount of surface available porosity can be ascertained by evaluating the differences between the PyC density measured using mercury porosimetry and a sink-float technique. Mercury does not wet the carbon and at low pressure does not tend to penetrate the porosity, while the liquid solution used in the sink-float measurement does. Thus, the difference between the two densities will increase with increasing available porosity (Ref. 5-10).

The unirradiated OPYC coating densities of the fertile particles were determined using a sink-float technique. The bulk density of the OPyC coatings was subsequently remeasured by mercury porosimetry, and the results are given in Table 5-6. The amount of surface-connected porosity or the difference in density $\left(\rho_{\mathrm{SF}}-\rho_{\text {bulk }}\right)$ determined for the unirradiated OPyC coatings is plotted in Fig. 5-12 as a function of coating rate. The amount of surface-connected porosity increases with increasing coating rate for the propylene and acetylene-propylene OPyC coatings, which is consistent with previous observations (Ref. 5-10).

The amount of inter-growth feature porosity in PyCs is not significantly altered by irradiation-induced densification (Ref. 5-6). Consequenty, the levels of fission gas released from the fertile particles should be a function of the amount of surface-connected porosity in the unirradiated coatings. However, when the fission gas release data were analyzed as a function of the calculated OPyC porosity, a clear relationship between the two sets of data could not be established. The lack of correlation might be attributed to an increase in porosity of the more anisotropic 
OPyC coatings during irradiation. During irradiation to high fast neutron fluences, anisotropic PyCs exhibit a turnaround in the densification and begin to expand. This expansion or decrease in density is attributed to tearing and cracking of the microstructural growth features of the PyC (Ref. 5-6). The development of these cracks increases the porosity in the $\mathrm{PyC}$ and consequently would result in an increase in fission gas release regardless of the initial level of surface-connected porosity in the oPyC coating. The permeability of the BISO coatings evaluated in this study might be attributed to a combination of the initial surface-connected porosity and an increase in porosity due to microstructural cracking after irradiation to the severe fast neutron fluences ranging from 10.7 to 12.1 $\times 10^{21} \mathrm{n} / \mathrm{cm}^{2}$ (E > $\left.0.18 \mathrm{MeV}\right)_{\mathrm{HTGR}}$.

Four of the fertile particle batches which exhibited permeable OPyC coatings (Table 5-6) were also tested in bonded fuel rods. Fuel rods containing batches 6542-01-020 and 6542-02-020 exhibited levels of fission gas release similar to those of the unbonded samples after irradiation to nominally the same conditions. However, rods which contained batches 6542-09-010 and 6542-01-010 exhibited two orders of magnitude lower fission gas release than the unbonded particle samples. The reason for the better retention of fission gas in the particles irradiated in the bonded fuel rods is not clear. It is possible that impregnation of the surface of the OPyC coating with pitch during fuel rod fabrication and the subsequent carbonization reduce the permeability of the OPyC coating.

\subsection{Mixed-Gas OPyC Coatings}

The OPyC coatings of Fort St. Vrain TRISO fuel particles and of BISO $\mathrm{ThO}_{2}$ particles irradiated in previous evaluation tests were deposited from propylene. However, to fabricate coated particles in a 240 -mm-diameter coater, it is necessary to deposit OPyC coatings from acetylene and propylene mixed in the proper proportions to avoid severe thermal transients during the coating operation. Data have previously been obtained on mixedgas OPyC coatings irradiated to moderate fast neutron fluences. BISO 
coated carbide fuel particles with OPyC coatings deposited from mixed gas exhibited good irradiation performance in capsules F-27 and F-28 (Ref. 5-11) and in Peach Bottom Core 2 (Ref. 5-12).

Five BISO coated fertile particle batches with OPYC coatings deposited from mixed gas were tested as unbonded particle samples in capsule P13S to evaluate the performance of these coatings upon irradiation to peak LHTGR fast neutron fluences. The OPyC coatings of the remaining BISO fertile particle batches tested in capsules $P 13 R$ and $P 13 S$ were deposited from propylene.

Results of the visual examination and fission gas release measurements made on the fertile unbonded particle samples are summarized in Table 5-4. The irradiation performance of the mixed-gas OPyC coatings was good. OPyC coating failure levels determined by visual examination ranged from 0 to $1.0 \%$ after irradiation to severe fast neutron fluences from 10.8 to $11.7 \times 10^{21}$ $\mathrm{n} / \mathrm{cm}^{2}$ (E $\left.>0.18 \mathrm{MeV}\right)_{\mathrm{HTGR}}$. Equivalent in-service failure levels calculated from fission gas release data for four of the batches ranged from 0 to $1.4 \%$, which indicated the coatings were relatively impermeable. A high level of fission gas release was measured for the other particle batch (6542-21-015) even though no OPyC coating failure was observed during the visual examination. The high fission gas release was attributed to the permeability of the low-density $\left(1.73 \mathrm{~g} / \mathrm{cm}^{3}\right)$ OPyC coating. Within the range of coating parameters tested, the irradiation performance of BISO OPyC coatings deposited from mixed gas was comparable to that of coatings deposited from propylene.

\subsection{Buffer Coating Performance}

The purpose of a low-density buffer coating immediately adjacent to the fuel kernel is to attenuate fission recoils and to provide a void volume to accommodate fission gases and fuel kernel swelling. The thickness and density of buffer coatings required for BISO particles irradiated to peak conditions are interrelated and are a function of kernel diameter and 
OPyC coating design. BISO coated ThO 2 particles with buffer coating thicknesses ranging from 39 to $87 \mu \mathrm{m}$ and densities ranging from 0.95 to $1.25 \mathrm{~g} / \mathrm{cm}^{3}$ were tested in capsules $\mathrm{P} 13 \mathrm{R}$ and P13S.

The buffer coatings densified during irradiation and appeared slightly anisotropic when viewed under polarized light. Densification resulted from a combination of irradiation-induced PyC shrinkage and fuel kernel swelling. Cracked buffer coatings were observed in most particle batches examined metallographically, as indicated in Table 4-36. Cracking of the buffer coating is not considered detrimental to the performance of BISO coatings unless the cracks propagate into the OPyC coating. This phenomenon, an example of which is shown in Fig. 4-107, was observed only in a couple of particles. In many particles the buffer coating cracked while the oPyC coating remained intact, as shown in Fig. 4-106. In most instances where the OPyC coating failed during irradiation, the cracks propagated into the buffer coating. Because of the interdependence of the buffer and OPyC coating failure, it was not possible to quantitatively evaluate buffer coating performance relative to coating thickness and density. The effects of these variables can be dealt with quantitatively only by using a model which can calculate free void volume in the buffer coating and for a specific coating design can calculate stress levels in the OPyC coating as a function of irradiation parameters. The results from capsules $\mathrm{P} 13 \mathrm{R}$ and $\mathrm{P} 13 \mathrm{~S}$ and from previous irradiation experiments (Ref. 5-2) will be analyzed using a BISO particle performance model to establish the buffer coating thickness and density limits for the LHTGR fuel specifications.

Buffer coatings are deposited from acetylene using argon as a diluent carrier gas. One particle batch (6542-12-025) was tested to determine if there were any detrimental irradiation effects from using $\mathrm{N}_{2}$ as the buffer carrier gas. The buffer coatings deposited using $\mathrm{N}_{2}$ carrier gas (Fig. 4-108) appeared similar to coatings deposited using argon as the carrier gas. Although a significant fraction of the buffer coatings deposited using $\mathrm{N}_{2}$ were cracked, no apparent differences between the coatings applied using argon and those applied using $\mathrm{N}_{2}$ carrier gas could be ascertained by metallographic examination. 


\subsection{Seal Coat}

A thin, high-density, impermeable PyC seal coat was applied between the buffer and IPyC coatings of Fort St. Vrain carbide fuel. The purpose of this coat was to prevent hydrolysis of the carbide kernels during subsequent handling and storage of incompletely coated fuel particles and to allow the buffer and IPyC coatings to decouple during irradiation. Since $\mathrm{ThO}_{2}$ fuel kernels are not subject to hydrolysis, a protective seal coat over the buffer coating is not required. From the standpoint of irradiation performance, it has not been established whether the buffer and OPyC coatings need to be decoupled by a seal coat during irradiation if the buffer coating is deposited from acetylene. Previous irradiation results have indicated that buffer coatings deposited from acetylene form a weaker bond with the OPyC coating than buffer coatings deposited from propylene, which was used for Fort St. Vrain fertile particles.

Al1 but two BISO particle batches tested in capsules P13R and P13S had seal coats. During irradiation the highly anisotropic seal coats cracked and densified, leaving numerous small gaps at the buffer/OPyC coating interface, as shown in Fig. 4-104. The breakup of the seal coat effectively decoupled the buffer and OPyC coating, as shown in Fig. 4-106, and did not initiate any cracks in the adjacent buffer or OPyC coating.

Particle batches 6542-11-015 and 6542-18-015 were tested without seal coats and exhibited OPyC coating failure levels of 0.4 and $0 \%$ after irradiation to fast neutron fluences of 11.5 and $11.4 \times 10^{21} \mathrm{n} / \mathrm{cm}^{2}$ ( $\mathrm{E}>$ $0.18 \mathrm{MeV}$ HTGR, respectively. The OPyC failure in batch 6542-11-015 was attributed to the relatively high anisotropy rather than the absence of a seal coat. Representative photomicrographs of particles from batch 6542-18-015 are shown in Fig. 4-105. The absence of a seal coat did not have any visible effect on the irradiation behavior of the BISO coatings. In approximately $3 \%$ of the particles, the buffer coating delaminated and shrank away from the OPYC coating. This indicates that buffer coatings 
deposited from acetylene may not form a strong bond with the OPyC coating. However, the strength of the buffer/OPyC coating bond is also a function of the buffer coating density. If a seal coat were not present, lowerdensity buffer coatings would be more susceptible to penetration of carbon during the deposition of the OPyC coating, which would make the buffer/OPyC coating interface less distinct.

In summary, a seal coat effectively decoupled the buffer and OPyC coating during irradiation. This retarded but did not completely eliminate propagation of cracks from the buffer into the OPyC coating. Two BISO $\mathrm{ThO}_{2}$ particle batches without seal coats performed satisfactorily during irradiation to severe fast neutron exposures. The absence of a seal coat did not have any apparent detrimental effect on BISO coating irradiation performance.

\subsection{Coating Design}

Results from capsules $\mathrm{P} 13 \mathrm{R}$ and $\mathrm{P} 13 \mathrm{~S}$ indicate that for the particle designs tested, OPyC coating properties such as coating rate or anisotropy are more important for particle survival than OPyC coating thickness. For example, particle batches 6542-16-010 and 6542-17-010 had the same nominal kernel diameters and buffer coating parameters; however, there was a large difference $(55 \mu \mathrm{m}$ versus $122 \mu \mathrm{m})$ in the OPyC coating thickness of the two batches. After irradiation to similar conditions, the batches exhibited similar failure levels of 2.6 and $5.4 \%$. The observed failure was attributed to the effects of high anisotropy of the coatings because the OPyC coating rate of both particle batches was less than $4.0 \mu \mathrm{m} / \mathrm{min}$. As discussed previously in this section, a significant increase in OPyC coating failure was observed for particle batches with OPyC coatings deposited at coating rates less than $4.0 \mu \mathrm{m} / \mathrm{min}$ owing to the increase in anisotropy at the lower deposition rates. In particle batches with OPyC coatings deposited at rates greater than $4.0 \mu \mathrm{m} / \mathrm{min}$, no coating failure was observed that could be attributed to variations in coating design, although a much narrower range of OPyC coating thicknesses was tested. Irradiation data from 
capsules P13R and P13S and previous screening tests (Ref. 5-13) will be analyzed using a BISO particle performance model to establish the range of coating thicknesses acceptable for LHTGR BISO fertile particles.

\subsection{Faceted and Nonround Particles}

Three batches of $\mathrm{ThO}_{2}$ BISO particles were tested to determine the effect of faceted coatings and nonround coatings on irradiation performance. The faceted particles were characterized by their multifaced coatings. Normally, the particles appeared like dodecahedrons (see Fig. 4-28). Faceted coatings predominantly occur when large batch loads and/or low levitating gas flow rates are used. The nonround particles tested were caused by badly misshapen kernels. This batch was produced from a kernel charge which had a high fraction of cracked kernels. An example of these particles can be seen in Fig. 4-99. Each of these batches was tested in the unbonded particle cells, and particles from batch 6542-20-035 were also tested in six fuel rods.

To date, quantitative techniques have not been developed to evaluate the fuel particle failures observed. However, these techniques are scheduled to be ready before firm specifications are set limiting the fraction of misshapen kernels (and/or coated particles) and faceted coatings. These results show a need for such specifications, since between 0 and $5.4 \%$ particle failures were observed in the fuel rod and unbonded particle tested at $\sim 1000^{\circ} \mathrm{C}$. Only one sample, batch $6542-20-035$, was tested at temperatures higher than $1000^{\circ} \mathrm{C}$, and it was irradiated in the thermal-cycled cel1. Particle failures on the order of $25 \%$ were observed for this batch.

\subsection{TRISO $\mathrm{ThO}_{2}$ Performance}

A TRISO coated $\mathrm{ThO}_{2}$ particle is the LHTGR backup fertile particle design and is also being considered for use in Fort St. Vrain reload segments. One TRISO coated $\mathrm{ThO}_{2}$ particle batch (6252-00-025) was tested in capsule P13S for comparative purposes. 
The particle batch exhibited good performance during irradiation to a severe fast neutron exposure of $11.5 \times 10^{21} \mathrm{n} / \mathrm{cm}^{2}(E>0.18 \mathrm{MeV})_{\text {HTGR }}$. Total coating and OPyC failure levels of 0 and $0.4 \%$, respectively, were observed during the visual examination, and the postirradiation fission gas release was equivalent to a failure level of $0.2 \%$. It should be noted, however, that the batch was irradiated at a relatively low temperature of $860^{\circ} \mathrm{C}$.

Representative photomicrographs of particle batch 6252-00-025 taken during the metallographic examination are shown in Fig. 4-109. The PyC coatings appeared relatively isotropic under polarized light. The IPyC coating debonded from the SiC layer in approximately $3.0 \%$ of the particles, and $10.8 \%$ of the particles had cracked buffer coatings. A reaction zone was observed on the inner surface of the SiC coating of one particle, as shown in Fig. 4-110. The IPyC coating was intact, although a portion of the cracked buffer coating pulled out during polishing. The reaction was not attributed to metallic fission products, since the irradiation temperature was low and signs of metallic fission products were not observed in the adjacent IPyC coating. A similar attack of the SiC coating was also observed in TRISO $\mathrm{ThO}_{2}$ particles irradiated in capsule $\mathrm{P} 13 \mathrm{P}$ at $1230^{\circ} \mathrm{C}$. The reaction probably resulted from oxidation since oxidation of the SiC coating had been previously observed in irradiated TRISO $\mathrm{UO}_{2}$ particles and unirradiated TRISO ( $\mathrm{Th}, \mathrm{U})_{2}$ particles during high-temperature thermal gradient annealing studies.

5.1.2.3. Thermochemical Stability. BISO coated fertile particles are not as susceptible to degradation by metallic fission products as BISO and TRISO coated fissile particles (Ref. 5-2). Radiographs of TRISO UC 2 and BISO ThO $_{2}$ particles irradiated in capsules P13R and P13S at temperatures of $1075^{\circ}$ and $950^{\circ} \mathrm{C}$ are shown in Fig. 4-125. Evidence of diffusion of metallic fission products from the $\mathrm{UC}_{2}$ fuel kernels can be observed; however, the BISO $\mathrm{ThO}_{2}$ particles do not have any localized concentrations of fission products in the PyC coatings. No evidence of metallic fission product degradation of $\mathrm{BISO} \mathrm{ThO}_{2}$ particles was observed in the fuel rod specimens 
irradiated at temperatures up to $1310^{\circ} \mathrm{C}$. Although the fertile particle BISO coatings allow a controlled diffusive release of volatile metallic fission products, such as cesium and strontium, the quantities are not sufficient to result in degradation of the PyC coatings.

No evidence of $\mathrm{ThO}_{2}$ fuel kernel migration was observed in capsule $P 13 R$ and $P 13 S$ fuel specimens. If kernel migration had occurred, elongation of the fuel kernel or thinning of the PyC coating on the hot side of the particles would have been detected. As discussed in Section 5.1.1.3, $\mathrm{UC}_{2}$ fuel kernel migration was observed in fuel rods irradiated in cell 5 at average centerline temperatures ranging from $1225^{\circ}$ to $1335^{\circ} \mathrm{C}$. The absence of $\mathrm{ThO}_{2}$ kernel migration in the P13R and P13S fuel specimens can be explained on the basis of a delay in the onset of kernel migration which has been observed in postirradiation heating experiments of low burnup samples (Ref. 5-14). Toward the end of the irradiation when the delay time was exceeded and migration was expected, the irradiation temperatures were too low for detectable migration to occur. Characterization of the temperature dependence of $\mathrm{ThO}_{2}$ migration in a manner which accounts for the delay in onset of migration has not been completed. Therefore, predicted migration distances for $\mathrm{ThO}_{2}$ kernels were not calculated using the CAPTEM code.

\subsubsection{Effects of High-Temperature Transients}

During the first year of operation under normal LHTGR steady-state operating conditions, 50 vol \% of the fuel will experience peak centerline temperatures of $\leq 1150^{\circ} \mathrm{C}$ and only 1 vol \% will experience peak centerline temperatures of $\geq 1425^{\circ} \mathrm{C}$. Fuel temperatures gradual1y drop with increasing age, and in the fourth year of operation they are approximately $150^{\circ} \mathrm{C}$ lower than during the first year. However, load-following transients result in increased localized fuel temperatures (Ref. 5-15). Therma1cycling conditions during load-following are dependent on the maximum allowable region outlet temperature, load-following range, and reactor 
design. In the core design described in Ref. 5-15 for the 1160-MW(e) LHTGR, load-following results in power and fuel temperature increases toward the center of the core. The peak fuel temperatures that were expected for the 100-60-100 load-following transient ranged from $1680^{\circ} \mathrm{C}$ at beginning-of-life to $1375^{\circ} \mathrm{C}$ at an end-of-life fluence of $8.0 \times 10^{21} \mathrm{n} / \mathrm{cm}^{2}$ $(E>0.18 \mathrm{MeV})_{\text {HTGR }}$ (Ref. 5-16). However, subsequent improvements in core loading patterns and rod programming have substantially reduced the temperatures expected for the 100-60-100 load-following transient.

Previous irradiation capsules have been operated at a constant design temperature (Ref. 5-2). Capsule P13S was the first attempt to simulate temperature cycling that results from load-following and/or control rod pattern changes in the LHTGR. Cell 1 of capsule P13S, which contained five fuel rods, was designed to be thermal cycled from a nominal operating temperature of $1075^{\circ} \mathrm{C}$ to $1600^{\circ} \mathrm{C}$ once every GETR fuel cycle. Identical fuel rods were irradiated in cell 1 of capsule P13R at a constant design temperature of $1075^{\circ} \mathrm{C}$ for comparative purposes. In addition to this test, eight fuel rods were heated to temperatures ranging from $1300^{\circ}$ to $1600^{\circ} \mathrm{C}$ in the TRIGA facility to evaluate the effects of high-temperature transients on severely irradiated fuel.

5.1.3.1. In-pile Thermal Cycling. Decalibrated temperatures of the P13S cell 1 control thermocouple are plotted in Fig. 5-13 as a function of fast neutron fluence. Thermal cycles, where thermocouple data were obtained at the peak of the cycle, are represented by the spikes. Also shown is a temperature envelope of the LHTGR peak fuel temperature versus fast fluence that were assumed to occur under load-following conditions (Ref. 5-16). The fuel temperatures obtained in P13S cell 1 during thermal cycling were representative of the peak LHTGR fuel temperatures expected, except during the latter part of the irradiation, when the P13S fuel temperatures were approximately $100^{\circ} \mathrm{C}$ higher.

The irradiation parameters and fuel failure levels, determined by postirradiation fission gas release measurements, for the fuel rods tested in cell 1 of capsules $P 13 R$ and P13S are given in Table 5-7. The same fuel 
specimens were tested in both cells, and the specimens were irradiated under similar conditions. The only difference was that the P13S fuel rods were thermal cycled 24 times to temperatures $200^{\circ}$ to $400^{\circ} \mathrm{C}$ higher than the nominal irradiation temperature. The effects of thermal cycling clearly resulted in increased fuel failure in all specimens during irradiation. However, the effect was most pronounced in fuel rods which contained out-ofspecification fuel. The $1 \mathrm{~A}$ and $1 \mathrm{D}$ fuel rods, which contained severely faceted fuel particles (1A) and fertile particles with the OPyC coating applied at a low deposition rate (1D), exhibited the highest failure levels of 22.0 and $24.0 \%$, respectively. Faceting and 1 ow OPyC coating deposition rates also resulted in relatively high failure levels of 4.8 and $2.2 \%$ in the non-thermal-cycled fuel rods (1A and 1D) tested in capsule P13R.

Fuel rods $1 \mathrm{~B}$ and $1 \mathrm{C}$ contained fuel particles more representative of LHTGR design requirements. These rods exhibited low average failure levels of 0.6 and $0 \%$, respectively, after irradiation to peak LHTGR fluence conditions in capsule P13R. After thermal cycling in P13S, the average in-service particle failure levels in rods $1 \mathrm{~B}$ and $1 \mathrm{C}$ were 1.1 and $2.2 \%$, respectively. If all the measured fission gas is assumed to have been released from failed fertile fuel, then the maximum fertile particle failure levels in rods $1 \mathrm{~B}$ and $1 \mathrm{C}$ were 1.3 and $2.4 \%$. These failure levels are below a failure level of approximately $7.5 \%$ predicted for $B I S O \mathrm{ThO}_{2}$ particles that experience a thermal transient to $1550^{\circ} \mathrm{C}$ after irradiation to a fast neutron fluence of $8.0 \times 10^{21} \mathrm{n} / \mathrm{cm}^{2}(\mathrm{E}>0.18 \mathrm{MeV})$ HTGR (Ref. 5-17). Because of the low fission rates in the fissile particles, a comparable fissile failure level was not determined.

5.1.3.2. Postirradiation Heat Treatment. After irradiation eight fuel rods were heated to temperatures ranging from $1300^{\circ}$ to $1600^{\circ} \mathrm{C}$ in the TRIGA facility (see Section 4.4.3). The results of the fission gas release measurements made on these fuel rods are given in Tables $4-19$ and 4-20 and Figs. 4-35 through 4-42. Six of the fuel rods did not experience any additional fuel failure during heating to temperatures $100^{\circ}$ to $300^{\circ} \mathrm{C}$ higher than their average irradiation temperature. This was evidenced by the absence of an increase in fission gas release during the heat treatment other than the 
increase resulting from the R/B temperature dependence. However, all five rods heated to a maximum temperature of $1600^{\circ} \mathrm{C}$ did experience additional fuel failure as a result of the heat treatment. Because of the possible contribution of the residual fission gas inventory from successive measurements to the final $R / B$ level, induced fuel failure could only be treated quantitatively in three of the fuel rods when the final $R / B$ measurement was made several days after the heat treatment. The results of the fission gas release measurements made on these three rods are given in Table 5-8.

Particle failure levels were calculated from the $R / B$ data by assuming a fractional release of $5.0 \times 10^{-3}$ for failed fuel. The results indicate that a significant level of particle failure was induced during the $1600^{\circ} \mathrm{C}$ heat treatment in the TRIGA facility. Fuel rod 7161-004-03-6, which was thermal cycled in-pile, also exhibited a significant increase in fuel failure during the heat treatment. Fuel rods 7161-004-14-5 and 7161-004-17-5 contained the same fissile and fertile particle batches as rod 7161-004-02-5, which was thermal cycled in-pile (Table 5-7). Total particle failure levels in the two fuel rods after heating to $1600^{\circ} \mathrm{C}$ in the TRIGA facility were 8.3 and 5.8\%, while fuel rod 7161-004-02-5 exhibited a failure level of only $1.1 \%$ after being thermal cycled in-pile. It is not clear why the postirradiation heat treatment resulted in such a significant increase in fuel failure relative to the in-pile thermal cycling.

\subsection{FUEL ROD IRRADIATION PERFORMANCE}

\subsubsection{Integrity}

A large number of pitch-bonded fuel rods have exhibited satisfactory performance in previous capsule irradiation experiments. The integrity of bonded fuel rods was found to be independent of irradiation temperature and fast neutron fluence within the realm of LHTGR operating conditions (Ref. 5-2).

The irradiation performance of bonded fuel rod specimens tested in capsules P13R and P13S was good. Very 1ittle irradiation-induced damage 
was observed, even in fuel rods irradiated to a fast neutron fluence of $12.4 \times 10^{21} \mathrm{n} / \mathrm{cm}^{2}$ (E $\left.>0.18 \mathrm{MeV}\right)_{\text {HTGR }}$, which is $55 \%$ beyond the peak LHTGR design fast neutron exposure. The visual appearance and amount of particle debonding observed in the fuel rods are summarized in Tables 4-1 and 4-2. The type of fuel rod fabrication process (hot-injection and slug-injection) and the firing process variables tested $\left(1500^{\circ}\right.$ and $1800^{\circ} \mathrm{C}$ firing temperatures, firing in argon or $\mathrm{N}_{2}$ atmosphere, and carbonization in $\mathrm{H}-451$ graphite tubes or packed beds of alumina) did not influence fuel rod irradiation performance. The postirradiation integrity of all the fuel rods fabricated or fired under these conditions was good.

The fuel rods irradiated in P13S cell 5 at a design temperature of $1500^{\circ} \mathrm{C}$ were in relatively good condition, although the rods appeared somewhat weak around the edges, especially those in positions $5 \mathrm{C}$ and $5 \mathrm{D}$, which exhibited slight circumferential cracking on the ends. Fuel rods tested in P13S cell 1, which was thermal cycled from a nominal operating temperature of $1075^{\circ} \mathrm{C}$ to temperatures ranging from $1500^{\circ}$ to $1650^{\circ} \mathrm{C}$ twenty-four times during capsule operation, were in good condition. These results indicate that high-temperature operation or thermal cycling to high temperatures is not detrimental to fuel rod integrity.

\subsubsection{Influence of Product and Process Variables}

5.2.2.1. Cure-in-P1ace. The majority of the rods were fired in $\mathrm{H}-451$ graphite tubes to simulate the cure-in-place process. Two fuel rods were fired in packed beds of alumina for comparative purposes.

The postirradiation integrity of the cure-in-place fuel rods was excellent. Seven of the rods exhibited slight matrix cracking and particle debonding, but this was attributed to the matrix variables tested in these rods rather than to the fabrication process. These results are consistent with other capsule tests which also demonstrated the excellent irradiation stability of cure-in-place fuel rods (Refs. 5-18, 5-19). 
5.2.2.2. Shim Particles. LHTGR fuel rods contain from 25.3 to 42.7 vol \% coated fuel particles. The remaining portion of the total particle packing volume ( 34.7 to 17.3 vol \%) is made up of uncoated graphite shim particles. The graphite shim particles used in the $\mathrm{P} 13 \mathrm{R}$ and $\mathrm{P} 13 \mathrm{~S}$ fuel rods were comminuted, bulk, near-isotropic graphite supplied by candidate vendors for near-isotropic HTGR graphite. The GLCC 1099 shim impregnated with furfuryl alcohol was used in 32 fuel rods. Four rods contained nonimpregnated GLCC 1099 shim, and the remaining two rods contained impregnated Lonza shim particles.

Fuel rods fabricated with GLCC 1099 shim particles (impregnated or nonimpregnated) which contained medium to high (20 to 36 vol \%) shim contents exhibited excellent integrity after irradiation to fast neutron fluences up to $12.3 \times 10^{21} \mathrm{n} / \mathrm{cm}^{2}$ ( $E>0.18 \mathrm{MeV}$ ) $\mathrm{HTGR}^{\cdot}$ However, six fuel rods fabricated with Lonza shim particles or with low (10 to 15 vol \%) shim contents exhibited slight matrix cracking and particle debonding. Most of the particle debonding occurred predominantly on one end of the rods. This indicates that the matrix content at the end of the rod was not adequate to provide a good matrix/particle bond rather than that the matrix itself was weak.

In the hot-injection process, the matrix (pitch plus filler) is injected into the particle bed from the bottom end of the rod. During the fabrication of the $P 13 R$ and $P 13 S$ fuel rods, it was difficult to achieve proper filtration of the matrix into the particle beds; consequently, a matrix inhomogeneity from one end of the rod to the other often existed. A matrix-poor region at one end of a rod would be more susceptible to particle debonding. Matrix nonuniformity could have been more prevalent in the rods containing low shim contents and Lonza shim. The Lonza shim was more porous than the GLCC 1099 shim and would therefore be expected to absorb more pitch during the injection process, which could result in a matrix-poor region at one end of the rod. The rods with lower shim contents had a higher particle packing fraction. This lower void space would restrict the flow of matrix during the injection process. 
These observations indicate the particle debonding observed on the ends of some of the fuel rods resulted from improper filtration of the matrix into the particle bed during rod fabrication, rather than from a characteristic of the type of shim particle used in the rods. The matrix injection problem during fabrication of fuel rods has been eliminated for subsequent capsule tests by using additives to decrease the viscosity of the matrix during injection.

5.2.2.3. Matrix Type. Fuel rods fabricated with natural-flake graphite filler and $A 240$ petroleum pitch binder have been irradiated successfully to peak LHTGR fast neutron fluences in previous capsules (Ref. 5-18). However, in some of the rods tested, high levels of OPyC coating failure occurred as a result of excessive matrix/coating interaction due to the strong bond formed between the matrix binder and the OPyC coatings. Formation of the strong bond was attributed to the porous surface texture of the OPyC coatings and the high pitch coke content of the matrix. A matrix blend of 30 wt \% 6353 natural-flake graphite filler, 65 wt \% A240 petroleum pitch binder, and 5 wt \% SC011 additive was used in 34 of the rods in capsules $\mathrm{P} 13 \mathrm{R}$ and $\mathrm{P} 13 \mathrm{~S}$. The remaining six rods contained Lonza KS-44 graphite filler and $\mathrm{SC003}$ or SC027 matrix additives. The volatile matrix additives were incorporated to improve the ease of fuel rod fabrication and to reduce the fuel rod pitch coke content after firing.

No evidence of matrix/coating interaction was observed in any of the $\mathrm{P} 13 \mathrm{R}$ and $\mathrm{P} 13 \mathrm{~S}$ fuel rods examined metallographically. No differences were observed between the matrices containing the 6353 natural-flake or the Lonza KS-44 graphite filler. The higher fraction of particle debonding observed in some of the rods was attributed to inadequate matrix injection rather than the matrix type, as discussed in Section 5.2.2.2.

The good irradiation stability of the P13R and P13S fuel rods indicates that the matrix combinations tested are suitable for LHTGR fuel rods. For these matrix combinations, pitch coke yields of 22 to $39 \%$ and levels of matrix macroporosity ranging from 26 to 43 vol \% are adequate to ensure good fuel rod integrity during irradiation to peak LHTGR fast neutron 
exposures. Nonuniform injection of the matrix material during fuel rod fabrication can result in loss of integrity and subsequent particle debonding.

5.2.2.4. Firing Conditions. Al1 fuel rods were carbonized by heating to $1000^{\circ} \mathrm{C}$ at $10^{\circ} \mathrm{C}$ per minute in an induction furnace in a nitrogen atmosphere. Thirty rods were high-fired at $1800^{\circ} \mathrm{C}$ in an argon atmosphere. The remaining 10 rods were either fired at $1800^{\circ} \mathrm{C}$ in a nitrogen atmosphere or at $1500^{\circ} \mathrm{C}$ in an argon atmosphere. Neither the lower firing temperature of $1500^{\circ} \mathrm{C}$ nor the nitrogen atmosphere had any apparent effect on the irradiation stability of the fuel rods. However, dimensional change measurements revealed that the fuel rods fired at $1500^{\circ} \mathrm{C}$ shrink less than companion rods fired at $1800^{\circ} \mathrm{C}$ (see Fig. 4-33).

\subsection{METALLIC FISSION PRODUCT BEHAVIOR}

From the analysis of the gamma-ray spectroscopy results of the fuel rods, graphite crucibles, and unbonded particle samples, the magnitude of metallic fission product release and redistribution in the capsule has been determined and a qualitative evaluation of fuel homogeneity in the rods made. A value for the fractional release of Cs-137 from failed fuel in the rods and unbonded fissile particle samples has also been estimated.

\subsubsection{Fuel Rod Homogeneity}

Fuel loadings in the rods were fairly homogeneous, as evidenced by the relatively uniform $\mathrm{Cs}-137$ and $\mathrm{Zr}-95$ fission product profiles measured in the rods shown in Figs. 4-43 through 4-46. There was evidence, however, of small localized regions of fuel inhomogeneity in some of the fuel rods. Regions of high fuel concentrations, the majority of which occurred near the ends of the fuel rods, are indicated by peaks in the fission product profiles. No correlation was obtained between these regions of slightly higher fuel concentration and fuel performance as assessed by metallographic examination, fission gas release measurements, or visual examination. 


\subsubsection{Release from Failed Fue1}

5.3.2.1. Fuel Rods. The measured and calculated fission product isotope inventories of the fuel rods that were gamma counted are given in Tables 4-25 and 4-26. The two fission product isotope inventories are in agreement within $\pm 20 \%$, which indicates that metallic fission product release from the fuel rods during irradiation was low. However, the best measure of fission product release from the fuel rods was obtained by evaluating the difference between the measured and calculated $\mathrm{Cs}-137 / \mathrm{Zr}-95$ ratios. This is because (1) $\mathrm{Cs}-137$ is more mobile than $\mathrm{Zr}-95$, so that a low measured ratio indicates cesium 1oss, and (2) by taking a ratio of the two fission product activities, the uncertainty associated with calculating the theoretical inventory is removed. The difference between the calculated and measured Cs-137/Zr-95 ratios given in Tables 5-9 and 5-10 indicates that cesium loss from the fuel rods was low. The only rods which exhibited a measurable cesium loss were those tested in $\mathrm{P} 13 \mathrm{R}$ position $1 \mathrm{C}$ and $\mathrm{P} 13 \mathrm{~S}$ positions $1 \mathrm{~A}$ and $1 \mathrm{D}$. The cesium losses for these rods were $10.0,9.5$, and $15.8 \%$, respectively.

A measure of the cesium loss was also obtained by examining the fuel rod graphite crucibles. Relative Cs-137 profiles obtained for all the graphite crucibles are shown in Figs. 4-47 and 4-48. The regions of the graphite crucible adjacent to the fuel rods tested in $\mathrm{P} 13 \mathrm{~S}$ positions $1 \mathrm{~A}$ and $1 \mathrm{D}$ showed a high Cs-137 activity, as would be expected since these rods exhibited a relative high level of fuel failure. Cesium concentrations in the other graphite crucibles were relatively low. The graphite crucible adjacent to the fuel rod in P13R position $1 \mathrm{C}$ also exhibited a low activity, which indicates that cesium loss from the $1 \mathrm{C}$ fuel rod was probably not as high as the $10 \%$ value given above. The low fission gas release ( $R / B$ $\mathrm{Kr}-85 \mathrm{~m}=1.0 \times 10^{-6}$ ) obtained for this rod, which indicates a fuel failure level of $0 \%$, supports this conclusion.

Most of the metallic fission product release observed was attributed to release from failed particles rather than diffusive release from intact BISO coated particles. This conclusion is supported by comparison of the measured cesium release of individual fuel rods with the fuel failure 
levels determined from the fission gas release measurements (see Section 4.4.2, Tables 4-15 and 4-16). Fuel rods tested in P13S positions $1 \mathrm{~A}$ and 1D, which exhibited significantly higher levels of cesium release than the other rods, had average fuel failure levels of 22.0 and $24.0 \%$, which were significantly higher than fuel failure levels observed in the other rods. The cesium release from $\mathrm{P} 13 \mathrm{~S}$ cell 5 (indicated by the graphite crucible cesium activity) was higher than the release from P13R cell 5, which is consistent with the higher level of fuel failure observed in P13S cel1 5 than in P13R cell 5. Fuel rod 2A of capsule P13R exhibited a higher cesium release and also a higher level of fuel failure than the other rods tested in cell 2 .

From the above results, the fractional release of $\mathrm{Cs}-137$ from failed fuel in the P13S $1 \mathrm{~A}$ and $1 \mathrm{D}$ fuel rods was estimated. Fractional Cs-137 release values were calculated for failed fuel in the rods by dividing the percent of cesium lost from the fuel rods determined from the measured and calculated Cs-137/Zr-95 ratios (Table 5-10) by the failure fractions determined from the fission gas release measurements (Table 4-16). These results are summarized in Table 5-11. The values of 0.43 and 0.66 probably underestimate the actual fractional $\mathrm{Cs}_{s}-137$ release values from failed fuel for two reasons: (1) the total $\mathrm{Cs}-137$ inventory in the bonded fuel rods was used in the calculations; however, a portion of the cesium released from the failed fuel has been sorbed by the graphite matrix; and (2) the measured Cs-137/Zr-95 ratios in Table 5-10 of fuel rods that did not exhibit any cesium loss are systematically higher than the calculated ratios by 5 to $10 \%$. If the measured ratios were decreased by $10 \%$, the cesium 10 s from rods $1 \mathrm{~A}$ and $1 \mathrm{D}$ would be $17.8 \%$ and $22.2 \%$, which would correspond to a fractional Cs-137 release from failed fuel of 0.81 and 0.93 , respectively.

\subsubsection{Unbonded Particles. Because the unbonded particle batches were} gamma-counted through a 2-in.-thick lead shield, a reliable calibration for quantitative analysis could not be made. Instead, a qualitative evaluation of the metallic fission product retention of each particle batch was made by comparing the measured and calculated $\mathrm{Cs}-137 / \mathrm{Zr}-95$ ratios. 
The Cs-137/Zr-95 ratios obtained for each fertile particle batch examined are given in Tables 4-32 and 4-33. The BISO and TRISO coated fertile particle batches did not exhibit any measurable cesium loss. The measured $\mathrm{Cs}-137 / \mathrm{Zr}-95$ isotope ratios were within $10 \%$ of the calculated theoretical ratio; however, the measured ratios were systematically higher. Reasons for this discrepancy are discussed in section 4.5.3. The fertile particle batches exhibited failure levels ranging from 0 to $10.2 \%$ (see Tables 4-4 and 4-5). No cesium release was observed, even in particle batches containing failed particles, within the experimental uncertainties of the measurements. These results indicate that the irradiation temperatures, which ranged from $830^{\circ}$ to $1010^{\circ} \mathrm{C}$, were low enough to prevent the diffusive release of cesium from the $\mathrm{ThO}_{2}$ particles even if failure occurred during the 6200-hr irradiation. These results are in accord with cesium release levels predicted for these samples using the FIPER code, which is used for calculating the release of metallic fission products into the primary coolant circuit of an LHTGR (Ref. 5-14).

The unbonded fissile particle batches were gamma-counted using the same procedure, and the $\mathrm{Cs}-137 / \mathrm{Zr}-95$ ratios obtained for each particle batch are given in Tables 4-30 and 4-31. Comparison of the measured and calculated fission product ratios revealed that some of the batches lost up to $20 \%$ of their cesium inventory. Since the measurement uncertainty in the $\mathrm{Cs}-137 / \mathrm{Zr}-95$ ratio is $\pm 10 \%$, a comparison was made only between the cesium loss and failure level in particle batches that exhibited significant failure. In Table 5-12, the cesium loss is compared with the failure level of these particle batches determined from the fission gas release measurements. To evaluate the fractional release of $\mathrm{Cs}-137$ from failed fissile fuel, the percent Cs-137 loss was plotted as a function of failure fraction (see Fig. 5-14). The slope of this curve (least-squares fit forced through zero) is 0.70 , which gives the fractional release of $\mathrm{Cs}-137$ from failed fissile particles irradiated in the temperature range of $1015^{\circ}$ to $1075^{\circ} \mathrm{C}$. This value is in good agreement with the normalized values of 0.81 and 0.93 for the fractional Cs-137 release from failed fuel in the two bonded rods. Although there is a large measurement uncertainty associated with these values, the data indicate that the fractional cesium release is 
high. It should be noted that in calculating metallic fission product release in an LHTGR core, the assumption is made that $100 \%$ of the metallic fission product inventory in failed fissile fuel is released to the fuel rod matrix (Ref. 5-20).

\subsubsection{Fission Product Transport}

An approximate cesium mass balance was obtained by comparing the Cs-137 isotope inventories observed in the fuel rods and graphite crucibles. Because of the large uncertainty associated with the gamma-counting data and the low cesium activity levels observed in most of the graphite crucibles, only cel1 1 of P13S, which had the highest cesium release, was analyzed. Fuel rods $1 \mathrm{~A}$ and $1 \mathrm{D}$ were the only rods in this cell which exhibited measurable cesium loss (see Section 5.3.2.1). The calculated Cs-137 losses from rods $1 \mathrm{~A}$ and $1 \mathrm{D}$ were 9.5 and $15.8 \%$, which corresponds to $1.9 \times 10^{4} \mu \mathrm{Ci}$ and $3.2 \times 10^{4} \mu \mathrm{Ci}$, respectively. The measured cesium activity of the P13S graphite crucibles is given in Table 4-29. The total Cs-137 activity of the cell 1 crucible (spectrum numbers 1 through 7 in Table 4-29) was $4.3 \times 10^{4}$ $\mu \mathrm{Ci}$, which represents $84.0 \%$ of the total $\mathrm{Cs}-137$ lost from fuel rods $1 \mathrm{~A}$ and $1 \mathrm{D}$.

The fact that most of the cesium released from the fuel rods was present in the graphite crucibles would be expected. Cesium released from the fuel rods is sorbed in the graphite and transported primarily by a surface or bulk diffusion mechanism rather than by gas phase transport. The graphite crucible operated at an average temperature of $760^{\circ} \mathrm{C}$ and a maximum temperature of $1140^{\circ} \mathrm{C}$ during irradiation. The mean migration distance of cesium in the graphite crucible calculated using a diffusion coefficient of $1.6 \times 10^{-9} \mathrm{~cm}^{2} / \mathrm{sec}$ at $760^{\circ} \mathrm{C}$ would be $2.7 \mathrm{~mm}$, which is slightly less than one-half the thickness of the graphite crucible wall.

\section{REFERENCES}

5-1. Scott, C. B., and D. P. Harmon, "Postirradiation Examination of Capsule F-30," Genera1 Atomic Report GA-A13208, Apri1 1, 1975. 
5-2. Harmon, D. P., and C. B. Scott, "Development and Irradiation Performance of LHTGR fuel," ERDA Report GA-A13173, General Atomic, October 31, 1975.

5-3. Koss, P., and K. Wallish, "Optical Determination of the Anisotropy of Pyrocarbon," in Proceedings of International Carbon Conference, Baden-Baden, Germany, June 25-30, 1972, pp. 256-258.

5-4. Kaae, J. L., "Relations Between the Structure and Mechanica1 Properties of Fluidized-Bed Pyrolytic Carbons," Carbon 9, 291 (1971).

5-5. Kaae, J. L., "Effect of Irradiation on the Mechanical Properties of Isotropic Pyrolytic Carbons," Gulf General Atomic Report Gulf-GAA12061, May 1972.

5-6. Kaae, J. L., "Irradiation-Induced Microstructural Changes in Isotropic Pyrolytic Carbons," Carbon $\underline{57}, 82$ (1975).

5-7. Gulden, T. D., "Mechanical Properties of Polycrystalline B-SiC," J. Am. Ceram. Soc. 52 , 587 (1969).

5-8. Harmon, D. P., J. T. Ganley, and O. M. Stansfield, "HTGR Fuel Product Specification, Issue B," ERDA Report GA-A13464, General Atomic, January 1976.

5-9. Stansfield, O. M., C. B. Scott, and J. Chin, "Kernel Migration in Coated Carbide Fuel Particles," Nucl. Technol. 25 , 517 (1975).

5-10. Kaae, J. L., "Microstructures of Isotropic Pyrolytic Carbons," Carbon 13, 55 (1975).

5-11. "Public Service Company of Colorado 330-MW(e) High-Temperature GasCooled Reactor Research and Development Program, Quarterly Progress Report for the Period Ending December 31, 1969," USAEC Report GA-9875, Gulf General Atomic, January 30, 1970.

5-12. Harmon, D. P., C. B. Scott, and W. J. Scheffel, "Postirradiation Examination of Peach Bottom Core-2 Fuel Element C11-07," General Atomic Report GA-A12450, August 6, 1974.

5-13. Scott, C. B., and D. P. Harmon, "Evaluation of Coated $\mathrm{ThO}_{2}$ Fuel Particles Irradiated in Capsules HT-12 Through HT-15 and HT-17 Through HT-19," ERDA Report GA-A13557, General Atomic, to be issued.

5-14. "HTGR Fuels and Core Development Program Quarterly Progress Report for the Period Ending February 29, 1976," ERDA Report GA-A13804, Genera1 Atomic, March 31, 1976. 
5-15. Shenoy, A. S., and D. W. McEachern, "HTGR Core Thermal Design Methods and Analysis," General Atomic Report GA-A12985, December 31, 1974.

5-16. Smith, G. L., private communication, General Atomic Company, March 1, 1976.

5-17. Smith, C. L., "Fuel Particle Behavior Under Normal and Transient Conditions," USAEC Report GA-A12971, General Atomic, October 1, 1974.

5-18. Scott, C. B., and D. P. Harmon, "Postirradiation Examination of Capsules HRB-4, HRB-5, and HRB-6," ERDA Report GA-A13267, General Atomic, November 28, 1975.

5-19. "HTGR Fue1s and Core Development Program Quarterly Progress Report for the Period Ending August 31, 1975," ERDA Report GA-A13592, General Atomic, September 30, 1975.

5-20. Alberstein, D., P. D. Smith, and M. J. Haire, "Metallic Fission Product Release from the HTGR Core," General Atomic Report GA-A13258, May 15, 1975. 


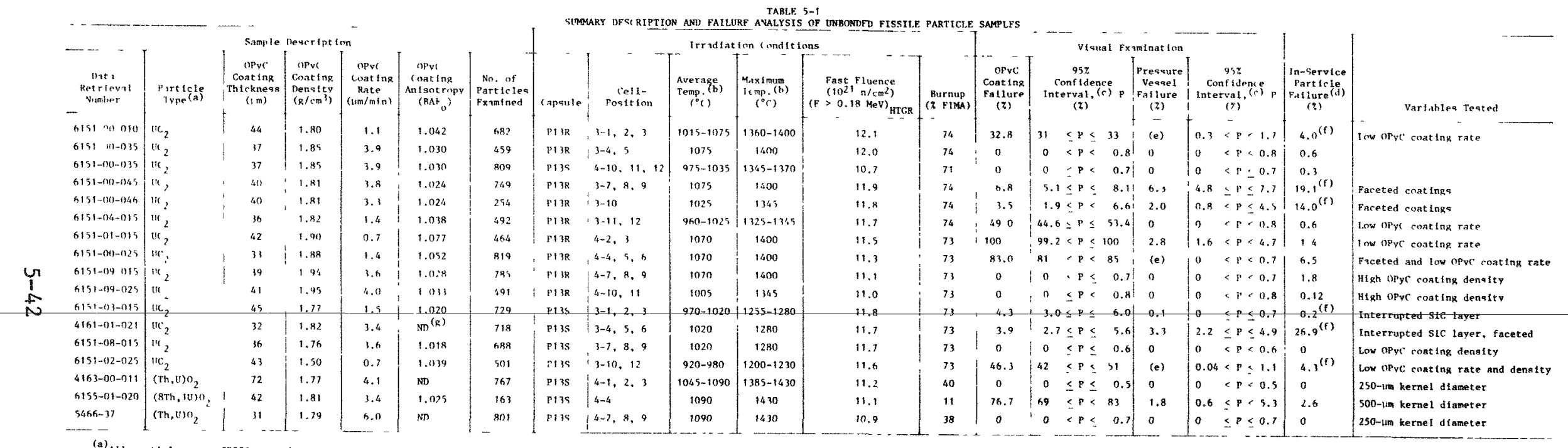

(a) ${ }_{\mathrm{All}}$ particles were rRTSO coted.

(b) Range of time averaged and maximum fuel hed centerline temperatures.

(c) An Infinite sample population with aztributes of the particle batch teqted to these conditions will have a failure level at the $95 \pi$ confidence level bounded by this interval.

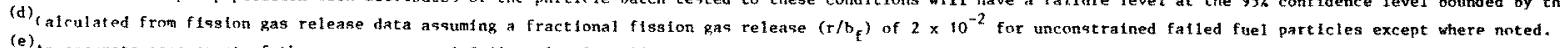

not be made during the viman exped substrate.

(8) 
TABLE 5-2

COMPARISON OF FISSILE PARTICLE OPYC AND TOTAL COATING FAILURE LEVELS

\begin{tabular}{|c|c|c|c|c|c|c|}
\hline \multirow[b]{2}{*}{$\begin{array}{c}\text { Data Retrieval } \\
\text { Number }\end{array}$} & \multirow{2}{*}{$\begin{array}{c}\text { SiC Coating } \\
\text { Substrate } \\
\text { Number (a) }\end{array}$} & \multirow{2}{*}{$\begin{array}{c}\text { Average } \\
\text { Temperature } \\
\left({ }^{\circ} \mathrm{C}\right)\end{array}$} & \multirow[b]{2}{*}{$\begin{array}{l}\text { Burnup } \\
(\% \text { FIMA) }\end{array}$} & \multirow{2}{*}{$\begin{array}{c}\text { Fast Fluence } \\
\left(10^{21} \mathrm{n} / \mathrm{cm}^{2}\right) \\
(\mathrm{E}>0.18 \mathrm{MeV})_{\text {HTGR }}\end{array}$} & \multicolumn{2}{|c|}{ Coating Failure (\%) } \\
\hline & & & & & $\mathrm{opyC}^{(\mathrm{b})}$ & $\operatorname{Tota1}{ }^{(c)}$ \\
\hline $6151-00-035$ & \multirow{4}{*}{$5862-1115$} & 1025 & 74 & 12.0 & 0 & 0.6 \\
\hline $6151-00-035$ & & $1045-1090$ & 71 & 10.7 & 0 & 0.3 \\
\hline $6151-08-015$ & & 980 & 73 & 11.6 & 0 & 0 \\
\hline $6151-02-025$ & & $970-1020$ & 73 & 11.6 & 46.3 & 4.3 \\
\hline $6151-09-015$ & \multirow{2}{*}{ S862-1095 } & 1005 & 73 & 11.1 & 0 & 1.8 \\
\hline $6151-01-015$ & & $935-1010$ & 73 & 11.5 & 100 & 1.4 \\
\hline $6151-09-025$ & \multirow{2}{*}{$5862-975$} & 1070 & 73 & 11.0 & 0 & 0.1 \\
\hline $6151-00-010$ & & $960-1025$ & 74 & 12.1 & 32.8 & 4.0 \\
\hline
\end{tabular}

(a) The same SiC substrate number indicates one particle batch through deposition of the SiC 1ayer. The batch was then split, and different oPyC coatings were applied.

(b) OPyC coating failure level was determined by visual examination.

(c) Total coating failure level was calculated from fission gas release data. 
TABLE 5-3

ANALYSIS OF METALLIC FISSION PRODUCT ATTACK OF SiC COATING IN TRISO UC ${ }_{2}$ PARTICLES

\begin{tabular}{|c|c|c|c|c|c|c|c|c|c|}
\hline $\begin{array}{c}\text { Particle Batch } \\
\text { Data Retrieval } \\
\text { Number }\end{array}$ & $\begin{array}{c}\text { Fuel Rod } \\
\text { Data } \\
\text { Retrieval } \\
\text { Number } \\
(7161-004-)\end{array}$ & Capsule & Position & $\begin{array}{l}\text { Average } \\
\text { Fue1 Rod } \\
\text { Temp. (a) } \\
\quad\left({ }^{\circ} \mathrm{C}\right)\end{array}$ & $\begin{array}{l}\text { Particle } \\
\text { I.D. } \\
\text { Number (b) } \\
(\text { I 7440-) }\end{array}$ & $\begin{array}{c}\text { Particle } \\
\text { Location }(\mathrm{c}) \\
(\mathrm{cm})\end{array}$ & $\begin{array}{l}\text { Average } \\
\text { Particie } \\
\text { Temp. } \\
\left({ }^{\circ} \mathrm{C}\right)\end{array}$ & $\begin{array}{l}\text { Particle } \\
\text { Temp. } \\
\text { Gradient } \\
\left({ }^{\circ} \mathrm{C} / \mathrm{cm}\right)\end{array}$ & $\begin{array}{l}\text { Reaction } \\
\text { Depth } \\
(\mu \mathrm{m})\end{array}$ \\
\hline $6151-00-035$ & $07-5$ & P13R & $2 B$ & 1055 & $\begin{array}{l}343 \\
340\end{array}$ & $\begin{array}{l}0.2200 \\
0.3876\end{array}$ & $\begin{array}{l}1037 \\
1000\end{array}$ & $\begin{array}{l}161 \\
283\end{array}$ & $\begin{array}{r}2.5 \\
<1.0\end{array}$ \\
\hline $6151-00-035$ & $08-13$ & P13R & $2 \mathrm{C}$ & 1045 & $\begin{array}{l}350 \\
353 \\
356 \\
363 \\
368 \\
371\end{array}$ & $\begin{array}{l}0.4431 \\
0.5290 \\
0.4587 \\
0.3979 \\
0.1487 \\
0.4511\end{array}$ & $\begin{array}{r}981 \\
954 \\
977 \\
994 \\
1038 \\
979\end{array}$ & $\begin{array}{r}287 \\
343 \\
297 \\
258 \\
96 \\
292\end{array}$ & $\begin{array}{r}<1.0 \\
<1.0 \\
<1.0 \\
1.5 \\
<1.0 \\
<1.0\end{array}$ \\
\hline $6151-00-035$ & $18-5$ & P13S & $5 \mathrm{C}$ & 1335 & $\begin{array}{l}403 \\
406 \\
411 \\
416\end{array}$ & $\begin{array}{l}0.2300 \\
0.4838 \\
0.1053 \\
0.4835\end{array}$ & $\begin{array}{l}1321 \\
1274 \\
1332 \\
1274\end{array}$ & $\begin{array}{r}121 \\
254 \\
55 \\
254\end{array}$ & $\begin{array}{l}3.0 \\
5.5 \\
1.5 \\
4.0\end{array}$ \\
\hline & & & & & $\begin{array}{l}419 \\
422 \\
428 \\
431 \\
434 \\
437 \\
609\end{array}$ & $\begin{array}{l}0.1950 \\
0.1378 \\
0.5915 \\
0.5715 \\
0.1725 \\
0.3730 \\
0.1253\end{array}$ & $\begin{array}{l}1325 \\
1330 \\
1243 \\
1249 \\
1327 \\
1298 \\
1331\end{array}$ & $\begin{array}{r}102 \\
72 \\
311 \\
300 \\
91 \\
196 \\
66\end{array}$ & $\begin{array}{r}3.0 \\
4.0 \\
3.0 \\
3.0 \\
4.5 \\
1.5 \\
14.0\end{array}$ \\
\hline $6151-08-015$ & $19-5$ & P13S & $5 \mathrm{D}$ & 1325 & $\begin{array}{l}316 \\
319 \\
322 \\
331 \\
333 \\
335\end{array}$ & $\begin{array}{l}0.3259 \\
0.2269 \\
0.2731 \\
0.5961 \\
0.4345 \\
0.3895\end{array}$ & $\begin{array}{l}1299 \\
1312 \\
1307 \\
1237 \\
1278 \\
1288\end{array}$ & $\begin{array}{l}161 \\
112 \\
135 \\
294 \\
215 \\
192\end{array}$ & $\begin{array}{l}4.0 \\
6.0 \\
6.0 \\
6.0 \\
7.5 \\
6.5\end{array}$ \\
\hline
\end{tabular}

(a) Time-averaged fuel rod centerline temperature.

(b) Identification number of laboratory photograph of fuel particle.

(c) Distance of particle from fuel rod centerline. 


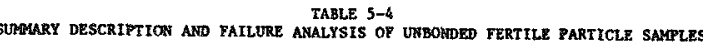

\begin{tabular}{|c|c|c|c|c|c|c|c|c|c|c|c|c|c|c|c|c|}
\hline \multicolumn{7}{|c|}{ Sample Description } & \multicolumn{6}{|c|}{ Ir radiat ion Condstions } & \multicolumn{2}{|c|}{ Visual Examination } & \multirow[b]{2}{*}{$\begin{array}{c}\begin{array}{c}\text { In-Service } \\
\text { Particle } \\
\text { Paitiure } \\
\text { (z) }\end{array} \\
(\bar{z})\end{array}$} & \multirow[b]{2}{*}{ Vartablea Teated } \\
\hline 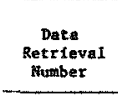 & $\begin{array}{c}\text { Buffer } \\
\text { Th1ckness } \\
\text { (htas) }\end{array}$ & 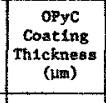 & 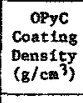 & 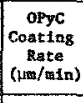 & $\begin{array}{c}\text { OPyc } \\
\text { Coating } \\
\text { Antisortropy } \\
\text { (BAF })\end{array}$ & $\begin{array}{c}\begin{array}{c}\text { No. of } \\
\text { Particlea } \\
\text { Tested }\end{array} \\
\end{array}$ & Capsule & $\begin{array}{c}\begin{array}{c}\text { Cell- } \\
\text { Position }\end{array} \\
\end{array}$ & $\begin{array}{c}\text { Average } \\
\text { Tewp. } \\
\left({ }^{\circ} \mathrm{C}\right) \\
\end{array}$ & 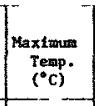 & 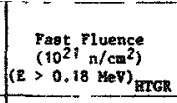 & 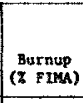 & $\begin{array}{c}\text { Tokal } \\
\text { costing } \\
\text { Fartlure } \\
\text { (z) } \\
\text { (z) }\end{array}$ & $\begin{array}{c}958 \\
\text { conftenge } \\
\text { Interval (f) } \\
(z)\end{array}$ & & \\
\hline 4252-06-018 & 78 & 77 & 1.82 & 5.8 & 1.029 & 840 & P13R & $3-1,2$ & 960 & 1055 & 12.1 & 5.1 & 0.1 & $0.01 \leq P \leq 0$. & 6.0 & \\
\hline $6342-01-020$ & 81 & 74 & 1.82 & 2.7 & 1.028 & 1004 & P13R & $3-3,4$ & 1010 & 1105 & 12.0 & 5.0 & 7.8 & $6.5 \leq P \leq 9$. & 0.9 & Low opyc coating rate \\
\hline $6342-01-010$ & 79 & 85 & 1.80 & 10.0 & 1.028 & 455 & P13R & $3-5$ & 1010 & $1 \operatorname{sos}$ & 12.0 & 5.0 & 0.2 & $0.04 \leq \leq 1$. & 60 & High opyc coating rate \\
\hline $6542-25-015$ & 99 & 76 & $\cdot .82$ & 10.8 & $\mathbb{N D}^{(d)}$ & 539 & 8138 & $4-11$ & 940 & 1050 & 10.9 & 4.1 & 1.7 & $0.9 \leq P \leq 3$. & & High oPyc coating rate, nonroumd kernels \\
\hline $6542-02-020$ & 87 & 72 & 1.91 & 8.5 & D & 1072 & PI3R & $3-7 \quad 8$ & 1010 & 1105 & 11.9 & 4.9 & 0 & $0 \leq \mathbb{0} \leq 0$. & 0.2 & \\
\hline $6542-02-020$ & 87 & 12 & 1.91 & 8.5 & No & 1149 & P13R & $4-5.6$ & 1000 & 1115 & 11.3 & 4.4 & 0 & $0 \quad \leq P \leq 0$. & 0.17 & \\
\hline $6542-02-030$ & 79 & 74 & 1.89 & 2.2 & 1.062 & 1148 & P13R & $3-9,10$ & $970-1010$ & $1065-110$ & 11.8 & 6.8 & 10.2 & $10.0 \leq P \leq 10$. & 16.5 & Low OPyC costing rate \\
\hline $6542-09-010$ & 84 & 25 & 1.93 & 5.0 & 1.042 & 998 & P13R & $3-11,12$ & $895-970$ & $975-1065$ & 11.9 & 4.8 & 0 & $0 \leq P \leq 0$. & 0 & \\
\hline $6542-11-015$ & 87 & ${ }^{74}$ & 1.83 & 2.6 & ND & 492 & P13R & $4-2$ & 1000 & 1115 & 11.5 & 4.5 & 0.4 & $0.1 \leq p \leq 1$ & & No seal coat \\
\hline $6542-19-015$ & 81 & $n$ & 1.78 & 7.6 & 1.026 & 495 & P13s & $4-7$ & 950 & 1085 & 10.9 & 3.7 & 0.6 & $0.2 \leq \mathbf{P} \leq 1$. & 12.0 & \\
\hline $6542-19-016$ & 82 & 71 & 1.78 & 7.0 & 1.026 & 502 & P135 & $4-8$ & 950 & 1085 & 10.9 & 3.7 & 0.2 & $0.04 \leq \mathrm{P} \leq 1$. & & \\
\hline $6542-18-015$ & 84 & 70 & 1.84 & 6.5 & st & 953 & P13R & $4-3,4$ & 1000 & 1115 & 11.4 & 4.5 & 0 & $0 \leq P \leq 0$. & 0 & No seal cont \\
\hline $6542-20-035$ & so & 83 & 1.84 & 3.6 & no & 1012 & PI3R & $4-7,8$ & 1000 & 1115 & 11.2 & 4.3 & 0.6 & $0.2 \leq \leq \leq 1$ & 3.5 & paceted coat1agg \\
\hline $6542-20-025$ & 76 & 81 & 1.82 & 3.5 & ND & 1040 & P13R & $4-9,10$ & $940-1000$ & $\mid 050-111$ & 11.1 & 4.2 & 1.0 & $0.6 \leq P \leq 1$ & 1.9 & Faceted contings \\
\hline 4252-06-010 & 85 & 76 & 1.82 & 5.8 & 1.029 & 872 & p13s & $3-1,2$ & 925 & 1035 & 11.8 & 4.3 & 0 & $0 \quad \leq p \leq 0$ & 0 & \\
\hline $6542-22-015$ & 85 & 81 & 1.80 & 4.0 & 1.020 & 979 & P13s & $3-3,4$ & 955 & 1075 & 11.7 & 4.3 & 1.0 & $0.5 \leq \leq \leq 1$. & 1.4 & Mitxed-gas opyc coating \\
\hline $6542-22-025$ & 81 & so & 1.81 & 7.6 & 1.028 & 964 & p13s & $3-5,6$ & 955 & 1075 & 11.7 & 4.2 & 0.3 & $0.1 \leq P \leq 0$ & 1.2 & Mixed-gas OPyC eoat Ing \\
\hline $6342-23-025$ & 82 & 73 & 1.89 & 5.8 & $\mathrm{~km}$ & 992 & p13s & $3-7,8$ & 955 & 1075 & 11.7 & 4.2 & 0 & $0 \leq P \leq 0$ & 0 & Mixed-gas opyc coating \\
\hline $6542-24-015$ & 86 & 83 & 1.94 & 6.6 & 1.032 & 942 & P13s & $3-9,10$ & $925-955$ & 1035-1075 & 11.5 & 4.1 & 0 & $0 \leq \leq 0$ & 0 & High-dens Ity mixed-gas opyc coatimg \\
\hline $6252-00-035$ & 60 & $\$ 2$ & 1.81 & a.2 & 1.015 & 528 & P135 & $3-12$ & 860 & 980 & 11.5 & 4.1 & o & $0 \leq P \leq 1$ & 0.2 & Triso costad \\
\hline $6542-17=010$ & 44 & 122 & 1.86 & 2.8 & sD & 458 & P13s & $4-1$ & 910 & 1040 & 11.2 & 3.9 & 2.6 & $1.5 \leq 7 \leq 4$ & & Coating deatga, tow opyc coat Ing resa \\
\hline 6582-16-010 & 39 & 55 & sp & 3.7 & א & 349 & P13s & $4-4$ & 950 & 1085 & 11.1 & 3.8 & 3.4 & $3.5 \leq 8 \leq 8$ & 23.5 & Coating denign \\
\hline $4252-02-010$ & 83 & 73 & 1.83 & 4.0 & 1.032 & 925 & P13s & $4-2,3$ & 950 & 1085 & 11.2 & 3.9 & o & $0 \leq P \leq 0$ & 5.5 & \\
\hline 6542-21-016 & 81 & 84 & 173 & 8.0 & 1.020 & 508 & P13s & $4-9$ & 950 & 1085 & 10.9 & 3.7 & 0 & $0 \leq p \leq 0$ & & Low OPyC coating density \\
\hline $6562-21-015$ & 79 & 82 & 1.73 & 7.8 & 1.020 & 513 & p13s & $4-10$ & 900 & 1025 & 90.8 & 3.6 & 0 & $0 \leq \leq 0$ & 70.5 & Low-density mixed-gas opyc costing \\
\hline 6542-12-023 & 84 & 70 & 1.79 & 6.7 & $\mathrm{~m}$ & 1214 & P13S & $4-11,12$ & $830-900$ & $940-1023$ & 10.7 & 3.6 & 0.2 & $0.1 \leq \mathbb{P} \leq 0$ & 1.0 & $\mathrm{~N}_{2}$ buffer carriet gasa \\
\hline
\end{tabular}

(a) Pallure of the OPyC cost ing of BISO particles or fallure of the ofyc and SiC coating of TRIs0 particles that would resule in the release of gaseous f1sasion producte.

(b) Aa infinite ample populas loa with sttributes of the particle batch tested to these conditions will have a fallure level at the 952 conf ideace level bound dod by ehis interval.

(c) Calculated from $\mathrm{f}$ lesion gas relesse data aseuming a fractional release $\left(x / h_{\mathrm{f}}\right)$ of $2 \times 10^{-2}$ for unconstrasned falled fuel particles.

(A) Mot deteraines. 
TABLE 5-5
SUMMARY DESCRIPTION AAD FAILURE ANALYSIS OF FERTLLE PARTICLES TESTED IN FUEL RODS

\begin{tabular}{|c|c|c|c|c|c|c|c|c|c|c|c|c|c|c|}
\hline \multicolumn{5}{|c|}{ Sample Description } & \multicolumn{5}{|c|}{ Irradiation Conditions } & \multicolumn{3}{|c|}{ Metallographic Examination } & \multirow[b]{2}{*}{$\begin{array}{c}\text { Maxinum } \\
\text { Fertile } \\
\text { Particle } \\
\text { Failure(c) } \\
(\%)\end{array}$} & \multirow[b]{2}{*}{ Variables Tested } \\
\hline $\begin{array}{l}\text { Ferrile Particle } \\
\text { Batch Data } \\
\text { Retrieval No. }\end{array}$ & \begin{tabular}{|} 
Fue1 Rod Data \\
Retriveval No. \\
(7161-004-)
\end{tabular} & $\begin{array}{l}\text { OPyC } \\
\text { Coating } \\
\text { Density } \\
\left(\mathrm{g} / \mathrm{cm}^{3}\right)\end{array}$ & \begin{tabular}{|c|} 
opyc \\
Coating \\
Rate \\
$(\mu \mathrm{m} / \mathrm{min})$
\end{tabular} & \begin{tabular}{|c|} 
OPyc \\
Coating \\
Anisotropy \\
(BAF)
\end{tabular} & $\begin{array}{l}\text { Capsule- } \\
\text { Position }\end{array}$ & $\begin{array}{l}\text { Average } \\
\text { Temp. (a) } \\
\left({ }^{\circ} \mathrm{C}\right)\end{array}$ & 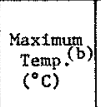 & $\begin{array}{c}\text { Fast FIuence } \\
\left(10^{21} \mathrm{n} / \mathrm{cm}^{2}\right) \\
(\mathrm{E}>0.18 \mathrm{MeV})_{\mathrm{HTGR}}\end{array}$ & $\begin{array}{l}\text { Burnup } \\
(\% \text { FIMA) }\end{array}$ & $\left|\begin{array}{l}\text { Number of } \\
\text { Particles } \\
\text { Examined }\end{array}\right|$ & $\begin{array}{c}\text { Pressure } \\
\text { Vesse1 } \\
\text { Failure } \\
\text { (\%) }\end{array}$ & $\begin{array}{l}95 \% \\
\text { Confidence } \\
\text { Interval, } \\
\text { (\%) }\end{array}$ & & \\
\hline $6542-20-035$ & $01-5$ & 1.84 & 3.6 & (d) & P13R-1A & 1115 & 1340 & 7.3 & 3.5 & $\mathrm{NE}^{(e)}$ & - & -- & 5.4 & \\
\hline $6542-20-035$ & $06-5$ & 1.84 & 3.6 & (d) & P13R-2A & 1050 & 1225 & 11.8 & 5.5 & 157 & 1.8 & $0.2 \leq p \leq 9.8$ & 5.3 & \\
\hline $6542-20-035$ & $25-5$ & 1.84 & 3.6 & (d) & $P 13 R-68$ & 1000 & 1155 & 3.6 & 0.6 & $\mathrm{NE}$ & -- & - & 0.3 & \\
\hline $6542-20-035$ & $01-7$ & 1.84 & 3.6 & (d) & P13S-iA & 1030 & $1545(\mathrm{f})$ & 7.0 & 3.2 & 135 & 51.9 & $43.5 \leq P \leq 60.1$ & 24.9 & Faceted coatings \\
\hline $6542-20-035$ & $11-6$ & 1.84 & 3.6 & (d) & P13S-2A & 1040 & 1170 & 11.5 & 4.6 & NE & -- & - & 3.2 & \\
\hline $6542-20-035$ & $30-5$ & 1.84 & 3.6 & (d) & $\mathrm{P} 13 \mathrm{~S}-6 \mathrm{~A}$ & 1085 & 1250 & 5.3 & 1.1 & $\mathrm{NE}$ & -- & -- & 0 & \\
\hline $6542-02-020$ & $02-6$ & 1.91 & 8.5 & (d) & P13R-1B & 1050 & 1225 & 8.1 & 3.9 & NE & -- & - & 0.6 & \\
\hline $6542-02-020$ & $05-6$ & 1.91 & 8.5 & (d) & P13R-1E & 1125 & 1305 & 9.9 & 4.8 & $\mathrm{NE}$ & -- & -- & 0 & \\
\hline $6542-02-020$ & $07-5$ & 1.91 & 8.5 & (d) & P13R-2B & 1050 & 1225 & 12.0 & 5.6 & 161 & 0 & $0 \leq P \leq 2.3$ & 0.3 & \\
\hline $6542-02-020$ & $17-5$ & 1.91 & 8.5 & (d) & P13R-5B & 1225 & 1460 & 9.0 & 2.8 & NE & -- & -- & 0.7 & \\
\hline $6542-02-020$ & $24-5$ & 1.91 & 8.5 & (d) & P13R-6D & 1020 & 1175 & 4.1 & 0.7 & NE & -- & - & 0.5 & \\
\hline $6542-02-020$ & $02-5$ & 1.91 & 8.5 & (d) & P13S-1B & 975 & $1470^{(f)}$ & 7.7 & 3.4 & $\mathrm{NE}$ & -- & -- & 1.3 & \\
\hline $6542-02-020$ & $05-5$ & 1.91 & 8.5 & (d) & P13S-1E & 1035 & $1575^{(f)}$ & 9.6 & 4.1 & NE & -- & - & 4.9 & \\
\hline $6542+02-020$ & $14-5$ & 1.91 & 8.5 & (d) & P13s-2D & 985 & 1100 & 12.0 & 4.6 & NE & -- & $\therefore$ & 0.2 & \\
\hline $6542-02-020$ & $17-6$ & 1.91 & 8.5 & (d) & P13S-5B & 1330 & 1605 & 8.7 & 2.6 & $\mathrm{NE}$ & - & -- & 2.2 & \\
\hline $6542-02-020$ & $27-5$ & 1.91 & 8.5 & (d) & P13S-6B & 1045 & 1215 & 4.8 & 1.0 & $\mathrm{NE}$ & - & -- & 0 & \\
\hline $6542-01-010$ & $03-5$ & 1.80 & 10.0 & 1.028 & $P 13 R-1 C$ & 1065 & 1235 & 8.7 & 4.2 & (g) & -- & -- & 0 & \\
\hline $6542-01-010$ & $08-13$ & 1.80 & 10.0 & 1.028 & $P 13 R-2 C$ & 1045 & 1195 & 12.2 & 5.6 & 144 & 0 & $0 \leq P \leq 2.6$ & 0.3 & \\
\hline $6542-01-010$ & $10-5$ & 1.80 & 10.0 & 1.028 & P 13R-2E & 965 & 1110 & 12.4 & 5.5 & $\mathrm{NE}$ & -- & - & 0.8 & \\
\hline $6542-01-010$ & $18-6$ & 1.80 & 10.0 & 1.028 & $P 13 R-5 C$ & 1235 & 1475 & 8.5 & 2.6 & $\mathrm{NE}$ & -- & - & 0 & \\
\hline $6542-01-010$ & $23-5$ & 1.80 & 10.0 & 1.028 & P13R-6C & 1005 & 1160 & 4.5 & 0.8 & 76 & 0 & $0 \leq P \leq 4.8$ & 0 & $\begin{array}{l}\text { High oPyC } \\
\text { coating rate }\end{array}$ \\
\hline $6542-01-010$ & $03-6$ & 1.80 & 10.0 & 1.028 & P13S-1C & 995 & $1505^{(f)}$ & 8.5 & 3.7 & NE & - & $\cdots$ & 26.3 & \\
\hline $6542-01-010$ & $13-6$ & 1.80 & 10.0 & 1.028 & P13S-2C & 975 & 1090 & 18.9 & 4.7 & 175 & 0 & $0 \leq P \leq 2.1$ & 0.7 & \\
\hline $6542-01-010$ & $18-5$ & 1.80 & 10.0 & 1.028 & P13s-5C & 1335 & 1625 & 8.4 & 2.4 & 165 & 0 & $0 \leq P \leq 2.3$ & 0.6 & \\
\hline $6542-01-010$ & $28-13$ & 1.80 & 10.0 & 1.028 & P13S-6C & 1050 & 1215 & 4.4 & 0.8 & 112 & 0 & $0 \leq P \leq 3.3$ & 0 & \\
\hline $6542-01-020$ & $04-6$ & 1.82 & 2.7 & 1.028 & P13R-1D & 1115 & 1290 & 9.3 & 4.5 & NE & -- & -- & 2.4 & \\
\hline $6542-01-020$ & $09-5$ & 1.82 & 2.7 & 1.028 & P13R-2D & 1035 & 1180 & 12.3 & 5.6 & $\mathrm{NE}$ & -- & -- & 8.3 & \\
\hline $6542-01-020$ & $16-5$ & 1.82 & 2.7 & 1.028 & P13R-5A & 1285 & 9515 & 9.4 & 3.1 & (g) & -- & -- & 0.9 & Low OPyC \\
\hline $6542-01-020$ & $04-5$ & 1.82 & 2.7 & 1.028 & P13S-1D & 1035 & $1555^{(f)}$ & 9.1 & 3.9 & NE & -- & -- & 4.9 & coating rate \\
\hline $6542-01-020$ & $16-6$ & 1.82 & 2.7 & 1.028 & P13s-5A & 1375 & 1645 & 9.2 & 2.8 & 89 & 5.6 & $2.1 \leq P \leq 12.6$ & 4.1 & \\
\hline $6542-01-020$ & $26-5$ & 1.82 & 2.7 & 1.028 & $P(3 \mathrm{~S}-6 \mathrm{~A}$ & 1085 & 1250 & 5.3 & 1.1 & NE & - & - & 0 & \\
\hline $6542-01-020$ & $29-5$ & 1.82 & 2.7 & 1.028 & P13S-6D & 1015 & 1215 & 3.9 & 0.7 & NE & -- & -- & 0 & \\
\hline $6542-19-015$ & $19-7$ & 1.78 & 7.6 & 1.026 & P13R-5D & 1225 & 1480 & 8.2 & 2.4 & 206 & 0.5 & $0.1 \leq p \leq 2.7$ & 0.2 & \\
\hline $6542-19-015$ & $21-5$ & 1.78 & 7.6 & 1.026 & P1 3R-6A & 1065 & 1220 & 5.4 & 1.1 & NE: & -- & - & 0 & \\
\hline $6542-19-015$ & $15-5$ & 1.78 & 7.6 & 1.026 & P13S-2E & 960 & 1095 & 12.4 & 4.6 & 36 & 0 & $0 \leq P \leq 9.9$ & 2.1 & coating density \\
\hline $6542-19-015$ & $19-5$ & 1.78 & 7.6 & 1.026 & P13S-5D & 1325 & 1610 & 7.9 & 2.2 & 194 & 5.7 & $3.2 \leq P \leq 9.9$ & 1.7 & \\
\hline $6542-09-010$ & $20-6$ & 1.93 & 5.0 & 1.042 & P $13 R-5 E$ & 1280 & 1590 & 7.7 & 2.1 & 144 & 0.7 & $0.1 \leq \mathrm{P} \leq 3.8$ & 0.1 & \\
\hline $6542-09-010$ & $22-5$ & 1.93 & 5.0 & 1.042 & $\mathrm{P} \mathbf{3} \mathrm{3R}-6 \mathrm{~B}$ & 1055 & 1215 & 5.0 & 1.0 & NE & -- & -- & 0 & High OPyC \\
\hline $6542-09-010$ & $12-5$ & 1.93 & 5.0 & 1.042 & P135-2B & 1010 & 1135 & 11.7 & 4.6 & 129 & 0.8 & $0.1 \leq \mathrm{P} \leq 4.3$ & 0 & coating densitv \\
\hline $6542-09-010$ & $20-5$ & 1.93 & 5.0 & 1.042 & P13S-5E & 1350 & 1650 & 7.5 & 2.0 & 247 & 0 & $0 \leq P \leq 1.5$ & 3.3 & \\
\hline
\end{tabular}

(b) Maximum fuel rod centeriine temperature during irradiation.

(b) Maximum fuel rod centerline temperature during irradiation.

(c) Calculated assuming a fractional release $\left(r / b_{f}\right)$ of $5 \times 10^{-3}$ for falled fuel particles in a constrained geometry and that all the fisston gas released came from falled fertile particles. (d) Not determined.

(e) $\mathrm{NE}=$ not examined.

(f) Thermal cycled in-pile.

(g) Metallography was performed after the fuel rod was heated to $1600^{\circ} \mathrm{C}$ in TRIGA. 
TABLE 5-6

FERTILE PARTICLE OPYC COATING DENSITY

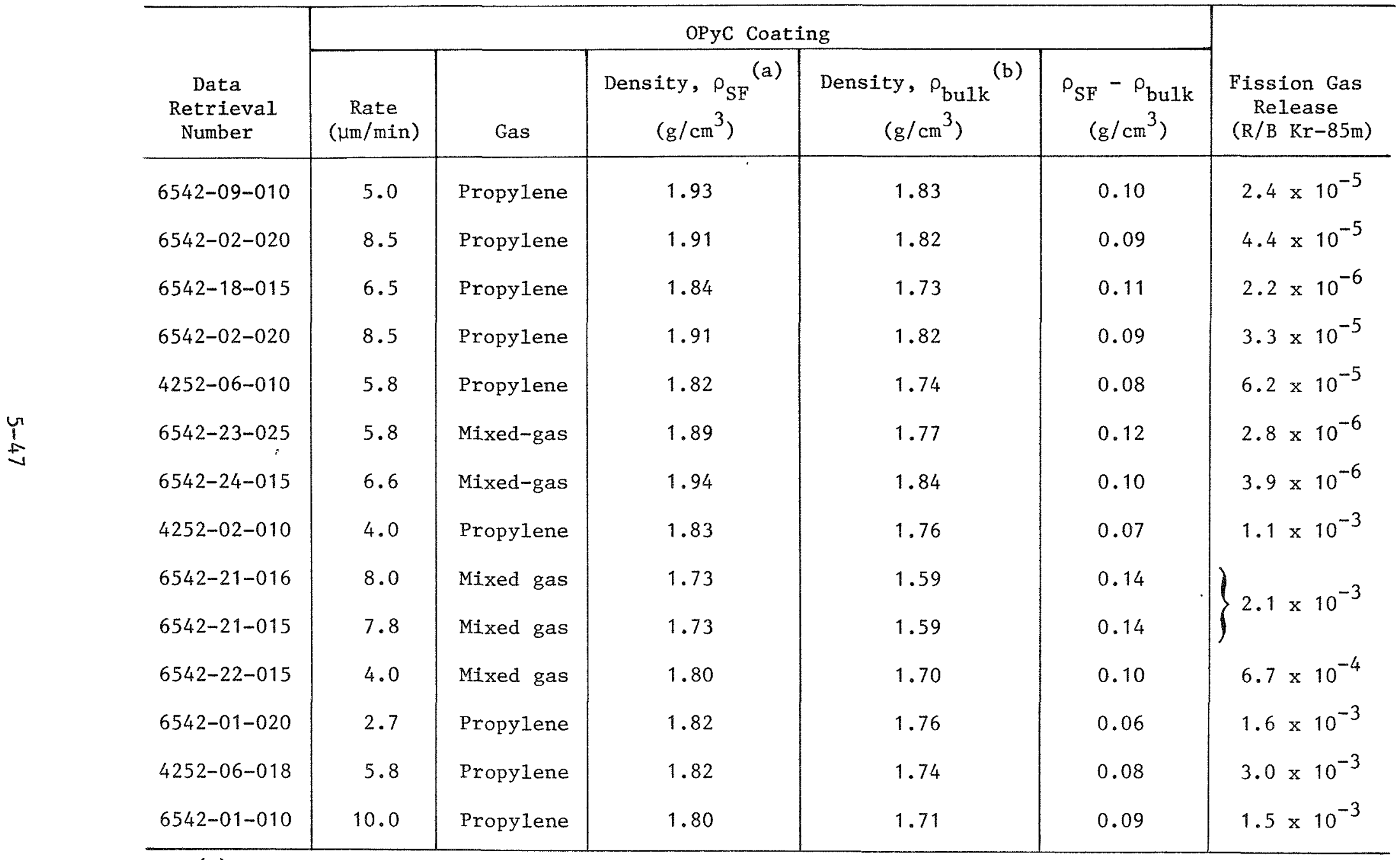

(a) Unirradiated OPyC coating density determined by a sink-float technique.

(b) Unirradiated OPyC coating density determined by low-pressure mercury intrusion. 
TABLE 5-7

IRRADIATION PARAMETERS AND FUEL FAILURE LEVELS IN FUEL RODS TESTED IN CELL 1 OF CAPSULES P13R AND P13S

\begin{tabular}{|c|c|c|c|c|c|c|c|c|c|c|}
\hline \multirow{2}{*}{$\begin{array}{l}\text { Fuel Rod Data } \\
\text { Retrieval No. } \\
\text { (7161-004-) }\end{array}$} & \multirow{2}{*}{$\begin{array}{l}\text { Capsule- } \\
\text { Position }\end{array}$} & \multirow{2}{*}{$\begin{array}{l}\text { Average } \\
\text { Temp. } \\
\left({ }^{\circ} \mathrm{C}\right)\end{array}$} & \multirow{2}{*}{$\begin{array}{l}\text { Maximum } \\
\text { Temp. } \\
\left({ }^{\circ} \mathrm{C}\right)\end{array}$} & \multirow{2}{*}{$\begin{array}{c}\text { Fast Fluence } \\
\left(10^{\left.21 \mathrm{n} / \mathrm{cm}^{2}\right)}\right. \\
(E>0.18 \mathrm{MeV}) \\
\text { HTGR }\end{array}$} & \multicolumn{2}{|c|}{ Burnup (\% FIMA) } & \multirow{2}{*}{$\begin{array}{c}\mathrm{R} / \mathrm{B} \\
\text { Failed } \\
\text { Fuel(a) }\end{array}$} & \multirow{2}{*}{$\begin{array}{l}\text { In-Service } \\
\text { Particle } \\
\text { Failure (b) } \\
\quad(\%)\end{array}$} & \multirow{2}{*}{$\begin{array}{l}\text { Maximum } \\
\text { Fertile } \\
\text { Particle } \\
\text { Failure (b,c) } \\
\quad(\%)\end{array}$} & \multirow[b]{2}{*}{ Remarks } \\
\hline & & & & & Fissile & Fertile & & & & \\
\hline $01-7$ & $\mathrm{P} 135-1 \mathrm{~A}$ & 1030 & (d) & 7.0 & 70 & 3.2 & $1.1 \times 10^{-3}$ & 22.0 & 24.9 & Faceted fuel particles \\
\hline $02-5$ & P13S-1B & 975 & (d) & 7.7 & 71 & 3.4 & $5.7 \times 10^{-5}$ & 1.1 & 1.3 & \\
\hline $03-6$ & P13S-1C & 995 & (d) & 8.5 & 71 & 3.7 & $1.1 \times 10^{-4}$ & 2.2 & 2.4 & $\begin{array}{l}\text { Fertile particles had } \\
\text { a high OPyC coating } \\
\text { rate. }\end{array}$ \\
\hline $04-5$ & P13S-1D & 1035 & (d) & 9.1 & 72 & 3.9 & $1.2 \times 10^{-3}$ & 24.0 & 26.3 & $\begin{array}{l}\text { Fertile particles had } \\
\text { a low opyc coating } \\
\text { rate. }\end{array}$ \\
\hline $06-5$ & $P 13 S-1 E$ & 1035 & (d) & 9.6 & 39 & 4.1 & $2.2 \times 10^{-4}$ & 4.4 & 4.9 & $\begin{array}{l}\text { (Th, U) } \mathrm{O}_{2} \text { fissile } \\
\text { particles }\end{array}$ \\
\hline $01-5$ & P13R-1A & 1115 & 1340 & 7.3 & 71 & 3.5 & $2.4 \times 10^{-4}$ & 4.8 & 5.4 & Faceted fuel particles \\
\hline $02-6$ & P13R-1B & 1050 & 1225 & 8.1 & 72 & 3.9 & $2.9 \times 10^{-5}$ & 0.6 & 0.6 & \\
\hline $03-5$ & P13R-1C & 1065 & 1235 & 8.7 & 73 & 4.2 & $1.0 \times 10^{-6}$ & 0 & 0 & $\begin{array}{l}\text { Fertile particles had } \\
\text { a high opyc coating } \\
\text { rate. }\end{array}$ \\
\hline $04-6$ & P13R-1D & 1115 & 1290 & 9.3 & 73 & 4.5 & $1.1 \times 10^{-4}$ & 2.2 & 2.4 & $\begin{array}{l}\text { Fertile particles had } \\
\text { a low OPyC coating } \\
\text { rate. }\end{array}$ \\
\hline $05-6$ & P13R-1E & 1125 & 1305 & 9.9 & 40 & 4.8 & $2.2 \times 10^{-7}$ & 0 & 0 & $\begin{array}{l}\text { (Th, U) } \mathrm{O}_{2} \text { fissile } \\
\text { particles }\end{array}$ \\
\hline
\end{tabular}

${ }^{(a)} \mathrm{R} / \mathrm{B}$ for $\mathrm{Kr}-85 \mathrm{~m}$ measured at $1100^{\circ} \mathrm{C}$. $\mathrm{R} / \mathrm{B}_{\text {failed fuel }}=\mathrm{R} / \mathrm{B}_{\mathrm{EOL}}-\mathrm{R} / \mathrm{B}_{\text {contamination }}$.

(b) Calculated by assuming a fractional release of $5 \times 10^{-3}$ for failed fuel in a constrained geometry.

(c) Calculated by assuming all the fission gas release came from failed fertile particles.

(d) P13s cell 1 fuel rods were thermal cycled 24 times during irradiation. 
TABLE 5-8

FAILURE ANALYSIS OF FUEL RODS HEATED TO $1600^{\circ} \mathrm{C}$ IN THE TRIGA FACILITY

\begin{tabular}{|c|c|c|c|c|c|c|c|}
\hline \multirow{3}{*}{$\begin{array}{l}\text { Data Retrieva1 } \\
\text { Number } \\
(7161-004-)\end{array}$} & \multirow[b]{3}{*}{$\begin{array}{l}\text { Capsule- } \\
\text { Position }\end{array}$} & \multirow{2}{*}{\multicolumn{2}{|c|}{$\begin{array}{l}\text { Fission Gas Release } \\
\left(\mathrm{R} / \mathrm{B} \mathrm{Kr}-85 \mathrm{~m} \text { at } 1100^{\circ} \mathrm{C}\right)\end{array}$}} & \multicolumn{4}{|c|}{ Fuel Particle Failure ${ }^{(b)}(\%)$} \\
\hline & & & & \multicolumn{2}{|c|}{ Pre-HT (a) } & \multicolumn{2}{|c|}{ Post-HT $(\mathrm{a})$} \\
\hline & & Pre-HT $(\mathrm{a})$ & Post-HT $(a)$ & Total & $\begin{array}{l}\text { Maximum } \\
\text { Fertile (c) }\end{array}$ & Total & $\begin{array}{c}\text { Maximum } \\
\text { Fertile (c) }\end{array}$ \\
\hline $14-5$ & P13S-2D & $1.2 \times 10^{-5}$ & $4.2 \times 10^{-4}$ & 0.2 & 0.2 & 8.3 & 8.9 \\
\hline $03-6$ & P13S-1C & $1.1 \times 10^{-4}$ & $5.2 \times 10^{-4}$ & 2.2 & 2.4 & 10.4 & 11.4 \\
\hline $17-5$ & $P 13 R-5 B$ & $2 \times 10^{-5}$ & $2.9 \times 10^{-4}$ & 0.6 & 0.7 & 5.8 & 6.5 \\
\hline
\end{tabular}

(a) Pre-HT and post-HT indicate before and after heating to $1600^{\circ} \mathrm{C}$ in the TRIGA facility.

(b) Fuel failure levels were calculated from the fission gas release data (corrected for heavy metal contamination) by assuming fractional release of $5.0 \times 10^{-3}$ for failed fuel.

(c) Calculated by assuming all fission gas was released from failed fertile particles. 
TABLE 5-9

FISSION PRODUCT ISOTOPE RATIOS OF CAPSULE P13R FUEL RODS

\begin{tabular}{|c|c|c|c|c|c|}
\hline $\begin{array}{c}\text { Data } \\
\text { Retrieval } \\
\text { Number } \\
(7161-004-)\end{array}$ & $\begin{array}{l}\text { Capsule } \\
\text { Position }\end{array}$ & $\begin{array}{l}\text { Measured } \\
\frac{\mathrm{Cs}-137}{\mathrm{Zr}-95}\end{array}$ & $\begin{array}{l}\text { Calculated } \\
\frac{\mathrm{Cs}-137}{\mathrm{Zr}-95}\end{array}$ & $\underset{(\%)}{\text { Difference }}$ & $\begin{array}{c}\text { Measured }^{(b)} \\
\frac{\mathrm{Cs}-137}{\mathrm{Cs}-134}\end{array}$ \\
\hline $01-5$ & $1 \mathrm{~A}$ & 0.024 & 0.023 & +4.2 & 0.668 \\
\hline $02-6$ & $1 \mathrm{~B}$ & 0.022 & 0.022 & 0 & 0.603 \\
\hline $03-5$ & $1 \mathrm{C}$ & 0.020 & 0.022 & -10.0 & 0.590 \\
\hline $04-6$ & $1 \mathrm{D}$ & 0.022 & 0.022 & 0 & 0.577 \\
\hline $05-6$ & $1 E$ & 0.021 & 0.022 & -4.8 & 0.560 \\
\hline $16-5$ & $5 \mathrm{~A}$ & 0.022 & 0.021 & +4.5 & 0.618 \\
\hline $17-5$ & $5 B$ & 0.022 & 0.021 & +4.5 & 0.648 \\
\hline $18-6$ & $5 \mathrm{C}$ & 0.023 & 0.021 & +8.7 & 0.631 \\
\hline $19-7$ & $5 D$ & (c) & 0.021 & -- & (c) \\
\hline $20-6$ & $5 E$ & 0.022 & 0.021 & +4.5 & 0.681 \\
\hline $21-5$ & $6 \mathrm{~A}$ & 0.023 & 0.022 & +4.3 & 0.948 \\
\hline $22-5$ & $6 B$ & 0.022 & 0.022 & 0 & 1.008 \\
\hline $23-5$ & $6 c$ & 0.023 & 0.022 & +4.3 & 0.976 \\
\hline $24-5$ & $6 \mathrm{D}$ & 0.023 & 0.022 & +4.3 & 1.410 \\
\hline $25-5$ & $6 E$ & 0.025 & 0.022 & +12.0 & 1.142 \\
\hline
\end{tabular}

(a) Difference between measured and calculated $\frac{\mathrm{Cs}-137}{\mathrm{Zr}-95}$ ratio.

(b) A theoretical Cs-134 inventory was not computed.

(c) Fuel rod 7161-004-19-7 was not gamma-counted. 
TABLE $5-10$

FISSION PRODUCT ISOTOPE RATIOS OF CAPSULE P13S FUEL RODS

\begin{tabular}{l|c|c|c|c|c}
\hline $\begin{array}{c}\text { Data } \\
\begin{array}{c}\text { Retrieval } \\
\text { Number } \\
(7161-004-)\end{array}\end{array}$ & $\begin{array}{c}\text { Capsule } \\
\text { Position }\end{array}$ & $\begin{array}{c}\text { Measured } \\
\text { Cs-137 }\end{array}$ & $\begin{array}{c}\text { Calculated } \\
\text { Cs-137 }\end{array}$ & $\begin{array}{c}\text { Difference } \\
(\%)\end{array}$ & $\begin{array}{c}\text { Measured } \\
\text { (bs }-137\end{array}$ \\
\hline $01-7$ & 1A & 0.021 & 0.023 & -9.5 & 0.671 \\
$02-5$ & 1B & 0.022 & 0.022 & 0 & 0.650 \\
$03-6$ & 1C & 0.023 & 0.022 & +4.3 & 0.603 \\
$04-5$ & 1D & 0.019 & 0.022 & -15.8 & 0.544 \\
$05-5$ & 1E & 0.023 & 0.022 & +4.3 & 0.558 \\
$16-6$ & 5A & 0.022 & 0.021 & +4.5 & 0.765 \\
$17-6$ & 5B & 0.022 & 0.022 & 0 & 0.634 \\
$18-5$ & 5C & 0.023 & 0.021 & +8.7 & 0.601 \\
$19-5$ & 5D & 0.024 & 0.021 & +12.5 & 0.644 \\
$20-5$ & 5E & 0.021 & 0.021 & 0 & 0.715 \\
\hline
\end{tabular}

(a) Difference between measured and calculated $\frac{\mathrm{Cs}-137}{\mathrm{Zr}-95}$ ratio.

(b) A theoretical Cs-134 inventory was not computed. 
TABLE $5-11$

FRACTIONAL CS-137 RELEASE FROM FAILED FUEL IN FUEL RODS

\begin{tabular}{c|c|c|c}
\hline Rod I.D. & $\begin{array}{c}\text { Equivalent } \\
\text { Fue1 Failure } \\
(\%)\end{array}$ & $\begin{array}{c}\text { Cs-137 Loss } \\
(\%)\end{array}$ & $\begin{array}{c}\text { Fractional Cs-137 } \\
\text { Release From } \\
\text { Failed Fue1 }\end{array}$ \\
\hline P13S-1A & 22.0 & 9.5 & 0.43 \\
P13S-1D & 24.0 & 15.8 & 0.66 \\
\hline
\end{tabular}

(a) Percent of fissions occurring in failed fuel (fissile and fertile) calculated from fission gas release data.

TABLE $5-12$

COMPARISON OF Cs-137 LOSS WITH FISSILE PARTICLE FAILURE LEVELS

\begin{tabular}{c|c|c|c}
\hline $\begin{array}{c}\text { Batch Data } \\
\text { Retrieva1 No. }\end{array}$ & Capsule & $\begin{array}{c}\text { Particle } \\
\text { Failure } \\
(\%)\end{array}$ & $\begin{array}{c}\text { Cs-137 } \\
\text { Loss } \\
(\%)\end{array}$ \\
\hline $6151-00-025$ & P13R & 6.5 & 4.4 \\
$6151-00-010$ & P13R & 4.0 & 8.5 \\
$6151-00-045$ & P13R & 19.1 & 13.6 \\
$6151-00-046$ & P13R & 14.0 & 8.6 \\
$4161-01-021$ & P13S & 26.9 & 18.9 \\
\hline
\end{tabular}

(a) Particles were irradiated in the temperature range of $1015^{\circ}$ to $1075^{\circ} \mathrm{C}$. 


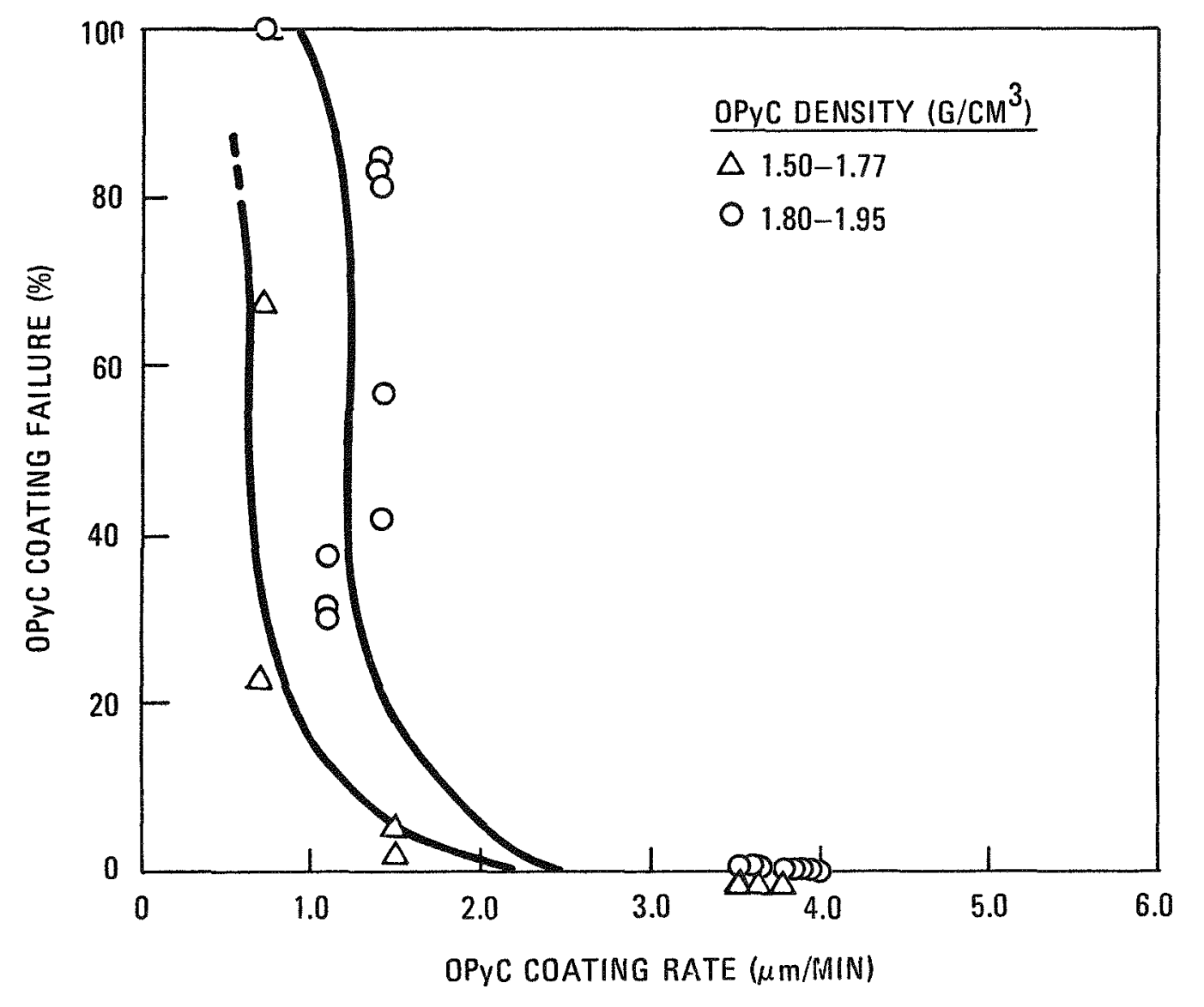

Fig. 5-1. OPyC coating failure versus coating rate and density for $\mathrm{UC}_{2}$ TRISO particles irradiated in capsules $\mathrm{P} 13 \mathrm{R}$ and $\mathrm{P} 13 \mathrm{~S}$ to fast neutron fluences of 10.7 to $12.1 \times 10^{21} \mathrm{n} / \mathrm{cm}^{2}$ ( $\mathrm{E}>0.18 \mathrm{MeV}$ ) HTGR at $1075^{\circ} \mathrm{C}$ (design) 


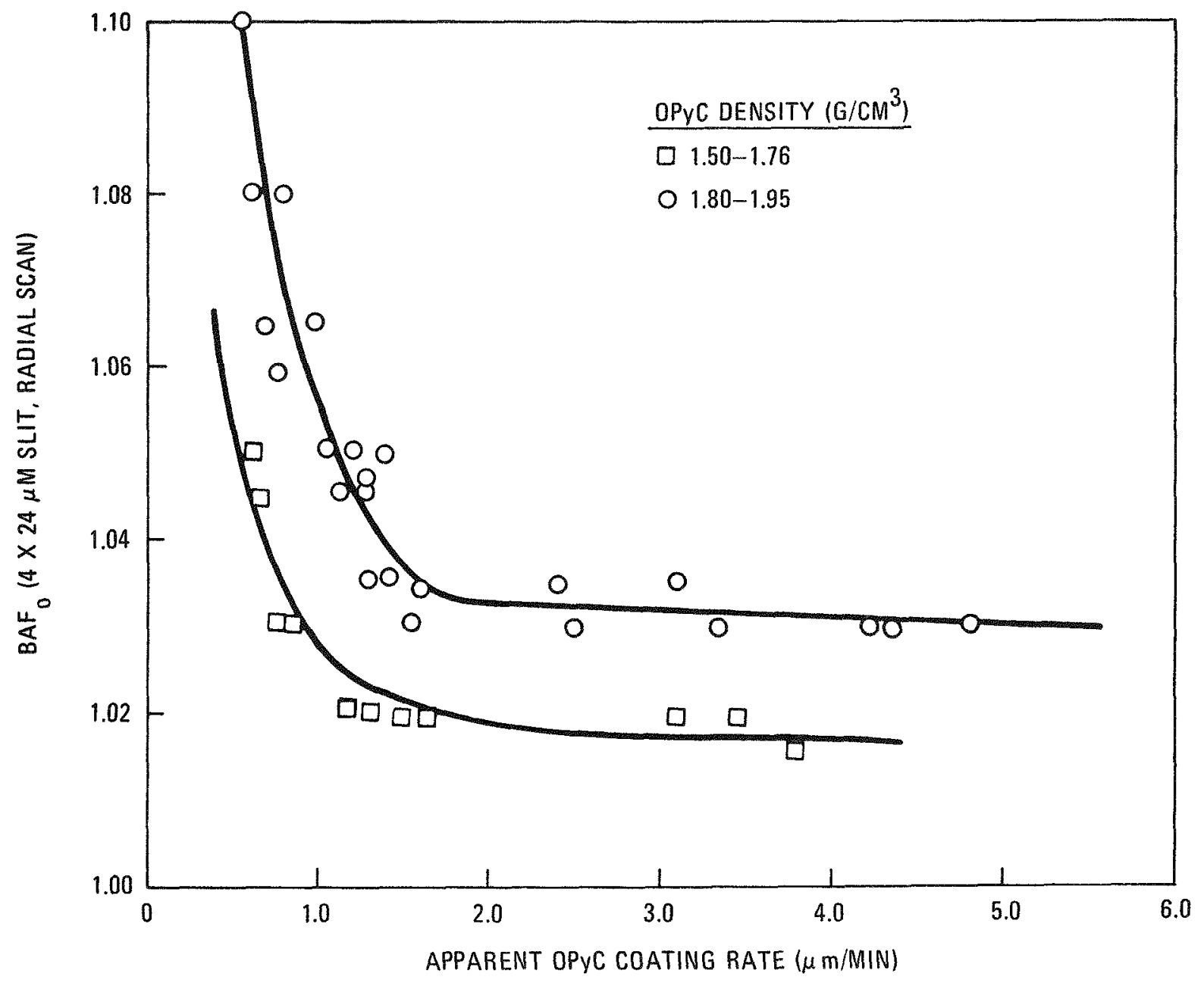

Fig. 5-2. Optical anisotropy measured on individual OPyC coatings of $\mathrm{UC}_{2}$ TRISO particles versus apparent coating rate. Anisotropy values were measured by using a $4 \times 24 \mu \mathrm{m}$ slit and radial scanning the coatings. A Seibersdorf unit was used and anisotropies are in $\mathrm{BAF}_{\mathrm{O}}$ units. 


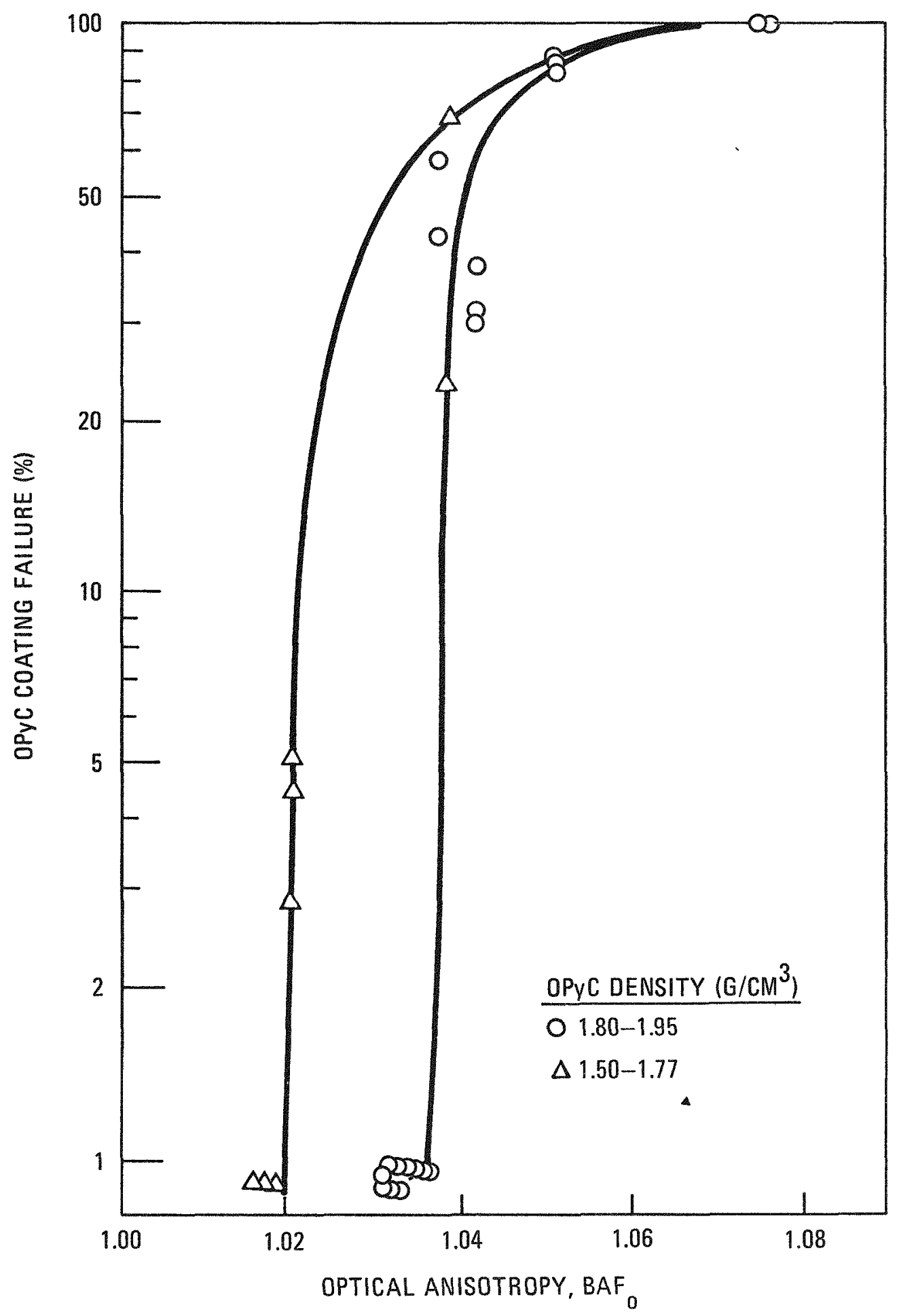

Fig. 5-3. OPYC coating failure versus optical anisotropy for $\mathrm{UC}_{2} \mathrm{TRISO}$ particles irradiated in unbonded beds at $1075^{\circ} \mathrm{C}$ to fast neutron fluences of 10.7 to $12.1 \times 10^{21} \mathrm{n} / \mathrm{cm}^{2}$ (E $>0.18 \mathrm{MeV}$ ) HTGR Optical anisotropies were measured by using a Seibersdorf unit and radially scanning the coatings with a $4 \times 24 \mu \mathrm{m}$ slit. 

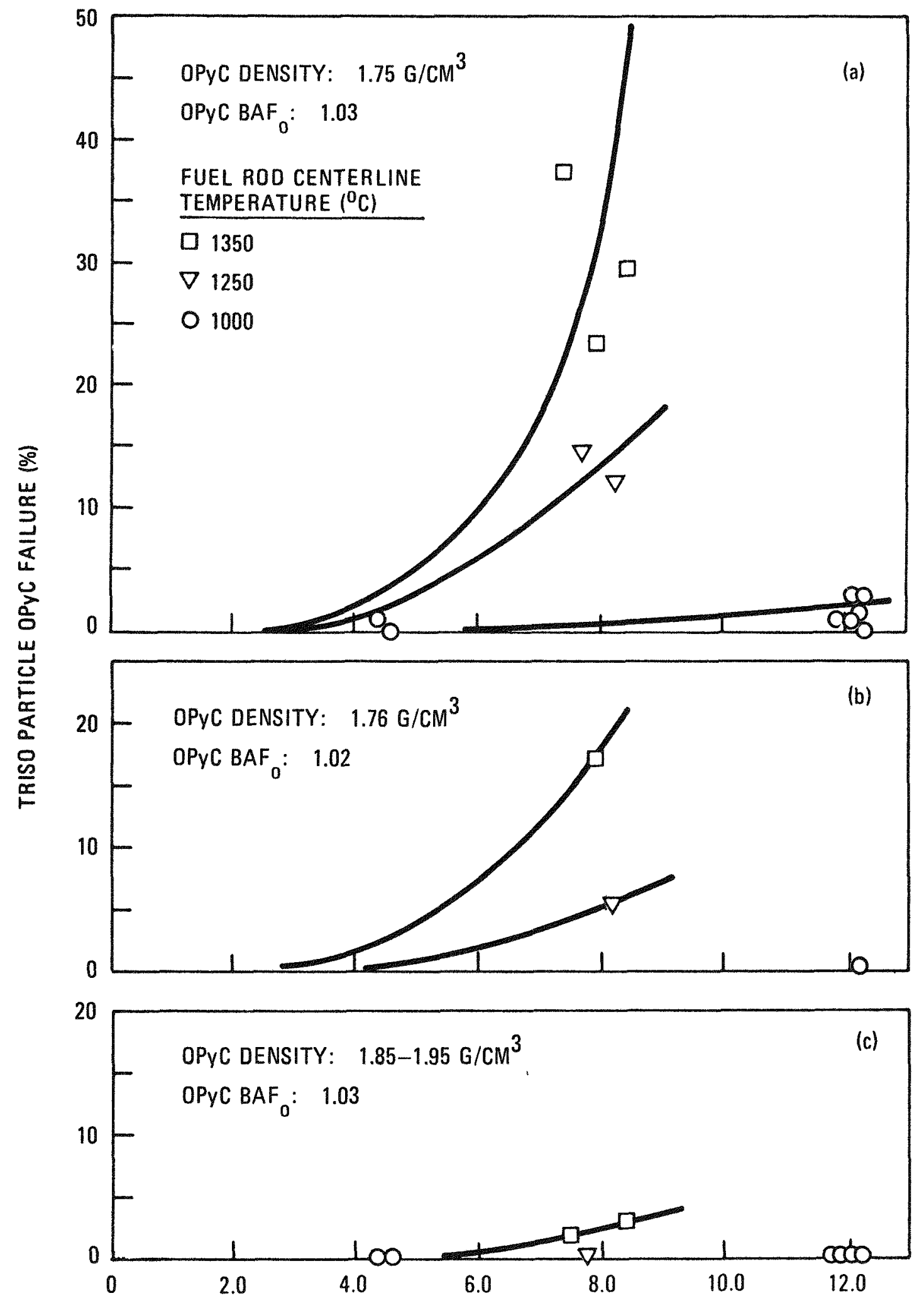

FAST NEUTRON FLUENCE $\left(10^{21} \mathrm{~N} / \mathrm{CM}^{2}\right)(\mathrm{E}>0.18 \mathrm{MeV})_{\mathrm{HTGR}}$

Fig. 5-4. OPyC performance on 600- $\mu \mathrm{m}$-diameter TRISO particles irradiated in $P 13 R$ and $P 13 S$ fuel rods 


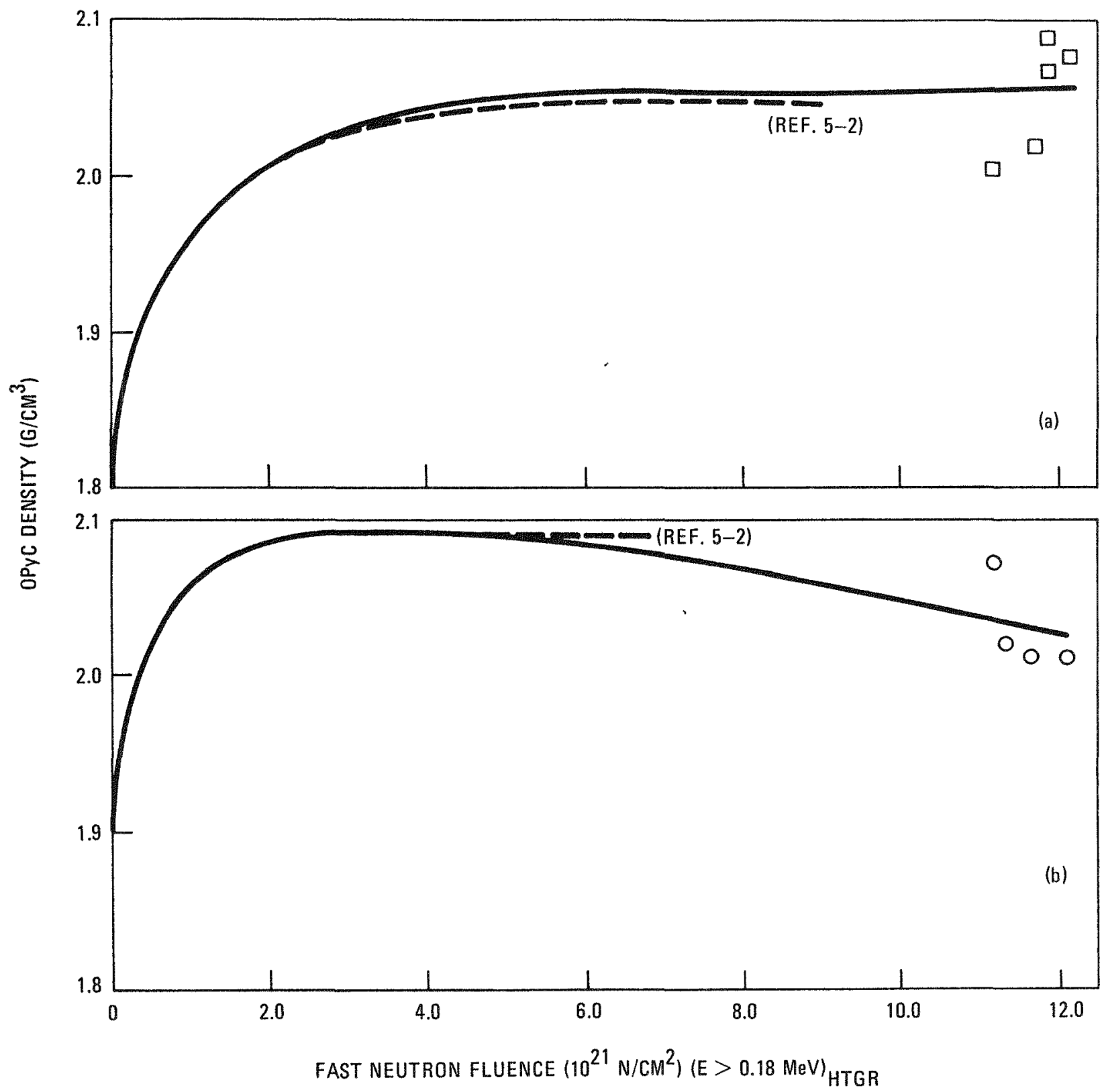

Fig. 5-5. Density change of OPyC coatings on TRISO fuel particles irradiated at $950^{\circ}$ to $1250^{\circ} \mathrm{C}$ : (a) initial densities of 1.76 to $1.82 \mathrm{~g} / \mathrm{cm}^{3}$ and (b) initial densities of 1.85 to $1.94 \mathrm{~g} / \mathrm{cm}^{3}$ 


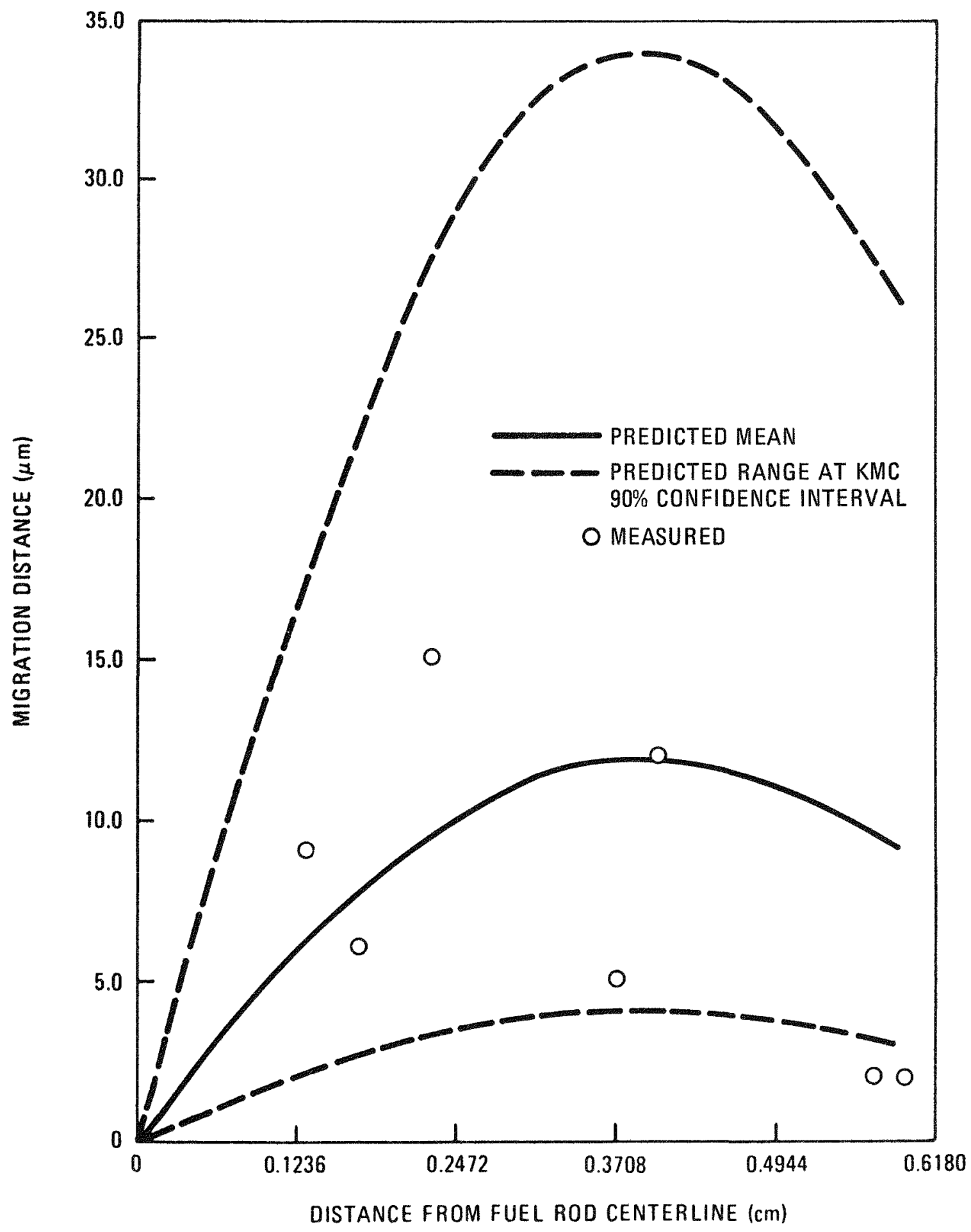

Fig. 5-6. Measured and predicted $\mathrm{UC}_{2}$ fuel kernel migration distance as a function of radial location in fuel rod 7161-004-18-5 irradiated in capsule P13S (position 5C) 


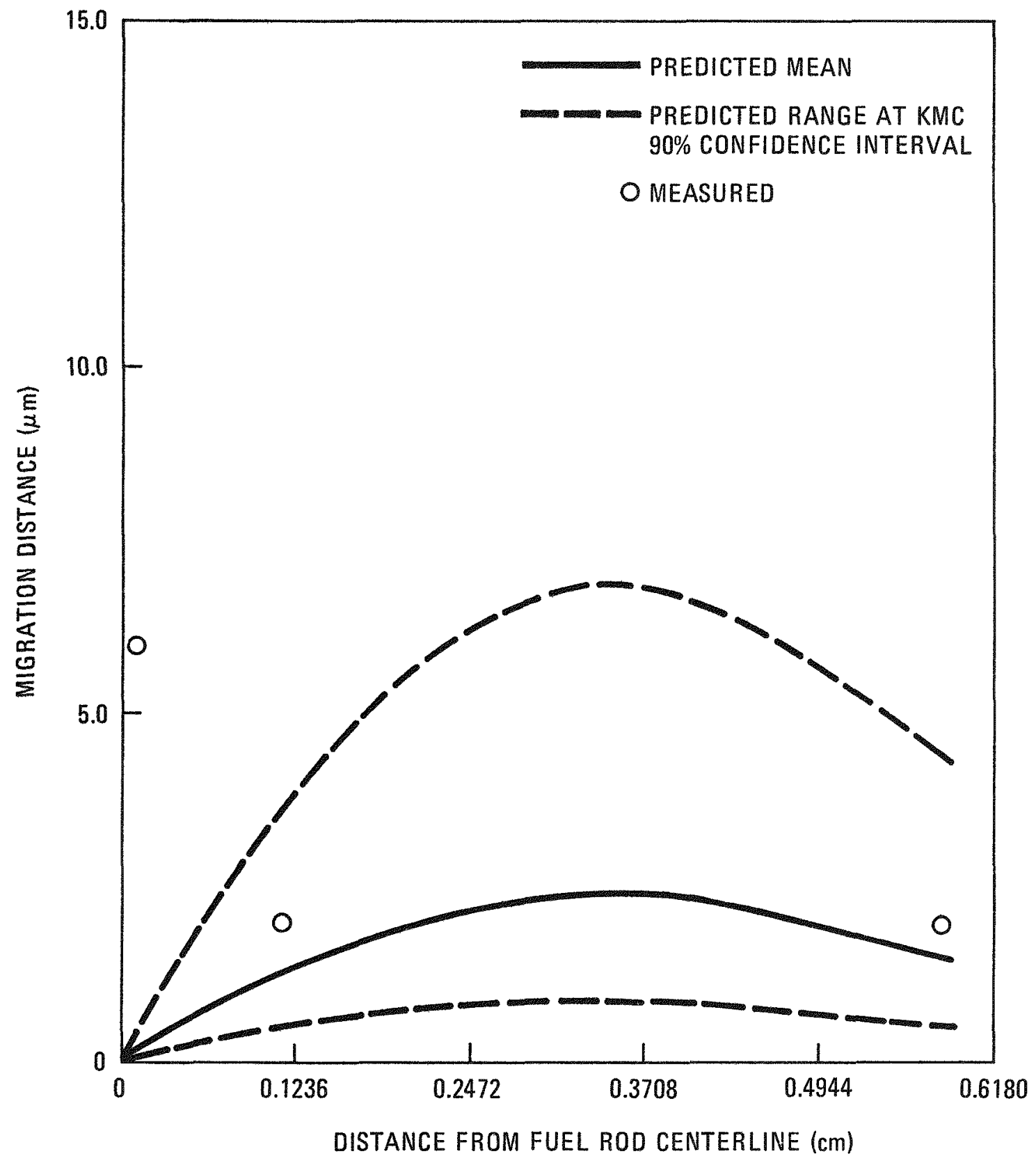

Fig. 5-7. Measured and predicted $\mathrm{UC}_{2}$ fuel kernel migration distance as a function of radial location in fuel rod 7161-004-19-7 irradiated in capsule P13R (position 5D) 


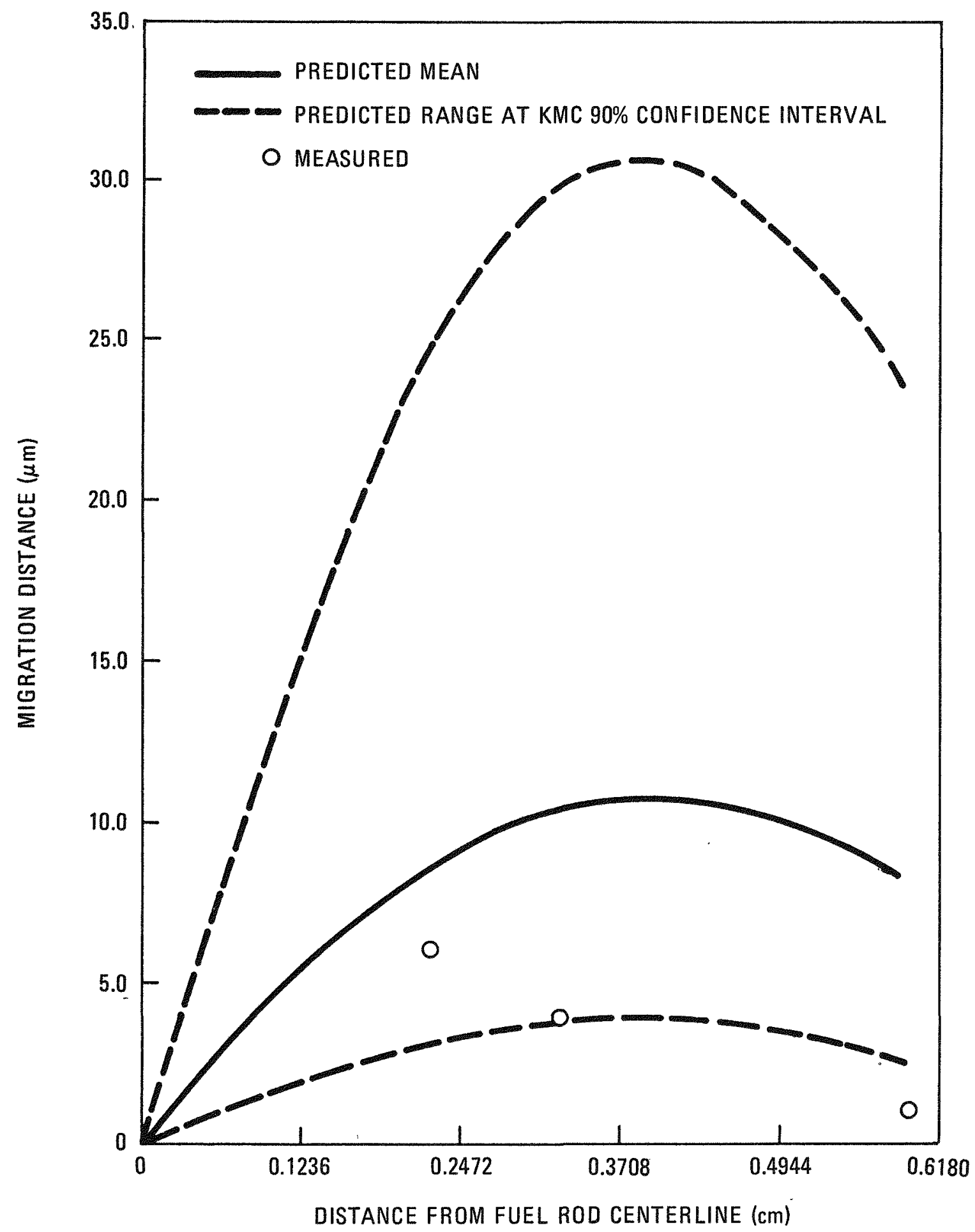

Fig. 5-8. Measured and predicted $\mathrm{UC}_{2}$ fuel kernel migration distance as a function of radial location in fuel rod 7161-004-19-5 irradiated in capsule P13S (position 5D) 


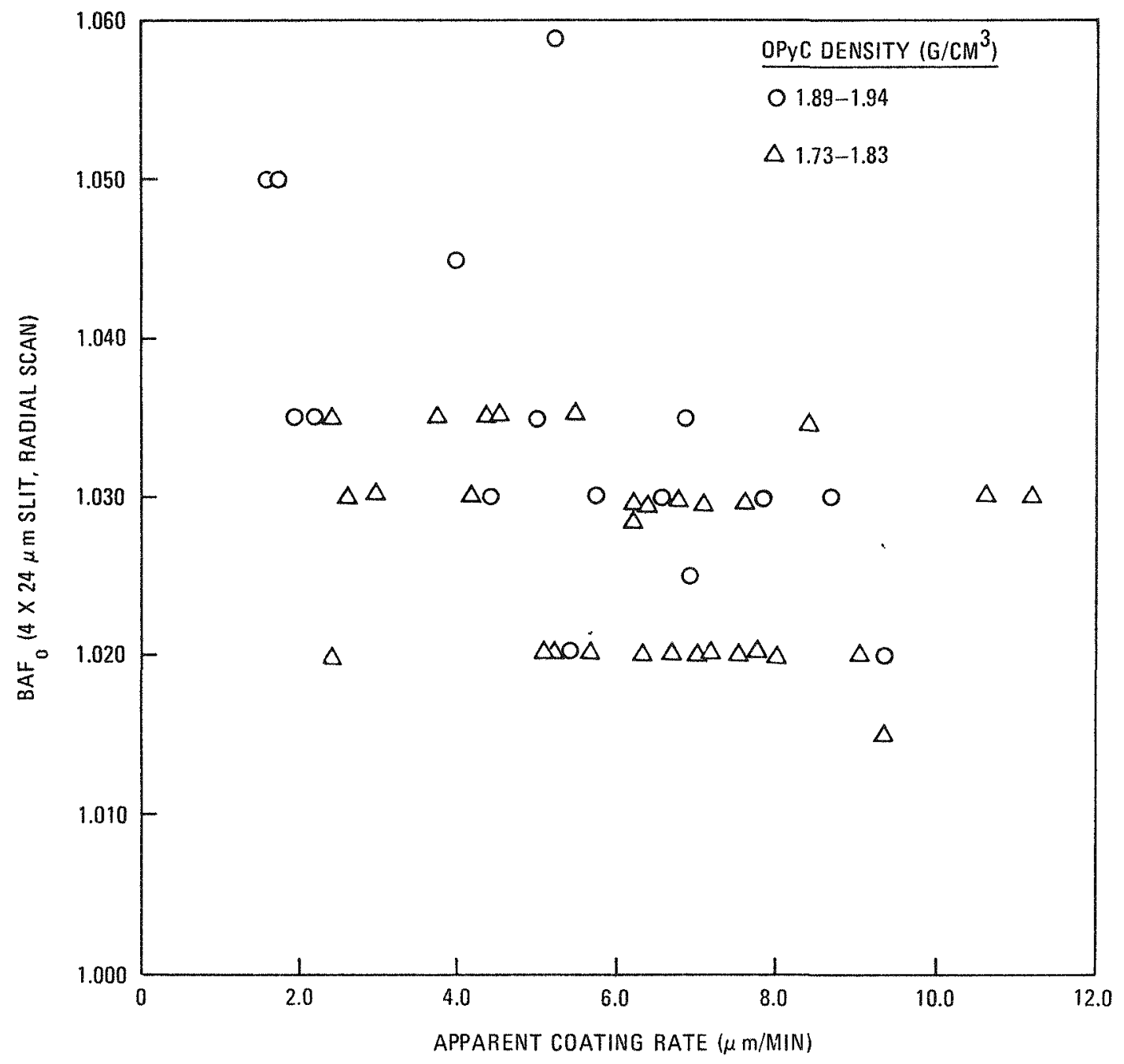

Fig. 5-9. Optical anisotropy measured on individual OPyC coatings of Tho BISO particles versus apparent coating rate. Optical anisotropy values were obtained by using a Seibersdorf unit and radially scanning the coatings with a $4 \times 24 \mu \mathrm{m}$ slit. Note the trend toward higher anisotropies as the coating rates decrease. Compare with Fig. 5-2. 

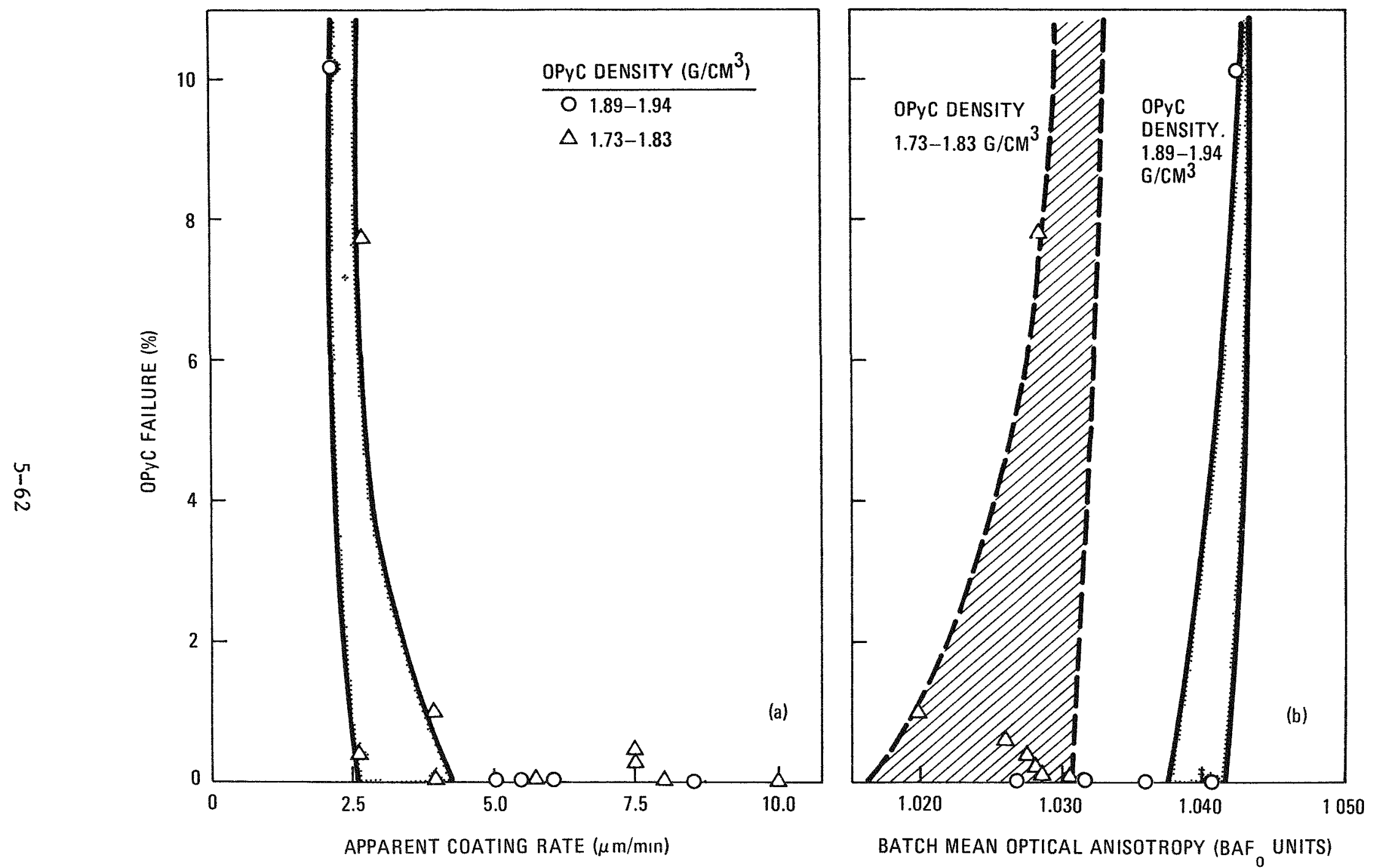

Fig. 5-10. OPyC coating failure of $\mathrm{ThO}_{2}$ BISO particles versus (a) apparent coating rate and (b) optical anisotropy $\left(\mathrm{BAF}_{\mathrm{O}}\right.$ ) for coatings of various densities. Particles were irradiated as unbonded samples in capsules $\mathrm{P} 13 \mathrm{R}$ and $\mathrm{P} 13 \mathrm{~S}$ at $\sim 1075^{\circ} \mathrm{C}$ to fast neutron fluences of 10.7 to $12.1 \times 10^{21} \mathrm{n} / \mathrm{cm}^{2}$ (E >0.18 MeV) HTGR $^{\circ}$ 

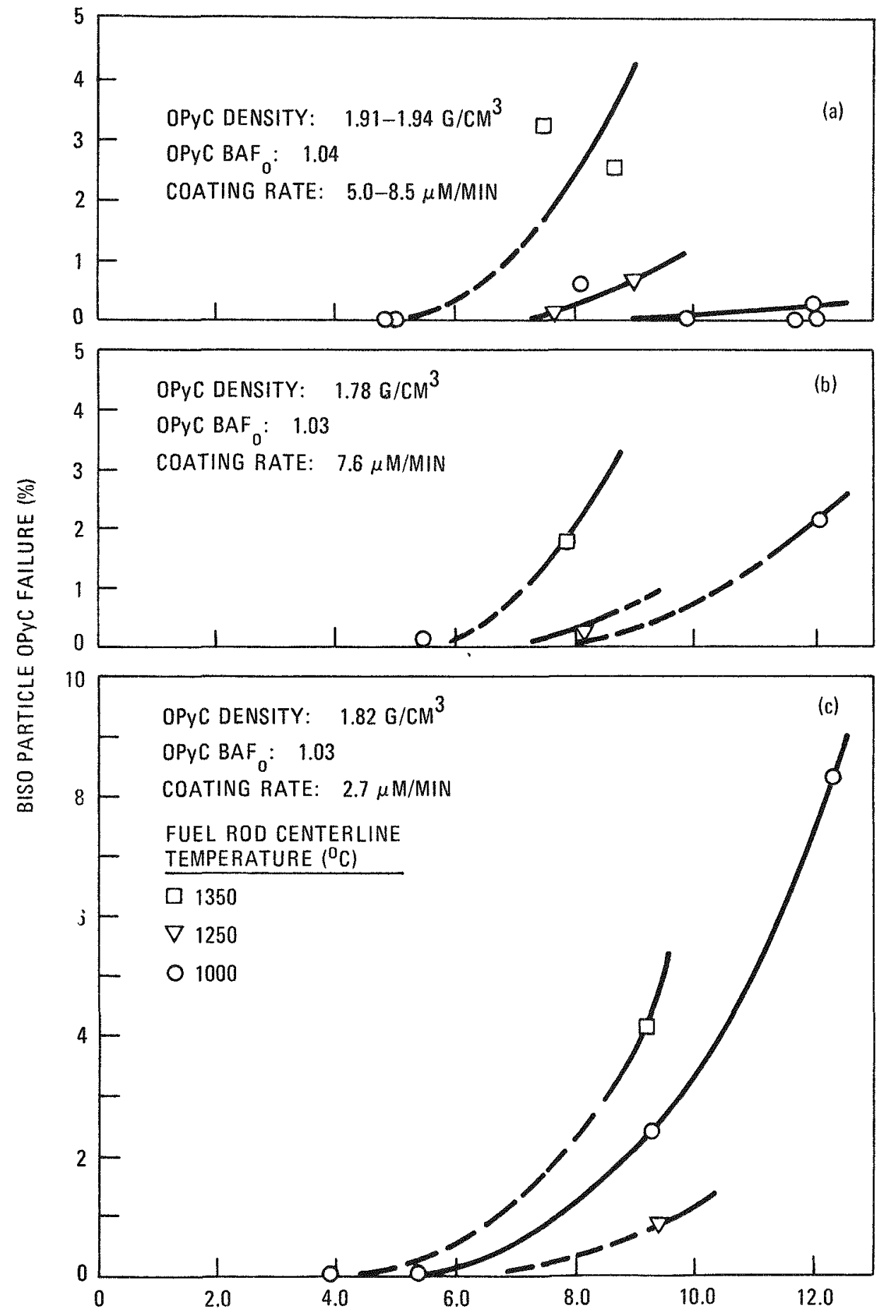

FAST NEUTRON FLUENCE $\left(10^{21} \mathrm{~N} / \mathrm{CM}^{2}\right)(\mathrm{E}>0.18 \mathrm{MeV})_{\mathrm{HTGR}}$

Fig. 5-11. Performance of OPyC coatings on $\mathrm{ThO}_{2}$ BISO particles irradiated in $\mathrm{P} 13 \mathrm{R}$ and $\mathrm{P} 13 \mathrm{~S}$ fuel rods. (a) High-density coatings deposited at medium to high coating rates; (b) low-density coatings deposited at high coating rates; and (c) low-density coatings deposited at low coating rates. 


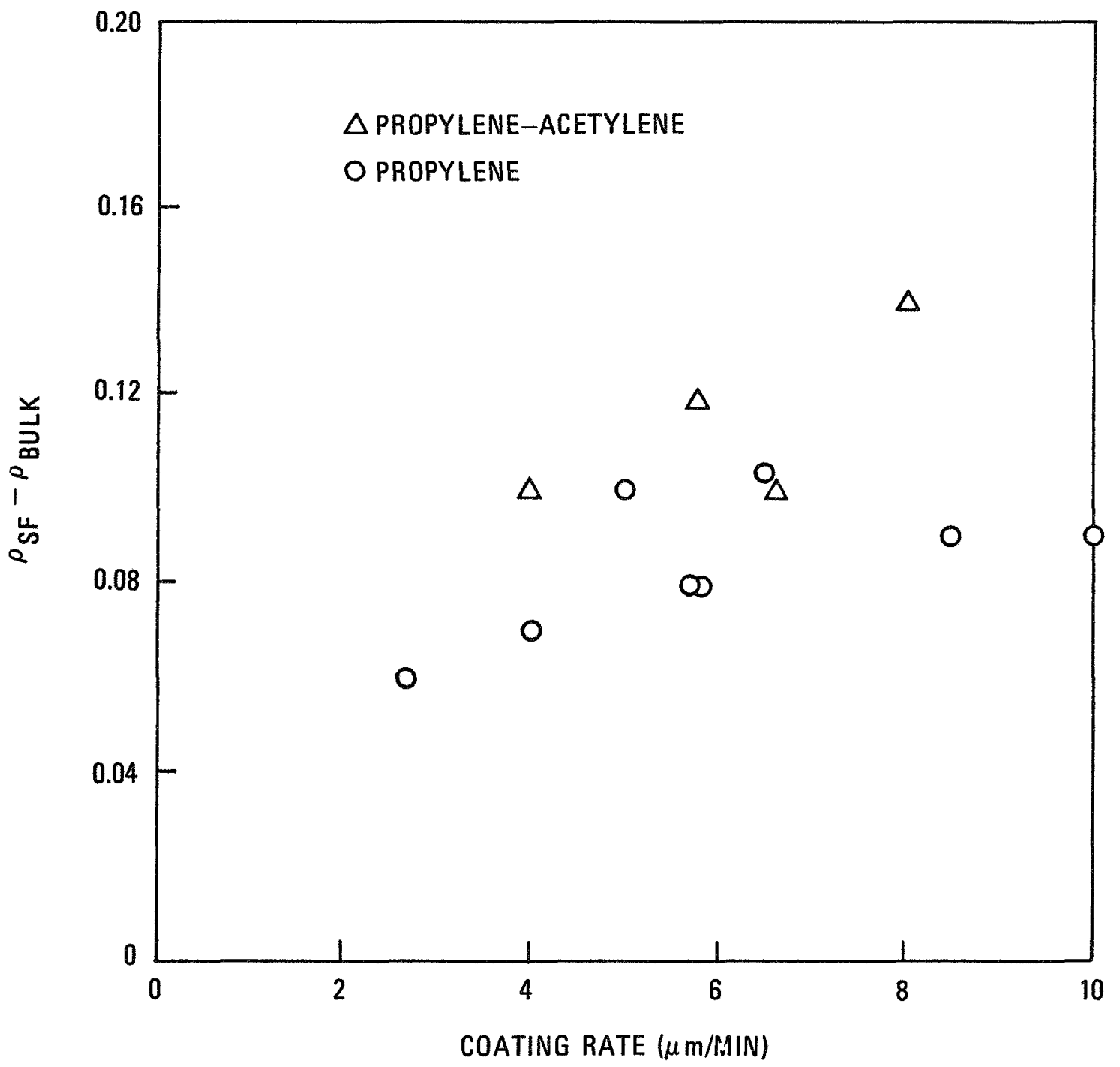

Fig. 5-12. Difference between sink-float and bulk density of fertile particle OPyC coatings as a function of coating rate 


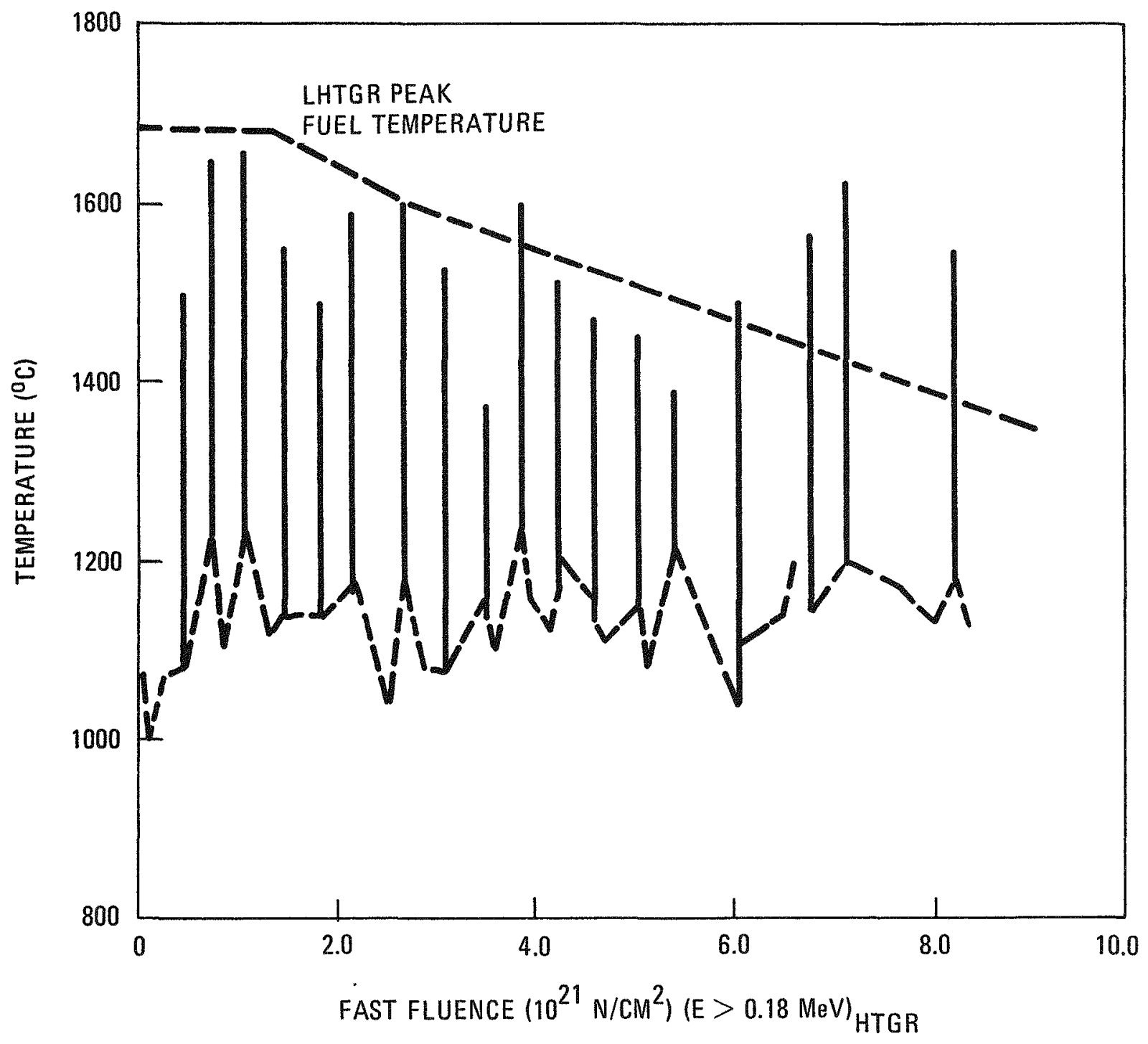

Fig. 5-13. Capsule P13S cell 1 decalibrated control thermocouple temperature versus fast neutron fluence. Also shown is the peak LHTGR fuel temperature envelope expected for load-following transients. 


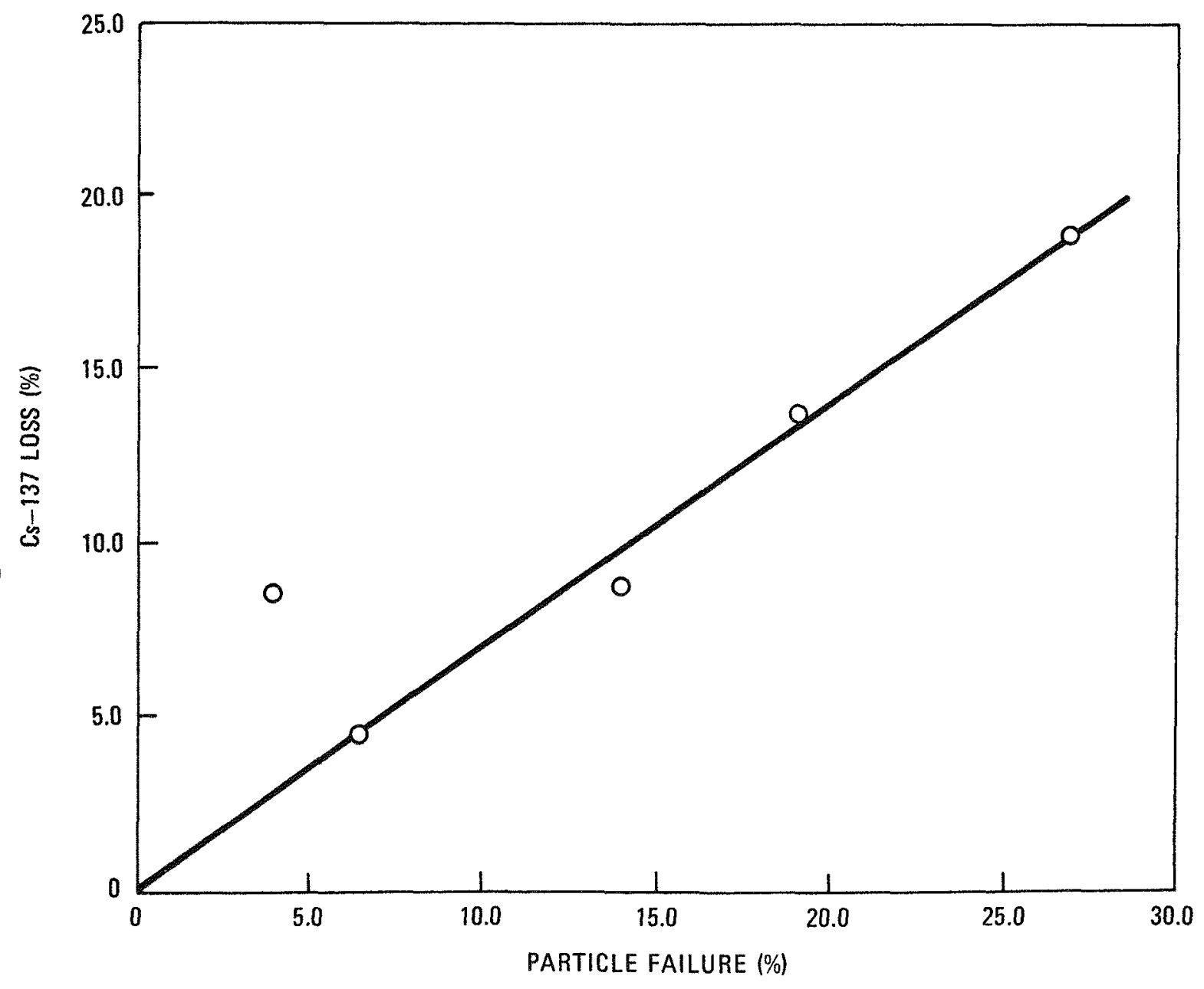

Fig. 5-14. Release of Cs-137 versus fissile particle failure level calculated from $R / B$ values 


\section{IMPLICATIONS FOR LHTGR FUEL DESIGN AND PERFORMANCE}

\subsection{FUEL PARTICLE IRRADIATION PERFORMANCE}

One of the primary goals of capsules P13R and P13S was to evaluate the performance of LHTGR reference-type fissile and fertile fuel particles during irradiation to LHTGR peak design conditions. A requirement of fuel performance derived from core design is that fuel meeting all requirements of particle design with no defective coatings shall retain at least one intact structural coating layer (i.e., retain gaseous fission products) on 99.5\% of all fuel particles at the $95 \%$ confidence level after irradiation at $1250^{\circ} \mathrm{C}$ to $8.0 \times 10^{21} \mathrm{n} / \mathrm{cm}^{2}$ (E $>0.18 \mathrm{MeV}$ ) ${ }_{\mathrm{HTGR}}$ and $/$ or $78 \% \mathrm{FIMA}$ (fissile) or $7.5 \%$ FIMA (fertile) for times up to 4 yr (Ref.6-1).

The irradiation performance of fissile particle batches with coating designs representative of the design requirements envisioned for the LHTGR is summarized in Table 6-1. The particle batches exhibited excellent stability during irradiation to peak burnups and fast neutron fluences which exceeded the LHTGR peak design exposure by 34 to $50 \%$. Visual examination and postirradiation fission gas release measurements revealed that no coating failure occurred in these particle batches with an upper $95 \%$ confidence level of $0.2 \%$. These particle batches also exhibited good performance in bonded rods; however, the failure levels could not be evaluated quantitatively. These results demonstrate that the fuel performance requirement discussed above is achieved with TRISO fissile particles having coating parameters representative of LHTGR design requirements.

These results are also consistent with LHTGR fuel performance models presented in Ref. 6-1. Methods for prediction of fuel performance given in Ref. 6-1 do not allow an evaluation of fuel failure levels at the severe 
fast neutron exposures achieved during the capsule P13R and P13S irradiation. However, these performance models predicted the particle batches given in Table 6-1 would have exhibited a failure level of $0.5 \%$ after reaching a fast neutron exposure of $8.0 \times 10^{21} \mathrm{n} / \mathrm{cm}^{2}$ (E $\left.>0.18 \mathrm{MeV}\right)_{\mathrm{HTGR}}$. These results indicate the performance models given in Ref, 6-1 are clearly conservative in this case.

Fertile particles with coating parameters representative of LHTGR design requirements also exhibited excellent irradiation performance under the severe conditions of capsules P13R and P13S, as shown in Table 6-2. No coating failure was observed during the visual examination, and all but two of the particle batches had OPYC coatings that were completely impermeable to the release of gaseous fission products. Two of the batches exhibited levels of fission gas release that were comparable to an in-serivce failure level of $0.2 \%$. These results were also consistent with fuel performance models which predict a failure level of $0.5 \%$ for these particle batches after irradiation to a fast neutron fluence of $8.0 \times 10^{21} \mathrm{n} / \mathrm{cm}^{2}$ (E > $>0.18$ MeV) ${ }_{\text {HTGR }}$.

The excellent irradiation performance of these fissile and fertile particle batches indicates that high levels of fuel particle integrity can be achieved during irradiation to peak LHTGR conditions by proper specification of the fuel particle design parameters and illustrates the conservative nature of the fuel performance models presented in Ref.6-1.

\subsection{PyC COATING BEHAVIOR}

The OPyC coating on HTGR fuel particles is one of the most important components of the fuel. In the fissile TRISO design, the OPyC performs several functions. It provides mechanical support to the SiC coating, which is the primary load-bearing layer; it protects the SiC from external impurity attack; and it also provides a means to mechanically bond the particles in the rod with the matrix. The OPYC on a BISO particle is, of course, the primary containment for fission gases and thus its survival is essential in preventing high release into the reactor coolant. 
Results from the $\mathrm{P} 13 \mathrm{R}$ and $\mathrm{P} 13 \mathrm{~S}$ capsules have provided a sound basis for a product specification for the OPyC 1ayer. The recomended limits for coating rate, anisotropy, and density are presented in Table 6-3. These results draw heavily upon the fissile particle tests, because with the TRISO design it is less difficult to interpret the results of PyC irradiation stability since parameters which influence the pressure vessel performance can be separated out in the analyses. This is not true with the BISO design since stress levels in the OPyC depend upon fission gas pressure as well as the irradiation-induced structural changes. However, it seems logical to conclude that the coatings exhibiting optimum performance in the TRISO design also will perform best in BISO particles--provided they are sufficiently impermeable to fission gases.

Data from these tests tend to support the above supposition and show that OPYC coatings having very low anisotropies with densities in the range of 1.85 to $1.95 \mathrm{~g} / \mathrm{cm}^{3}$ will survive to peak fluence at $1250^{\circ} \mathrm{C}$ with essentially no failure. Coatings with densities as $10 w$ as $1.80 \mathrm{~g} / \mathrm{cm}^{3}$ appear to have adequate performance on TRISO particles. However, the permeability of fission gases through coatings of these densities needs to be further investigated before the coatings can be recommended for use on the BISO particle.

Tests are now under way in capsules P13T (Ref, 6-2) and P13V (Ref. 6-3), which are designed to verify at the $95 \%$ confidence level that particles with these attributes will survive peak design conditions with $\mathbf{2 9 9 . 5 \%}$ of the particles intact. Demonstration of this goal is one of the primary objectives of the capsule irradiation program.

\subsection{FUEL KERNEL MIGRATION}

Fuel kernel migration is one of four phenomena that control fuel particle performance during irradiation at high temperatures (Ref .6-1). The HTGR core thermal design limits kernel migration to an acceptably low rate that will not result in significant coating degradation and subsequent 
coating failure. The same techniques that are utilized in core thermal design to predict the extent of fuel kernel migration were applied in the thermal allalysis of capsules P13R and P13S. There was excellent agreement between the predicted migration distances and the distances observed during the metallographic examination. These results give a high degree of confidence in the kernel migration coefficient design curves and the technique used in core thermal design to evaluate the extent of kernel migration during normal and transient core operation.

\subsection{FUEL ROD IRRADIATION PERFORMANCE}

The criteria used for evaluating fuel rod performance are as follows (Ref. 6-4):

1. To maintain integrity to full HTGR 1ifetime exposure.

2. To exhibit dimensional change compatible with the graphite fuel block so that no detrimental fuel-graphite mechanical interaction occurs.

3. To have bonding characteristics that do not cause fuel failure as a result of matrix-coating interaction.

4. To provide a heat transfer medium that will avoid excessive temperatures and thermal gradients in the fuel. Thermal conductivity shall be at least $4 \mathrm{Btu} / \mathrm{hr}-\mathrm{ft}-{ }^{\circ} \mathrm{F}$ to end of design life.

The postirradiation integrity of the bonded fuel rod specimens tested in capsules $\mathrm{P} 13 \mathrm{R}$ and $\mathrm{P} 13 \mathrm{~S}$ was excellent and demonstrated that the performance criteria of integrity and bonding characteristics noted above can be achieved using a variety of processing conditions and matrix components. Very little irradiation-induced damage was observed even in fuel rods thermal cycled during irradiation or irradiated to a fast neutron fluence of $12.4 \times 10^{21}$ $\mathrm{n} / \mathrm{cm}^{2}(\mathrm{E}>0.18 \mathrm{MeV})_{\mathrm{HTGR}}$, which is $55 \%$ beyond the peak LHTGR design fast 
neutron exposure. The different fuel rod matrix and shim materials tested exhibited satisfactory performance, and no evidence of interaction between the fuel rod matrix and fuel particle coatings was observed.

These results are consistent with previous capsule tests (Ref. 6-4) which indicate that the integrity of pitch-bonded fuel rods is independent of irradiation temperature and fast neutron fluence within the realm of LHTGR operating conditions.

\subsection{FUEL ROD DIMENSIONAL CHANGE BEHAVIOR}

Characterization of irradiation-induced fuel rod dimensional change is referenced for the proper thermal and mechanical design of LHTGR fuel elements. An evaluation of the radial clearance between the fuel rods and the fuel holes is necessary for core thermal analyses. Also, irradiationinduced fuel rod dimensional changes must be compatible with the dimensional change behavior of the graphite fuel elements (GLCC Grade H-451) to ensure that excessive stresses are not built up as a result of dimensional interferences between the fuel rod columns and graphite fuel element during reactor operation.

Data on irradiation strain of $\mathrm{H}-451$ graphite obtained from capsule irradiation tests have been statistically fit to produce dimensional change contours used in core design. These contours are a function of mean irradiation temperature and fast neutron fluence (Refs, 6-5 and 6-6). The irradiation strain data obtained from fuel irradiated in capsules P13R and P13S are compared with graphite dimensional change curves in Fig. 6-1. For comparison of fuel rod with graphite dimensional changes, graphite strain contours for temperatures approximately $200^{\circ}$ to $300^{\circ} \mathrm{C}$ below the fuel centerline temperature should be used.

Fuel rod diametral changes appear to be quite compatible with those of the graphite (radial direction) to and slightly beyond peak HTGR fast neutron fluences (i.e., $8 \times 10^{21} \mathrm{n} / \mathrm{cm}^{2}, E>0.18 \mathrm{MeV}$ ). Since a 0.08 - to $0.012-\mathrm{mm}$ 
radial gap typically exists between the rods and the fuel element block, interference between these two components would not be expected until fast neutron fluences in excess of $\sim 10^{22} \mathrm{n} / \mathrm{cm}^{2}(\mathrm{E}>0.18 \mathrm{MeV})_{\text {HTGR }}$ are reached, as was the case for capsule P13R and P13S rods.

As noted earlier in this report, the diametral dimensional changes measured for the $\mathrm{P} 13 \mathrm{R}$ and $\mathrm{P} 13 \mathrm{~S}$ fuel rods were approximately $45 \%$ less than predicted using current codes. A similar discrepancy had been noted from other tests of LHTGR rods (Ref, 6-7). An empirically derived correction factor is now being used in core design calculations, and this factor takes into account these P13R and P13S dimensiona1 data (Ref.6-4).

Data supplied for use in core design calculations have treated the rods as behaving isotropically. The results from these tests and from Peach Bottom test element irradiations (Ref. 6-8) have shown that fuel rods exhibit anisotropic dimensional changes, and at high fast fluences net expansion in the axial direction occurs. The cause for this anisotropic behavior has not been defined from these tests; however, recently reported results from a screening test of candidate materials indicate the graphite shim could be a contributing factor (Ref. 6-9). A detailed study of results from all of the most current tests of LHTGR fuel rods is now being undertaken to more thoroughly characterize the dimensional change mechanism. Input to core design calculations will be modified to reflect the anisotropic behavior of the rods.

REFERENCES

6-1. Smith, C. L., "Fuel Particle Behavior Under Normal and Transient Conditions," USAEC Report GA-A12971, General Atomic, October 1, 1974.

6-2. Young, C. A., and D. P. Harmon, "Preirradiation Report of Fue1 Materials for P13T Capsule Irradiation," ERDA Report GA-A13343, General Atomic, April 1976.

6-3. "HTGR Fuels and Core Development Program Quarterly Progress Report for the Period Ending August 31, 1975," ERDA Report GA-A13592, General Atomic, September 30, 1975. 
6-4. Harmon, D. P., and C. B. Scott, "Development and Irradiation Performance of LHTGR Fue1," ERDA Report GA-A13173, Genera1 Atomic, October $31,1975$.

6-5. Shenoy, A. S., and D. W. McEachern, "HTGR Core Thermal Design Methods and Analysis," General Atomic Report GA-A12985, December 31, 1974.

6-6. Price, R. J., and L. A. Beavan, "Final Report on Graphite Irradiation Test OG-2," ERDA Report GA-A13556, General Atomic, December 15, 1975.

6-7. Scott, C. B., and D. P. Harmon, "Postirradiation Examination of Capsules HRB-4, HRB-5, and HRB-6," ERDA Report GA-A13267, Genera1 Atomic, November 28, 1975.

6-8. "HTGR Fuels and Core Development Program Quarterly Progress Report for the Period Ending November 30, 1975," ERDA Report GA-A13737, Genera1 Atomic, December 31, 1975.

6-9. Johnson, W. R., et a1., "Postirradiation Examination of Capsules HT-24 and HT-25," ERDA Report GA-A13486, General Atomic, September 15, 1975. 
TABLE 6-1

PERFORMANCE OF LHTGR REFERENCE TYPE FISSILE PARTICLES

\begin{tabular}{c|c|c|c|c}
\hline Batch Number & $\begin{array}{c}\text { Number of } \\
\text { Particles } \\
\text { Tested (a) }\end{array}$ & $\begin{array}{c}\text { Pressure Vesse1 } \\
\text { Failure (b) } \\
(\%)\end{array}$ & $\begin{array}{c}95 \% \text { Confidence } \\
\text { Interva1(c), P } \\
(\%)\end{array}$ & $\begin{array}{c}\text { R/B Failure } \\
\text { Leve1 (d) } \\
(\%)\end{array}$ \\
\hline $6151-00-035$ & 459 & 0 & $0 \leq \mathrm{P} \leq 0.8$ & 0 \\
$6151-00-035$ & 809 & 0 & $0 \leq \mathrm{P} \leq 0.6$ & 0 \\
$6151-08-015$ & $\underline{688}$ & 0 & $0 \leq \mathrm{P} \leq 0.7$ & $\underline{0}$ \\
Tota1 & 1956 & 0 & $0 \leq \mathrm{P} \leq 0.2$ & 0 \\
\hline
\end{tabular}

(a) Particle samples were irradiated to fast neutron fluences of 10.7 to $12.0 \times 10^{21} \mathrm{n} / \mathrm{cm}^{2}$ ( $\mathrm{E}>0.18 \mathrm{MeV}$ ) $\mathrm{HTGR}$ and burnups of 72 to $74 \%$ FIMA at temperatures ranging from $975^{\circ}$ to $1075^{\circ} \mathrm{C}$.

(b) Determined by visual examination.

(c) The $95 \%$ confidence statement for the failure levels determined by visual examination means that an infinite population of particles with attributes of these batches tested to these conditions will have a failure level at the $95 \%$ confidence level bounded by this interval.

(d) Calculated from postirradiation fision gas release data by assuming a fractional release of $2 \times 10^{-2}$ for failed fuel in an unconstrained geometry. 
TABLE 6-2

PERFORMANCE OF LHTGR REFERENCE TYPE FERTILE PARTICLES

\begin{tabular}{|c|c|c|c|c|}
\hline Batch Number & $\begin{array}{l}\text { Number } \\
\text { Particles } \\
\text { Tested (a) }\end{array}$ & $\begin{array}{c}\text { Pressure Vesse1 } \\
\text { Failure (b) } \\
(\%)\end{array}$ & $\begin{array}{l}95 \% \text { Confidence } \\
\text { Interva1 }(\mathrm{c}), \mathrm{P} \\
(\%)\end{array}$ & $\begin{array}{l}\text { R/B failure } \\
\text { Leve1(d) } \\
(\%)\end{array}$ \\
\hline $6542-02-020$ & 1149 & 0 & $0 \leq \mathrm{P} \leq 0.5$ & 0.2 \\
\hline $6542-02-030$ & 1072 & 0 & $0 \leq P \leq 0.5$ & 0.2 \\
\hline $6542-09-010$ & 948 & 0 & $0 \leq P \leq 0.5$ & 0 \\
\hline $6542-18-015$ & 953 & 0 & $0 \leq P \leq 0.5$ & 0 \\
\hline $4252-06-010$ & 872 & 0 & $0 \leq P \leq 0.6$ & 0 \\
\hline $6542-23-025$ & 992 & 0 & $0 \leq P \leq 0.5$ & 0 \\
\hline $6542-24-015$ & 942 & $\underline{0}$ & $0 \leq P \leq 0.5$ & 0 \\
\hline Total & 6928 & 0 & $0 \leq \mathrm{P} \leq 0.1$ & 0.06 \\
\hline
\end{tabular}

(a) Particle samples were irradiated to fast neutron fluences of 11.3 to $11.9 \times 10^{21} \mathrm{n} / \mathrm{cm}^{2}$ ( $\mathrm{E}>0.18 \mathrm{MeV}$ ) HTGR and burnups of 4.1 to $4.9 \% \mathrm{FIMA}$ at temperatures ranging from $895^{\circ}$ to $1010^{\circ} \mathrm{C}$.

(b) Determined by visual examination.

(c) The $95 \%$ confidence statement for the failure levels determined by visual examination means that an infinite population of particles with attributes of these batches tested to these conditions will have a failure level at the $95 \%$ confidence level bounded by this interval.

(d) Calculated from postirradiation fission gas release data by assuming a fractional release of $2 \times 10^{-2}$ for failed fuel in a constrained geometry. 
TABLE 6-3

RECOMMENDED OPYC COATING ATTRIBUTE SPECIFICATIONS FOR HTGR FISSILE TRISO AND FERTILE BISO PARTICLES

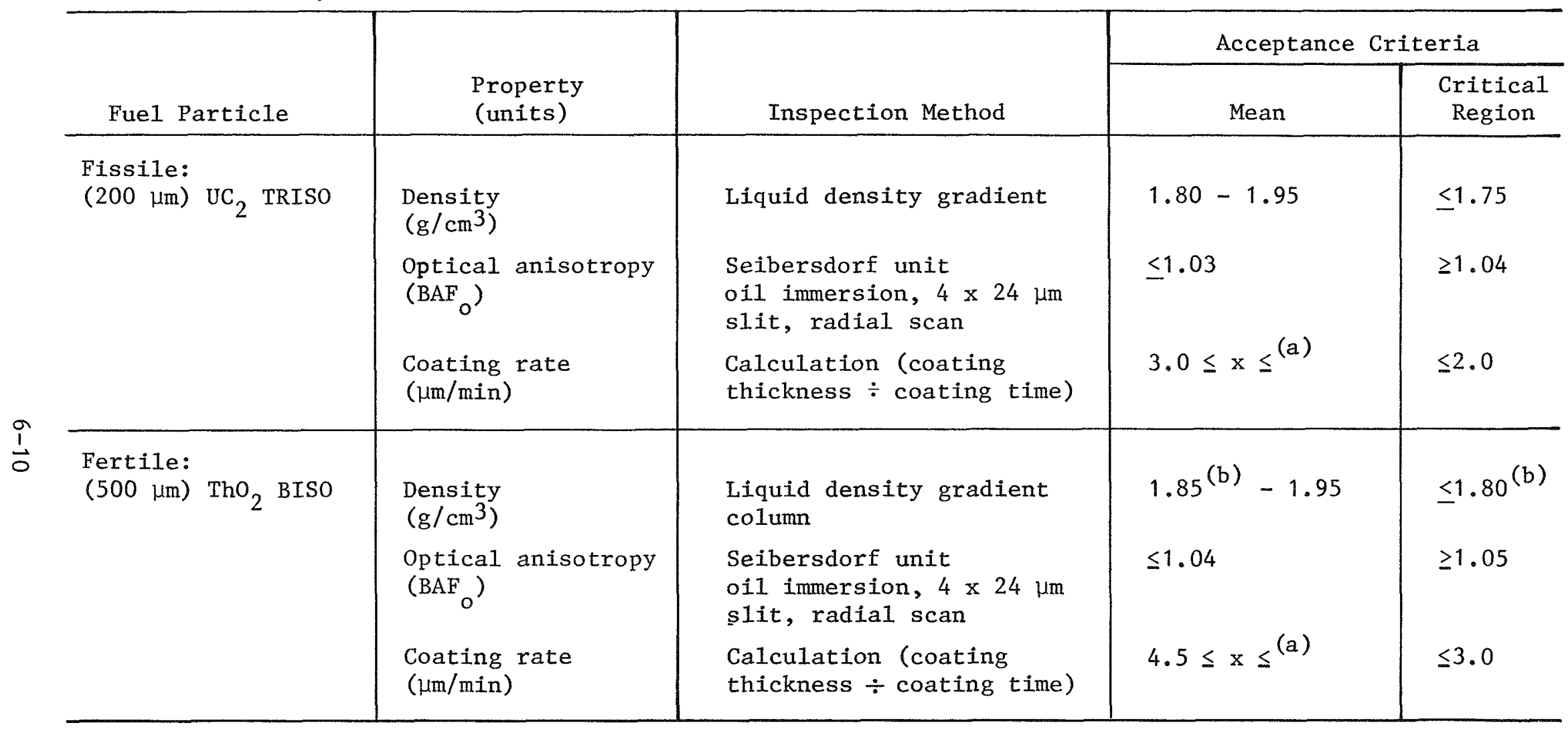

(a) The upper limit for the coating rate was not defined from these tests.

(b) The lower limit and critical region need to be defined by coating permeability measurements. 


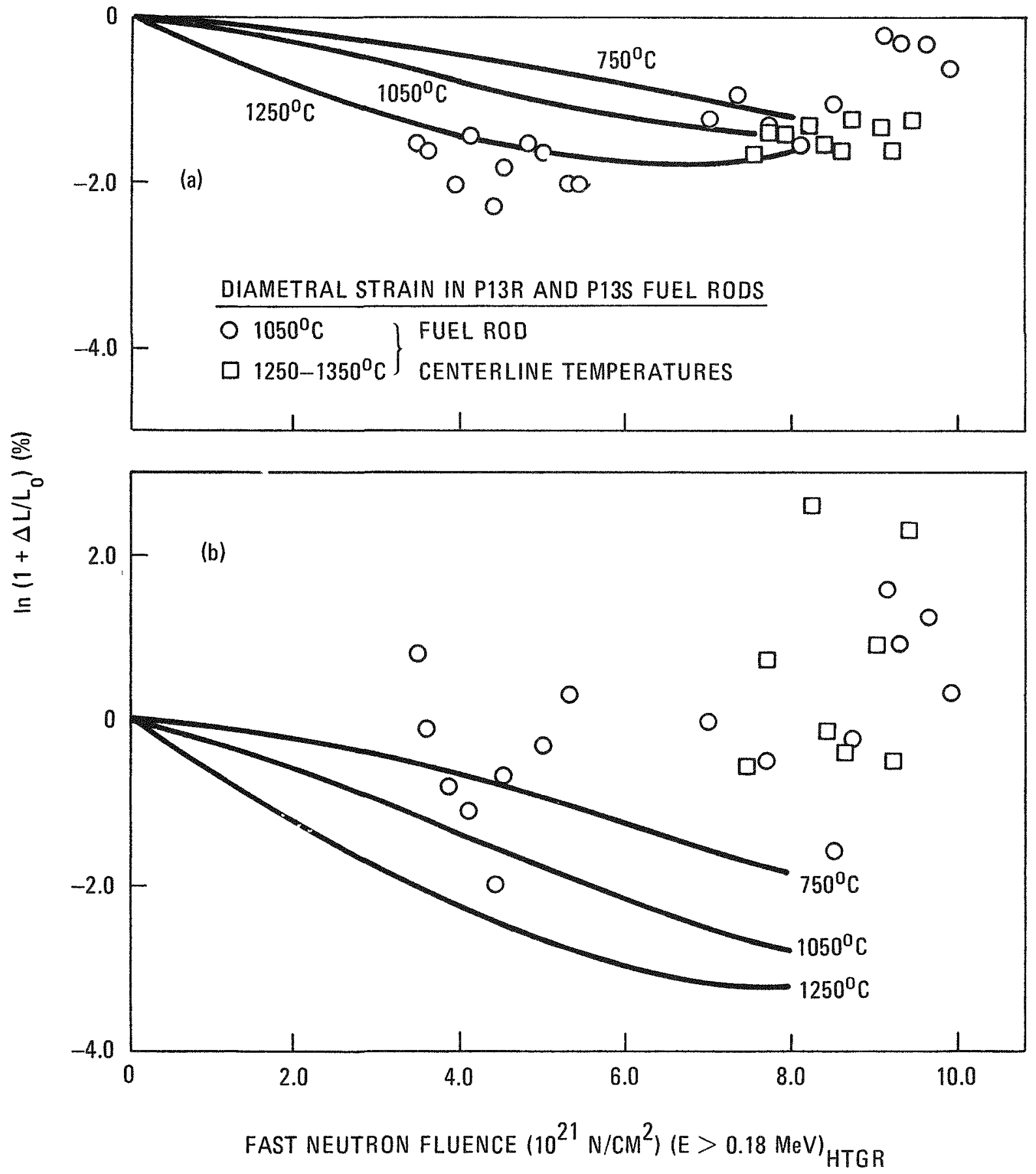

Fig. 6-1. Irradiation strain of H-451 graphite, (a) radial and (b) axial, as a function of fast fluence compared with measured P13R and P13S fuel rod dimensional changes 


\section{SUMMARY}

Capsules P13R and P13S demonstrated the integrity of LHTGR reference type fresh and alternate fuels over a wide range of irradiation conditions. Evaluation of these results with respect to specific fuel properties provided a substantial data base for the LHTGR Fuel Product Specification and performance models used in core design studies. A brief summary of the irradiation results is presented below:

1. Bonded fuel rods were successfully irradiated to fast neutron exposures of $12.4 \times 10^{21} \mathrm{n} / \mathrm{cm}^{2}$ (E $>0.18 \mathrm{MeV}$ ) ${ }_{\mathrm{HTGR}}$, which is $55 \%$ beyond the LHTGR peak design fast neutron fluence of $8.0 \times 10^{21}$ $\mathrm{n} / \mathrm{cm}^{2}$.

2. Thermal cycling to high temperatures did not adversely affect fuel rod integrity.

3. Postirradiation integrity of fuel rods fired at $1500^{\circ} \mathrm{C}$ or in a nitrogen atmosphere was comparable to that of fuel rods fired under the LHTGR reference conditions of $1800^{\circ} \mathrm{C}$ in an argon atmosphere. These results contribute to the qualification of a lower firing temperature and nitrogen cover gas.

4. Irradiation-induced diametral fuel rod dimensional changes were compatible with the dimensional change behavior of LHTGR nearisotropic moderator graphite.

5. Particle batches with coating designs representative of the design requirements envisioned for the LHTGR exhibited excellent irradiation performance. A number of fissile and fertile particle batches were irradiated without coating failure to fast neutron exposures which exceeded the LHTGR peak design exposure by 34 to $51 \%$. 
6. A large number of particle batches were tested with coating designs or particle attributes that were not representative of LHTGR design requirements. The irradiation performance of these samples provided important data required to establish fuel particle specification limits.

7. Fuel particles with severely faceted coatings, nonround fuel kernels, or interrupted SiC layers exhibited higher failure rates than similarly designed fuel particles that did not have these attributes.

8. Survival of OPyC coatings is dependent on anisotropy over a wide density range. However, once low OPyC coating anisotropies have been achieved, it becomes necessary to restrict the range of OPyC coating density to ensure survival at high irradiation temperatures.

9. In-pile thermal cycling to temperatures up to $1650^{\circ} \mathrm{C}$ resulted in increased fuel failure levels relative to fuel failure levels in fuel specimens that were not thermal cycled. The effects of thermal cycling on fuel particle integrity were the most pronounced in fuel particles having out-of-specification coating parameters. Fertile particles more representative of LHTGR design requirements exhibited failure levels well below the level predicted for BISO $\mathrm{ThO}_{2}$ particles that experience a thermal transient to $1550^{\circ} \mathrm{C}$ after irradiation to a fast neutron fluence of $8.0 \times 10^{21} \mathrm{n} / \mathrm{cm}^{2}$ $(\mathrm{E}>0.18 \mathrm{MeV})_{\mathrm{HTGR}^{\circ}}$

10. A number of BISO fertile particle batches were irradiated to fast neutron fluences up to $12.1 \times 10^{21} \mathrm{n} / \mathrm{cm}^{2}(\mathrm{E}>0.18 \mathrm{MeV})_{\mathrm{HTGR}}$ without any apparent coating failure. However, these batches exhibited varying levels of permeability to gaseous fission products (up to an $\mathrm{R} / \mathrm{B}$ of $2 \times 10^{-3}$ for $\mathrm{Kr}-85 \mathrm{~m}$ at $1100^{\circ} \mathrm{C}$ ). The opyC permeability was attributed to a combination of the intergrowth feature porosity after deposition and an increase in the surfaceconnected porosity due to irradiation-induced cracking of the PyC microstructure. 
11. Measured $\mathrm{UC}_{2}$ fuel kernel migration distances were in good agreement with migration distances predicted with techniques and design data used in core thermal design studies to evaluate the extent of fuel kernel migration in the LHTGR core during normal and transient operation.

12. An addition of $1.0 \mathrm{wt} \%$ thorium to $\mathrm{UC}_{2}$ kernels to improve the kernel sphericity did not alter the performance of the fuel relative to kernels without the thorium addition.

13. Deletion of the seal coat previously applied to fertile particle buffer coatings was shown to have no effect on coating irradiation performance. 
